SUMMARY OF SELECTED CHARACTERISTICS OF LARGE RESERVOIRS

IN THE UNITED STATES AND PUERTO RICO, 1988

By Barbara C. Ruddy and Kerie J. Hitt

U.S. GEOLOGICAL SURVEY

Open-File Report 90-163 
DEPARTMENT OF THE INTERIOR

MANUEL LUJAN, JR., Secretary

U.S. GEOLOGICAL SURVEY

Dallas L. Peck, Director

For additional information write to:

District Chief

U.S. Geological Survey

Box 25046, Mail Stop 415

Federal Center

Denver, CO 80225-0046
Copies of this report can

be purchased from:

U.S. Geological Survey

Books and Open-File Reports Section

Box 25425

Federal Center

Denver, CO 80225-0425 


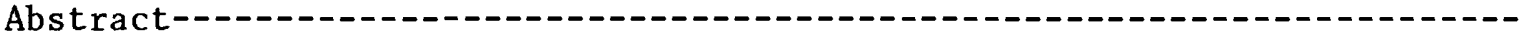

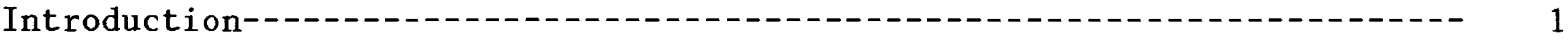

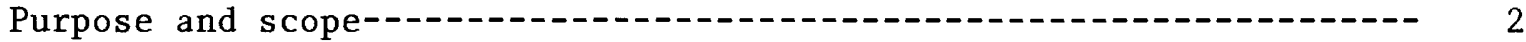

Availability of data------

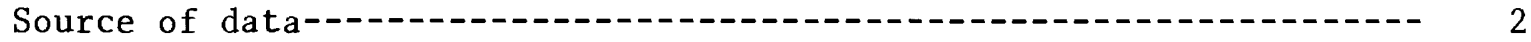

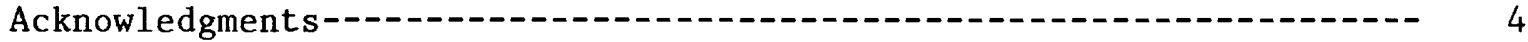

Summary of selected characteristics of large reservoirs-------------

Explanation of data---

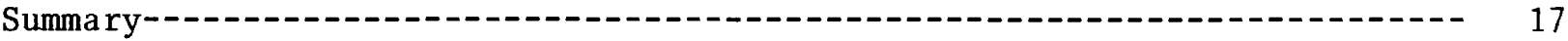

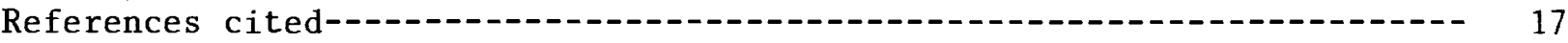

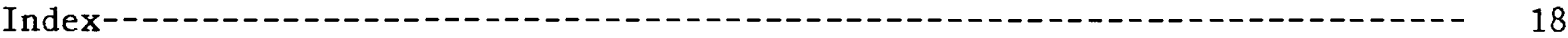

FIGURES

Figure 1. Map showing water-resources regions of the United States and

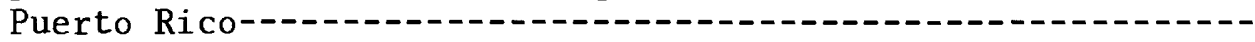

2. Graphs showing number and total normal capacity of reservoirs completed before 1920, during each decade from 1920 to 1979 , and from 1980 to 1988

3. Map showing locations of reservoirs that have normal capacities of at least 5,000 acre-feet or maximum capacities of at least 25,000 acre-feet in the United States and Puerto Rico--

TABLES

Table 1. Ranking of States by number of reservoirs and total normal capacity---n- 10

2. Large reservoirs in the United States that have a normal capacity of 2,000,000 acre-feet or more---------------- 12

3. Reservoirs in the United States and Puerto Rico that have a normal capacity of at least 5,000 acre-feet or a maximum capacity of at least 25,000 acre-feet---n-

\section{CONVERSION FACTORS}

For those readers who prefer to use metric (International System) units rather than inch-pound units, the conversion factors for terms used in this report are listed below:

\section{Multiply inch-pound unit} acre acre-foot

foot inch square mile

$$
\begin{aligned}
& B y \\
& 0.4047 \\
& 1,234 \\
& 0.3048 \\
& 2.54 \\
& 2.590
\end{aligned}
$$

To obtain metric unit hectare cubic meter meter centimeter square kilometer 


\section{SUMMARY OF SELECTED CHARACTERISTICS OF LARGE RESERVOIRS IN THE UNITED STATES AND PUERTO RICO, 1988}

By Barbara C. Ruddy and Kerie J. Hitt

\section{ABSTRACT}

This report presents selected characteristics of 2,728 reservoirs and controlled natural lakes located within the 50 States of the United States and the Commonwealth of Puerto Rico. Data are presented for reservoirs that have normal capacities of at least 5,000 acre-feet or maximum capacities of at least 25,000 acre-feet and that were completed as of January 1, 1988. Reservoir data include: location, maximum capacity, normal capacity, surface area, drainage area, year completed, and use.

The normal capacities of the 2,728 reservoirs and controlled natural lakes total more than 467 million acre-feet, and the combined surface areas for 2,362 or those reservoirs total almost 16 million acres. More than 9 percent (259) of the reservoirs are located in California. The total normal capacity of reservoirs in California is more than 40 million acre-feet (about (8.6 percent of the total normal capacity for the United States and Puerto Rico), and the total surface area is almost 700,000 acres (about 4.4 percent of the listed total surface area for the United States and Puerto Rico).

\section{INTRODUCTION}

For centuries, reservoirs have been built to regulate water supplies for irrigation and municipal use, for flood control, and for power generation. Generally, reservoirs were constructed by various public and private agencies, and there was no national authority that regulated dam construction and development or that collected information about the dams and reservoirs. Before 1972, the number and condition of non-Federal dams had never been assessed, and the licensing and inspection of dams varied among the States. However, in 1972 , Congress established a national program for safety of dams (Public Law 92-367). The law defined "dam" as any artificial barrier that impounds or diverts water and is 25 feet or more in height or has an impounding capacity of 50 acre-feet or more. The purpose of the program was to guard the public against loss of life and property from dam failures by identifying potentially unsafe structures. In the late $1970^{\prime} \mathrm{s}$, the importance of the dam safety program was emphasized by the failure of the Teton Dam in Idaho (1976) and the Toccoa Dam in Georgia (1977).

Section 5(1) of Public Law 92-367 directed the Chief of Engineers of the U.S. Army Corps of Engineers to prepare an "inventory of all dams located in the United States." In 1982, the U.S. Army Corps of Engineers completed a computerized inventory of dams that contained information on approximately 66,000 structures. The U.S. Army Corps of Engineers stopped updating the computer file after satisfying the provisions of the dam safety act. Now (1989) the Federal Emergency Management Agency is responsible for compiling information about the safety of dams in the United States. 
The U.S. Geological Survey extracted and compiled data about large reservoirs in the United States from the U.S. Army Corps of Engineers national inventory of dams (1982) and other sources. This compilation has potential value in assessing the condition of the Nation's water supply, studying the water quality of lakes and reservoirs, estimating reservoir evaporation, and preparing basinwide water budgets.

\section{Purpose and Scope}

This report provides a consistent nationwide inventory of location, size, and other characteristics of large reservoirs and controlled natural lakes in the United States as of January 1, 1988. This report represents a synthesis of data from the U.S. Army Corps of Engineers national inventory of dams (1982), the report from Martin and Hanson (1966), and additional information from various Federal and State agenies. This report on selected characteristics of large reservoirs in the United States and the Commonwealth of Puerto Rico revises and supersedes the report by Martin and Hanson (1966).

\section{Availability of Data}

The data compiled for this report are maintained in an automated geographic information system and can be distributed in machine-readable form upon request. Any corrections or additions to the data that can be furnished by the readers of this report would be greatly appreciated. Please direct comments on or requests for the data to:

Assistant Chief Hydrologist for Water Assessment and Data Coordination U.S. Geological Survey

407 National Center

Reston, VA 22092

\section{Source of Data}

In 1981, the U.S. Geological Survey undertook a project to map the surface-water development in the United States (Hitt, 1985). Two of the elements depicted on the map are dams and reservoirs. Each of the Federal agencies involved in dam construction and maintenance had its own method of tracking and measuring the capacity of the structures under its jurisdiction. A unified approach to classifying the structures was necessary; therefore, the U.S. Geological Survey adopted the U.S. Army Corps of Engineers national inventory of dams (1982) as a consistent basis for compiling the map. Other advantages in using this inventory were that it was available in digital format, and it contained information about non-Federal and Federal dams.

Personnel compiling the map first chose to examine only structures that had at least 5,000 acre-feet of normal capacity. Studies have indicated that although impoundments smaller than 5,000 acre-feet greatly outnumber larger ones, the small reservoirs account for only about 2 percent of the total usable capacity of the Nation (Langbein, 1982). However, the limitation of 5,000 acre-feet excluded many flood-control structures because they have small 
normal capacities. Consequently, a second set of dams that had normal capacities of less than 5,000 acre-feet and maximum capacities of at least 25,000 acre-feet was retrieved from the U.S. Army Corps of Engineers national inventory of dams (1982). Most of the dams in this category were constructed primarily for prevention of floods and have a small permanent pool but a large flood-control capacity. Controlled natural lakes (hereinafter also referred to as "reservoirs") meeting the size requirements for reservoirs also were included. Imposing these size limits decreased the number of dams from 66,000 to about 2,700 .

The U.S. Geological Survey extracted the 2,728 structures from the U.S. Army Corps of Engineers national inventory of dams (1982) and created its own digital file of dams and reservoirs. From the data fields in the U.S. Army Corps of Engineers inventory, the following fields were selected for use in compiling the map of surface-water development: State, official name of dam, popular name of dam, name of impoundment, name of owner, normal capacity, maximum capacity, latitude, longitude, county code, water-resources region and basin codes, year of completion, number of navigation locks, installed hydroelectric generating capacity, purpose of dam, stream, and structural height. Values in these fields were transferred to the U.S. Geological Survey's file; values in the other fields in the U.S. Army Corps of Engineers inventory were not transferred.

The dams and reservoir file was reviewed and verified for the map of surface-water development. Sedimentation ponds, tailing ponds, and other types of industrial and mining impoundments were deleted because most of them are offstream developments that do not affect the streamflow regime. Multiple structures impounding the same reservoir also were deleted. Values in certain data fields (name of dam, name of impoundment, name of owner, normal capacity, maximum capacity, latitude, longitude) were reviewed and corrected by the U.S. Geological Survey. The values were checked against U.S. Geological Survey water-resources data reports and State inventories, where available. Nine other Federal agencies (U.S. Army Corps of Engineers, Tennessee Valley Authority, U.S. Bureau of Reclamation, U.S. Bureau of Indian Affairs, U.S. Fish and Wildlife Service, National Park Service, U.S. Soil Conservation Service, U.S. Forest Service, Federal Energy Regulatory Commission) and government agencies in five States also reviewed and corrected their parts of the U.S. Geological Survey's file. The revised digital file of dams and reservoirs was the source of the information on size, location, and owner of dams and reservoirs plotted on the map of surface-water development (Hitt, 1985).

After the map of surface-water development was published, the U.S. Geological Survey's dams and reservoirs file was additionally checked. The file was reviewed and updated during the preparation of the State articles for two water summary reports (U.S. Geological Survey, 1986, 1990). Location and size of dams and reservoirs were reviewed on maps of surface-water development in the State sections of U.S. Geological Survey (1986, fig. 2 in each State section). Sizes of dams and reservoirs were verified again for cumulative reservoir storage graphs in the State section of U.S. Geological Survey (1990, fig. $1 \mathrm{~B}$ in each State section). Missing structures were added to the file during both reviews. 
The file was enhanced further by adding surface area, drainage area, storage ratio, and more detailed basin information. The data on surface area were needed to make realistic estimates of reservoir evaporation. Evaporative loss from reservoirs is a concern because substantial quantities of water evaporate from reservoirs that would not have evaporated if the water had not been impounded. Annual free water-surface evaporation ranges from a low of 20 inches per year in northwestern Washington to a high of 100 inches per year in southeastern California (Farnsworth and others, 1982).

Information on surface area, drainage area, and storage ratios first were obtained from Martin and Hanson (1966). If the information was not available from Martin and Hanson, it then was obtained from the annual U.S. Geological Survey State water-data reports, from District offices of the U.S. Geological Survey, or from appropriate State agencies. Surface areas are available for about 87 percent of the reservoirs in the file.

\section{Acknowledgments}

Acknowledgment is made to the following agencies and organizations for their cooperation in making the reservoir data available: U.S. Army Corps of Engineers, U.S. Bureau of Reclamation, Tennessee Valley Authority, other Federal agencies, State agencies, and water and irrigation districts.

\section{SUMMARY OF SELECTED CHARACTERISTICS OF LARGE RESERVOIRS}

The reservoirs in the file were grouped by the water-resources regions that are shown in figure 1 . These regions, defined by Seaber and others (1987), generally correspond to major river basins. The number and total normal capacity of reservoirs developed in the United States and Puerto Rico before 1920, during each decade from 1920 to 1979 , and from 1980 to 1988 are shown by water-resources region in figure 2. The location of the reservoirs is shown in figure 3 . By 1988 , normal capacity of 2,728 reservoirs totaled about 467 million acre-feet. The surface area of 2,362 reservoirs totaled almost 16 million acres. Surface areas for 366 reservoirs were not available.

In the eastern United States (regions 01 and 02 ), reservoirs were developed to meet the municipal demands of growing cities during the last half of the century (Martin and Hanson, 1966). Reservoir development for hydroelectric power generation and flood control occurred in the $1950^{\prime} \mathrm{s}$ and $1960^{\prime} \mathrm{s}$ in parts of the central, southern, and southwestern United States (regions 03, 05, 08, and 13). Growth occurred later in parts of the western United States (regions $10,11,12,14,17$, and 18). Reservoirs in the western United States were needed to regulate the seasonal flows in rivers for irrigation supplies. Prior to 1920 , the increase in the number of reservoirs was almost entirely due to the Reclamation Act (Newlands) of 1902. The Reclamation Act provided Federal aid for building reservoirs and stimulated the construction of reclamation works in the arid West (Martin and Hanson, 1966). Large increases in reservoir storage also occurred after 1950 when Federal money was made available to construct dams. In the $1980^{\prime}$ s, few large-scale water projects have been constructed. Most of the economical sites for locating dams already have been developed, and the current (1988) emphasis primarily is on nonstructural measures for assuring water supplies and preventing flood damage. Development of small-scale projects, such as dams to generate lowhead hydroelectric power, could become important if energy costs rise. 


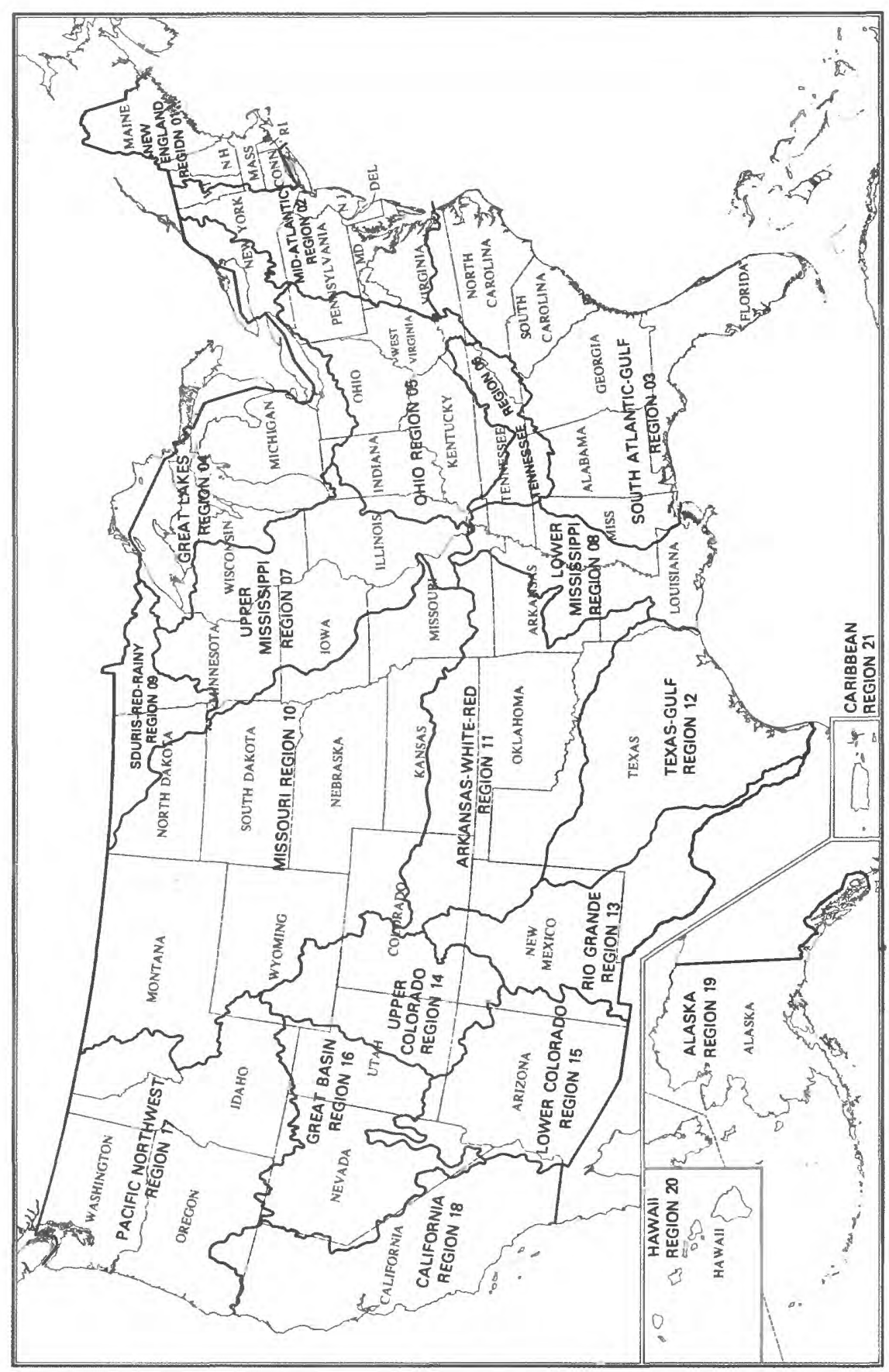

告 

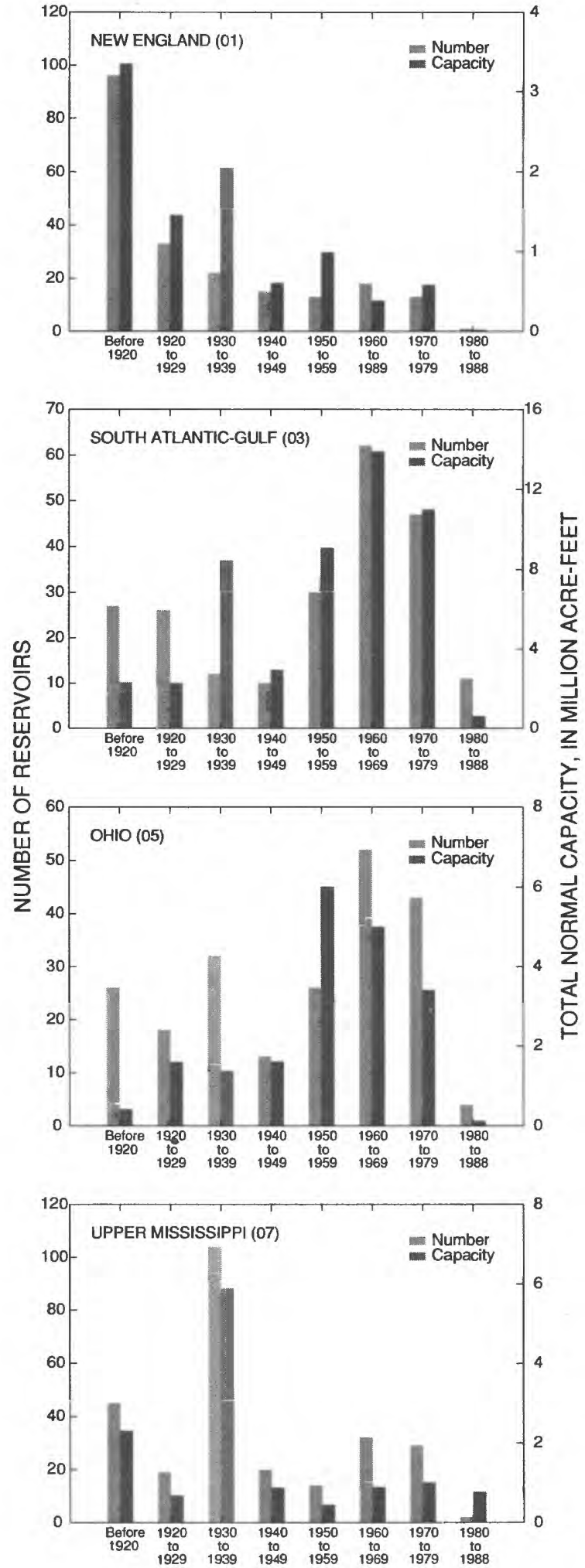
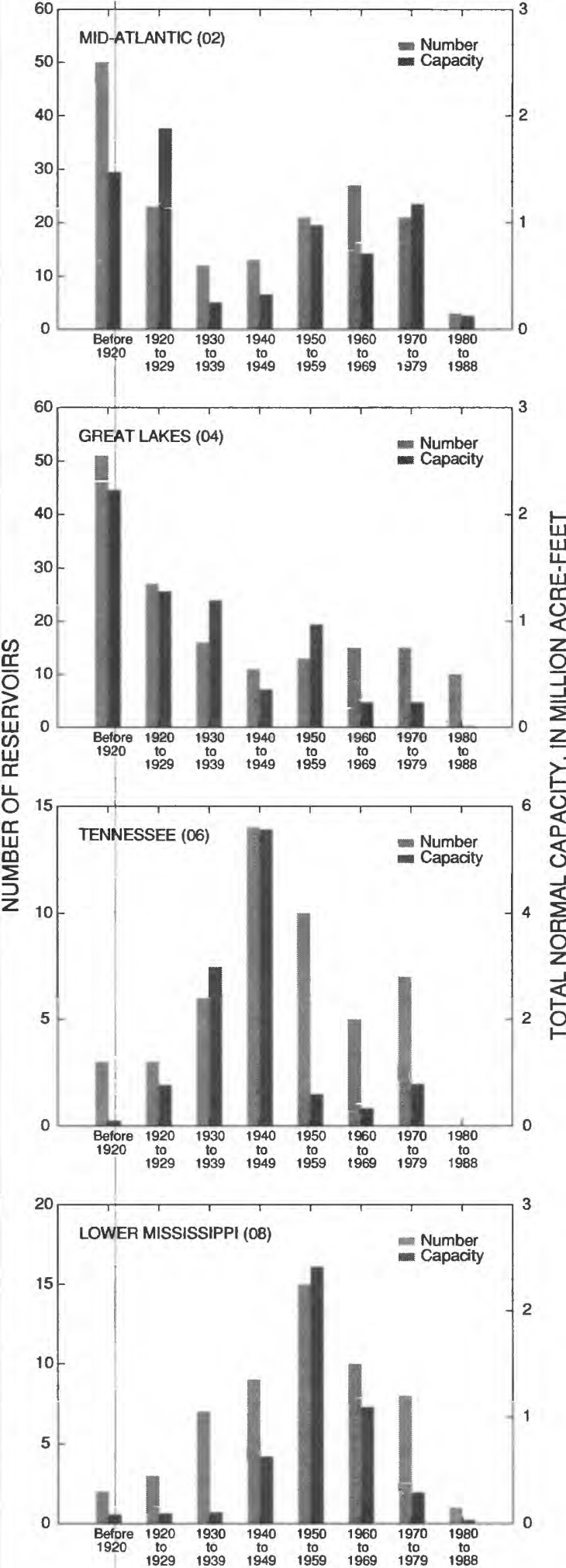

Figure 2.--Number and total normal capacity of reservoirs completed before 1920, during each decade from 1920 to 1979, and from 1980 to 1988. 

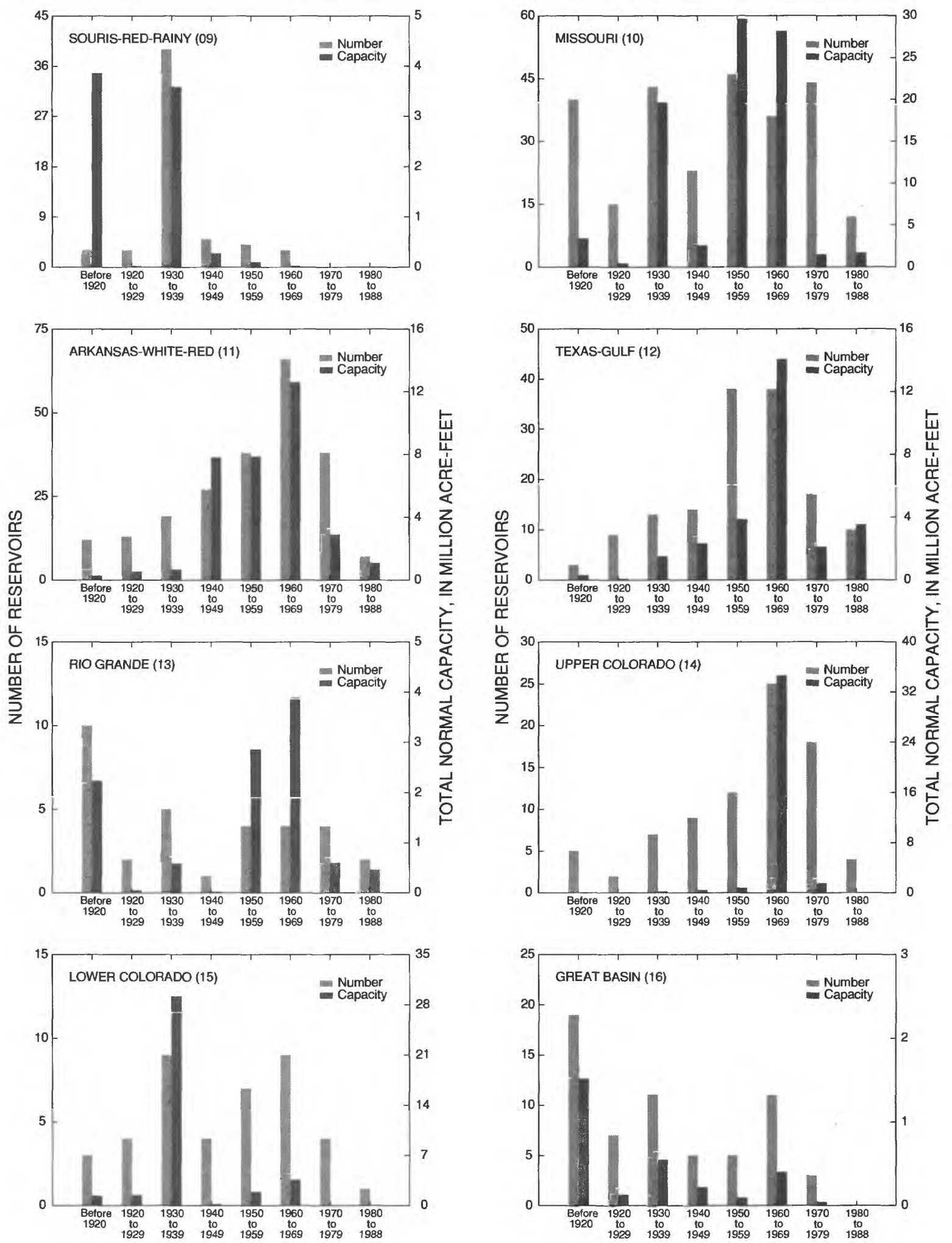

Figure 2.--Number and total normal capacity of reservoirs completed before 1920, during each decade from 1920 to 1979, and from 1980 to 1988--Continued. 

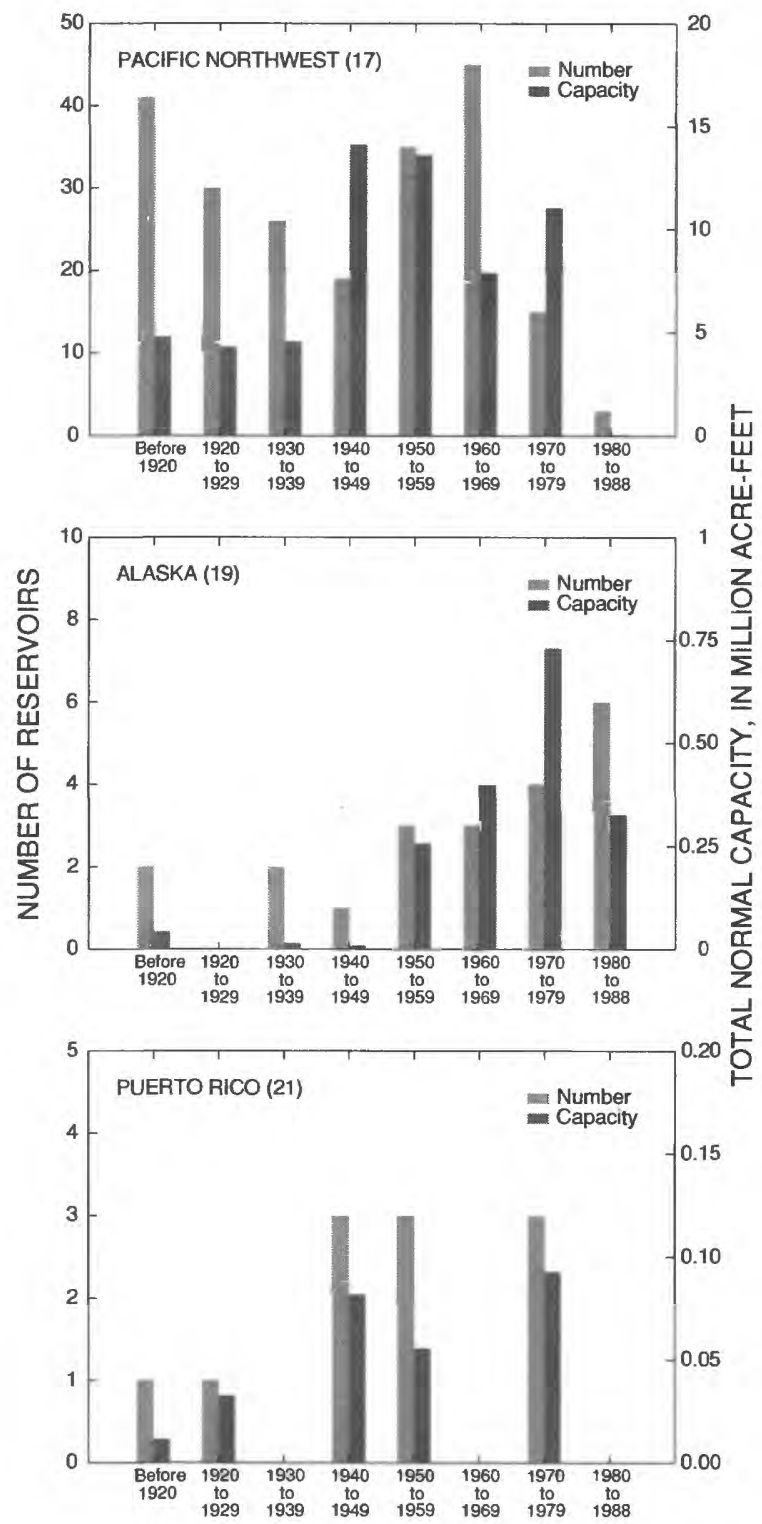
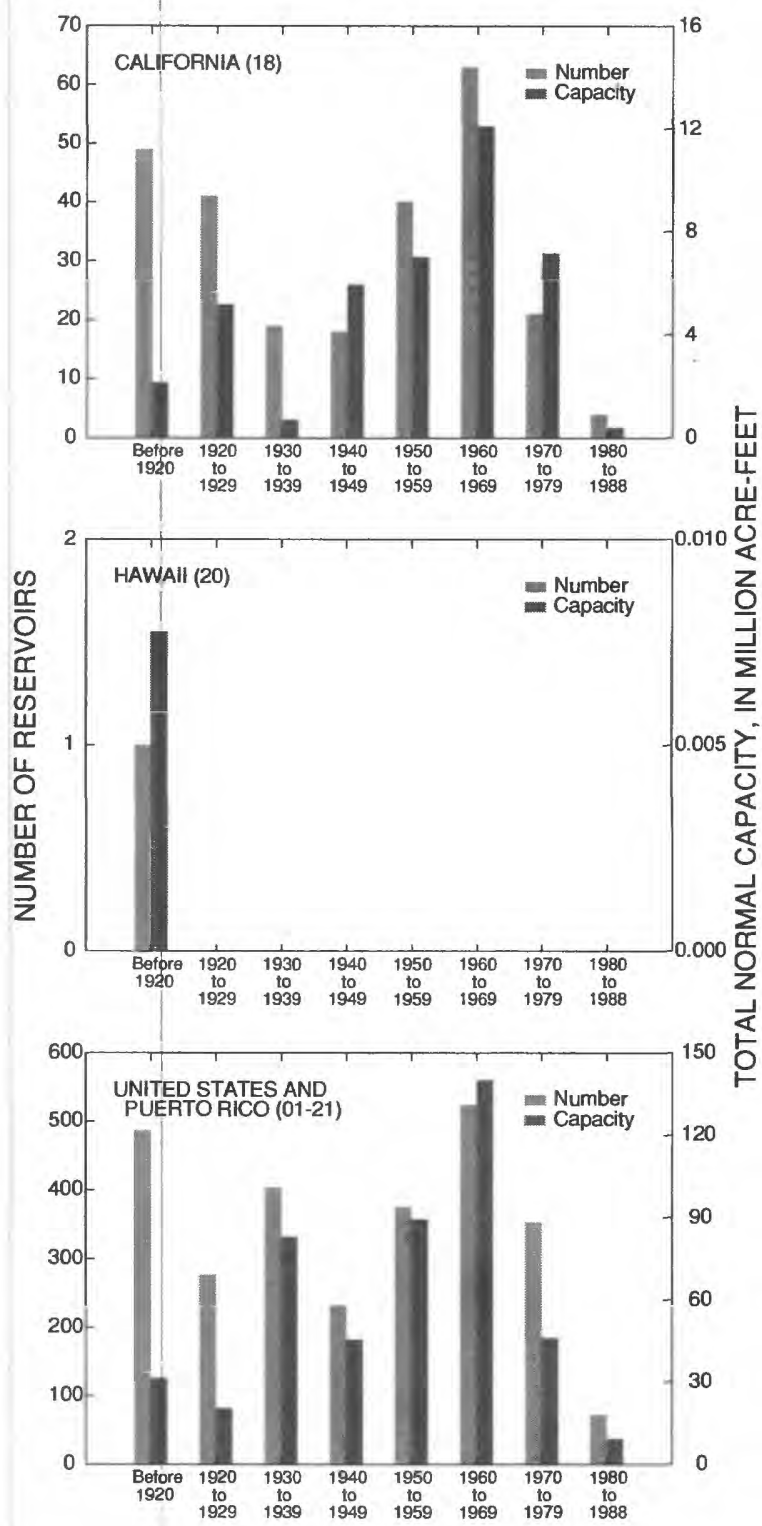

Figure 2.--Number and total normal capacity of reservoirs completed before 1920, during each decade from 1920 to 1979, and from 1980 to 1988--Continued. 


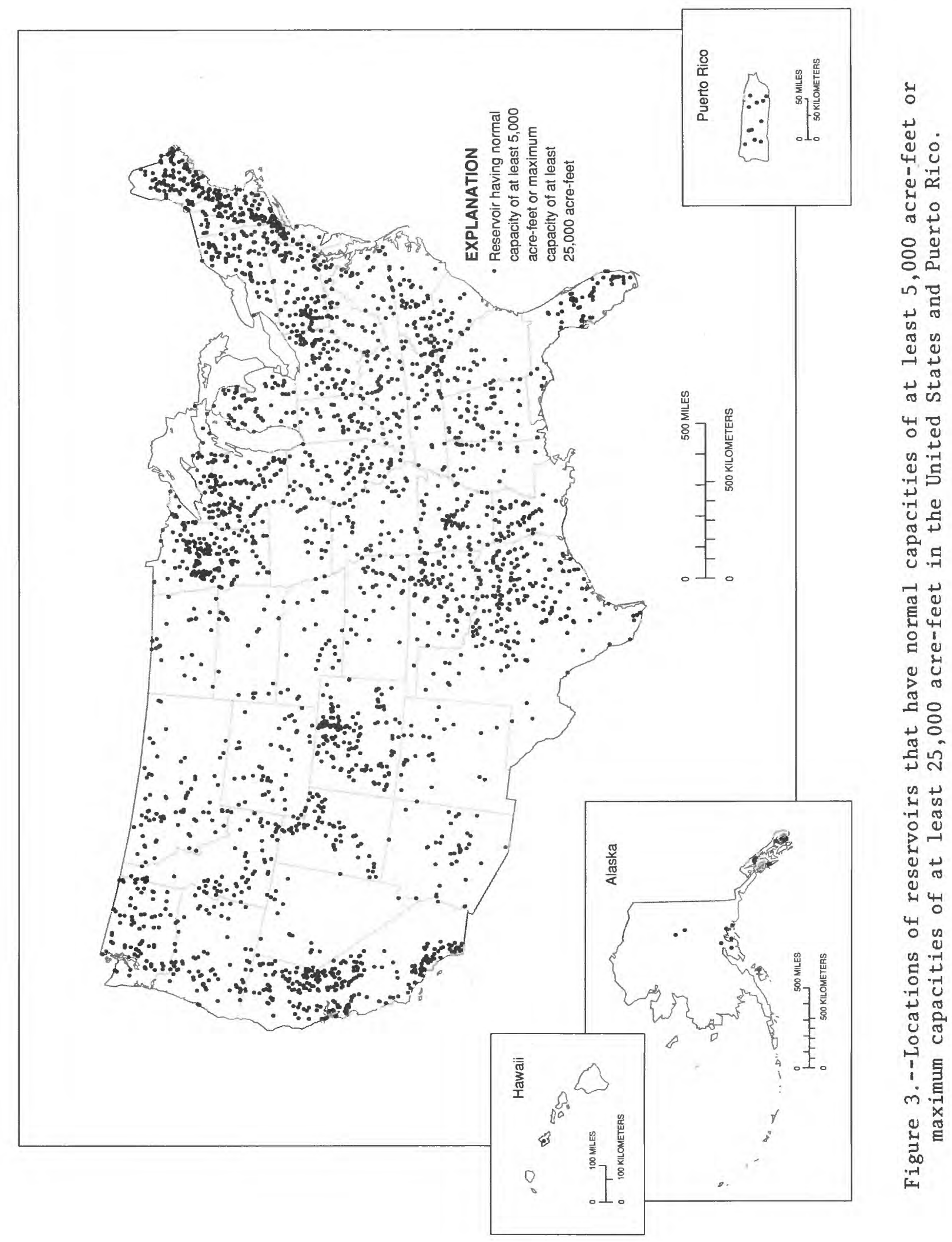


California outranks all other States in terms of number and total normal capacity of reservoirs (table 1 ). The 259 reservoirs located in California comprise about 9.5 percent of the total number of reservoirs and about

8.6 percent of the total normal capacity in the United States and Puerto Rico. Texas is second in terms of number of reservoirs. Texas has 182 reservoirs, which account for about 6.7 percent of the total number and about 7.0 percent of the total normal capacity. Many controlled natural lakes are located in Minnesota, Maine, and Wisconsin, which may explain why these States are ranked in the top 10 in terms of numbers of reservoirs. More large capacity reservoirs are located in the West than in the East because more land is available to be flooded, and the reservoirs are located in larger drainage basins. In the East, where the population density is greater, many small-capacity reservoirs are used for municipal water supplies.

Table 1.--Ranking of states by number of reservoirs and total normal capacity

[Number in parentheses is the number of reservoirs with no data for normal capacity]

\begin{tabular}{|c|c|c|}
\hline State & $\begin{array}{l}\text { Number of } \\
\text { reservoirs }\end{array}$ & $\begin{array}{c}\text { Total normal capacity } \\
\text { (acre-feet) }\end{array}$ \\
\hline $\begin{array}{l}\text { California } \\
\text { Texas } \\
\text { Minnesota } \\
\text { Colorado } \\
\text { Maine }\end{array}$ & $\begin{array}{l}259 \\
182 \\
161 \\
146 \\
109\end{array}$ & $\begin{array}{r}40,396,140 \\
32,710,139 \\
12,740,942 \\
6,348,681 \\
5,291,556\end{array}$ \\
\hline $\begin{array}{l}\text { New York } \\
\text { Wisconsin } \\
\text { Pennsylvania } \\
\text { Washington } \\
\text { Oklahoma }\end{array}$ & $\begin{array}{r}101 \\
97 \\
89 \\
81 \\
75\end{array}$ & $\begin{array}{r}6,142,347 \\
4,261,903 \\
3,822,008 \\
25,280,880 \\
14,316,084\end{array}$ \\
\hline $\begin{array}{l}\text { Michigan } \\
\text { Montana } \\
\text { Oregon } \\
\text { Idaho } \\
\text { Ohio }\end{array}$ & $\begin{array}{l}75 \\
70 \\
68 \\
63 \\
61\end{array}$ & $\begin{array}{r}2,511,295 \\
32,396,158 \\
9,716,508 \\
14,324,200 \\
1,324,025\end{array}$ \\
\hline $\begin{array}{l}\text { Arkansas } \\
\text { Florida } \\
\text { North Carolina } \\
\text { Utah } \\
\text { Illinois }\end{array}$ & $\begin{array}{l}60 \\
59 \\
59 \\
58 \\
53\end{array}$ & $\begin{array}{r}13,140,978 \\
14,875,870 \\
6,049,106 \\
6,806,612 \\
2,159,569\end{array}$ \\
\hline $\begin{array}{l}\text { Kentucky } \\
\text { Alabama } \\
\text { Wyoming } \\
\text { Louisiana } \\
\text { Missouri }\end{array}$ & $\begin{array}{l}51 \\
47 \\
45 \\
43 \\
39\end{array}$ & $\begin{array}{r}11,515,319 \\
8,612,040 \\
6,941,167 \\
5,908,979 \\
8,862,779\end{array}$ \\
\hline
\end{tabular}


Table 1.--Ranking of states by number of reservoirs and total normal capacity--Continued

\begin{tabular}{|c|c|c|}
\hline State & $\begin{array}{l}\text { Number of } \\
\text { reservoirs }\end{array}$ & $\begin{array}{c}\text { Total normal capacity } \\
\text { (acre-feet) }\end{array}$ \\
\hline Kansas & 37 & $6,918,641$ \\
\hline New Hampshire & 36 & 954,500 \\
\hline Tennessee & 35 & $8,126,402$ \\
\hline Arizona & 34 & $36,394,234$ \\
\hline Indiana & 33 & $1,082,776$ \\
\hline South Carolina & 32 & $11,038,984$ \\
\hline Virginia & 31 & $3,975,070$ \\
\hline Connecticut & 29 & 883,433 \\
\hline Iowa & 28 & $1,764,267$ \\
\hline Georgia & 25 (2) & $9,706,790$ \\
\hline New Mexico & 25 & $5,992,008$ \\
\hline Nebraska & 25 & $3,322,629$ \\
\hline North Dakota & 24 & $18,761,923$ \\
\hline West Virginia & 22 & 942,613 \\
\hline Alaska & 21 & $1,776,650$ \\
\hline Massachusetts & 21 & $1,710,319$ \\
\hline Mississippi & 20 & $1,071,017$ \\
\hline New Jersey & 20 & 404,092 \\
\hline South Dakota & 19 & $25,526,671$ \\
\hline Nevada & 16 & $28,989,833$ \\
\hline Vermont & 14 & 277,769 \\
\hline Maryland & 13 & 856,292 \\
\hline Puerto Rico & 11 & 274,215 \\
\hline Rhode Island & 4 & 136,206 \\
\hline Delaware & 1 & 6,300 \\
\hline Hawaii & 1 & 7,761 \\
\hline Total & $2,728 \quad(2)$ & $467,356,680$ \\
\hline
\end{tabular}

The list of reservoirs greater than 2,000,000 acre-feet in usable capacity compiled by Martin and Hanson (1966) and the reservoirs that have a normal capacity of 2,000,000 acre-feet or more listed in table 2 are not directly comparable. The terms "usable capacity" and "normal capacity" are not interchangeable. Usable capacity is defined as the volume, in acre-feet, normally available for release from a reservoir below the maximum controllable level (Martin and Hanson, 1966). Normal capacity is defined as the total storage space, in acre-feet, below the normal retention level, which includes dead storage but excludes flood control or surcharge storage. There are some reservoirs included in table 2 which were not included in the Martin and Hanson list, and there are some reservoirs included in the Martin and Hanson list which were not included in table 2 because of the difference in capacity definitions. 
Table 2.--Large reservoirs in the United States that have a normal capacity of 2,000,000 acre-feet or more

Name of reservoir

(name of dam)
State

$\begin{array}{cc}\text { Normal } & \text { Year } \\ \text { capacity } & \text { completed }\end{array}$

Lake Mead (Hoover)

Lake Powell (Glen Canyon)

Lake Oahe

Lake Sakakawea (Garrison)

Lake Fort Peck

Franklin D. Roosevelt (Grand Coulee)

Lake Koocanusa (Libby)

Shasta Lake

Toledo Bend

Levee 65 Borrow Canal (Structure 153)

Lake Cumberland (Wolf Creek)

Lake Murray

Lake Francis Case (Fort Randa11)

Flaming Gorge

(Oroville 1-048)

Amistad

Hungry Horse

Dworshak

Rainy Lake

Bull Shoals Lake

Sam Rayburn

International Falcon

Table Rock Lake

Lake Texoma (Denison)

Hartwell Lake

Clarks Hill Lake

Painted Rock

Wilson Lake

Claire Engle Lake (Trinity)

New Melones Lake

Eufaula Lake

L-29, Sec. 3 Borrow Canal

(Structure 334)

Lake Ouachita (Blakely Mountain)

Kentucky Lake

Elephant Butte

San Luis

(Don Pedro 68-007)

Lake of the Ozarks (Osage)

Nevada-Arizona
Arizona
South Dakota
North Dakota
Montana
Washington
Montana
California
Louisiana-Texas
Florida

$28,255,000$

1936

$27,000,000$

1966

$18,900,000$

1966

$18,300,000$

1953

$15,200,000$

1937

$9,386,000$

1942

$5,809,000$

1973

$4,552,000$

1945

$4,477,000$

1968

$4,147,000$

1938

Kentucky

South Carolina

South Dakota

Utah

California

$3,995,000$

1951

$3,952,000$

1930

$3,800,000$

1954

$3,787,000$

1964

$3,537,577$

1968

Texas

Montana

Idaho

Minnesota

Arkansas

$3,505,400$

1969

$3,468,000$

1953

$3,450,000$

1973

$3,312,000$

1909

$3,048,000$

1951

Texas

Texas

Missouri

Oklahoma-Texas

Georgia

$2,852,600$

1965

$2,767,400$

1954

$2,702,000$

1959

$2,643,300$

1944

$2,550,000$

1960

Georgia-

South Carolina

Arizona

Kansas

California

California

Oklahoma

$2,510,000$

1953

$2,491,700$

1960

$2,478,350$

1964

$2,448,000$

1962

$2,400,000$

1979

$2,330,000$

1964

Florida

$2,200,000$

1978

Arkansas

Kentucky

New Mexico

$2,151,100$

1953

$2,121,000$

1944

$2,110,298$

1916

California

$2,041,000$

1967

California

$2,030,000$

1971

1931 
Thirty-eight reservoirs in the United States have a normal capacity of 2,000,000 acre-feet or more (table 2). Martin and Hanson (1966) list 31 reservoirs that have a usable capacity of 2,000,000 acre-feet or more and were completed or under construction before 1963. There are nine reservoirs listed in table 2 that were not listed in Martin and Hanson (1966) and were completed after Martin and Hanson compiled their list. The development of reservoir storage between 1963 and 1988 was less than the development which occurred during the previous 16 years (1947 through 1962), when 22 large reservoirs were completed (Martin and Hanson, 1966). The 27 large reservoirs built after 1947 (table 2) generally were multipurpose reservoirs in public ownership. Only one of the recently completed large reservoirs, Painted Rock, is a single-purpose reservoir in public ownership. Painted Rock was constructed for flood control. The 38 large reservoirs (table 2) account for 46 percent of the total normal capacity of reservoirs listed in this report.

\section{EXPLANATION OF DATA}

Selected characteristics of reservoirs that have a normal capacity of at least 5,000 acre-feet or a maximum capacity of at least 25,000 acre-feet and that were completed as of 1988 are listed in table 3. Reservoirs are listed alphabetically by State and by name of reservoir. Reservoirs without names are listed alphabetically by name of dam before the reservoirs listed by name of reservoir. The elements of the table are discussed below. Most of the definitions are paraphrased from the inventory description of the U.S. Army Corps of Engineers national inventory of dams (1982). The table lists 1,166 more reservoirs than were published previously in Martin and Hanson (1966). These data are not totally complete because the information is being updated periodically. In some areas of the United States, reservoir capacity is reported in units other than acre-feet. The following conversions may be useful:

1 acre-foot $=0.3259$ million gallons;

1 acre-foot $=0.04356$ million cubic feet .

Name of reservoir--the official name of the lake or reservoir. Dashes indicate that the reservoir does not have a name. Usually the official name of the reservoir and the official name of the dam are the same. The latest legal name for the reservoir is listed.

Name of stream--the official name of the stream or river on which the dam is built. This stream or river also is the major source of water to the reservoir. If the stream does not have a name, it is indicated as a tributary to the river into which it flows. If the dam is located offstream, the name of the major contributing stream is listed, and the reservoir location is indicated as offstream.

Name of dam--the official name of the dam. Usually the official name of the dam is the same as the official name of the reservoir.

Owner--the name of the owner of the dam. The owner of the dam may not necessarily be the operator of the dam or the party legally responsible for the operation of the dam. The names of Federal agencies are abbreviated as follows: 
U.S. Department of Agriculture

Soil Conservation Service

Forest Service

USDA

SCS

USDA

FS

U.S. Department of Energy

Federal Energy Regulatory Commission

DOE

FERC

Tennessee Valley Authority

TVA

U.S. Department of the Interior

Fish and Wildlife Service

DOI

FWS

Bureau of Land Management

DOI

BLM

Bureau of Reclamation

DOI

BOR

Bureau of Indian Affairs

DOI

BIA

National Park Service

DOI

NPS

U.S. Department of Labor

Mine Safety and Health Administration

DOL

MSHA

U.S. Department of Defense
U.S. Army
U.S. Navy
U.S. Air Force
U.S. Marine Corps

DOD

USA

DOD

USN

DOD

USAF

DOD

USMC

U.S. Department of Justice

Bureau of Prisons

DOJ

BOP

U.S. Army Corps of Engineers

Lower Mississippi Valley Division

Memphis District

New Orleans District

St. Louis District

Vicksburg District

DAEN

LMV

DAEN

LMM

DAEN

LMN

DAEN

LMS

DAEN

LMK

Missouri River Division

DAEN

MRD

Kansas City District

Omaha District

DAEN

MRK

DAEN

MRO

New England Division

DAEN

NED

North Atlantic Division

DAEN

NAD

Baltimore District

DAEN

NAB

New York District

DAEN

NAN

Norfolk District

DAEN

NAO

Philadelphia District

DAEN

NAP 


\begin{tabular}{|c|c|c|}
\hline \multirow{2}{*}{$\begin{array}{l}\text { Name of Federal Agency } \\
\text { Name of Organization }\end{array}$} & \multicolumn{2}{|c|}{ Abbreviation } \\
\hline & Agency & Organization \\
\hline \multicolumn{3}{|c|}{ U.S. Army Corps of Engineers (continued) } \\
\hline North Central Division & DAEN & NCD \\
\hline Buffalo District & DAEN & $\mathrm{NCB}$ \\
\hline Chicago District & DAEN & $\mathrm{NCC}$ \\
\hline Detroit District & DAEN & NCE \\
\hline Rock Island District & DAEN & NCR \\
\hline St. Paul District & DAEN & NCS \\
\hline North Pacific Division & DAEN & NPD \\
\hline Alaska District & DAEN & NPA \\
\hline Portland District & DAEN & NPP \\
\hline Seattle District & DAEN & NPS \\
\hline Walla Walla District & DAEN & NPW \\
\hline Ohio River Division & DAEN & ORD \\
\hline Huntington District & DAEN & $\mathrm{ORH}$ \\
\hline Louisville District & DAEN & ORL \\
\hline Nashville District & DAEN & ORN \\
\hline Pittsburg District & DAEN & ORP \\
\hline Pacific Ocean Division & DAEN & POD \\
\hline South Atlantic Division & DAEN & $\mathrm{SAD}$ \\
\hline Charleston District & DAEN & SAN \\
\hline Jacksonville District & DAEN & SAJ \\
\hline Mobile District & DAEN & SAM \\
\hline Savannah District. & DAEN & SAS \\
\hline Wilmington District & DAEN & SAW \\
\hline South Pacific Division & DAEN & SPD \\
\hline Los Angeles District & DAEN & SPL \\
\hline Sacramento District & DAEN & SPK \\
\hline San Francisco District & DAEN & SPN \\
\hline Southwestern Division & DAEN & SWD \\
\hline Albuquerque District & DAEN & SWA \\
\hline Fort Worth District & DAEN & SWF \\
\hline Galveston District & DAEN & SWG \\
\hline Little Rock. District & DAEN & SWL \\
\hline Tulsa District & DAEN & SWT \\
\hline Others not listed above & USA & Name \\
\hline
\end{tabular}


Hydrologic unit--the hydrologic unit code indicating the drainage basin in which the reservoir is located. The eight-digit code is divided into four two-digit fields. The first two digits identify the water-resources region (shown in figure 1), the next two digits identify the subregion, the next two digits identify the accounting unit, and the last two digits identify the cataloging unit. Hydrologic units and the associated hydrologic-unit maps are described by Seaber and others (1987).

Latitude and longitude--the latitude and longitude of the dam at its maximum section are listed to the nearest degree, minute, and second. Latitudes and longitudes may vary by a few seconds depending on the source of the data and what point was identified as maximum section.

Capacity--the normal and maximum capacities of the reservoirs are listed in acre-feet. Normal capacity is defined as the total volume in a reservoir below the normal retention level, including dead storage, but excluding flood control or surcharge storage. The maximum capacity is defined as the total volume in the reservoir below the maximum attainable watersurface elevation and includes any surcharge storage. Surcharge storage is the storage above the total retention level. This storage increase can result from flashboards increasing the dam height and allowing temporary detention of a volume of flood water above the controllable pool level.

Surface area--the area, in acres, of the water surface of the reservoir. It usually is listed for normal capacity, but sometimes it is listed for maximum capacity or for an unknown storage. Surface area of a reservoir varies depending on the storage level of the reservoir.

Drainage area--the area measured in a horizontal plane, enclosed by a topographic divide, from which surface runoff from precipitation would normally drain. It is measured in square miles at the location of the dam. The drainage area of an offstream reservoir is not listed because the contributing drainage area has no relation to the inflow to the reservoir. The listed drainage areas account for what would normally drain into the reservoir due to topography; effects of transbasin diversions are not included.

Year completed--the year in which dam construction was completed. At some dams, reservoir storage began during construction, and therefore, the reservoir effect on streamflow may precede the year of dam completion.

Use--the major purposes or uses of the reservoir are listed with the primary use first. The other uses are secondary and may be a resulting benefit of dam construction. 


\section{SUMMARY}

Selected characteristics of 2,728 reservoirs and controlled natural lakes located within the 50 States of the United States and the Commonwealth of Puerto Rico are listed in this report. The data are from a digital file of dams and reservoirs developed by the U.S. Geological Survey. Reservoirs that have normal capacities of at least 5,000 acre-feet or maximum capacities of at least 25,000 acre-feet and that were completed as of January 1, 1988, are listed. Reservoir data include: location, maximum capacity, normal capacity, surface area, drainage area, year completed, and use.

The normal capacities of the reservoirs total more than 467 million acre-feet, and the combined surface areas for 2,362 of those reservoirs total almost 16 million acres. Reservoirs in California account for more than 9 percent (259) of the reservoirs listed and also account for more than 40 million acre-feet of normal storage and almost 700,000 acres of the listed surface area.

\section{REFERENCES CITED}

Farnsworth, R.K., Thompson, E.S., and Peck, E.L., 1982, Annual free water surface (FWS) evaporation--shallow lake, 1956-1970, map 3 in pocket, of Evaporation Atlas for the Contiguous 48 United States: Washington, D.C., National Oceanic and Atmospheric Administration Technical Report NWS 33, map scale $1: 4,800,000$.

Hitt, K.J., 1984 [1985], Surface-water and related land resources development in the United States and Puerto Rico: U.S. Geological Survey special map: scale $1: 3,168,000$.

Langbein, W.B., 1982, Dams, reservoirs, and withdrawals for water supply-historic trends: U.S. Geological Survey Open-File Report 82-256, 9 p.

Martin, R.0., and Hanson, R.L., 1966, Reservoirs in the United States: U.S. Geological Survey Water-Supply Paper 1838, 114 p.

Seaber, P.R., Kapinos, F.P., and Knapp, G.L., 1987, State Hydrologic Unit Maps: U.S. Geological Survey Water-Supply Paper 2294, 63 p.

U.S. Army Corps of Engineers, 1982: National inventory of dams database in card format (computer tape), available from National Technical Information Service, Springfield, VA 22162, 非ADA 118670.

U.S. Geological Survey, 1986, National water summary 1985--Hydrologic events and surface-water resources: U.S. Geological Survey Water-Supply Paper $2300,506 \mathrm{p}$.

U.S. Geological Survey, 1990, National Water Summary 1987--Hydrologic events and water supply and use: U.S. Geological Survey Water-Supply Paper $2350,553 \mathrm{p}$. [in press] 


\section{INDEX}

Page

Page

Alabama--_-

Montana-_..-. 163

Alaska---_-

Arizona----

Arkansas------

Nebraska--...-. 170

California--

Colorado--

Connecticut--.-

Delaware---_-- 79

Florida--_- 79

Georgia--_- 85

Hawaii-_-_._. 88

Idaho--

Illinois-- 94

Nevada-n-_-n 173

New Hampshire---_-_-_-n 175

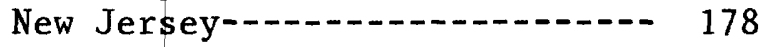

New Mexico-n-_-_- 180

New York-_..- 183

North Carolina--.---.-- 193

North Dakota--_-_-_-_-_- 199

Ohio--..-. 202

Oklahoma-_._...-. 208

Oregon-_-_- 215

Indiana-- 99

Pennsylvania--_-_-_-_- 222

Iowa--_- 103

Kansas--_- 106

Puerto Rico--.-_-_-_-_--- 231

Kentucky-n- 109

Rhode Island--.-- 232

South Carolina--..-_..- 233

Louisiana--_-_-_- 115

Maine---_-_-_-_- 119

South Dakota-n- 236

Tennessee---0.-- 238

Texas--_- 242

Maryland---.-.- 130

Utah--

Massachusetts---_-_-_---- 131

Michigan---:-D 134

Vermont-- 266

Virginia-- 267

Minnesota---_-_- 141

Mississippi-- 157

Washington-- 271

West Virginia--_- 279

Missouri-- 159

Wisconsin-- 281

Wyoming-_- 291 


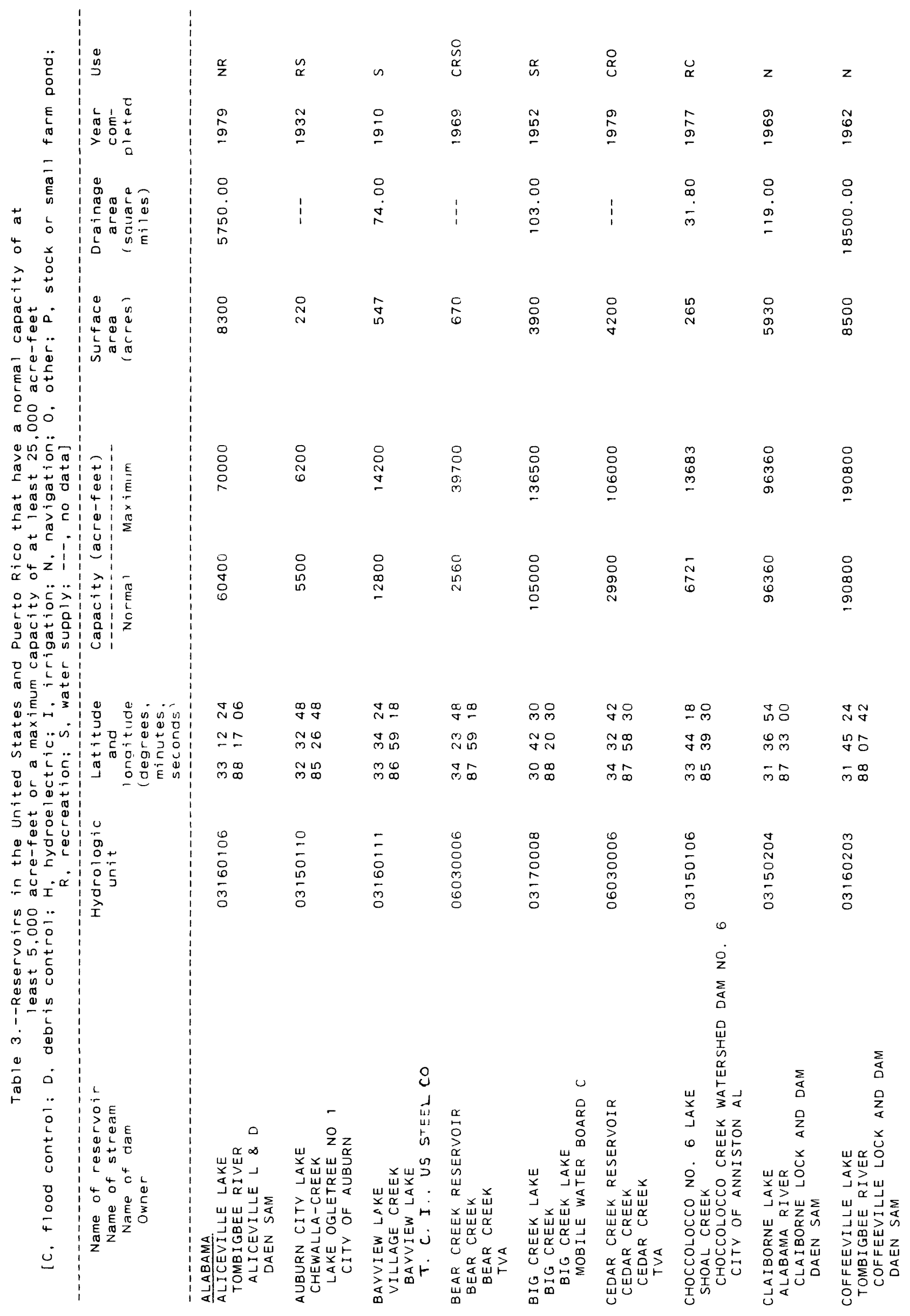




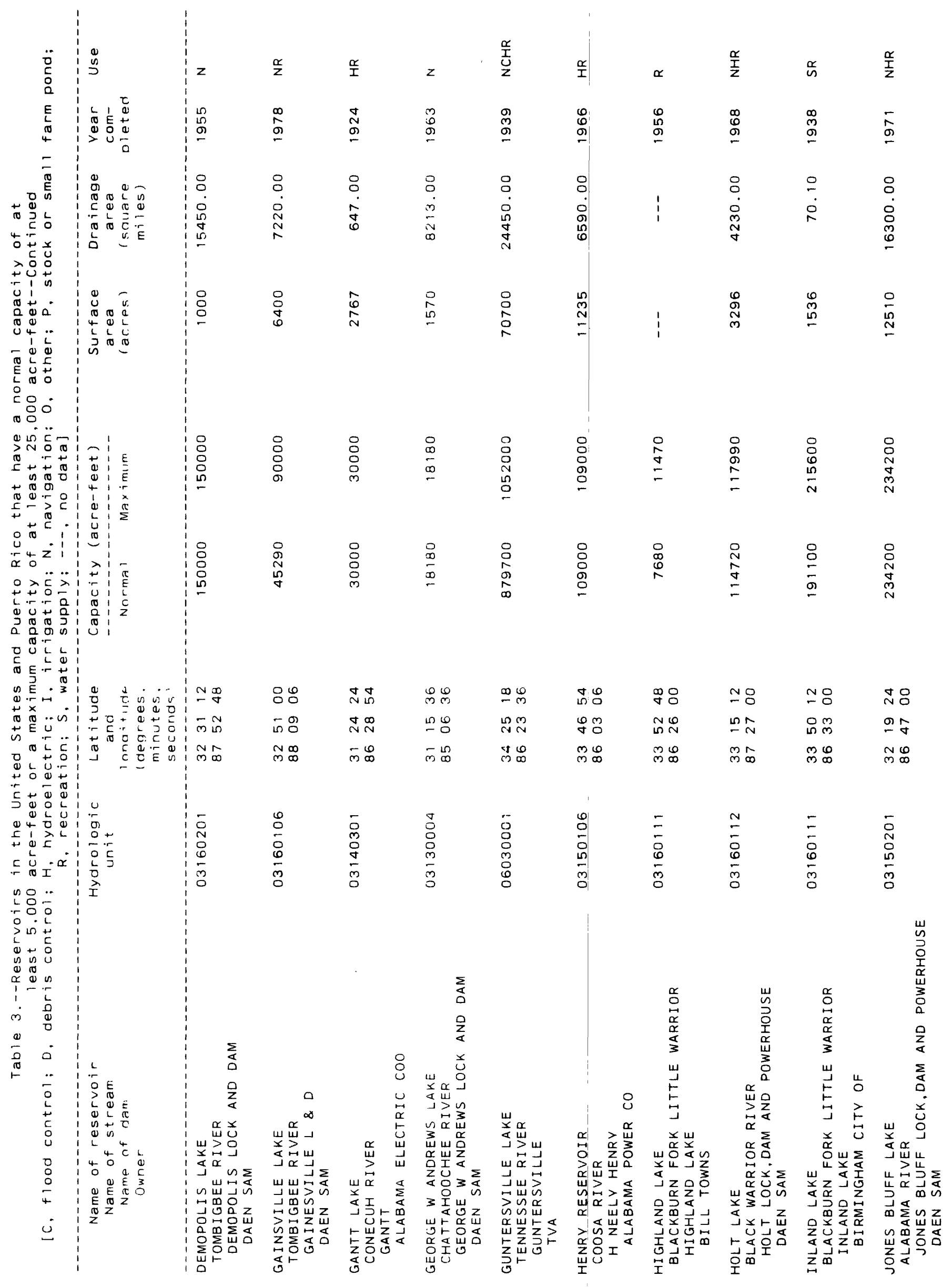




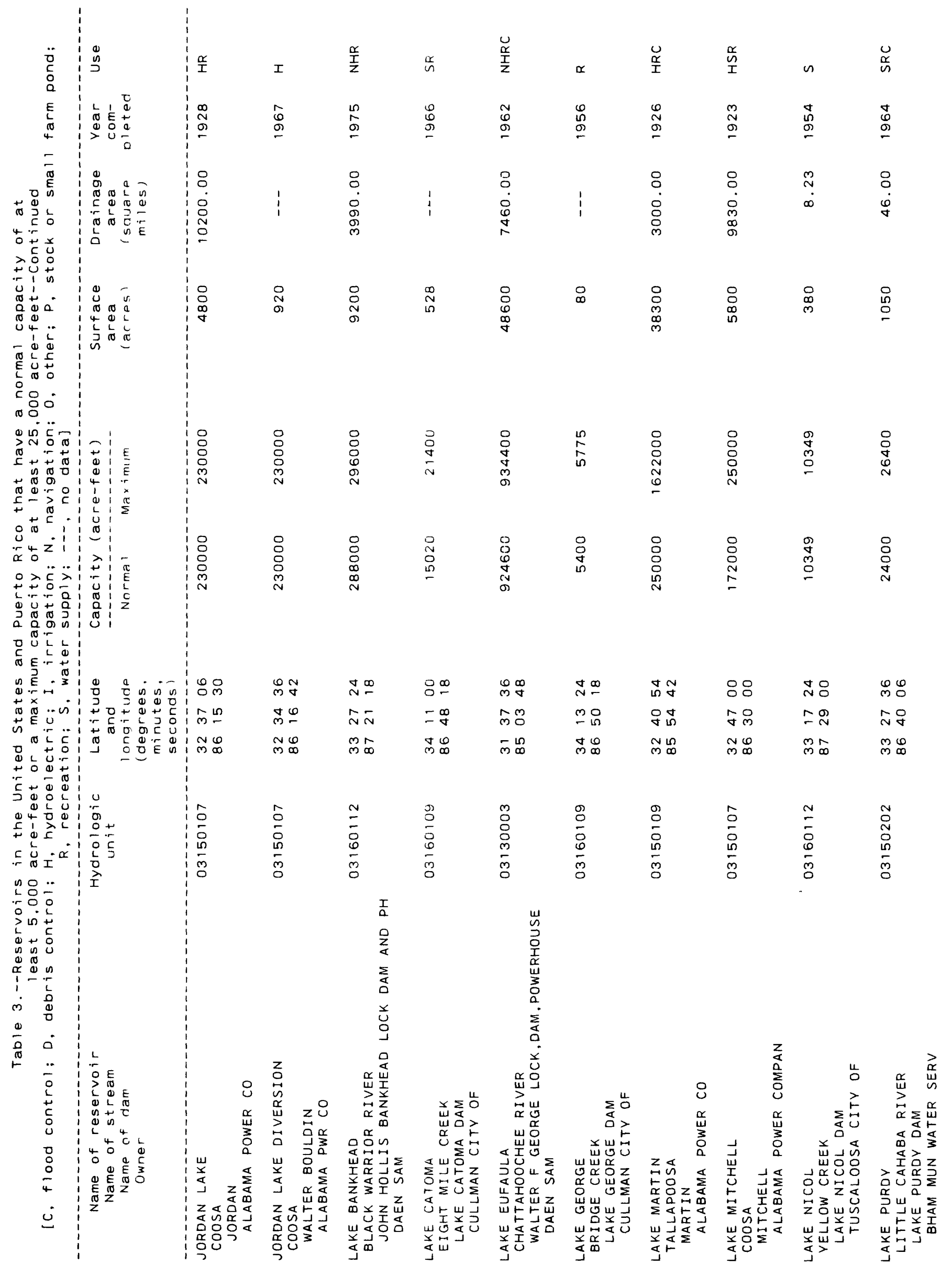




\begin{tabular}{|c|c|c|c|c|c|c|c|}
\hline 舀 & $\stackrel{\propto}{\underline{I}}$ & $\begin{array}{l}\stackrel{\alpha}{U} \\
\stackrel{I}{I}\end{array}$ & $\begin{array}{l}\text { O } \\
\text { ơ }\end{array}$ & $\begin{array}{l}\frac{\alpha}{U} \\
\text { I }\end{array}$ & $\stackrel{\alpha}{\sim}$ & $\stackrel{\propto}{I}$ & $\alpha$ \\
\hline ז̊ & $\frac{\nabla}{\sigma}$ & 's & $\begin{array}{l}\text { n } \\
\stackrel{0}{0}\end{array}$ & 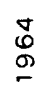 & $\stackrel{\text { Oే }}{\stackrel{-}{\sigma}}$ & $\stackrel{\stackrel{N}{N}}{\stackrel{D}{\sim}}$ & 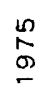 \\
\hline & 음 & 음 & & 임 & & $\therefore$ & \\
\hline $\begin{array}{l}1 \\
\vdots\end{array}$ & $\begin{array}{l}\dot{\circ} \\
\text { 。े }\end{array}$ & $\begin{array}{l}\dot{J} \\
\stackrel{J}{J}\end{array}$ & $\vdots$ & $\begin{array}{l}\dot{0} \\
\hat{N} \\
\hat{N}\end{array}$ & 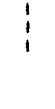 & $\underset{\stackrel{N}{N}}{\stackrel{\dot{D}}{N}}$ & $\begin{array}{l}1 \\
1 \\
1\end{array}$ \\
\hline 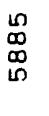 & $\begin{array}{l}0 \\
\hat{a} \\
\hat{\varphi}\end{array}$ & $\begin{array}{l}8 \\
\frac{O}{N} \\
\dot{n}\end{array}$ & $\begin{array}{l}0 \\
\stackrel{0}{0} \\
\stackrel{0}{2}\end{array}$ & $\begin{array}{l}\text { O } \\
\text { In } \\
-\end{array}$ & 邑 & 음 & $\vdots$ \\
\hline $\begin{array}{l}\circ \\
: \\
\therefore \\
0 \\
\infty\end{array}$ & $\begin{array}{l}\stackrel{\circ}{\circ} \\
\stackrel{\circ}{h} \\
\stackrel{0}{N}\end{array}$ & $\begin{array}{l}\stackrel{D}{ } \\
\text { O } \\
\stackrel{M}{O} \\
\stackrel{N}{N}\end{array}$ & 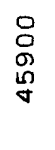 & \begin{tabular}{l} 
̊ \\
Ñ \\
\multirow{J}{0}{}
\end{tabular} & $\begin{array}{l}\text { ㅇ } \\
\text { N } \\
\text { ㅇ }\end{array}$ & $\begin{array}{l}\text { : } \\
\text { : }\end{array}$ & 吕 \\
\hline 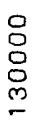 & $\begin{array}{l}\stackrel{\circ}{\circ} \\
\text { 吕 } \\
\stackrel{0}{N}\end{array}$ & 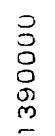 & $\begin{array}{l}\frac{O}{\circ} \\
\frac{0}{N}\end{array}$ & 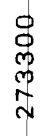 & 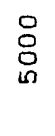 & $\begin{array}{l}\text { : } \\
: \\
\infty\end{array}$ & $\begin{array}{l}\text { O } \\
\text { Oे }\end{array}$ \\
\hline
\end{tabular}

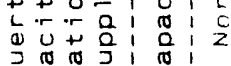

a o o w

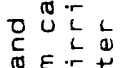

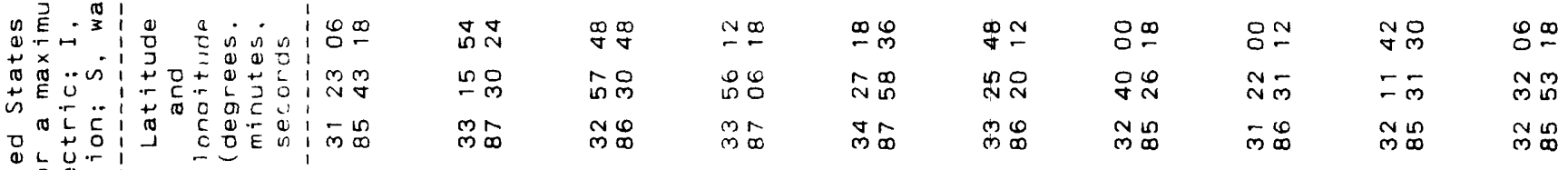

$\overline{5}+\frac{\pi}{0}$

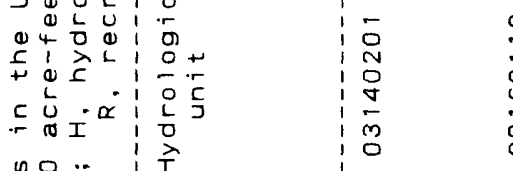

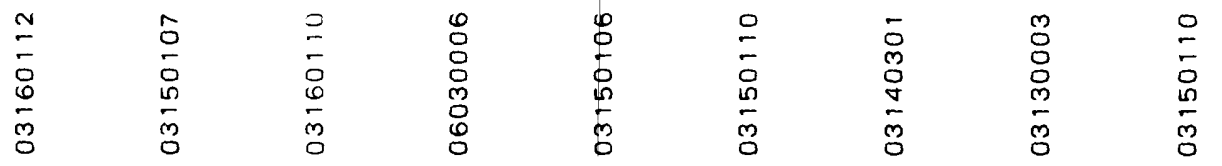

\section{2
.
0
2
2
0
0
0
0
0
1
1
$\dot{0}$
0
0
$\frac{0}{0}$
1 \\ $\frac{0}{0}$}

$>$ in

$\begin{array}{lll}0 & 0 & 0 \\ 0 & 0 & 0 \\ 0 & 0 & 0 \\ 1 & 0 & 0\end{array}$

1.
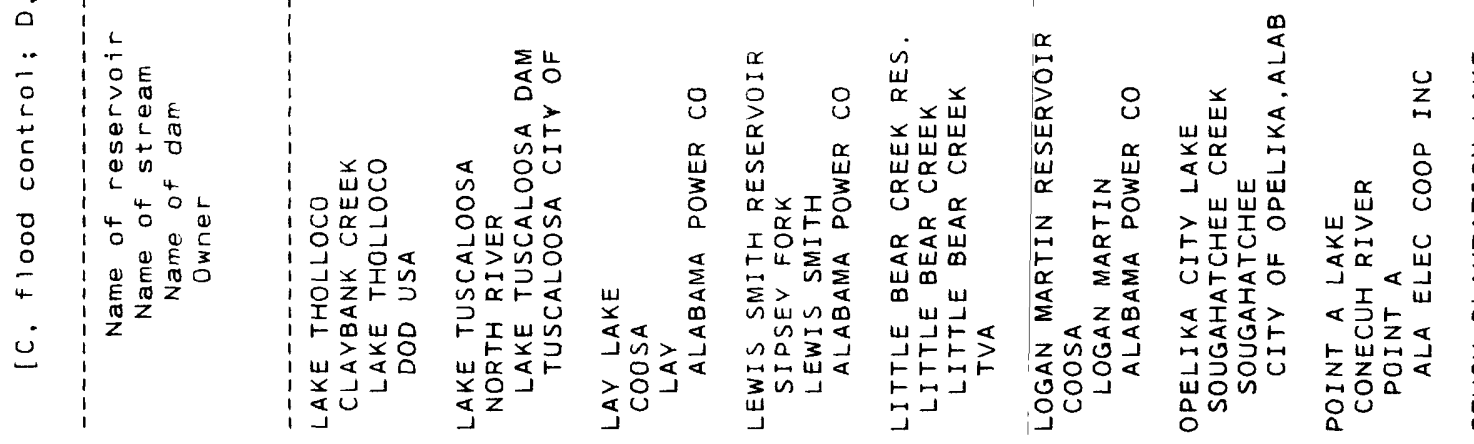

玄 


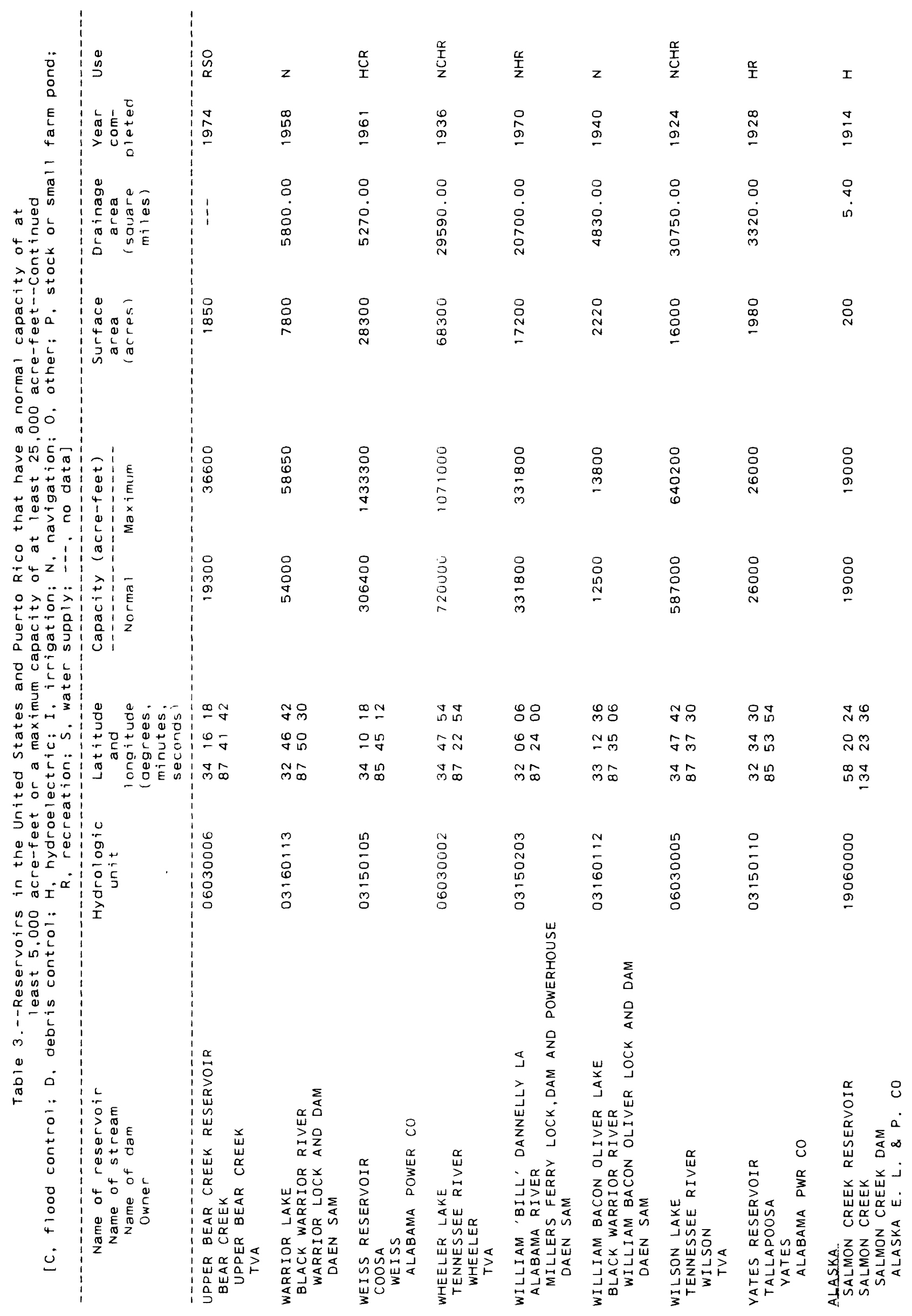




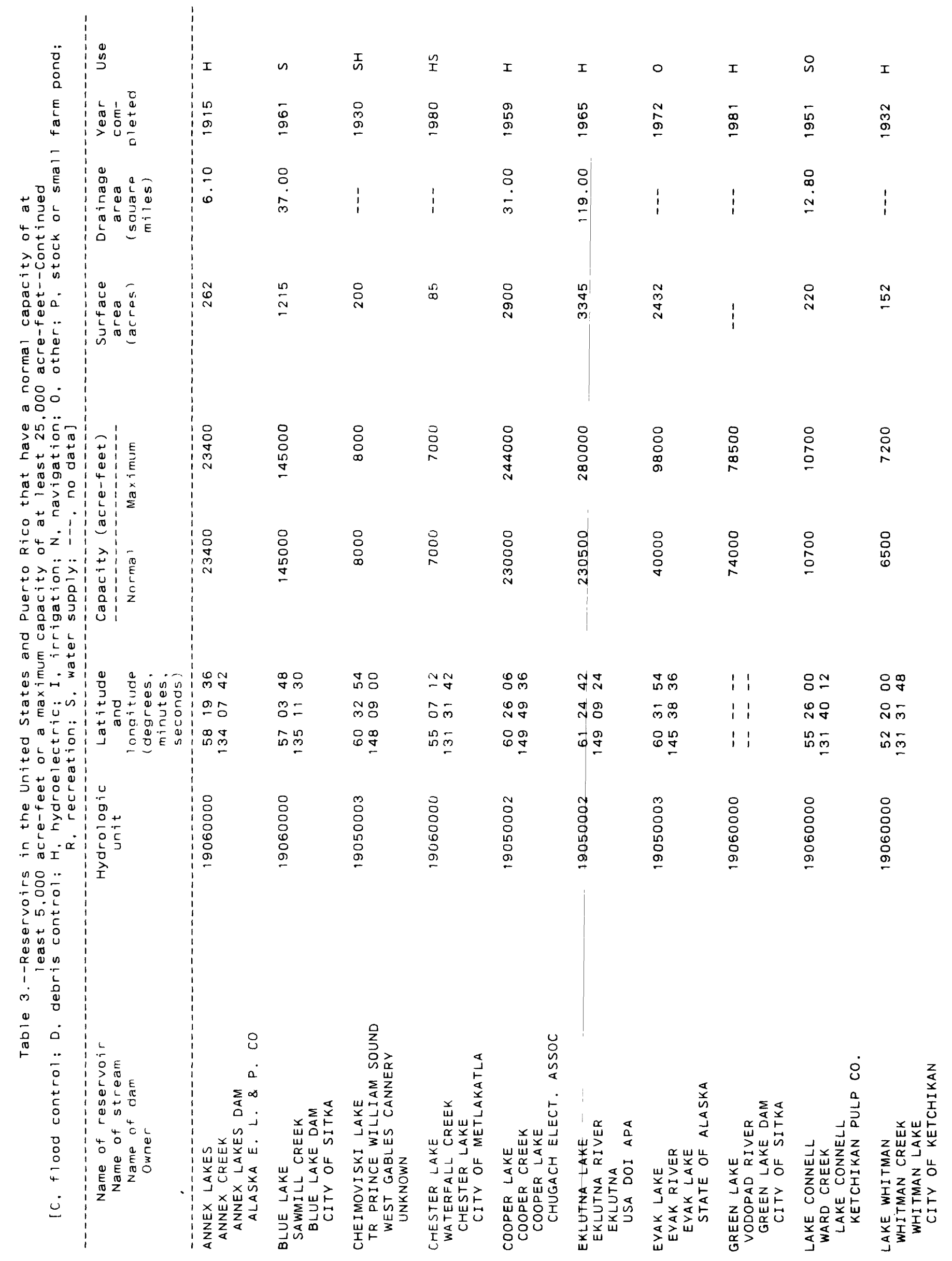




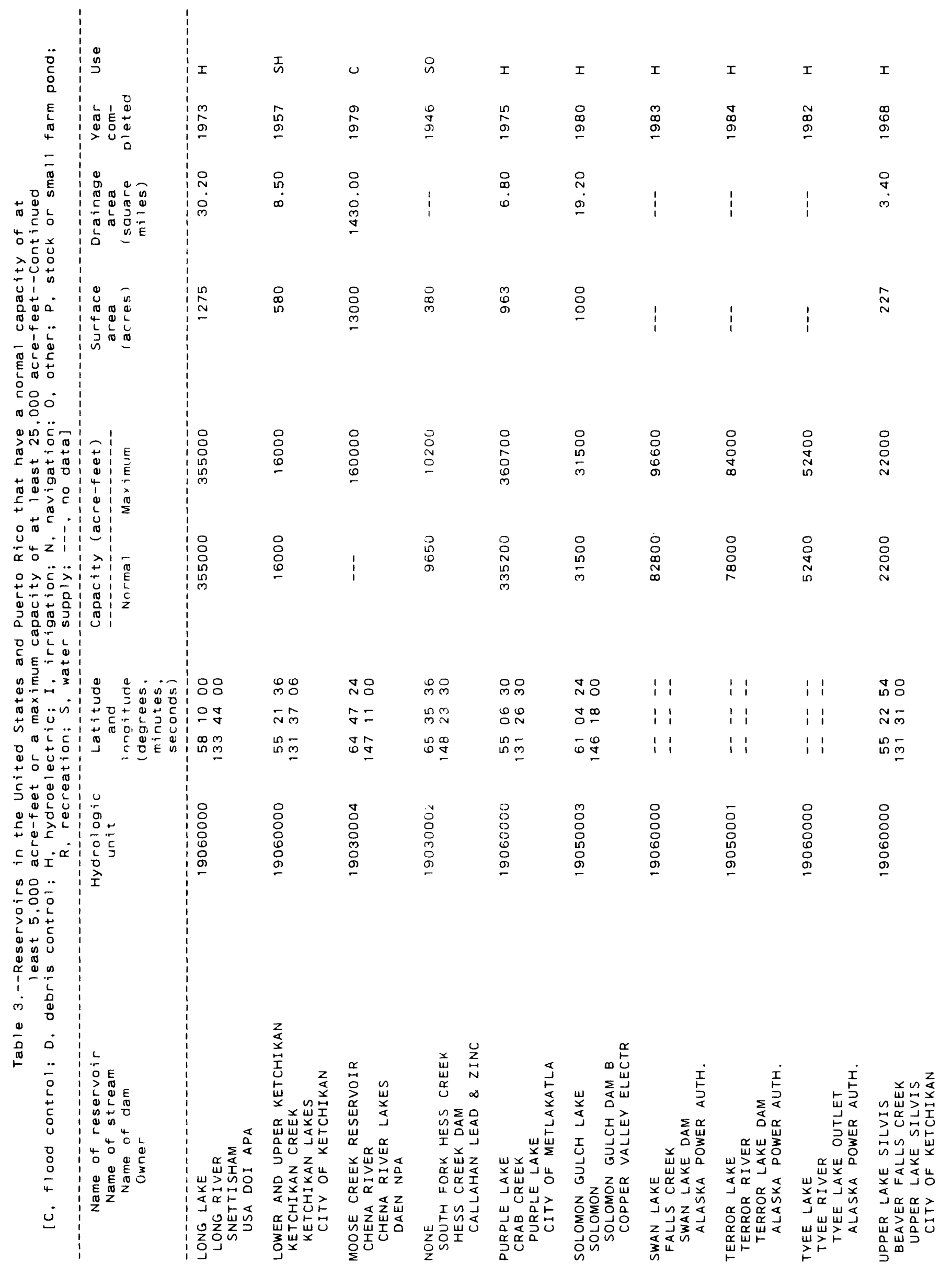




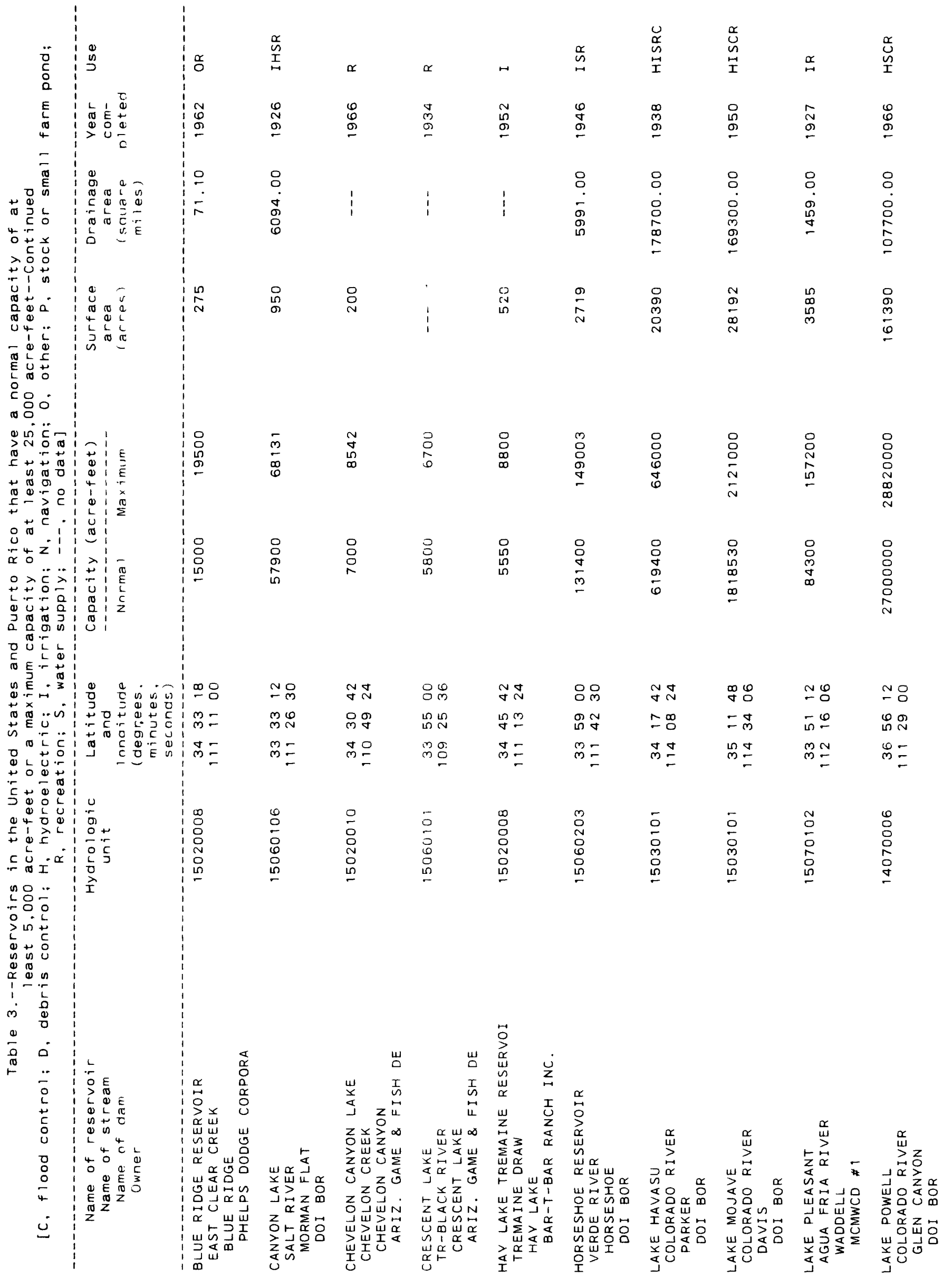




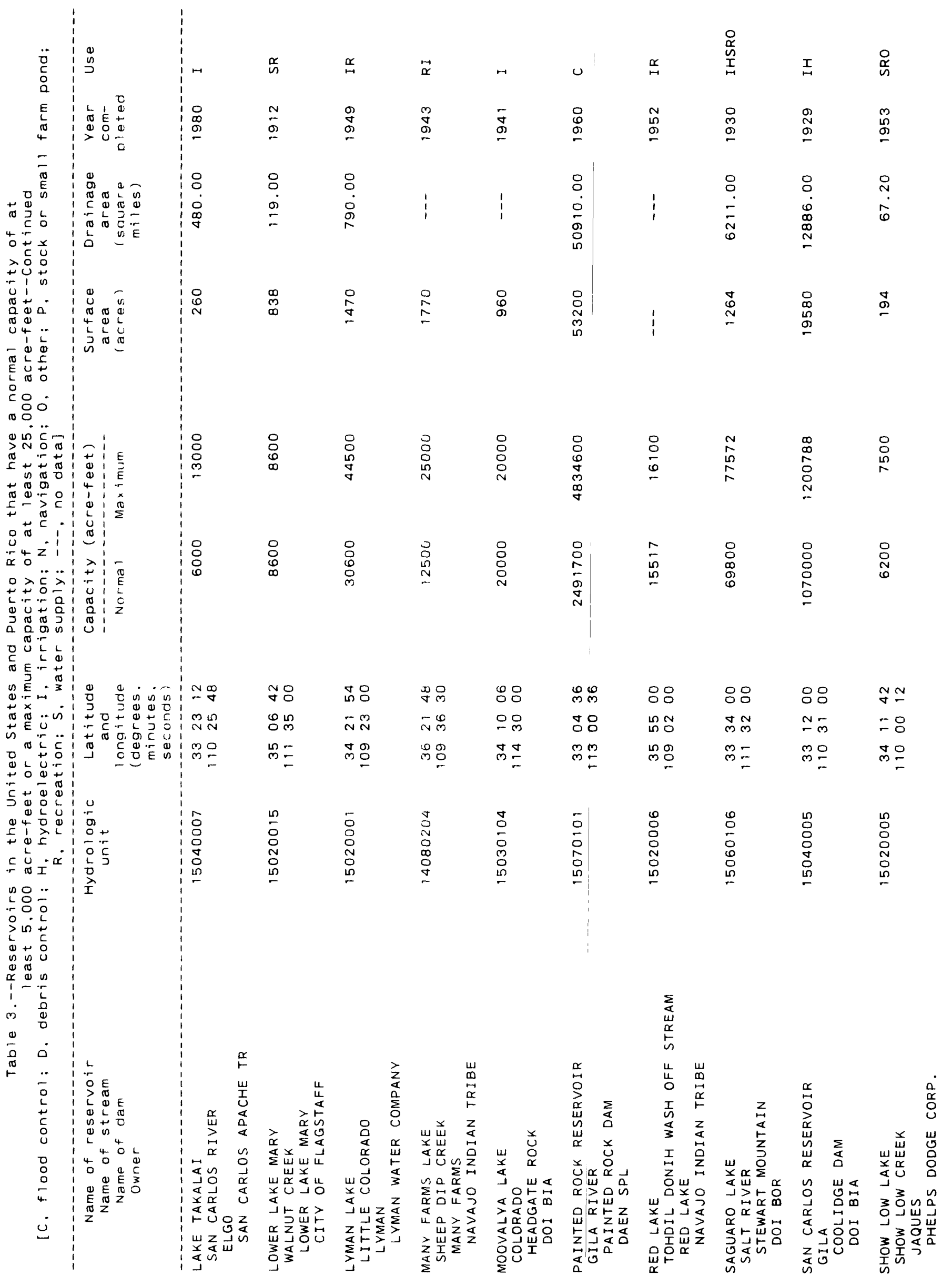




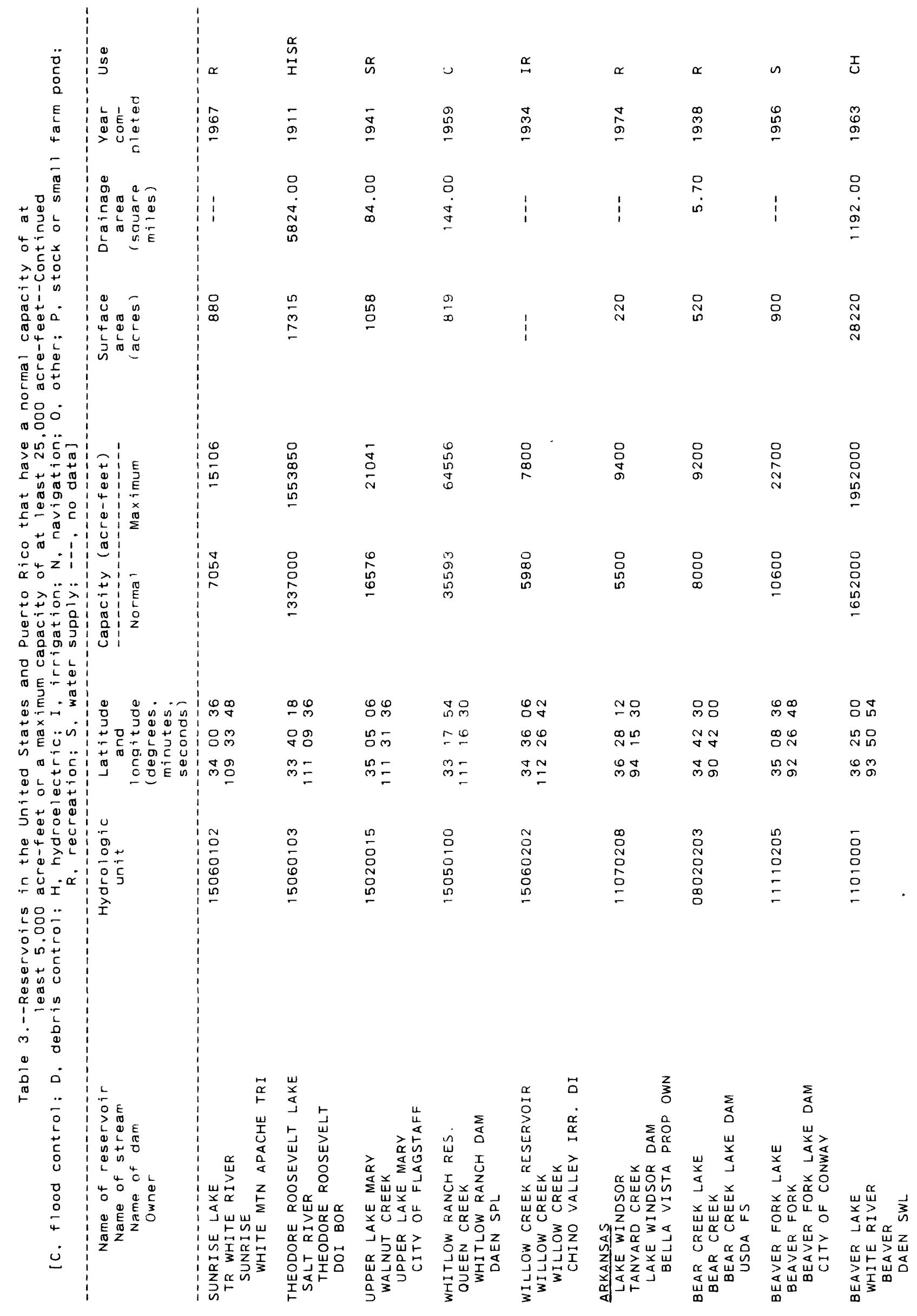




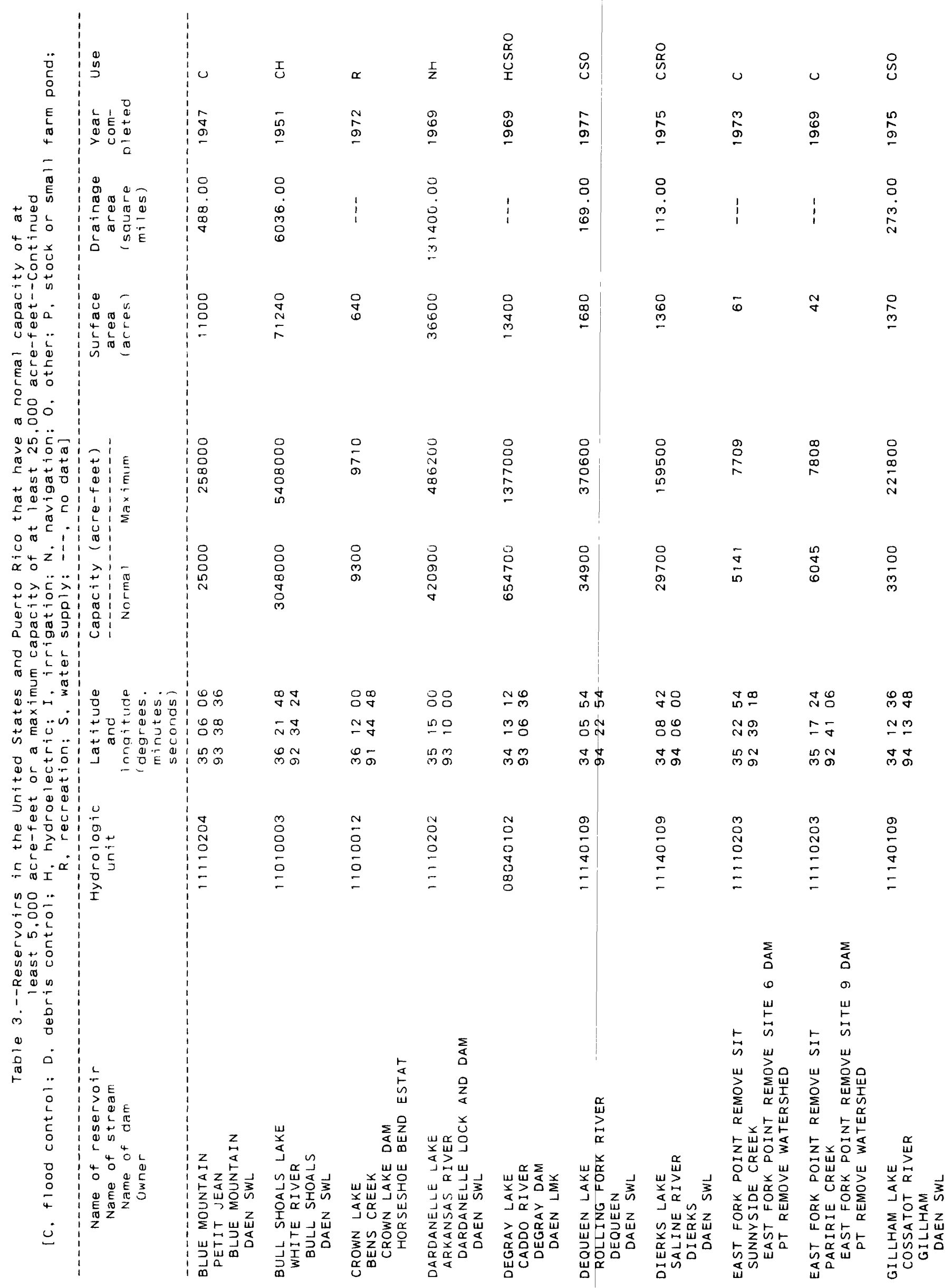




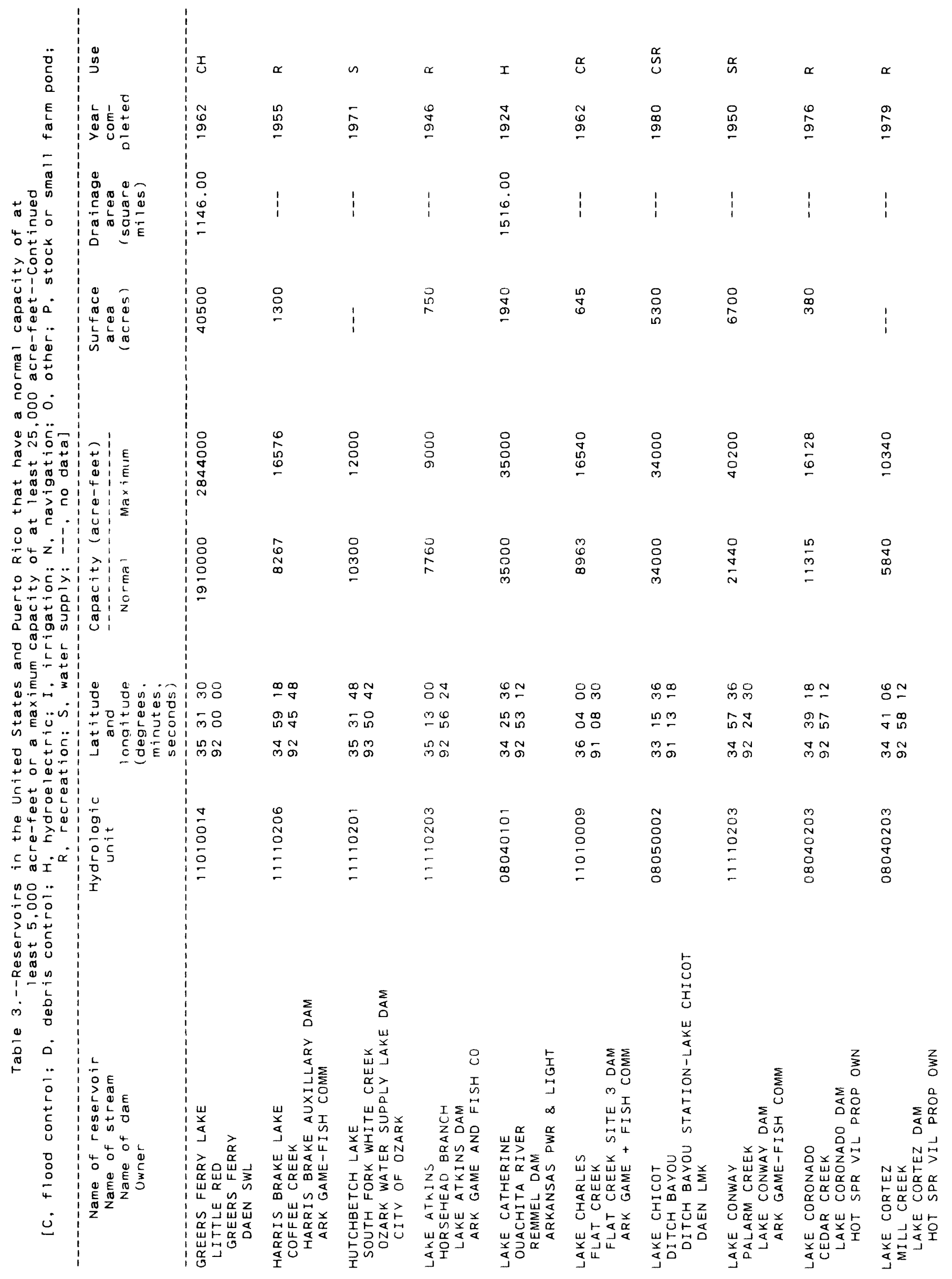




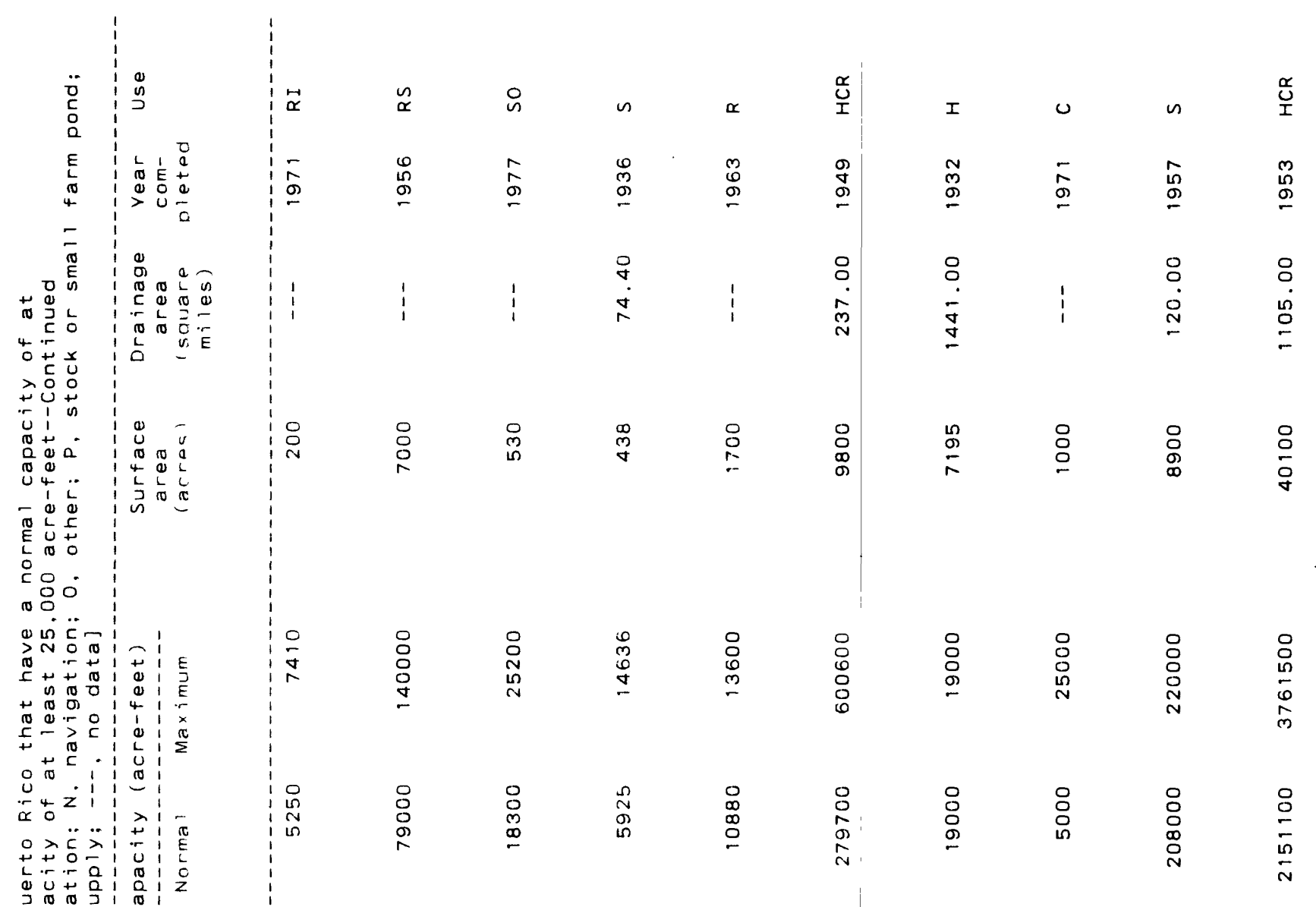

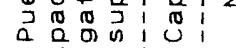

ס记

त E.r

a

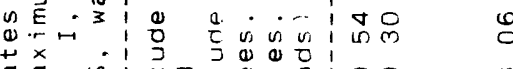

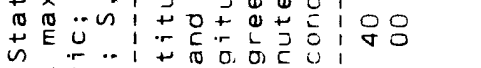

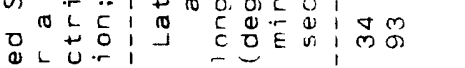

\begin{tabular}{|c|c|c|c|c|c|c|}
\hline 묘 & 造 & 일 & 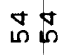 & అ్ల & 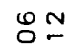 & $\stackrel{\infty}{-\infty}$ \\
\hline$\triangle N$ & $\stackrel{\infty}{m} \stackrel{\infty}{0}^{\infty}$ & Io & $\Phi \underset{\sim}{ }$ & $\stackrel{\oplus}{N O}$ & Б兄 & โ \\
\hline लM & ஜூ & $M \sim$ & लू & गे \% & ஹ゙ず & हె స్ \\
\hline
\end{tabular}

$\because 00$

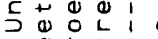

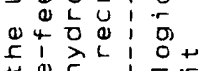

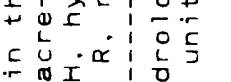

.

no.. I

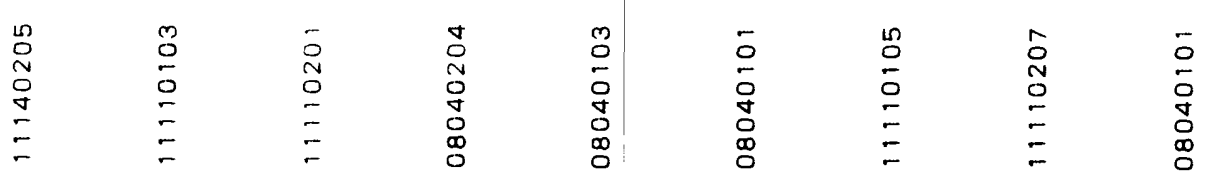

$>$ in

is

焉

\begin{tabular}{c|c|c}
1 & \\
1 & 0
\end{tabular}

은

ल ख

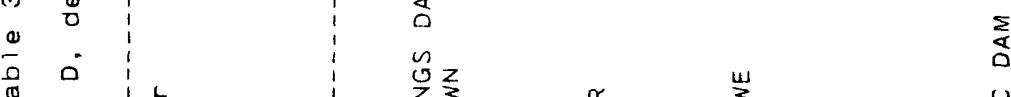

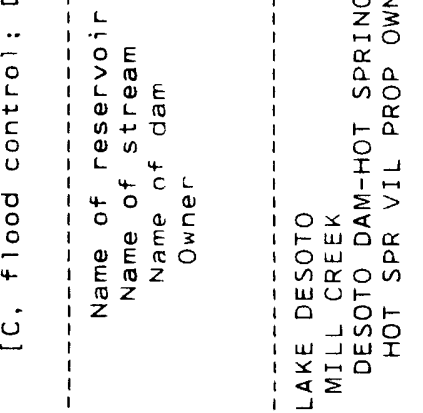

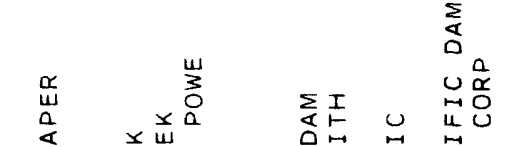

$\sum_{\substack{\alpha \\ \alpha}}^{\alpha}$

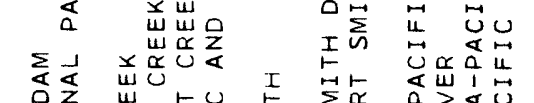

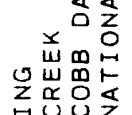

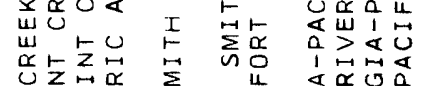

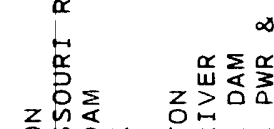

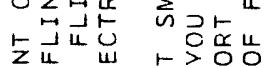

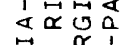

$\vec{\alpha} \supset>$ 昰

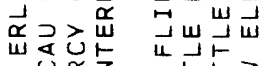

늠뚠

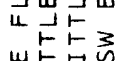

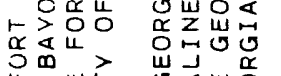

出茫结

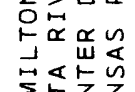

包出

岁吉出品

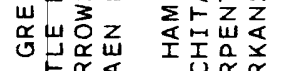

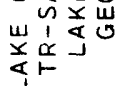

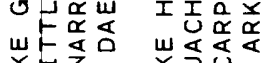

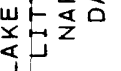

岸过造选 


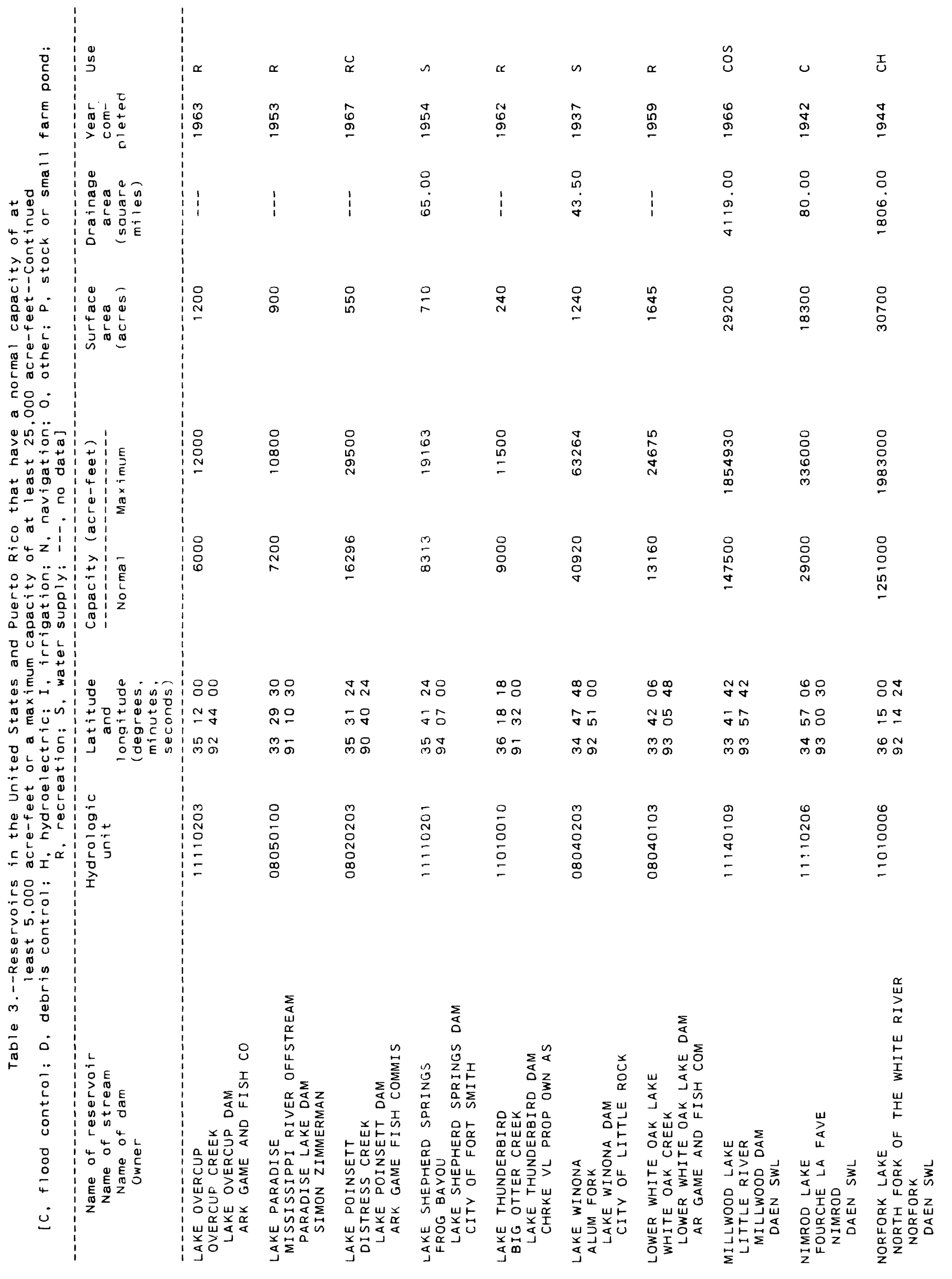




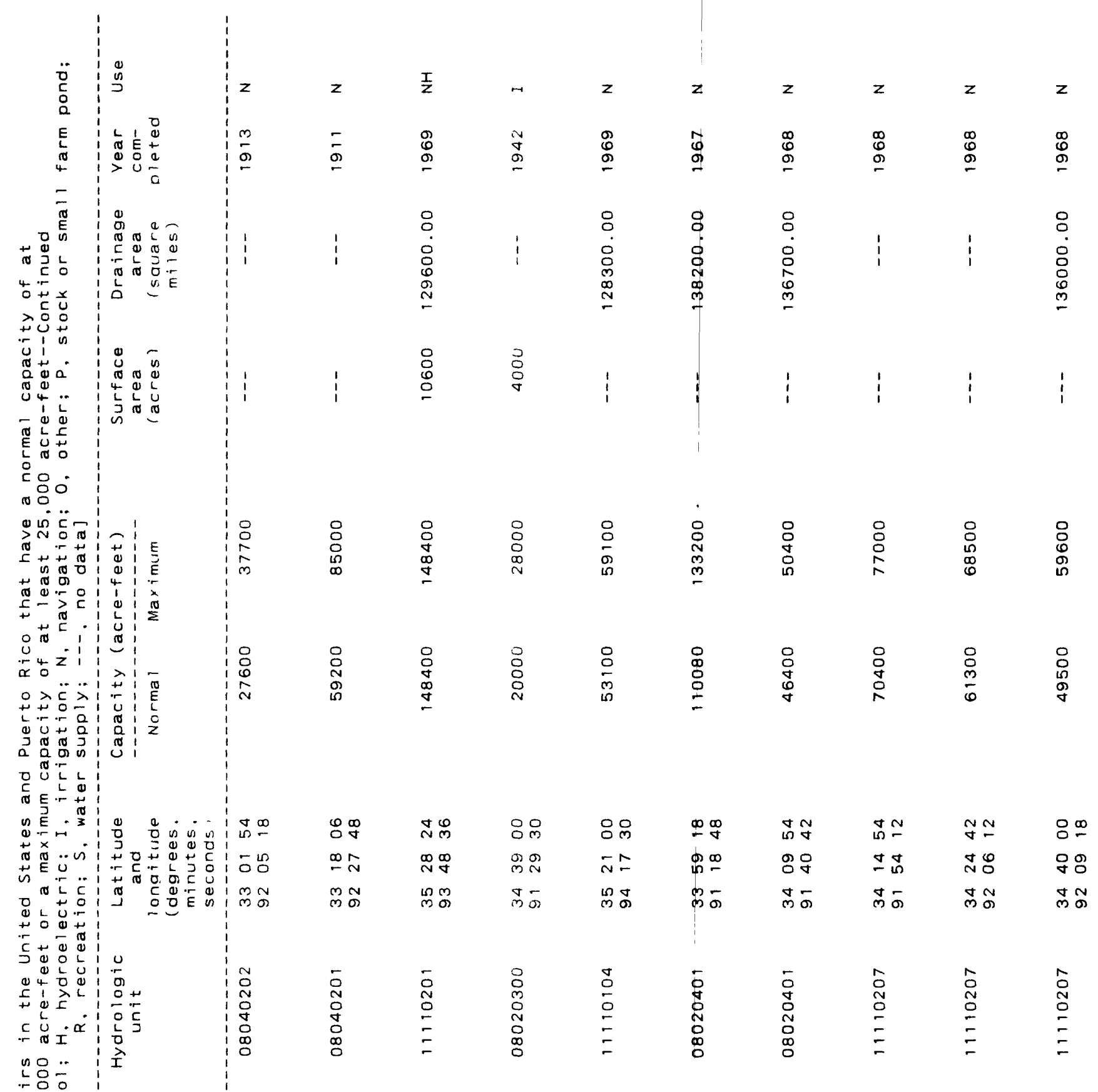




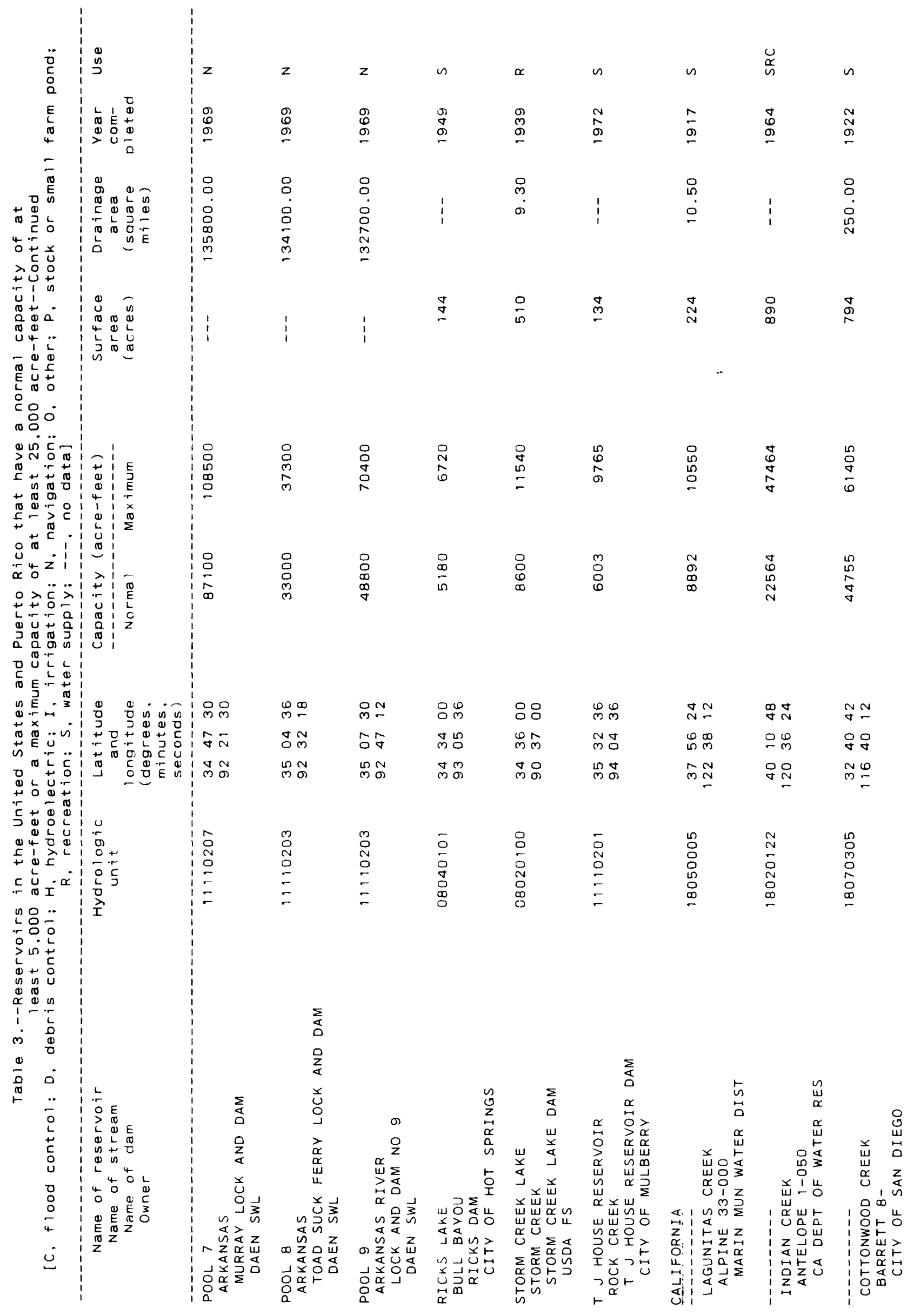




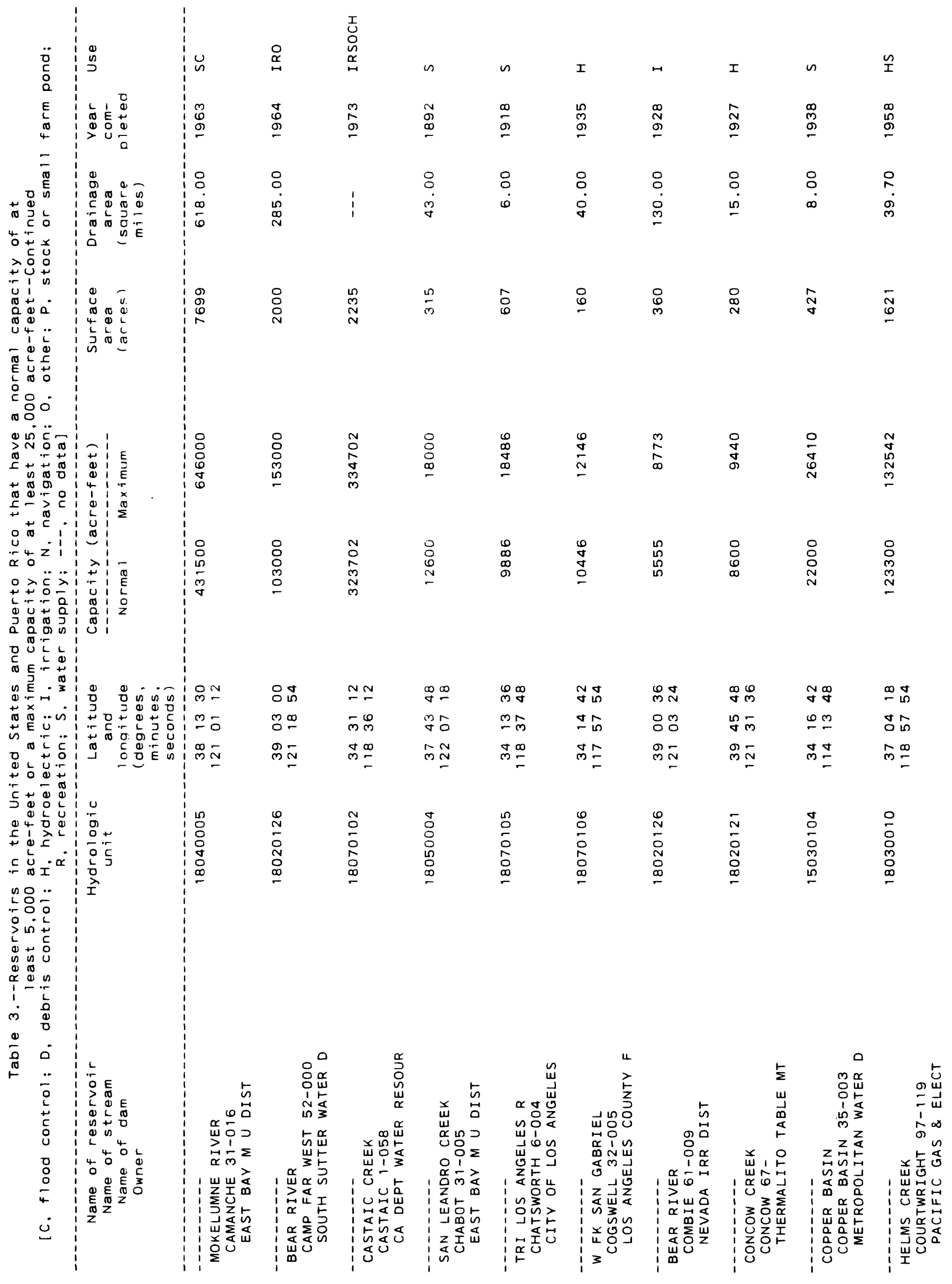




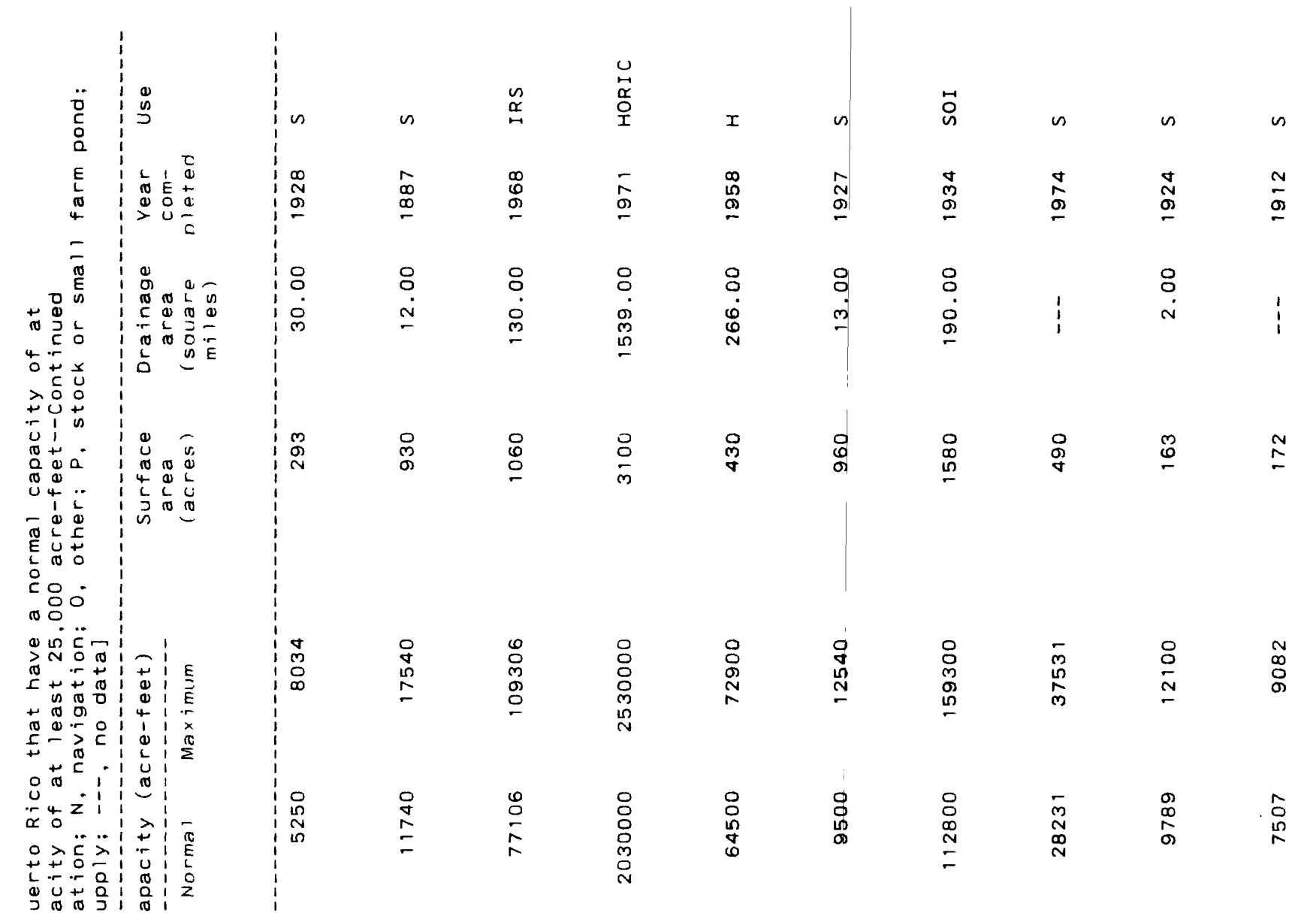

उ

ס放

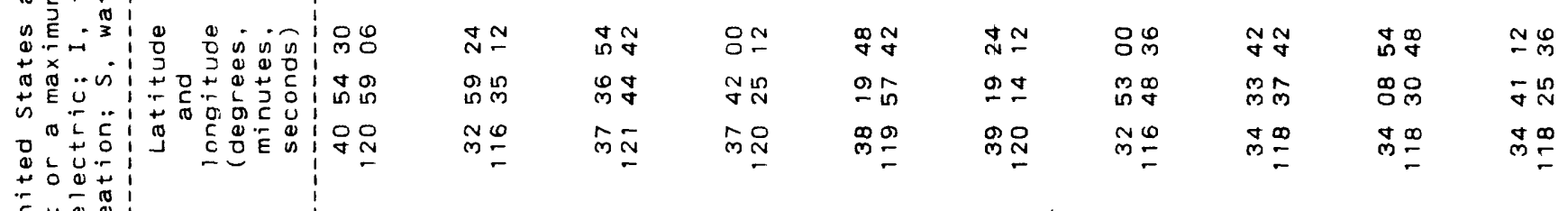
ᄃेta

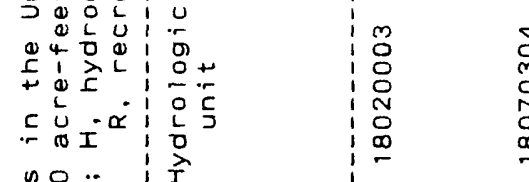

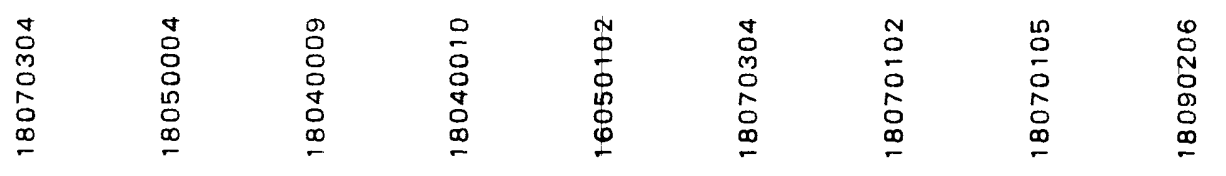

$$
\text { : }
$$
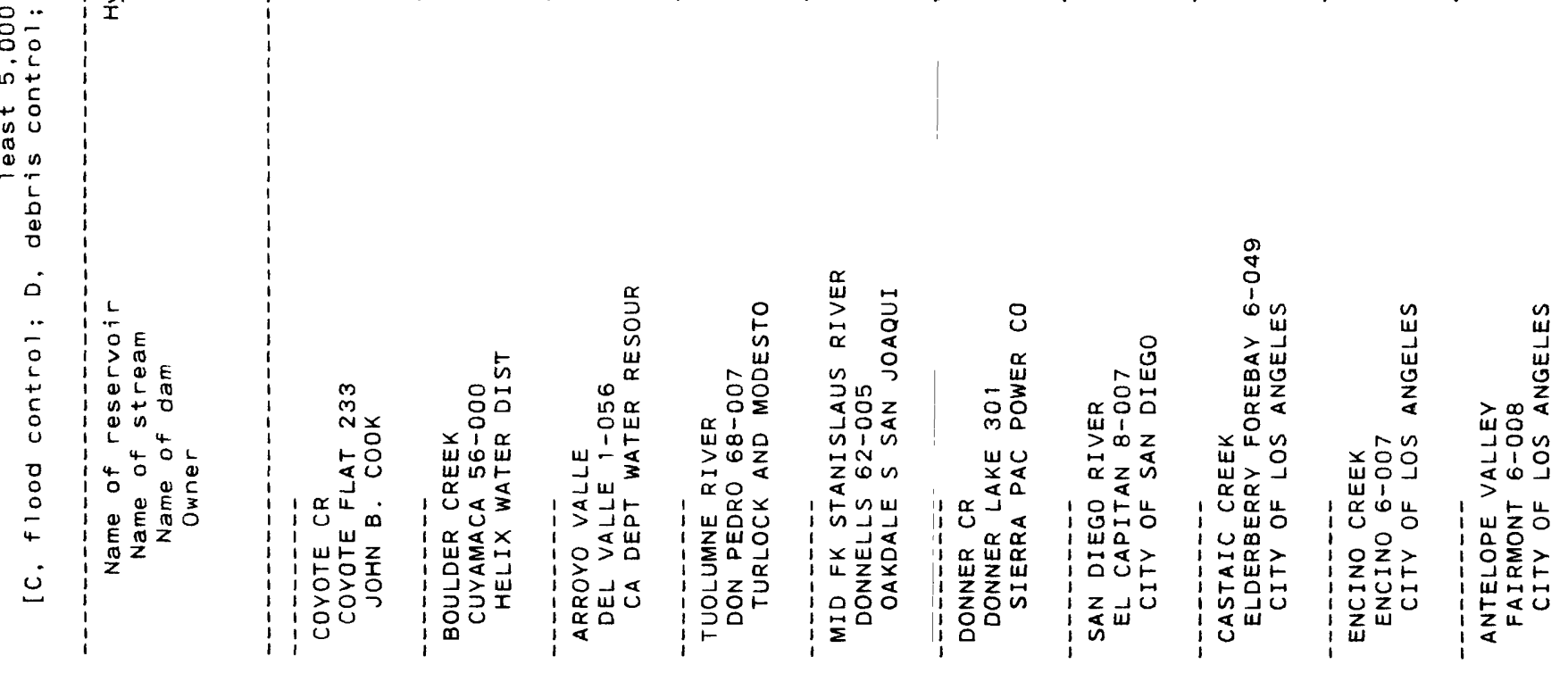


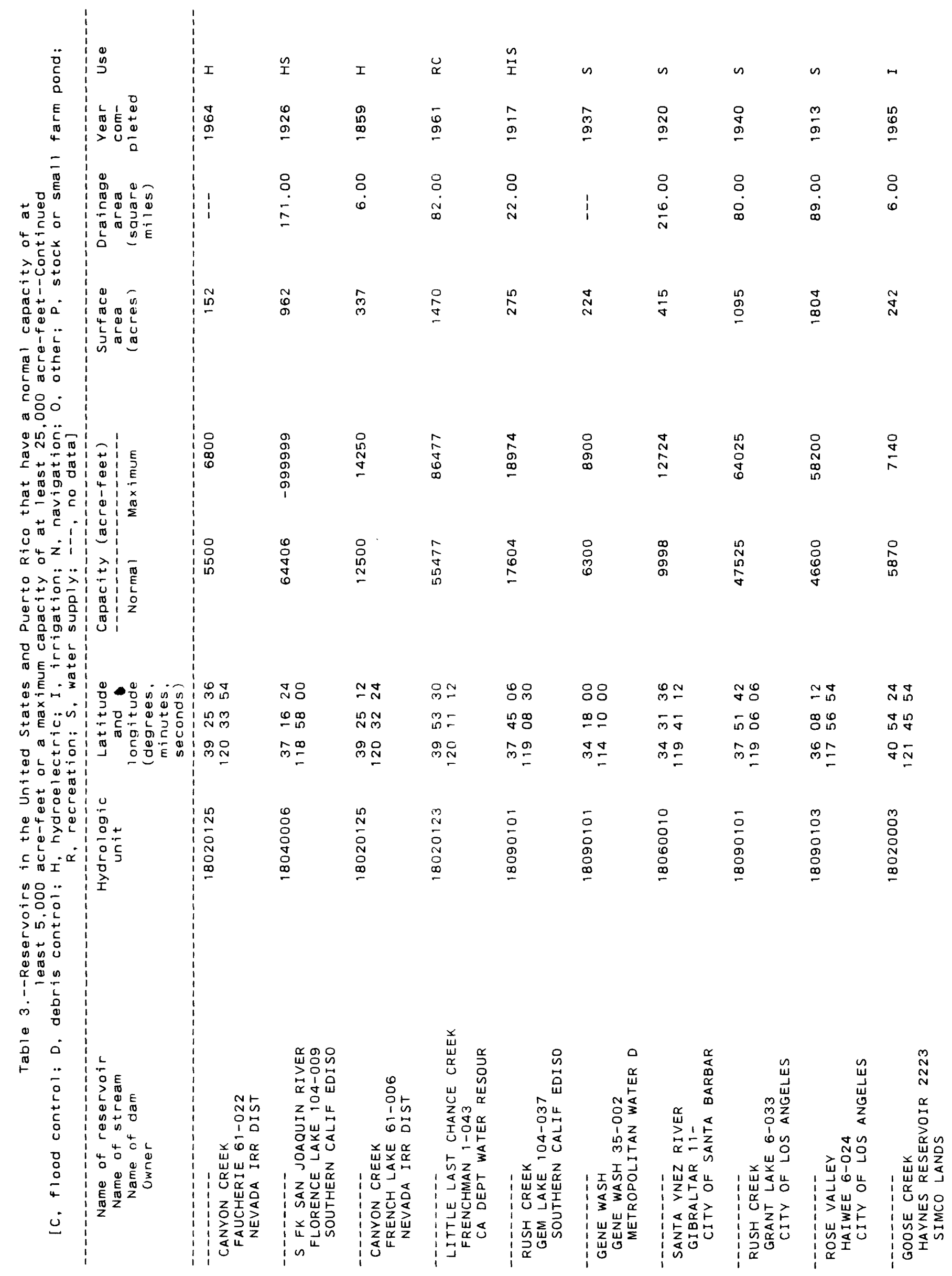




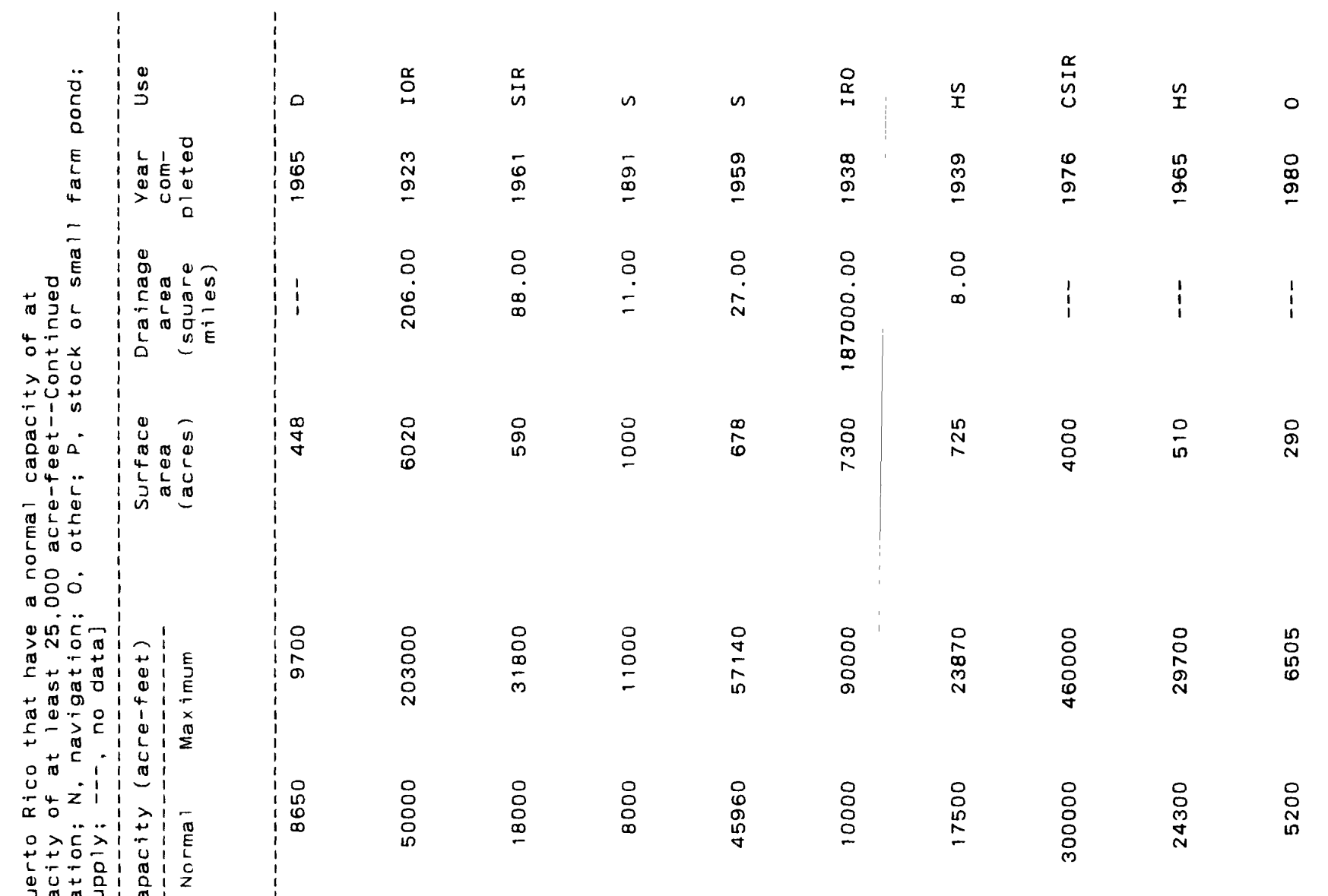

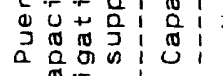

OO

D

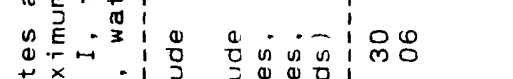

每...

出

0

$+$

$+\frac{1}{2}$

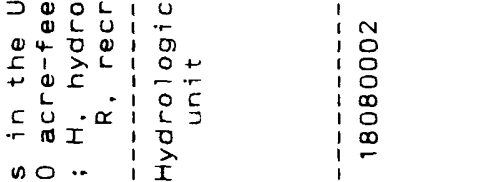

กั

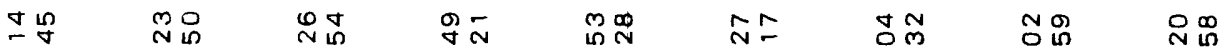

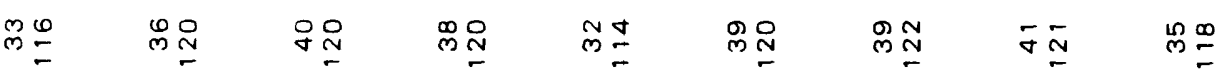

$0-1$

$\cos$

范品

更

-

$\circ$

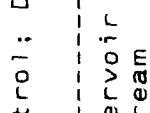

$\begin{array}{llll}0 & 0 \\ 0 & 0 & 0 \\ 0 & 0 & 0 \\ 0 & 0 & 0\end{array}$

: 1400

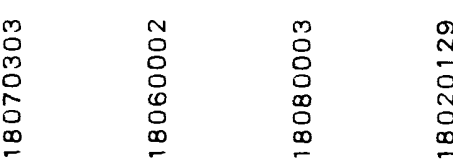

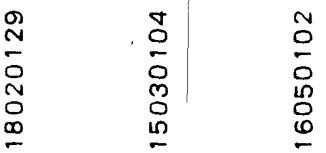

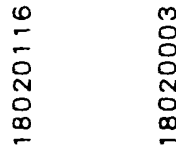

$\begin{array}{ll}\text { ON } & \text { N } \\ 0 & 0 \\ 0 & 0 \\ 0 & 0 \\ 0 & 0 \\ 0 & 0\end{array}$ 


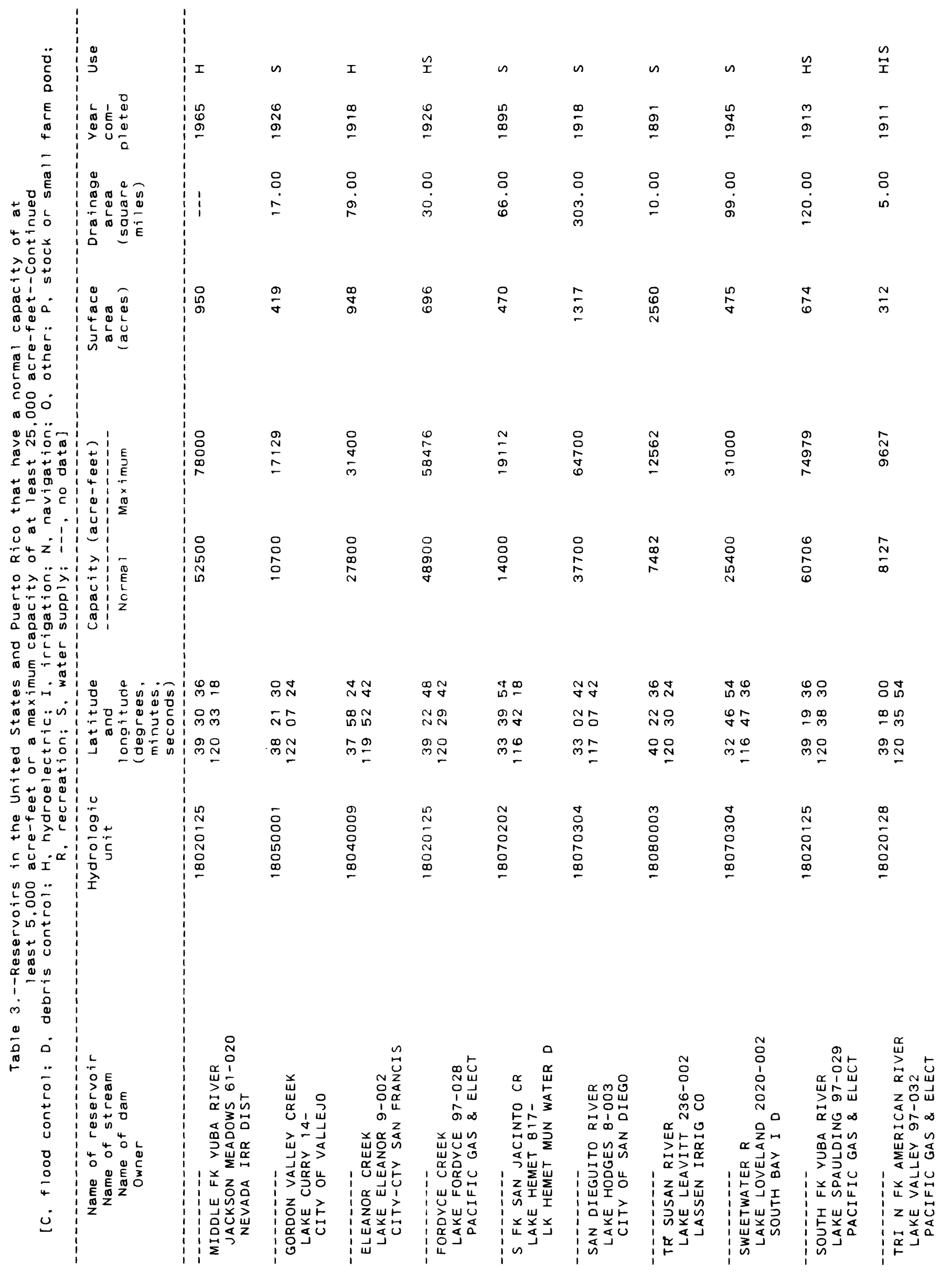




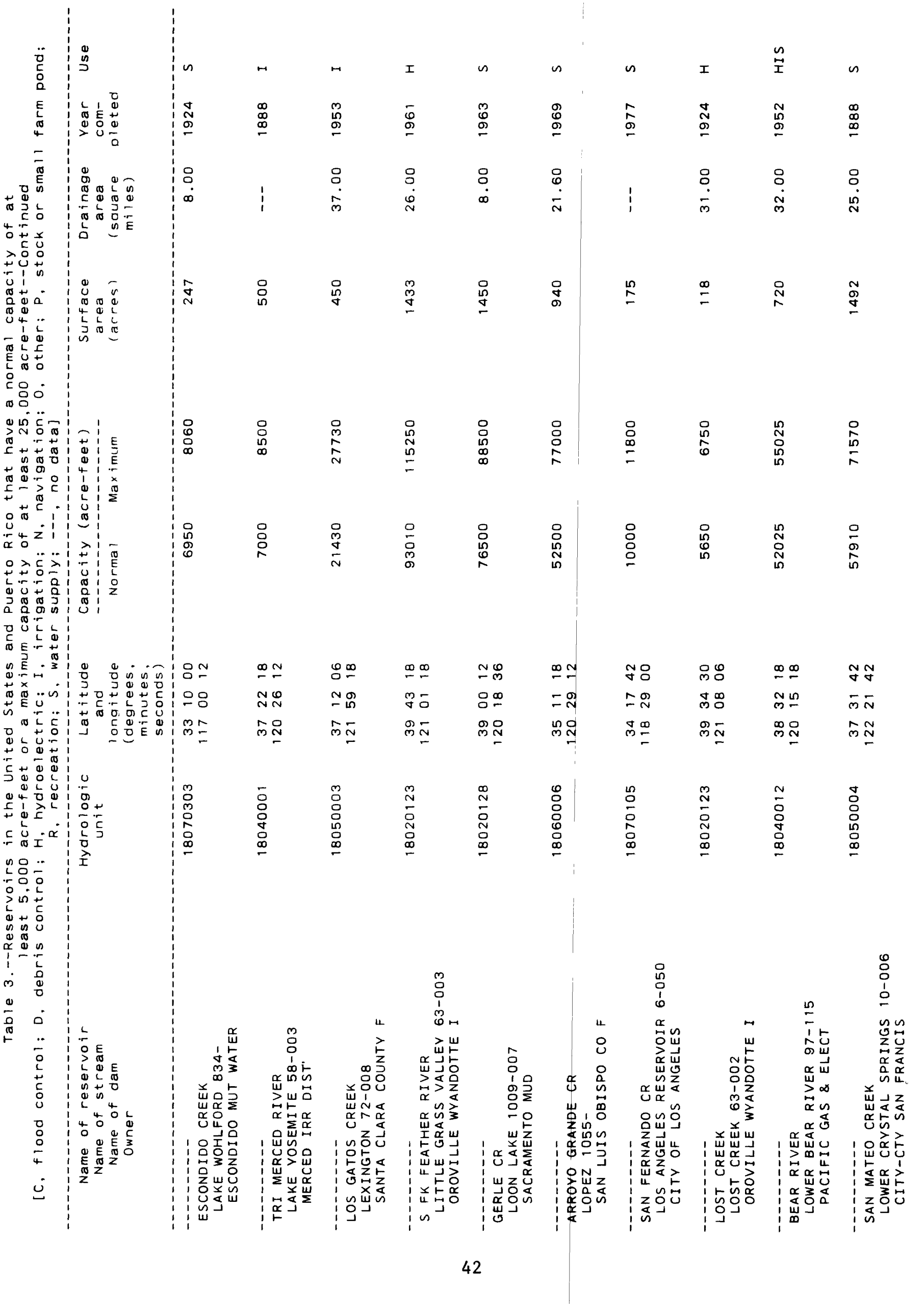




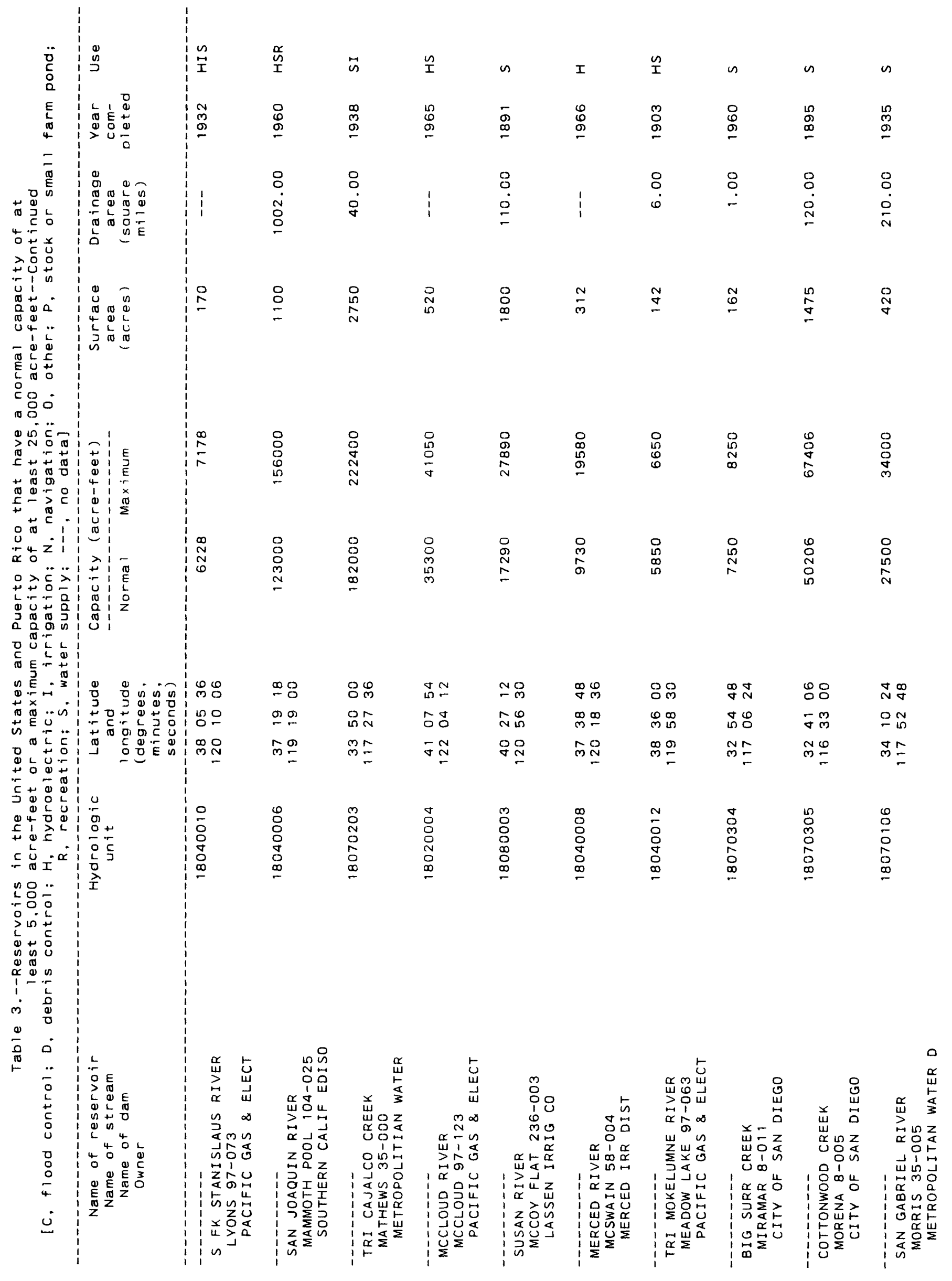




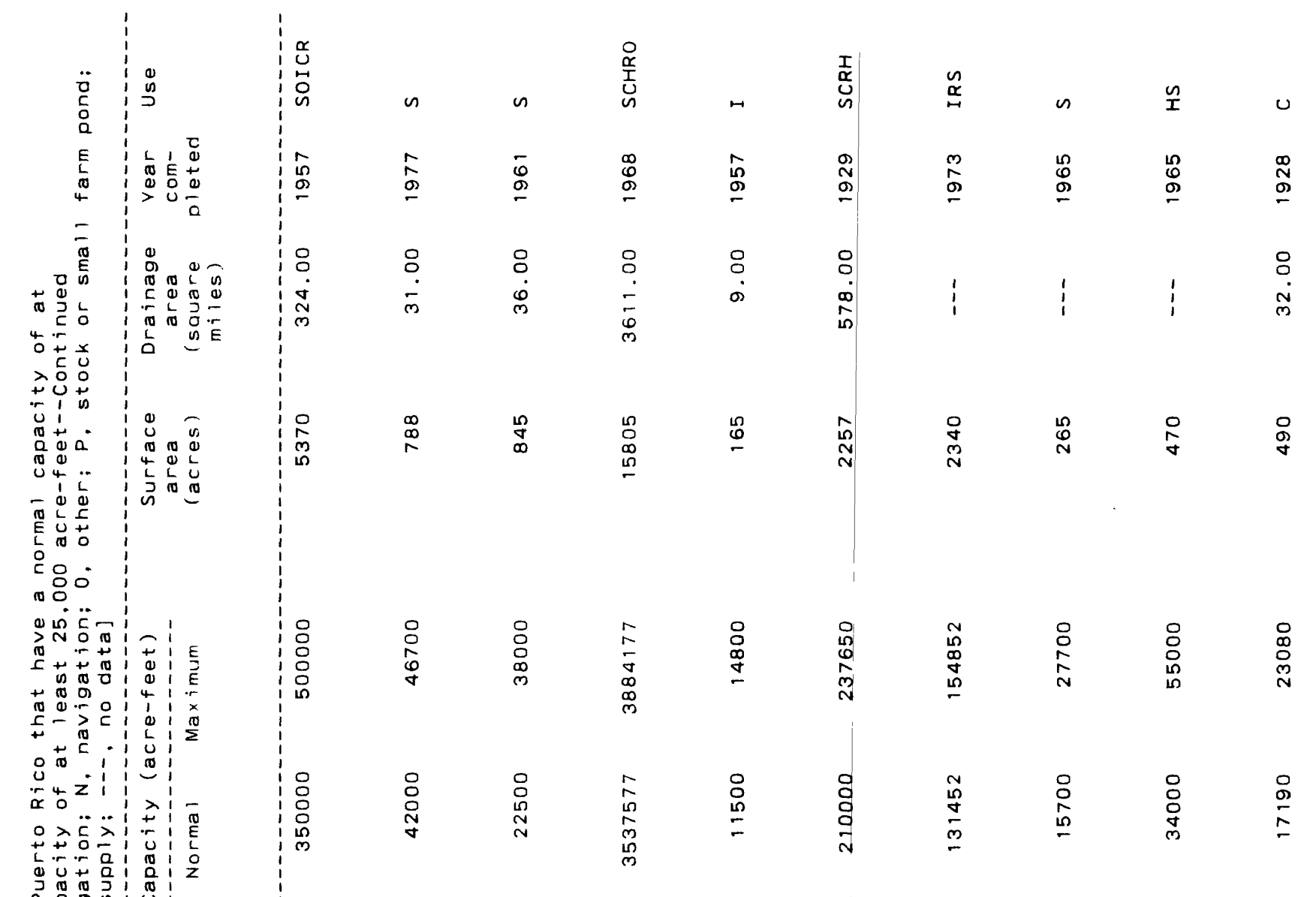

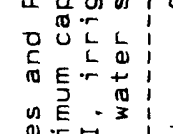

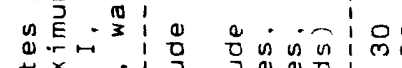

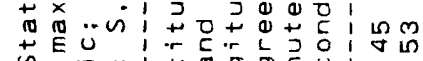

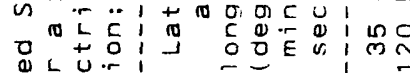

足然

ᄃํ⿺

\begin{tabular}{ll|l}
\hline & 0 \\
0 & 0 & 0 \\
0 & 0 & 0 \\
\hline & 0 & 0 \\
\hline
\end{tabular}

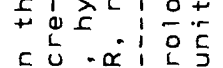

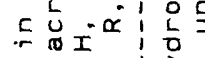

n:플

\begin{tabular}{|c|c|c|c|c|c|c|c|c|}
\hline$\stackrel{\infty}{\sim} \mathcal{\sim}$ & $\ddot{m} \cong$ & 电 & $\stackrel{\circ}{\circ}$ & ภ̇ঃ & 용요 & $\underset{N}{\perp}$ & $\stackrel{\infty}{\sim} \underset{\sim}{\sim}$ & 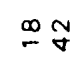 \\
\hline 过 \& & ปั & $\stackrel{N}{\sim} \stackrel{\infty}{\sim}$ & हम & $\stackrel{50}{-5}$ & $\overline{i n}=$ & 용요 & 응요 & $\stackrel{\operatorname{Ln}}{\circ} \stackrel{\infty}{q}$ \\
\hline$\hat{\sim} \stackrel{N}{N}$ & $\mathbb{m}_{\infty}^{\infty} \mathbb{N}$ & ウ̄̄ & $\dot{m} \bar{N}$ & mొD & $m=$ & $q \bar{N}$ & $P_{\bar{N}}$ & હ્ન \\
\hline
\end{tabular}

>

齐古通

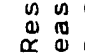

$1-r$

.

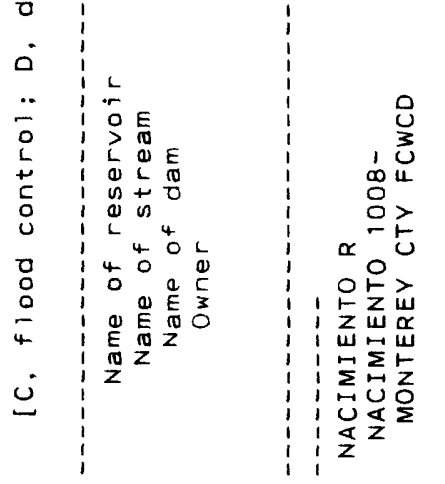

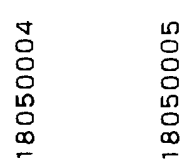

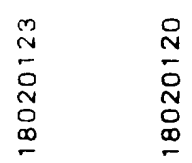

2
0
0
1
0
0
0

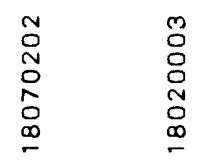

$\begin{array}{ll}3 & 0 \\ 0 & 0 \\ 0 & 0 \\ 0 & 0 \\ 0 & 0 \\ 0 & 0 \\ 0 & 0\end{array}$

$$
\begin{gathered}
- \\
0 \\
1 \\
\frac{1}{m}
\end{gathered}
$$

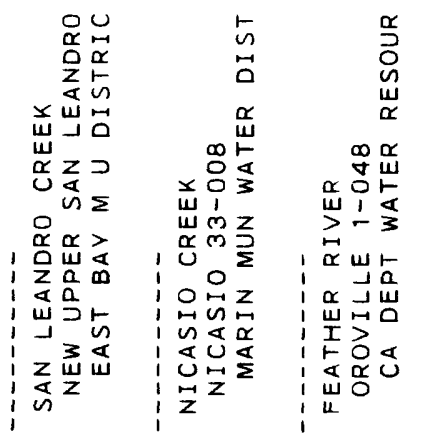

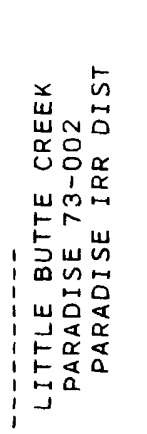

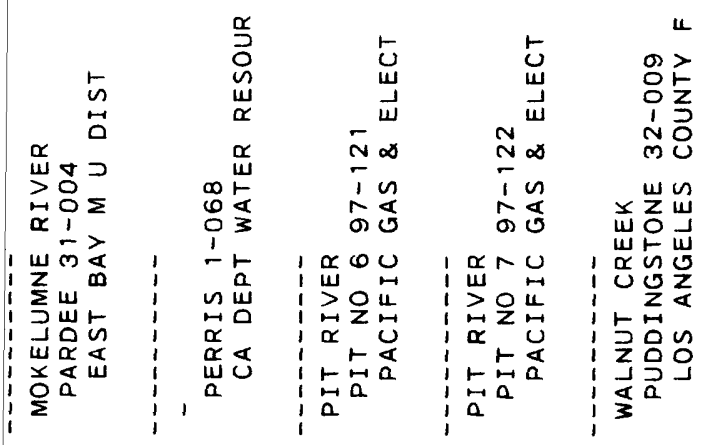




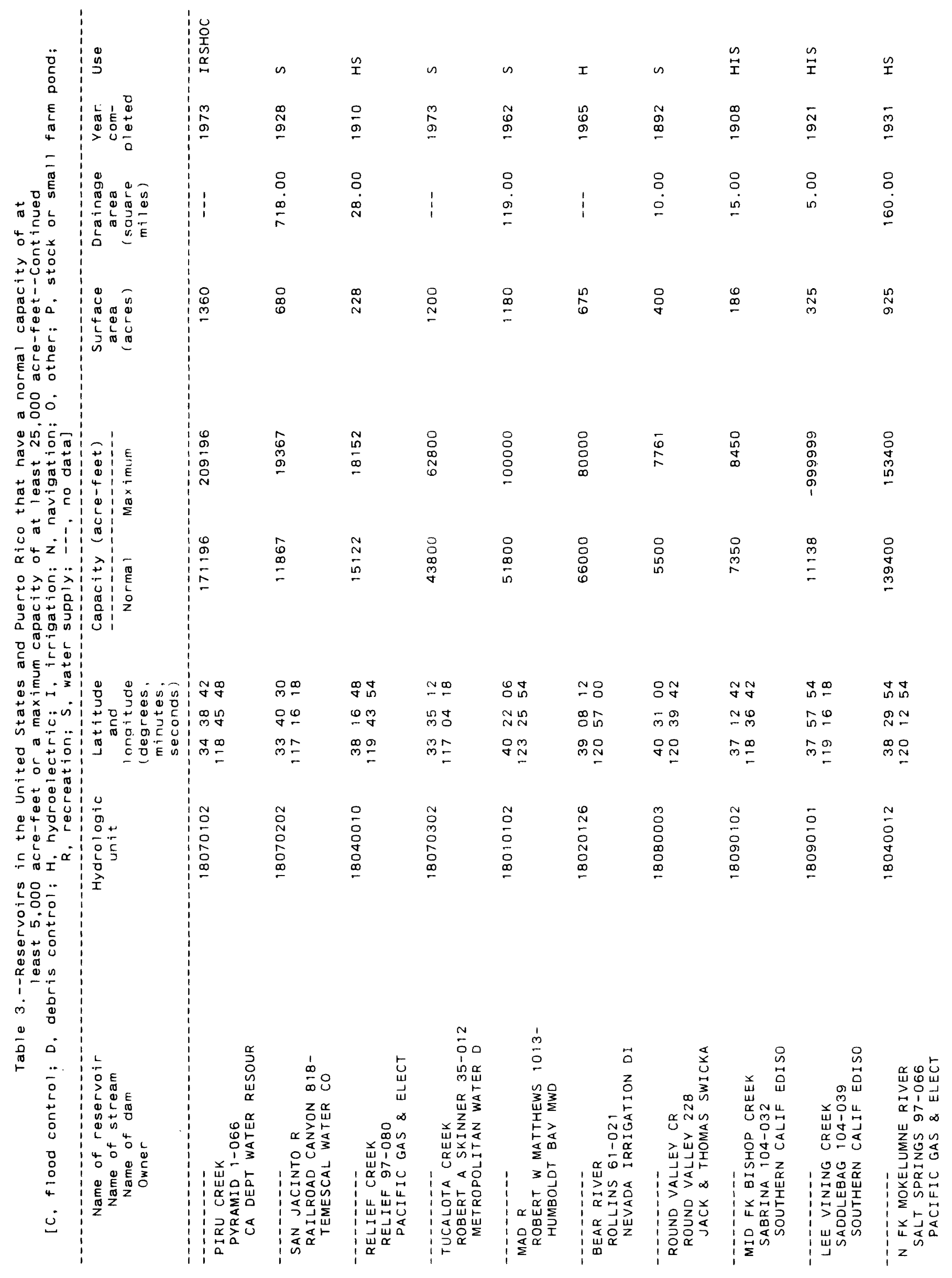




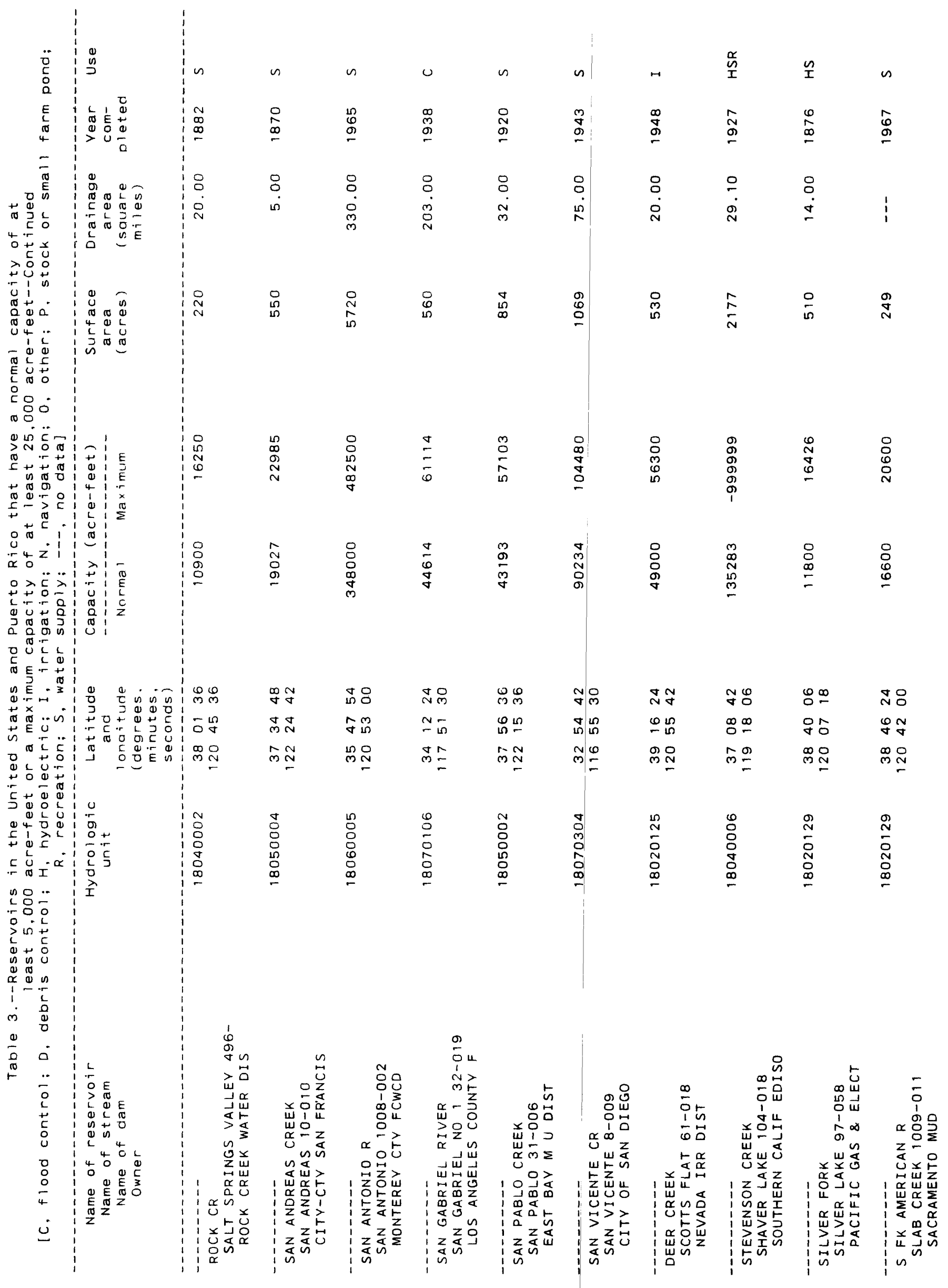




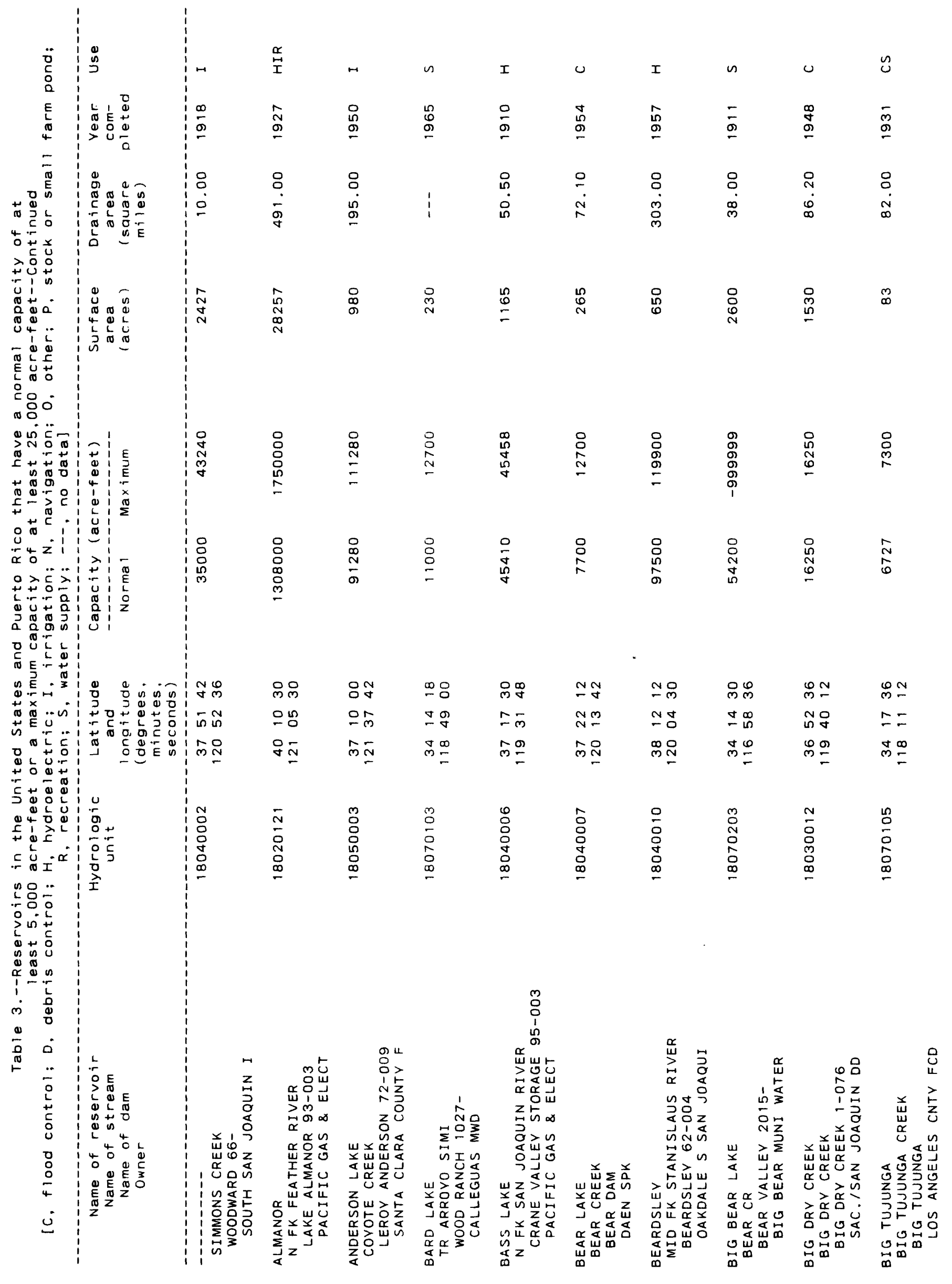




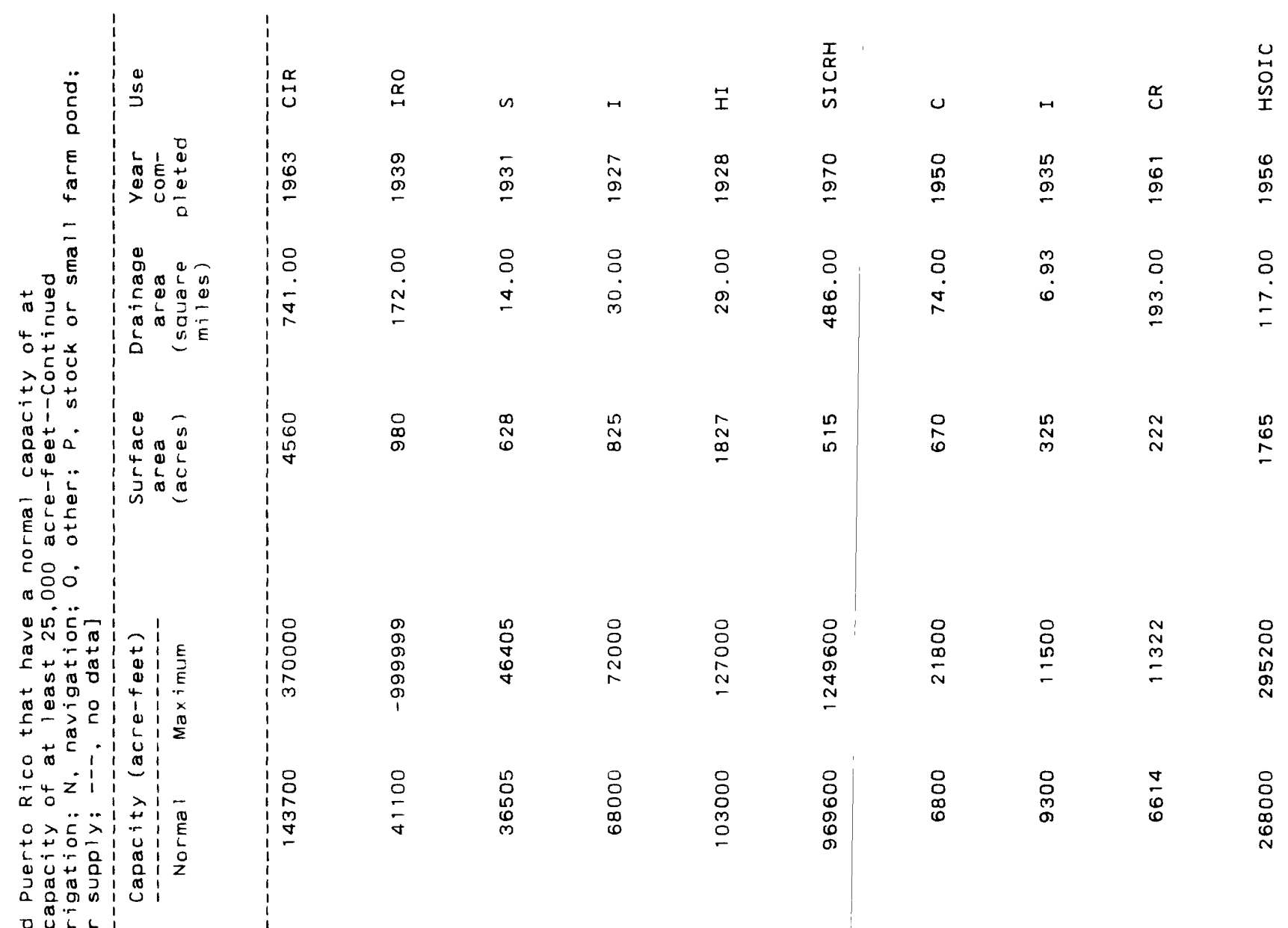

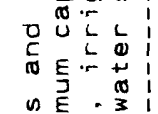

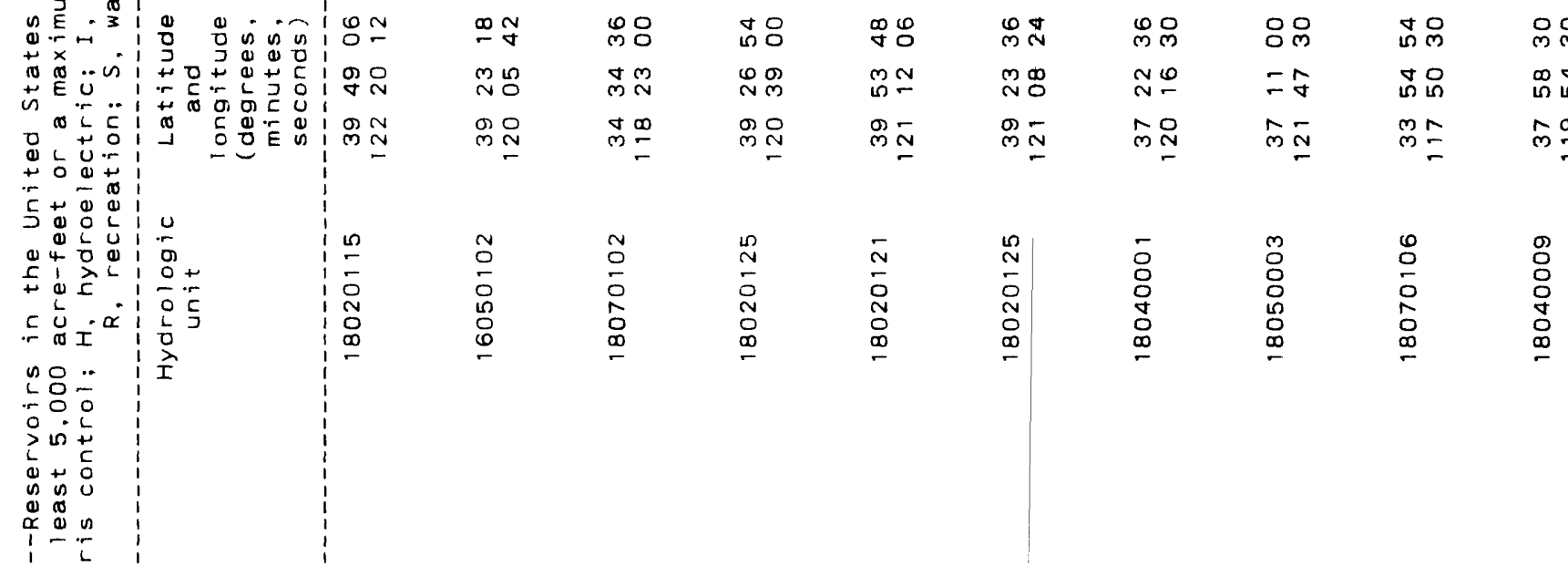

लं

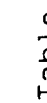




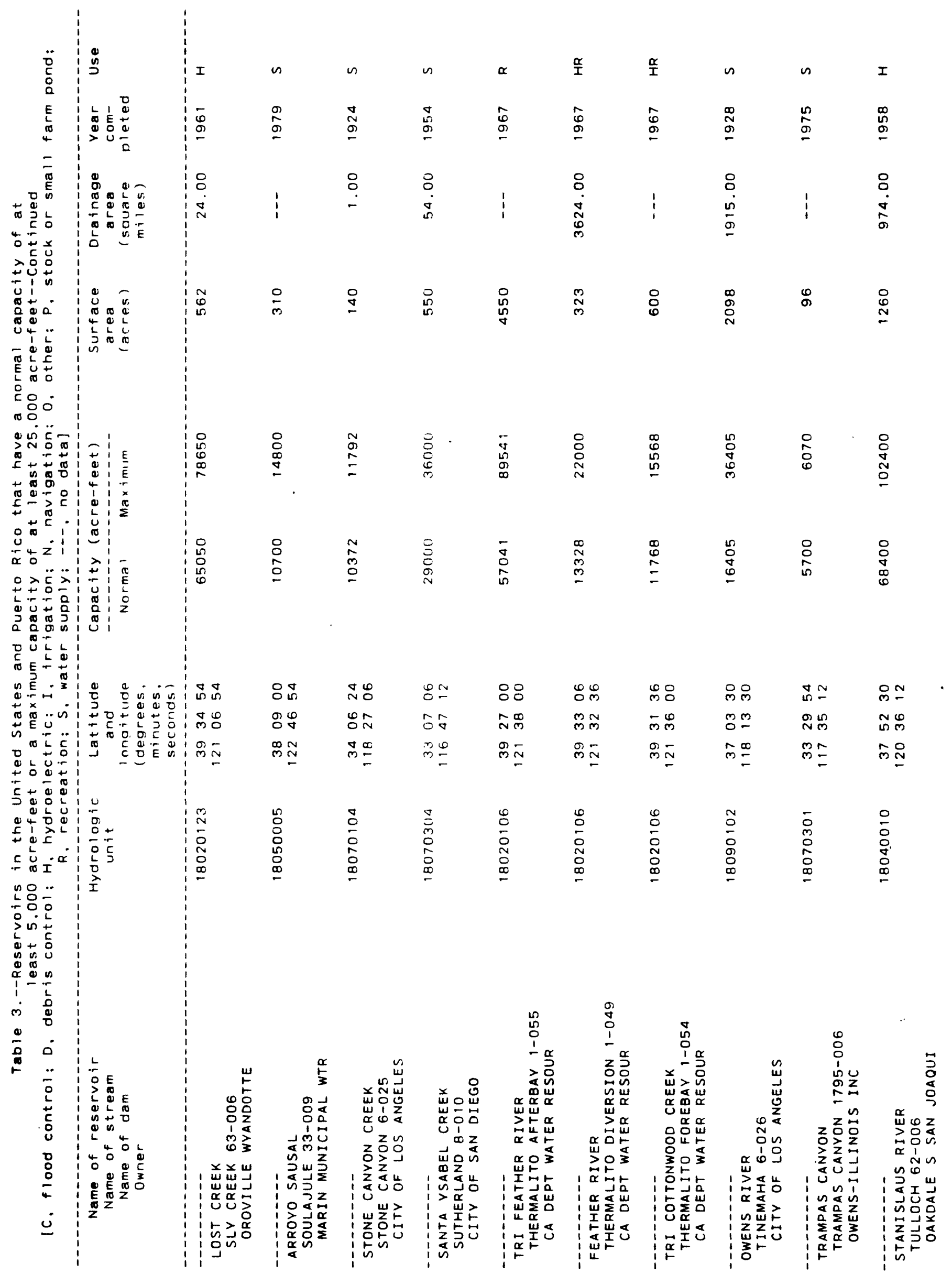




\begin{tabular}{|c|c|c|c|c|c|c|c|}
\hline $\begin{array}{l}\overrightarrow{0} \\
\tilde{\alpha} \\
\tilde{n}\end{array}$ & $\underline{\underline{I}}$ & $n$ & $n$ & u & $\rightarrow$ & $n$ & un \\
\hline $\begin{array}{l}3 \\
\text { o } \\
\text { I. }\end{array}$ & ¿ & $\begin{array}{l}\hat{\mathscr{L}} \\
\text { O }\end{array}$ & $\begin{array}{l}\mathscr{g} \\
\stackrel{2}{\alpha}\end{array}$ & $\begin{array}{l}0 \\
0 \\
\text { 9 }\end{array}$ & $\begin{array}{l}\mathscr{0} \\
\stackrel{0}{0} \\
\stackrel{2}{2}\end{array}$ & $\frac{N}{\hat{a}}$ & $\begin{array}{l}\stackrel{\circ}{\circ} \\
\stackrel{2}{2}\end{array}$ \\
\hline 응 & : & : & 8 & : & : & & 8 \\
\hline வ & $\dot{m}$ & $\dot{\bar{N}}$ & $\frac{\dot{m}}{m}$ & $\dot{\infty}$ & $\stackrel{\dot{\sim}}{\Xi}$ & $\mathrm{i}$ & $\dot{N}$ \\
\hline 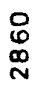 & ఫ్ & $\underset{\sim}{\stackrel{0}{\infty}}$ & $\begin{array}{l}\infty \\
\stackrel{0}{0}\end{array}$ & $\underset{\mathbb{\infty}}{\stackrel{0}{*}}$ & 只 & 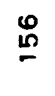 & 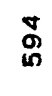 \\
\hline 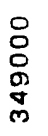 & $\begin{array}{l}\stackrel{n}{8} \\
\stackrel{8}{8}\end{array}$ & $\begin{array}{l}\text { 응 } \\
\text { 足 } \\
\text { m }\end{array}$ & $\begin{array}{l}\text { O } \\
\stackrel{0}{X} \\
0\end{array}$ & $\begin{array}{l}\text { O } \\
\text { o } \\
\stackrel{\text { N }}{\text { N }}\end{array}$ & $\begin{array}{l}\text { O } \\
\text { D } \\
\text { D } \\
\text { m }\end{array}$ & $\begin{array}{l}\stackrel{n}{N} \\
\stackrel{3}{0} \\
0\end{array}$ & $\frac{\hat{\sigma}}{\dot{\sigma}}$ \\
\hline 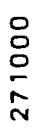 & 总 & $\begin{array}{l}\circ \\
\circ \\
\circ \\
\circ\end{array}$ & $\begin{array}{l}8 \\
8 \\
\frac{0}{10}\end{array}$ & $\begin{array}{l}\circ \\
8 \\
8 \\
0 \\
-2\end{array}$ & $\begin{array}{l}\text { 을 } \\
\text { 品 }\end{array}$ & $\begin{array}{l}\text { ¿े } \\
\text { Nু }\end{array}$ & $\begin{array}{l}\text { \& } \\
\stackrel{5}{0} \\
\delta\end{array}$ \\
\hline
\end{tabular}

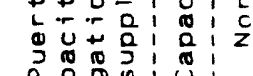

范。

कि $E \div$

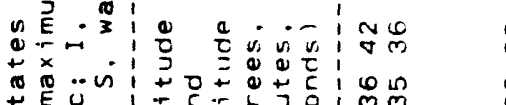

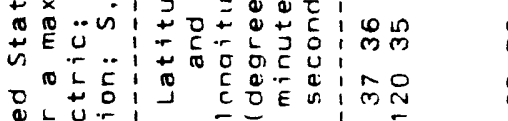

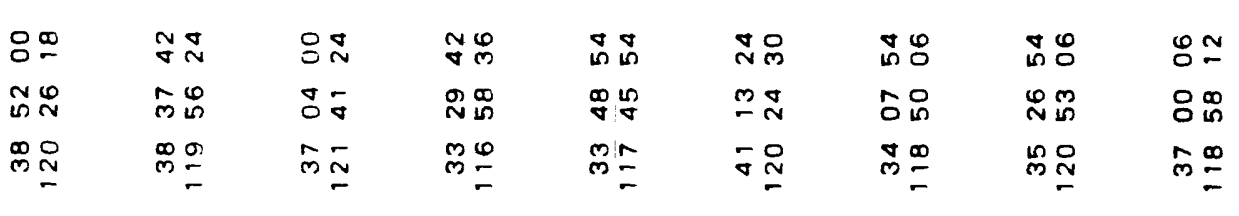

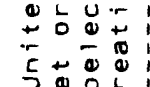

¿

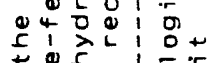

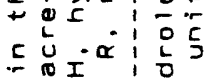

보요

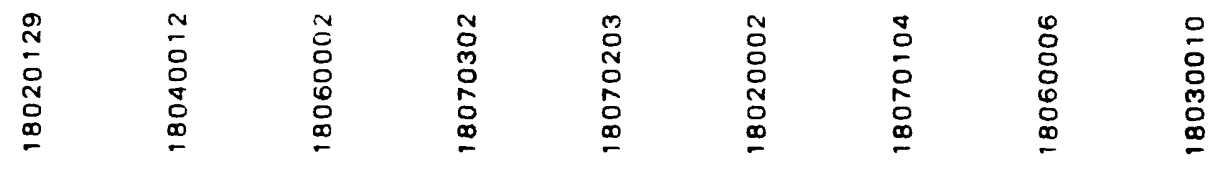

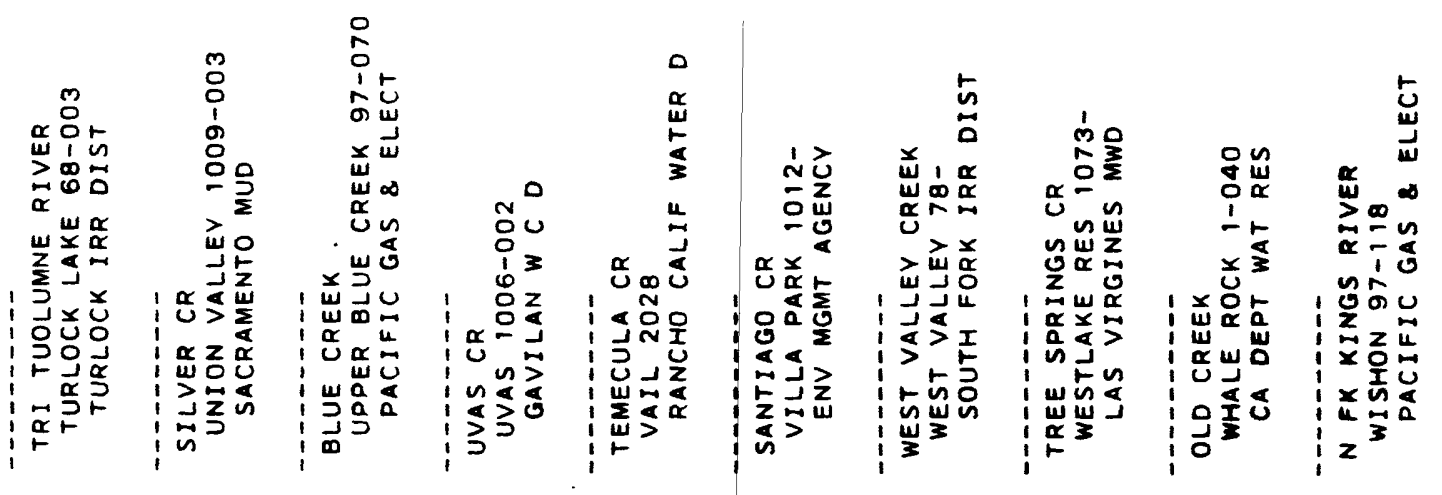




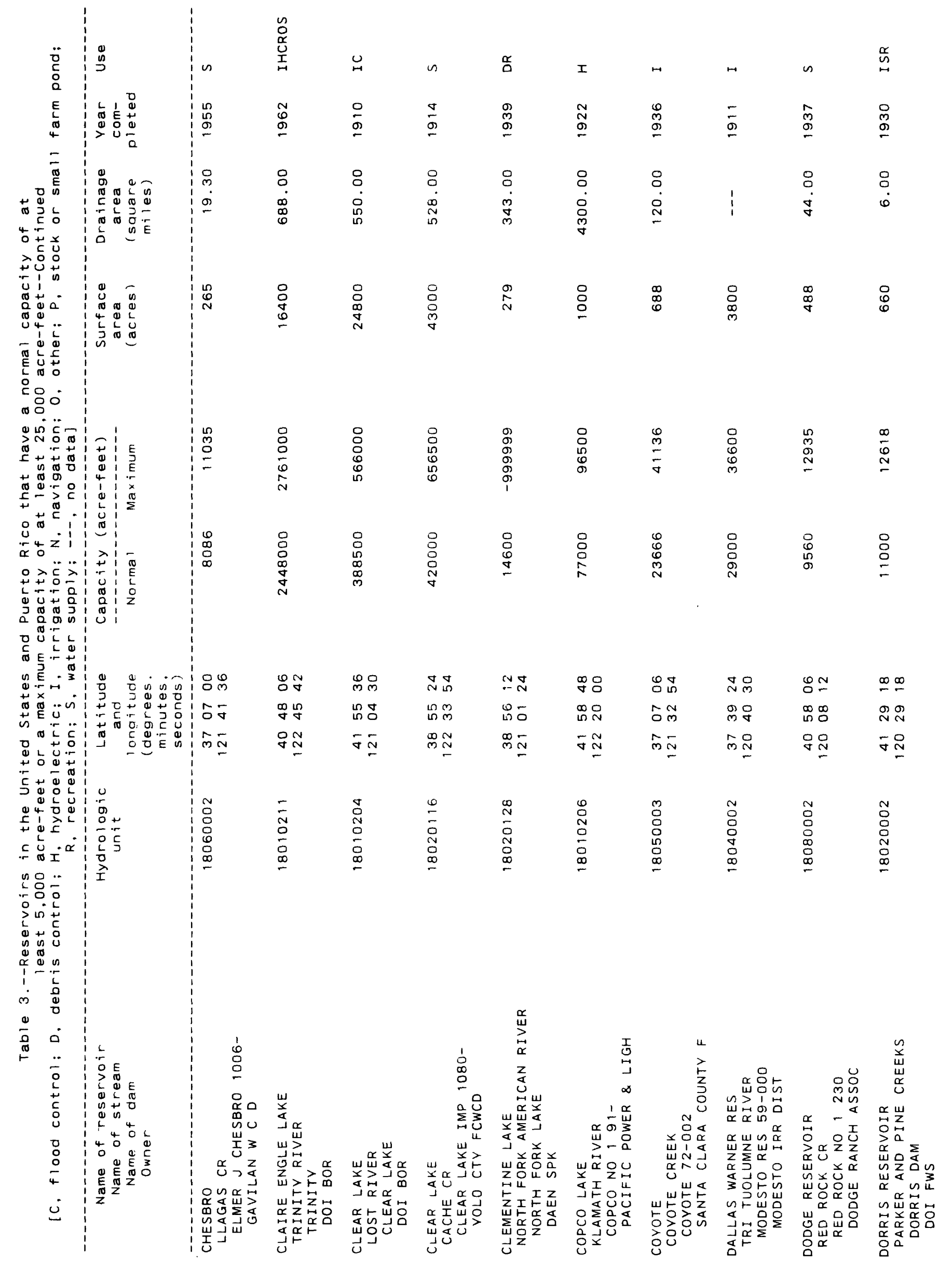




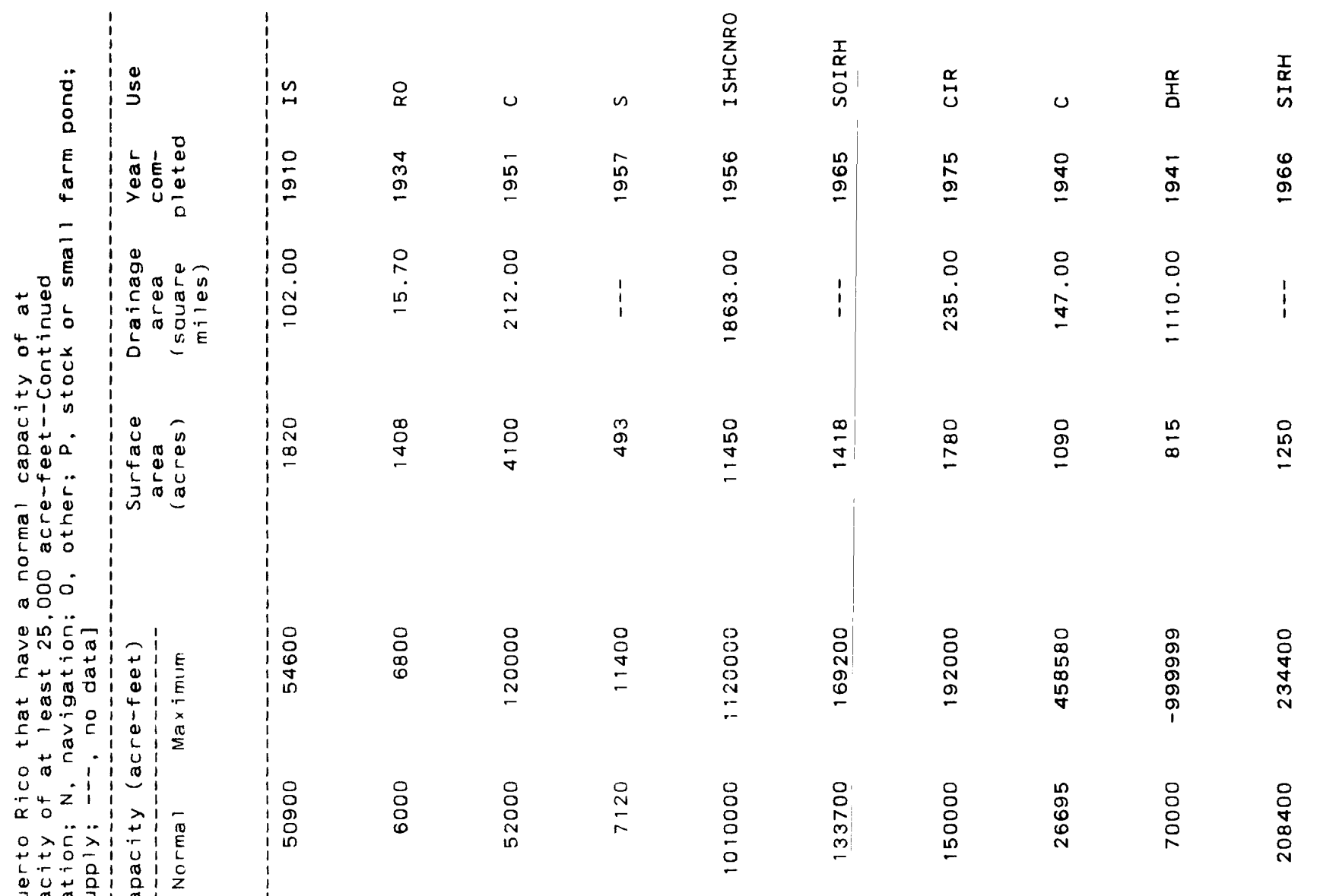

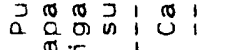

인ㄷㄴ

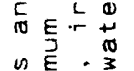

$\underset{\Theta}{\infty} \dot{H}^{3}$

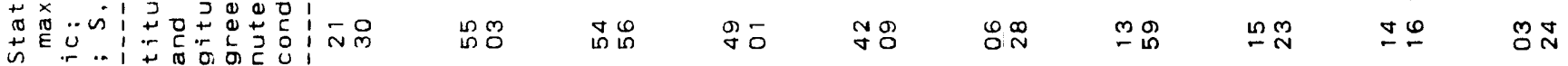

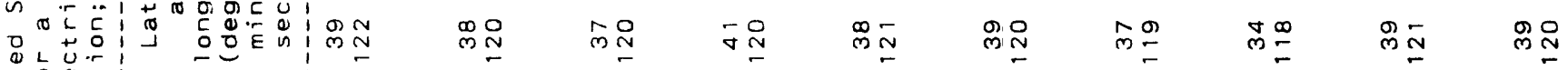

$\pm+\frac{0}{\infty}+\frac{\pi}{0}$

政

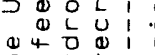

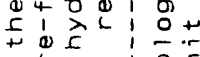

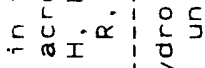

됴요

$>$ in

足

की

a

i)

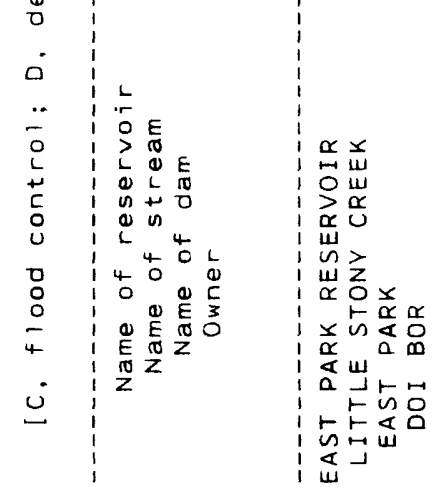

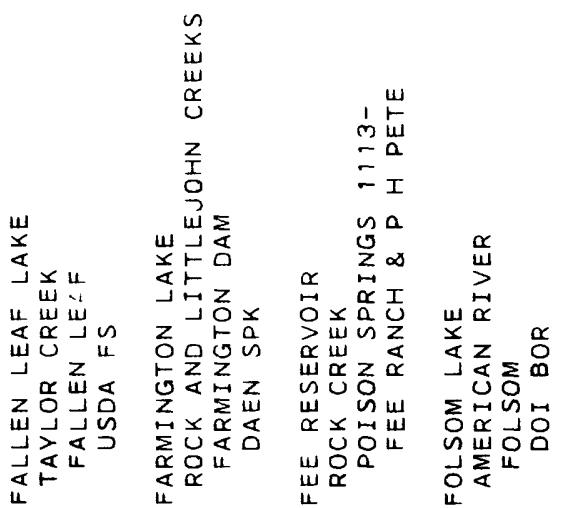

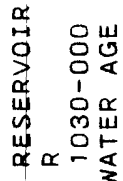

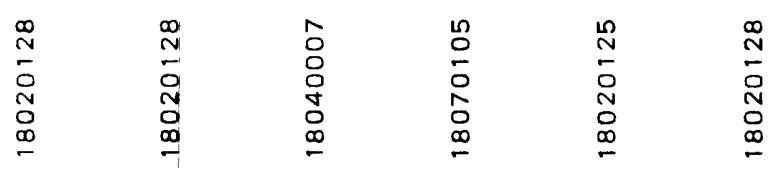

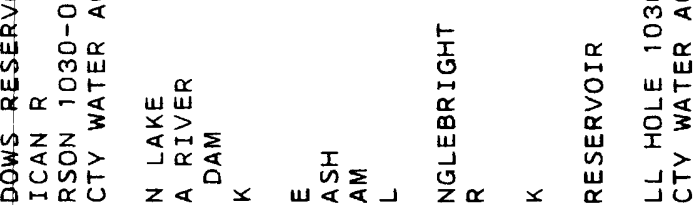

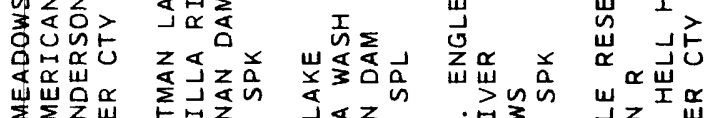

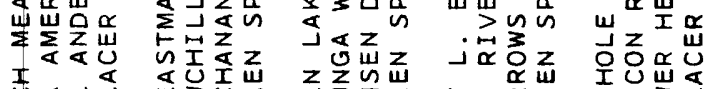

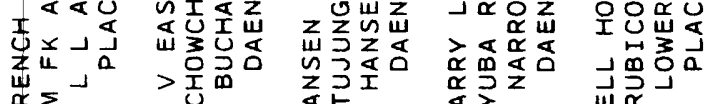

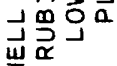




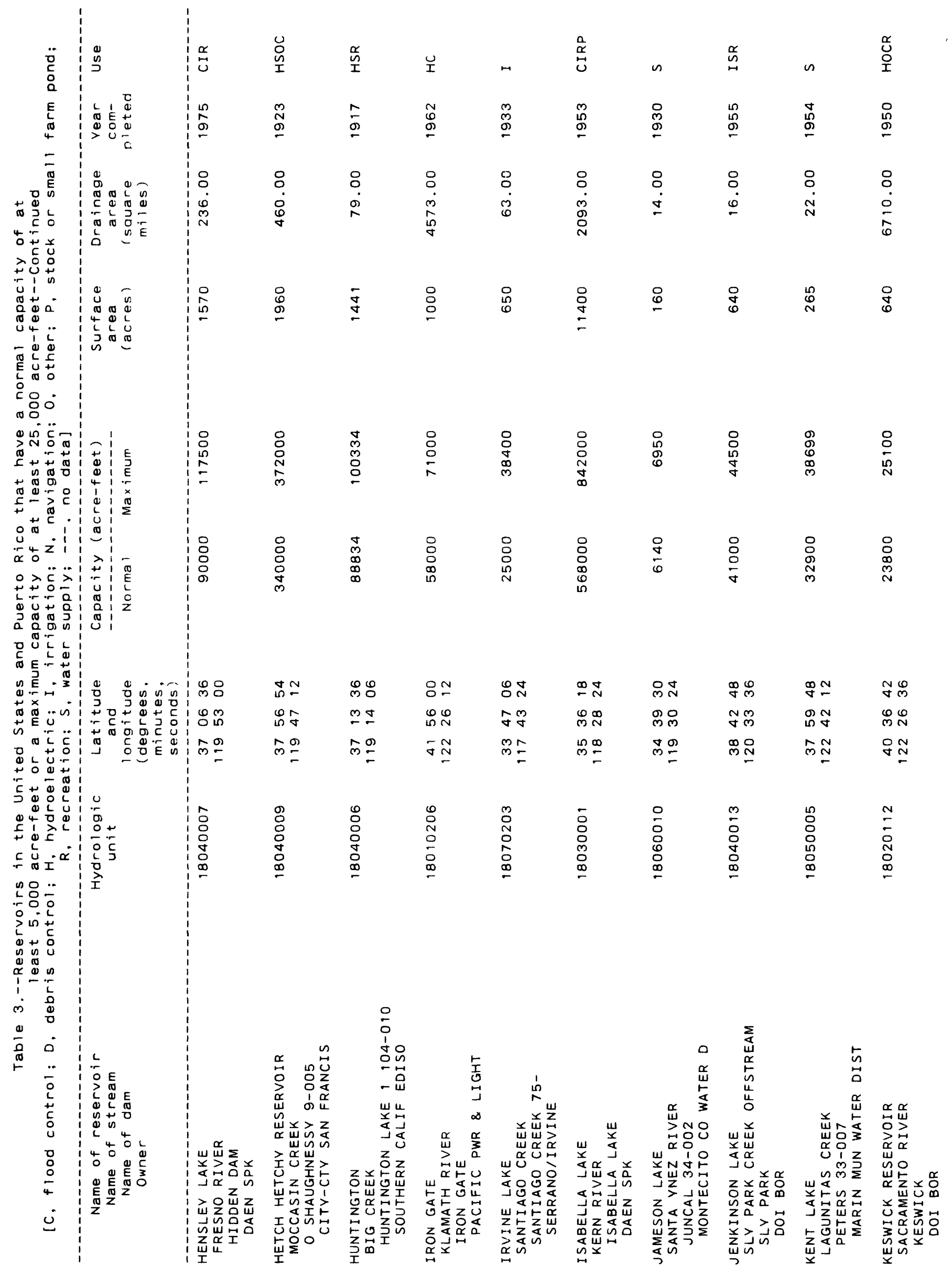




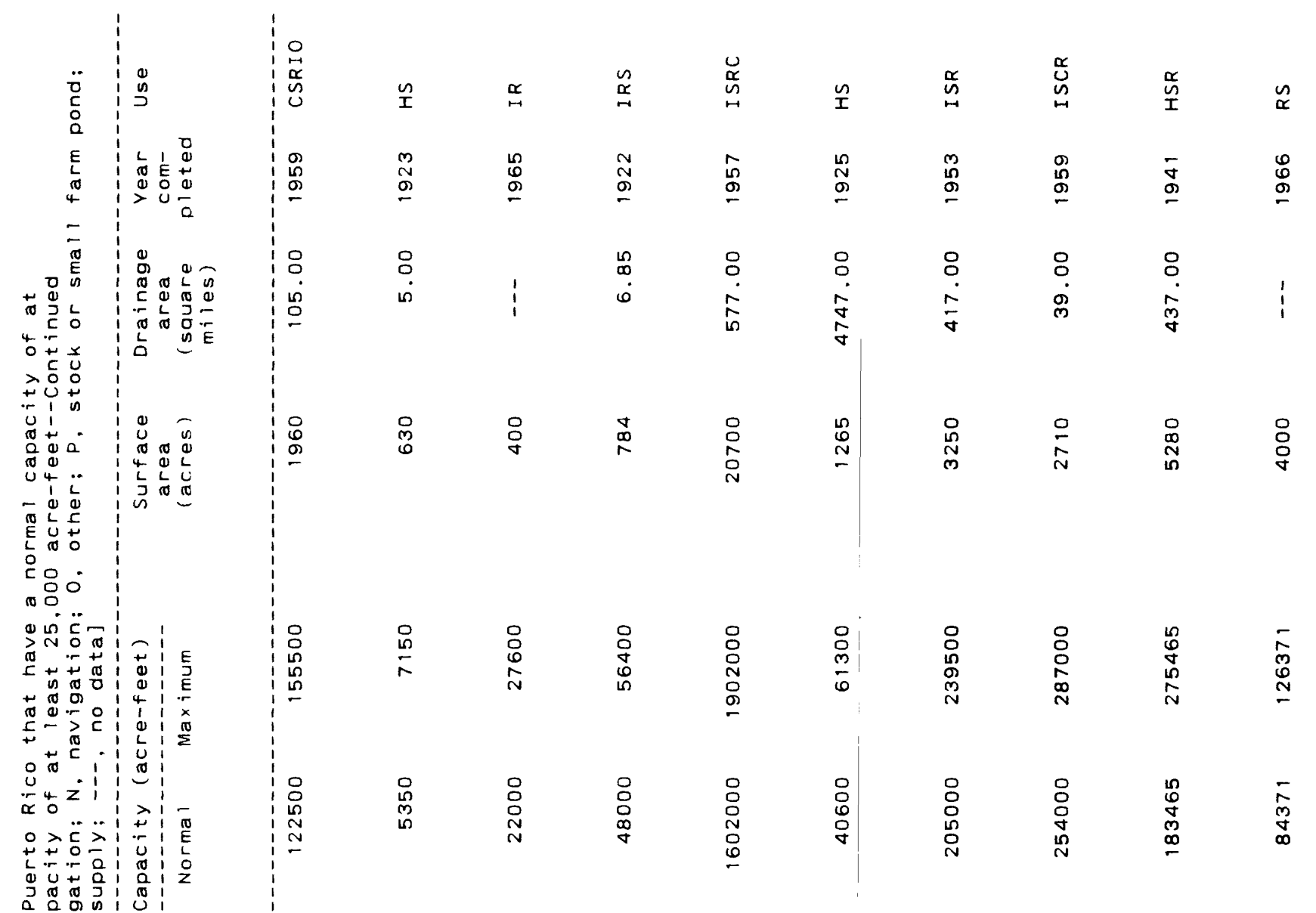

\begin{tabular}{|c|c|c|c|c|c|c|c|}
\hline ஜீః & $\stackrel{\infty}{\sim}$ & ₹ & $\underset{\sigma}{\infty} \underset{\sim}{\sim}$ & $\stackrel{\infty}{-}$ & ৪ষ্ & $\mathscr{\gamma} \underset{q}{\infty}$ & $\stackrel{\infty}{-\infty}$ \\
\hline โீ & $\stackrel{\infty}{\infty} \stackrel{m}{n}$ & 느으 & 용응 & - & 足 & $N$ & $\mathscr{M} \underset{\sim}{\sim}$ \\
\hline జ్ల $\underset{N}{ }$ & m & 於 & $\underset{m N}{\infty}$ & $\bar{\sigma} \bar{N}$ & வூ & मॄ. & $\hat{m} \stackrel{\infty}{=}$ \\
\hline
\end{tabular}

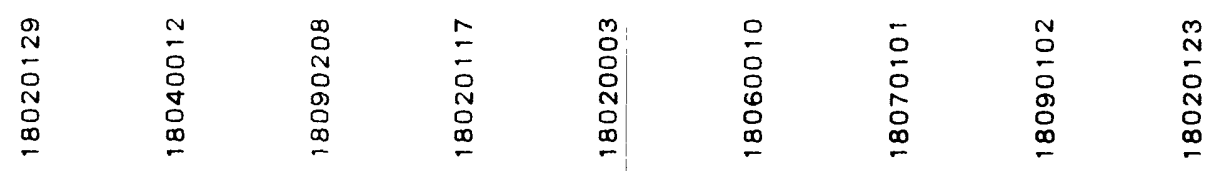

ه

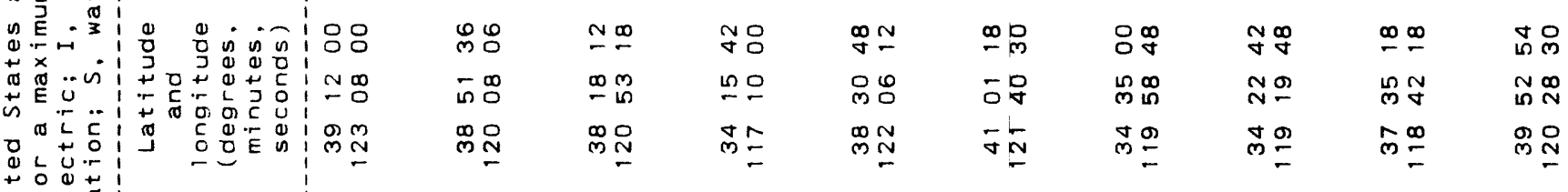

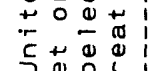

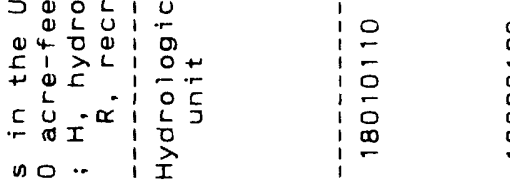

80

a

告告

1.

.
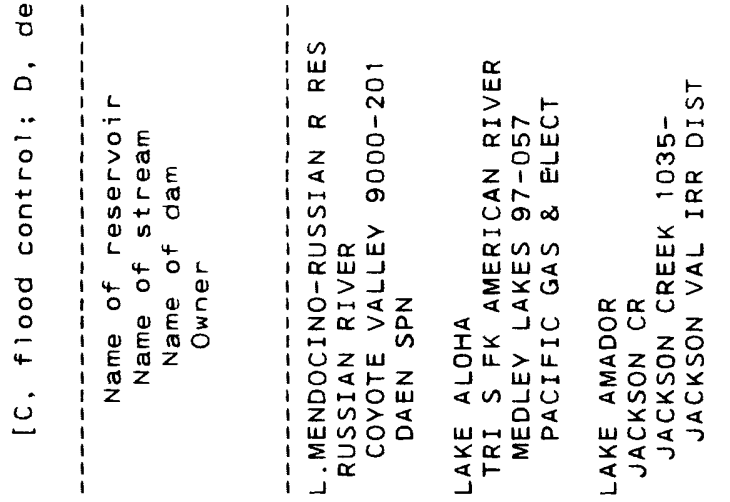

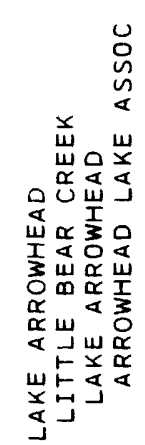

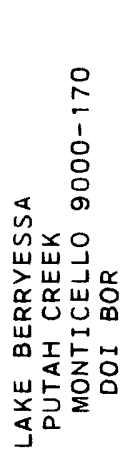

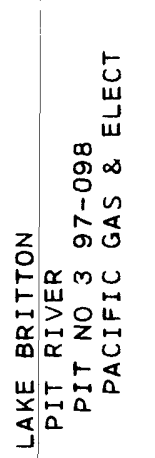
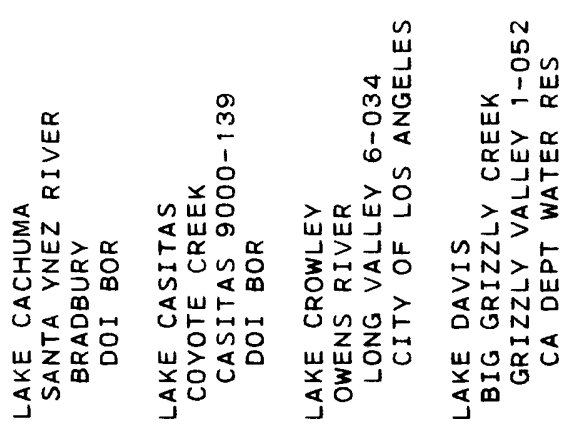


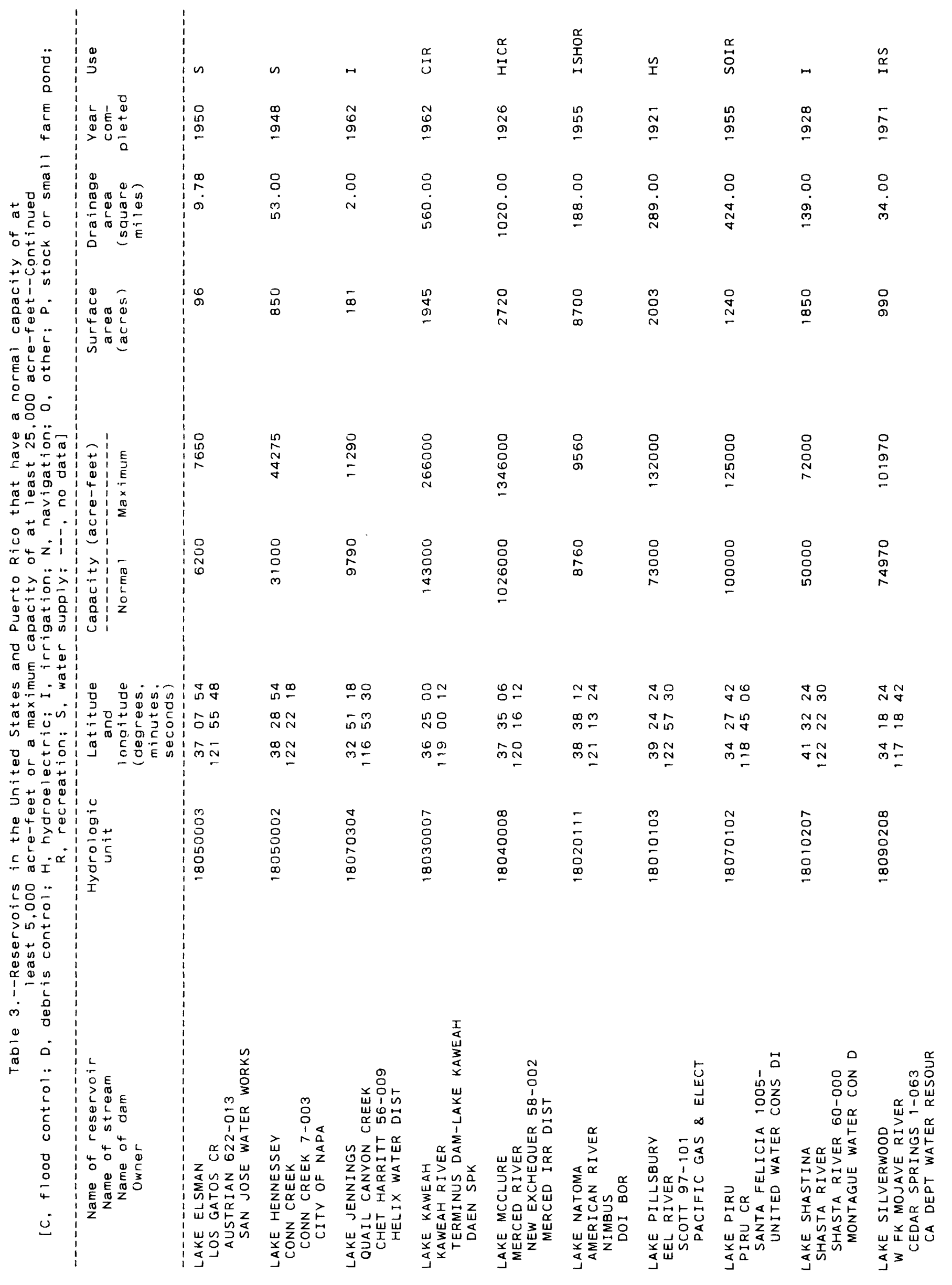




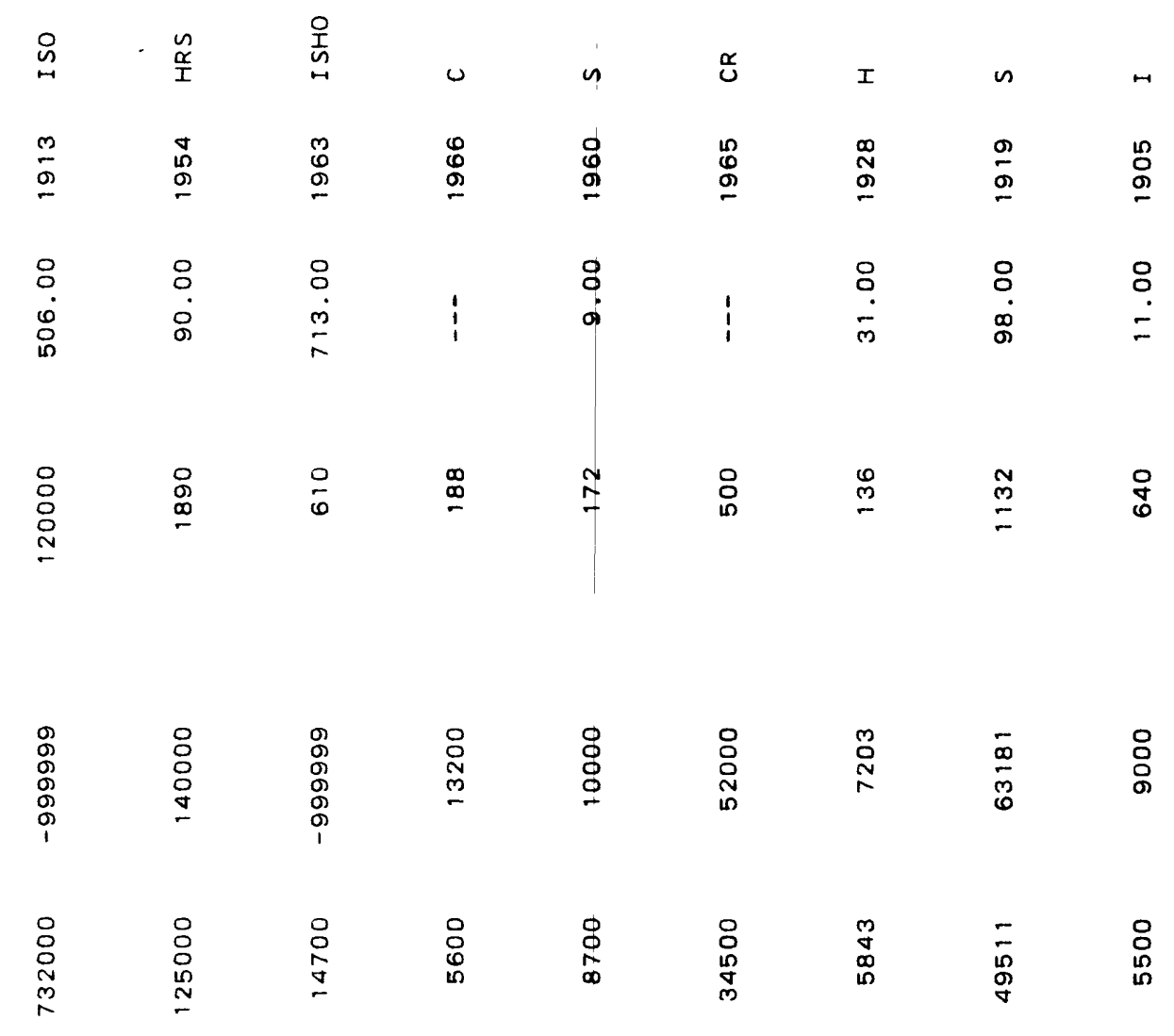

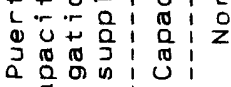

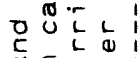

$\pi$

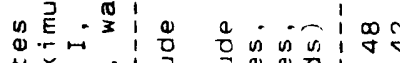

N

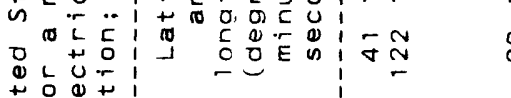

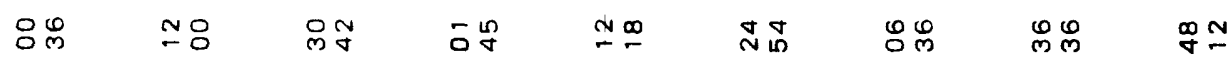

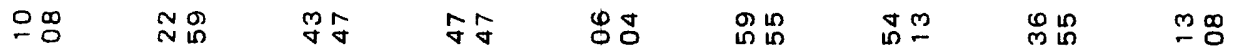

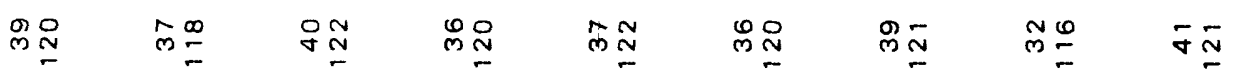

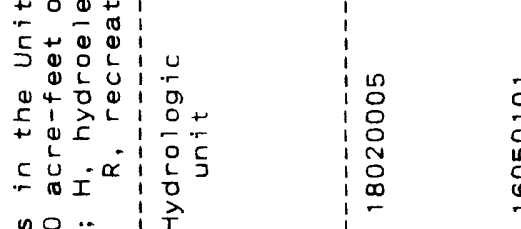

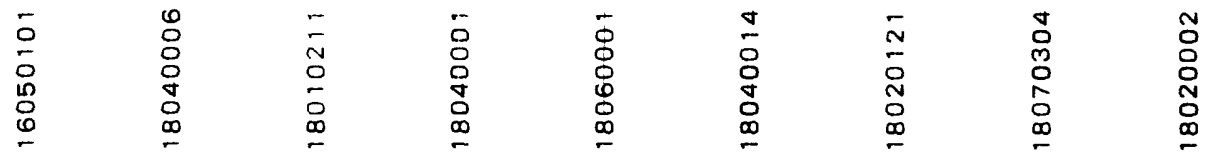

2
0
0
2
0
0
0
0
0
0
1
0
0
0
0
0
0
0
0

年 


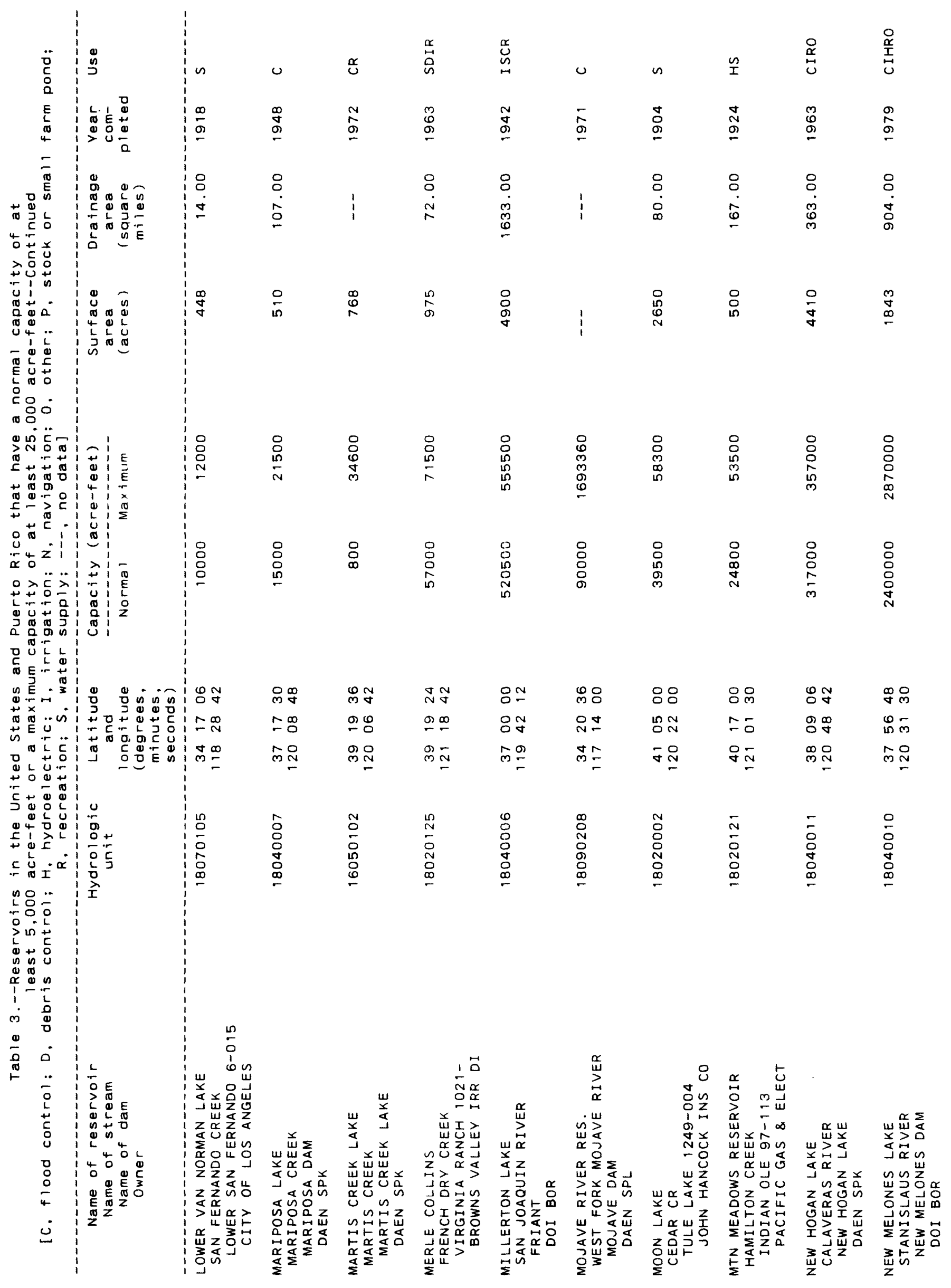




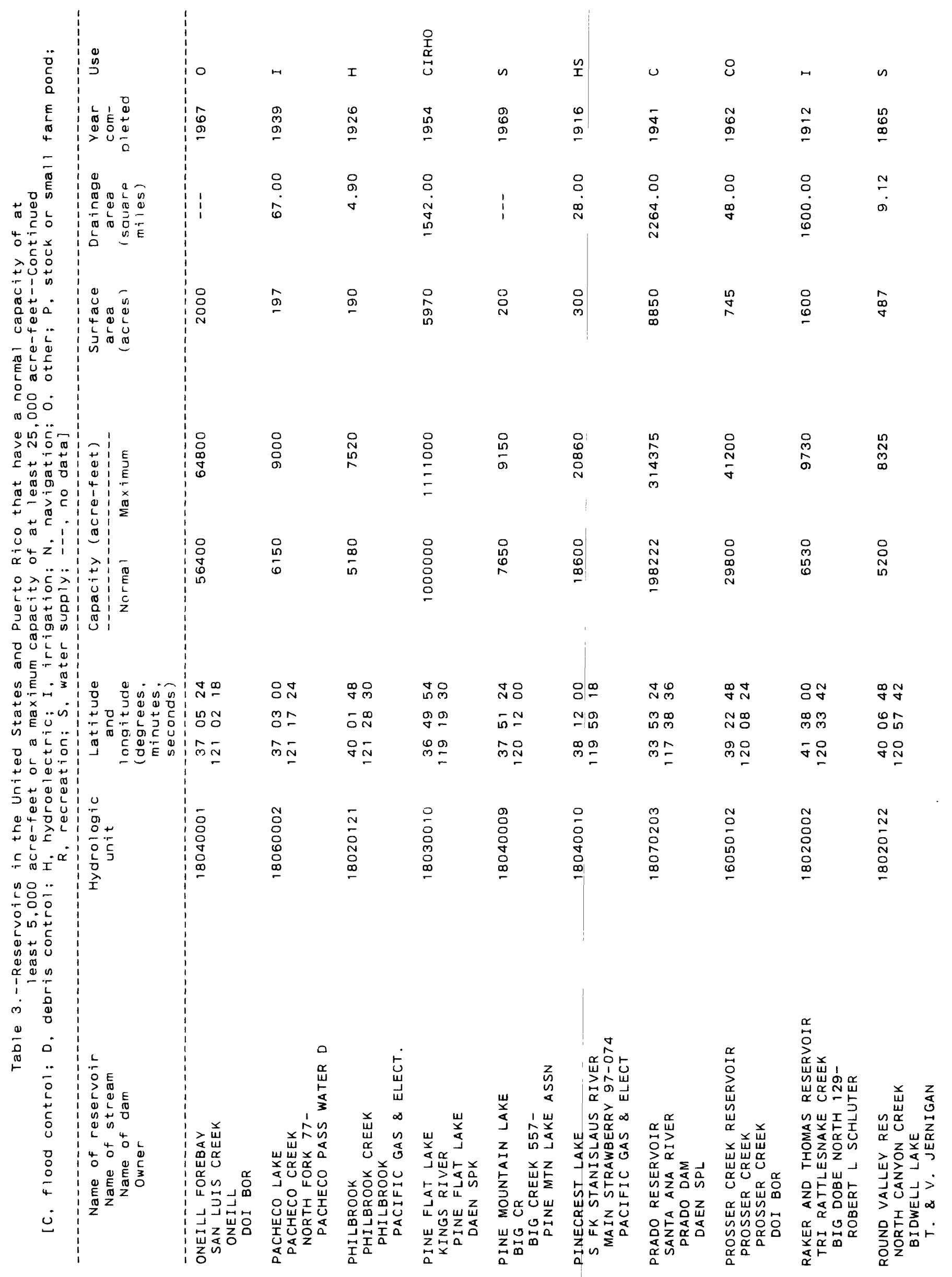




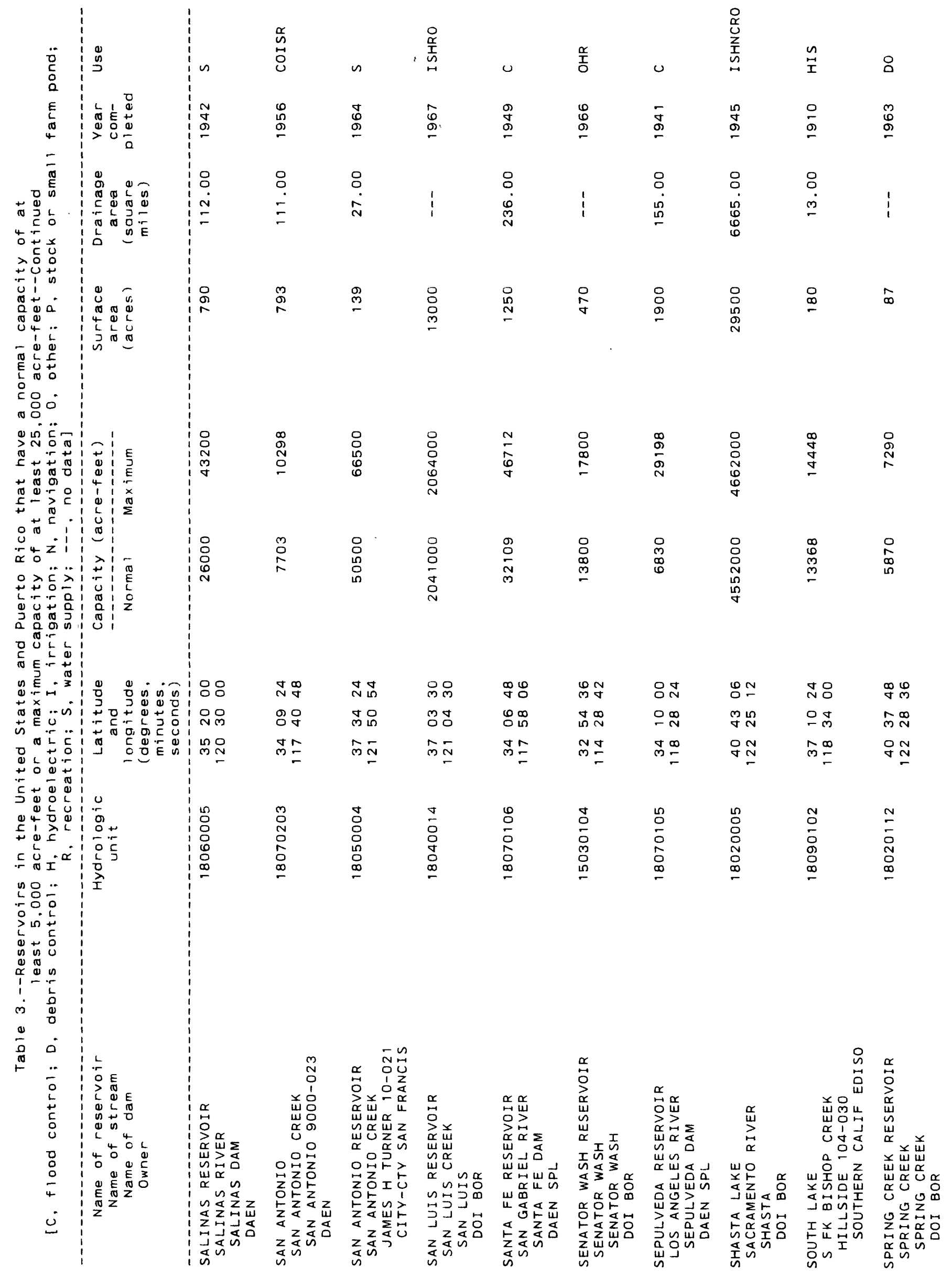




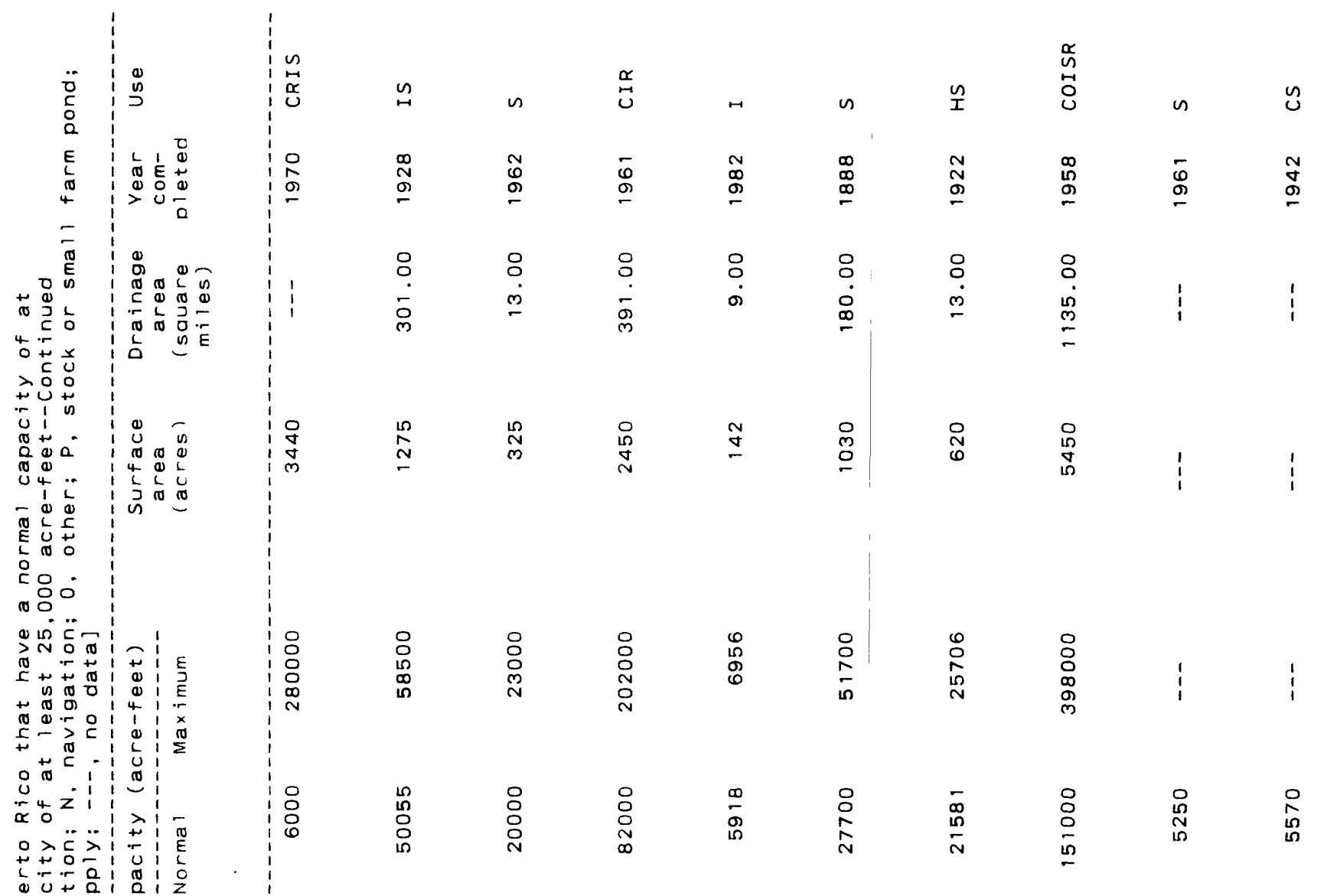

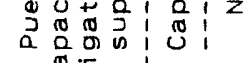

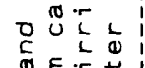

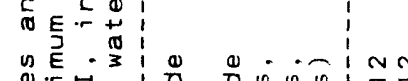

arn

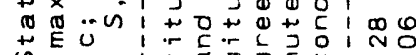

ज四芒:

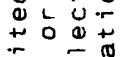

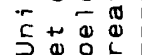

$\stackrel{5}{+}$

政

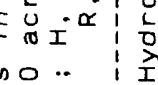

\begin{tabular}{|c|c|c|c|c|c|}
\hline 电芯 & $\simeq \simeq$ & 잉요 & 䑻 & 유 & స్ర \\
\hline & 范 & 贾品 & $\hat{o N}$ & $\overline{7} 8$ & 조 \\
\hline$=$ & 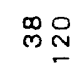 & $\stackrel{\mathscr{m}}{\stackrel{\infty}{=}}$ & ్ㅣㅁㅗ & $m !$ & mi \\
\hline
\end{tabular}

.

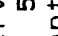

年

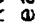

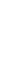

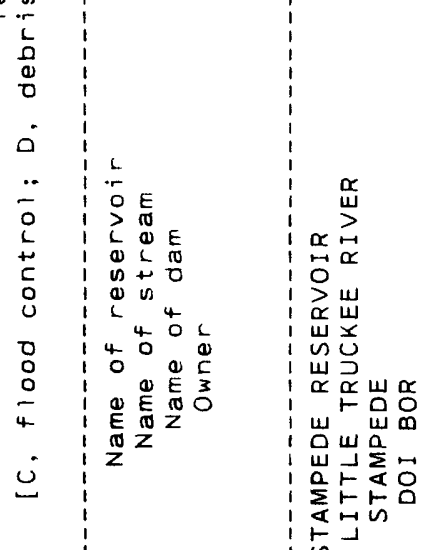

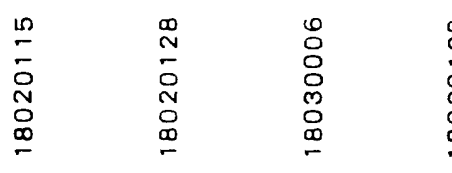

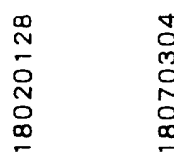

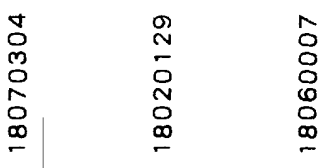

$\begin{array}{lll}0 & 0 \\ 0 & 0 \\ 0 & 0 \\ 0 & 0 \\ 0 & 0 \\ 0 & 0 \\ 0 & 0 \\ & 0\end{array}$

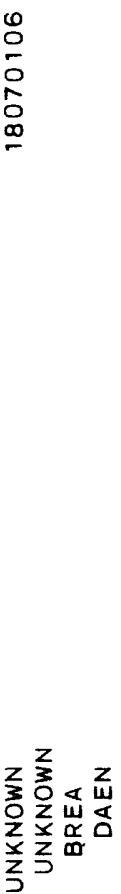




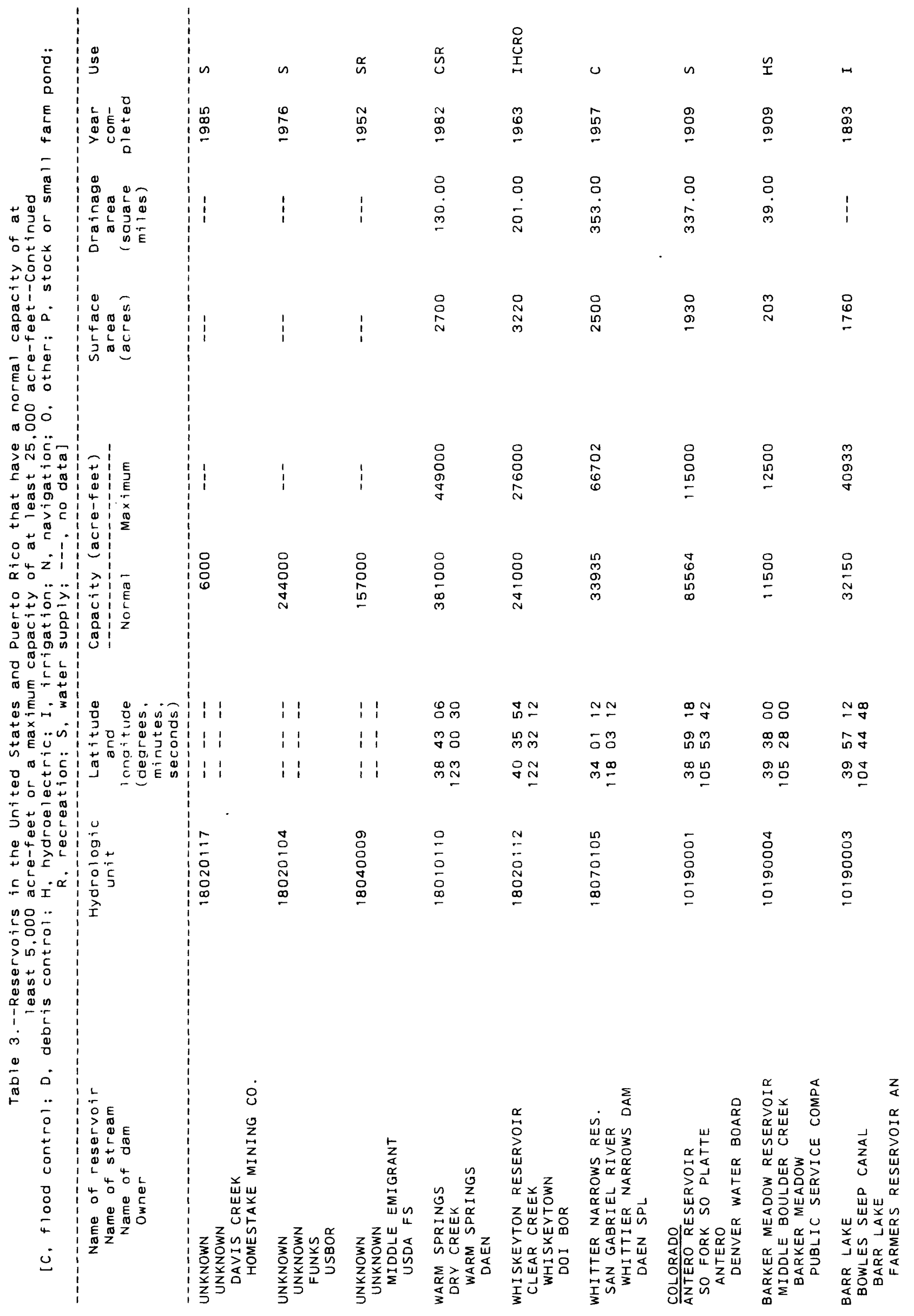




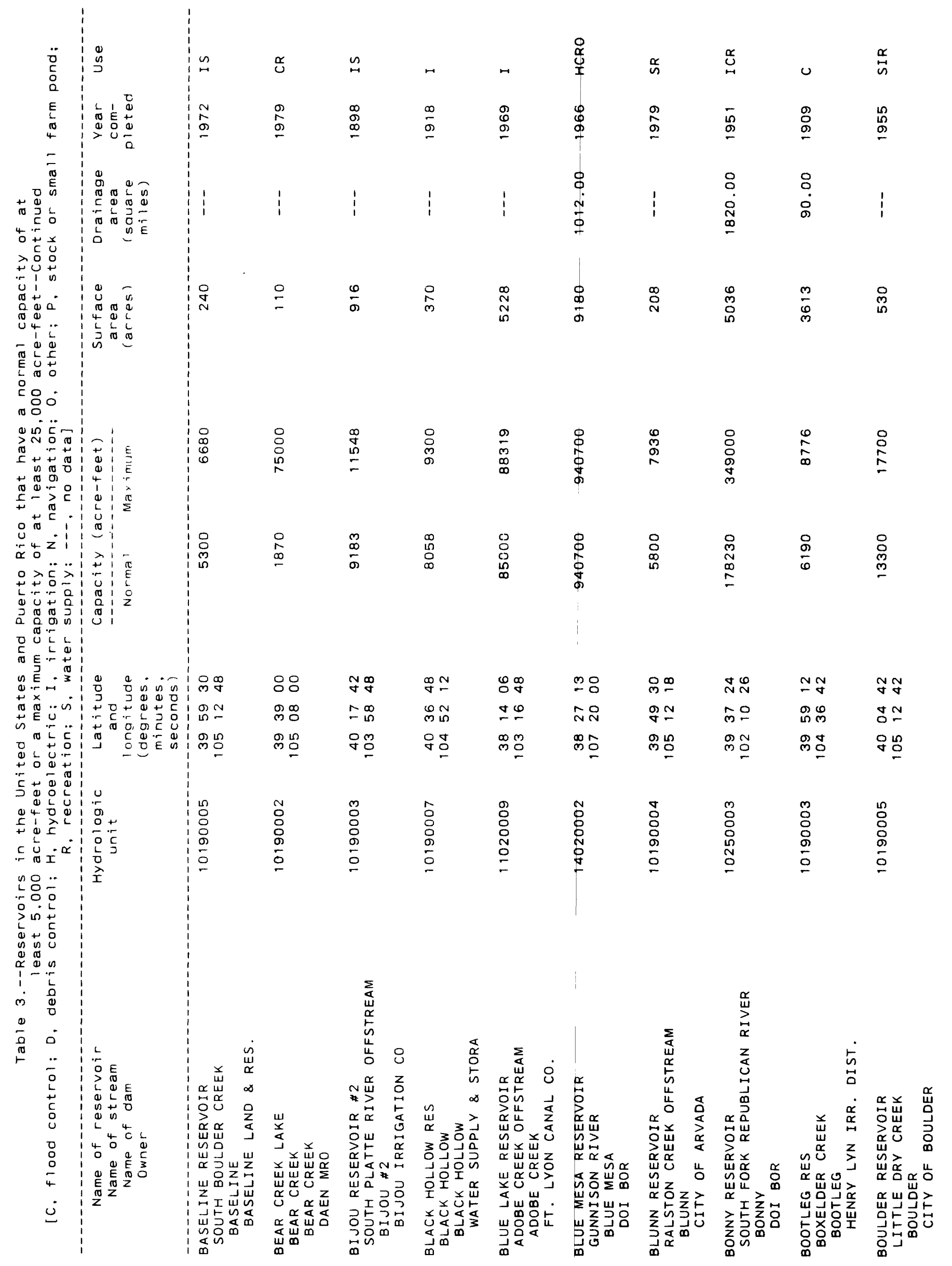




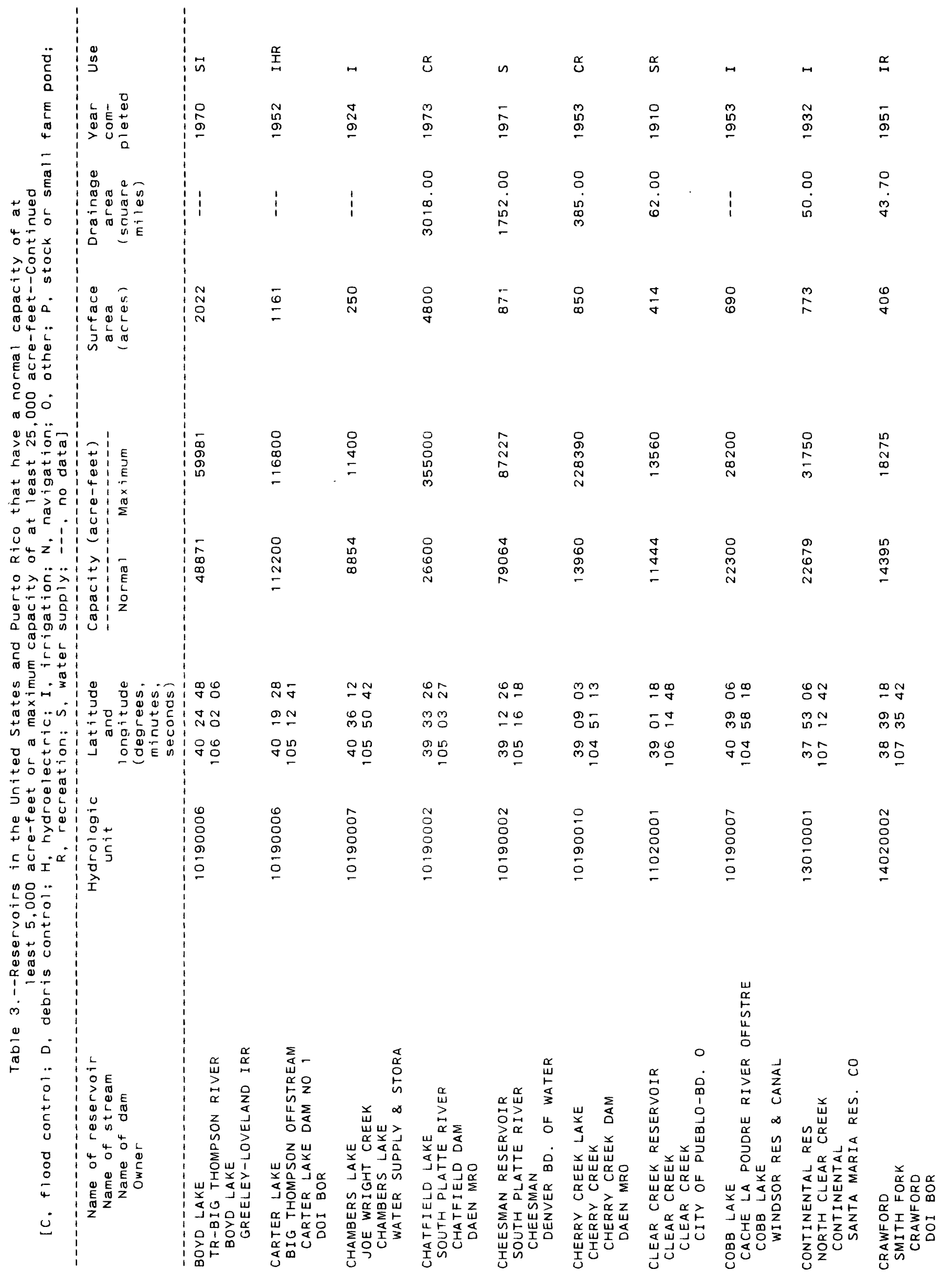




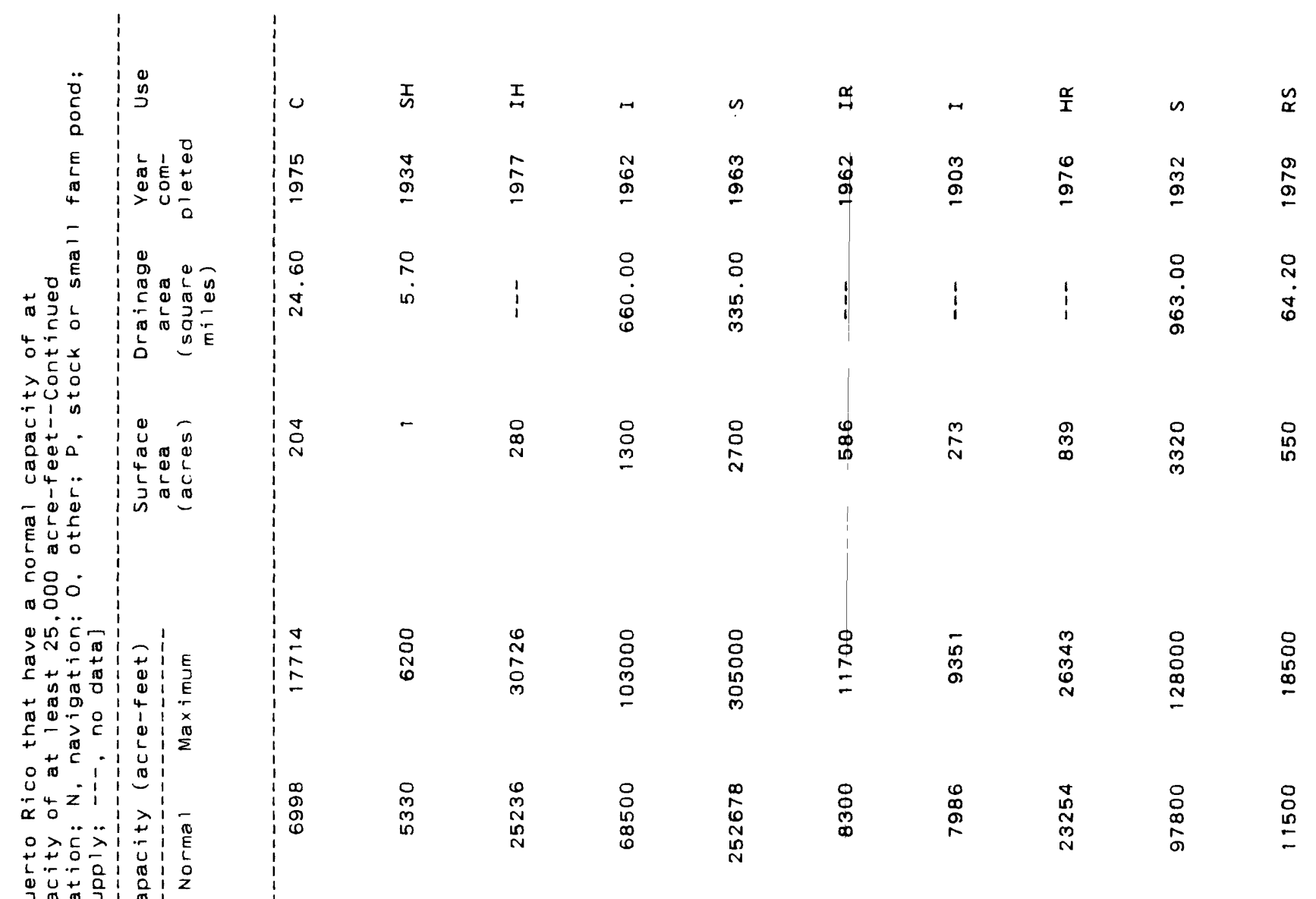

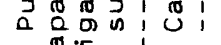

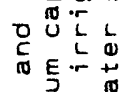

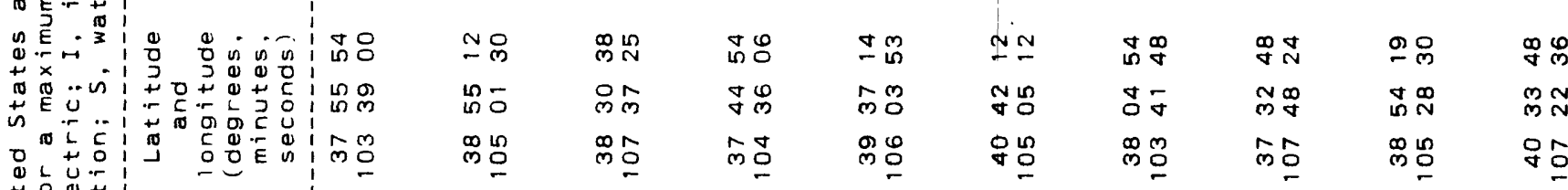

范紫葡

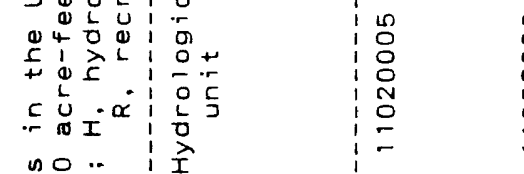

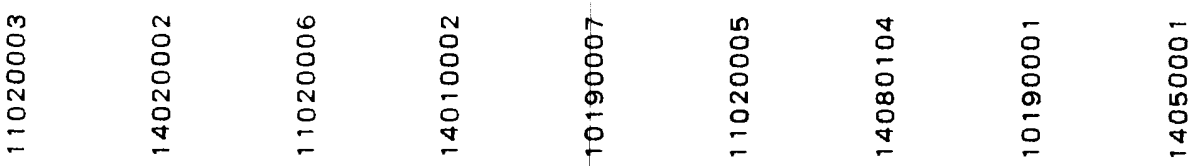

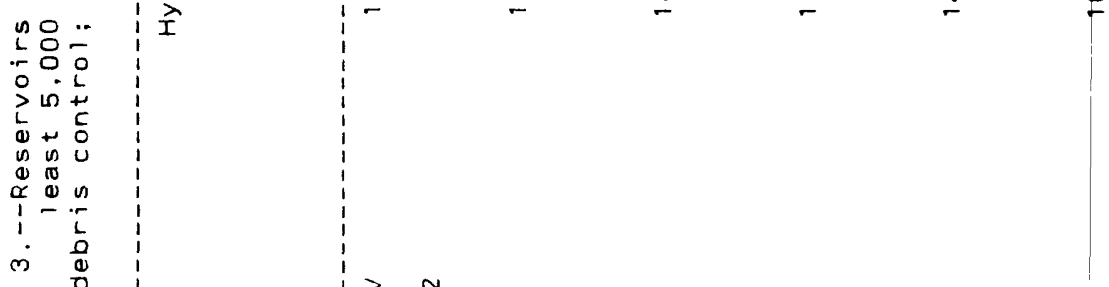

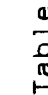
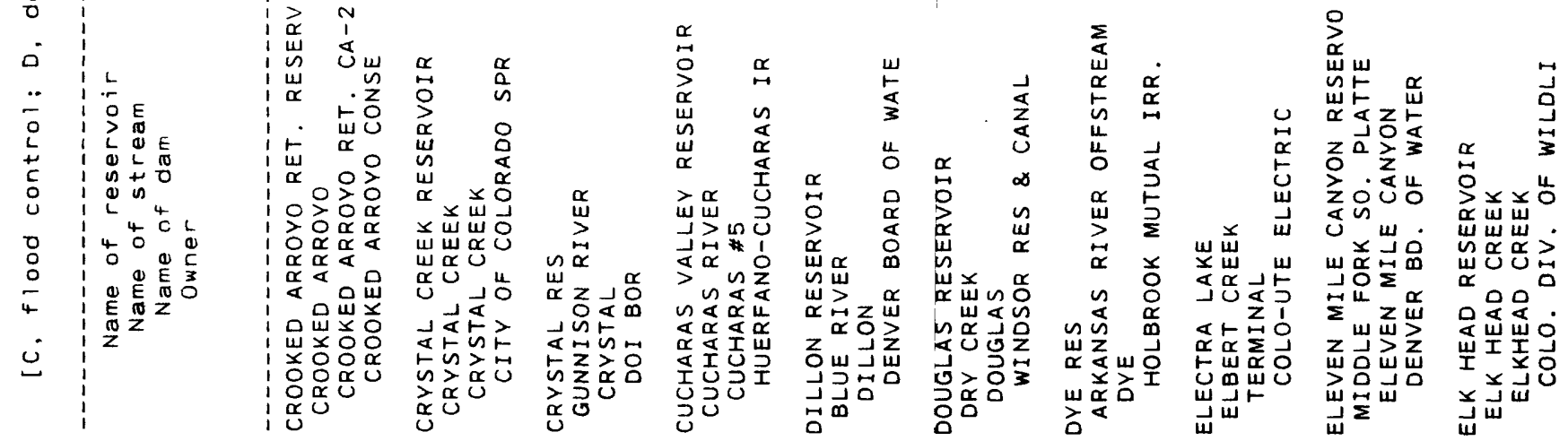


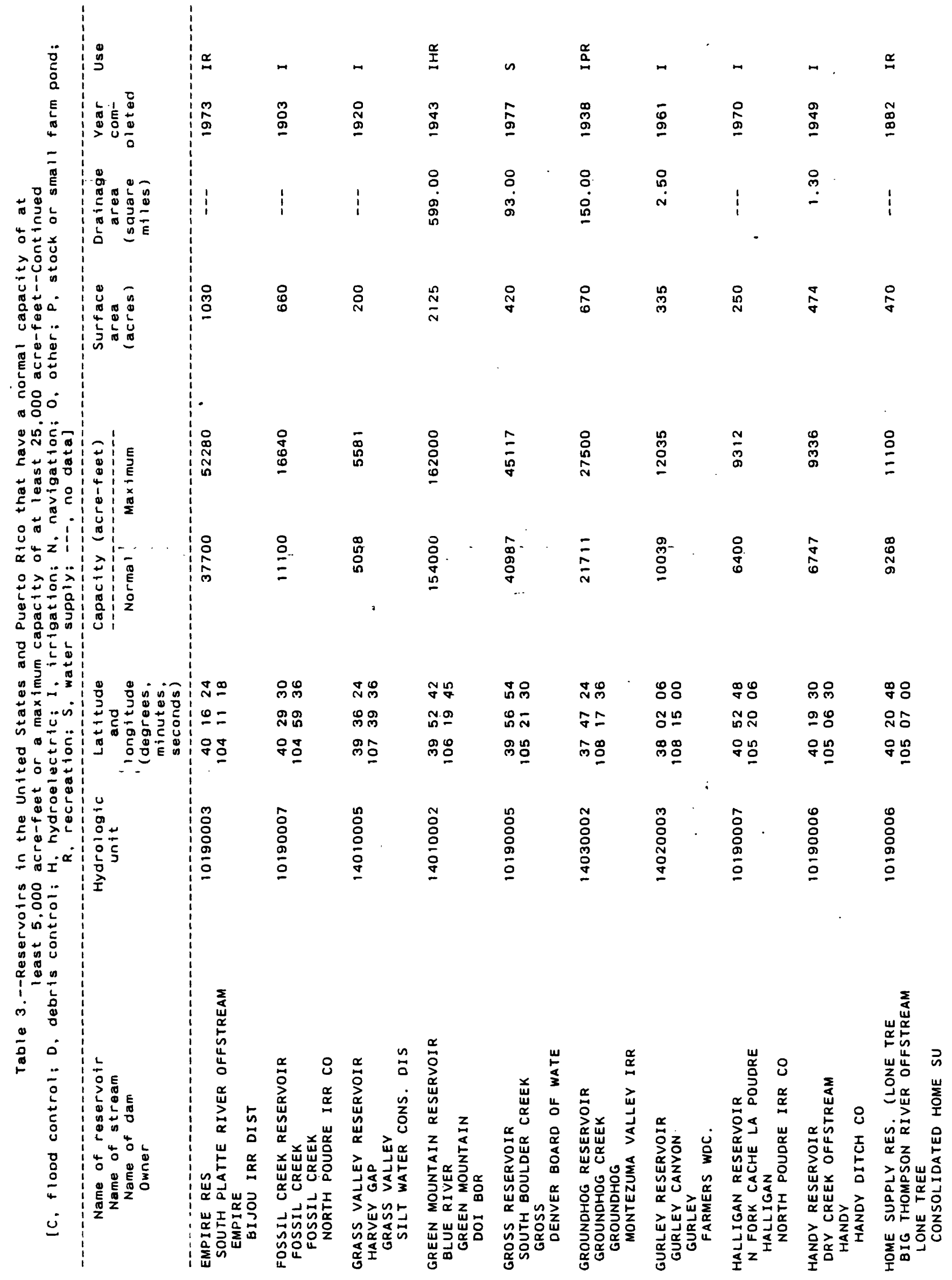




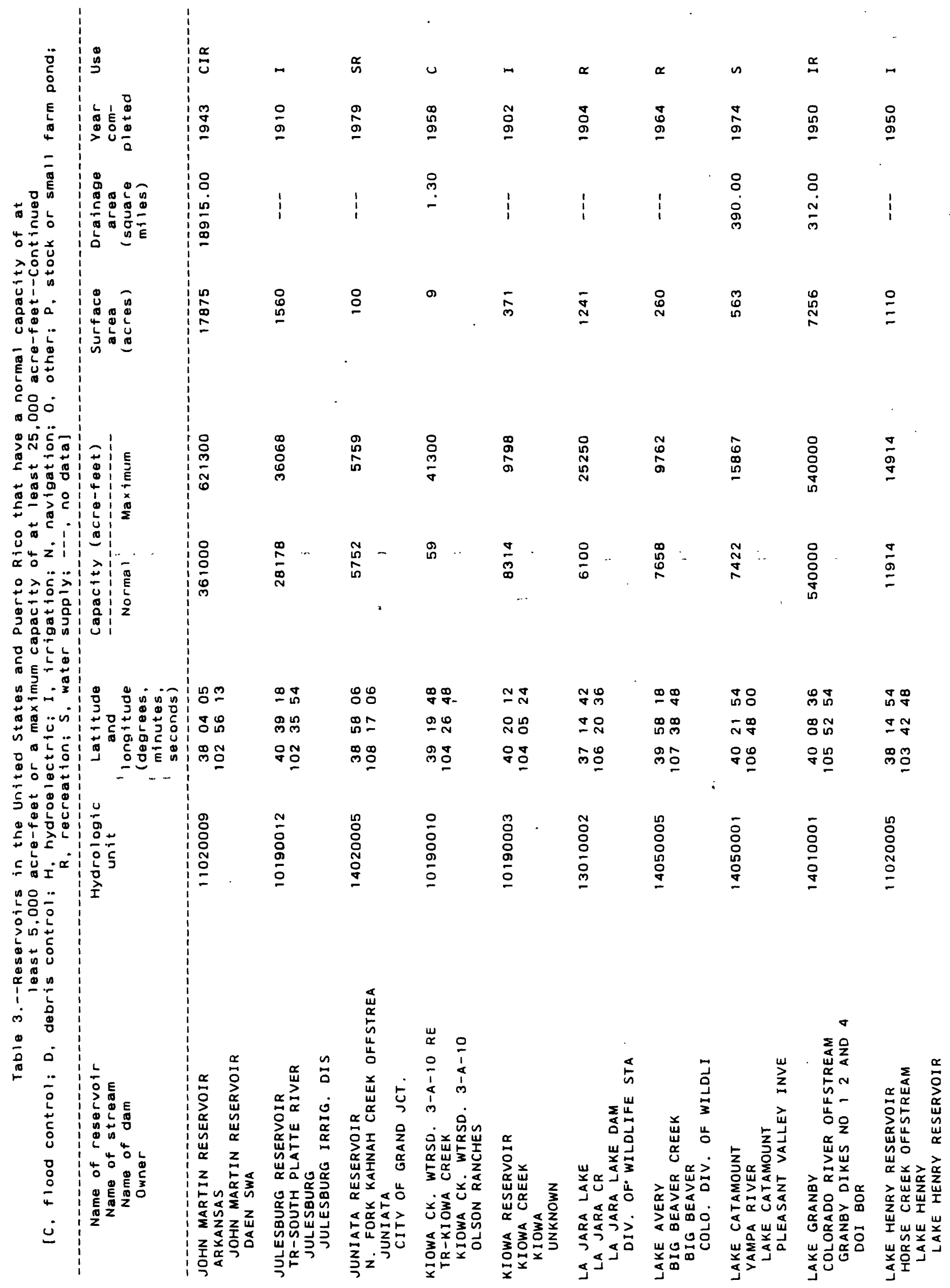




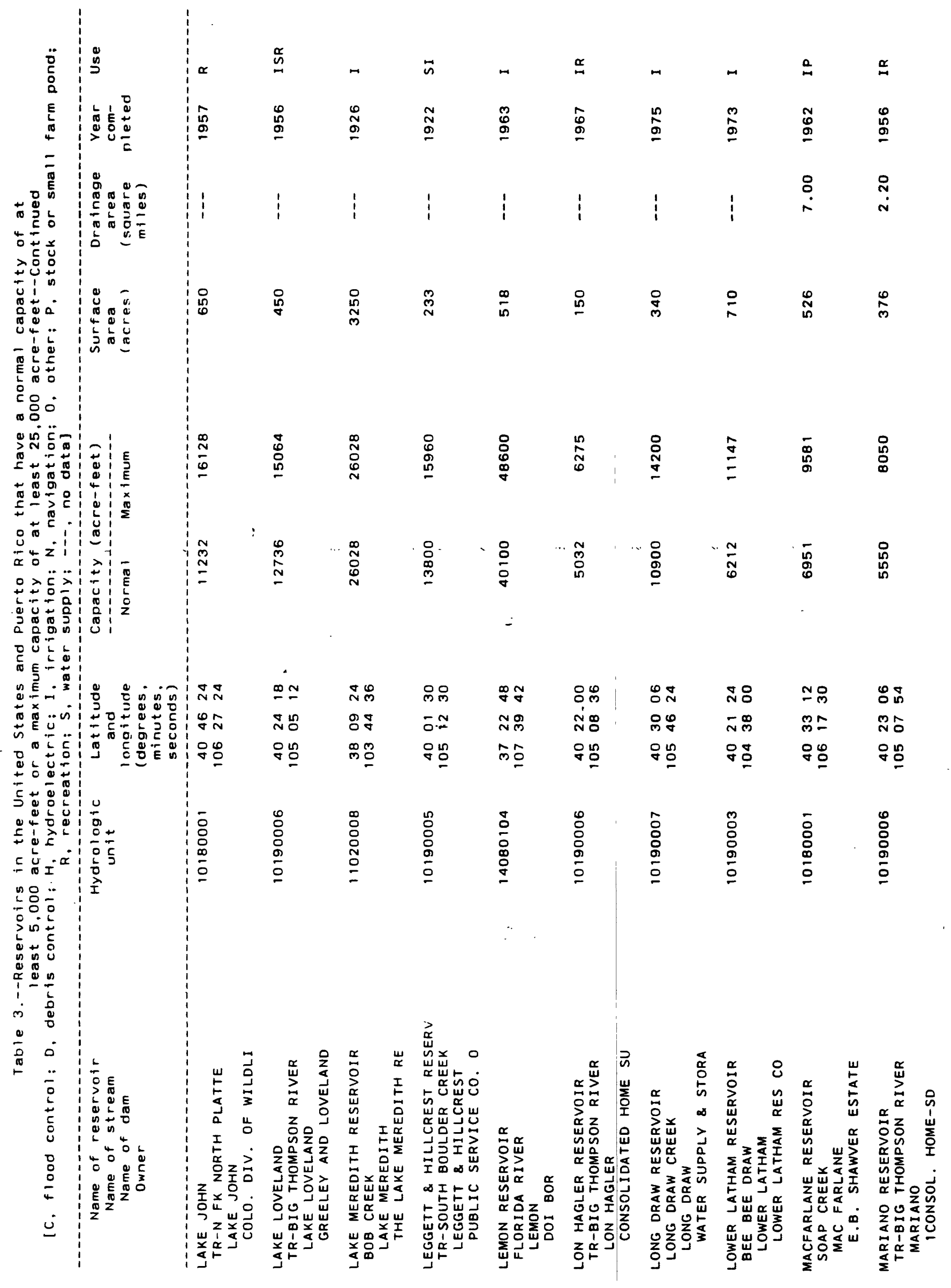




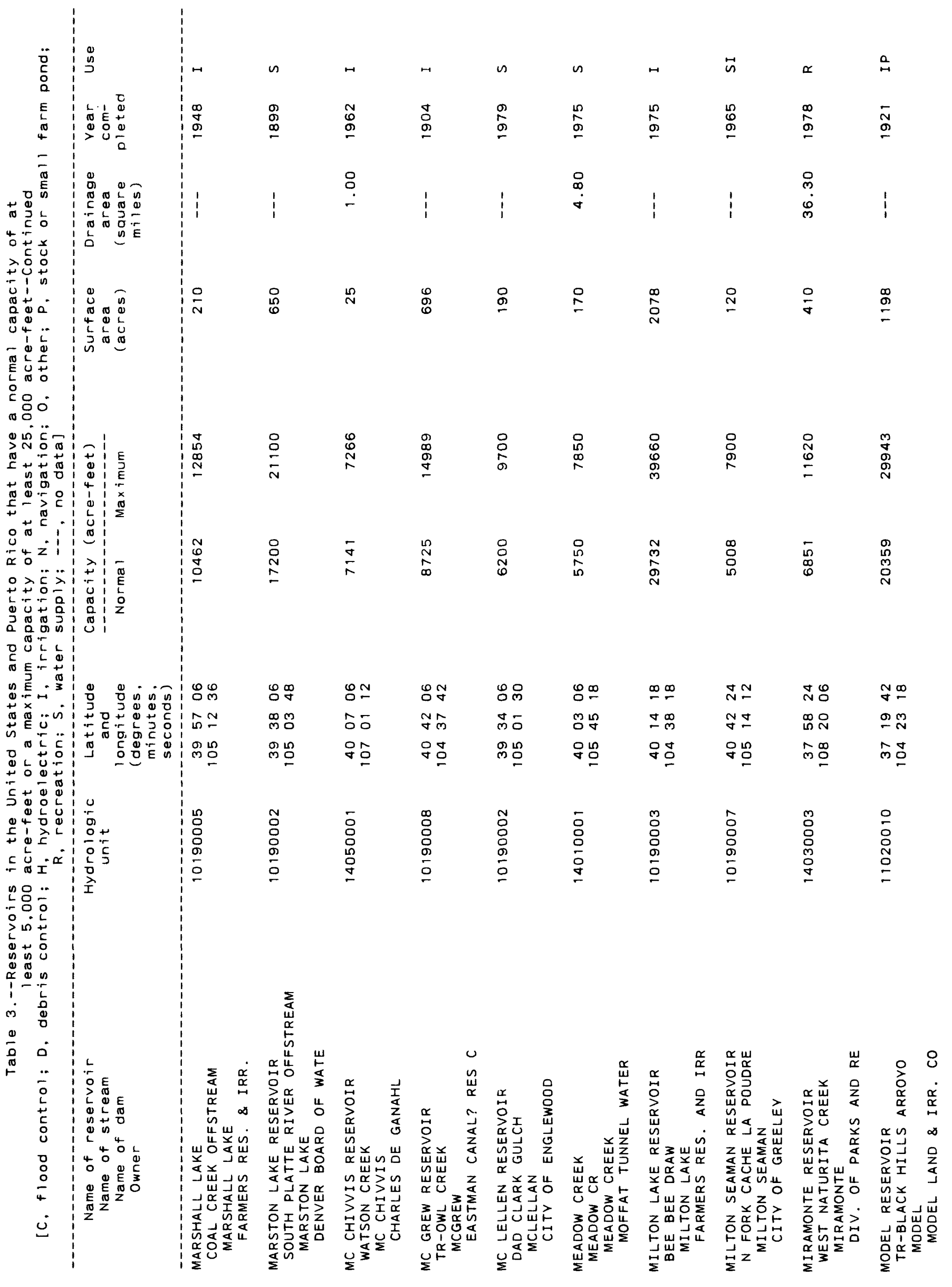




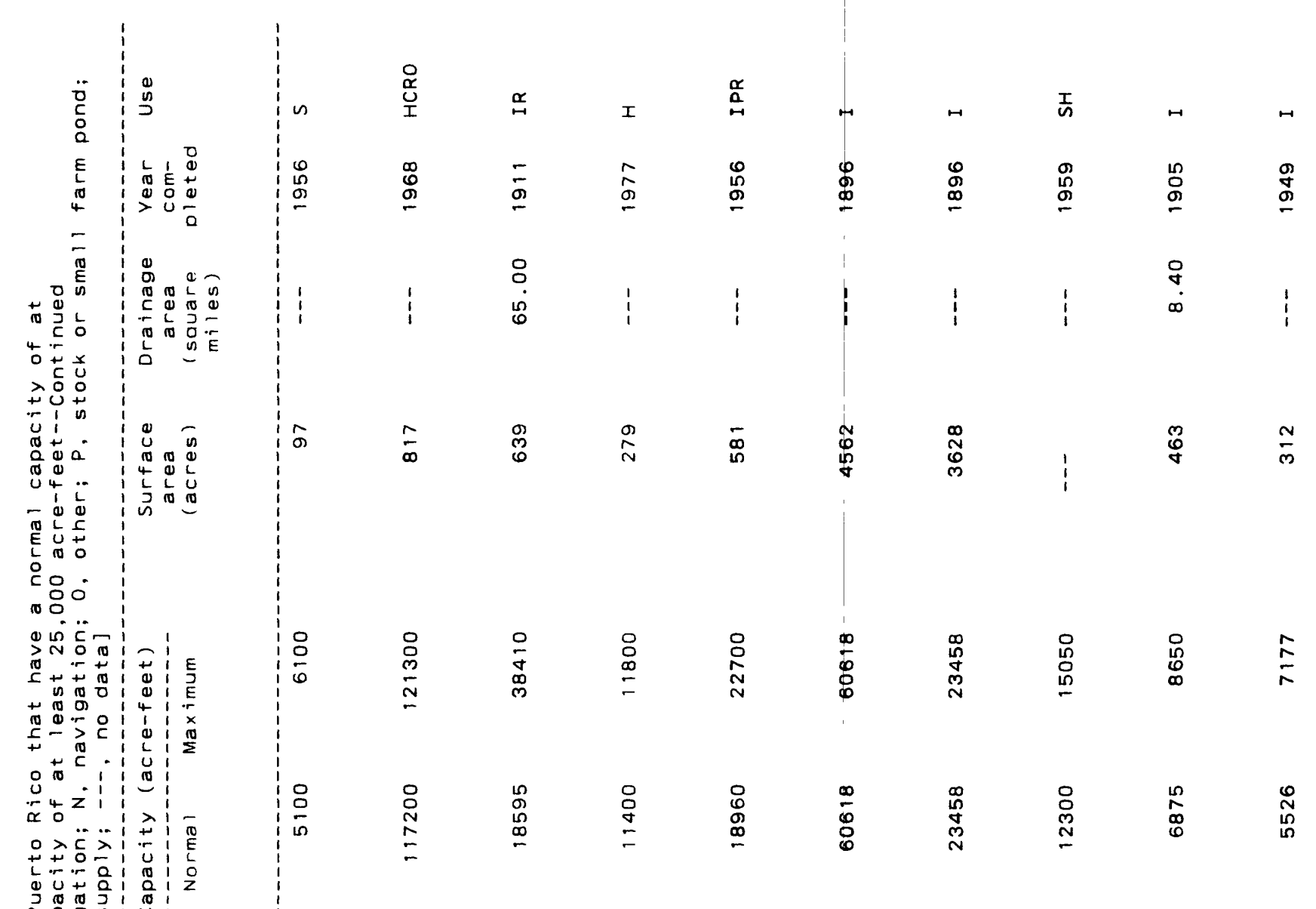

ว

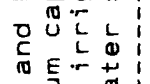

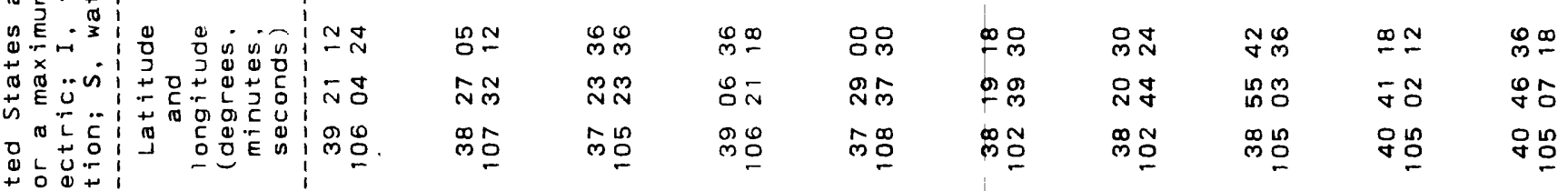

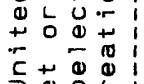

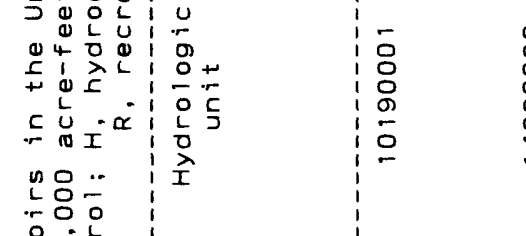

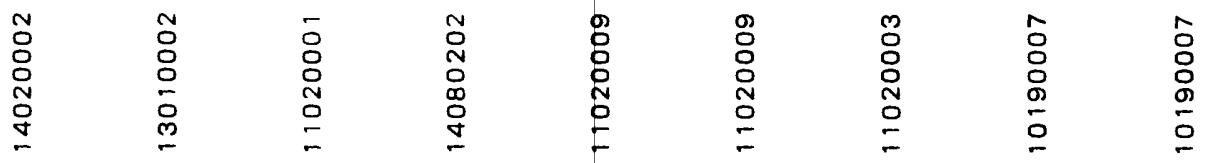

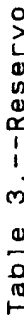

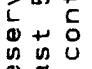

年

i $\frac{0}{0}$

$\frac{1}{10}$
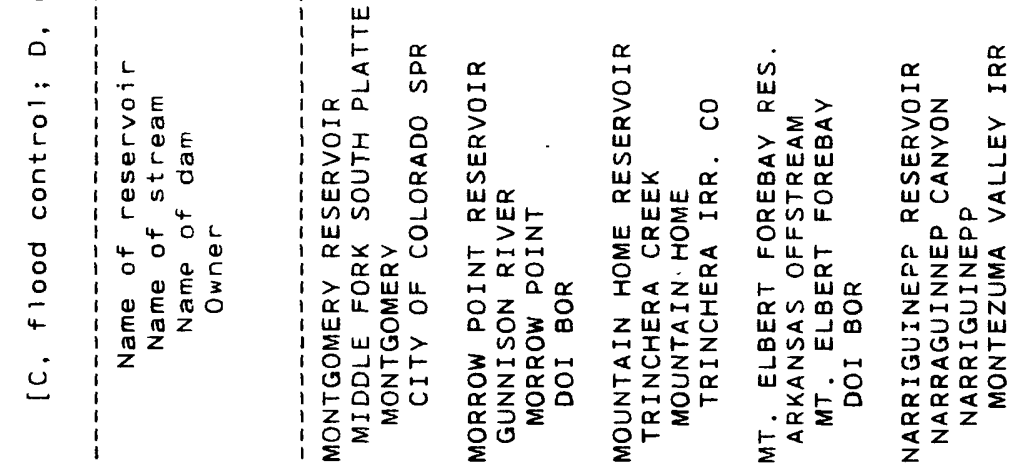

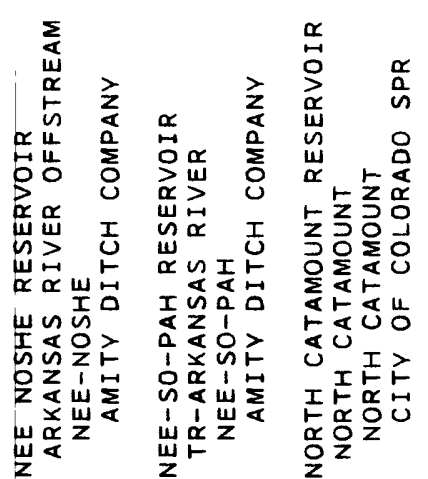

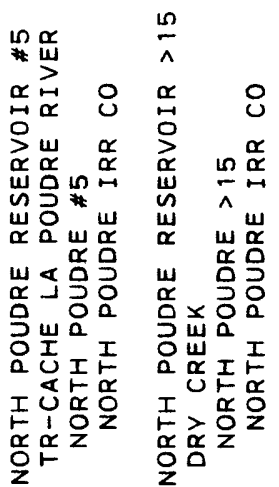




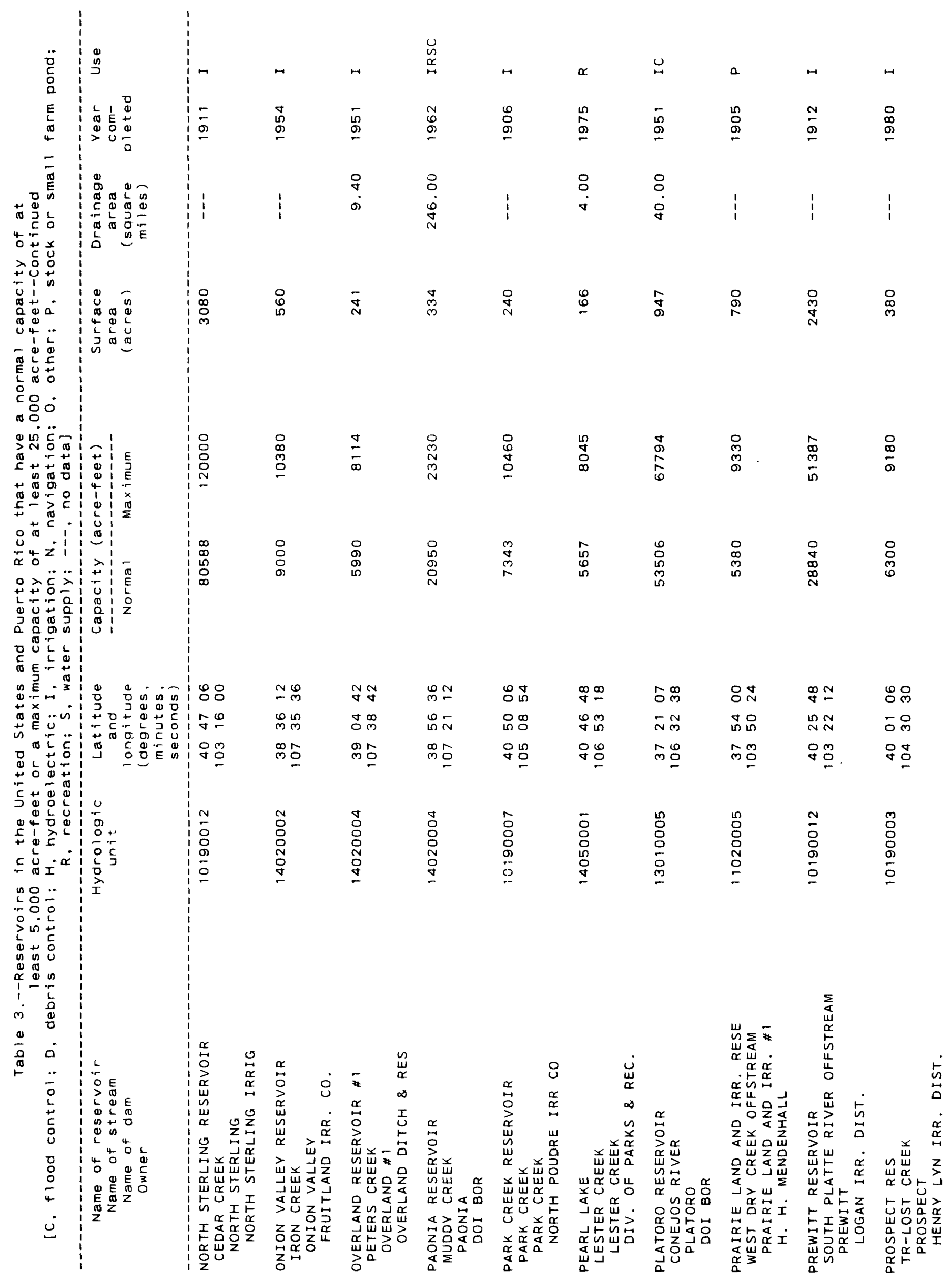




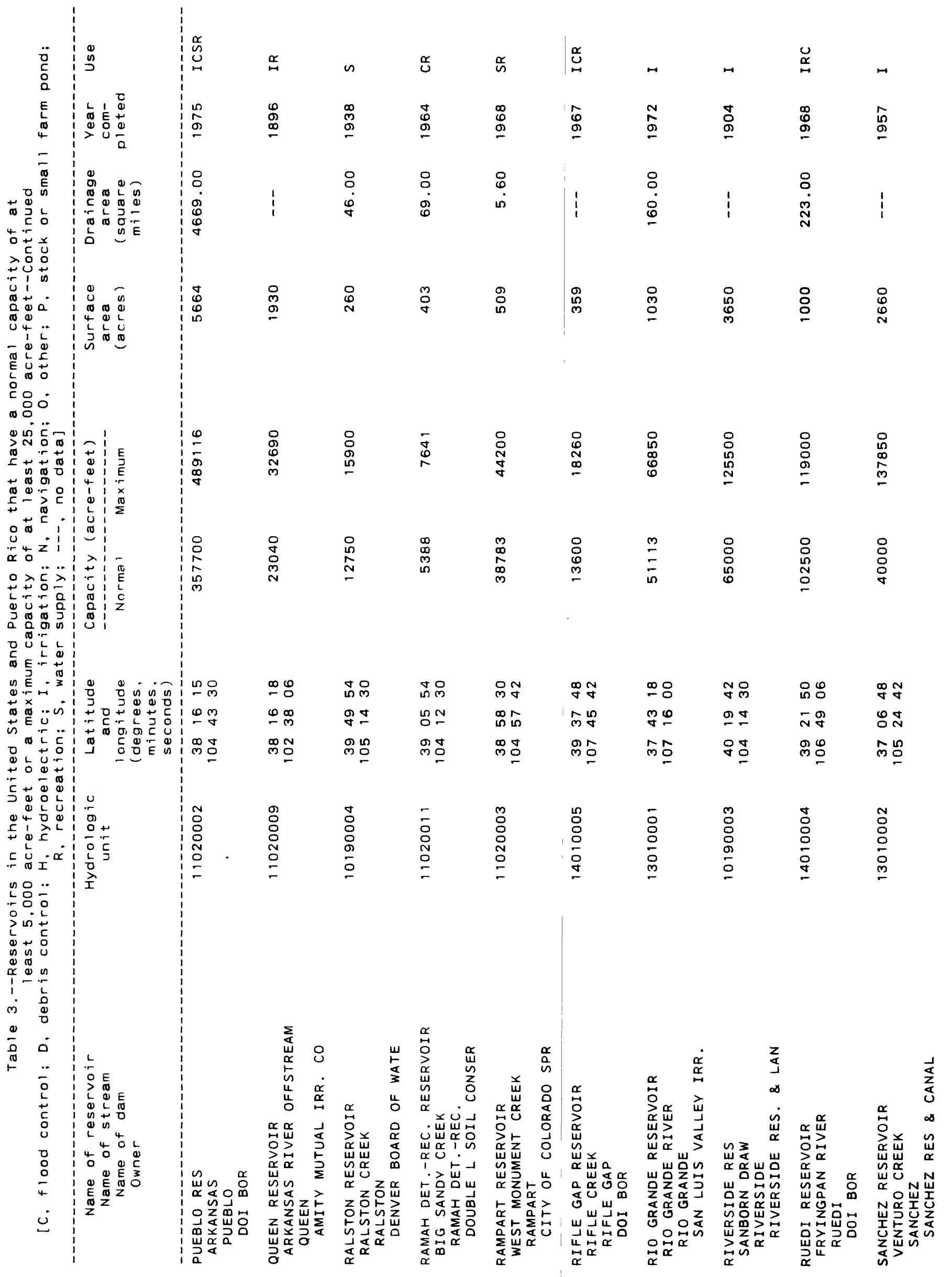




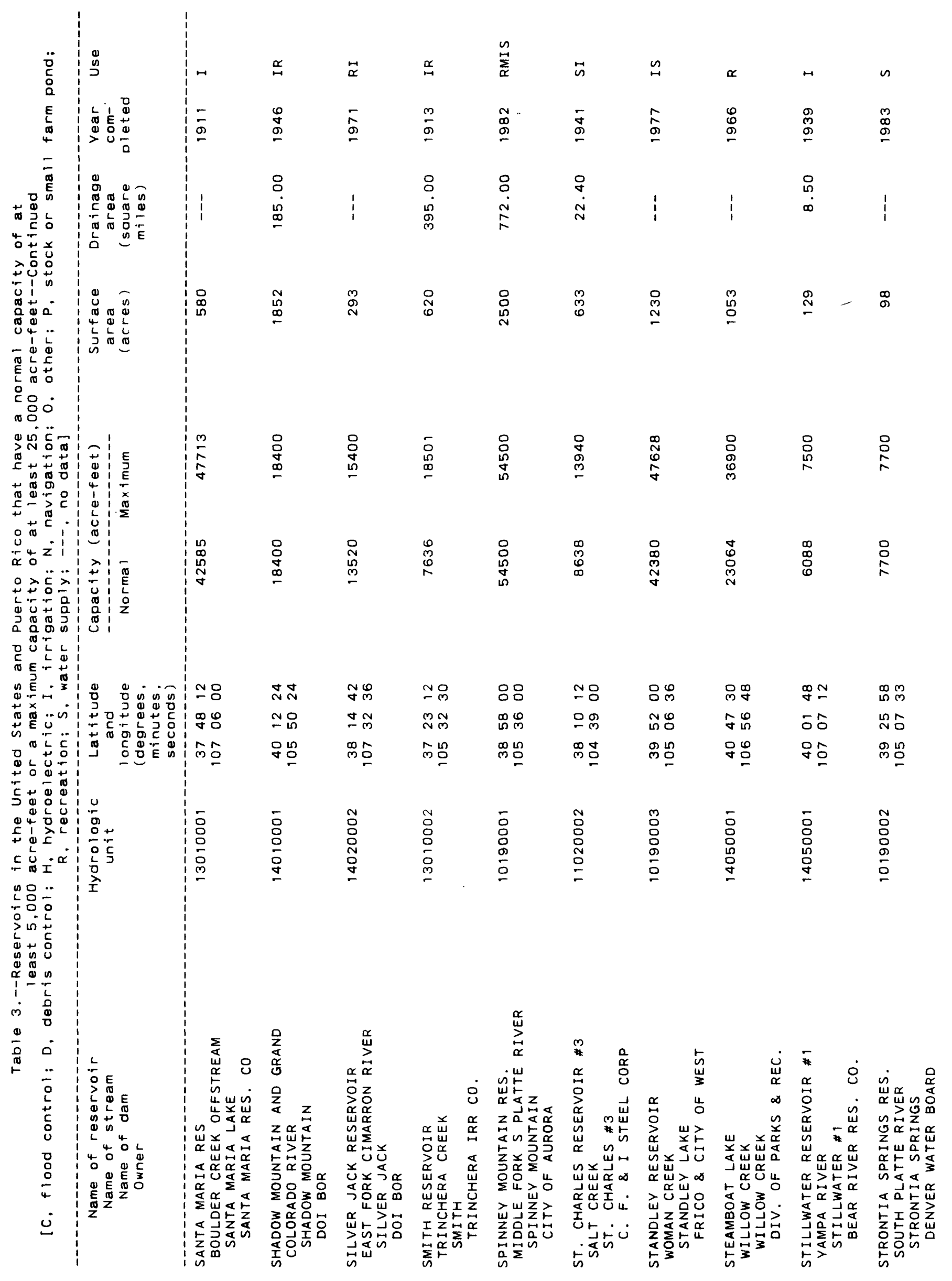




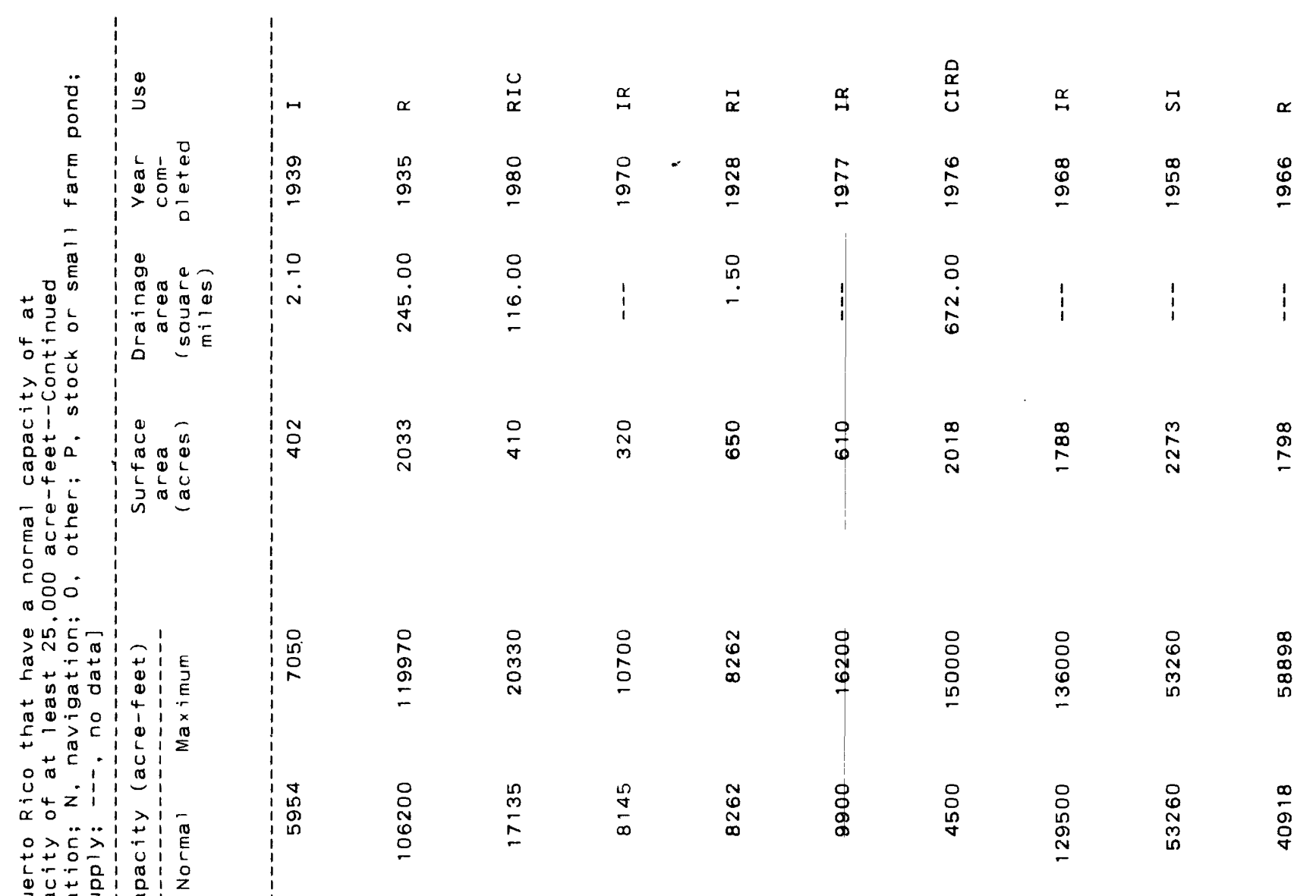

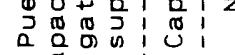

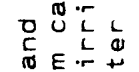

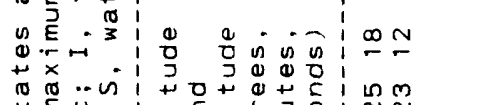

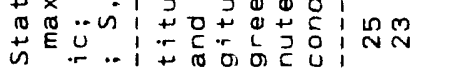

กN

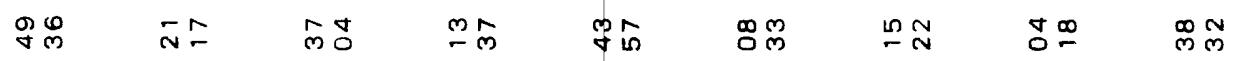

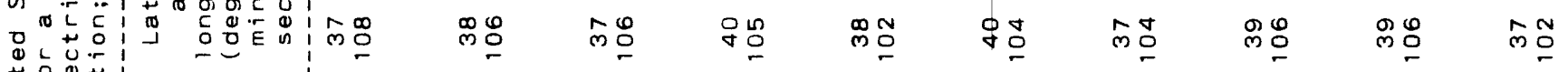

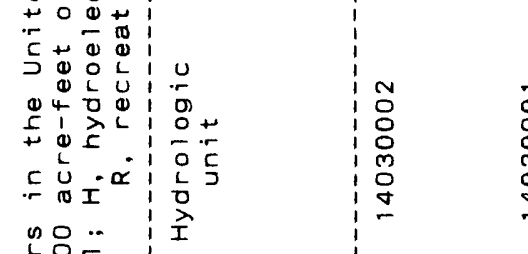

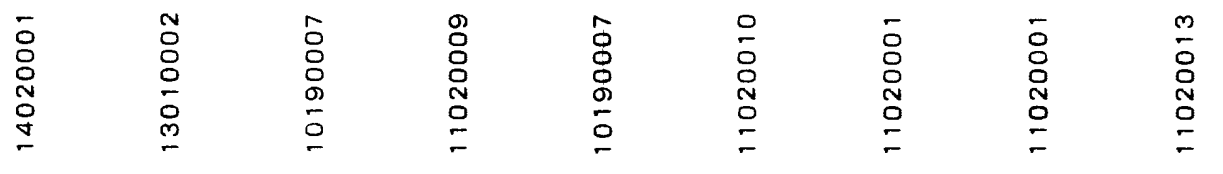

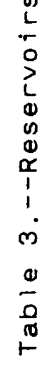

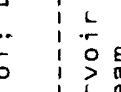

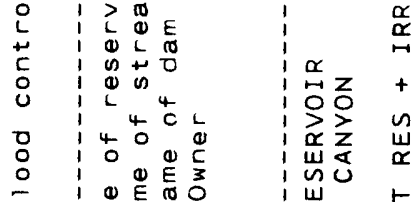

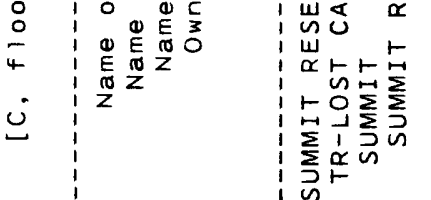

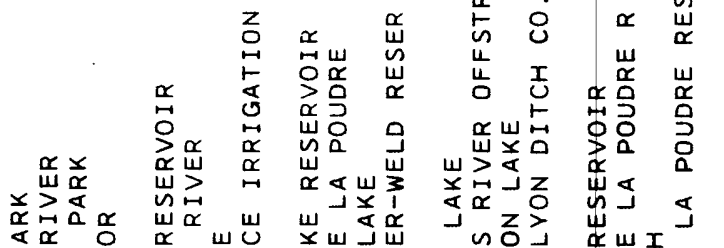

$\frac{x}{\alpha} \underset{x}{x} \frac{\alpha}{\alpha} \frac{\alpha}{0}$

a

w纯造

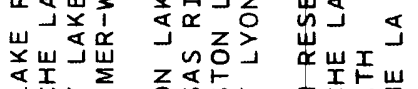

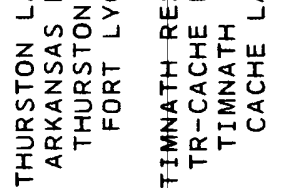

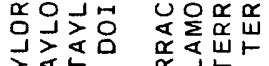

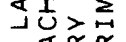

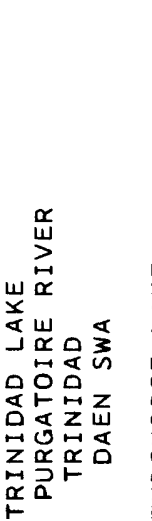

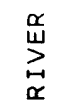

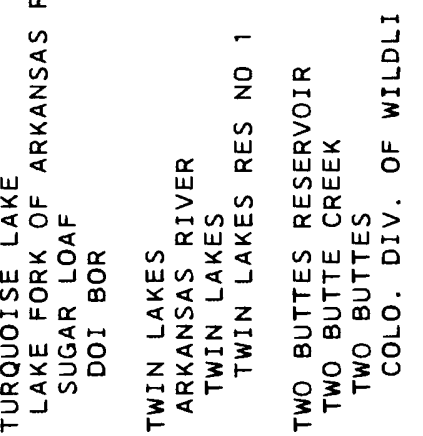




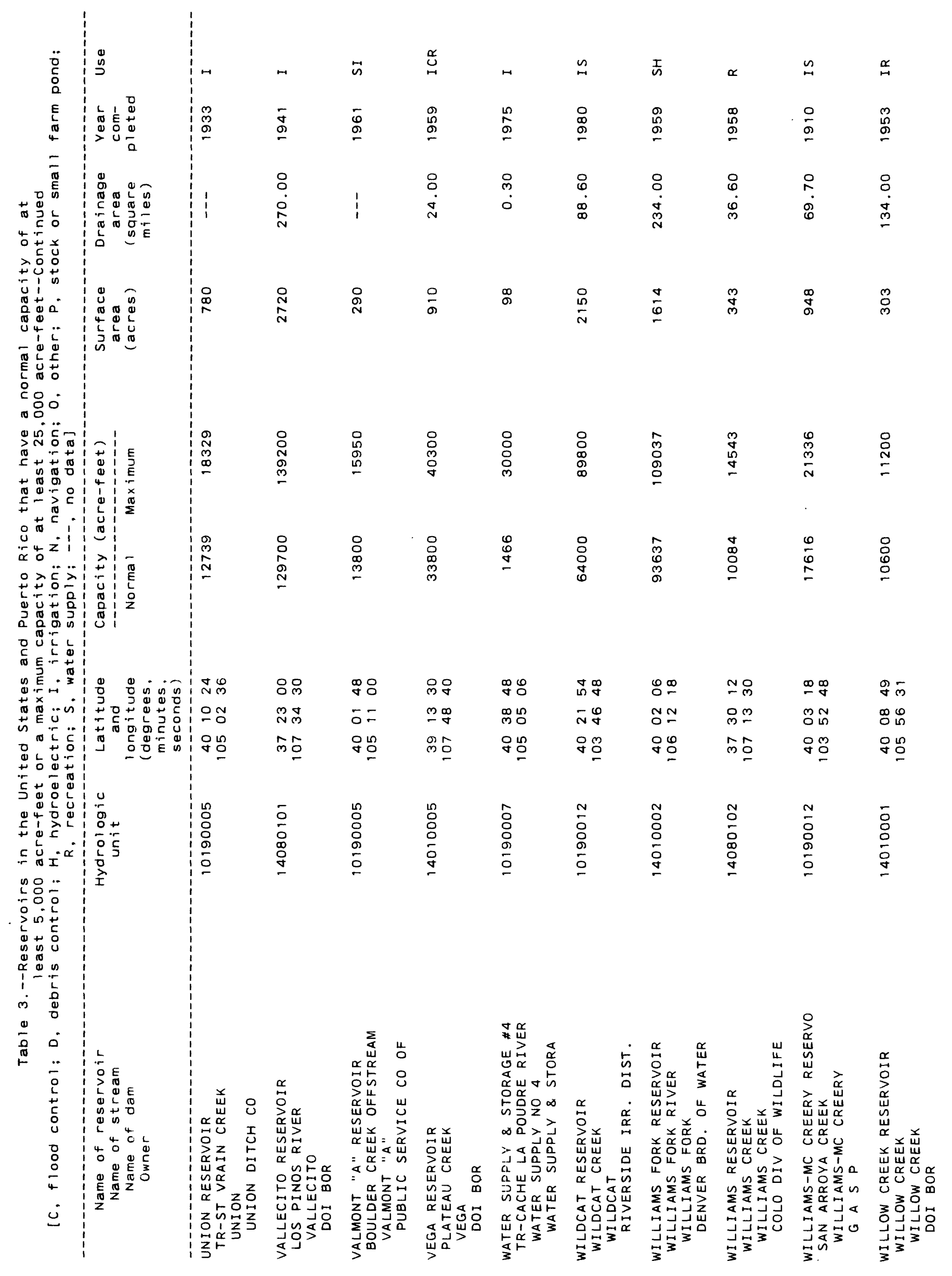




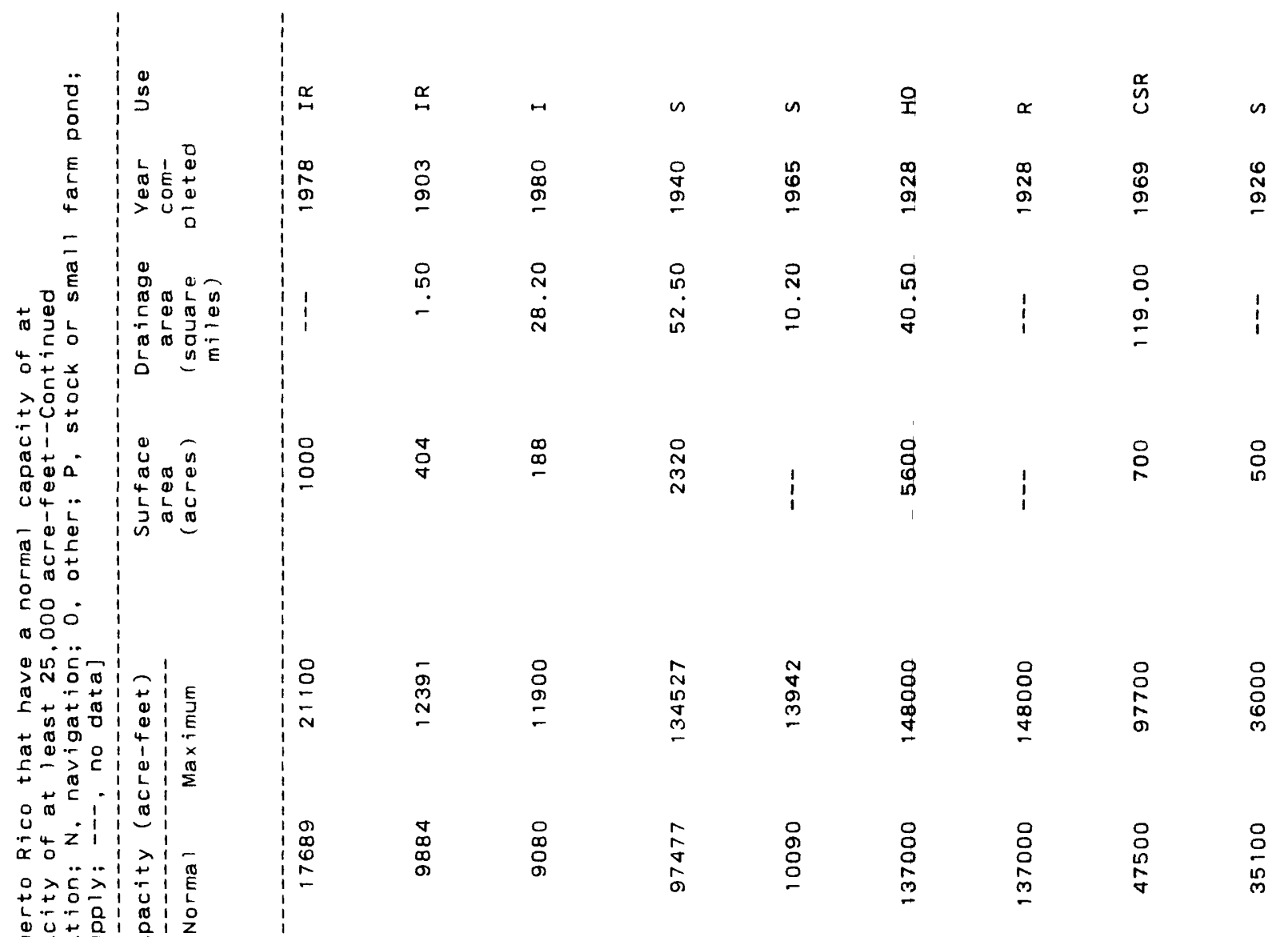

כ.

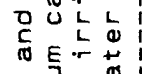

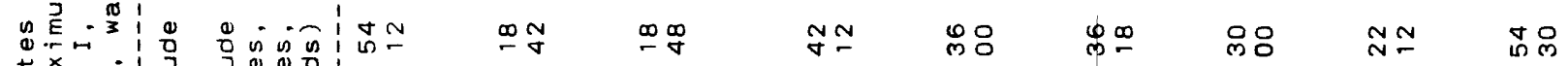

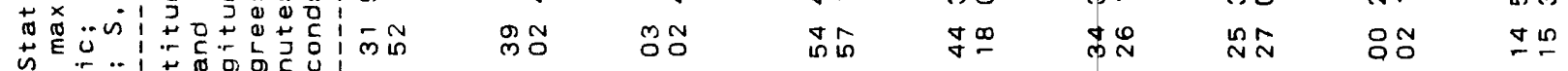

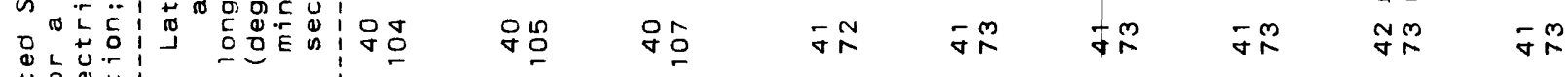

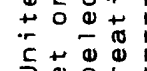

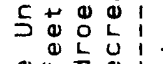

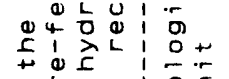

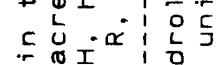

.

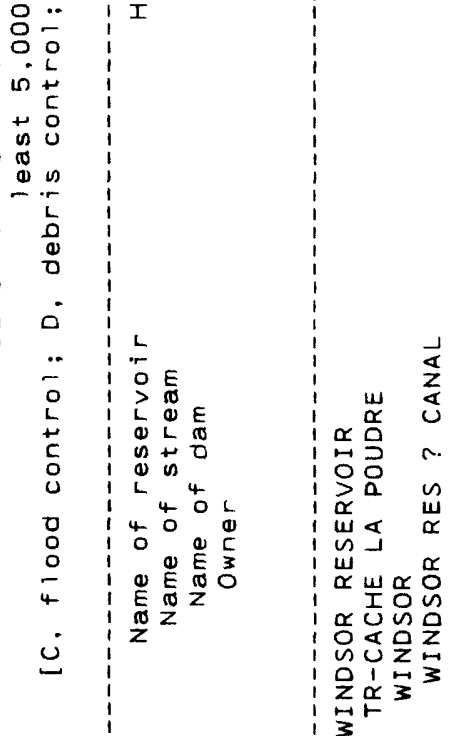

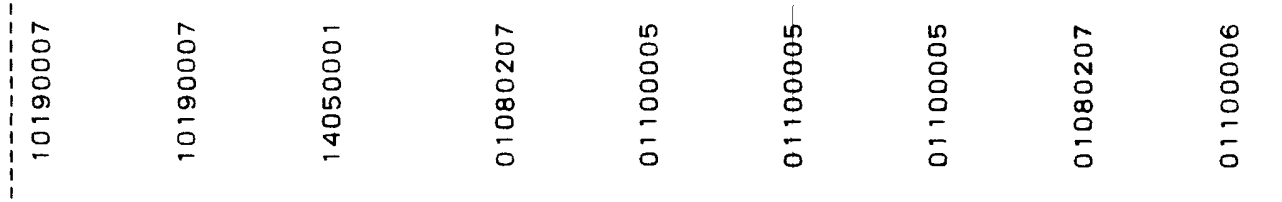

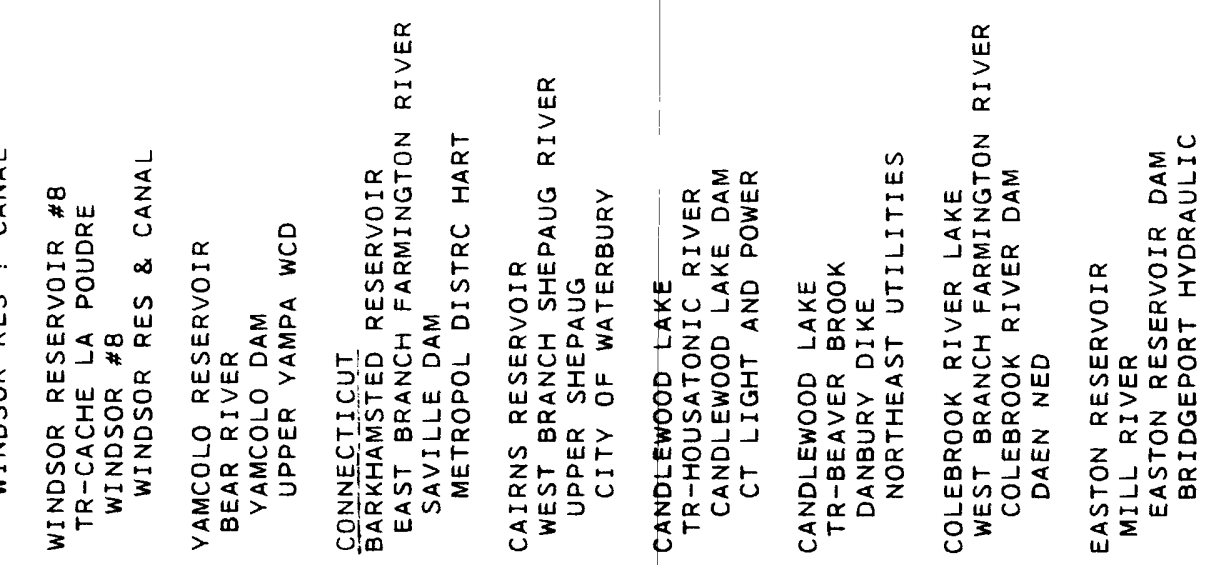




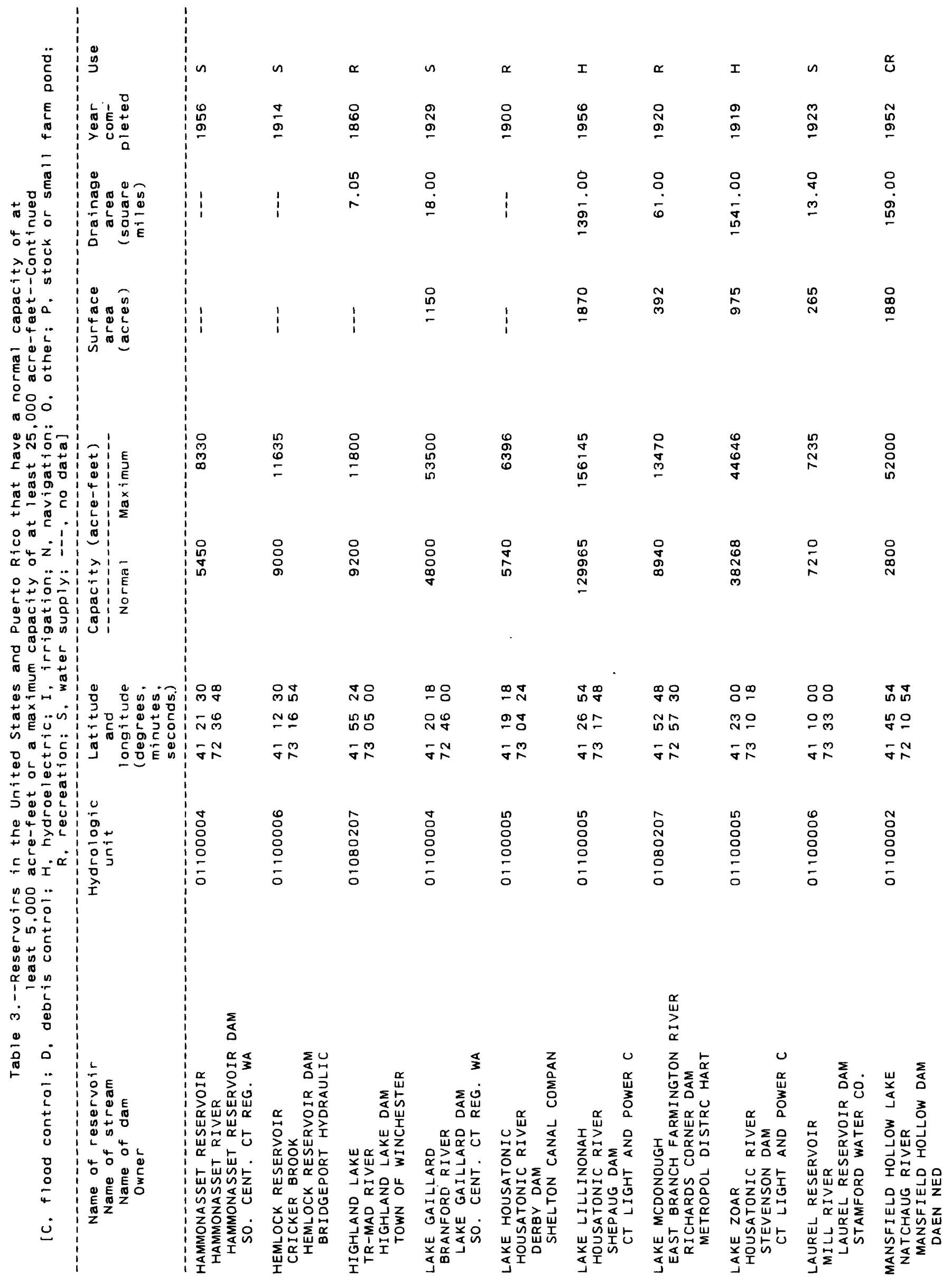




\begin{tabular}{|c|c|c|c|c|c|c|c|}
\hline in & in & s & $\propto$ & $n$ & $n$ & 0 & is \\
\hline$\frac{m}{\sigma}$ & $\stackrel{\infty}{\sigma}$ & $\stackrel{0}{\circ}$ & 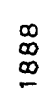 & $\bar{\sigma}$ & 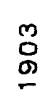 & $\begin{array}{l}: \\
\stackrel{\circ}{\circ}\end{array}$ & $\frac{0}{\sigma}$ \\
\hline$\stackrel{8}{?}$ & 8 & & & 8 & 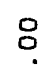 & 으. & \\
\hline$\infty$ & $\underset{m}{\sim}$ & 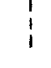 & 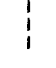 & ஜू & $\therefore$ & $\dot{\circ}$ & 1 \\
\hline 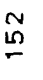 & $\underset{\infty}{i}$ & 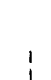 & $\underset{\infty}{\infty}$ & 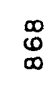 & 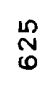 & 吕 & 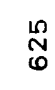 \\
\hline $\begin{array}{l}0 \\
0 \\
0 \\
0\end{array}$ & 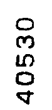 & 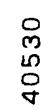 & $\begin{array}{l}\text { \&. } \\
\stackrel{\Delta}{0}\end{array}$ & \begin{tabular}{l} 
: \\
\multirow{N}{*}{}
\end{tabular} & $\begin{array}{l}\stackrel{0}{0} \\
\text { - }\end{array}$ & $\begin{array}{l}\text { : } \\
\text { J }\end{array}$ & 品 \\
\hline$\hat{\mathbf{s}}$ & 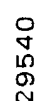 & 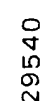 & $\begin{array}{l}\text { D } \\
\stackrel{1}{1}\end{array}$ & $\begin{array}{l}: \\
: \\
0\end{array}$ & $\begin{array}{l}: \\
:\end{array}$ & $\stackrel{n}{ }$ & $\frac{8}{1}$ \\
\hline
\end{tabular}

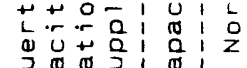

a o on

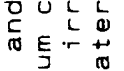

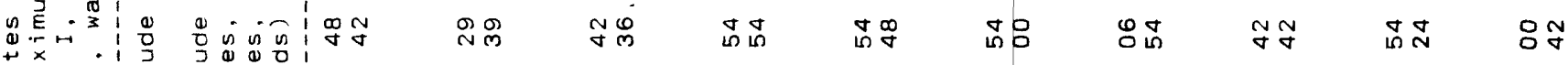

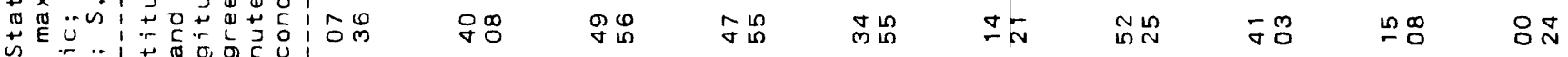

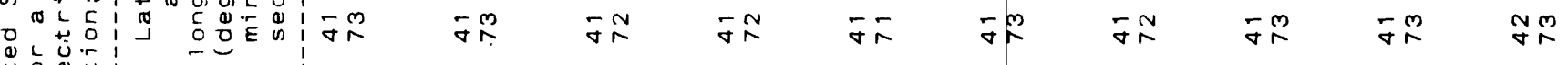

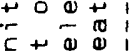

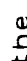

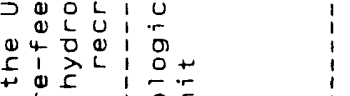

c

n을

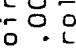

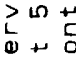

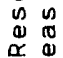

$1-$

ल

西

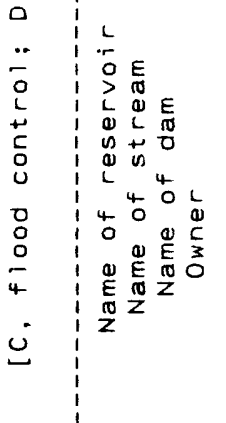

$\mid \begin{array}{ll}\circ & 0 \\ 8 & 8 \\ 0 & 0 \\ 0 & 0\end{array}$

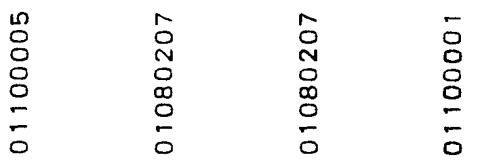

$\begin{array}{lll}: & \\ \vdots & 0 \\ 0 & 0 \\ 0 & 0 \\ 0 & 0 \\ 0 & 0\end{array}$

$\frac{2}{\circ}$

$\begin{array}{ll}\stackrel{0}{0} & \stackrel{0}{\circ} \\ \frac{0}{0} & \frac{0}{0} \\ \frac{0}{0} & 0\end{array}$

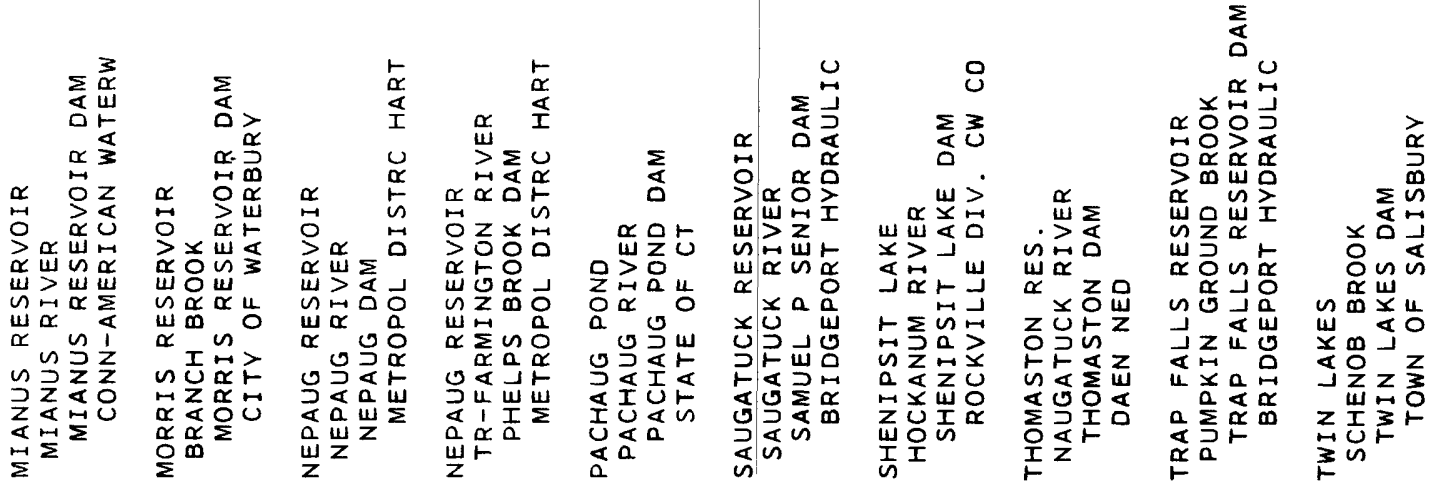




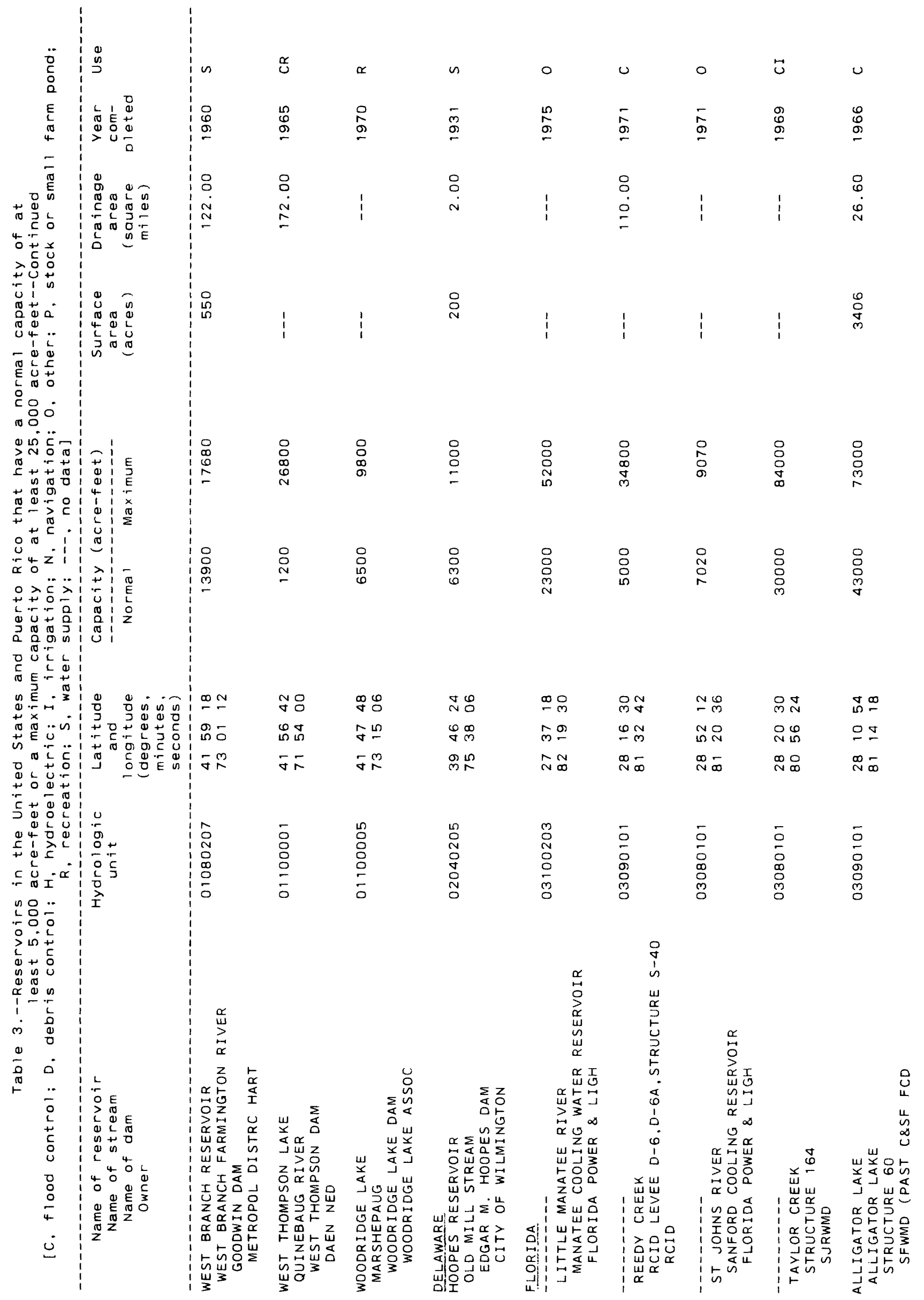




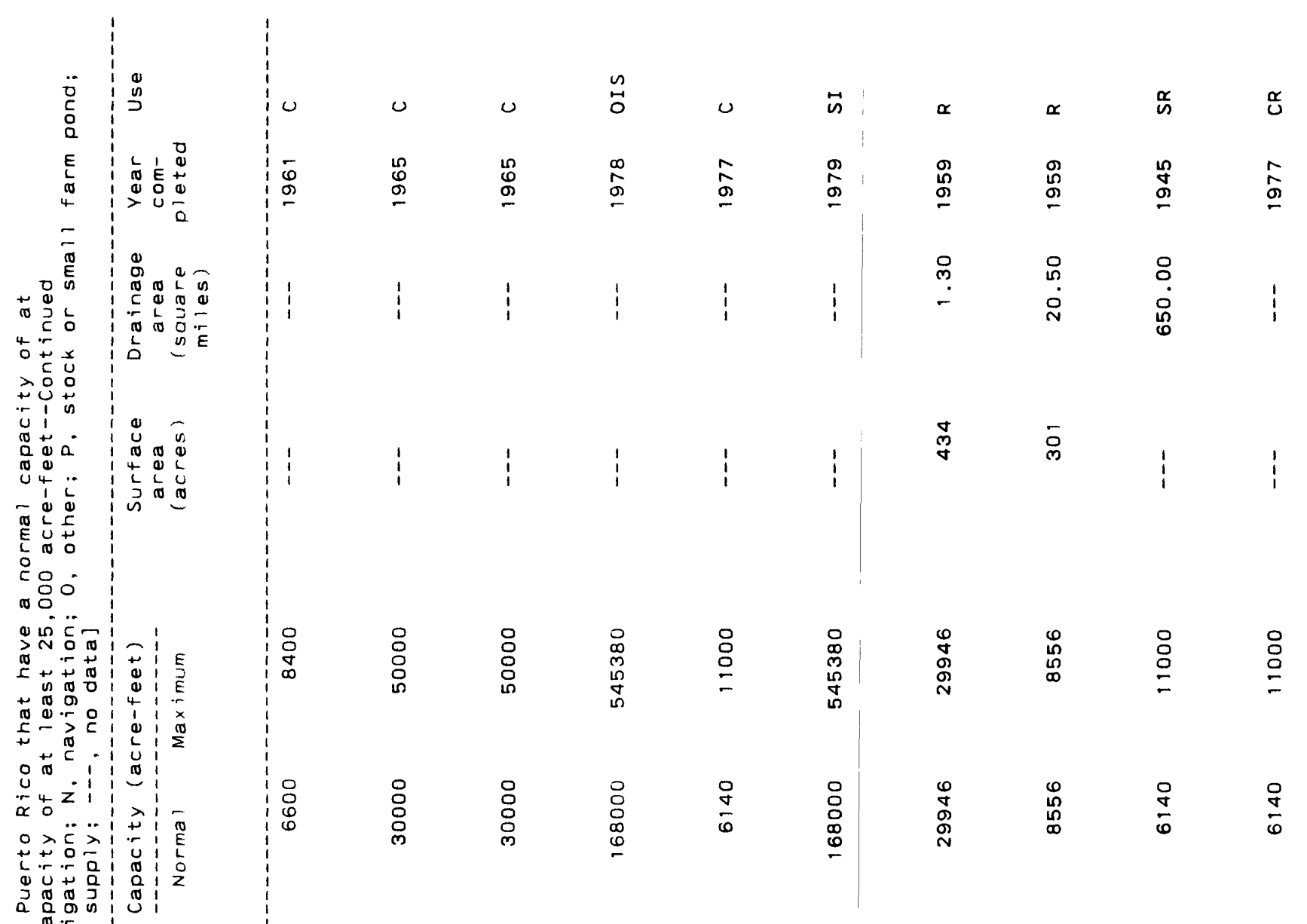

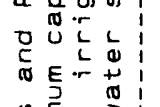

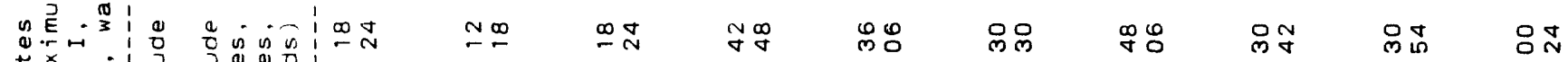

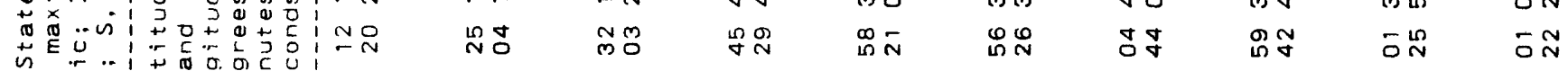

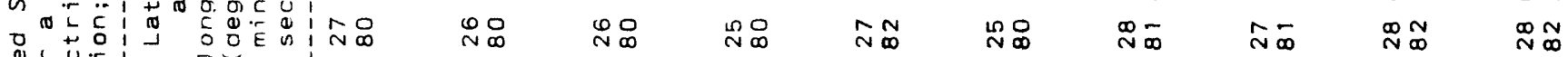

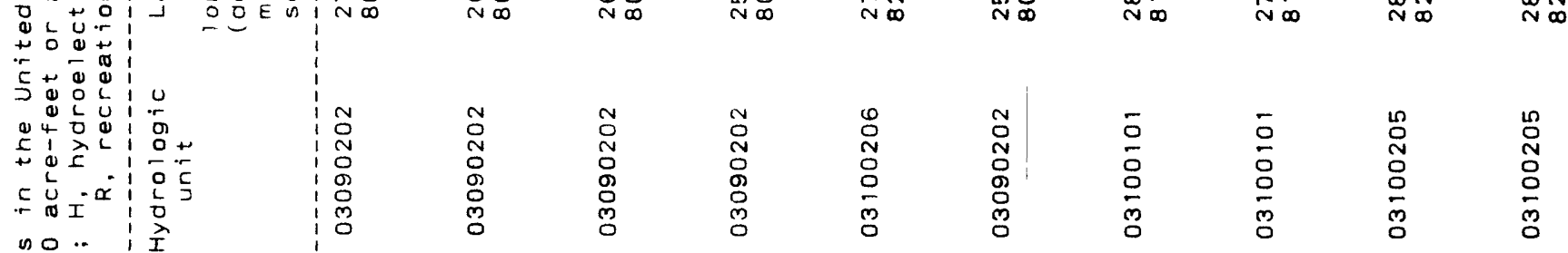

3 is

ब

(5)

$\alpha \underset{c}{0}$

i.

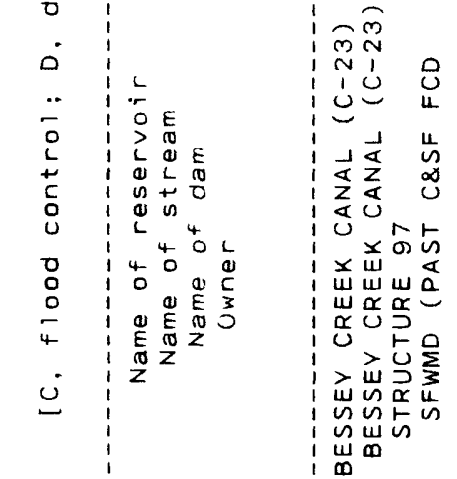

سב

ta $\quad \frac{1}{a}$

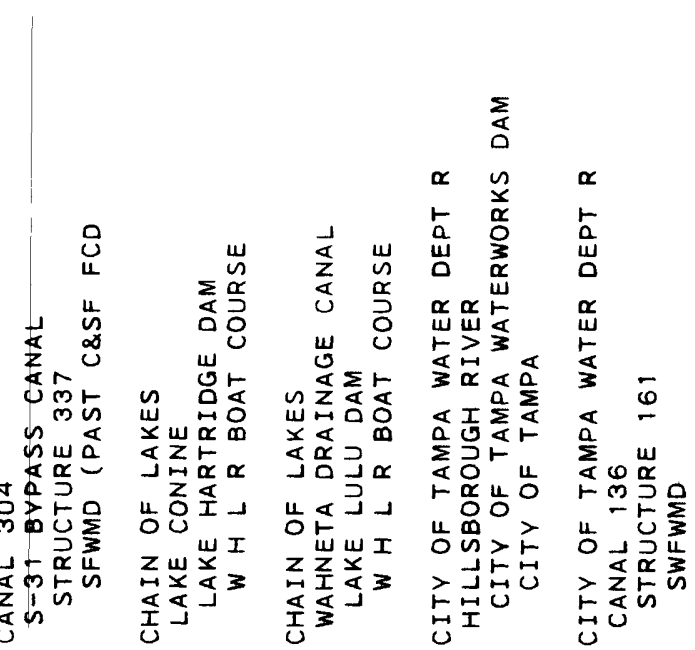




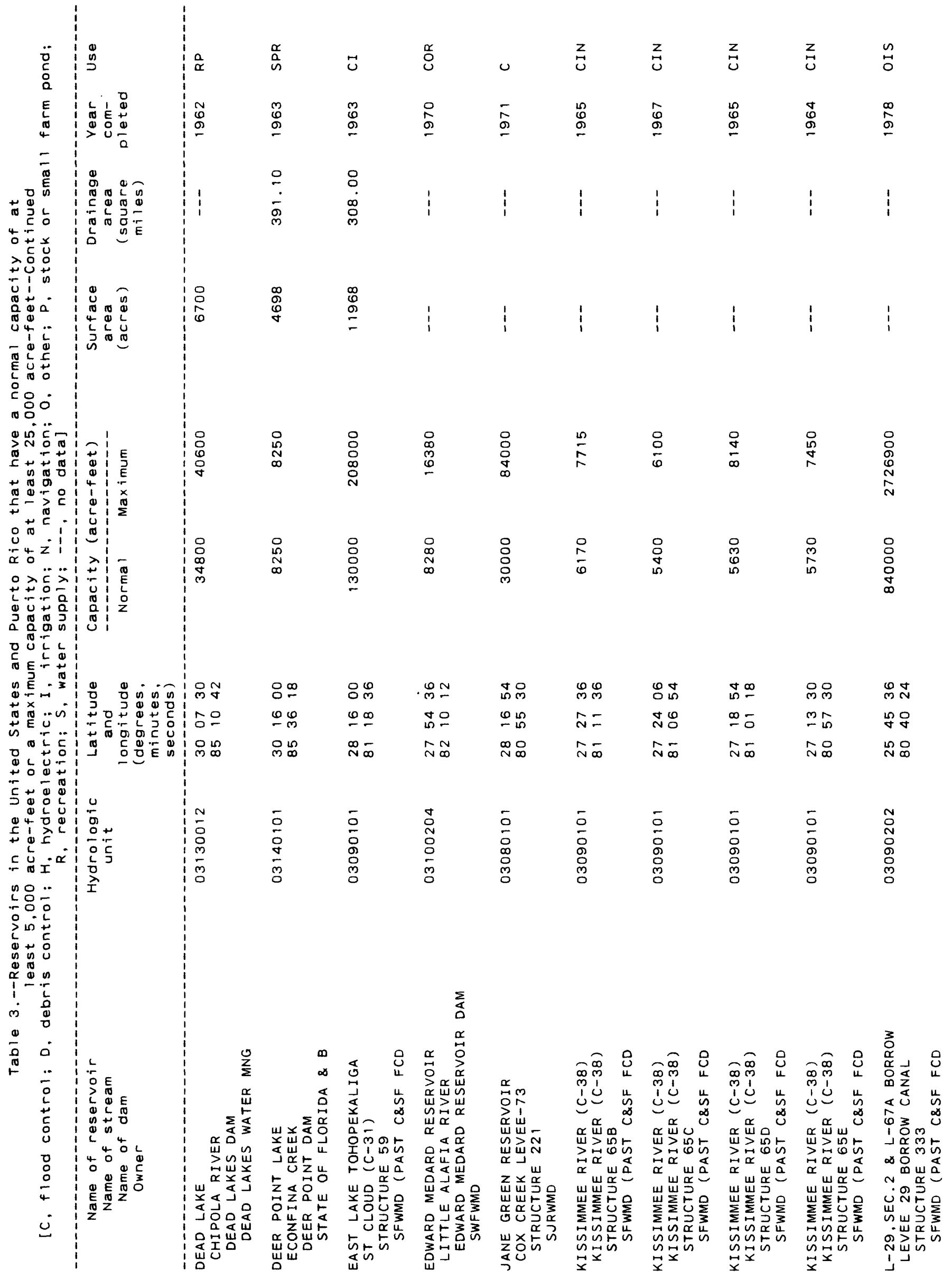




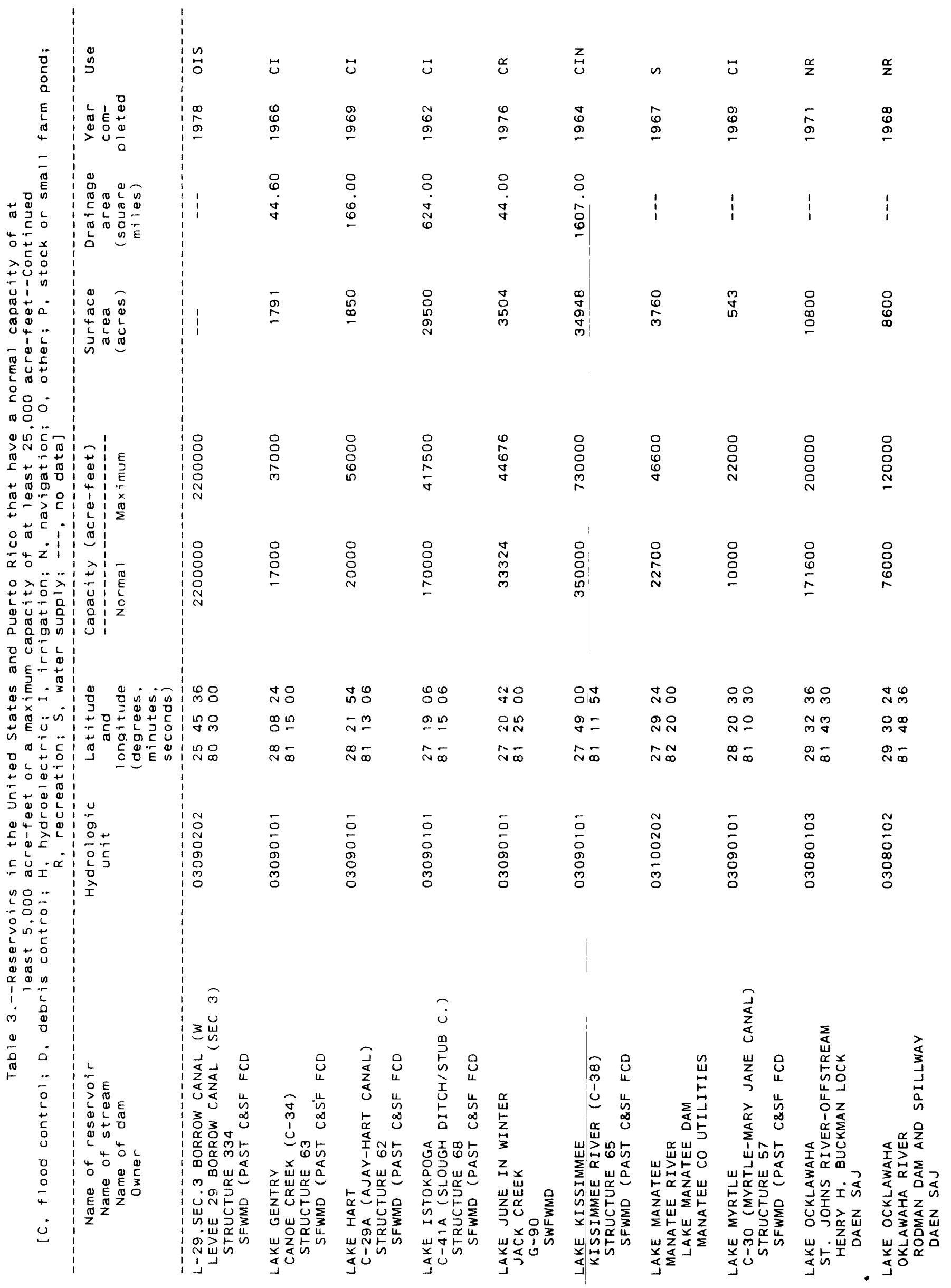




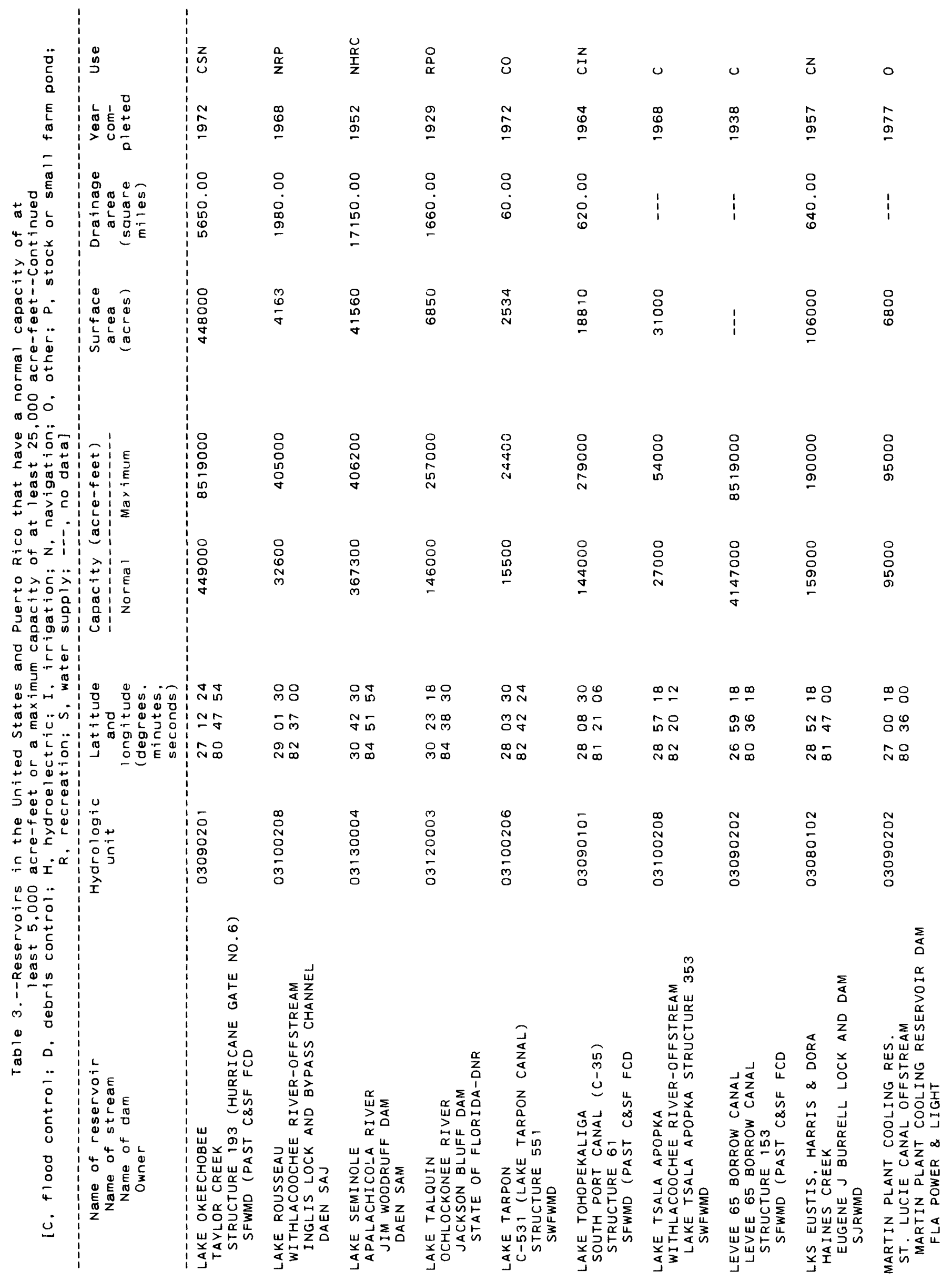




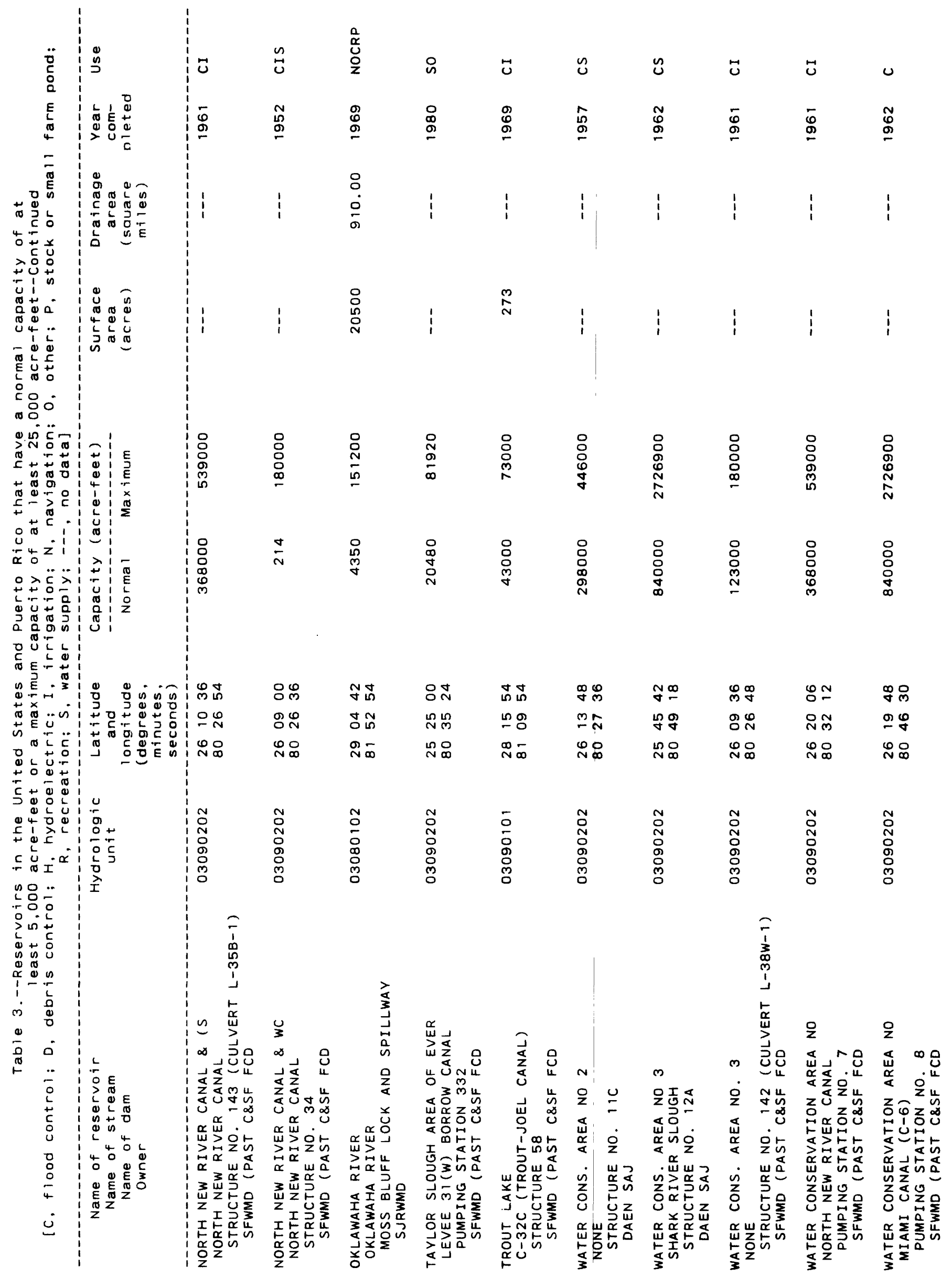




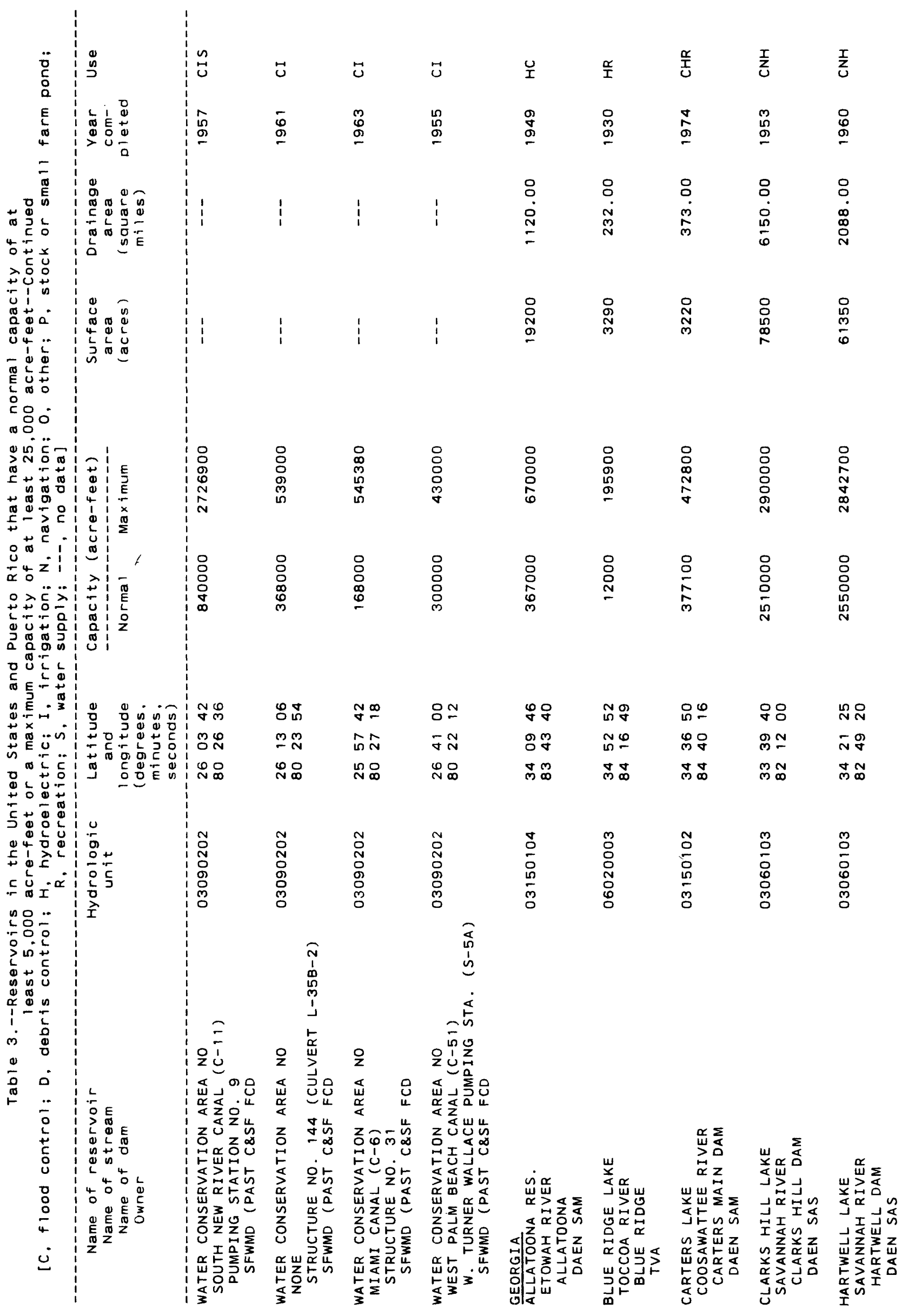



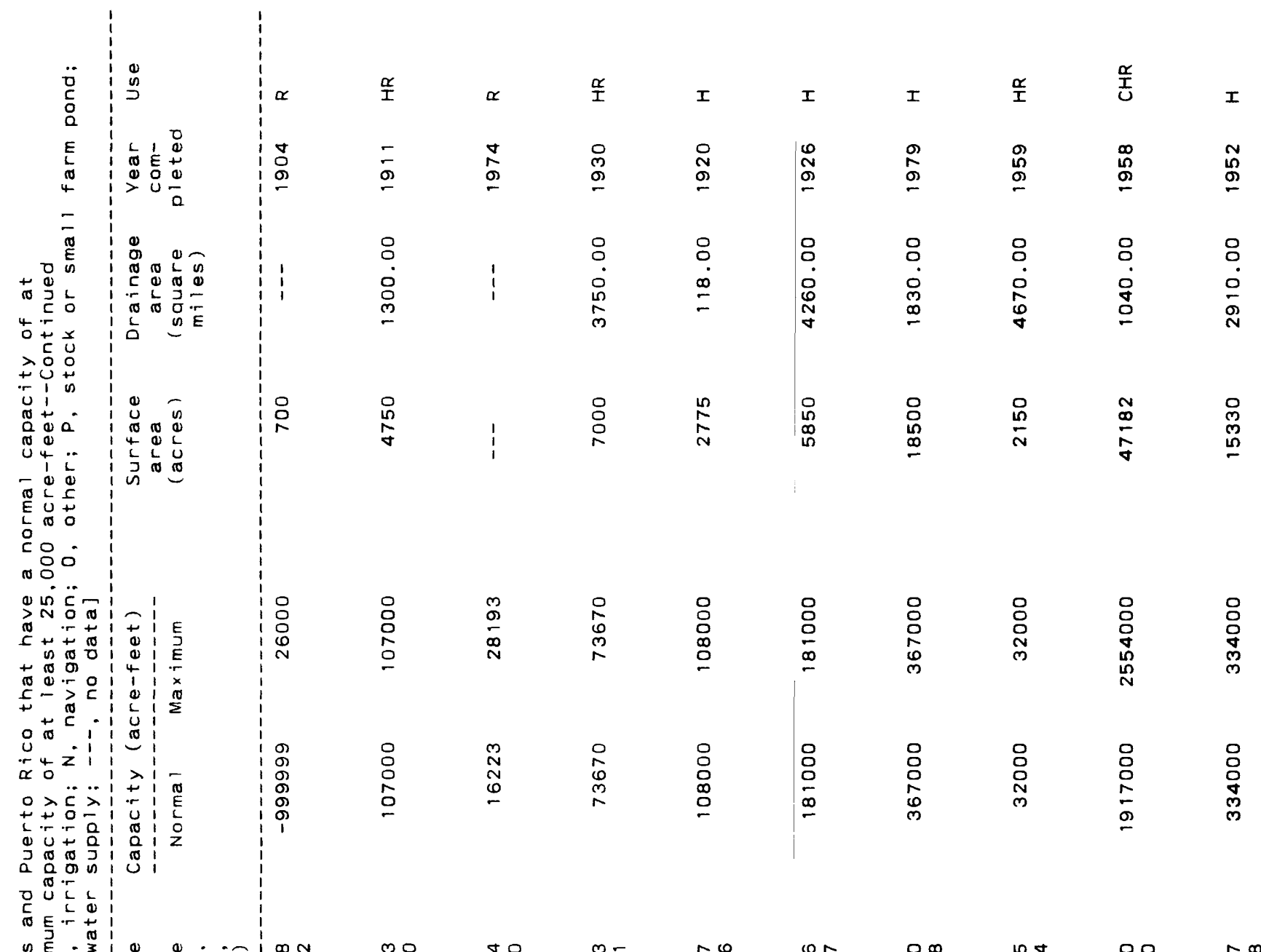

:

20ำ

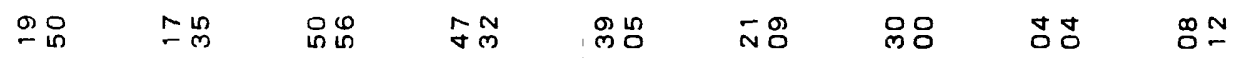

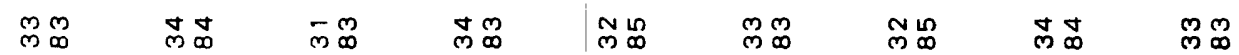

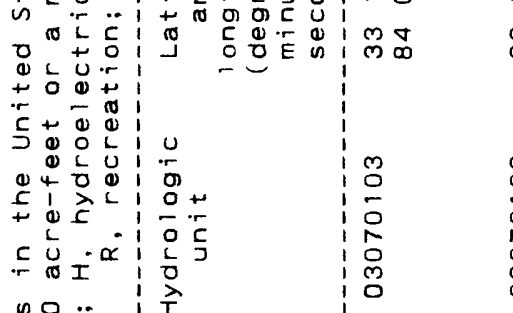

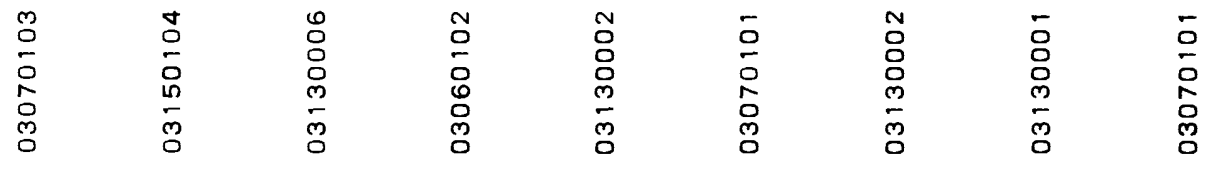

$$
\begin{aligned}
& n \\
& 2 \\
& 0 \\
& 0 \\
& 2 \\
& 0 \\
& 0 \\
& 0 \\
& 0 \\
& \vdots \\
& \vdots \\
& n \\
& 0 \\
& 0 \\
& 0 \\
& 10 \\
& 5
\end{aligned}
$$$$
80
$$$$
\text { in }+
$$$$
\underset{1}{1} \stackrel{0}{\infty}
$$$$
\frac{10}{10}
$$

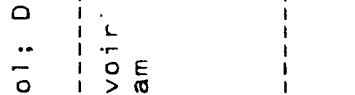

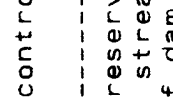

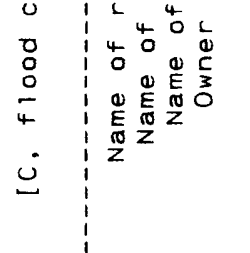
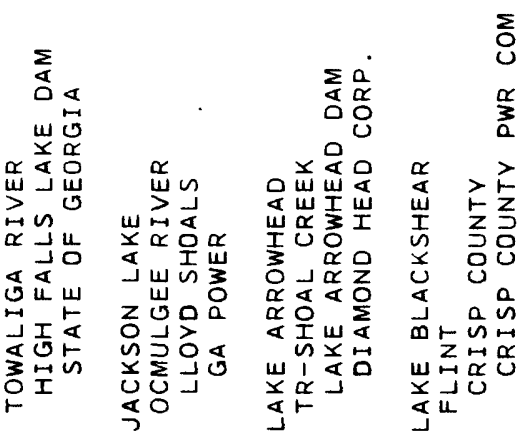

$\Sigma_{0}$
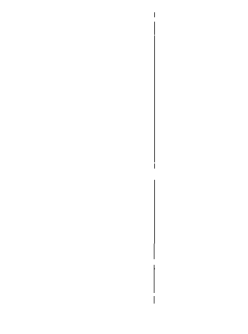


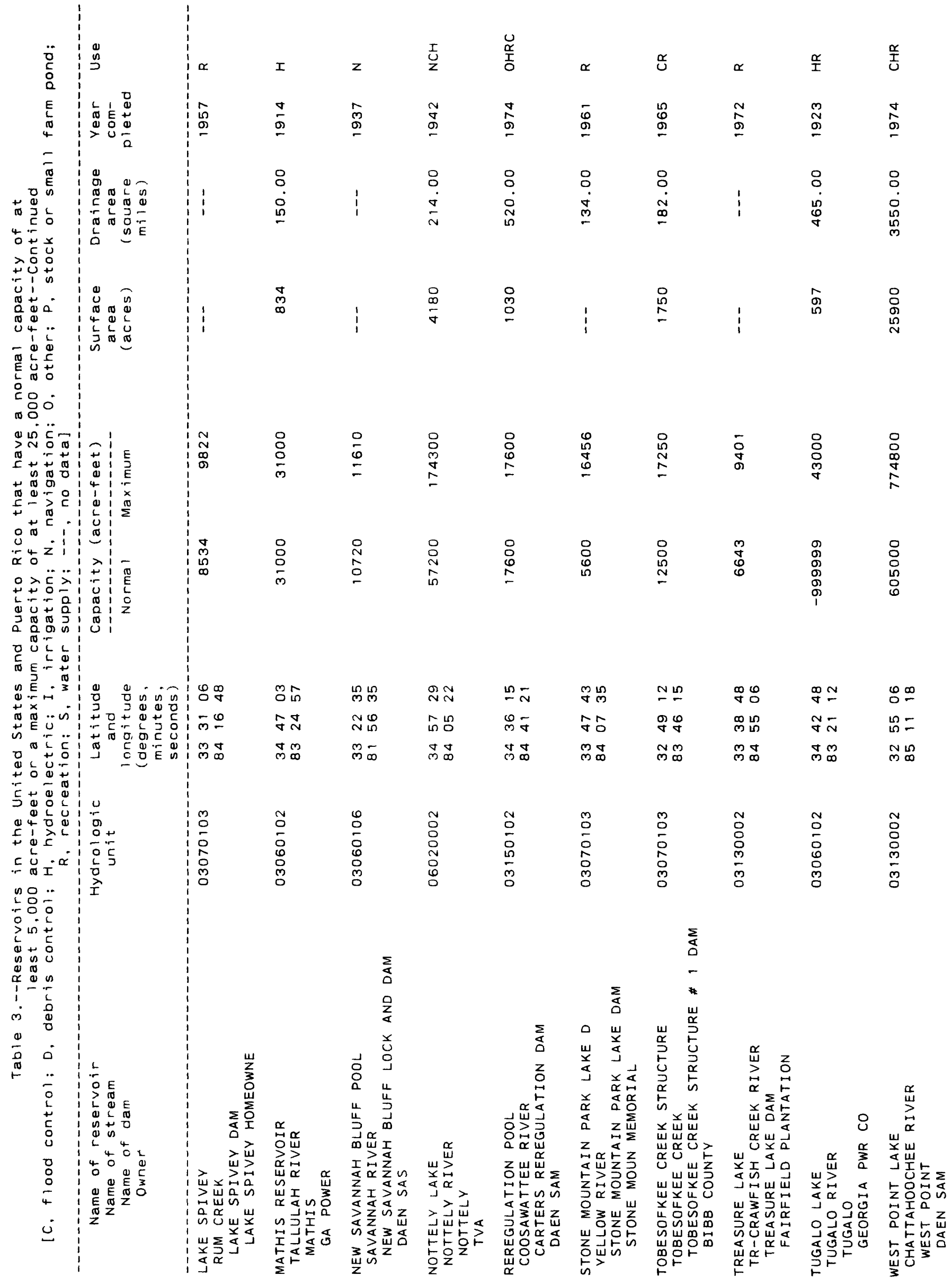




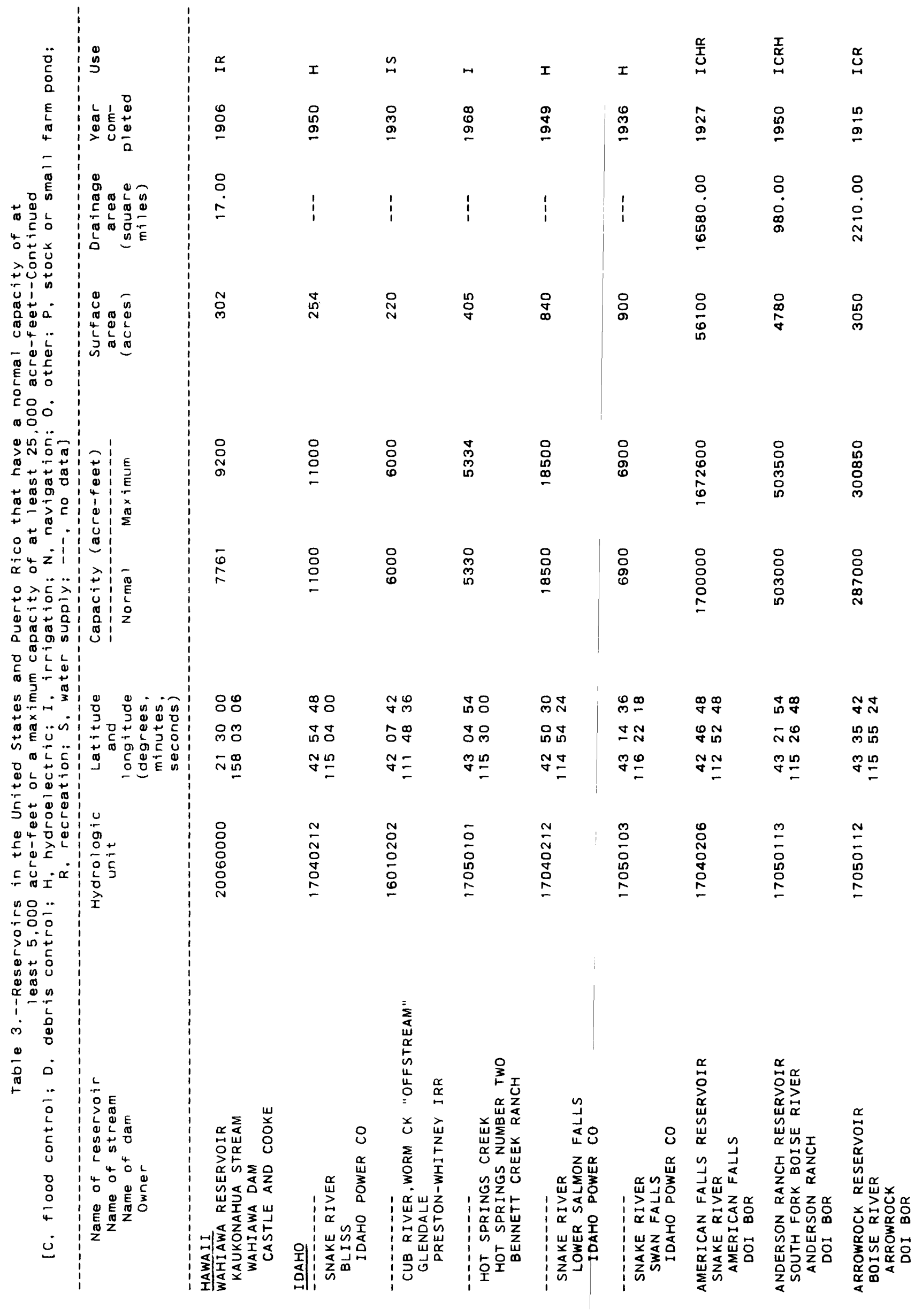




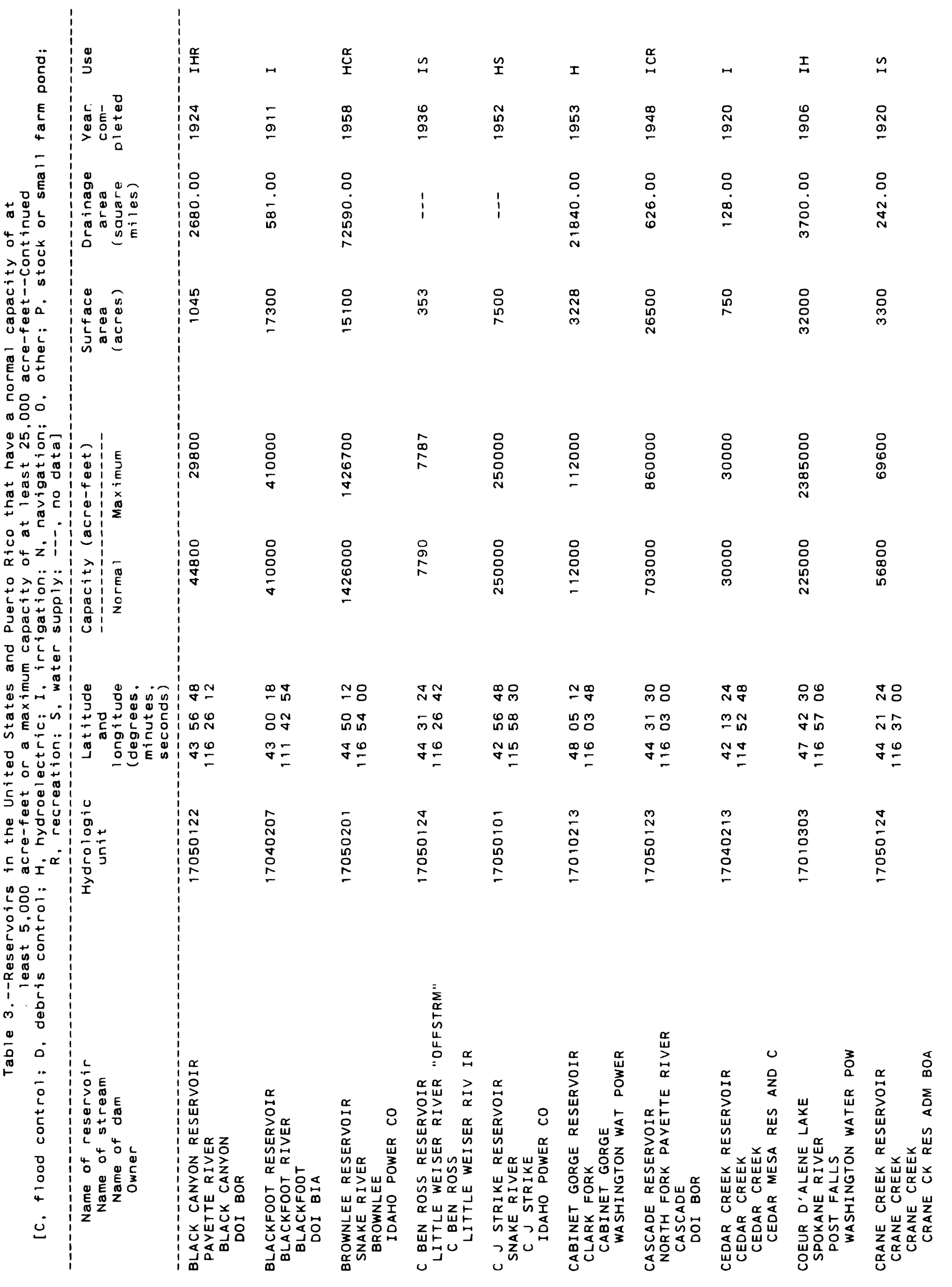




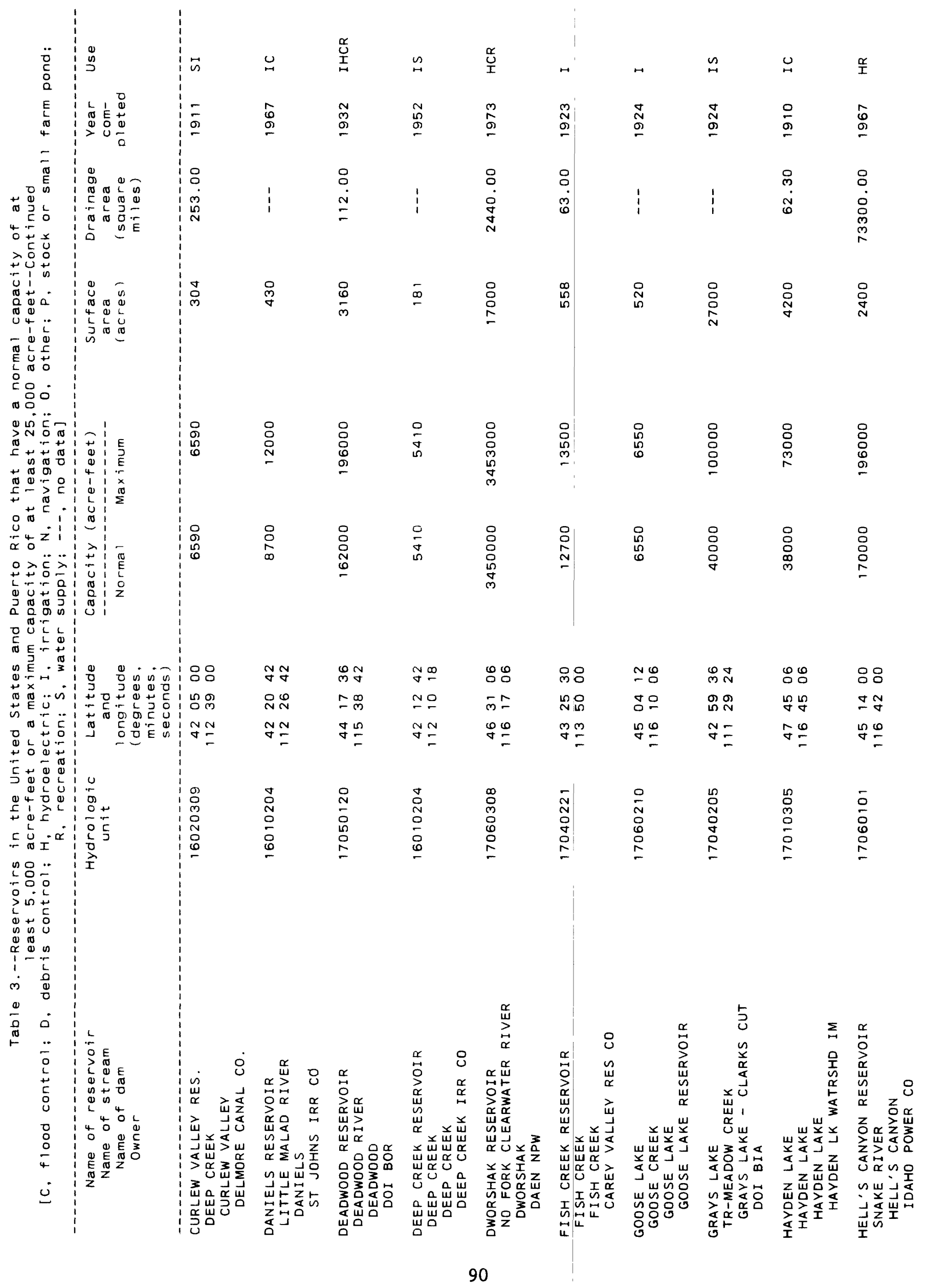




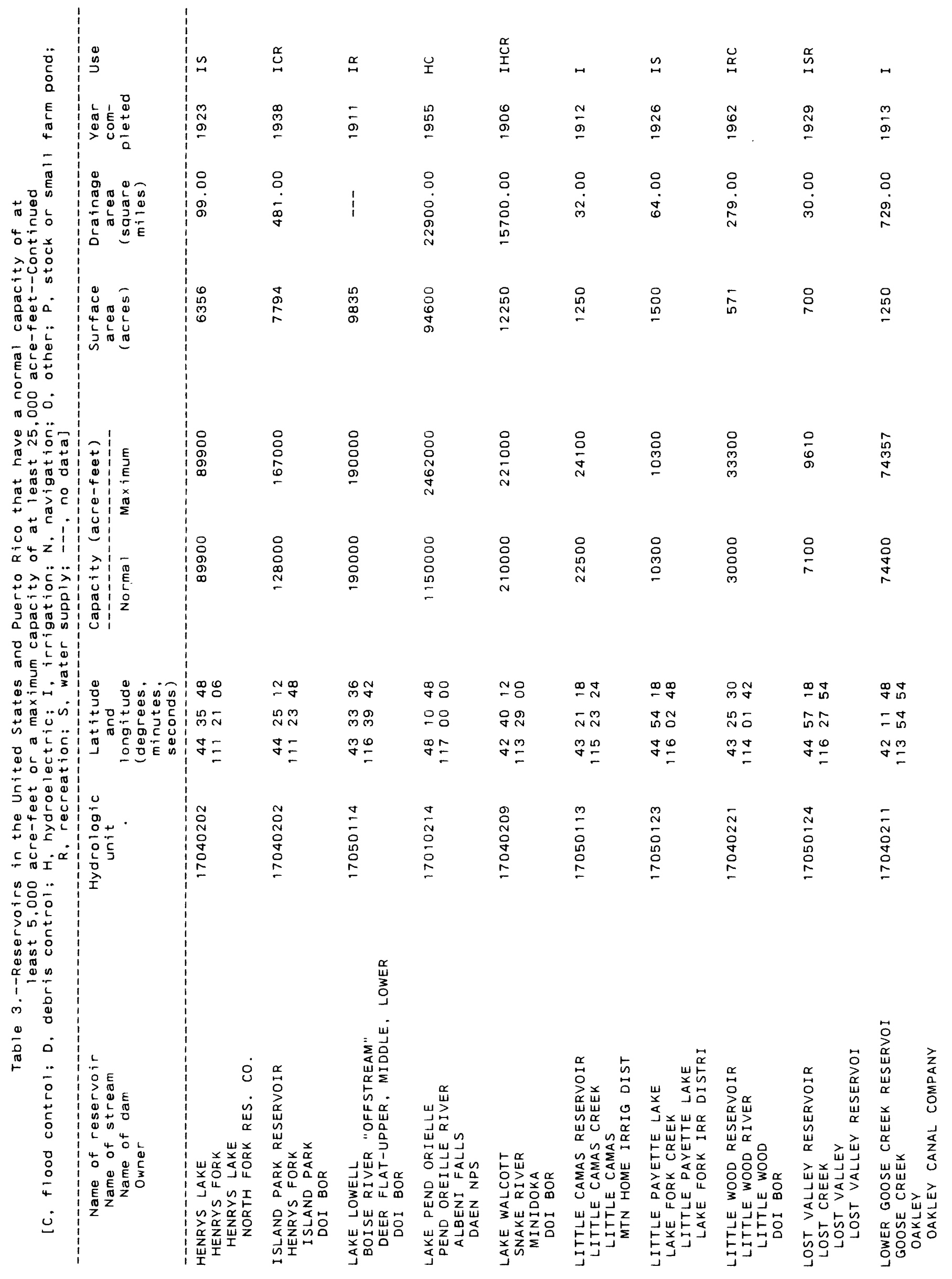




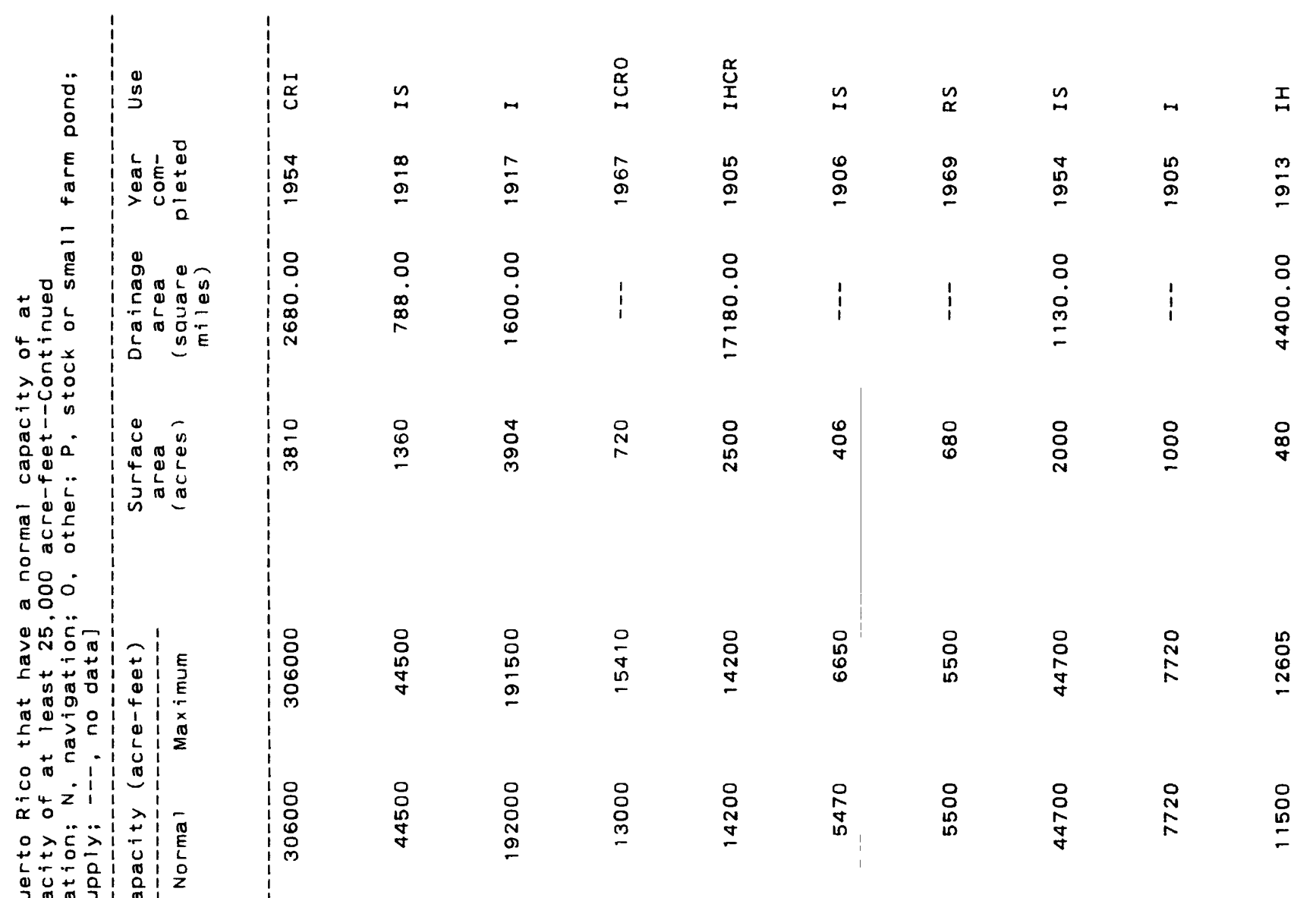

\begin{tabular}{|c|c|c|c|c|c|c|c|c|}
\hline$\stackrel{N}{N}$ & $\stackrel{\infty}{-}$ & 足 & $\stackrel{\nabla}{\sim} \underset{\sim}{\infty}$ & 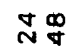 & ํㅛ & $\stackrel{\Delta}{N}$ & 용 & $\stackrel{M}{N}$ \\
\hline in & $\stackrel{\mathscr{D}}{=} \overline{\mathrm{N}}$ & N $_{\text {D }}^{m}$ & м’응 & 욤 & 응으 & กิ & 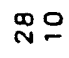 & $\mathscr{\sim}$ \\
\hline$\stackrel{m}{\square}$ & $\stackrel{m}{g}=$ & 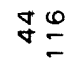 & $\underset{\forall}{\stackrel{V}{\Xi}}$ & $\stackrel{m}{g}$ & $\underset{\gamma}{\sim} \underline{\sigma}$ & $\stackrel{M}{g} \cong$ & $\mathcal{\nabla} \Xi$ & \\
\hline
\end{tabular}

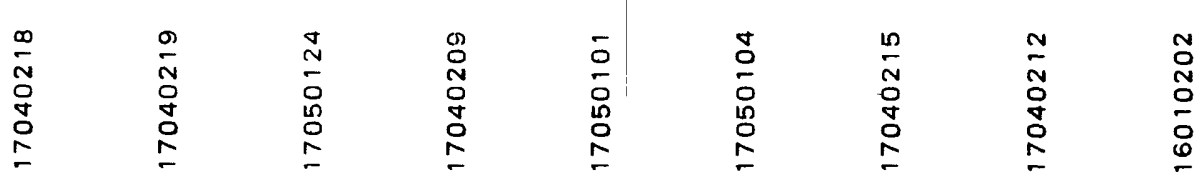

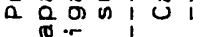

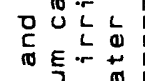

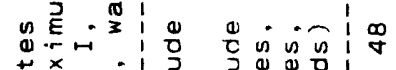

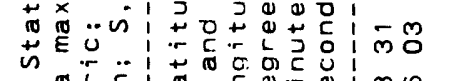

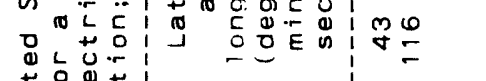

:

$-\pi$

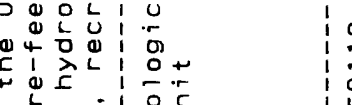

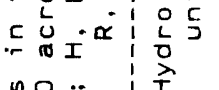

nᄋㅛ민

웅.

a)

is

年

i.

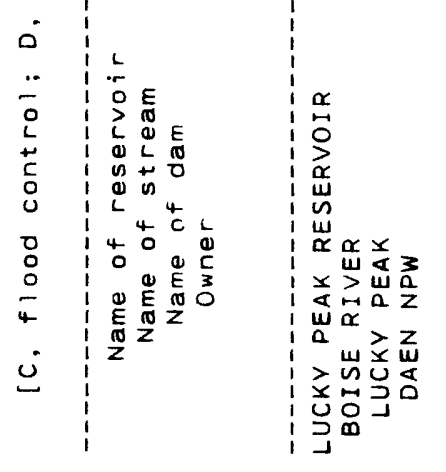

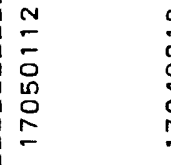

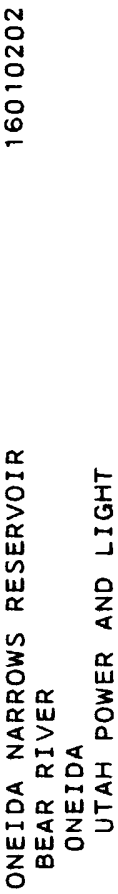




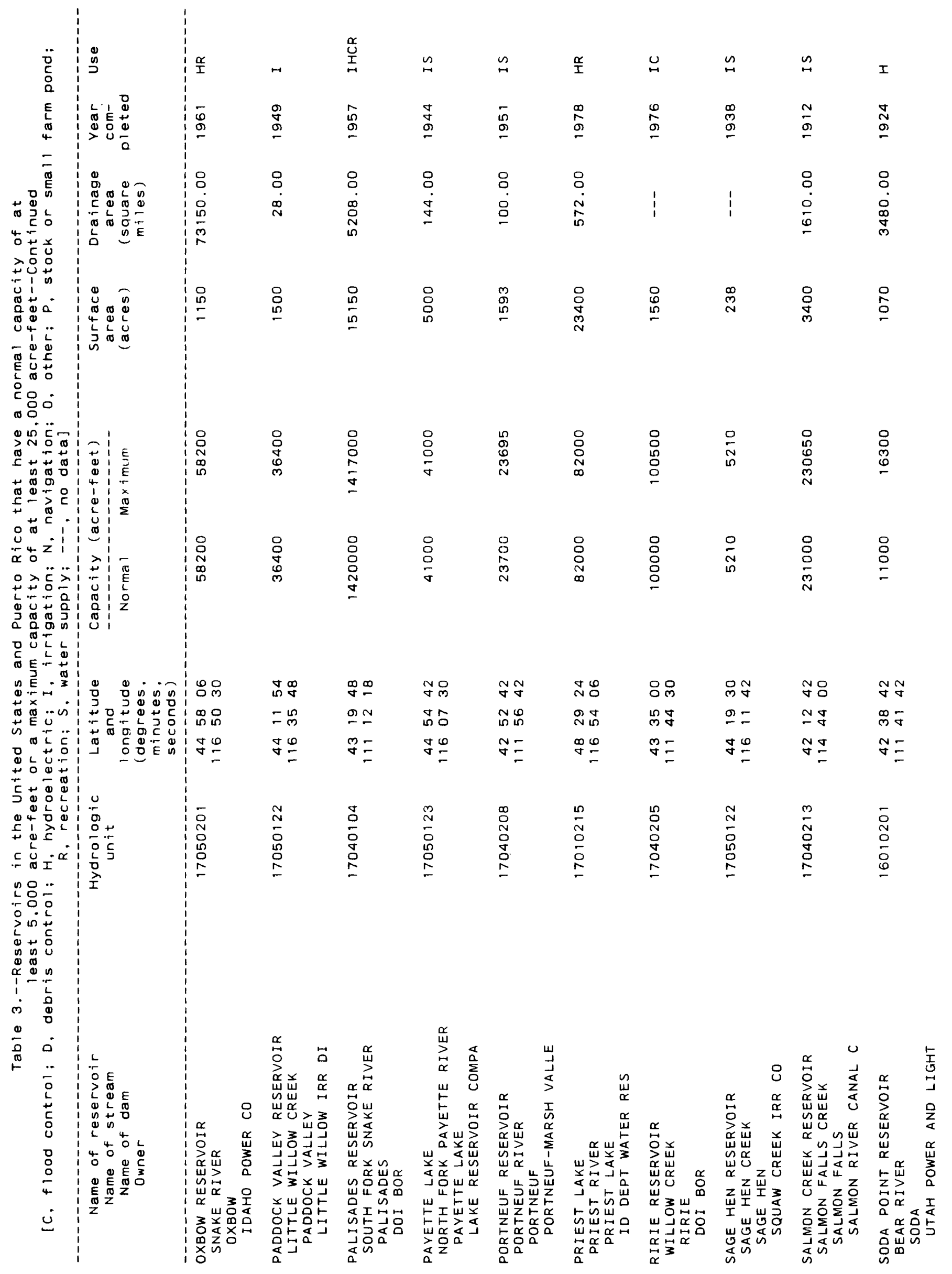




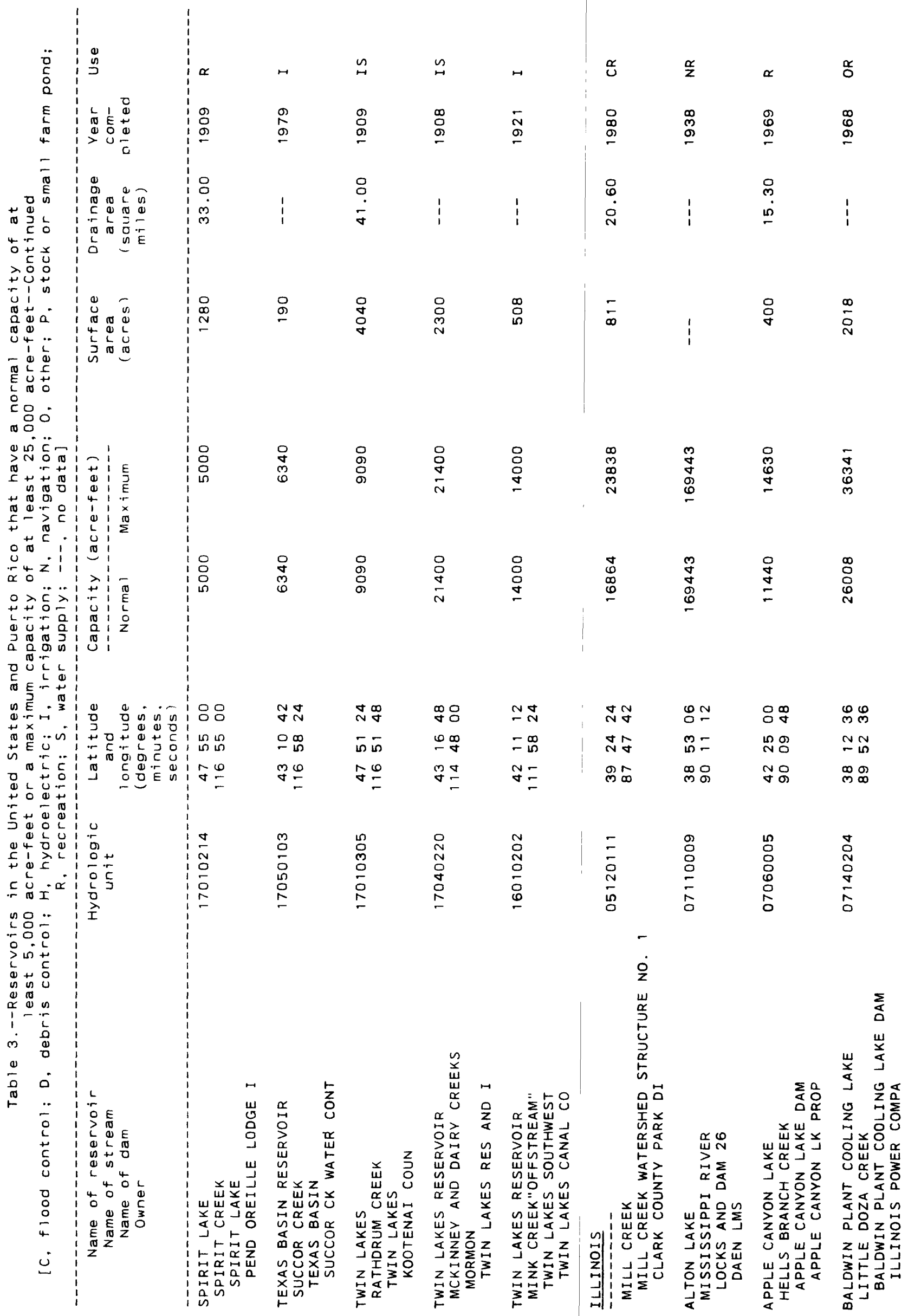




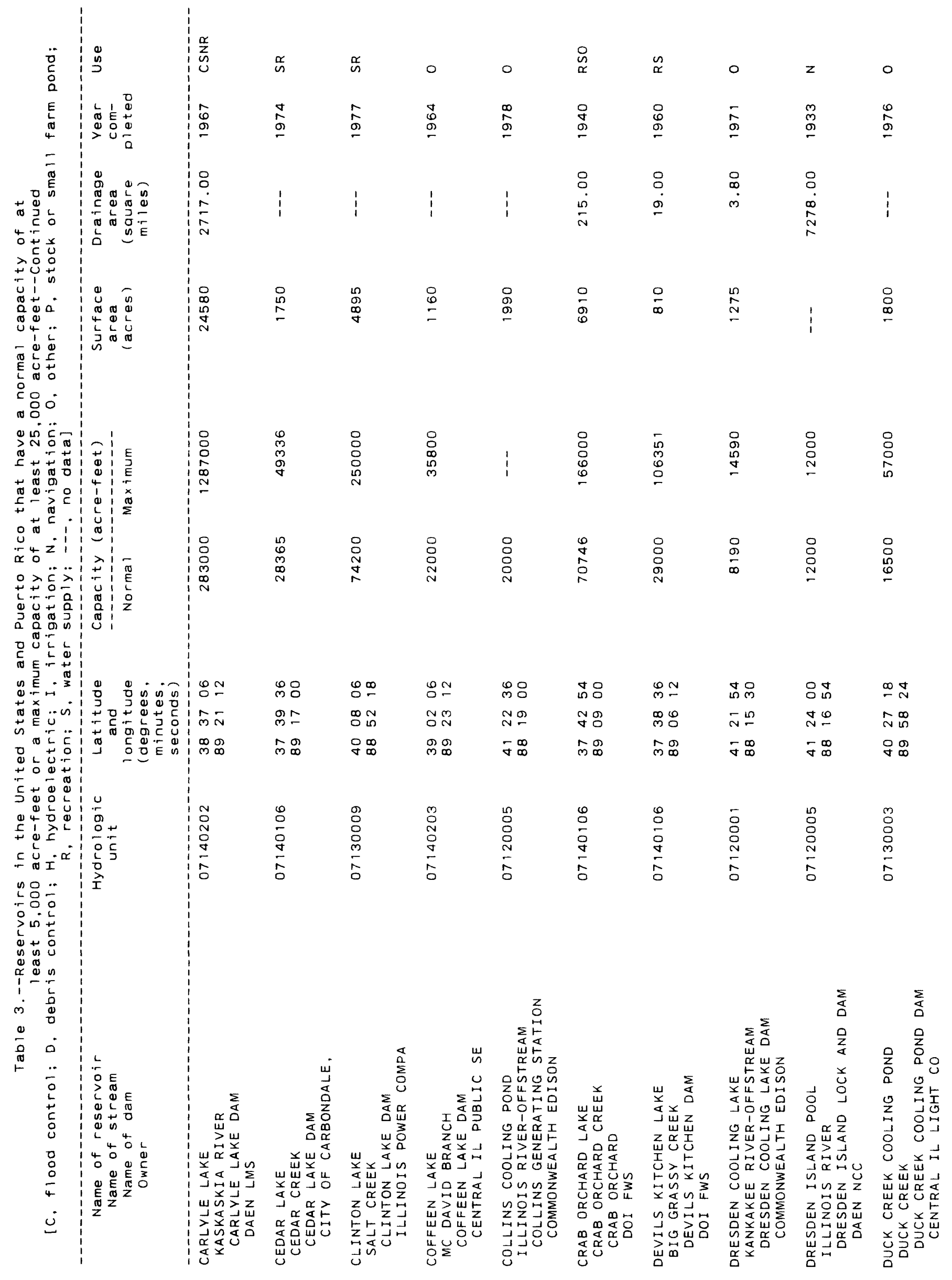




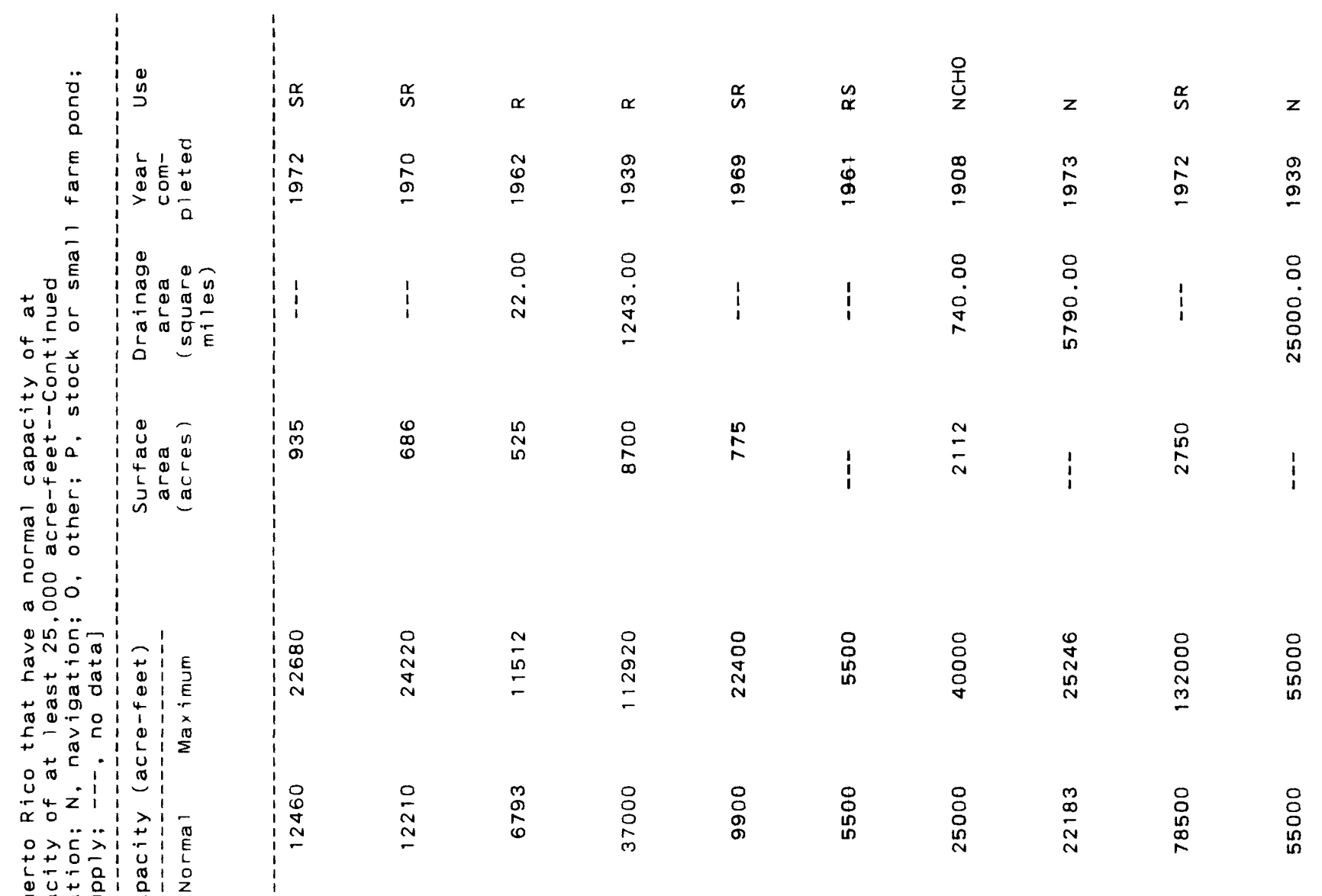

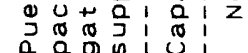

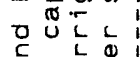

क $E$

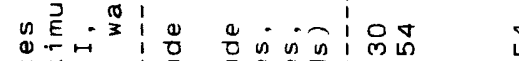

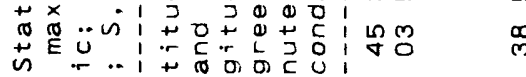

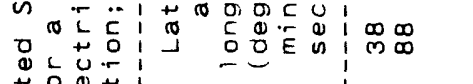

ก็ำ

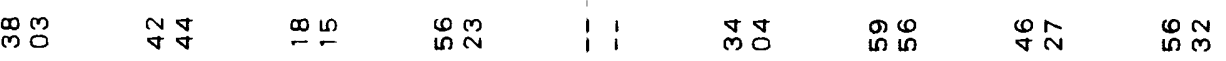

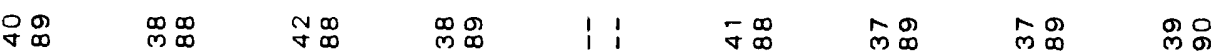

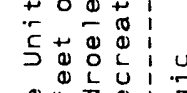

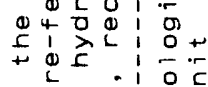

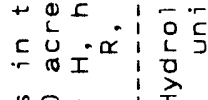

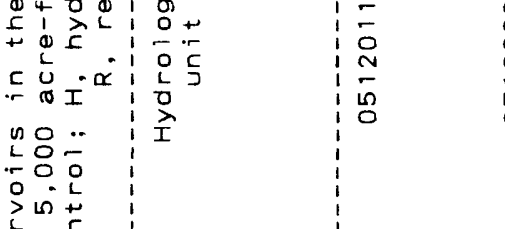

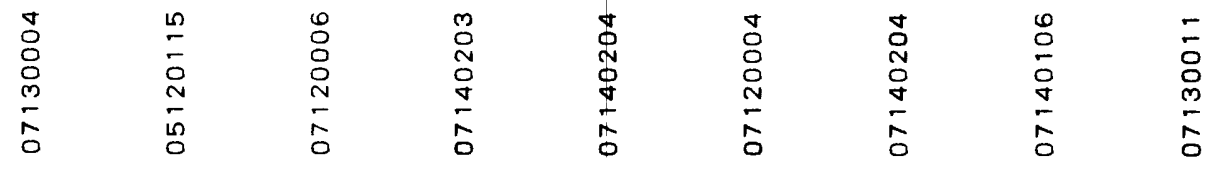
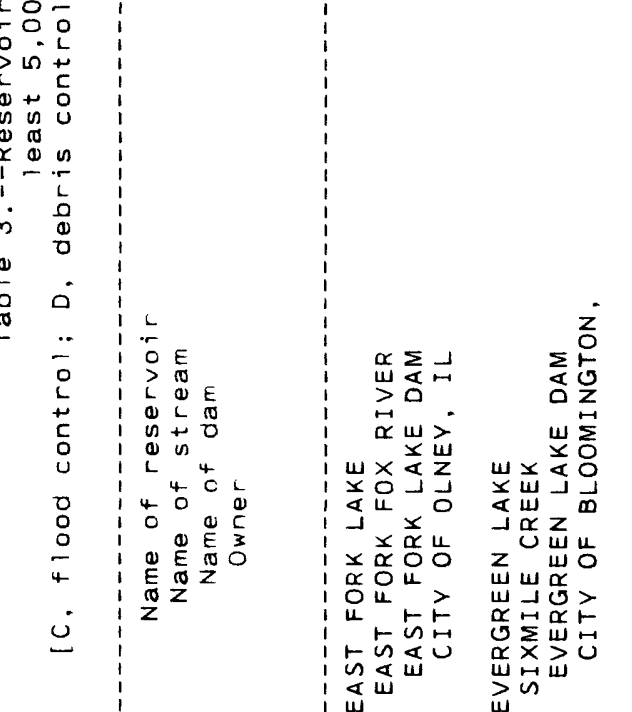


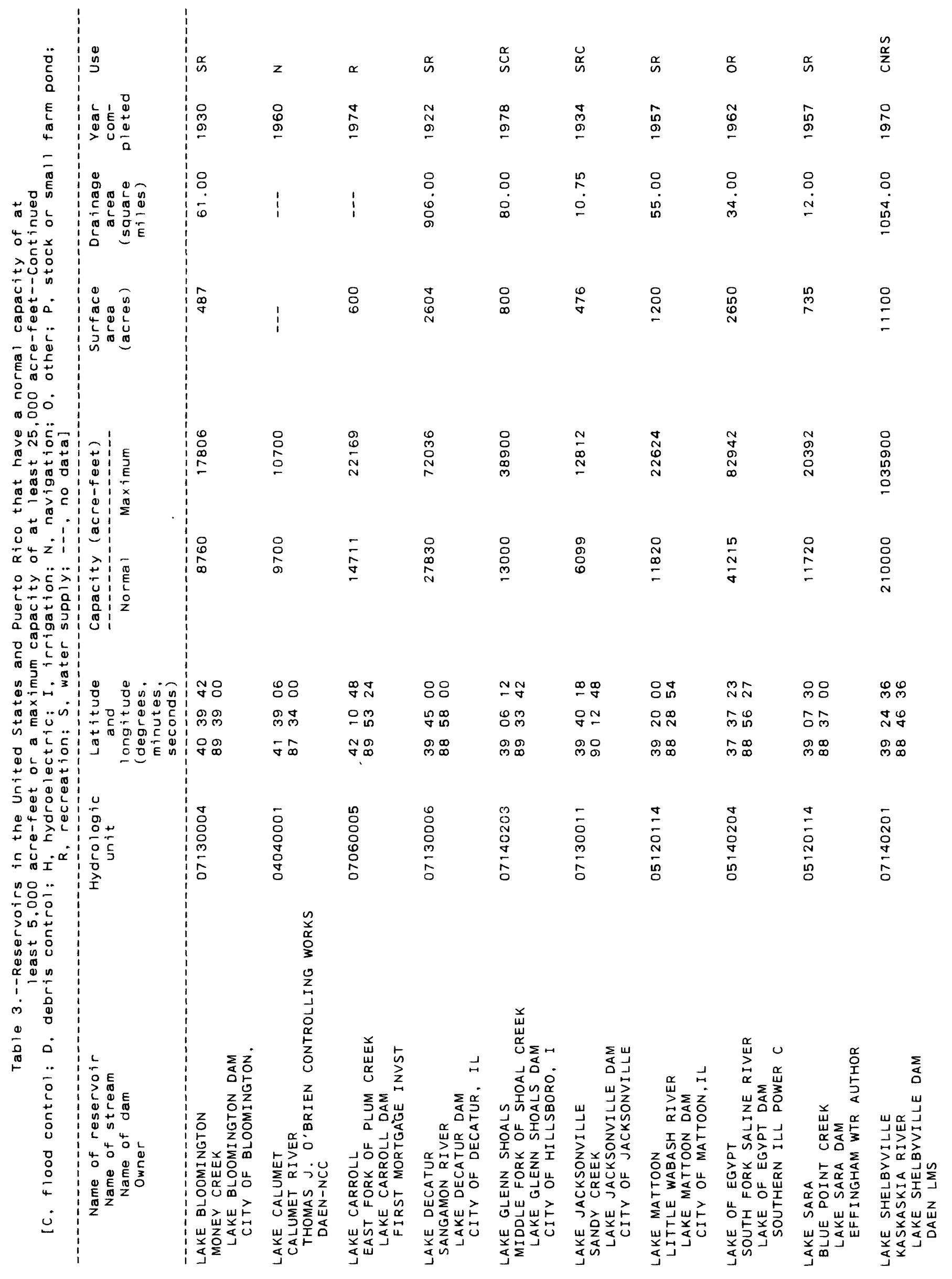




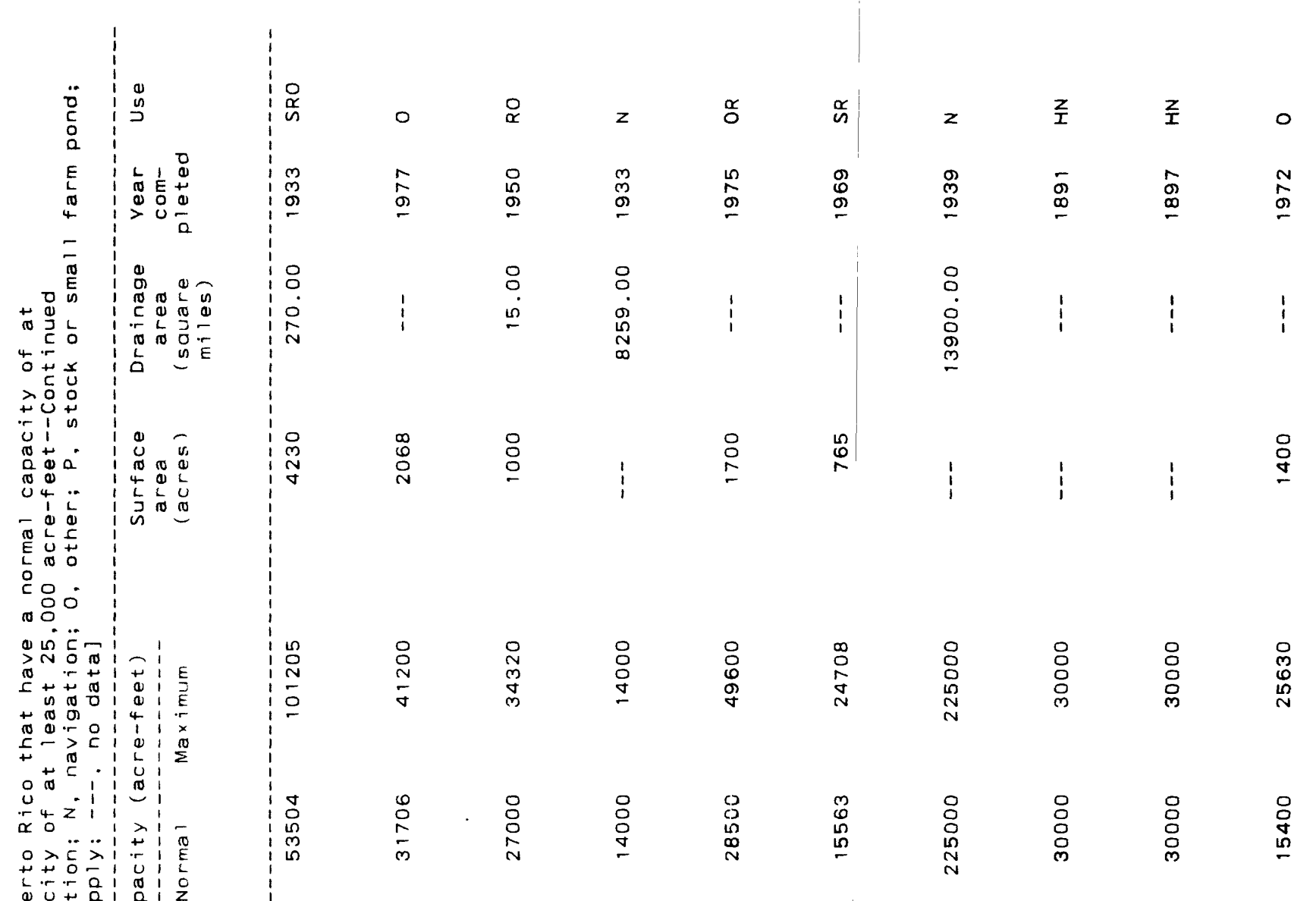

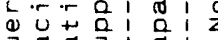

$\begin{array}{llllll}2 & \pi & 0 & 0 & 0 & 0 \\ 0 & 0 & 0 & 0\end{array}$

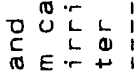

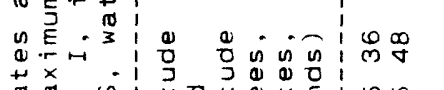

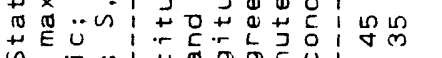

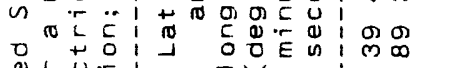

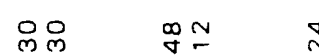

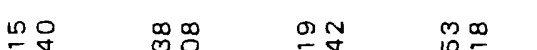

Tim

$80 \frac{10}{20}$

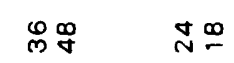

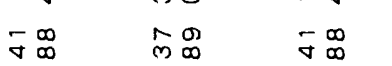

$\mathfrak{m}_{\infty}^{\infty}$

Ni

क्ले

윴

品 लूर

+0 व

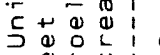

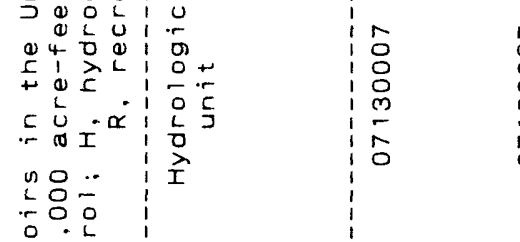

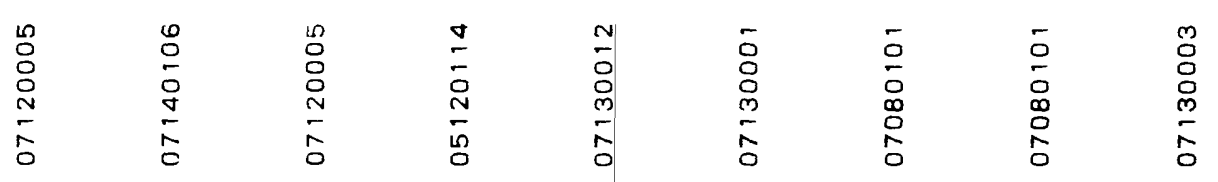

둥요

बे

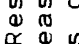

0
1
1

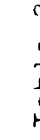

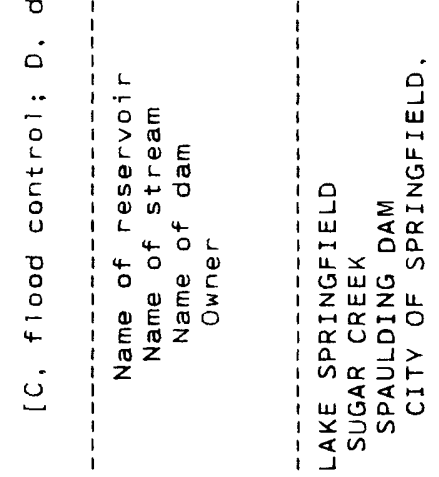<smiles>[C-][Co]</smiles>

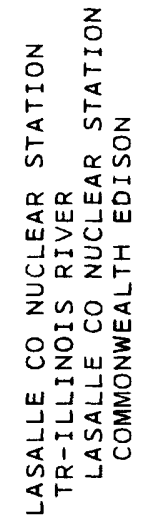

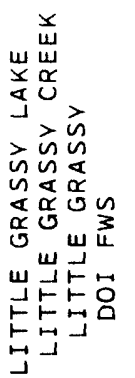
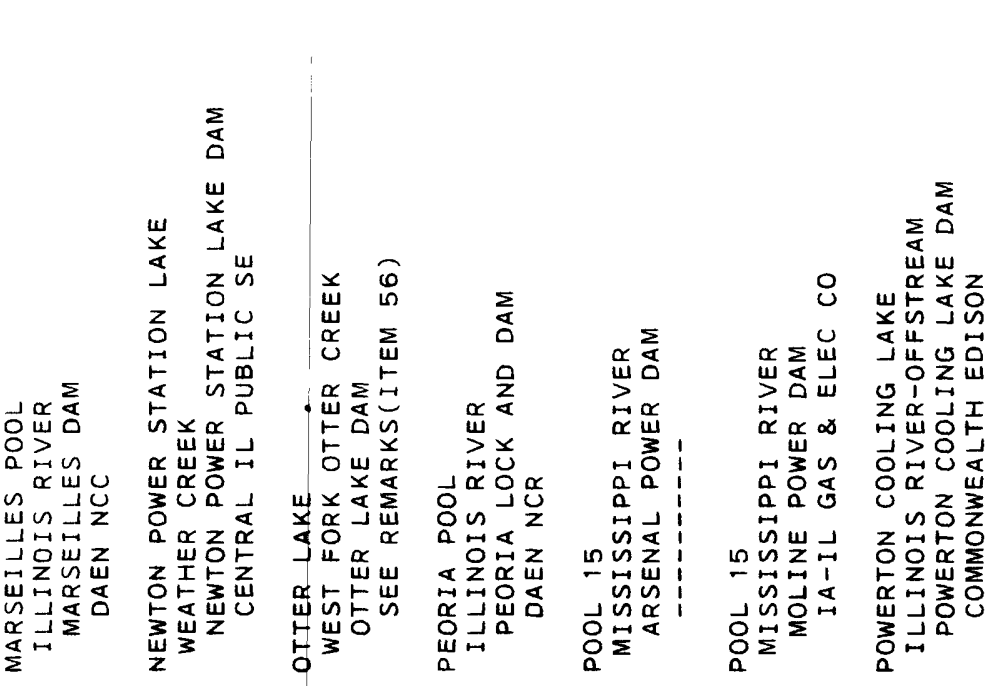


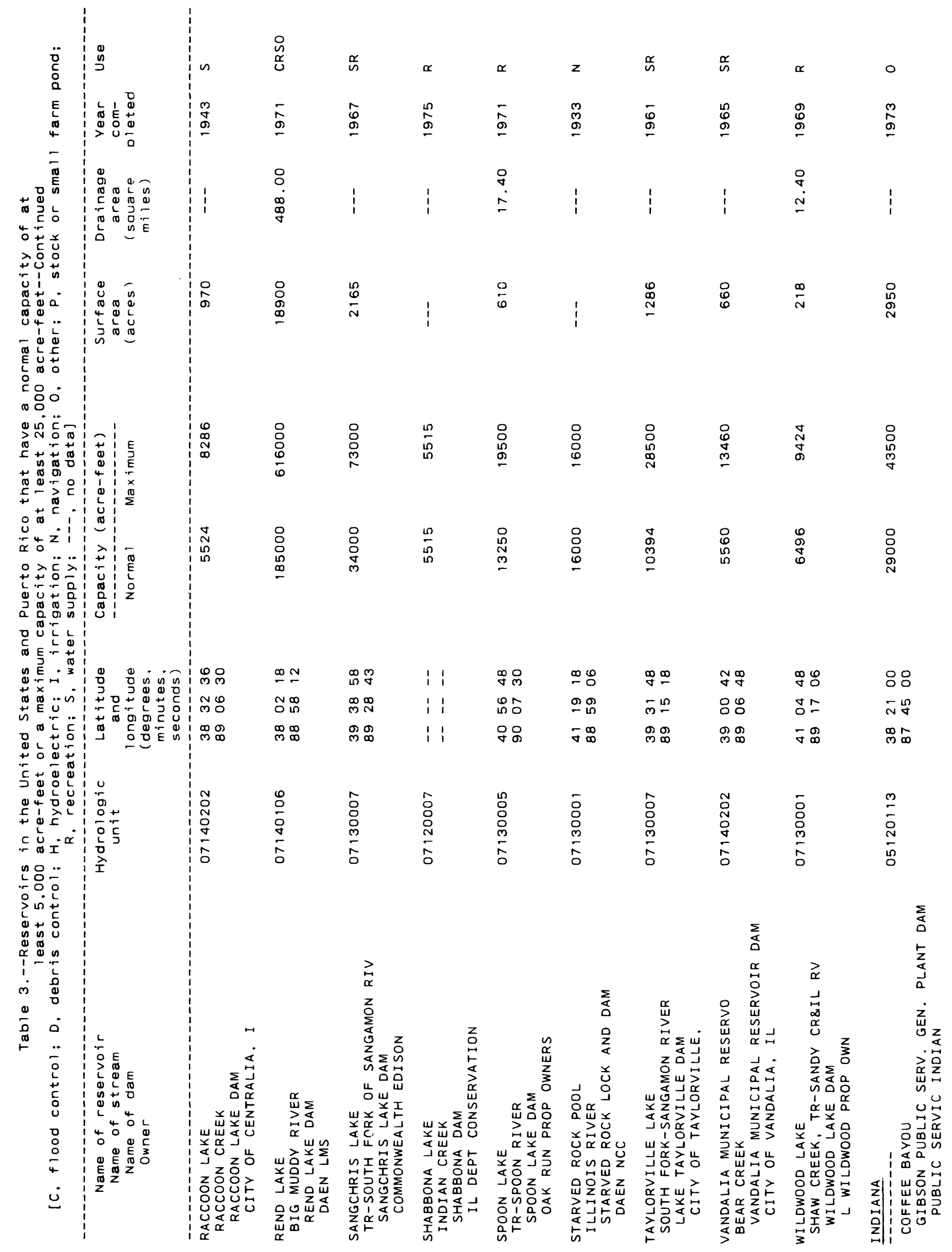




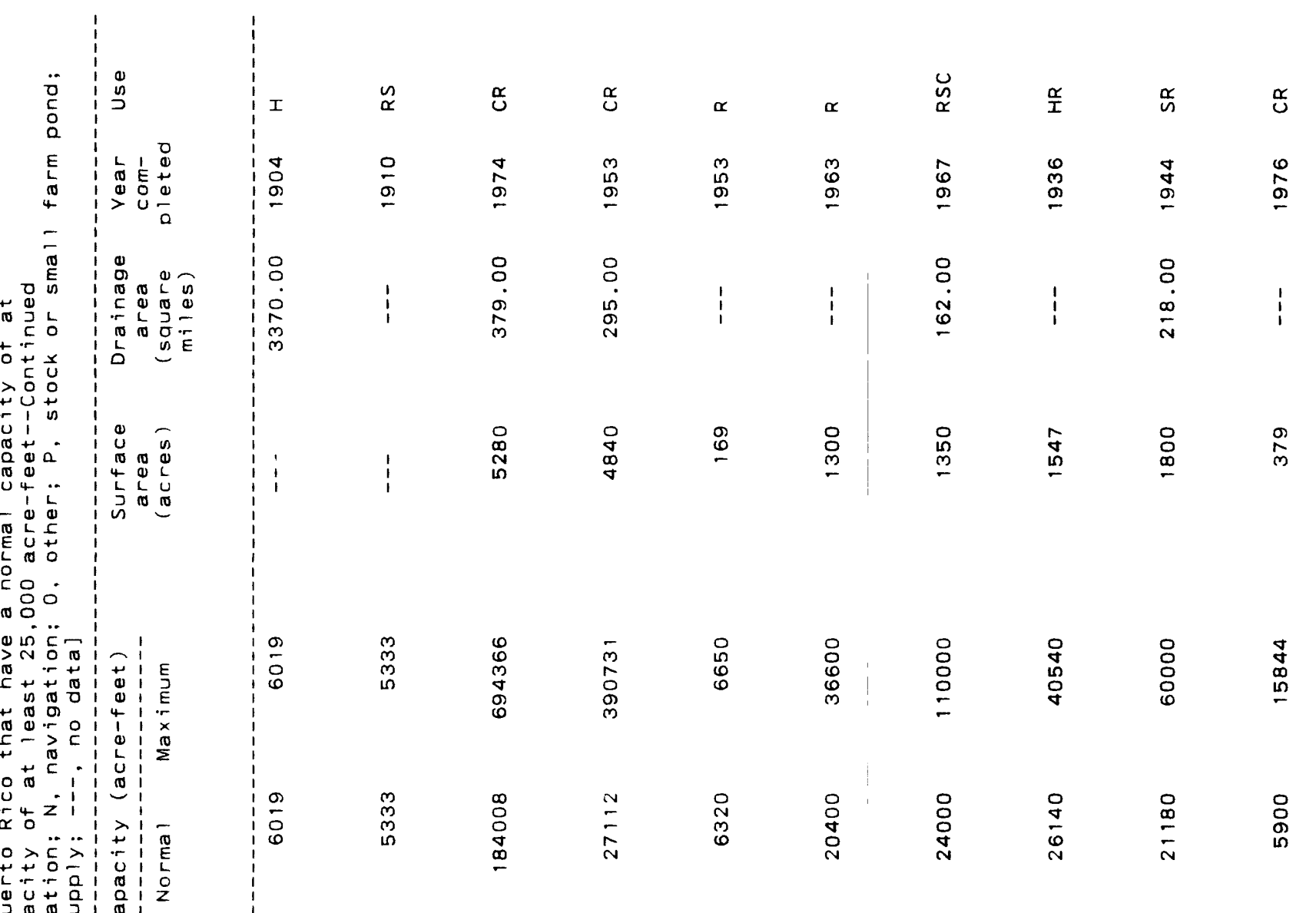

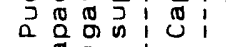

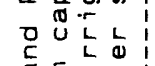

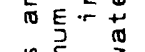

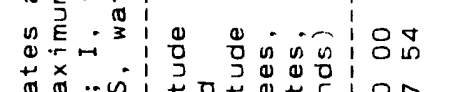

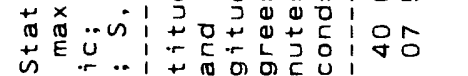
的芒芹

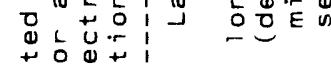
$\bar{c}+\frac{\pi}{0}$

a

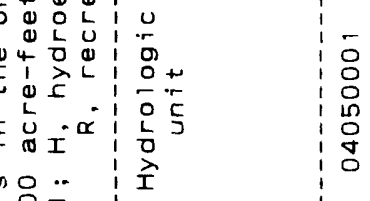

\begin{tabular}{|c|c|c|c|c|c|c|c|}
\hline$\cong \stackrel{N}{ }$ & 응 & $\cong 8$ & ஃ๐ & $\stackrel{\infty}{\infty}$ & $\simeq 8$ & N̊ & $\ddot{m} \simeq$ \\
\hline$\underbrace{\infty}_{\forall}$ & $\stackrel{2}{N}$ & 里员 & $\stackrel{\infty}{-} \mathscr{0}$ & Nom & $\underset{\sigma}{\infty}$ & ஜூற் & ํํำ \\
\hline$\underset{m}{\infty} \underset{\infty}{\infty}$ & م્ & $\ddot{m} \infty$ & $\ddot{m} \infty$ & 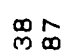 & றூை & $q_{\infty}^{\infty}$ & の \\
\hline
\end{tabular}

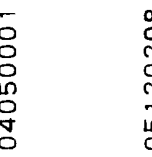

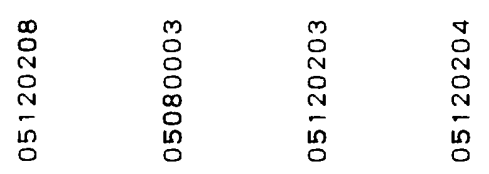

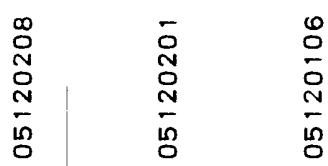

$\begin{array}{ll}\bar{D} & \stackrel{M}{N} \\ \stackrel{N}{N} & \stackrel{N}{N} \\ \frac{N}{n} & \frac{1}{0}\end{array}$

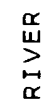
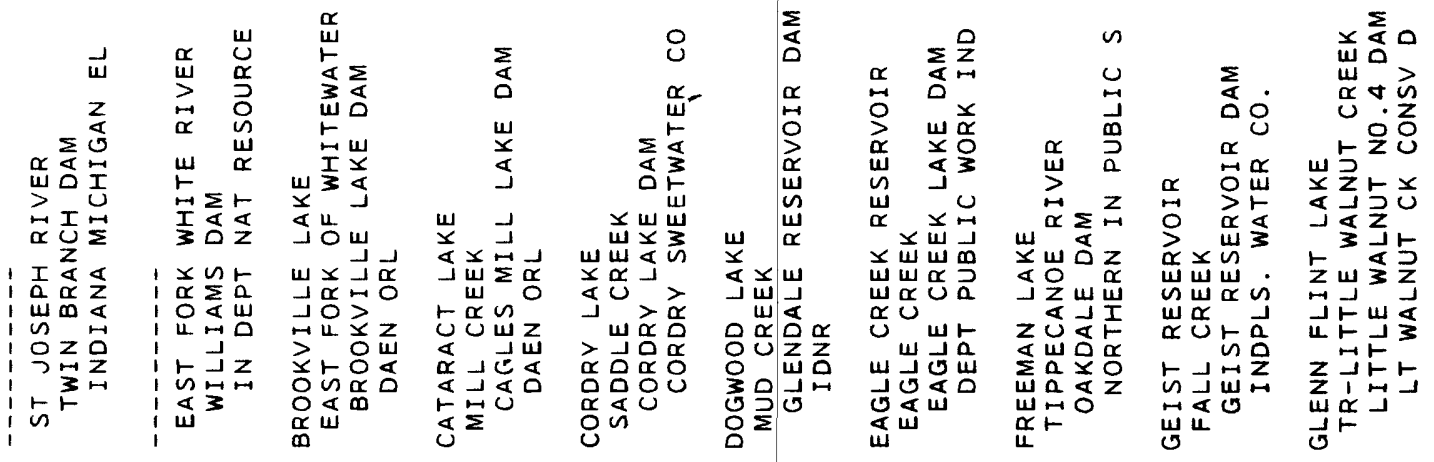


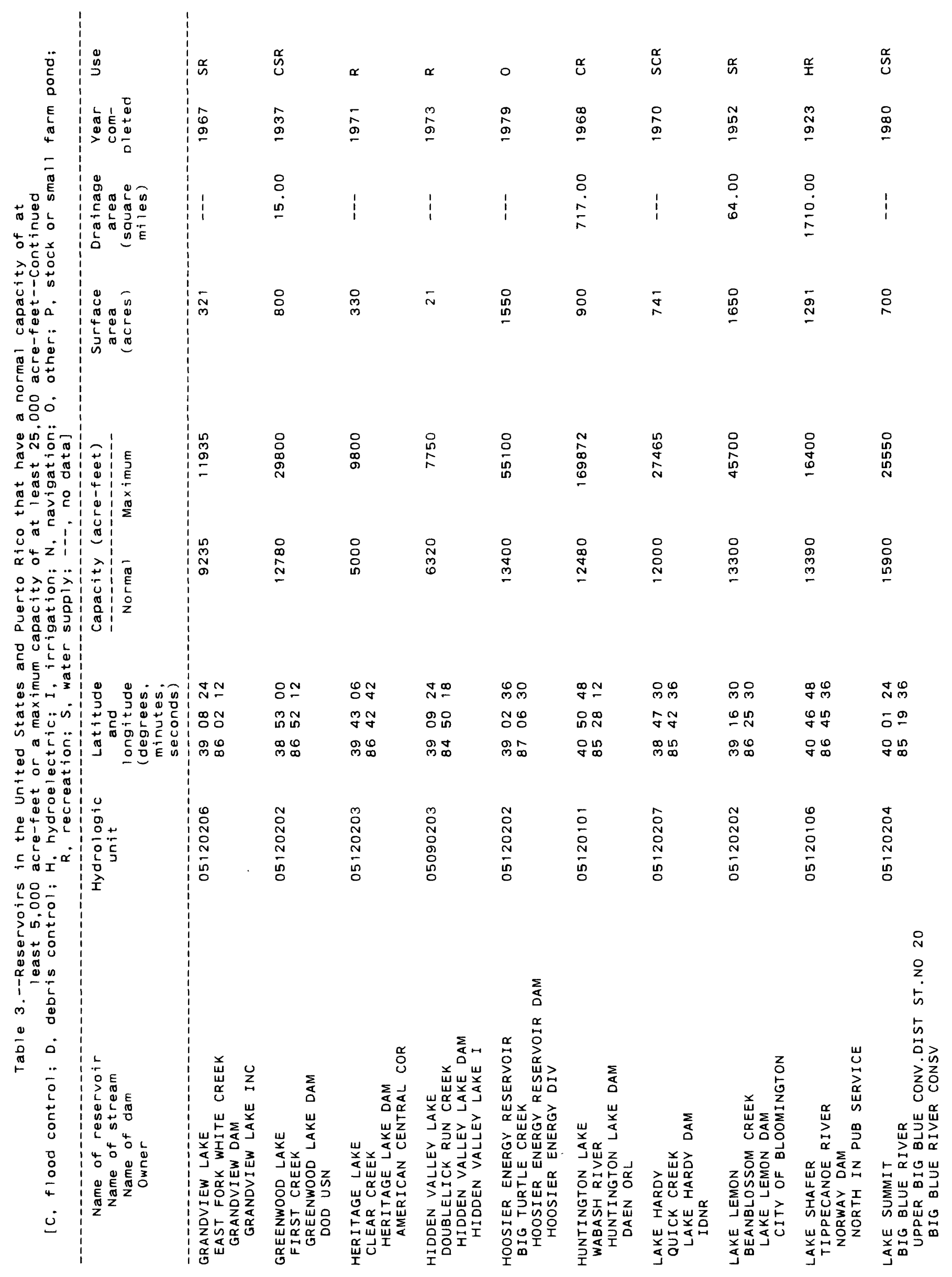




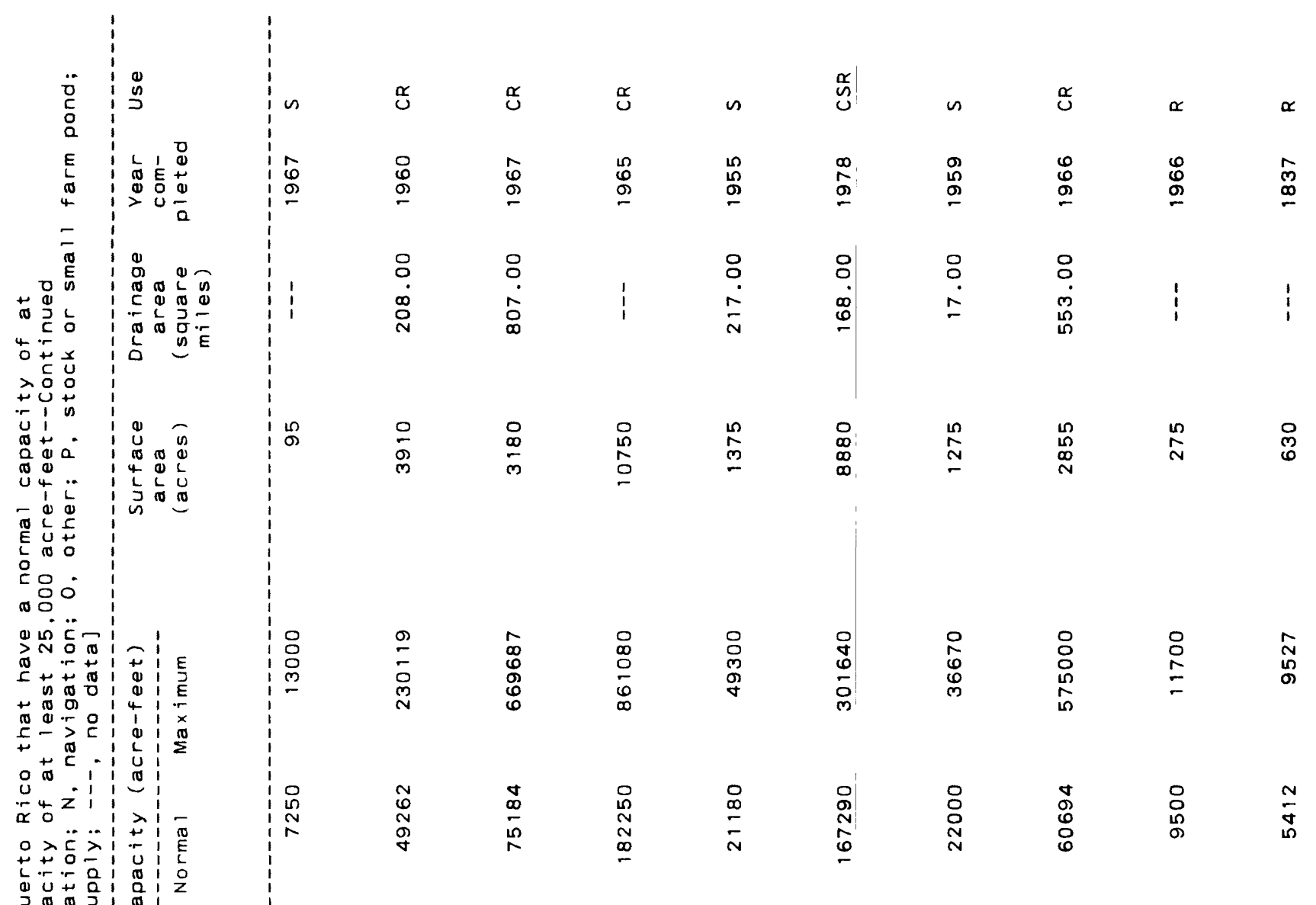

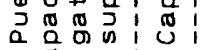

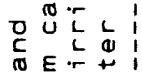

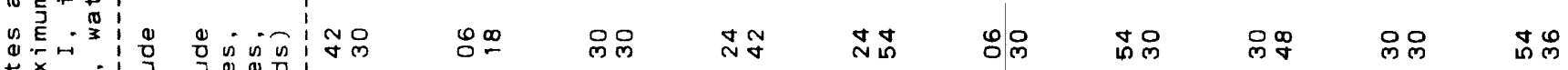

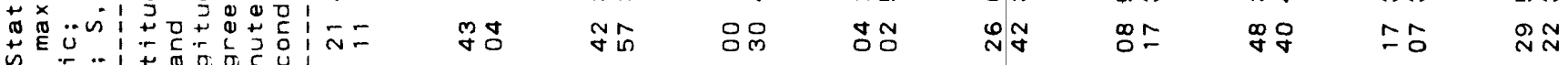

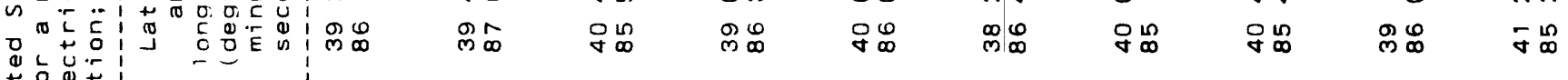

ᄃे

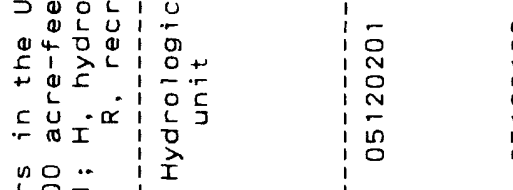

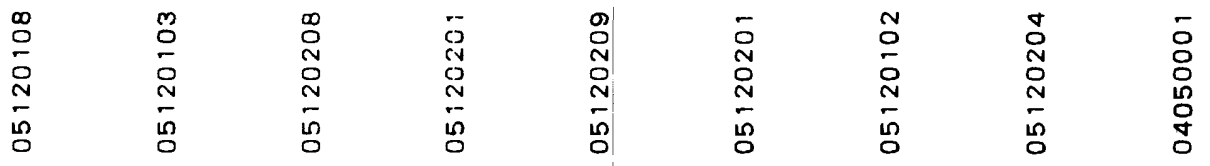



.

离落过

⿶凵

$\dot{m}$

$\frac{0}{\pi}$

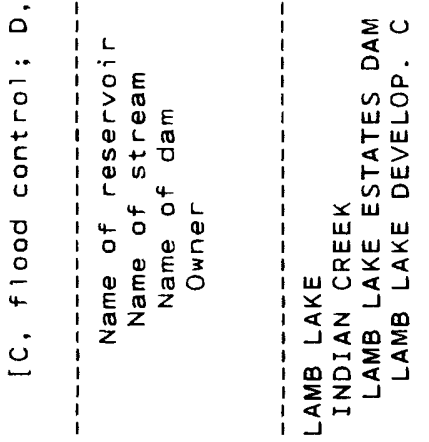

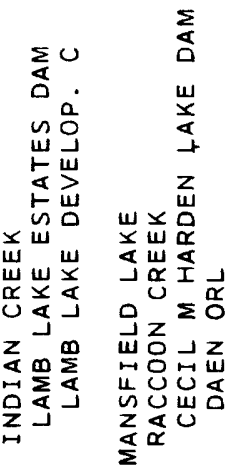

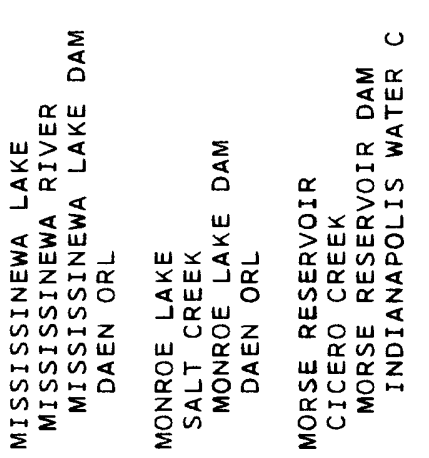

$u$

$\sum_{\alpha}^{\alpha} \sum_{0}^{\infty}$

$\underset{\substack{\alpha \\ \alpha}}{\stackrel{\sim}{u}}$

崖。

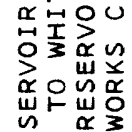

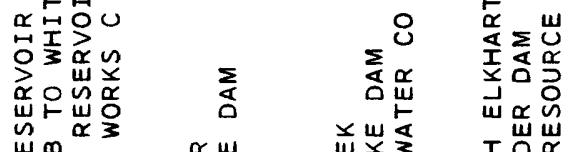

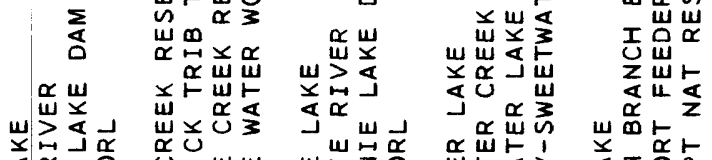

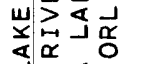

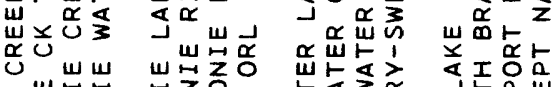

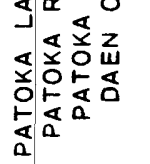

荺㟧

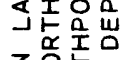




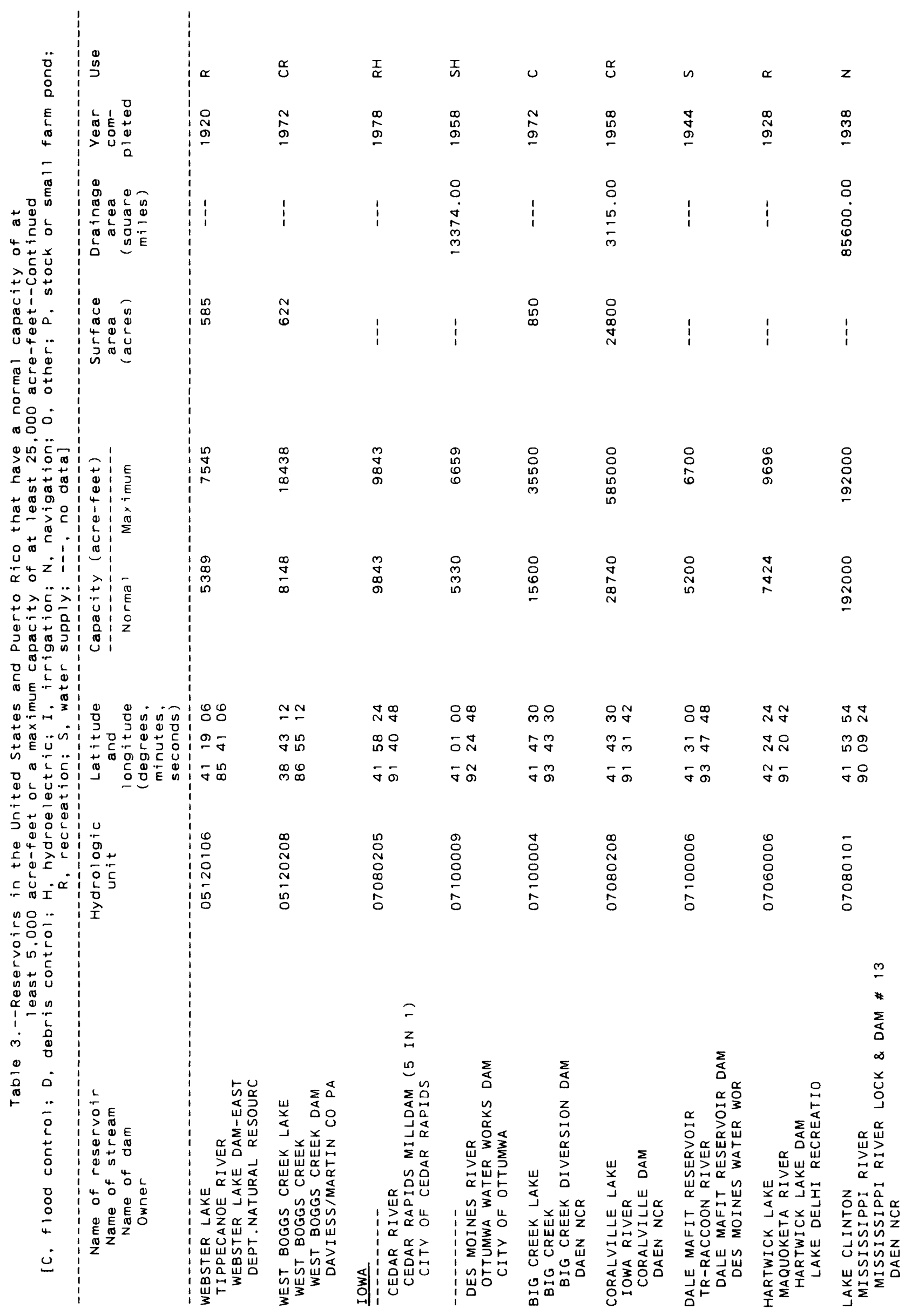




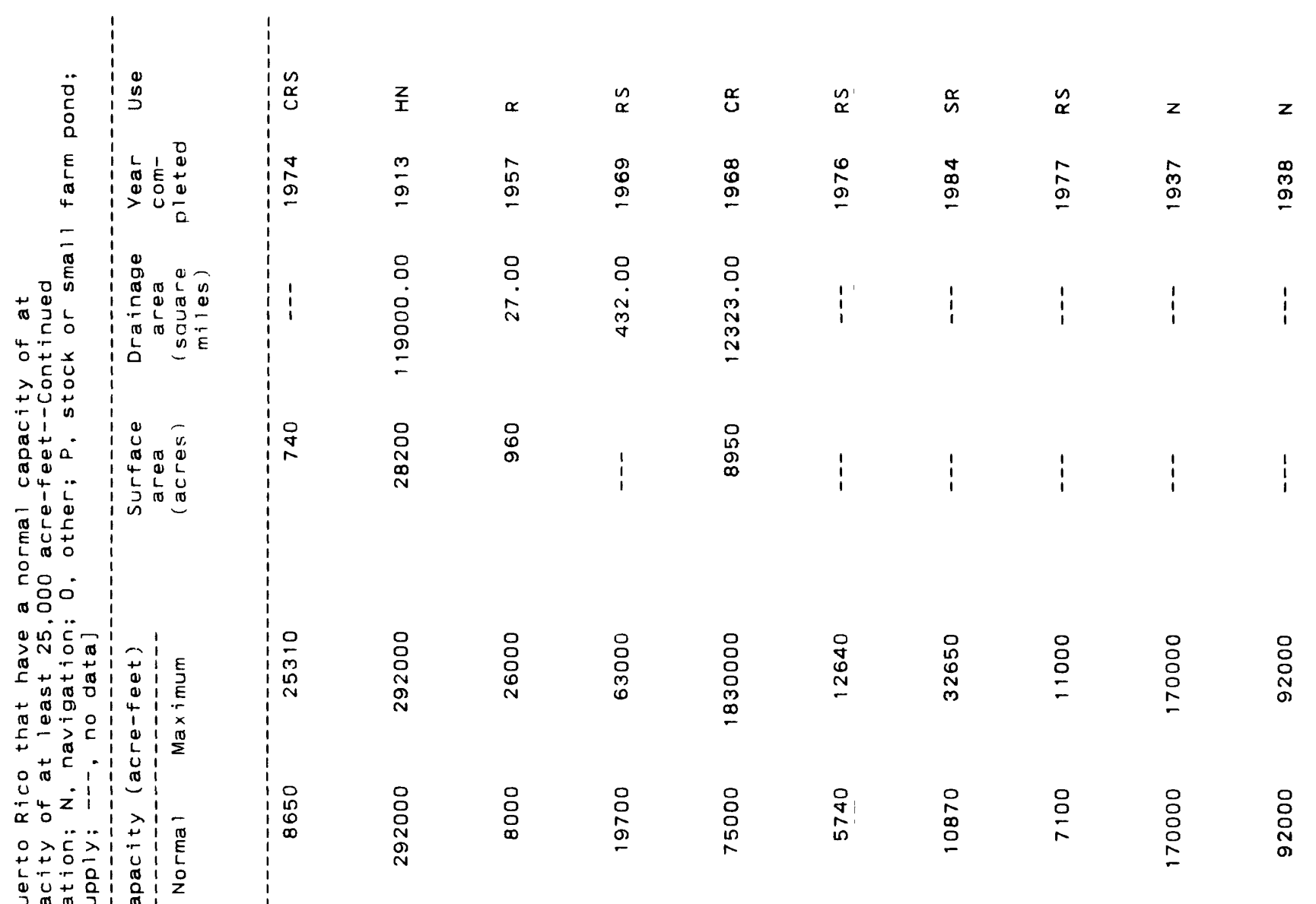

0 a

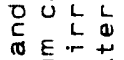

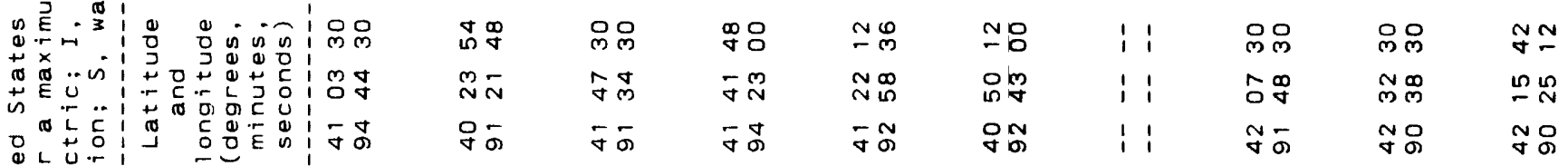

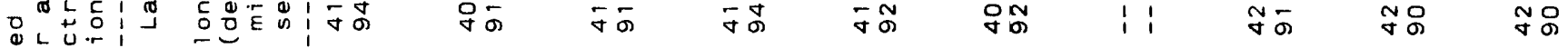

$\bar{c}+\frac{0}{0} \stackrel{0}{0}$

(

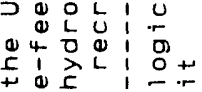

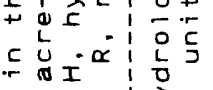

능..

s

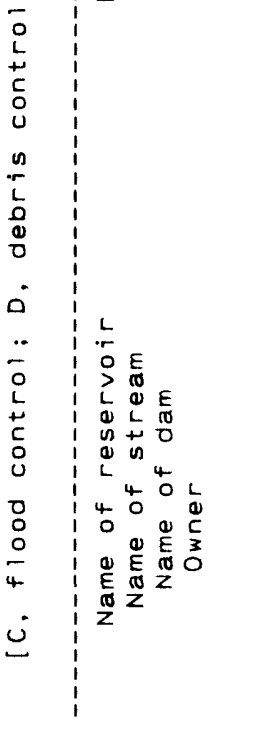

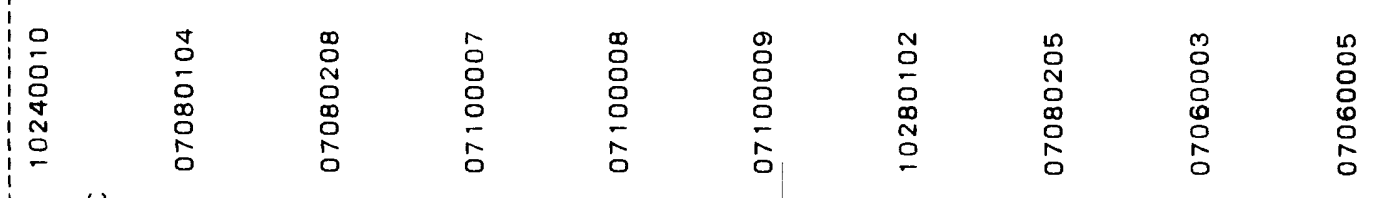
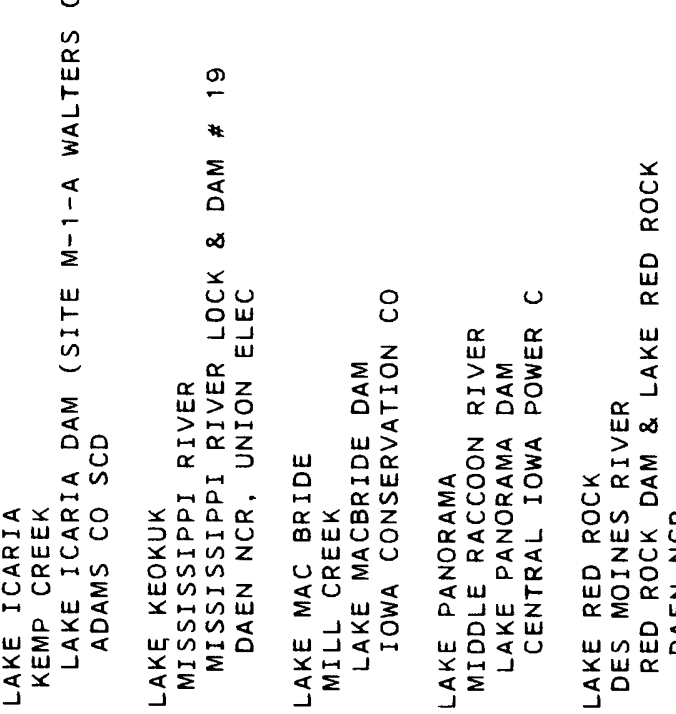


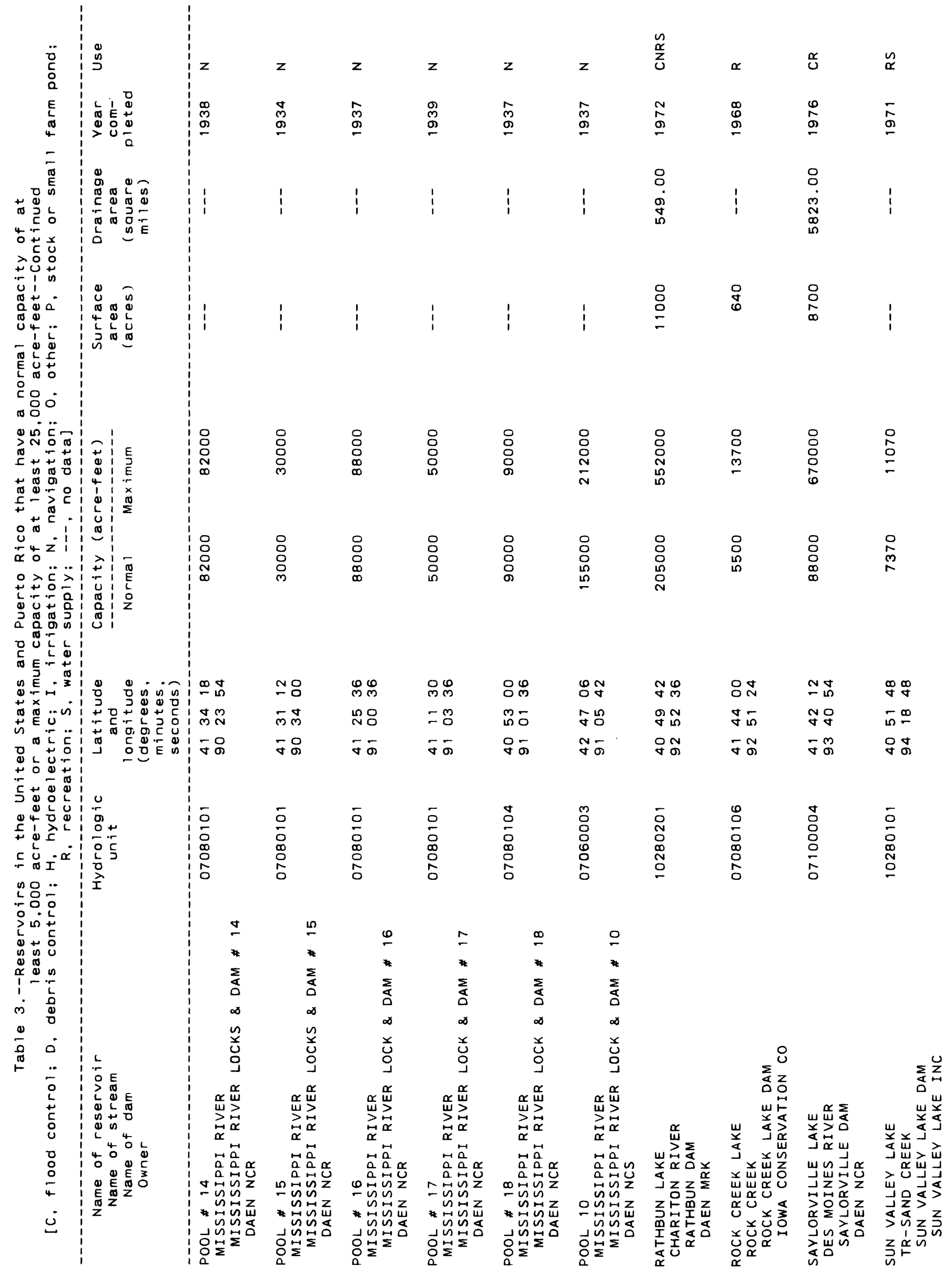




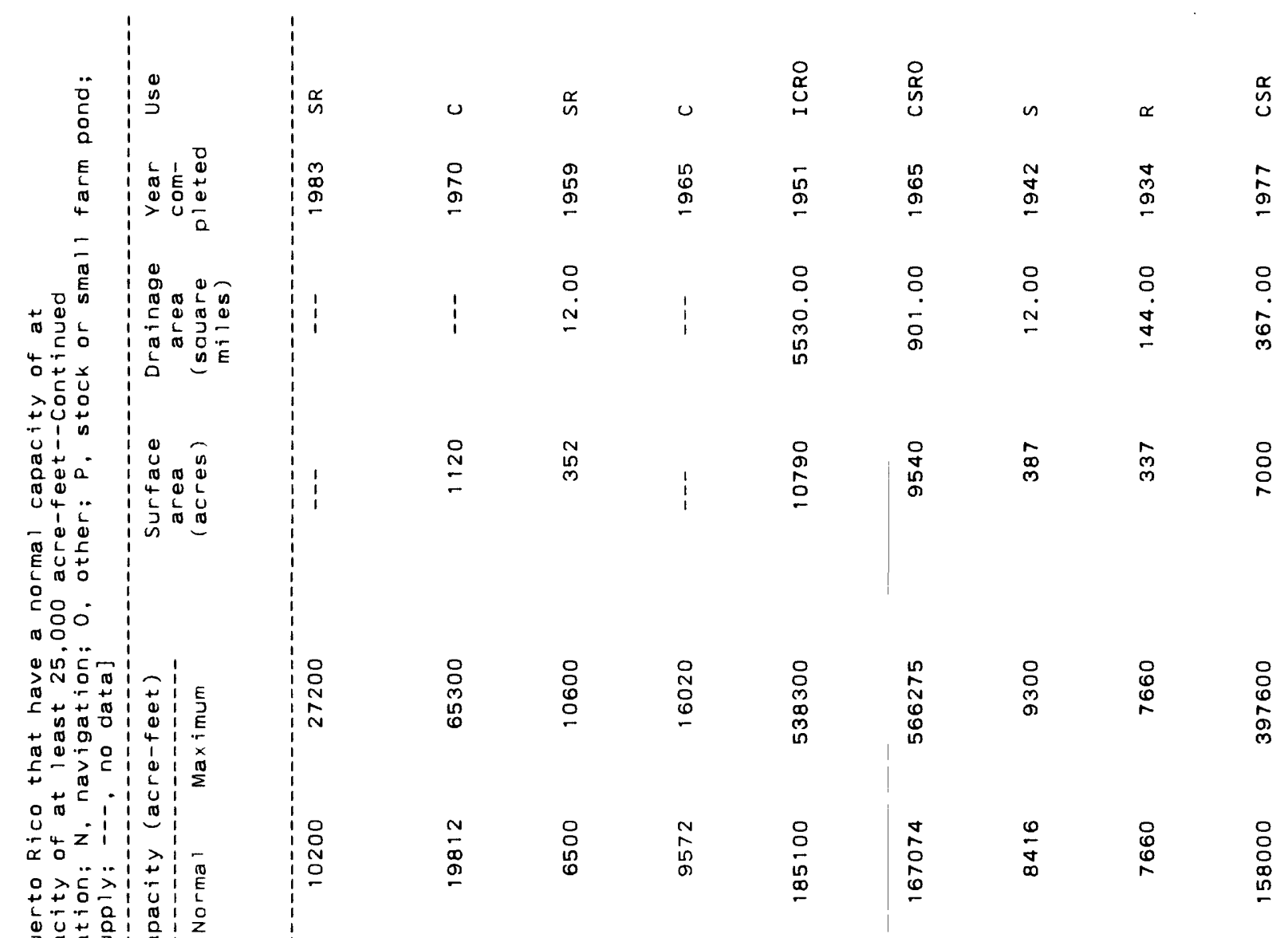

\begin{tabular}{|c|c|c|c|c|c|}
\hline$\stackrel{\Delta}{N}$ & ப̊ & $\stackrel{ \pm}{N}$ & ఱ్ల & "্লః & ถึ \\
\hline$\hat{\forall}$ & $\underline{m} ㅇ$ & $\hat{\sigma} \stackrel{m}{q}$ & $\stackrel{M}{\forall}$ & o & $N \underset{T}{O}$ \\
\hline ஸे & กิ & ల్లి & ले & ల్లి & ஸे \\
\hline
\end{tabular}

O०

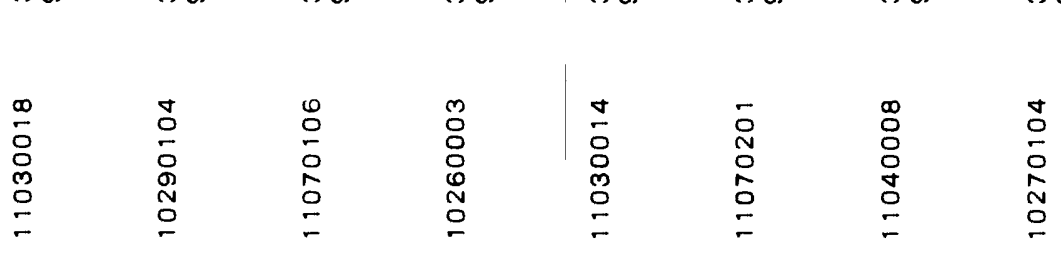
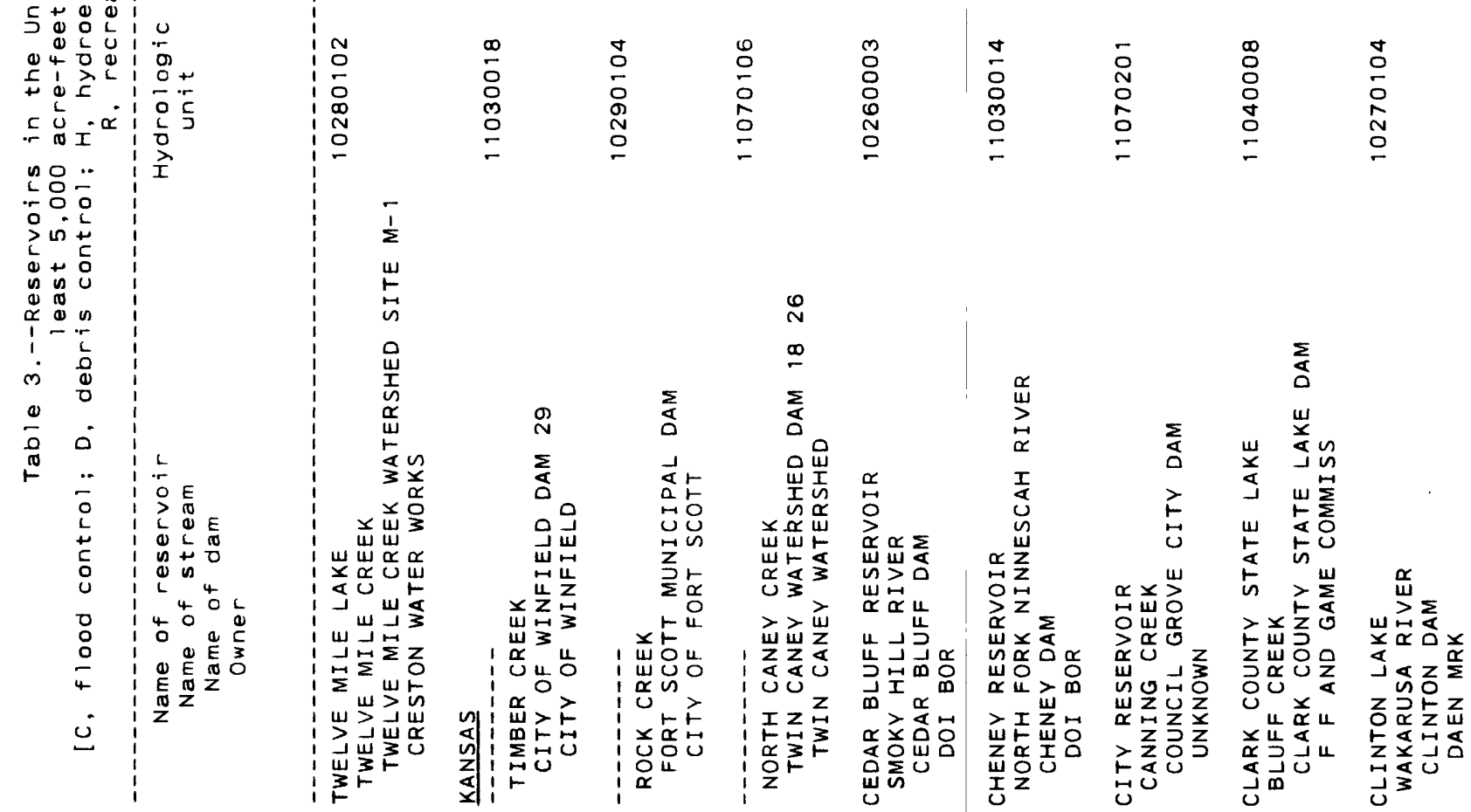


\section{See corrections to this table in the errata text file.}

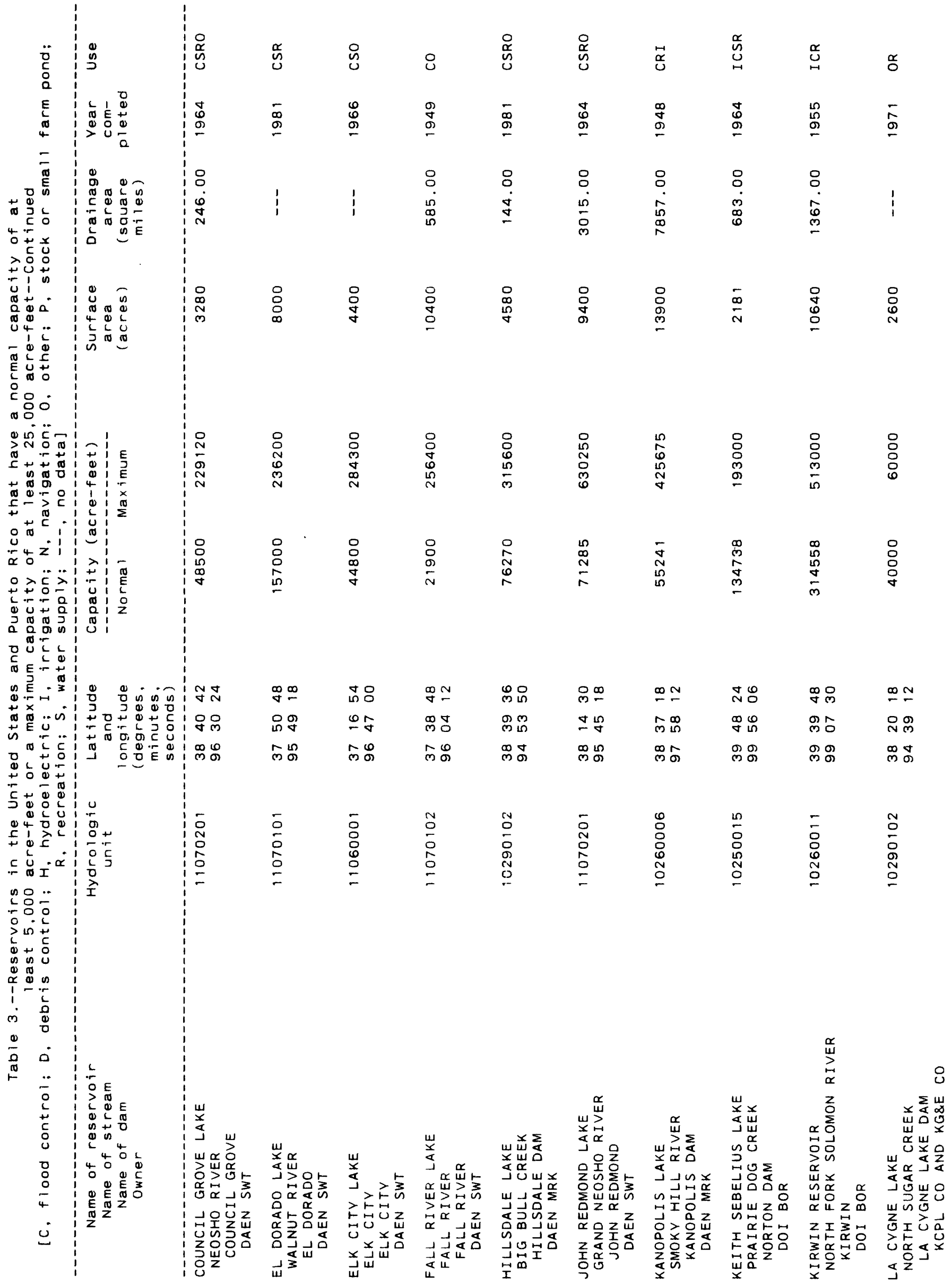


See corrections to this table in the errata text file.

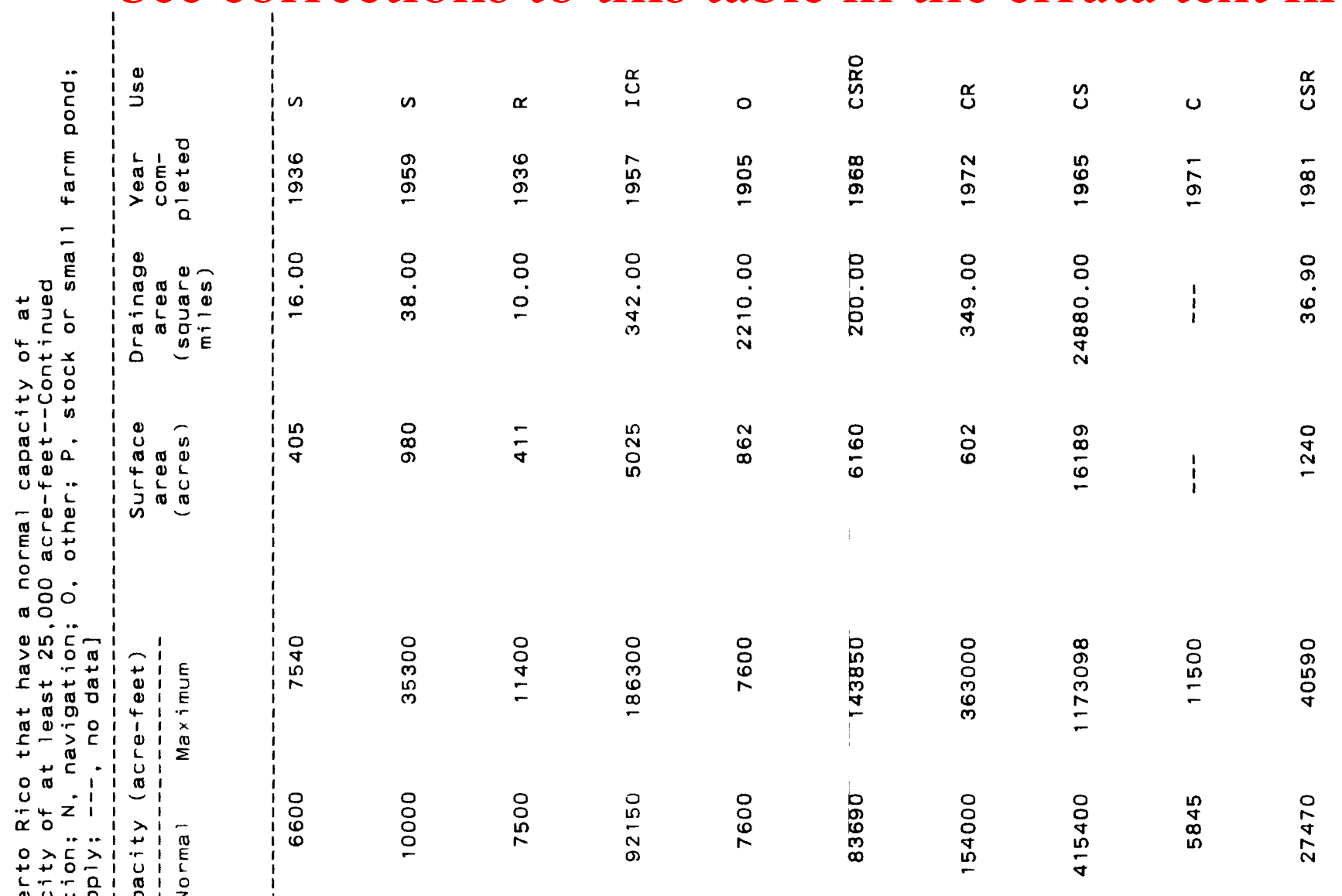

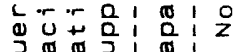

a O on

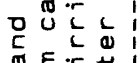

बह

ब.-

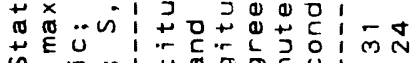

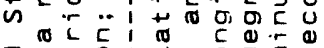

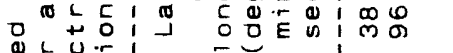

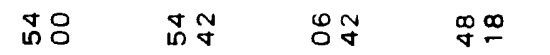

응

กิก

ㅇำ

ํํ ำ ำ

m

Nละ

윰

ำ

กำ ำ

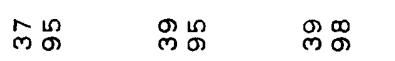

ले के

mô

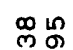

लळ

ํำ

$\stackrel{\infty}{N}$

ᄃ

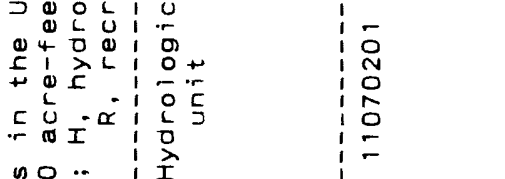

$\stackrel{n}{N}$
$\stackrel{1}{0}$
0
$=$

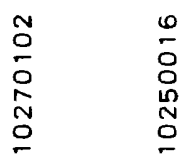

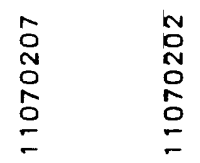

$\overline{0}$
$\frac{0}{2}$
N
0

$\stackrel{ }{\circ}$

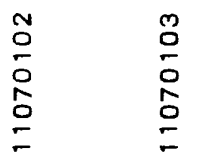

1

$a+c$

is

$10 \frac{n}{2}$

मे

-.

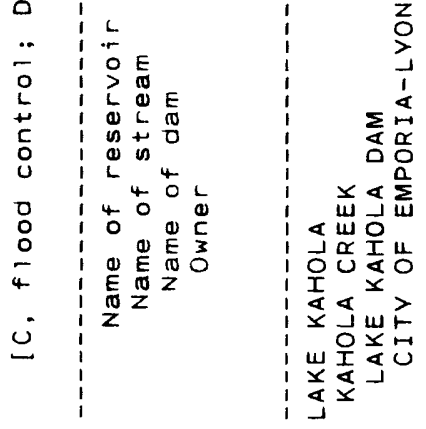

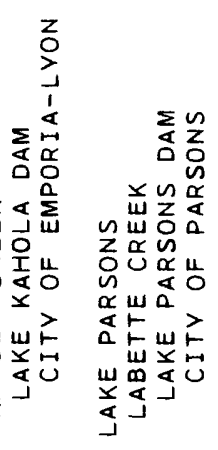

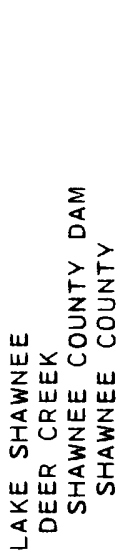

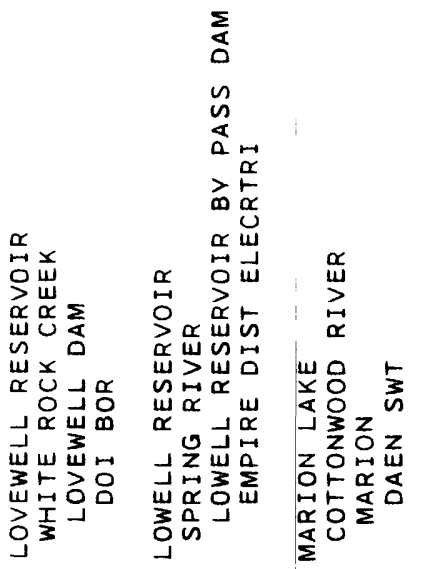
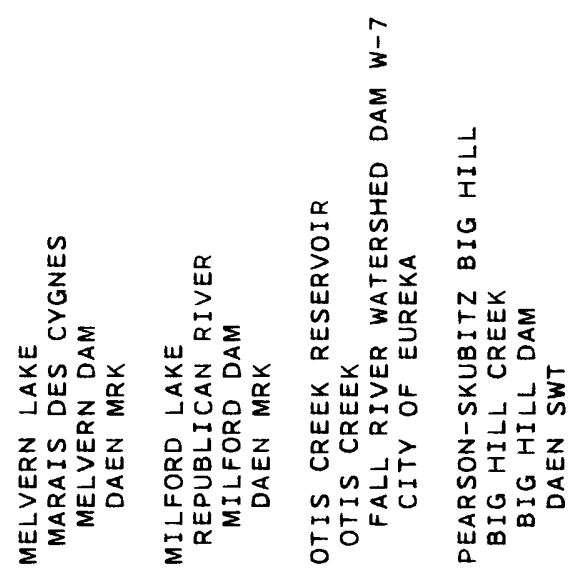
See corrections to this table in the errata text file.

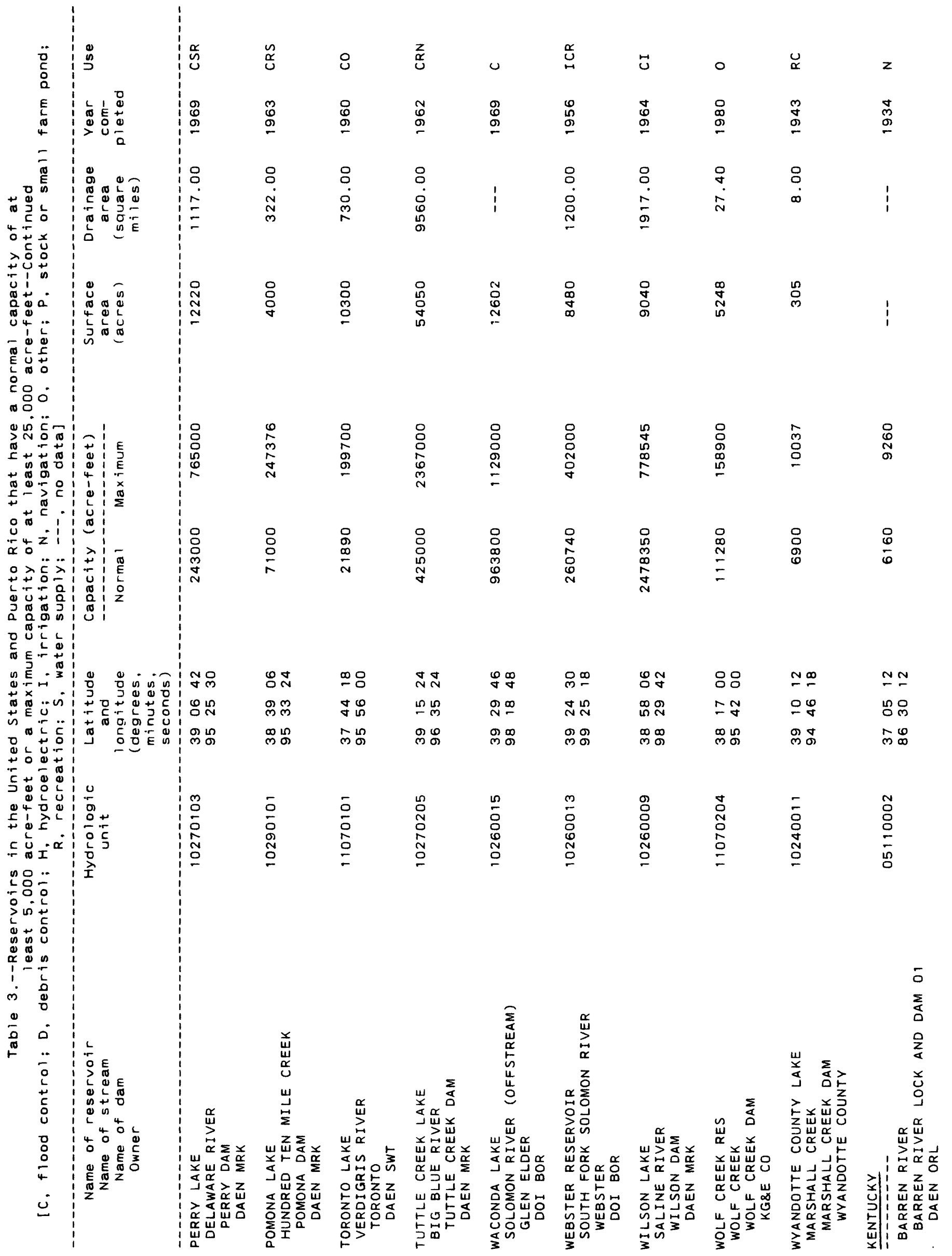




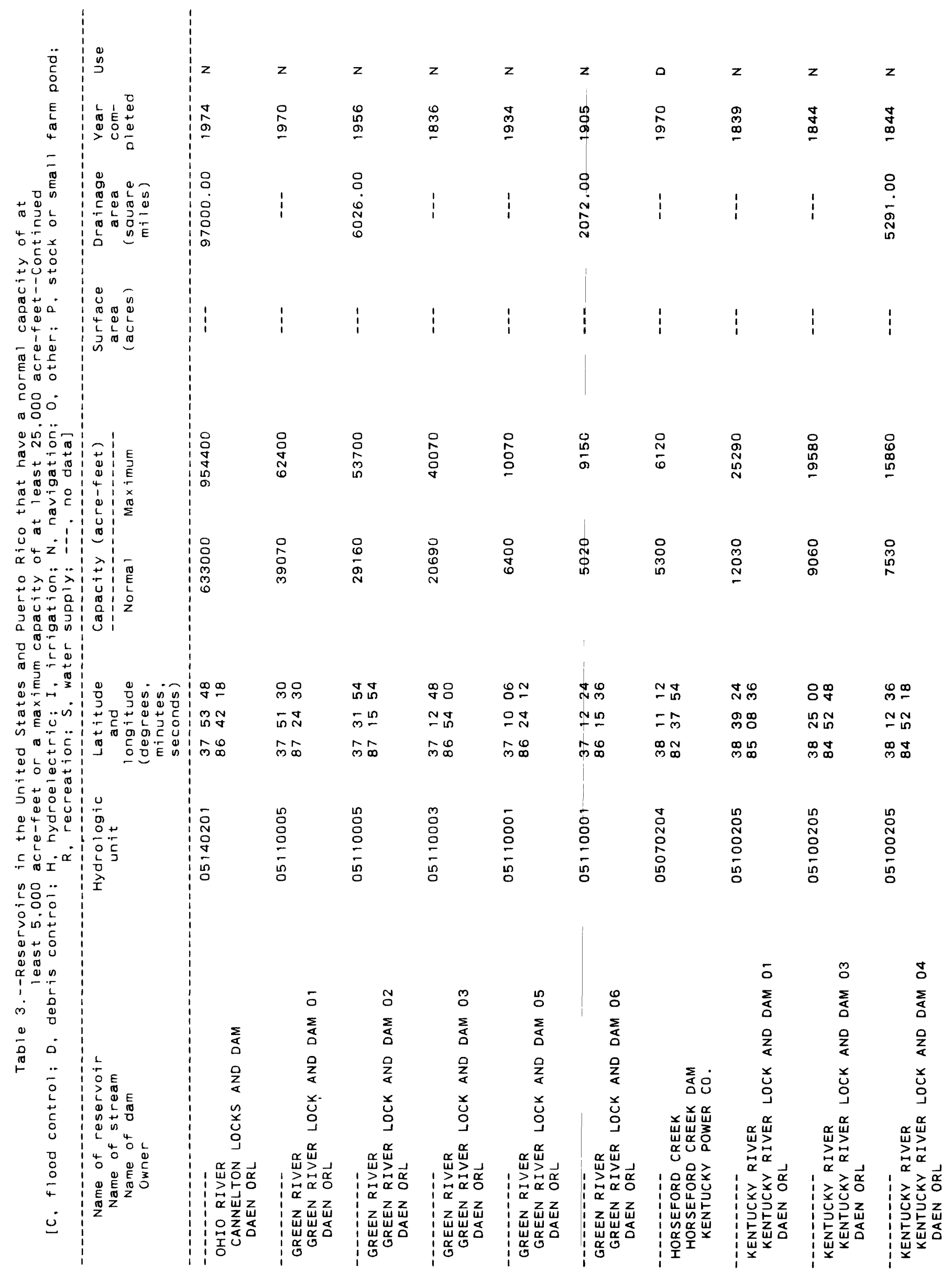




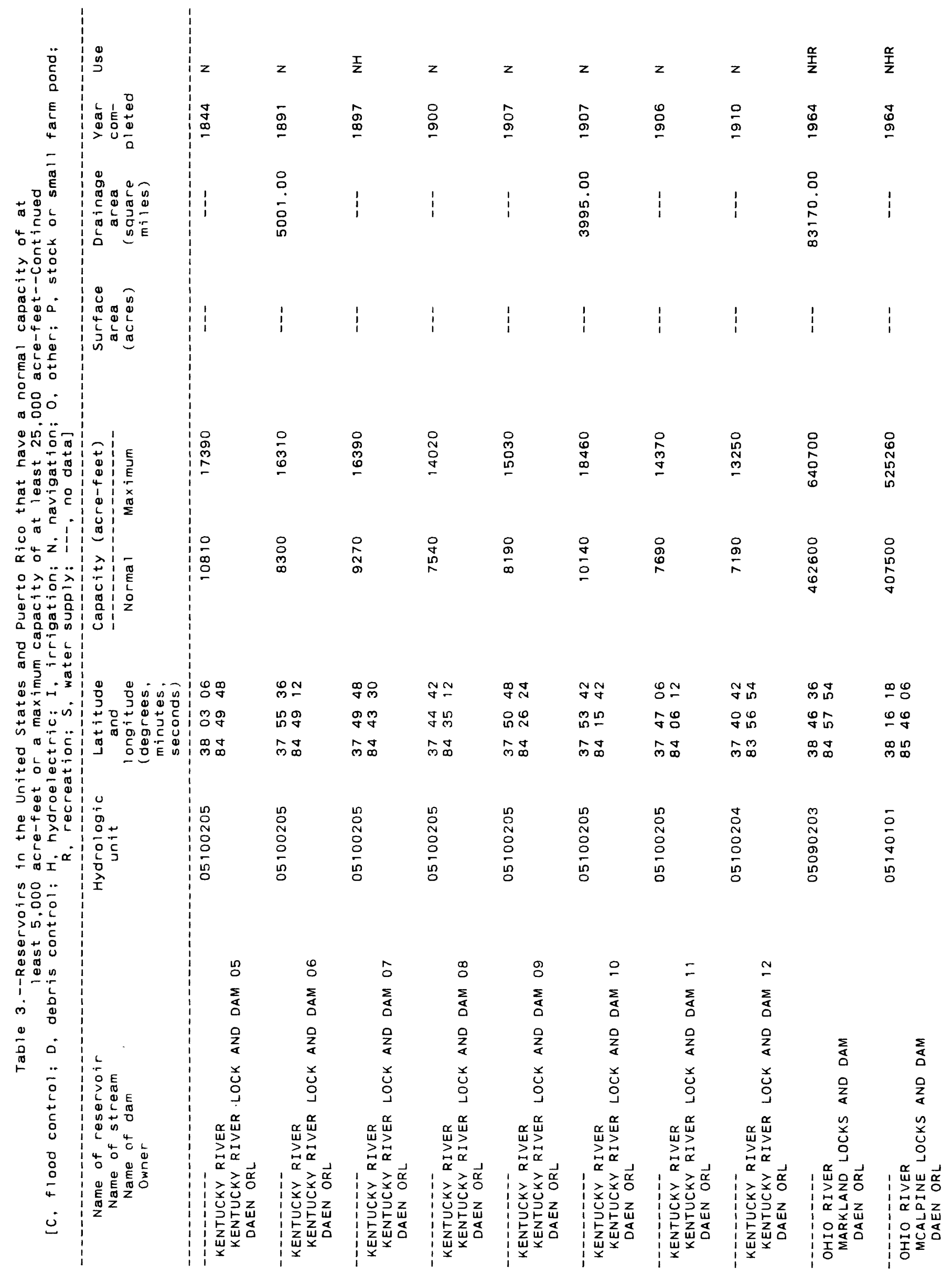




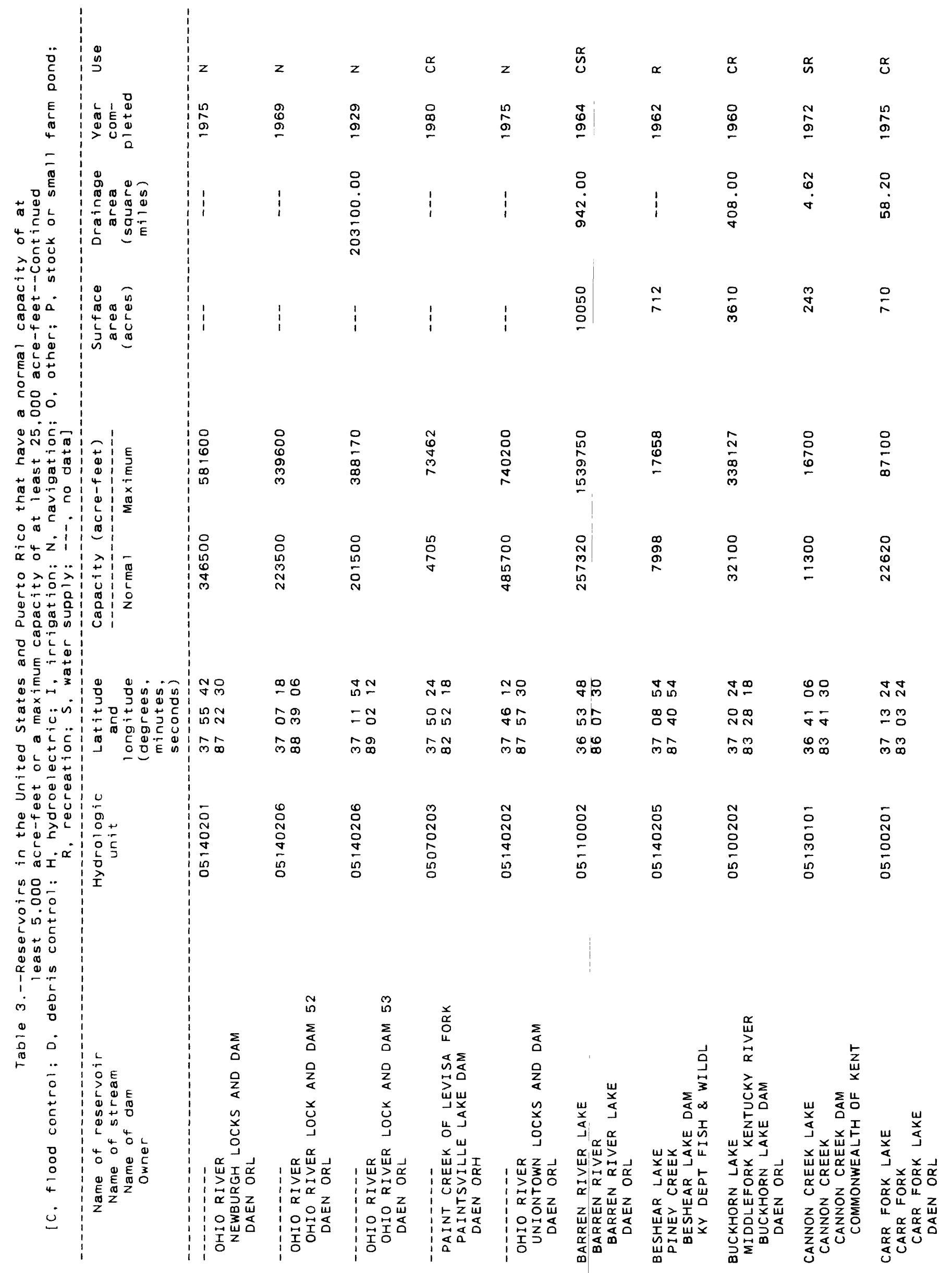




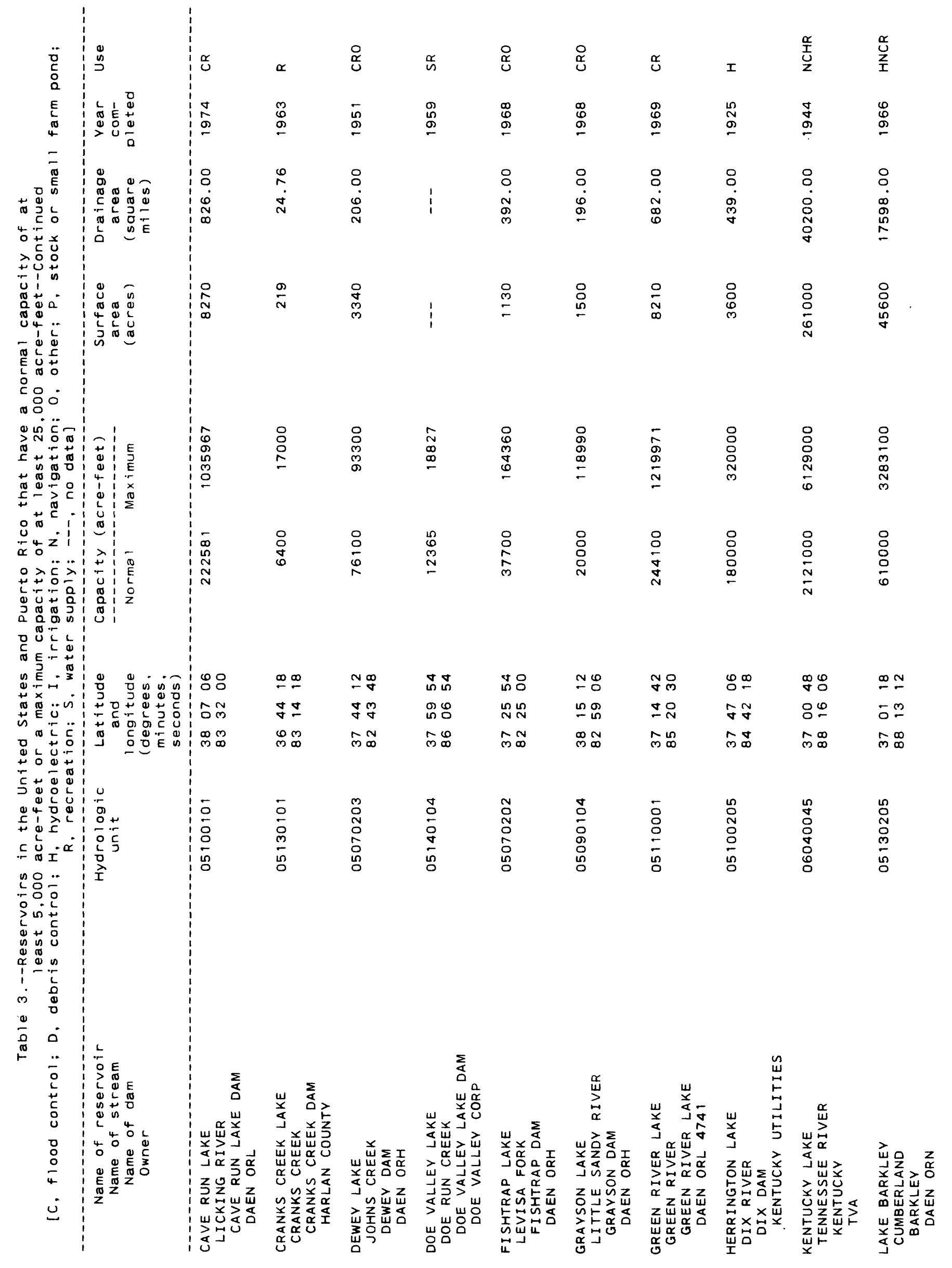




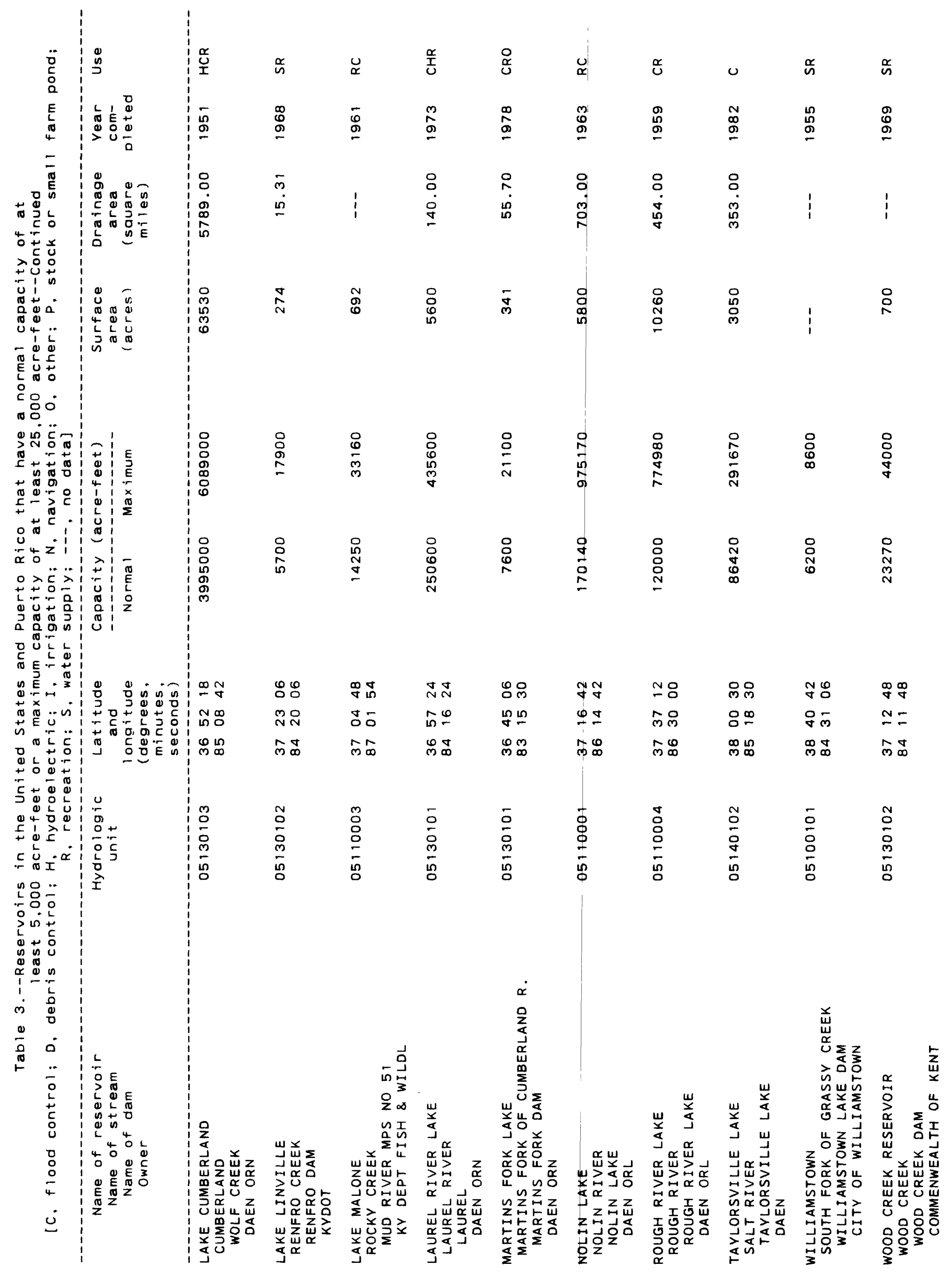




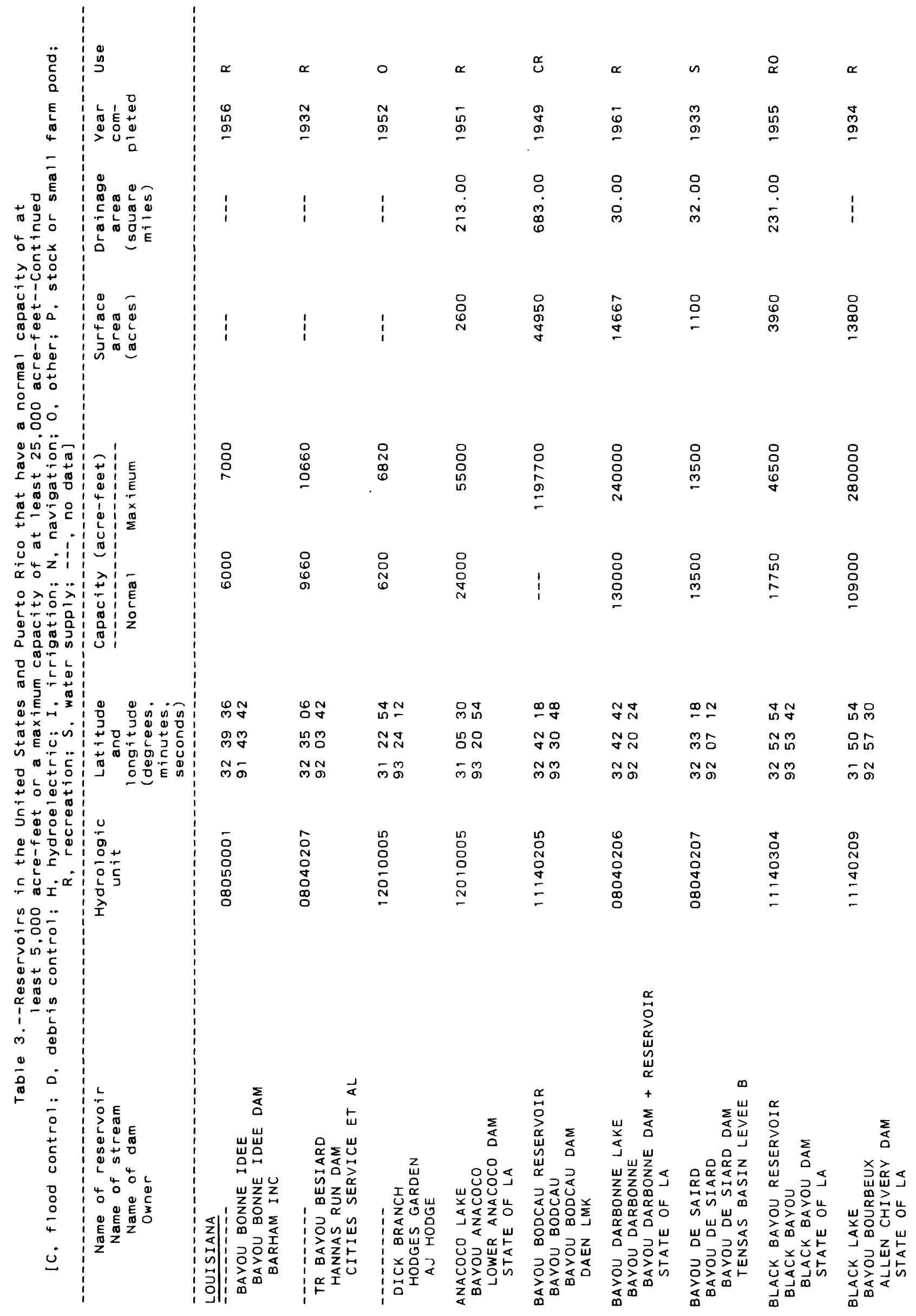




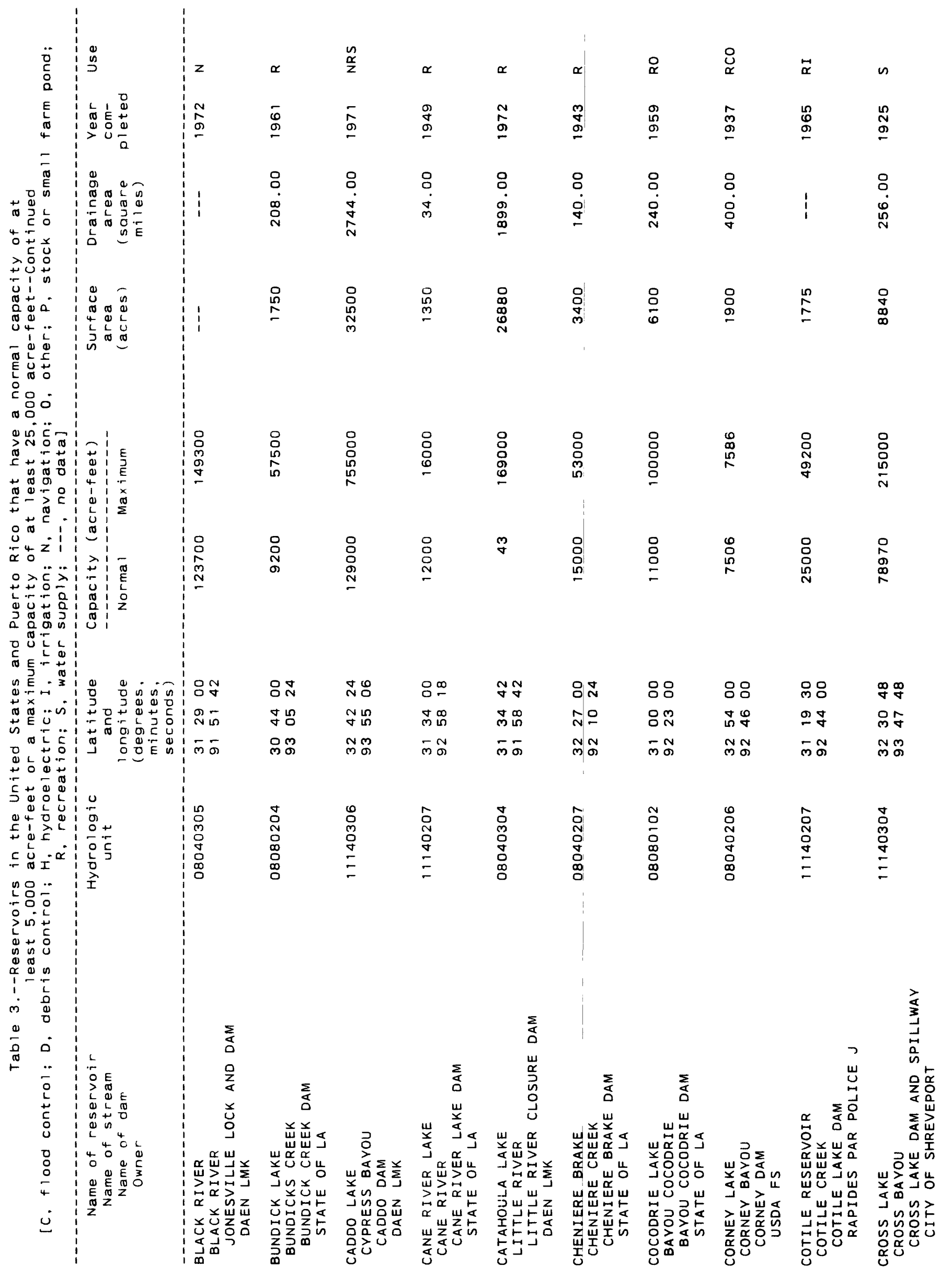




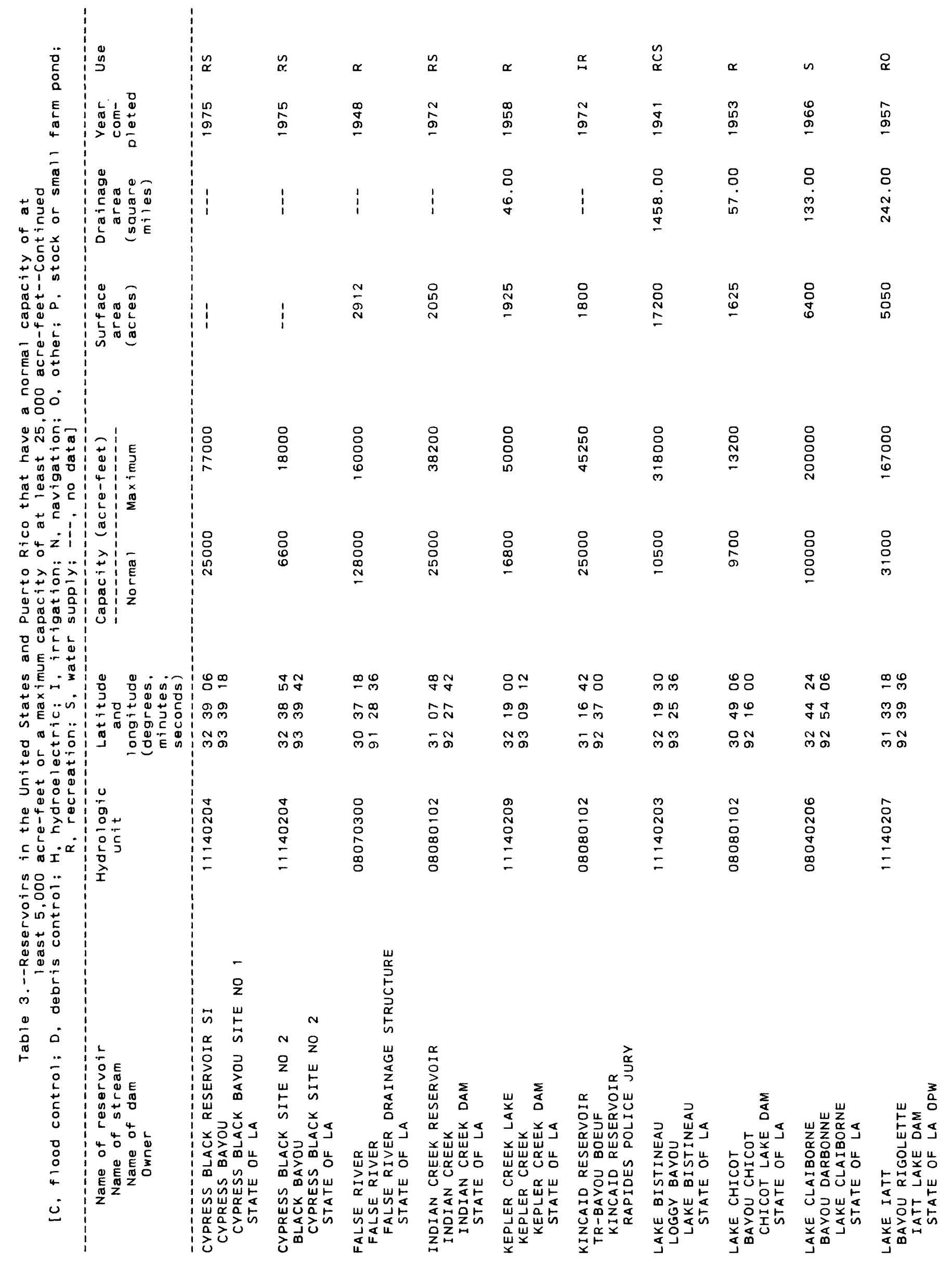




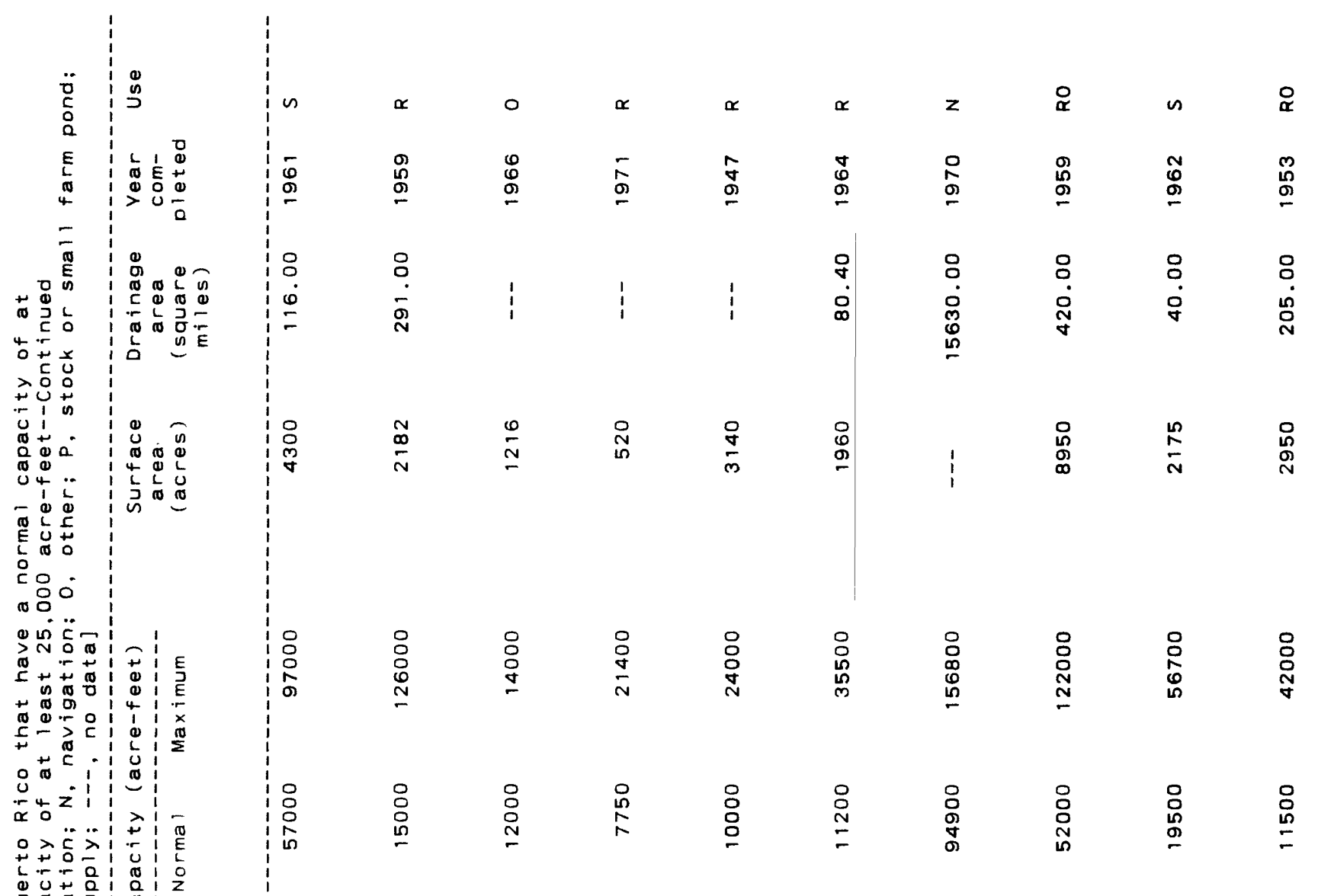

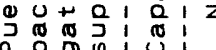

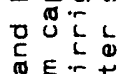

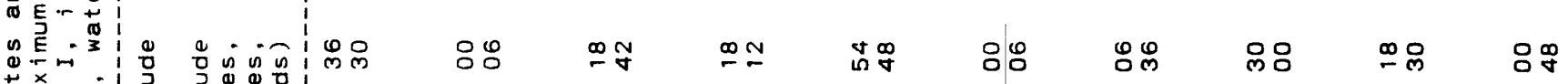

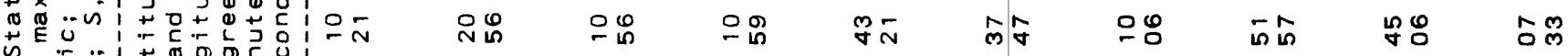

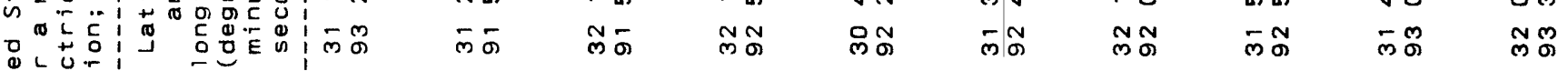

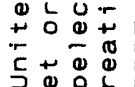

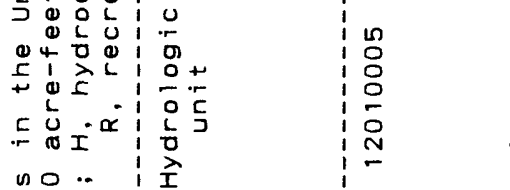

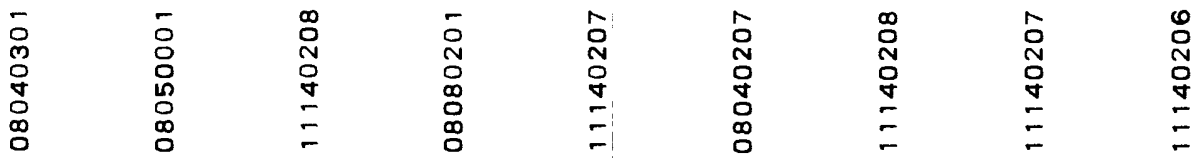

$>$

a

告

$\dot{0}$

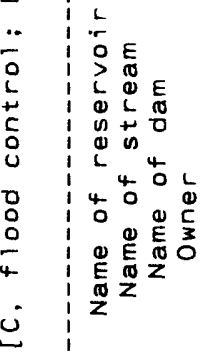

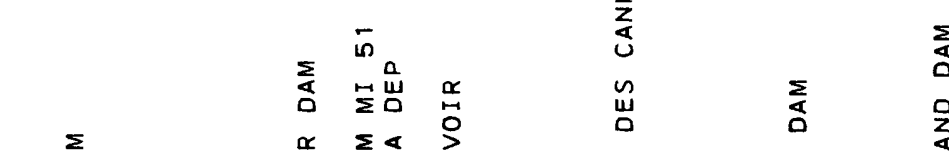

离

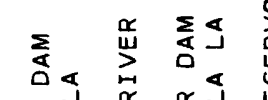

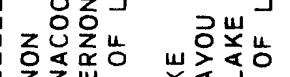

㐫的

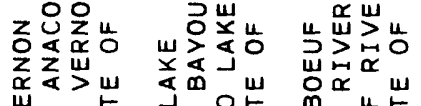

次品

ग出

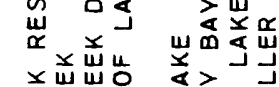

㟧

足

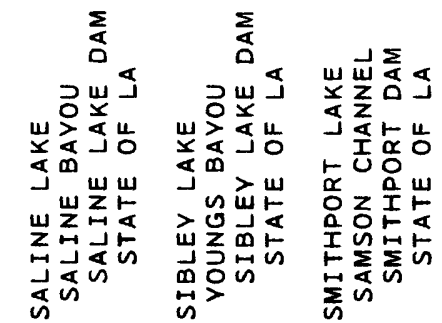




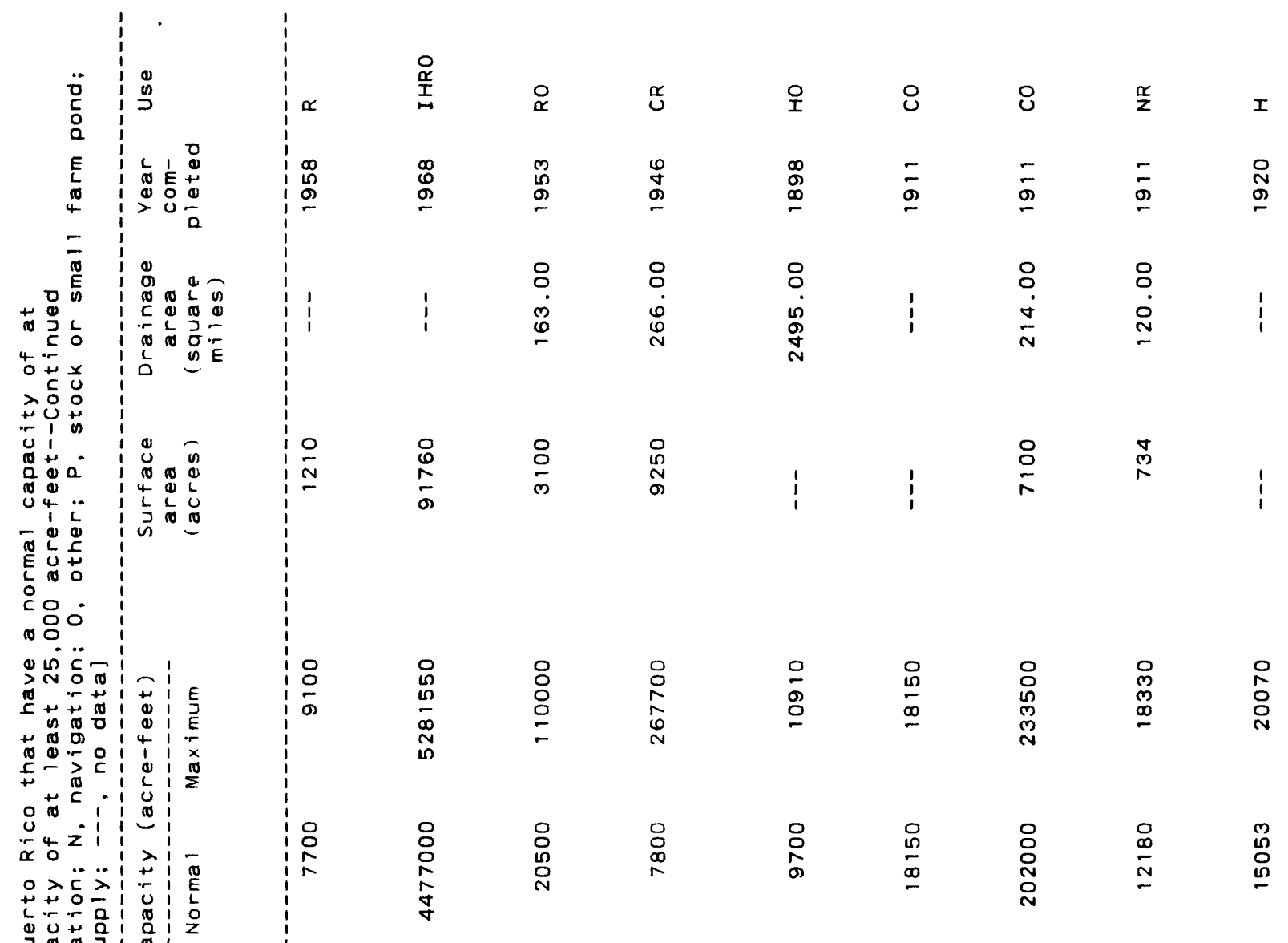

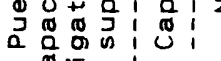

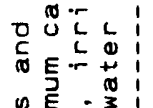

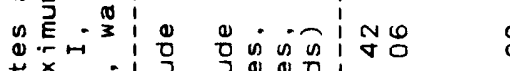

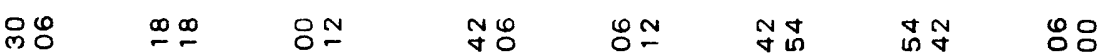

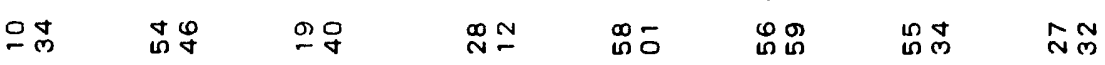

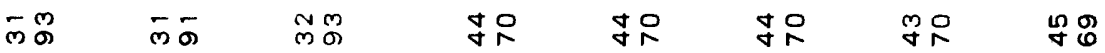

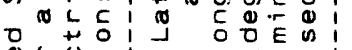
ㄷำ

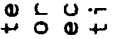

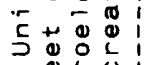

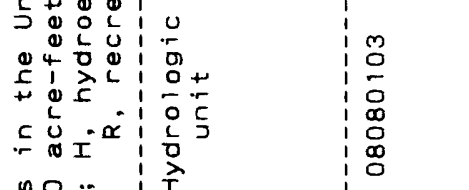

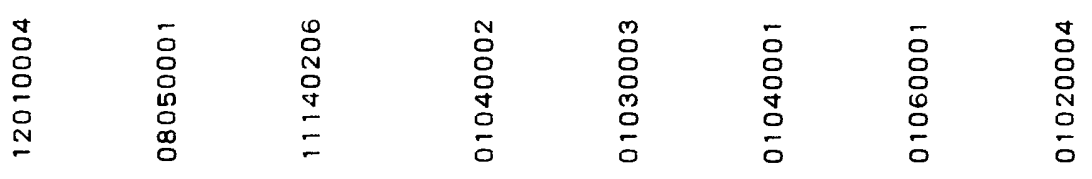
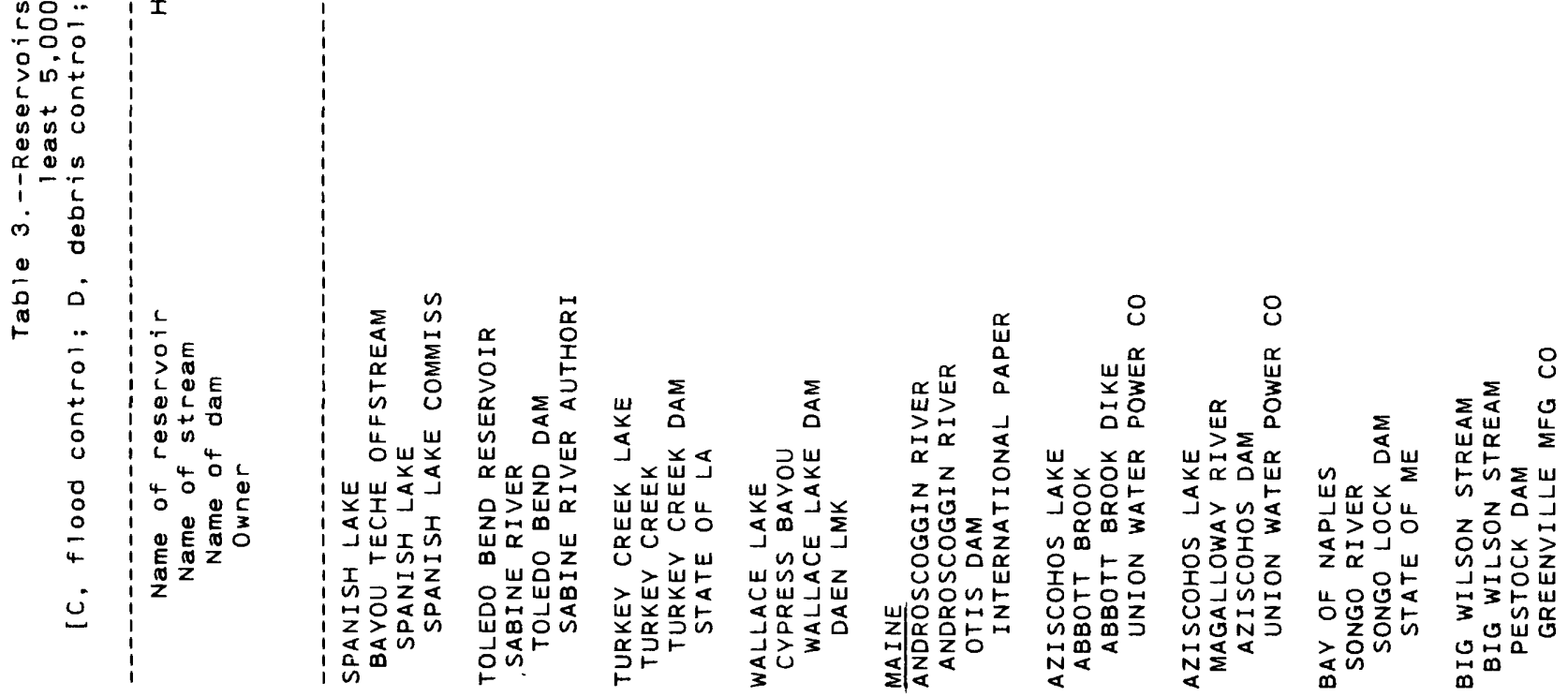


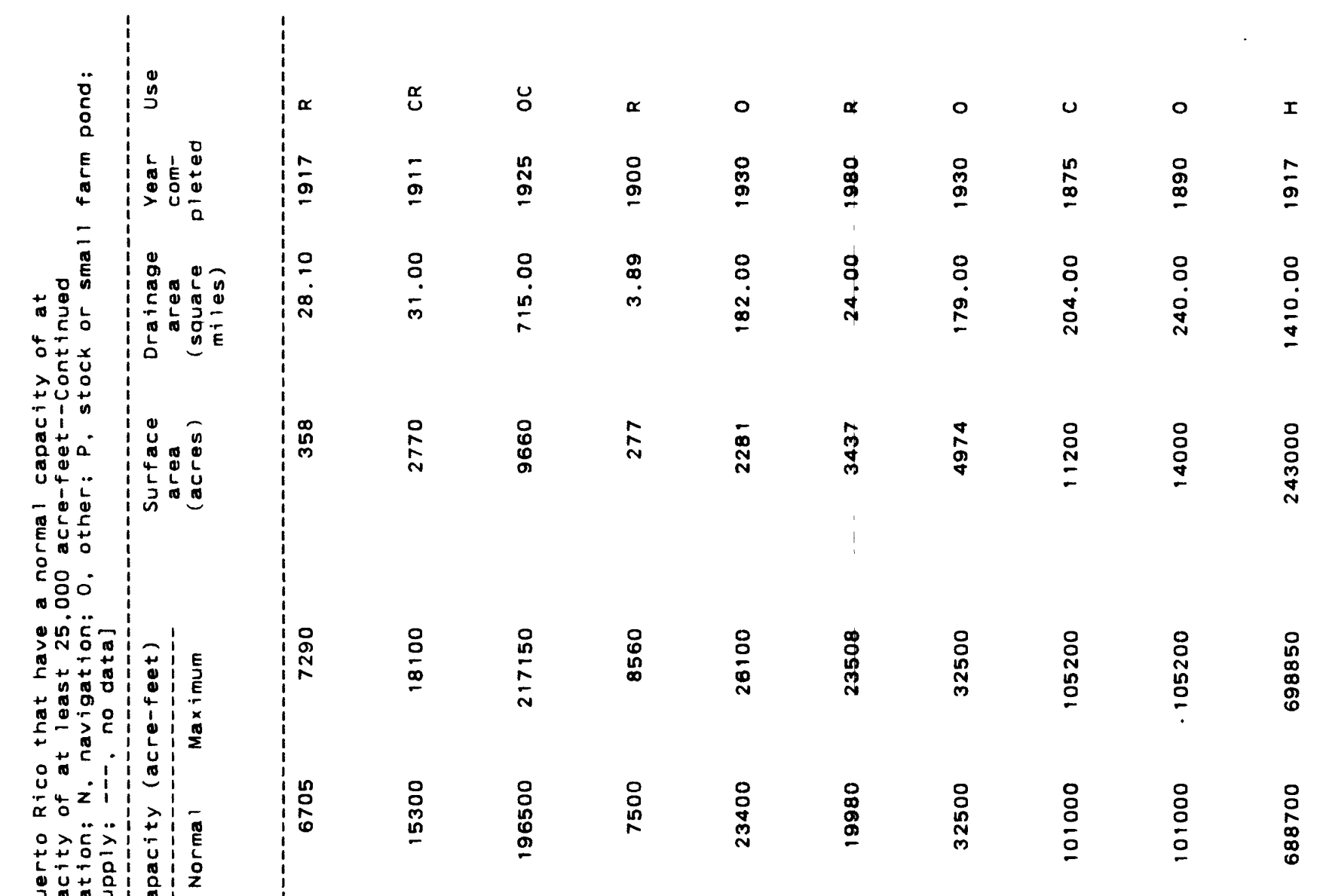

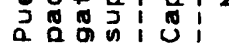

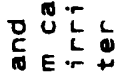

:

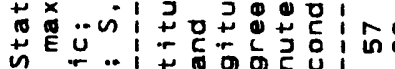

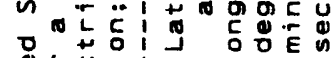

98

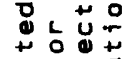

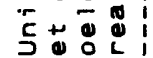

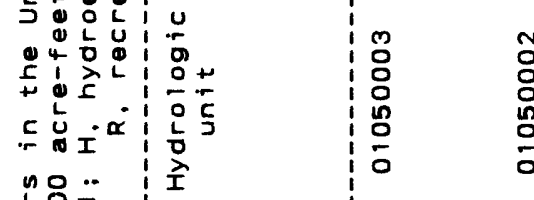

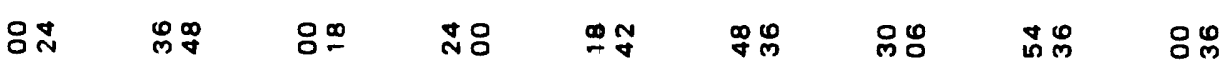

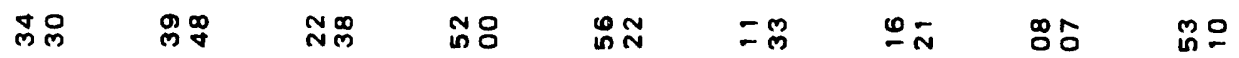

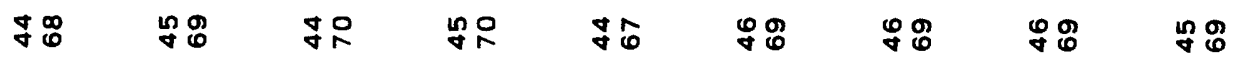

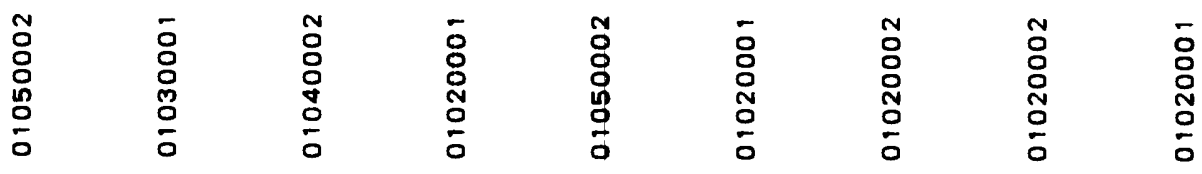

is

$\operatorname{lol} 2$

:

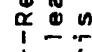

(6)

$\stackrel{\circ}{\circ}$
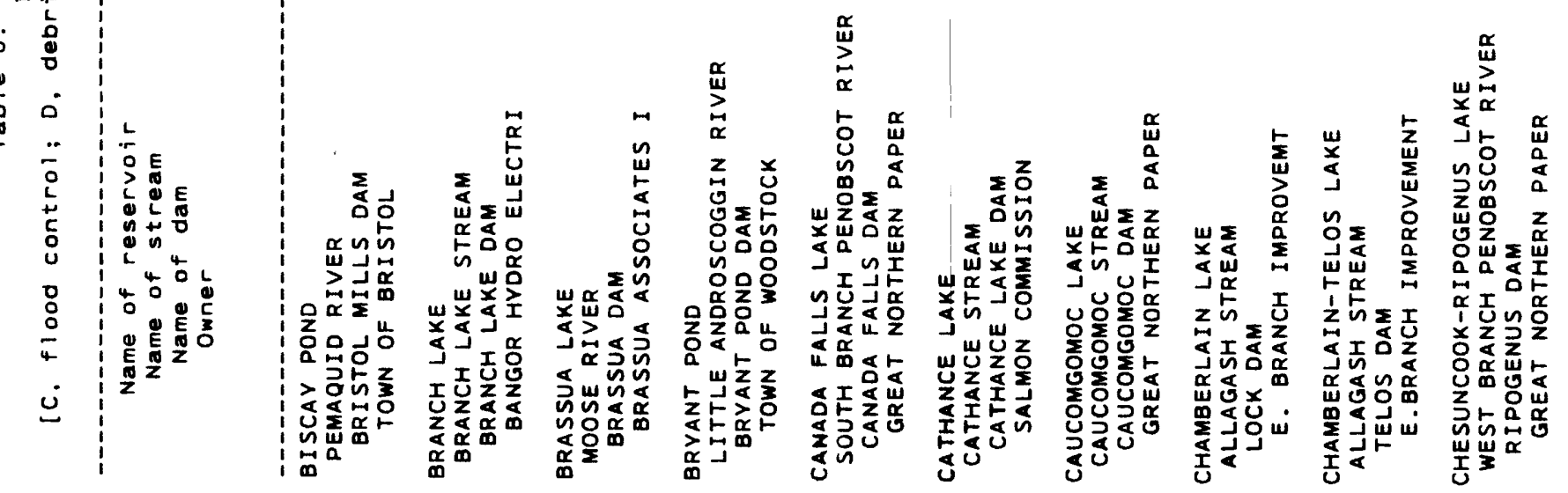


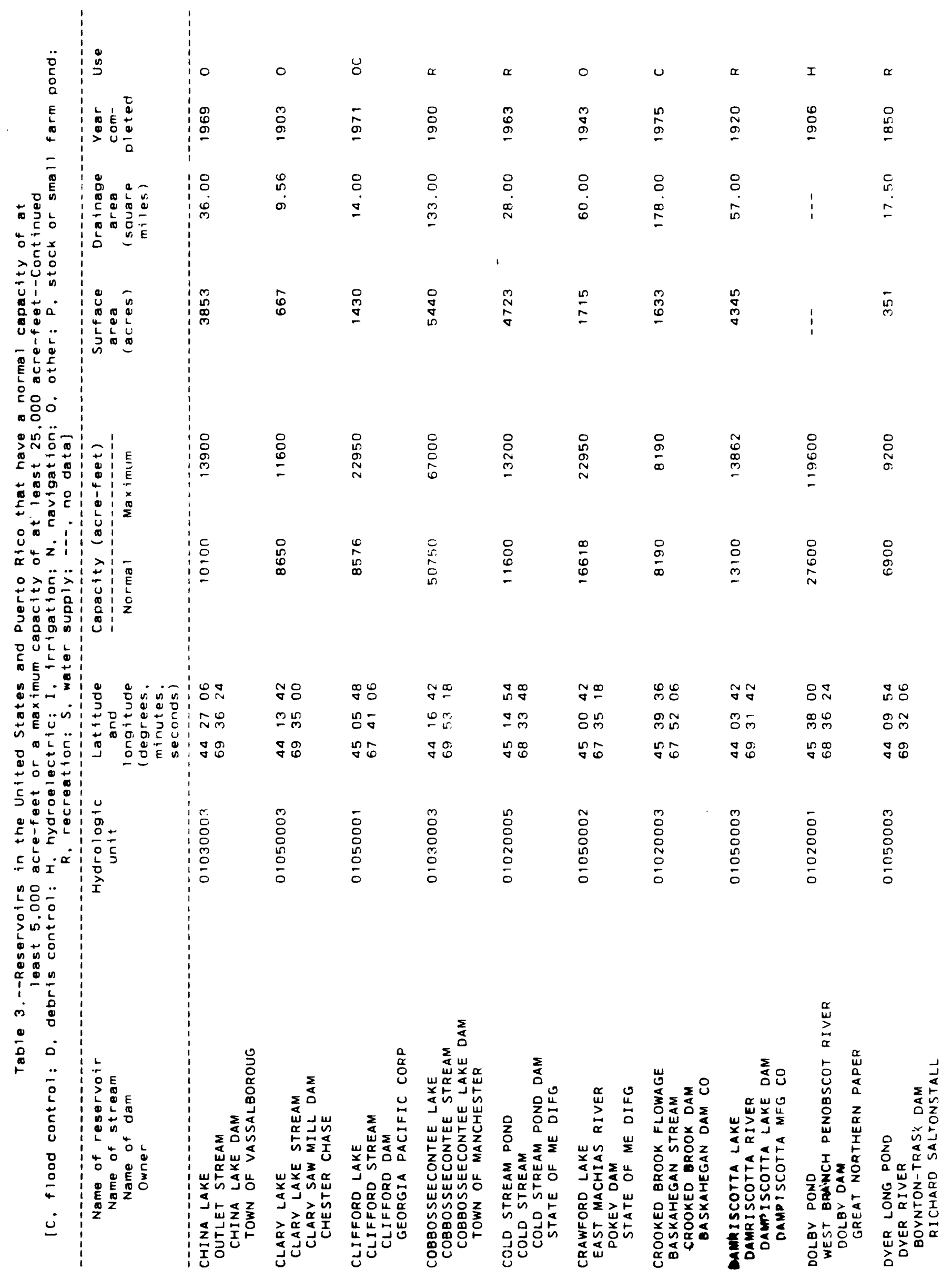




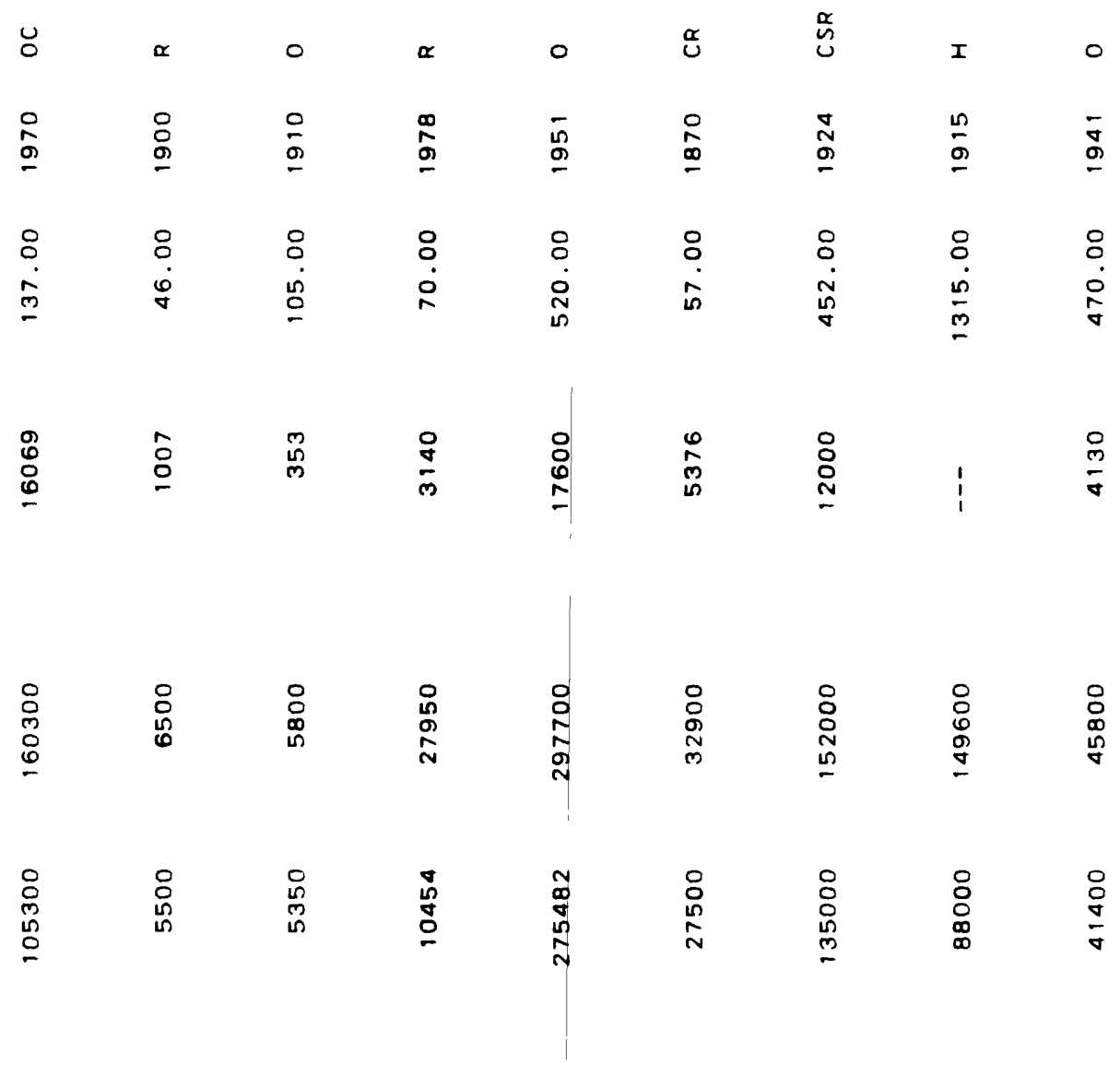

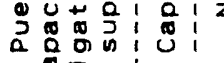

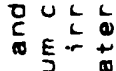

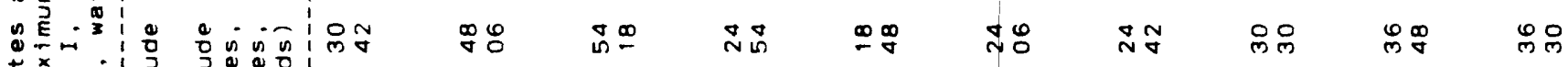

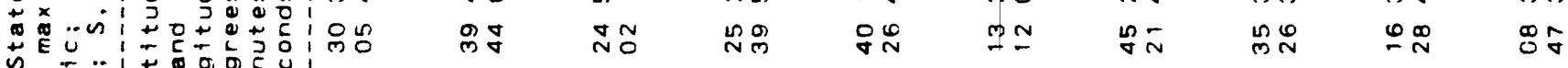

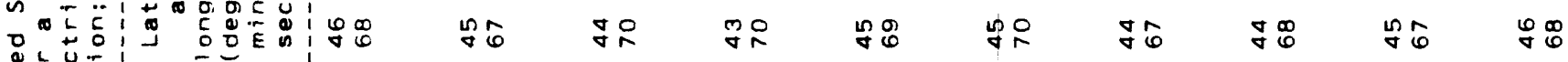

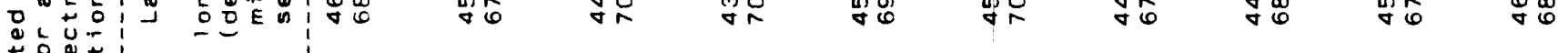
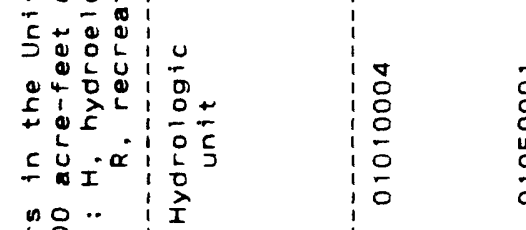

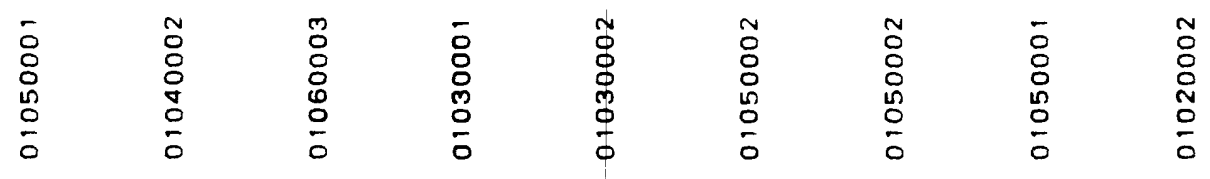

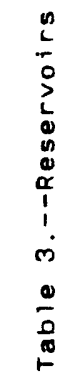

年

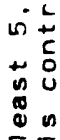

i
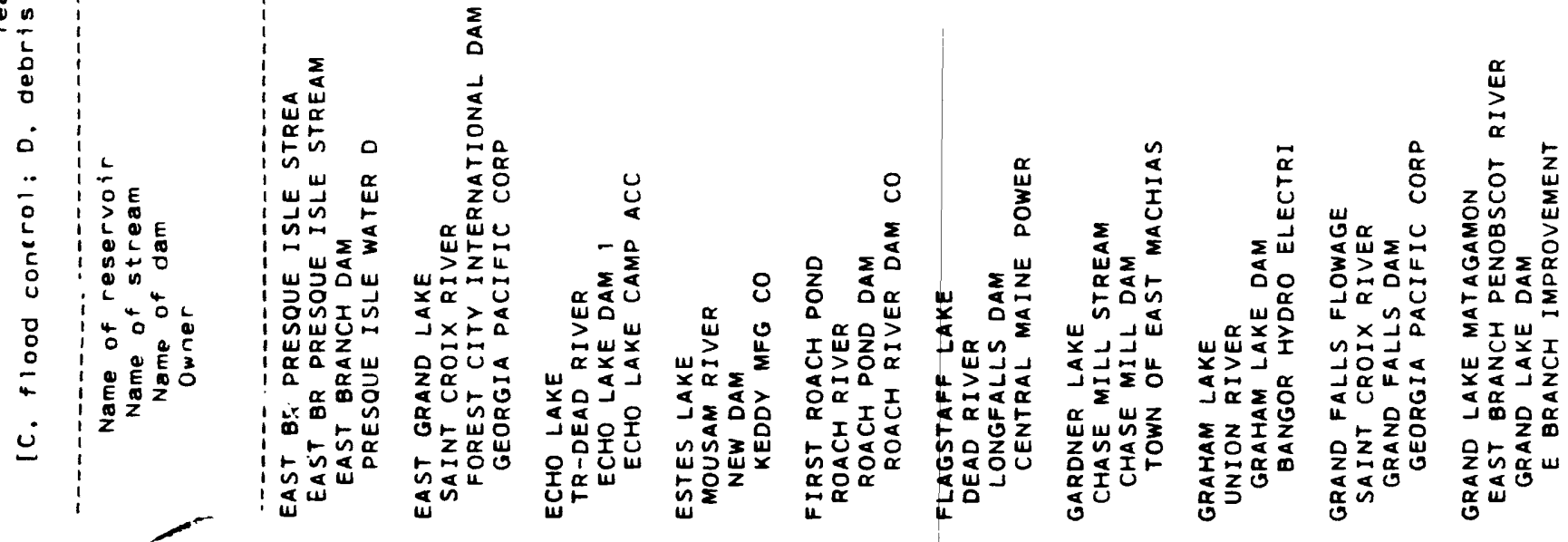


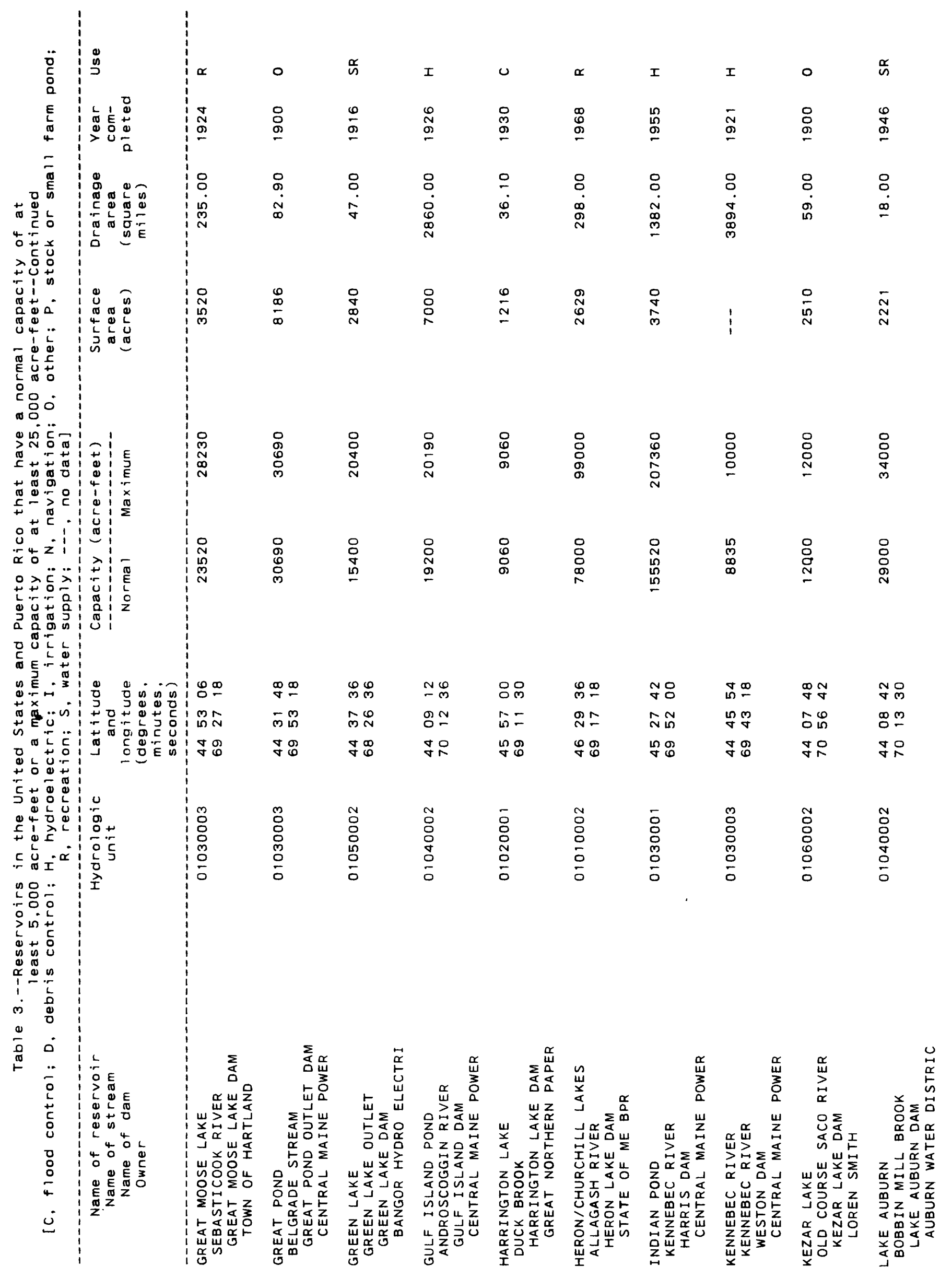




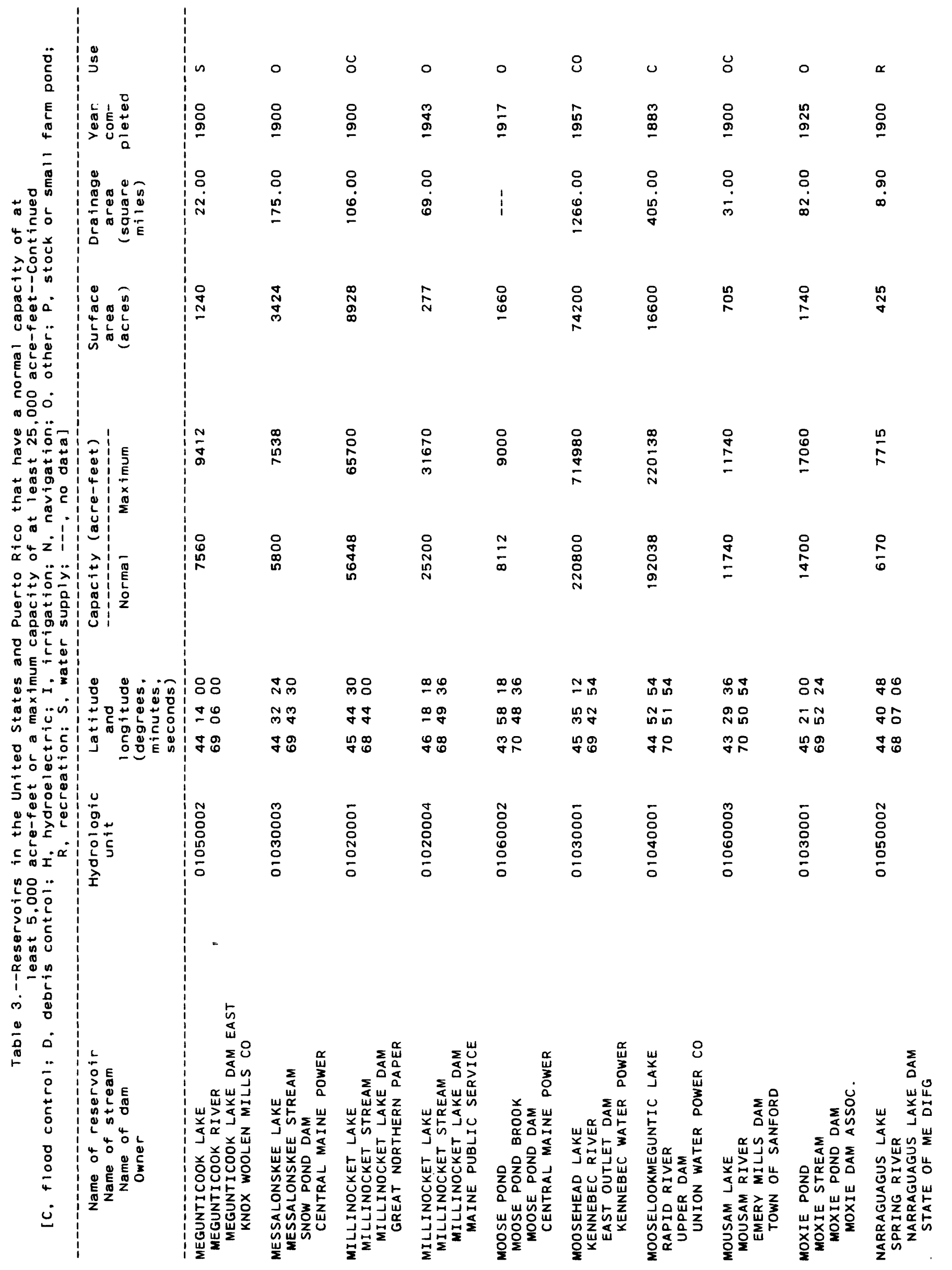




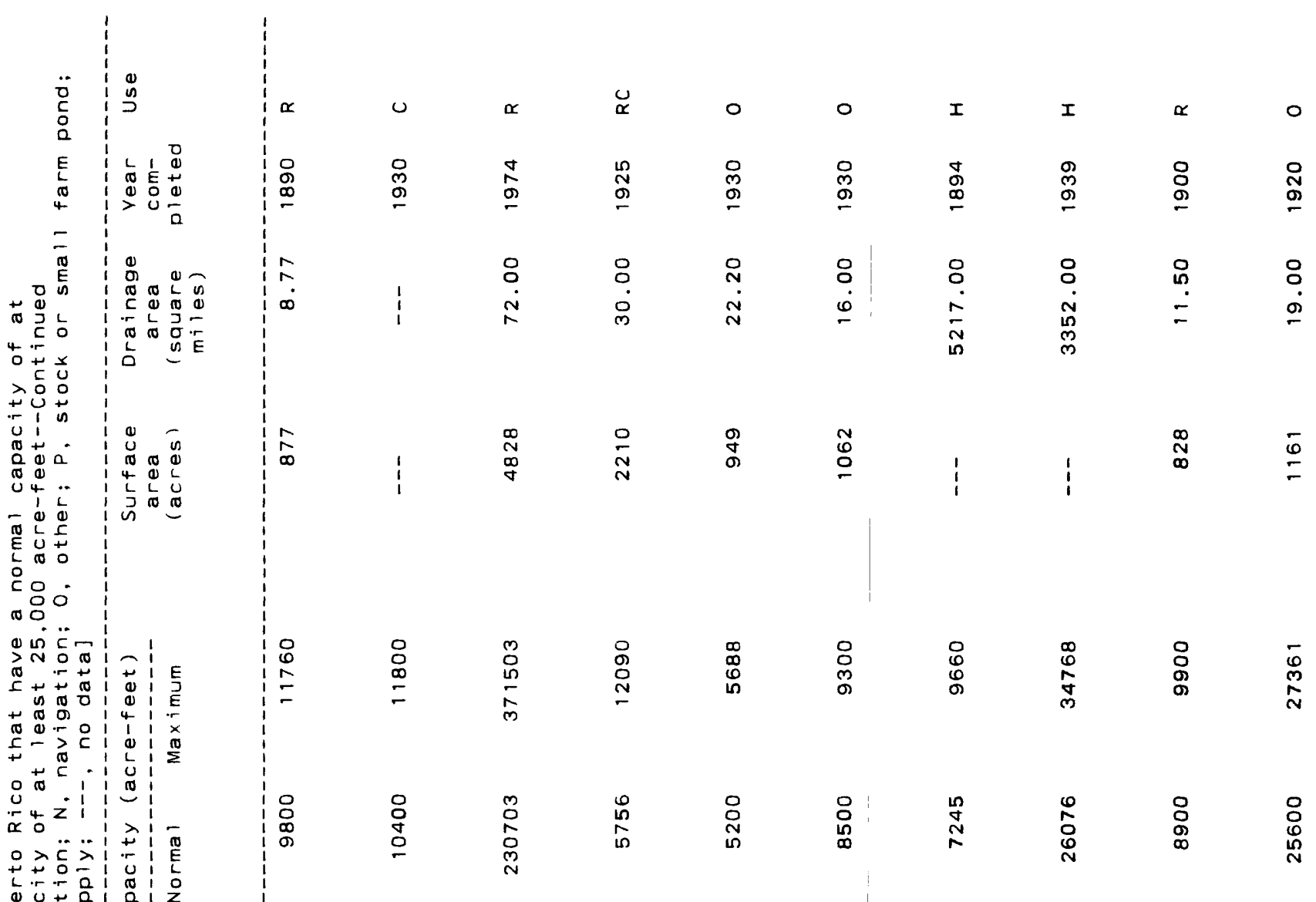

ब计告

\begin{tabular}{ll|l|l}
3 & $\pi$ & $\pi$ & 0 \\
0 & 0 & 0 & 0
\end{tabular}

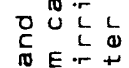

की है.

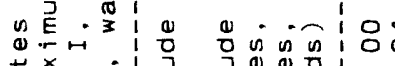

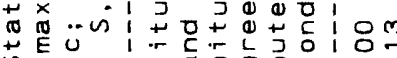

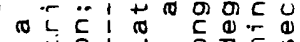

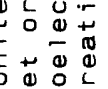

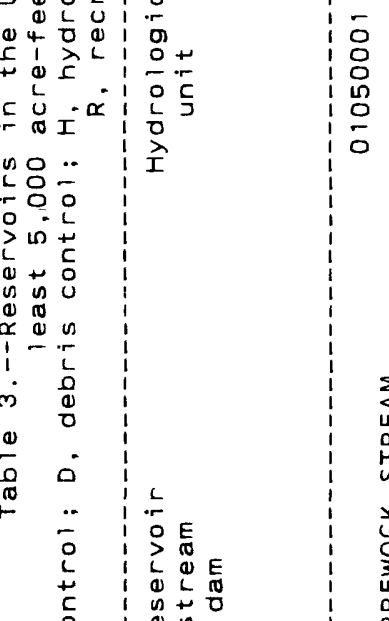

\begin{tabular}{|c|c|c|c|c|c|c|c|}
\hline$\simeq \cong$ & $\cong 8$ & ๐ & $\stackrel{\infty}{q} \underset{+}{\infty}$ & $\sim^{\infty}$ & ㅇ & $\simeq \stackrel{m}{0}$ & 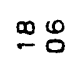 \\
\hline$\overline{0}$ & $\begin{array}{l}\infty \mathbb{N} \\
0\end{array}$ & 華 & 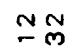 & 迈車 & 느요 & लే & भुज \\
\hline 98 & டி & mo & 웅 & ing & in $\infty$ & 过 & 寸 \\
\hline
\end{tabular}

$\begin{array}{ll} & \\ 1 & \\ 0 & 0 \\ 0 & 0 \\ 0 & 0 \\ 0 & 0 \\ 0 & 0 \\ 0 & 0\end{array}$

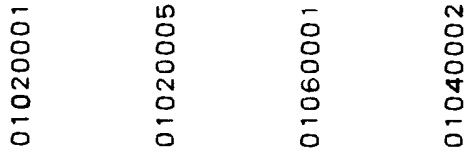

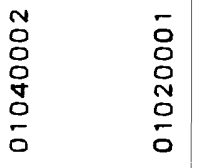

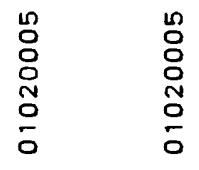

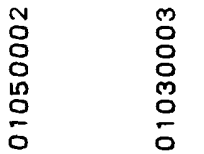

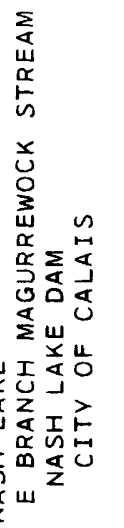

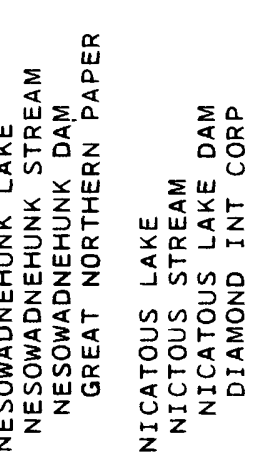
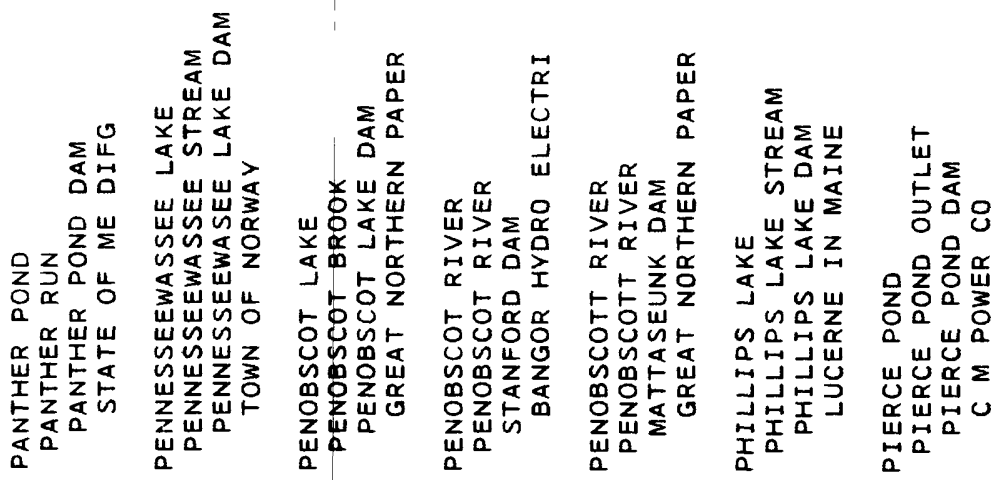


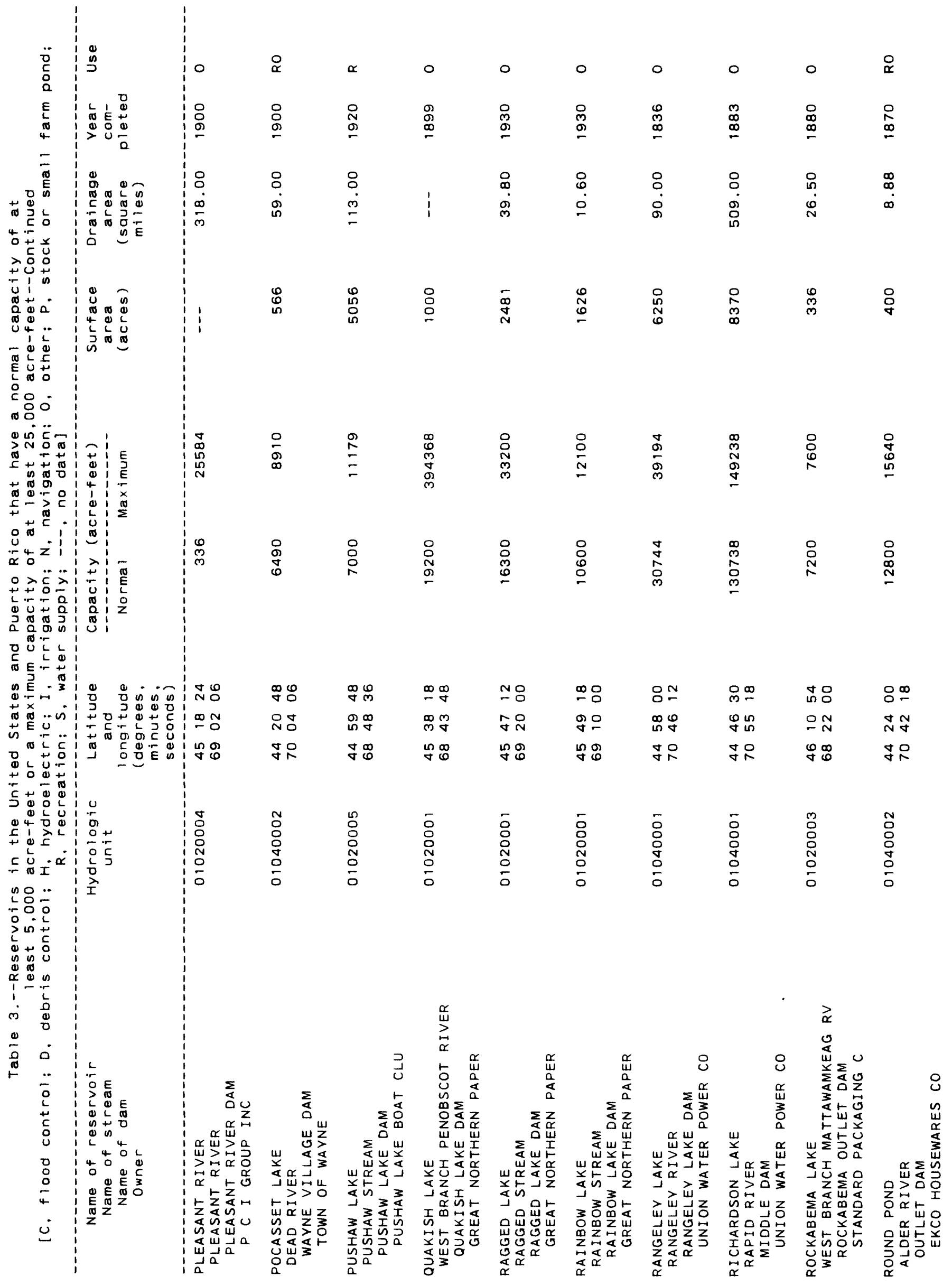




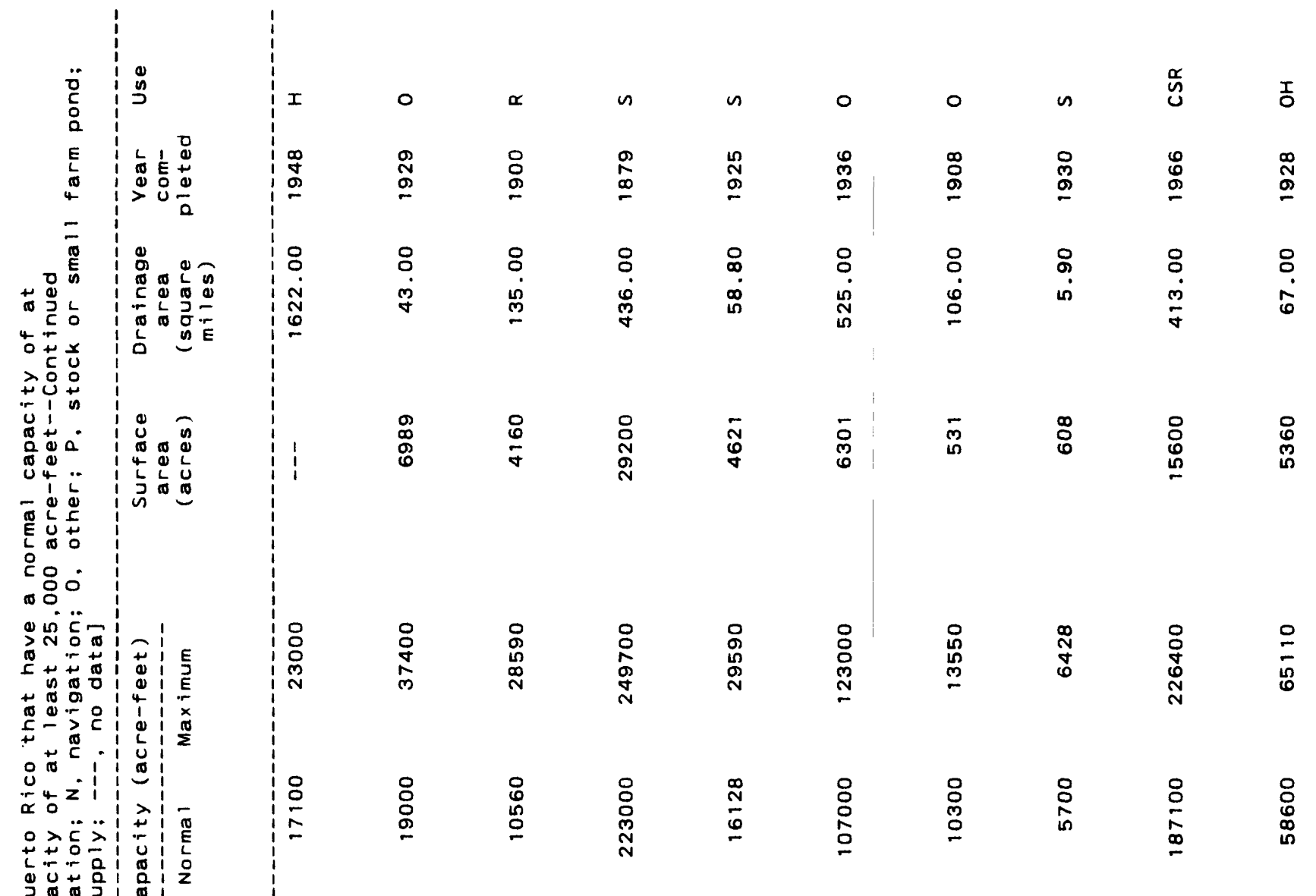

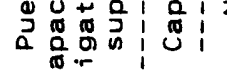

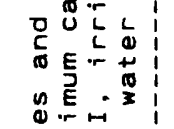

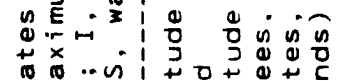

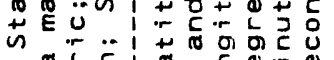

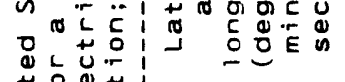

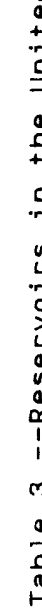

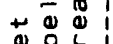

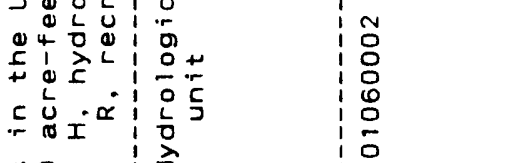

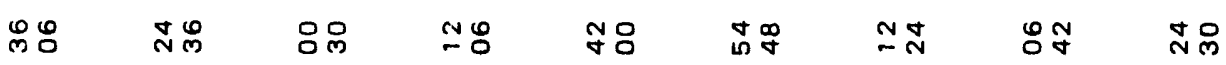

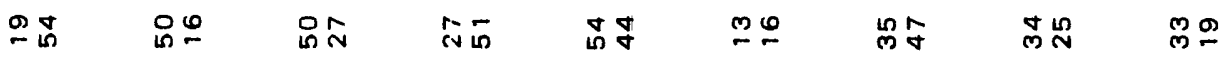

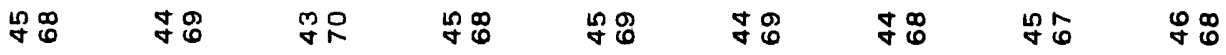

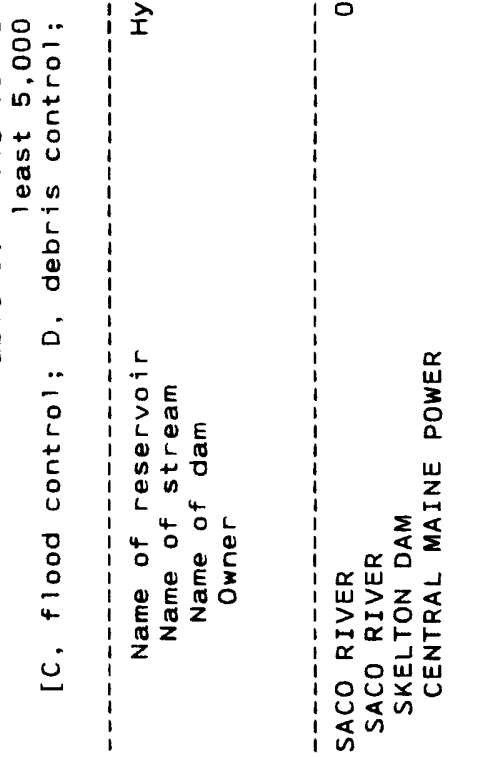

$\begin{array}{ll}\text { I } & \text { O } \\ \text { Oे } & 0 \\ \text { N } & 0 \\ 0 & 0 \\ 0 & 0\end{array}$

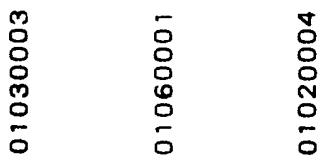

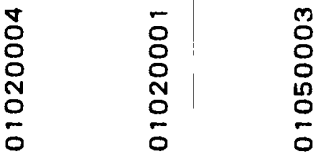

$\begin{array}{lll}\text { n } & - & \text { D } \\ 0 & 0 & 0 \\ \text { ก } & 0 & 0 \\ 0 & 0 & 0 \\ 0 & 0 & 0\end{array}$

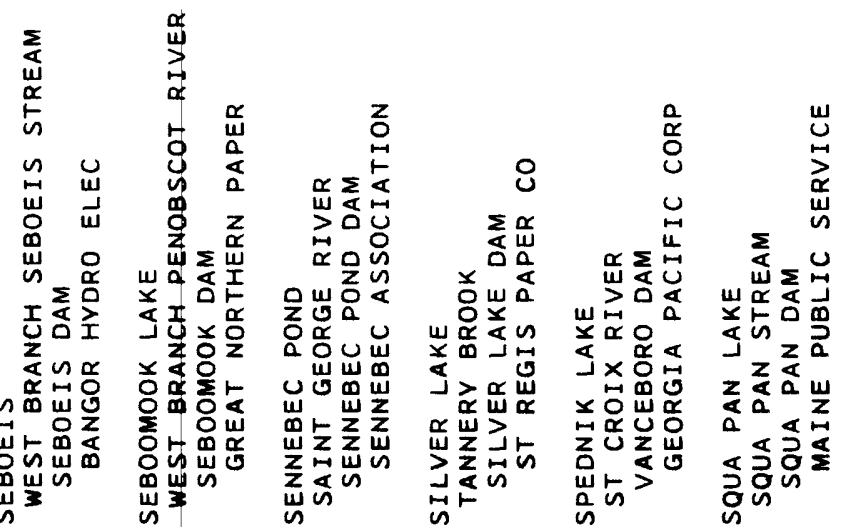




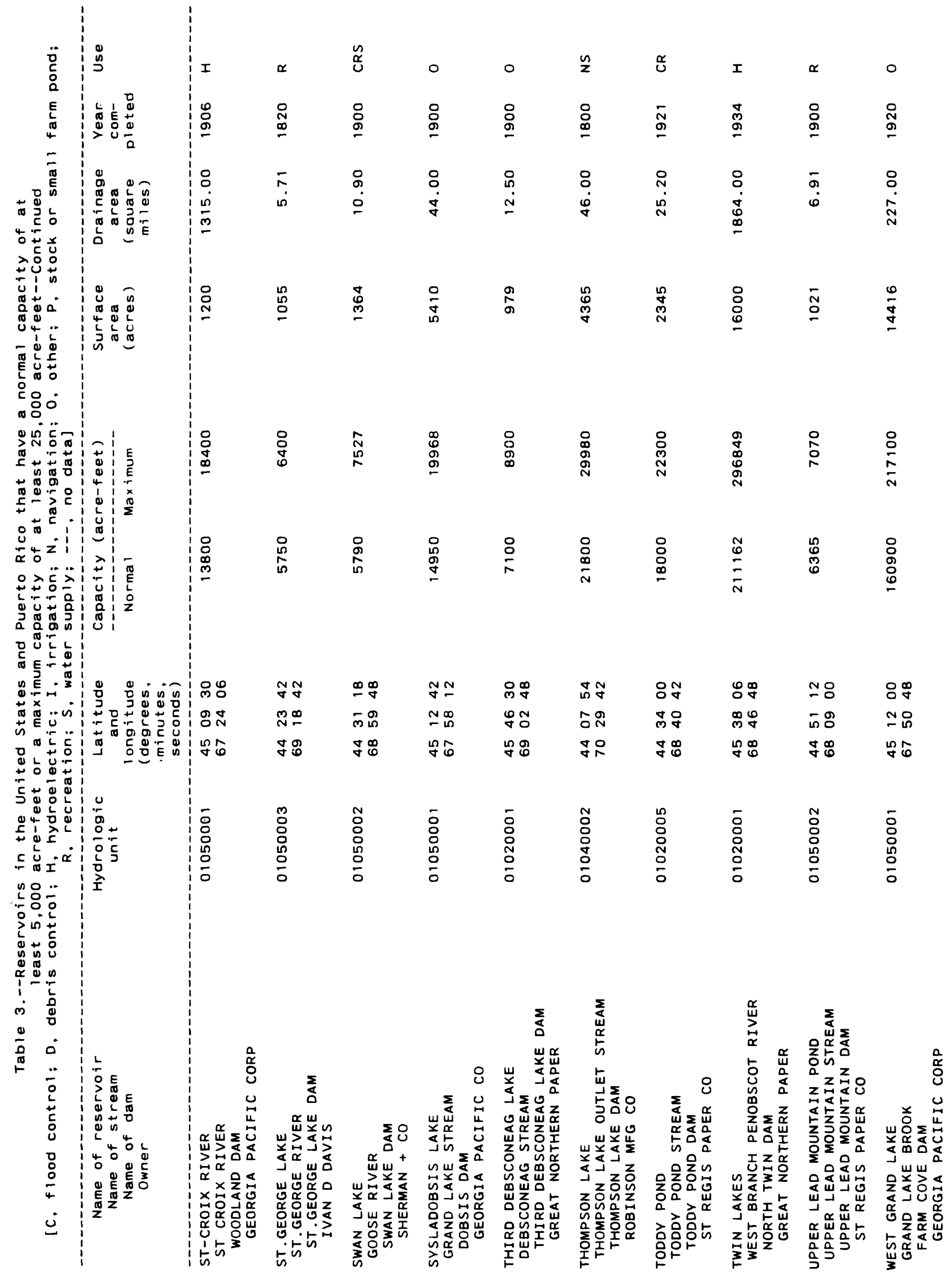




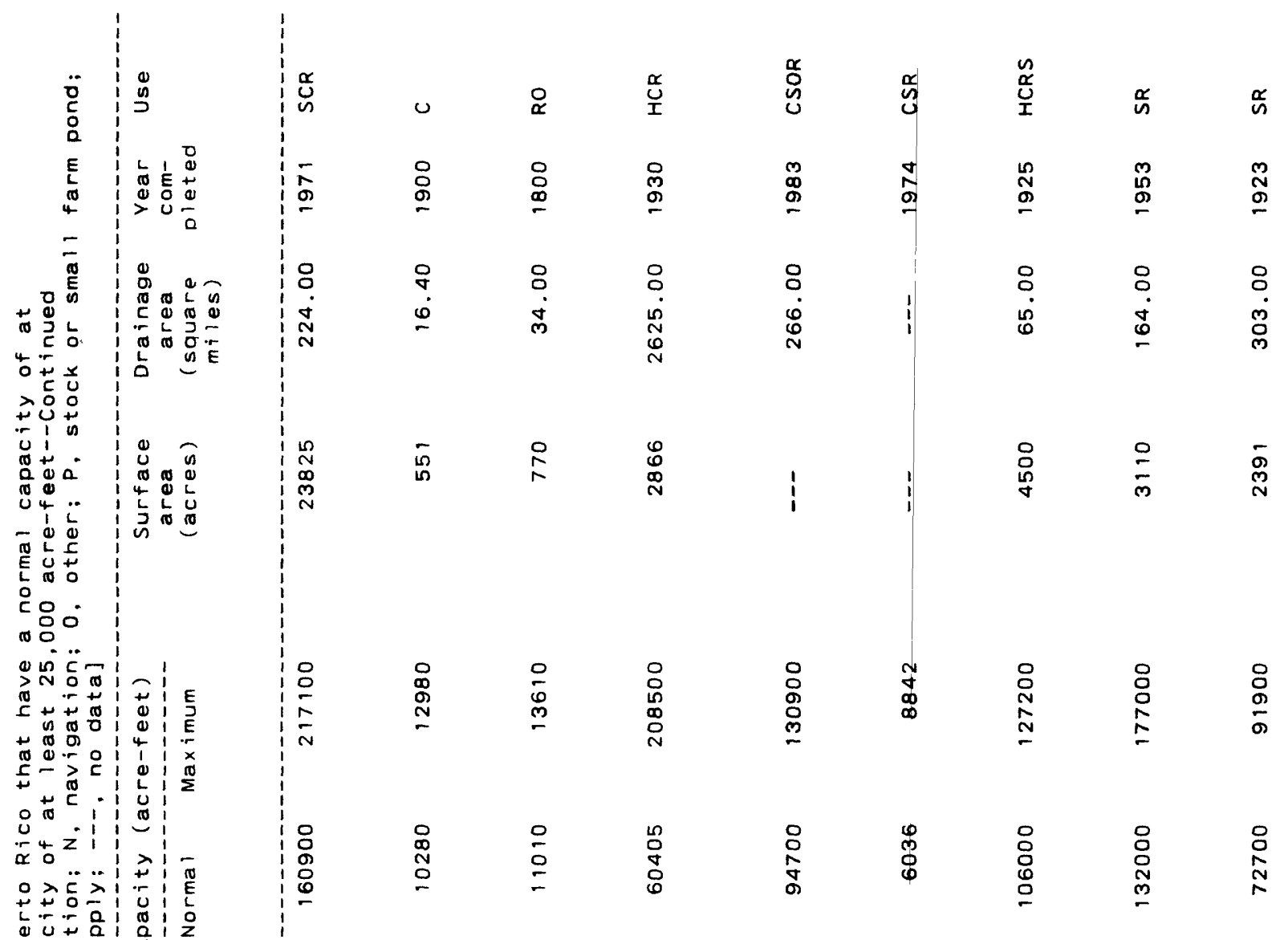

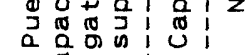

은

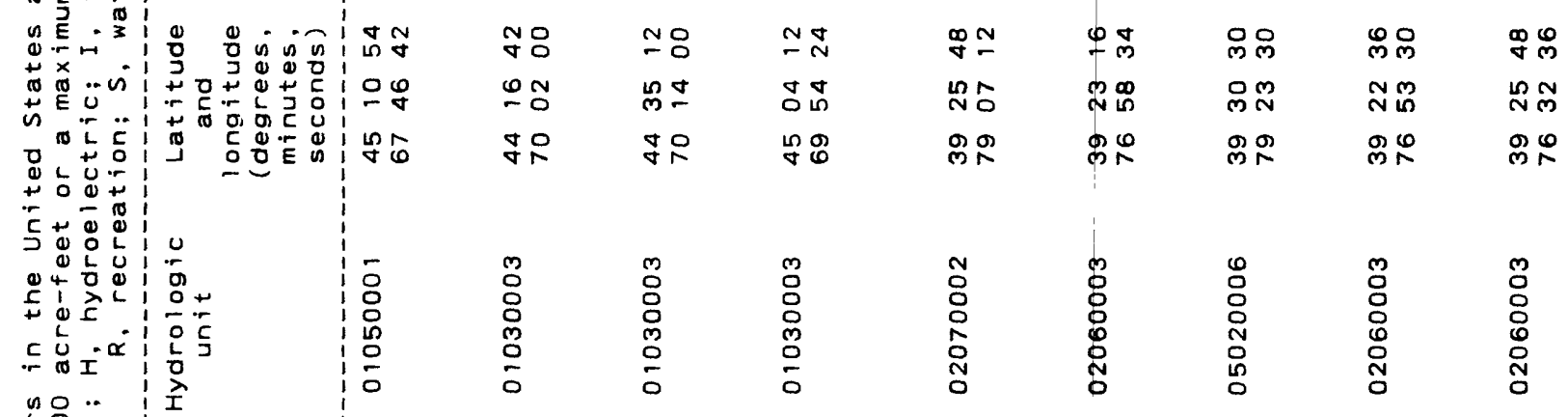

\section{.}

$>\ln$

ot

10

i

$\therefore$

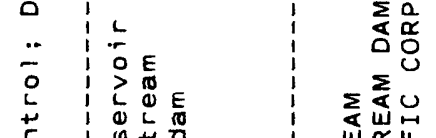

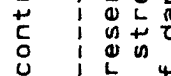

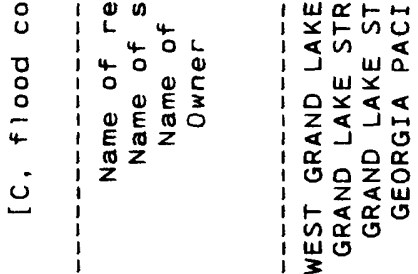
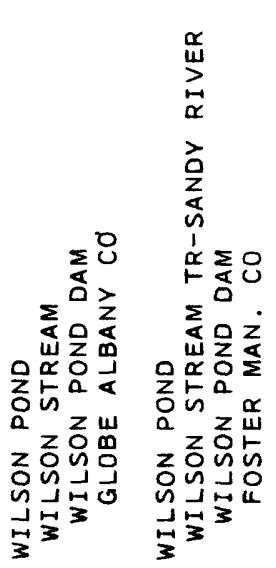

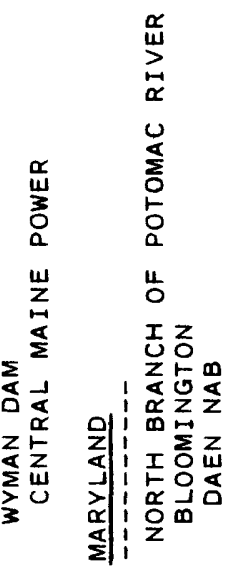

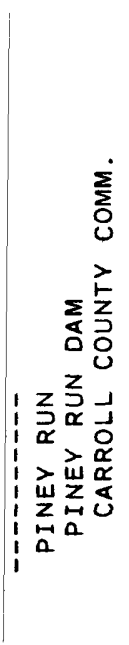

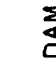

品

品

岃它号

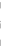

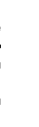

蛋

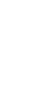

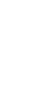




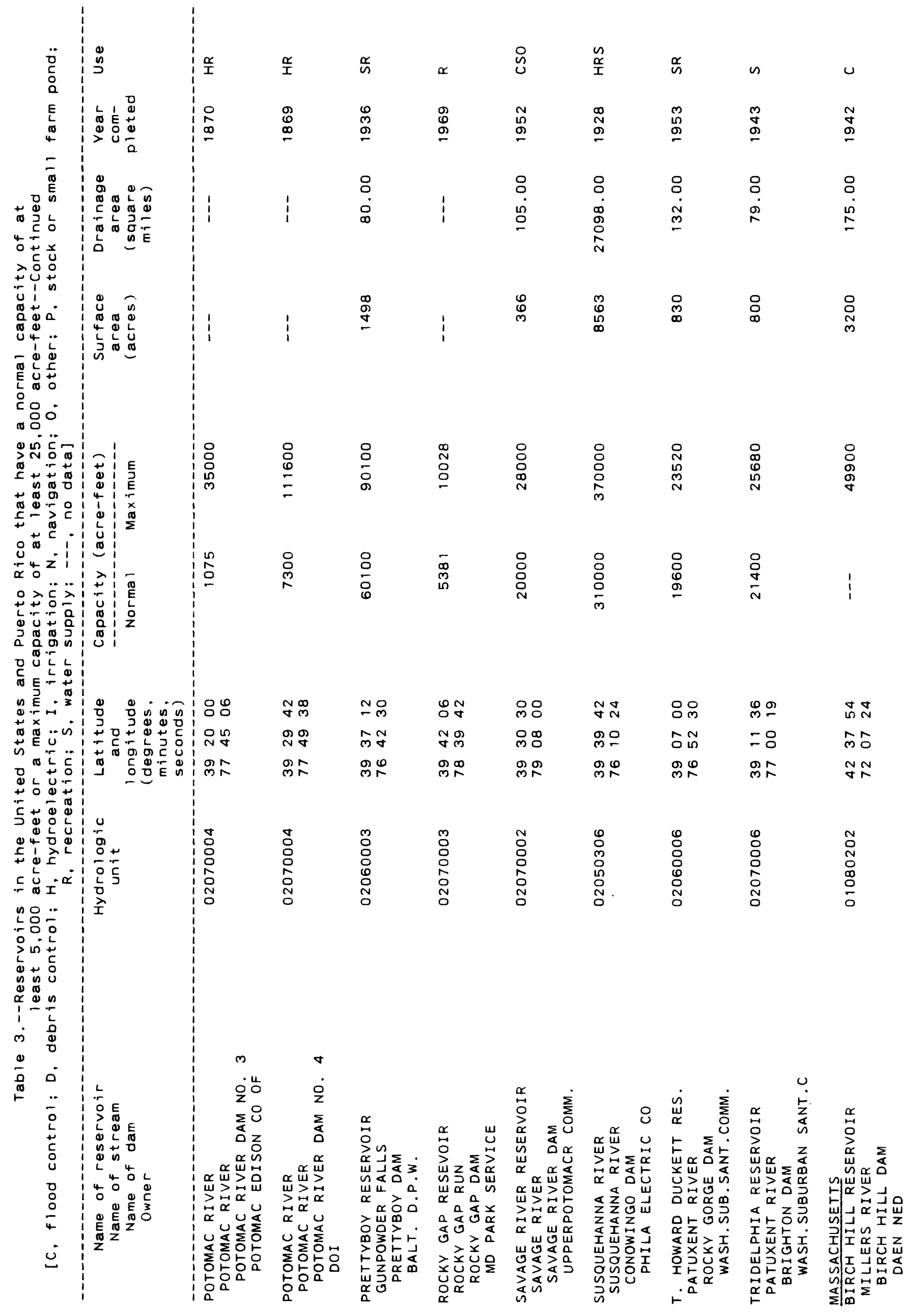




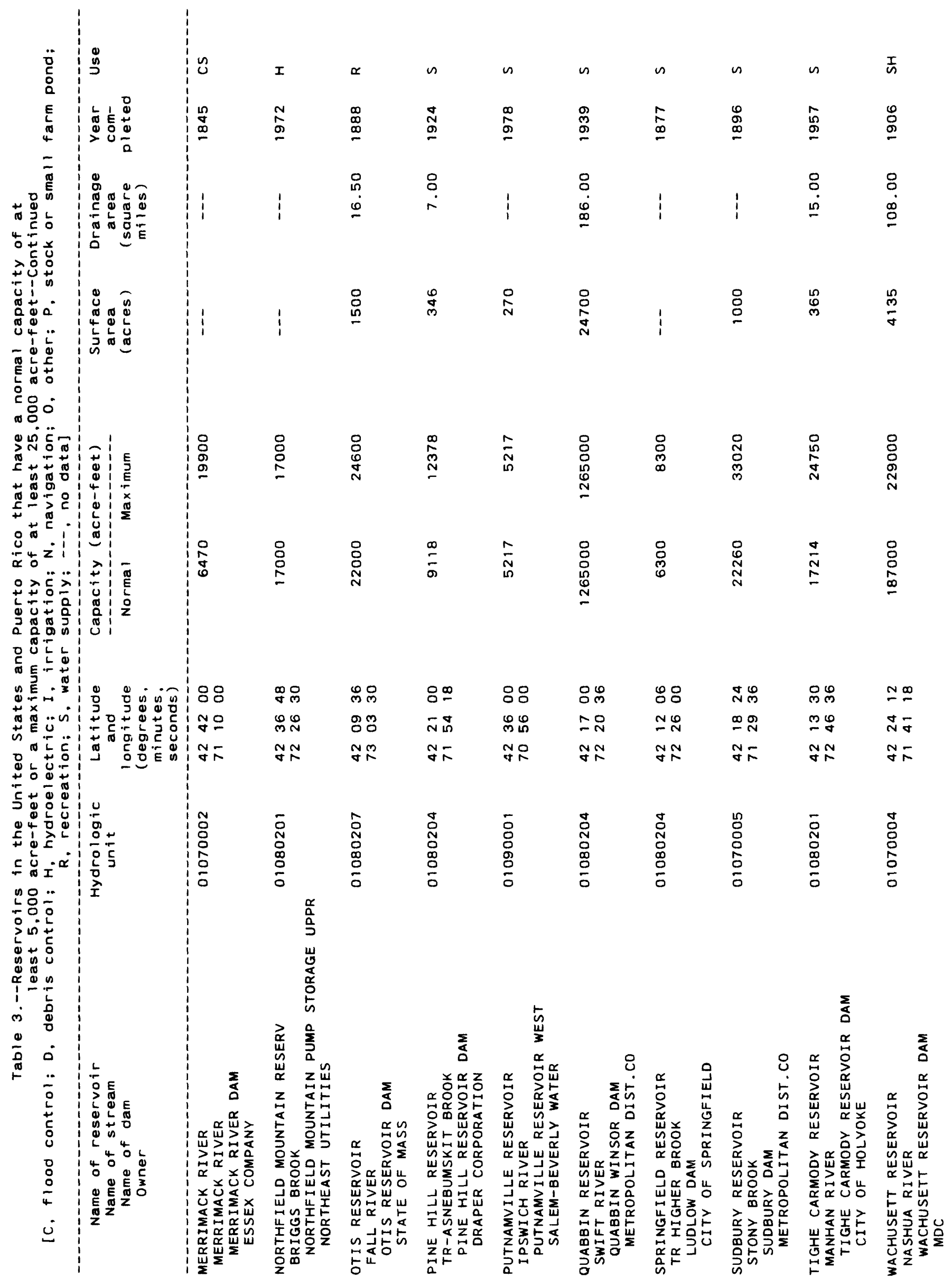




\begin{tabular}{|c|c|c|c|c|c|c|c|}
\hline$I$ & $I$ & $\underset{\alpha}{\frac{T}{Z}}$ & I & I & $I$ & I & $I$ \\
\hline$\frac{9}{9}$ & 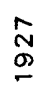 & $\frac{0}{0}$ & $\frac{3}{2}$ & 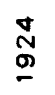 & $\frac{\Phi}{\Phi}$ & $\frac{N}{\sigma}$ & 官 \\
\hline 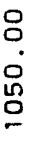 & 吕 & 1 & 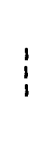 & 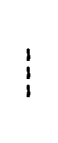 & 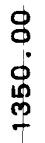 & 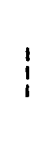 & $i$ \\
\hline 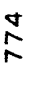 & 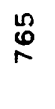 & 1 & $\vdots$ & $!$ & 1 & 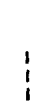 & 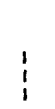 \\
\hline $\begin{array}{l}0 \\
\stackrel{0}{0} \\
0 \\
\\
N\end{array}$ & $\begin{array}{l}\stackrel{O}{n} \\
\stackrel{N}{N} \\
\stackrel{N}{-}\end{array}$ & $\begin{array}{l}\text { : } \\
\text { पे }\end{array}$ & $\begin{array}{l}\circ \\
\stackrel{\circ}{0} \\
0 \\
\infty\end{array}$ & $\begin{array}{l}\stackrel{0}{0} \\
0 \\
0\end{array}$ & 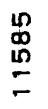 & $i$ & $\begin{array}{l}\stackrel{8}{\circ} \\
\text { 吕 }\end{array}$ \\
\hline 总 & $\begin{array}{l}\text { 吕 } \\
\text { م⿱ 口} \\
0\end{array}$ & 吕 & 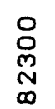 & $\begin{array}{l}\text { 足 } \\
\text { in }\end{array}$ & $\begin{array}{l}\text { 品 } \\
\text { 足 }\end{array}$ & $\begin{array}{l}\stackrel{\circ}{\circ} \\
\stackrel{\text { N }}{N}\end{array}$ & 总 \\
\hline
\end{tabular}

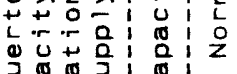

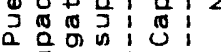

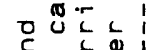

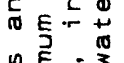

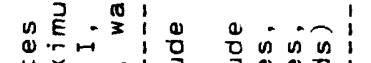

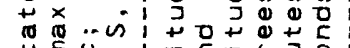

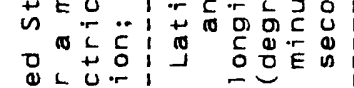

$\because 0 \%$

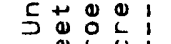

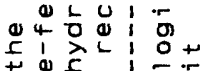

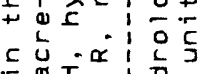

no..

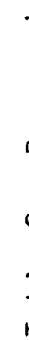

\begin{tabular}{|c|c|c|c|c|c|c|c|}
\hline$\stackrel{\infty}{\infty}$ & 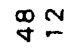 & ๕ั & $\mathcal{F} \mathcal{V}$ & $\stackrel{N}{N}$ & $\stackrel{\infty}{\rightarrow} \stackrel{\infty}{-\infty}$ & $\mathscr{\infty}$ & 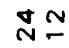 \\
\hline 욤 & 足 & $\stackrel{\infty}{\infty} \underset{N}{\infty}$ & MN & $\mathscr{q} \mathfrak{q}$ & ద్ర & กี & in \\
\hline$\stackrel{\sim}{\sim} \underset{\infty}{\infty}$ & $\stackrel{q}{\infty}$ & \&ু & $\stackrel{m}{\infty}$ & \& & $-\stackrel{\infty}{\infty}$ & ᄂ $\int_{\infty}^{\infty}$ & $\mathcal{F}_{\infty}$ \\
\hline 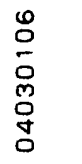 & $\begin{array}{l}\text { D } \\
0 \\
0 \\
\text { m } \\
\text { ơ } \\
\text { ó }\end{array}$ & 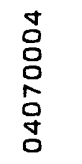 & $\begin{array}{l}\overline{0} \\
\vdots \\
\& \\
0 \\
0\end{array}$ & $\begin{array}{l}\infty \\
0 \\
0 \\
0 \\
0 \\
0 \\
0\end{array}$ & $\begin{array}{l}\overline{0} \\
8 \\
\circ \\
8 \\
8 \\
0\end{array}$ & $\begin{array}{l}\text { D } \\
0 \\
0 \\
0 \\
0 \\
0\end{array}$ & 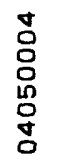 \\
\hline
\end{tabular}

$>$ in

政

年

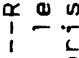

i
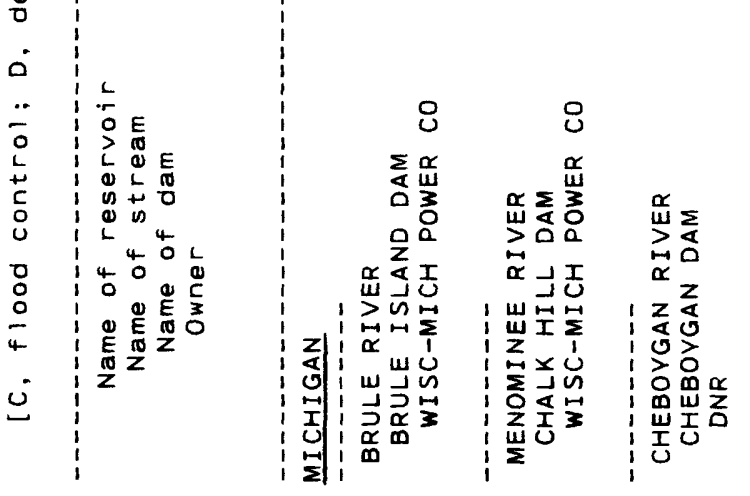

常 


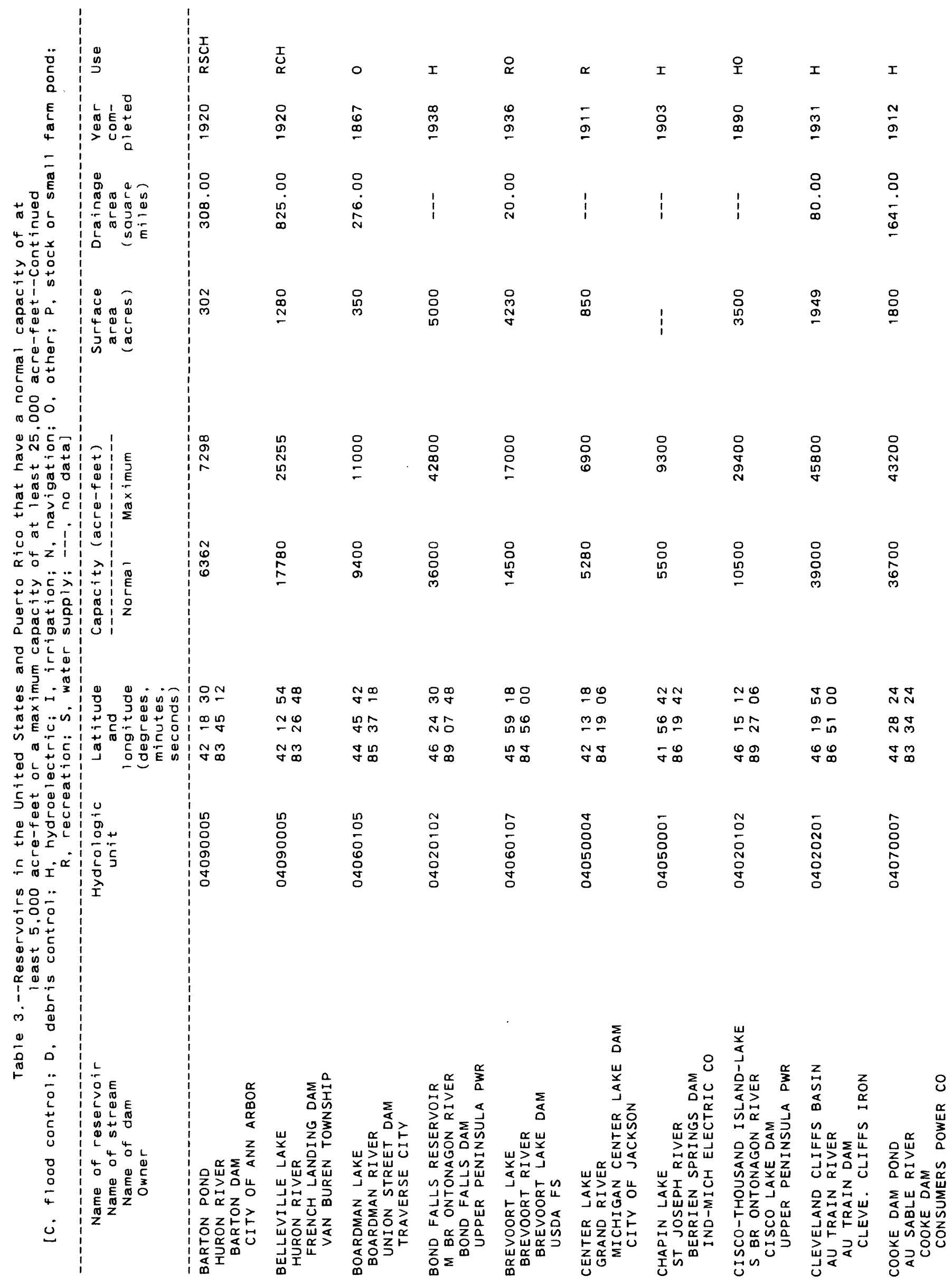




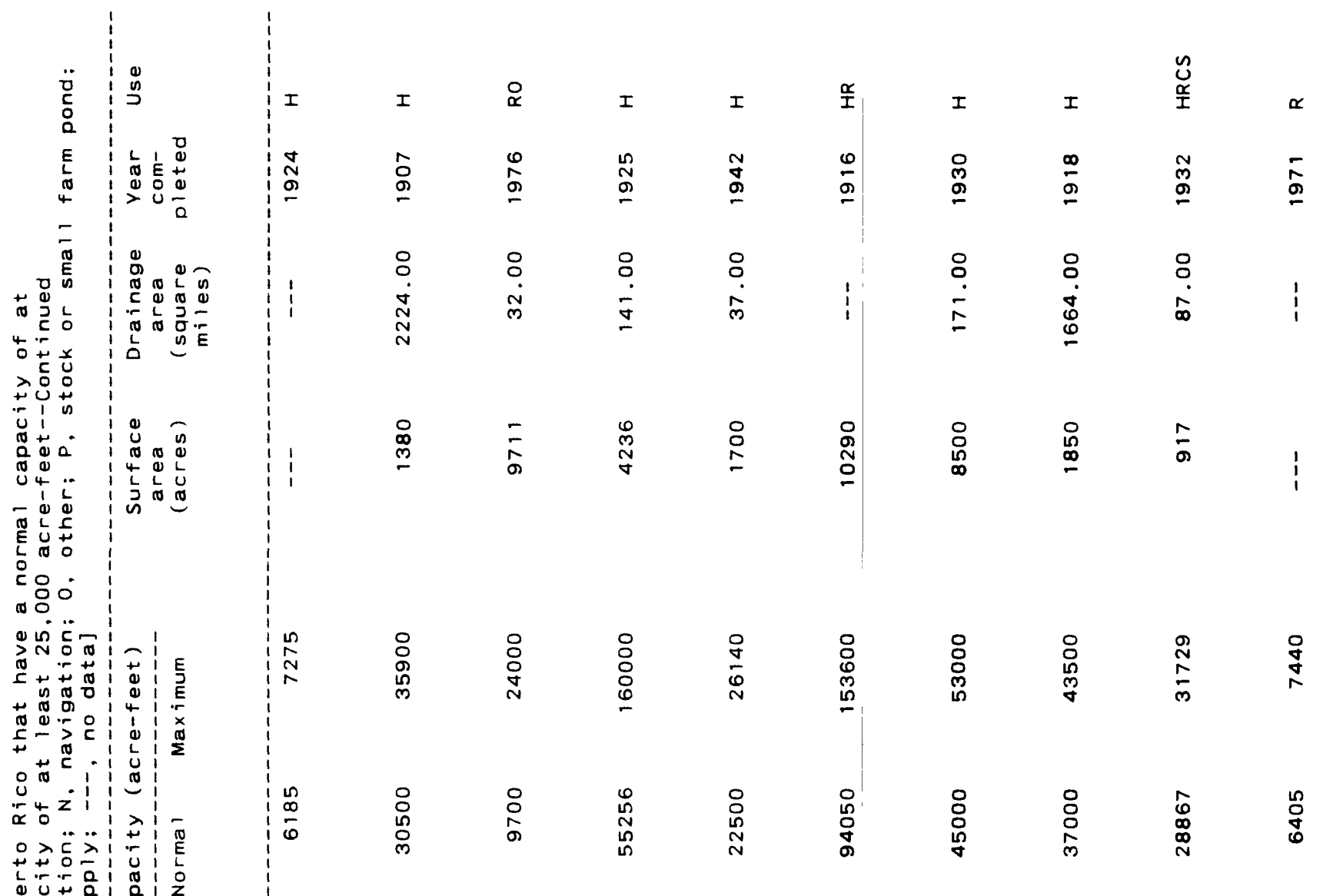

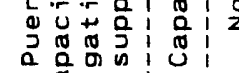

वUنे

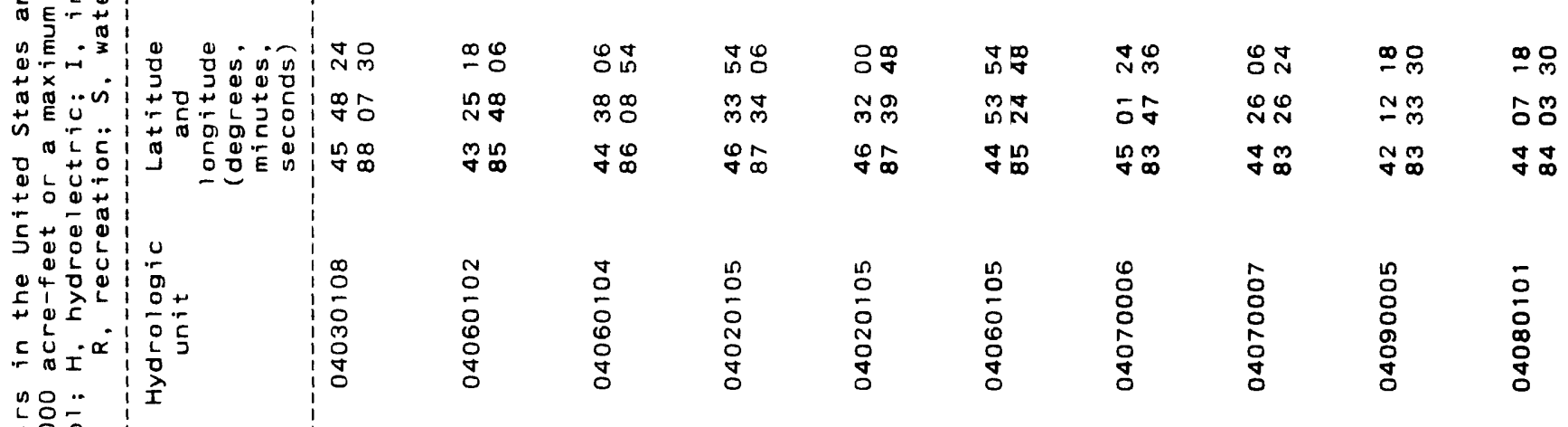

.

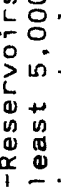

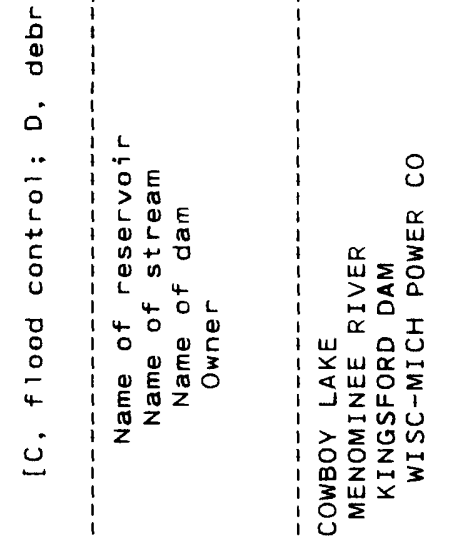

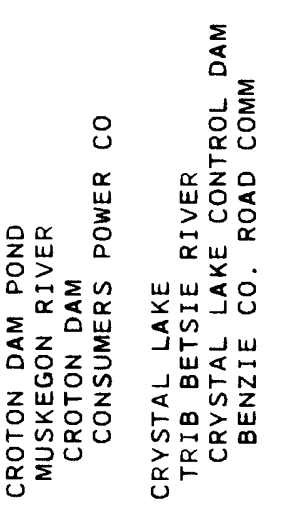
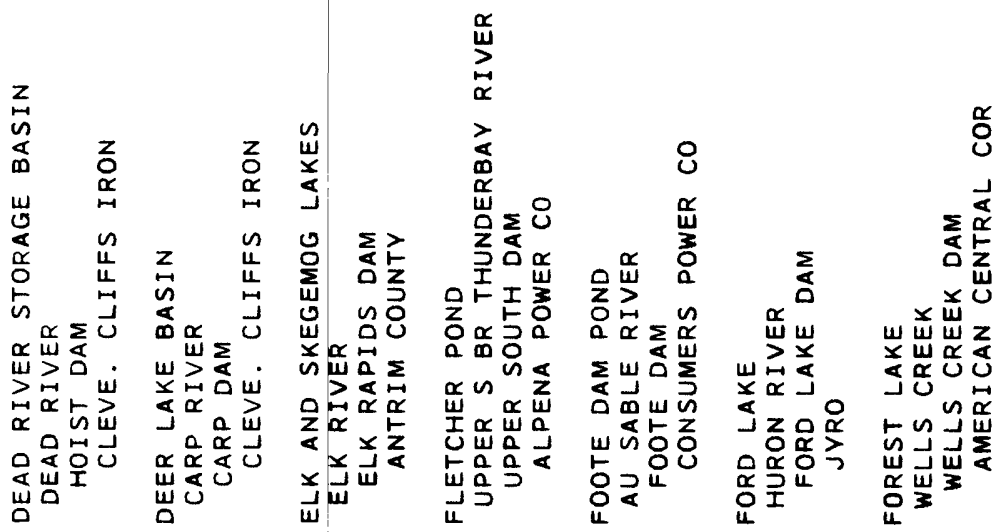


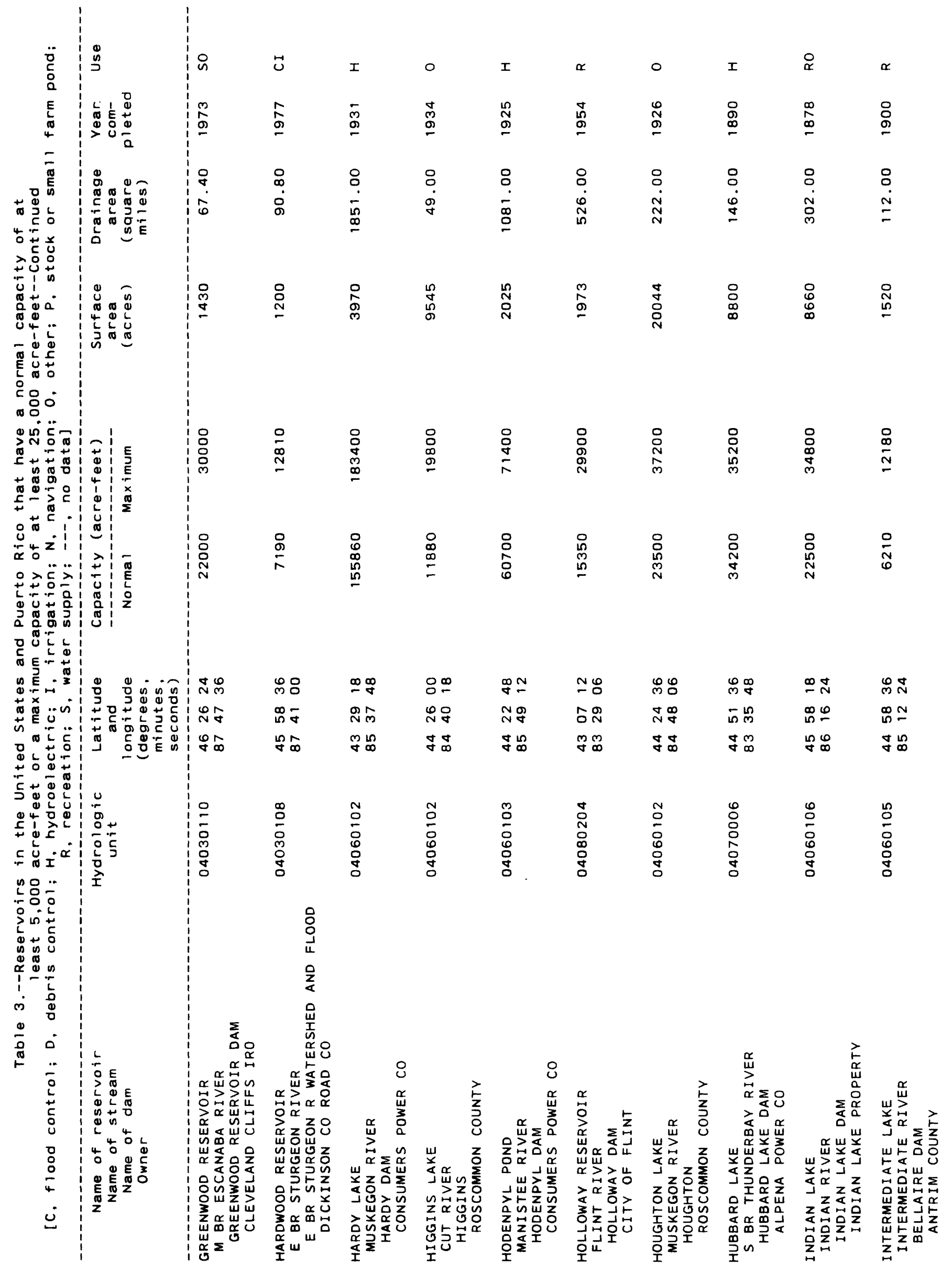




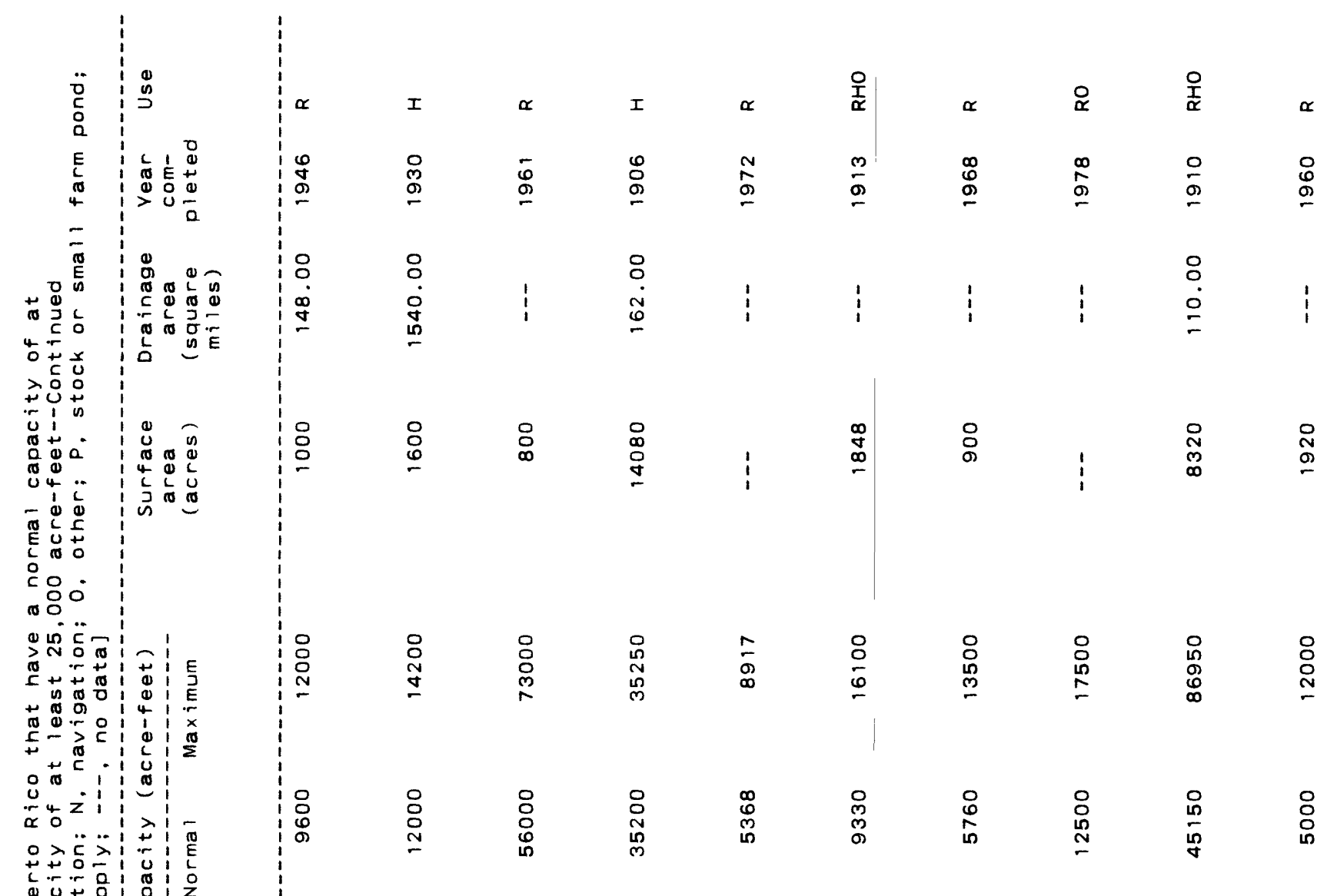

ou+ 0

उ

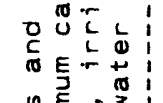

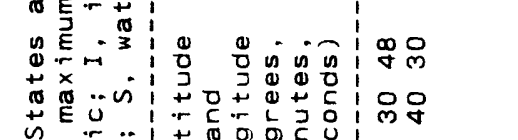

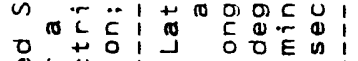

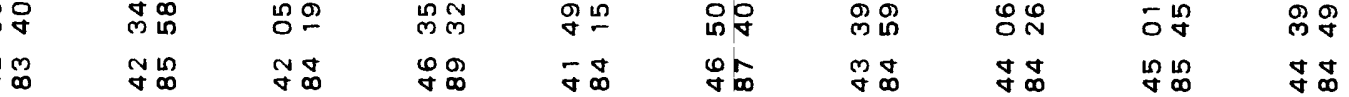

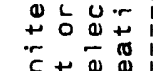

ᄃํ.

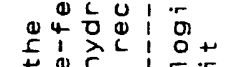

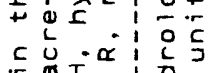

no‥

年

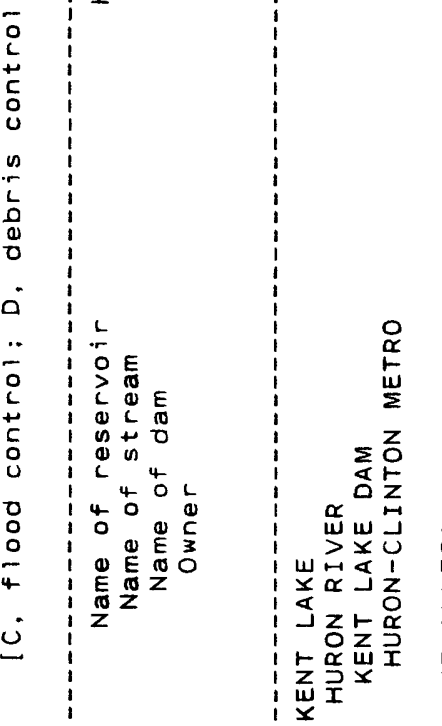

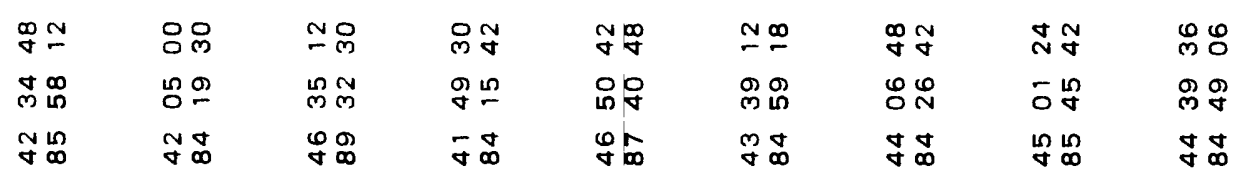

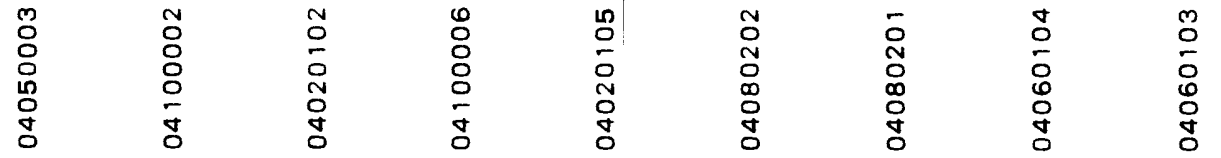

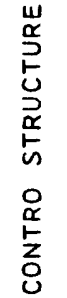




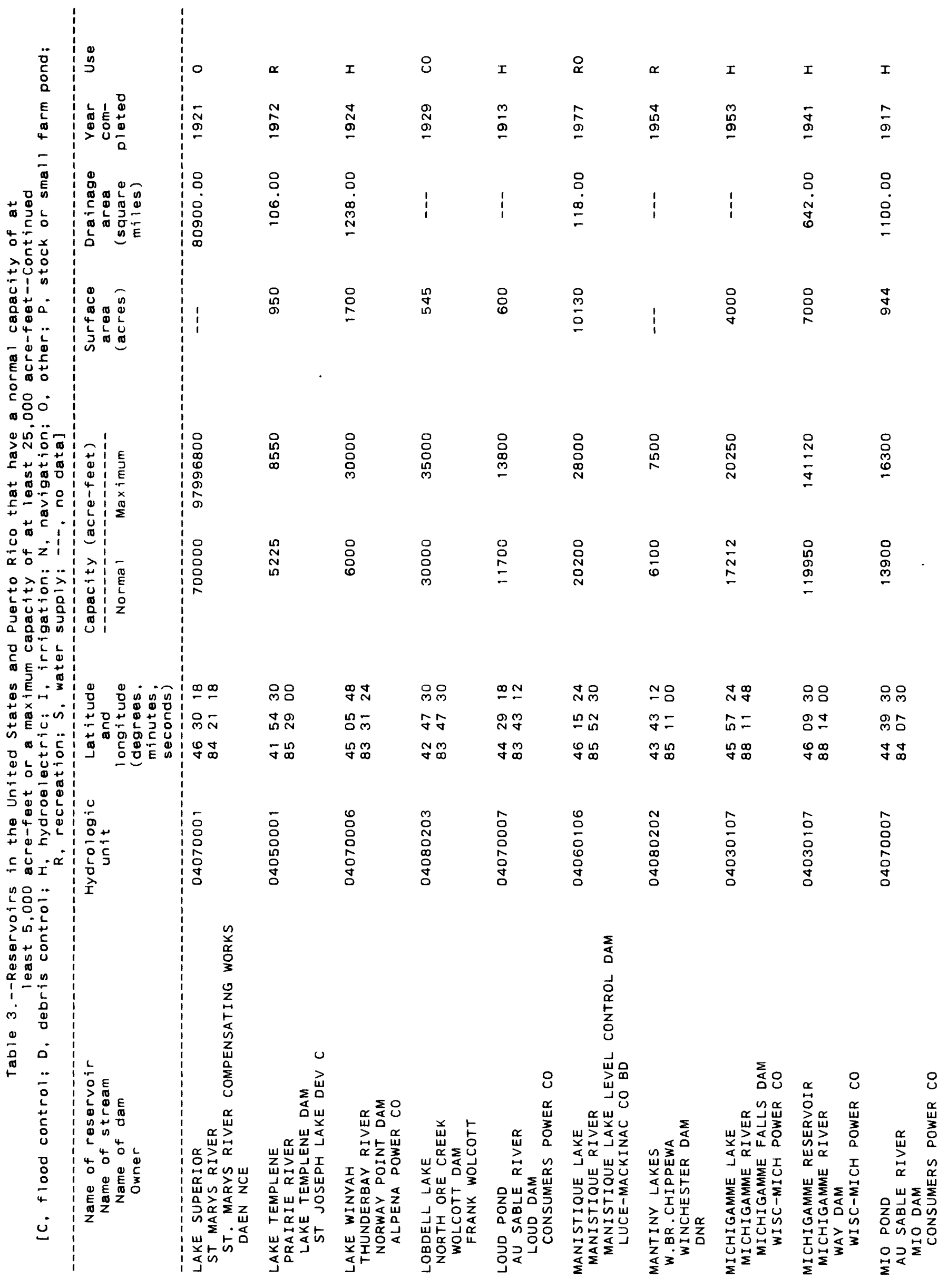




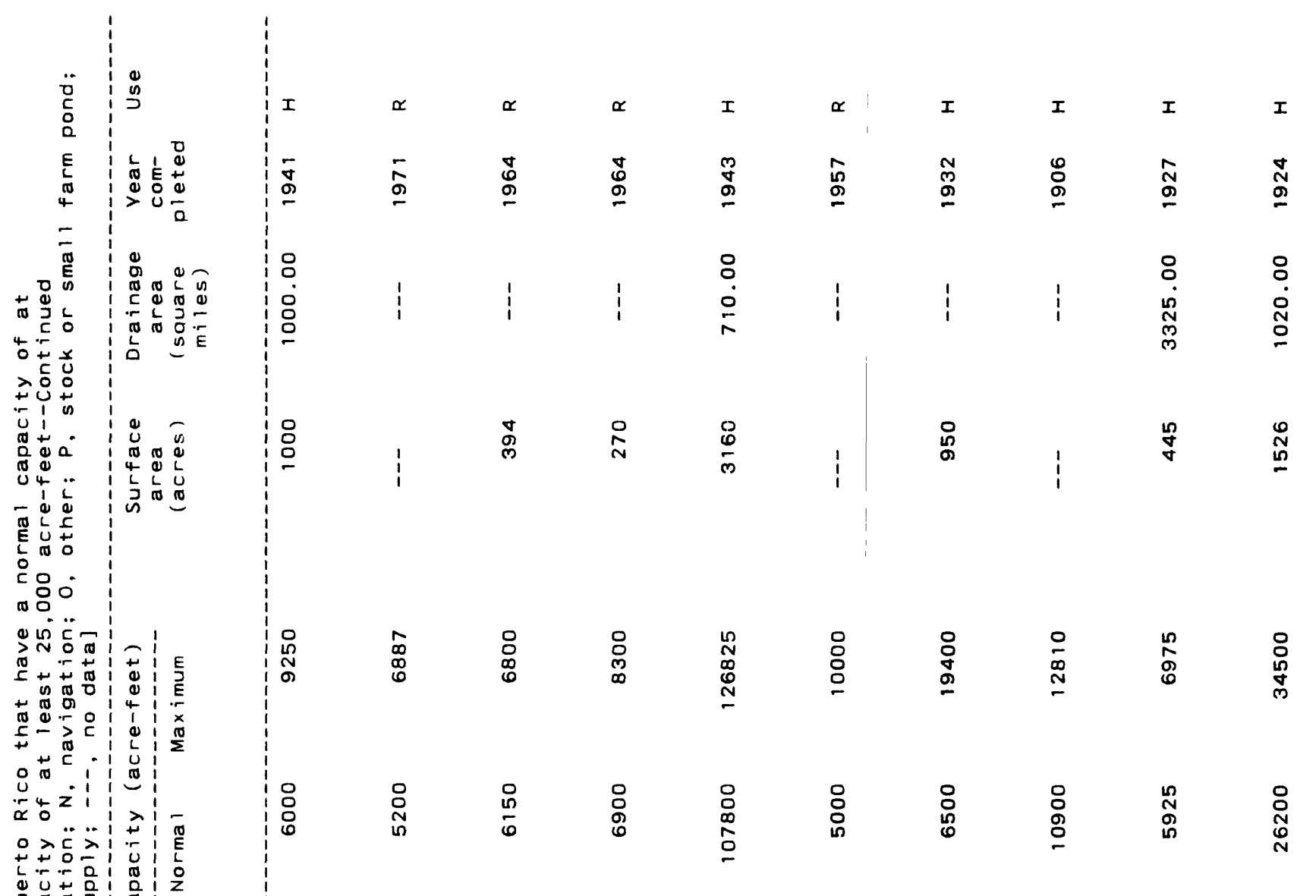

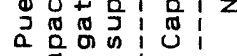

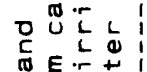

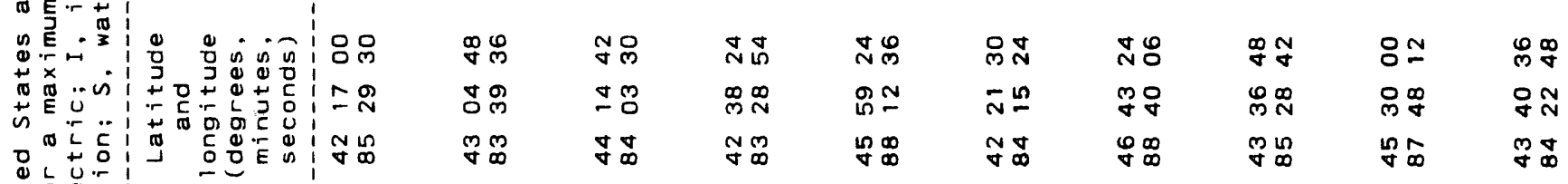

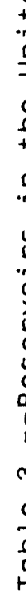

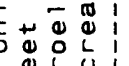

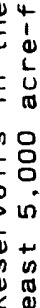

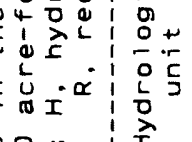

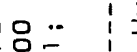

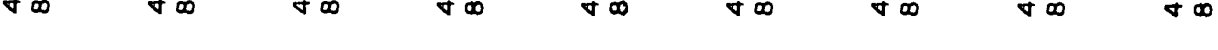

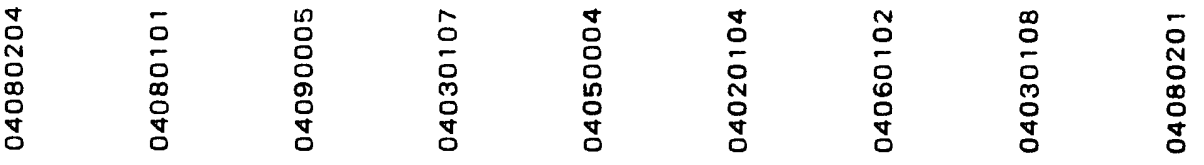

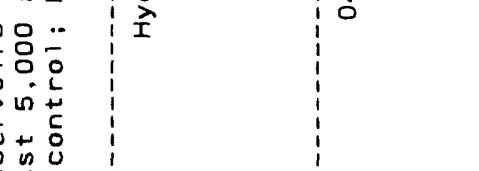

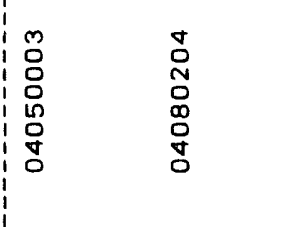
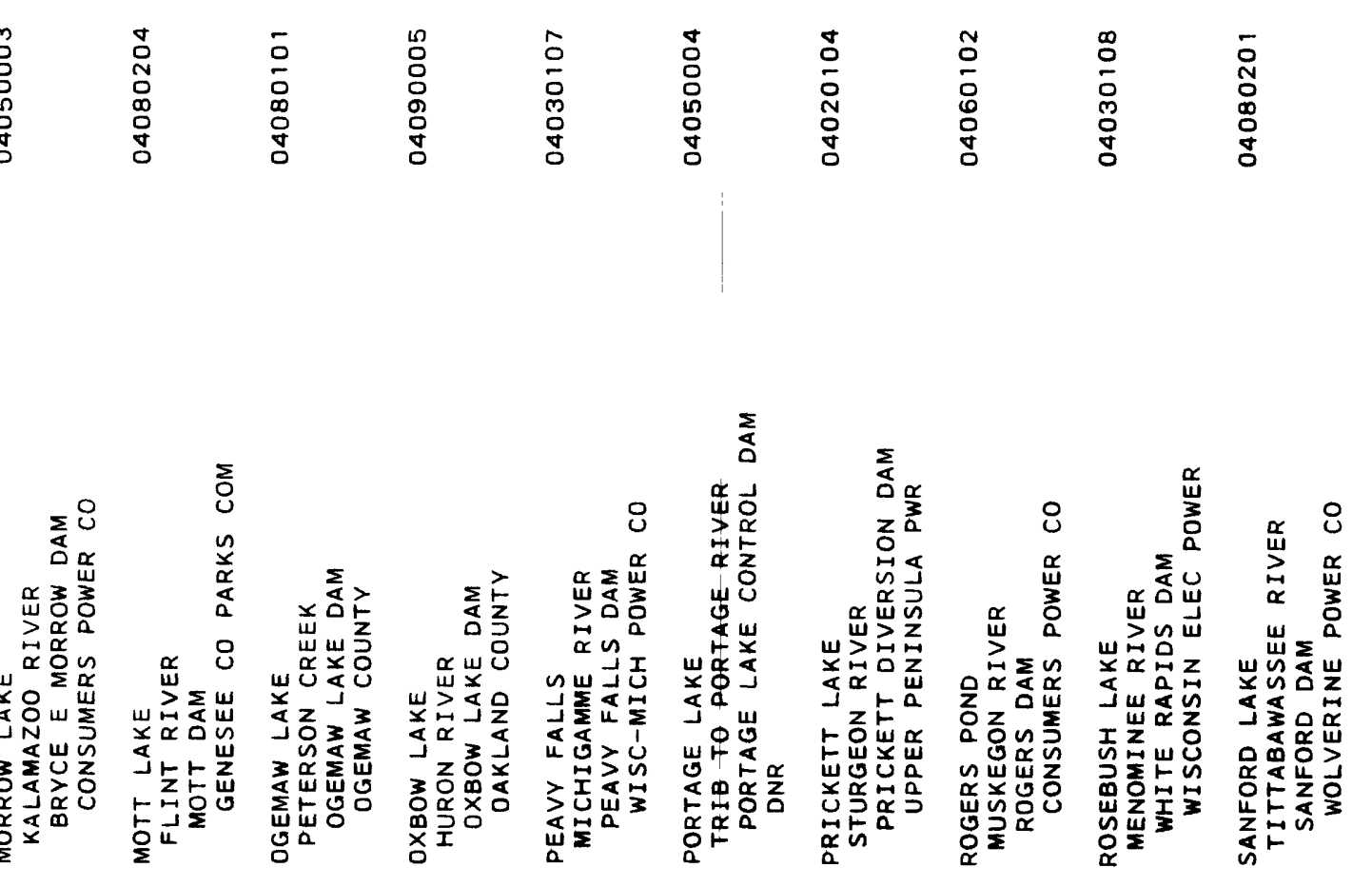


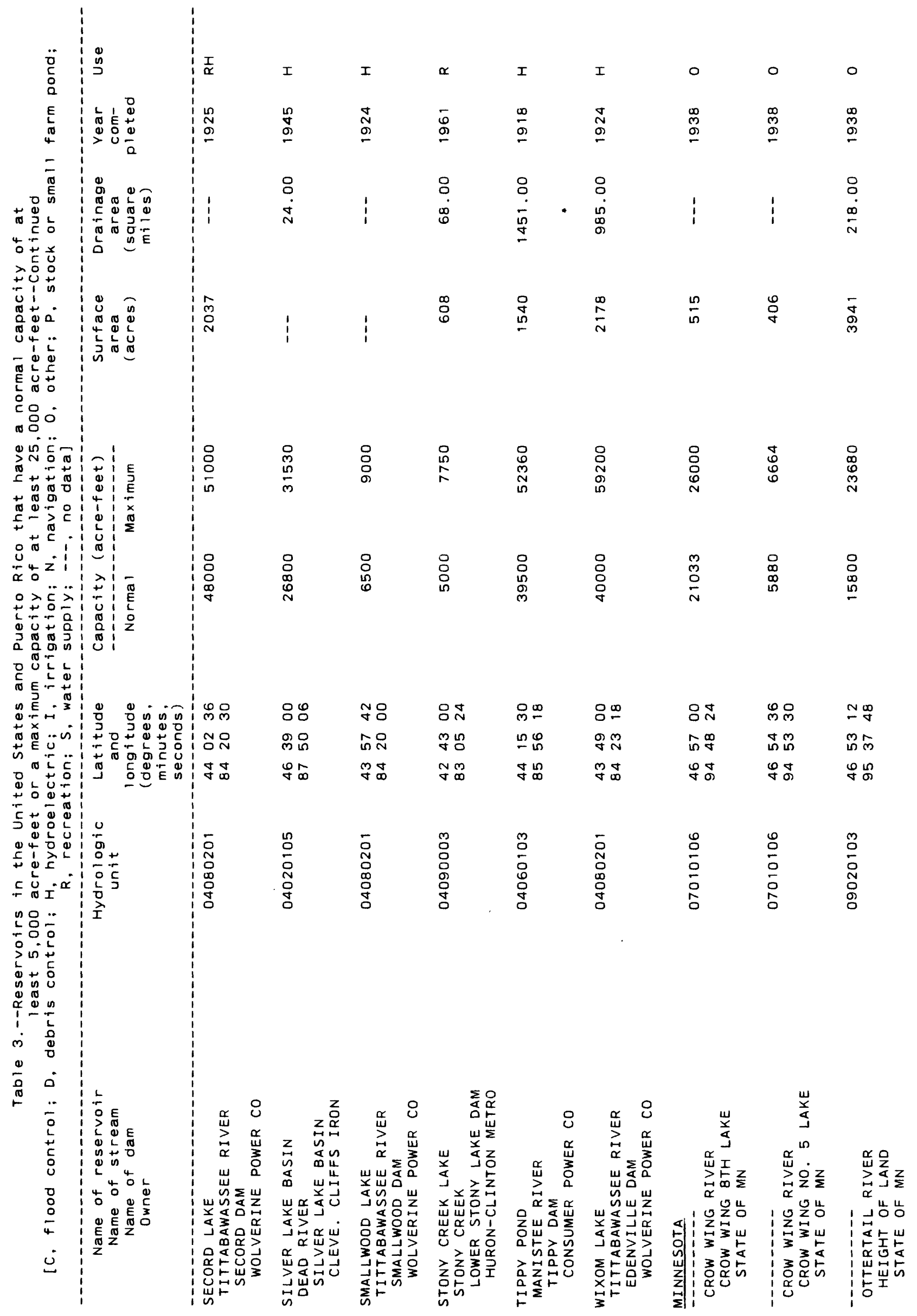




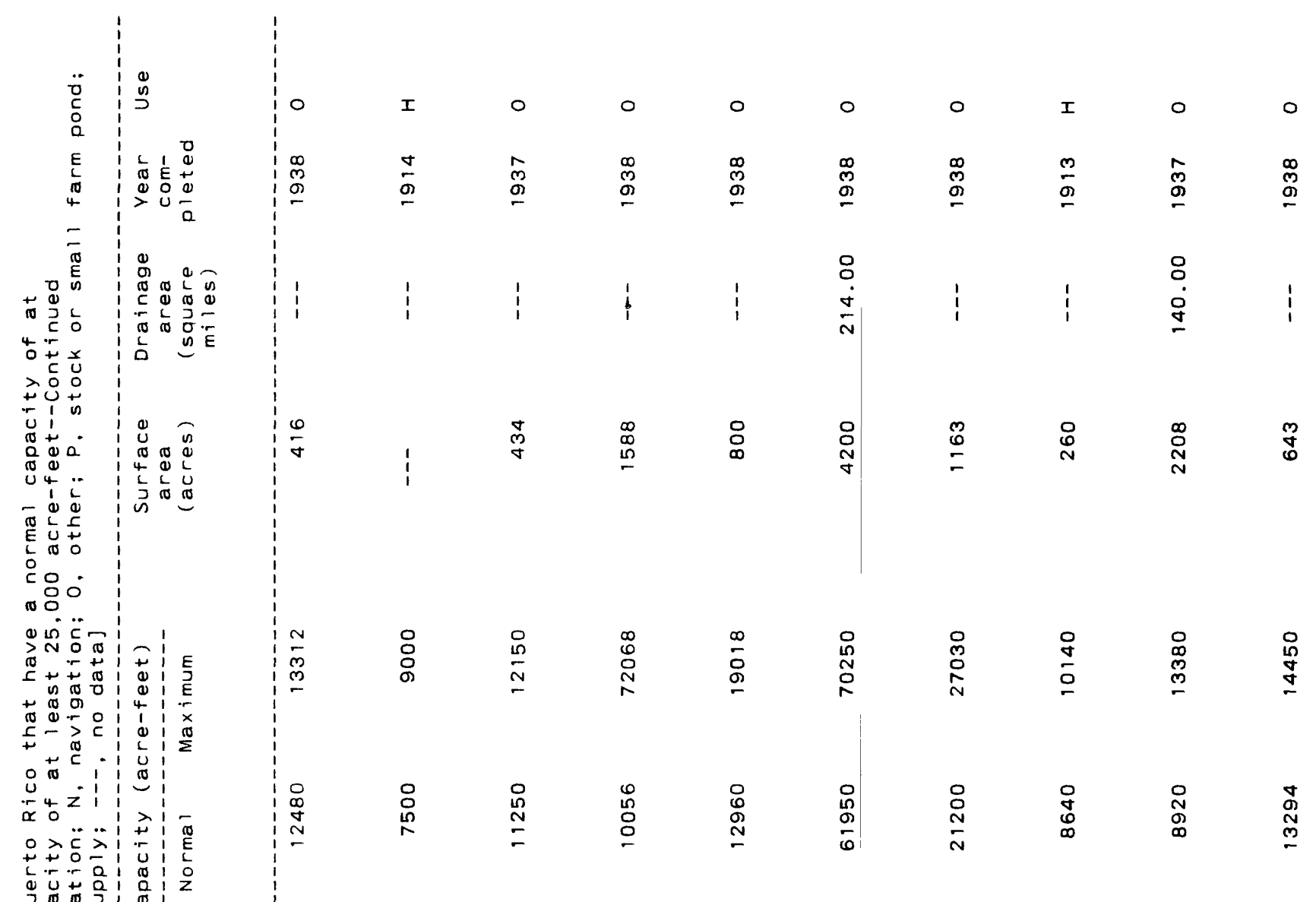

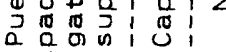

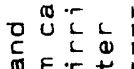

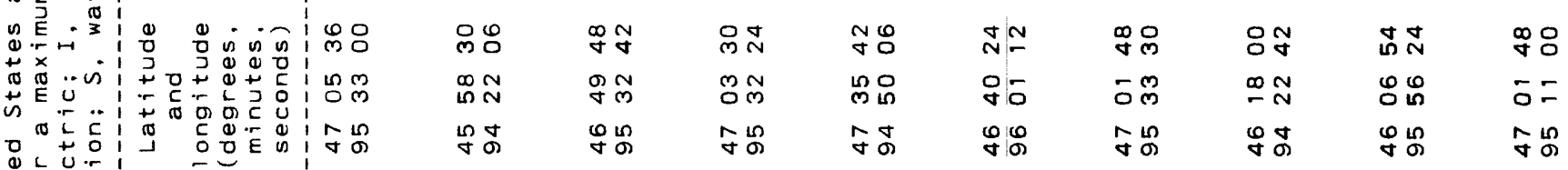

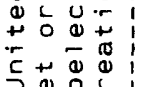

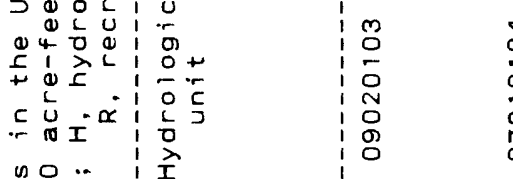

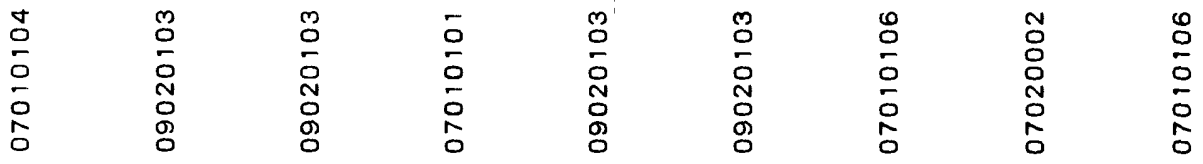

a

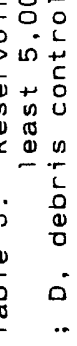

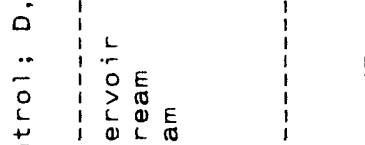

in

ᄂ 4 疻

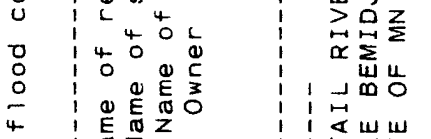

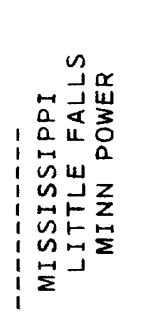

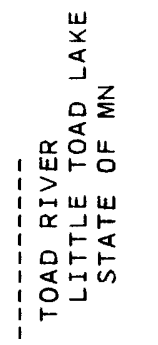

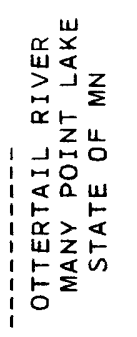

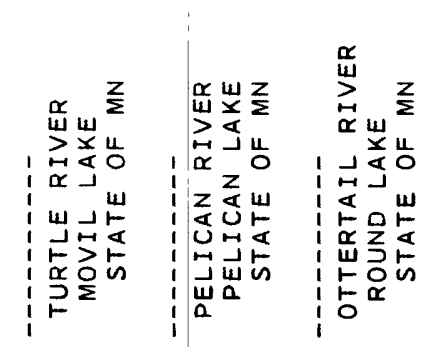

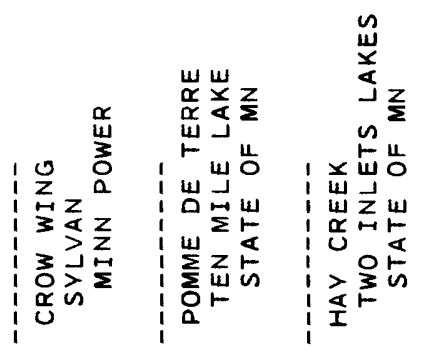




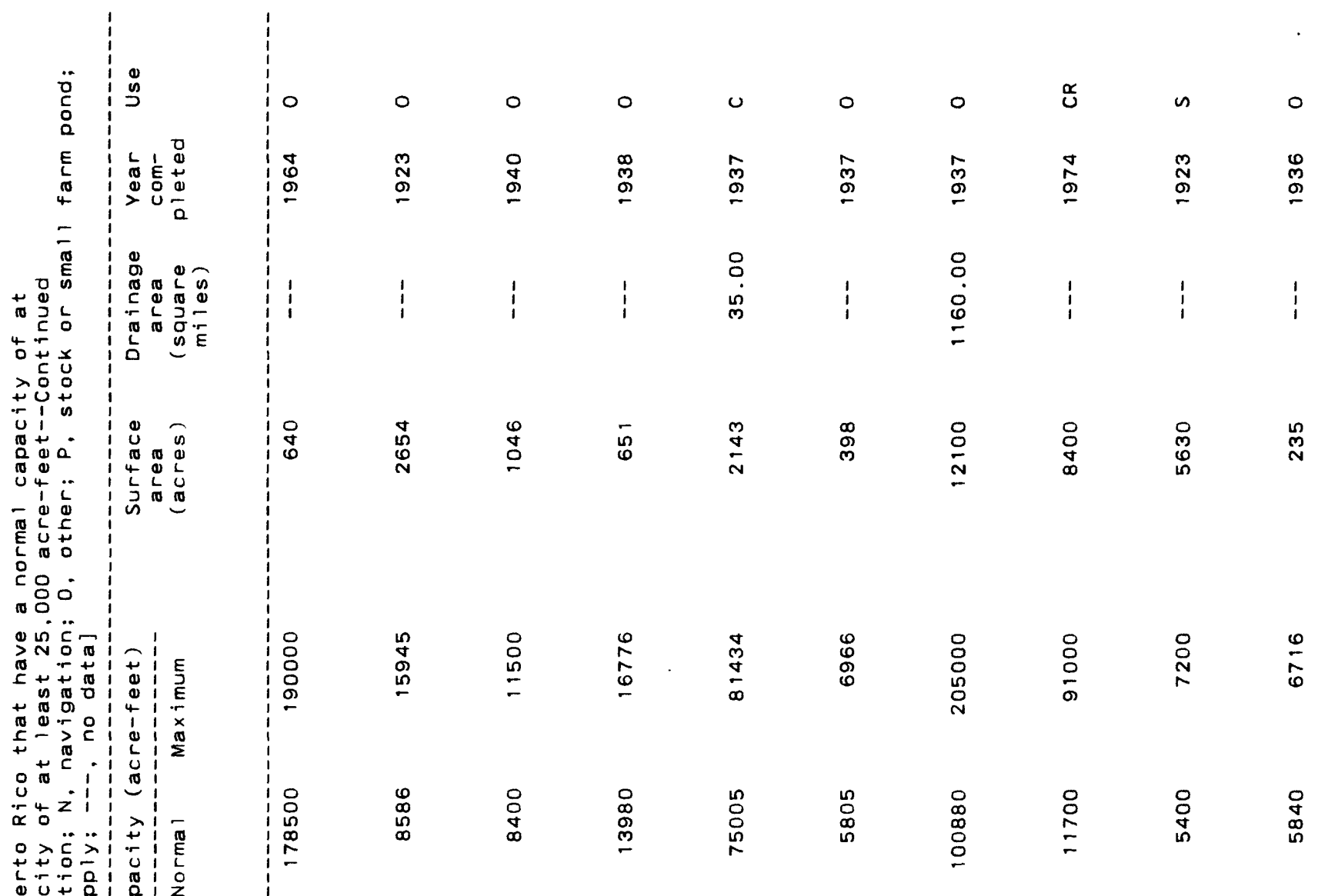

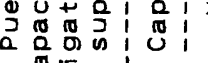

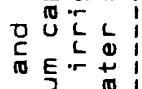

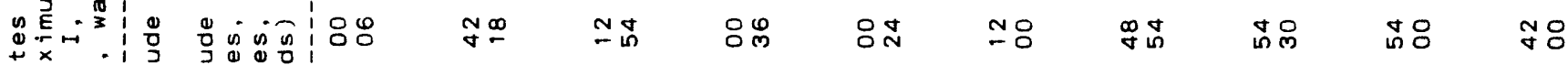

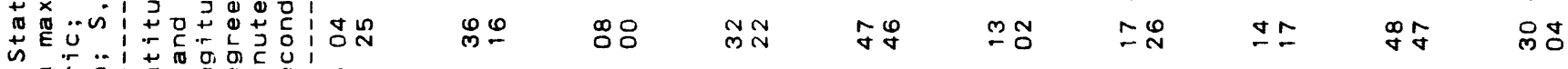

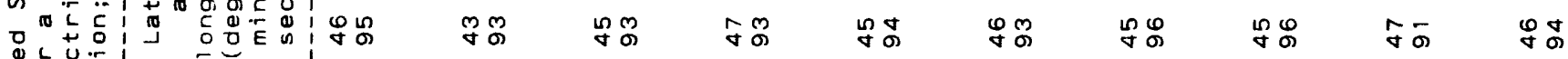

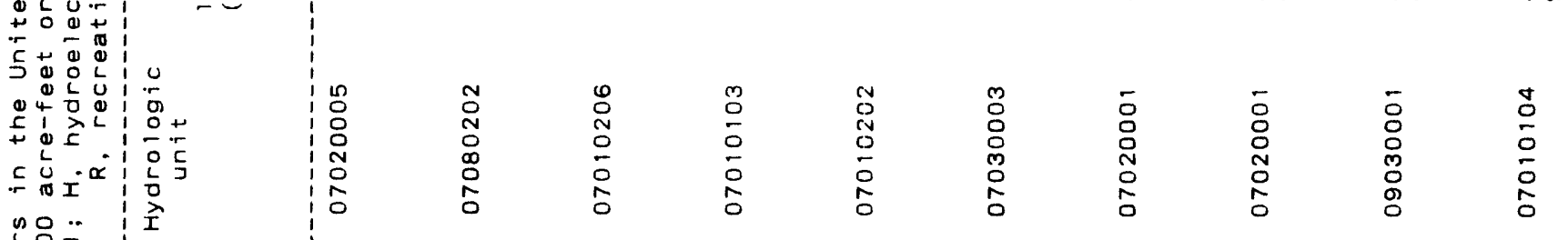

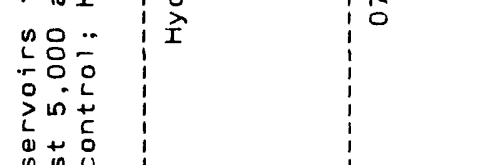

$\underset{\frac{a}{3}}{\frac{4}{3}}$

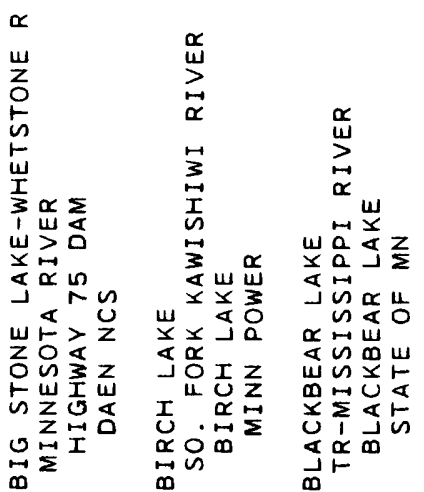




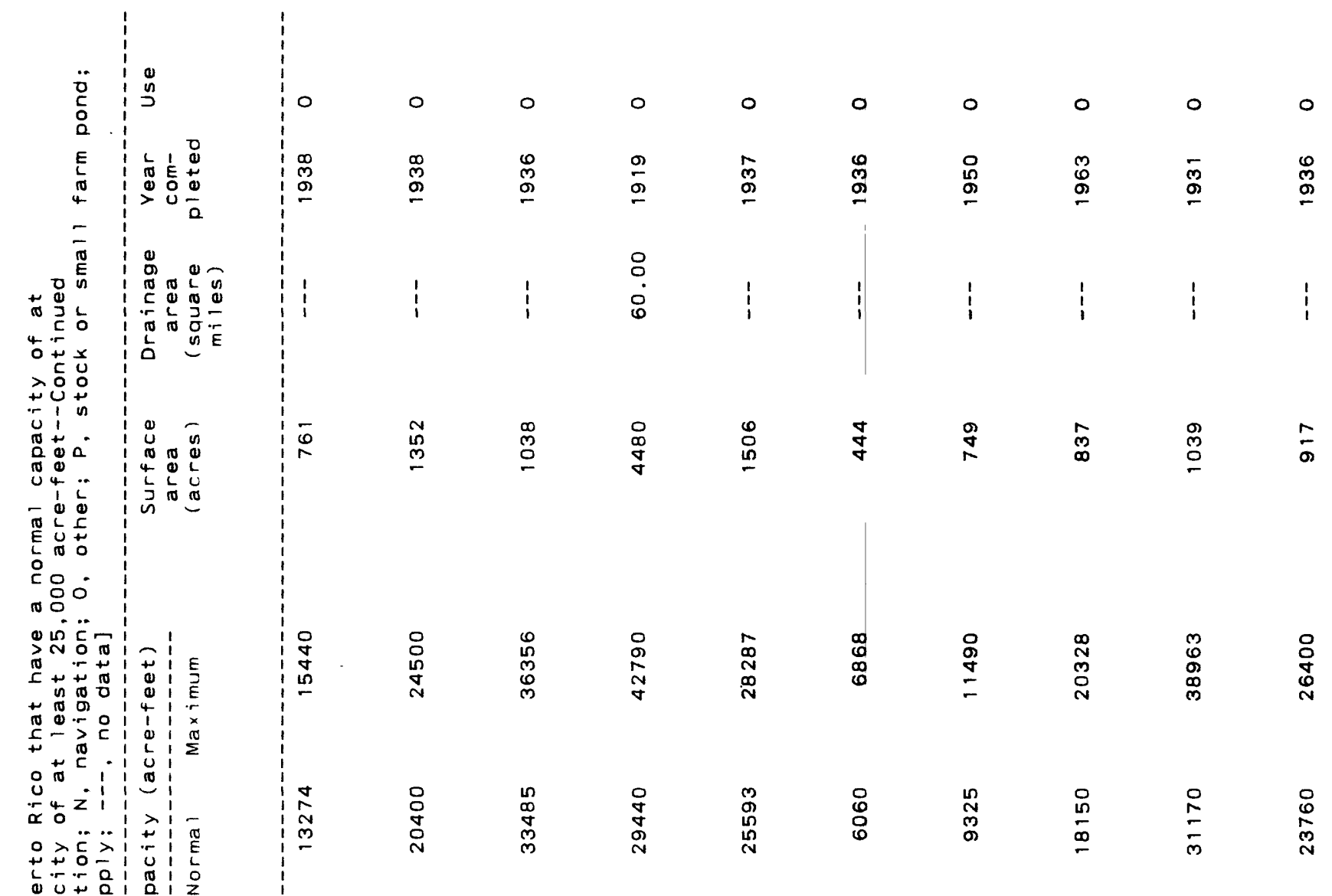

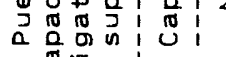

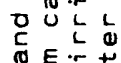

a $\vec{E} \cdot \frac{\pi}{3}$

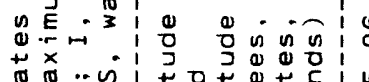

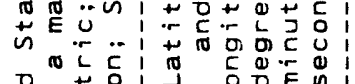

요

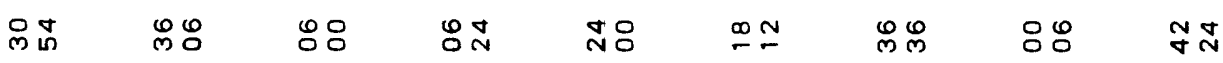

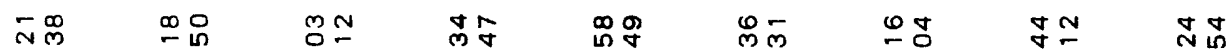

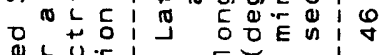

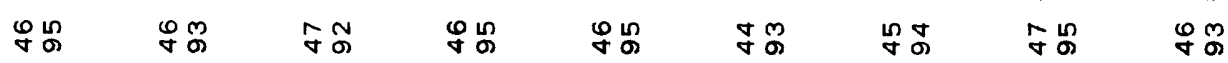

$\bar{c}+\frac{\pi}{0}$

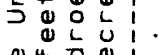

$\begin{array}{lllll}0 & 0 & 0 & 0 & 0 \\ \Sigma & 1 & 1 & 0\end{array}$

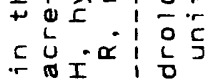

nᄋ‥

$\therefore 8 \div$

>

袋倠

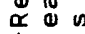

क

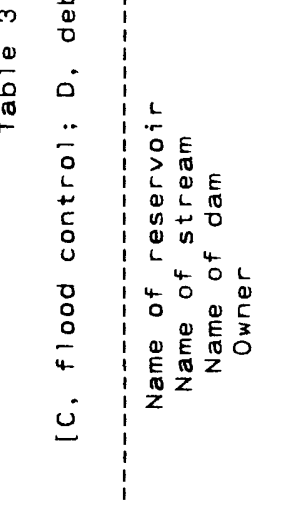

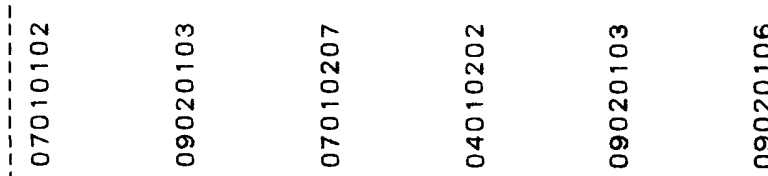

$\begin{array}{ll}0 & \text { N } \\ 0 & 0 \\ 0 & 0 \\ \text { I } & \text { N } \\ \text { O } & \text { O }\end{array}$

$m$
0
$N$
0
0
0
0

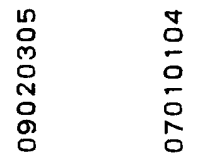

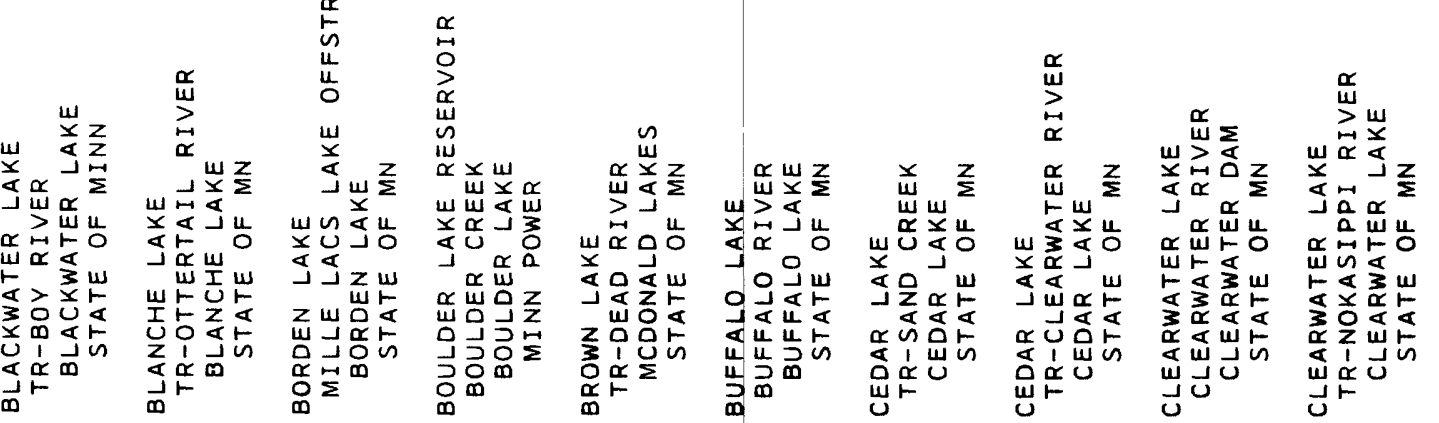




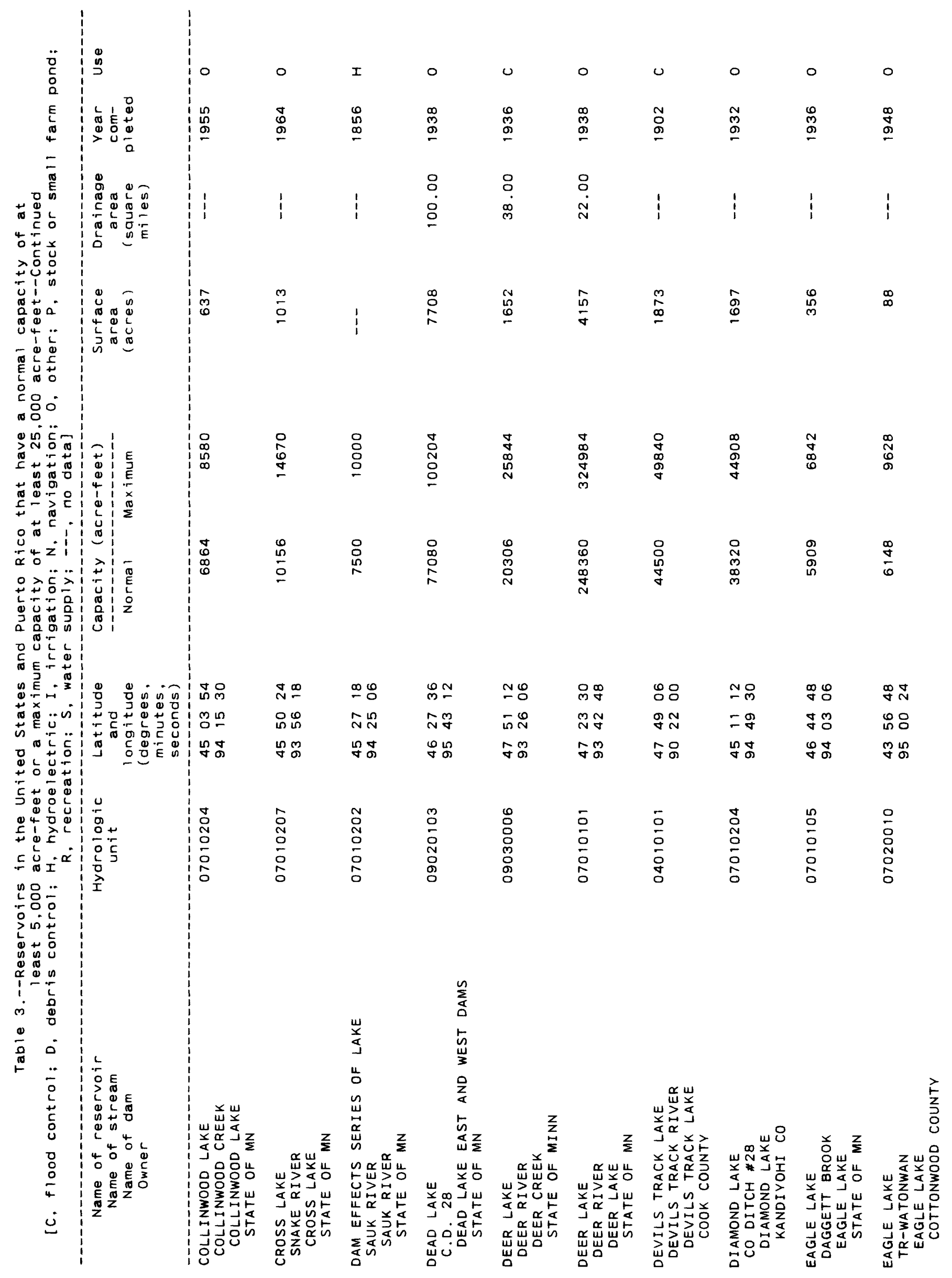




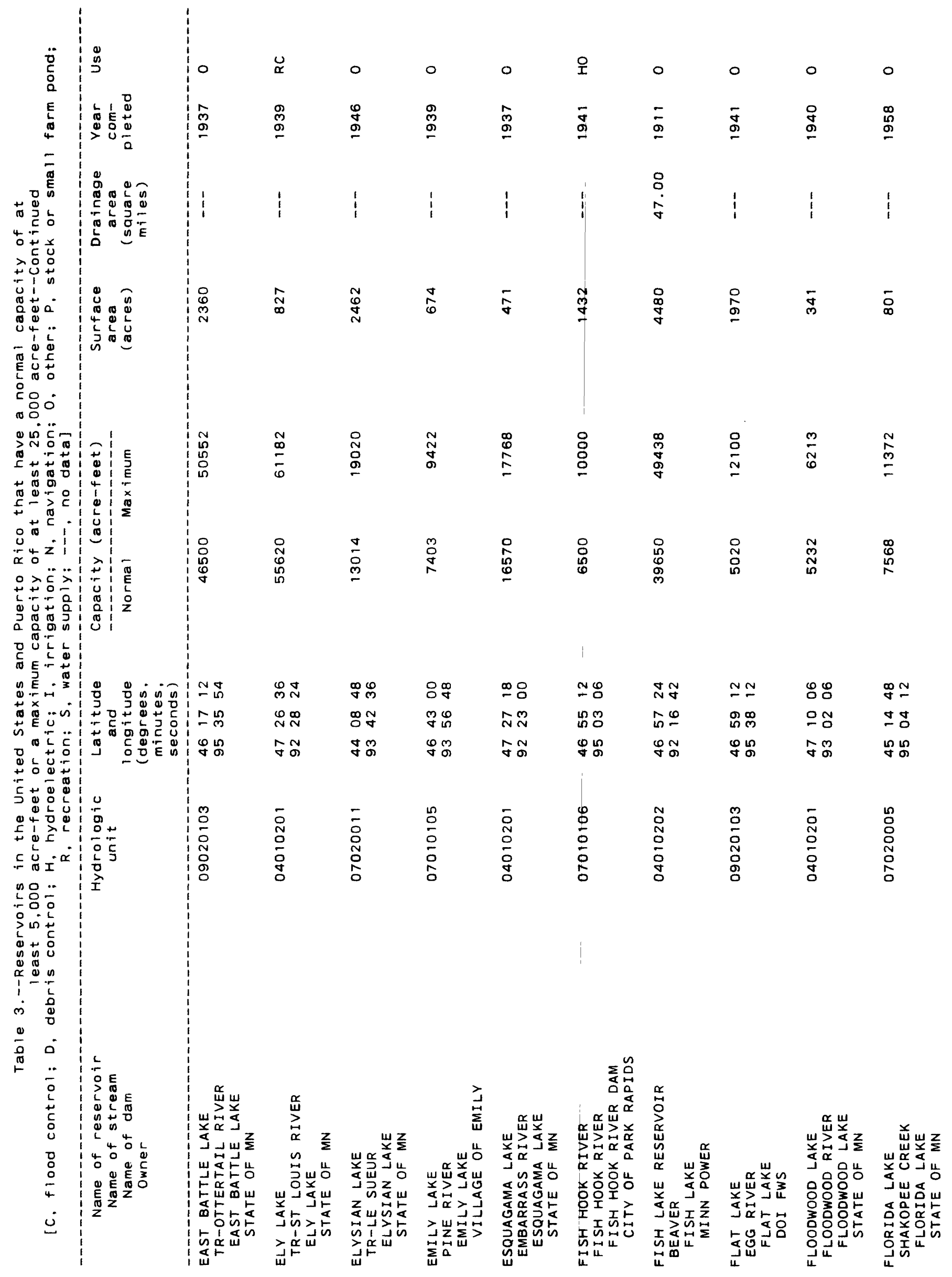




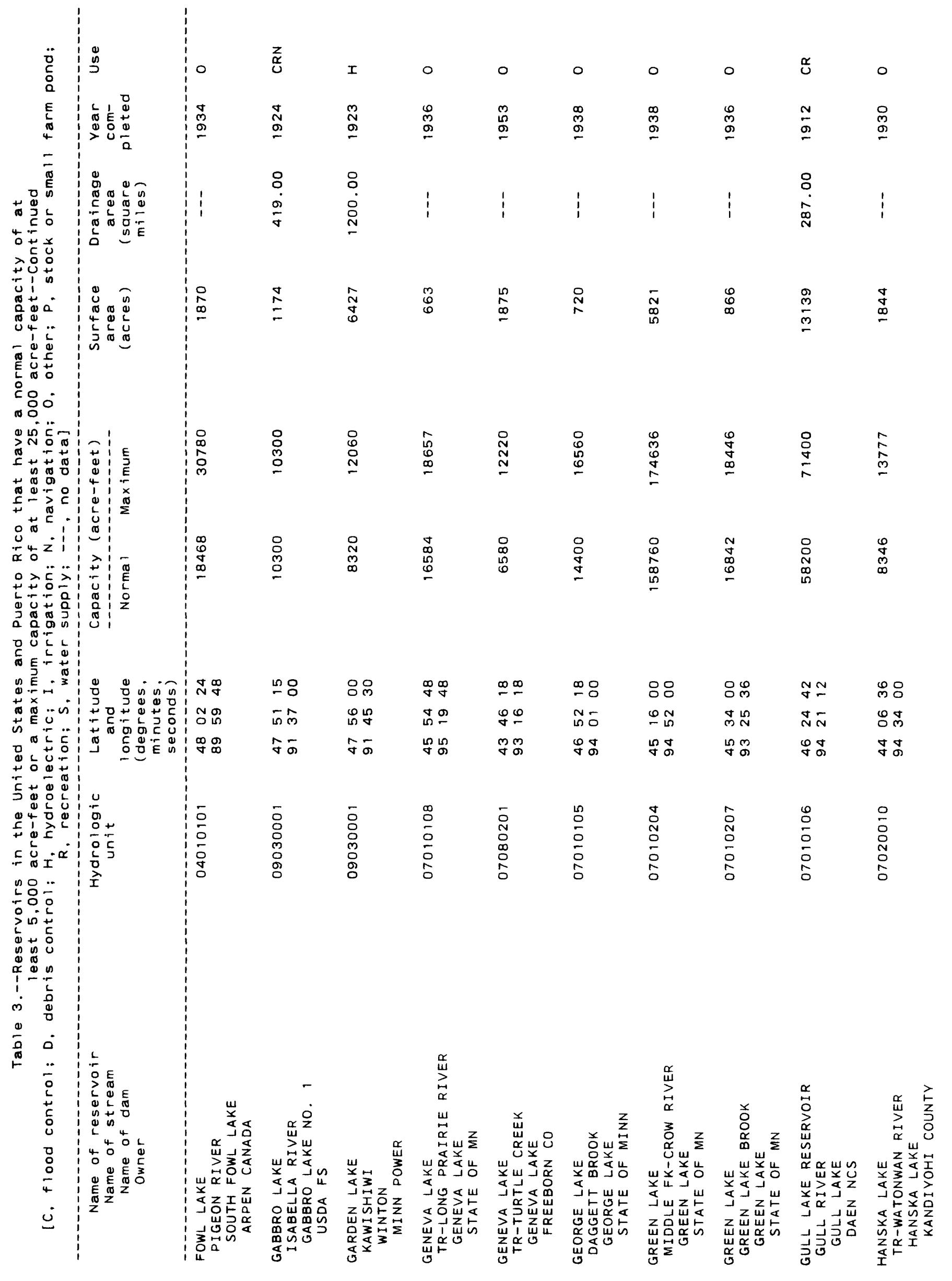




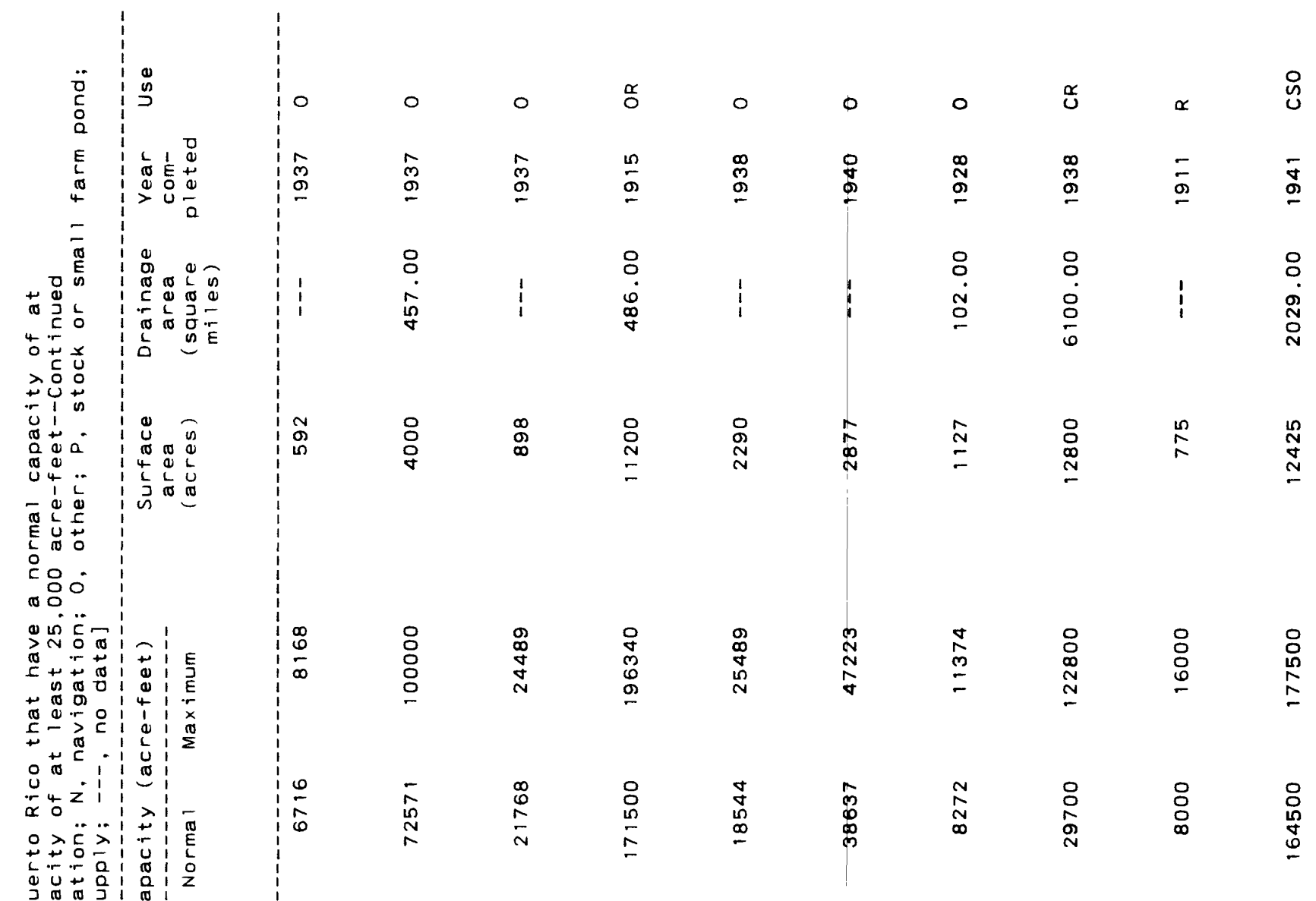

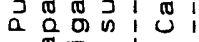

万俈亡

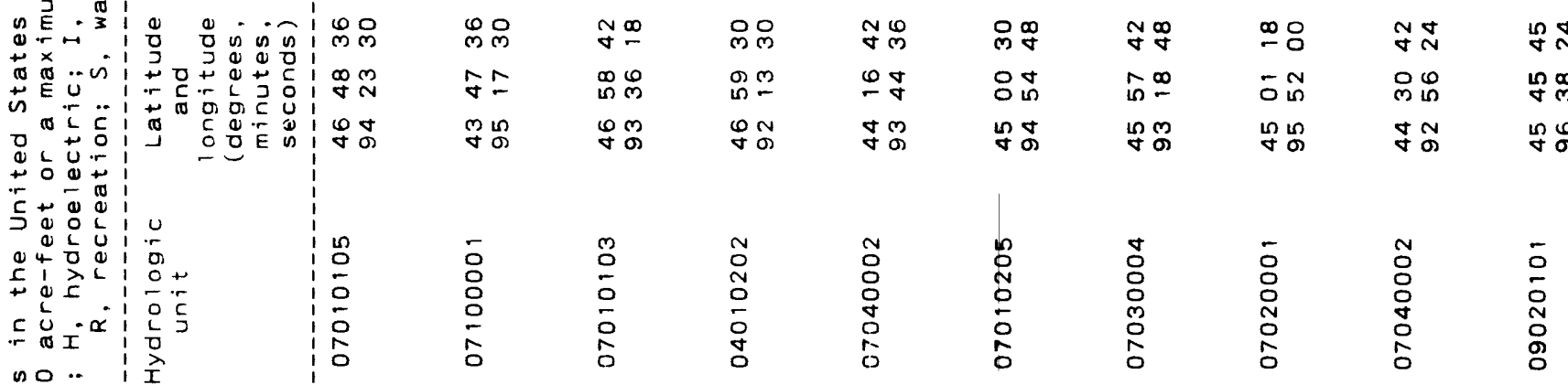$$
\begin{aligned}
& 2 \\
& 0 \\
& 0 \\
& 0 \\
& 0 \\
& 0 \\
& 0 \\
& 0 \\
& 0 \\
& 1 \\
& 1 \\
& 0 \\
& 0 \\
& 0 \\
& 0 \\
& 0 \\
& 10
\end{aligned}
$$$$
\text { i }
$$ 


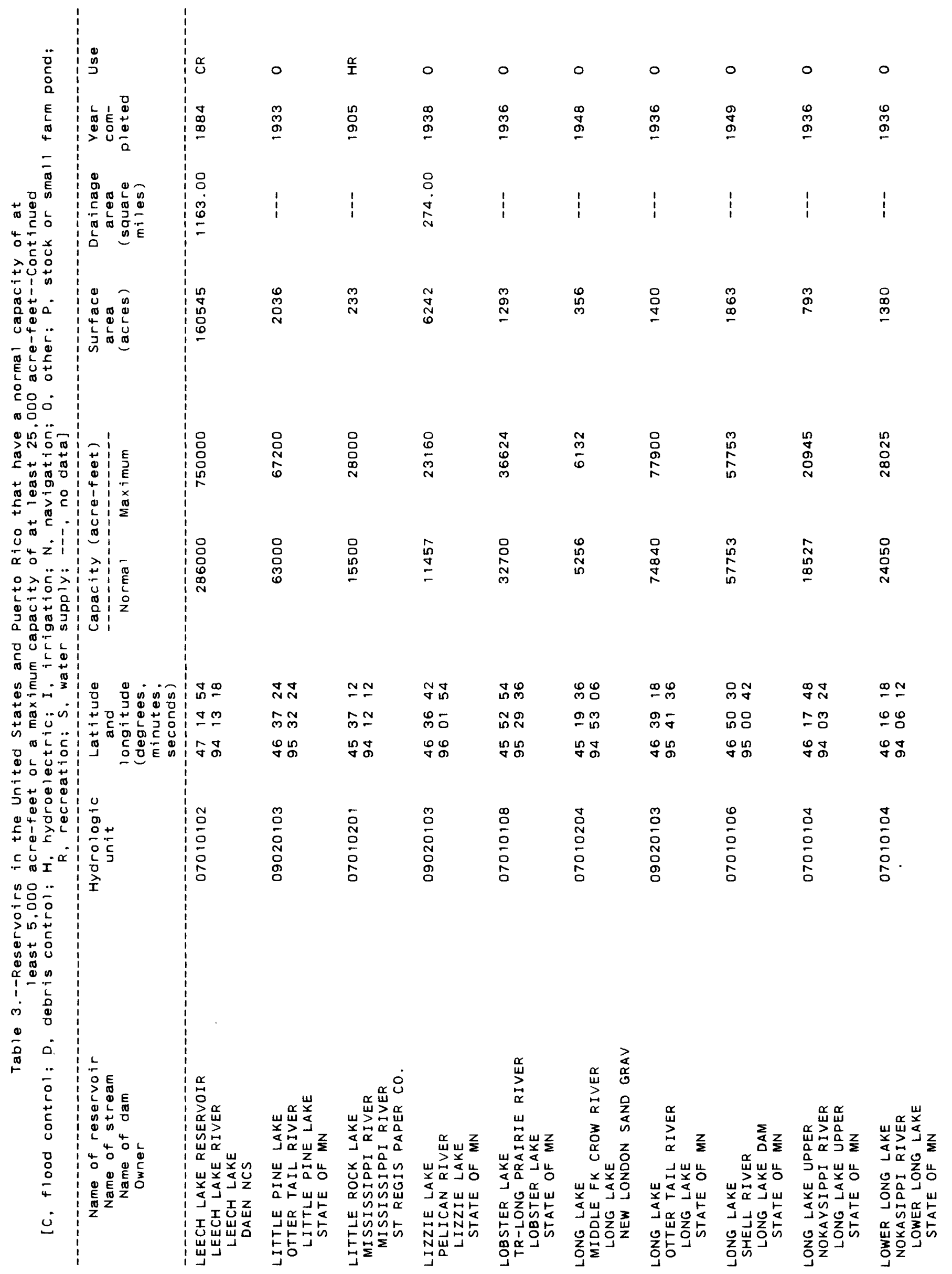




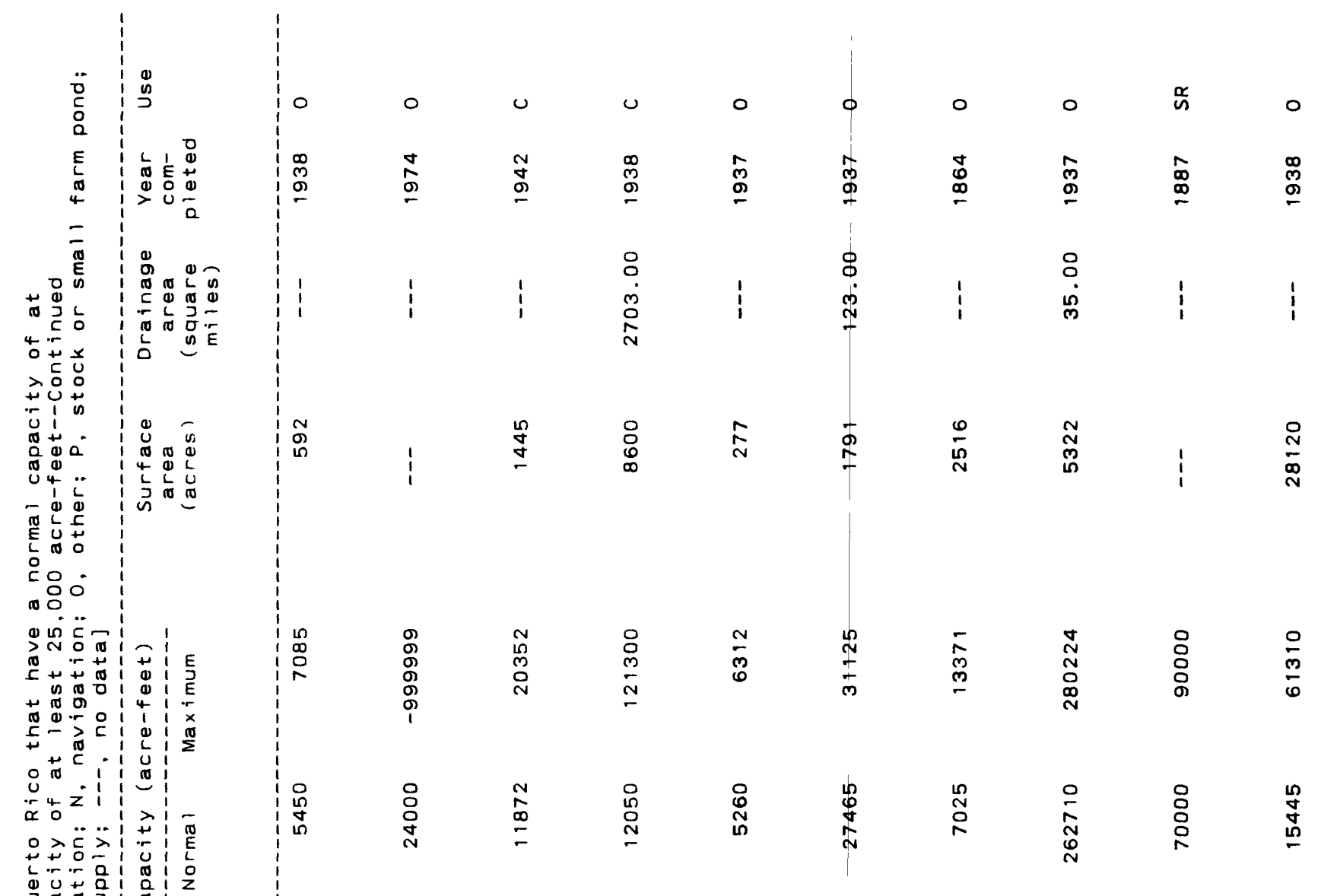

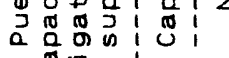

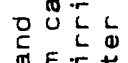

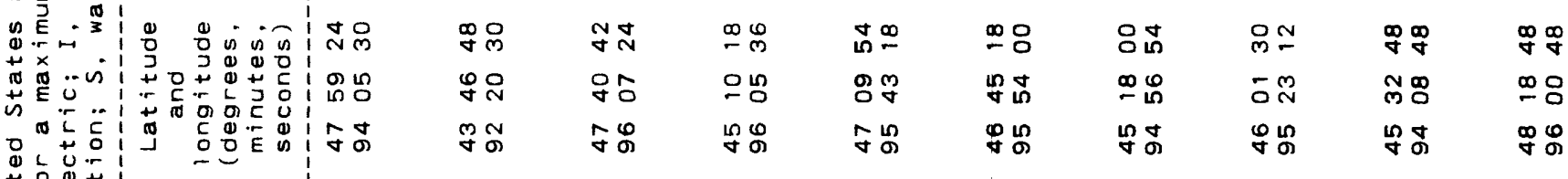

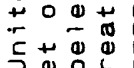

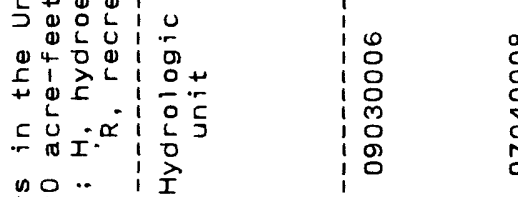

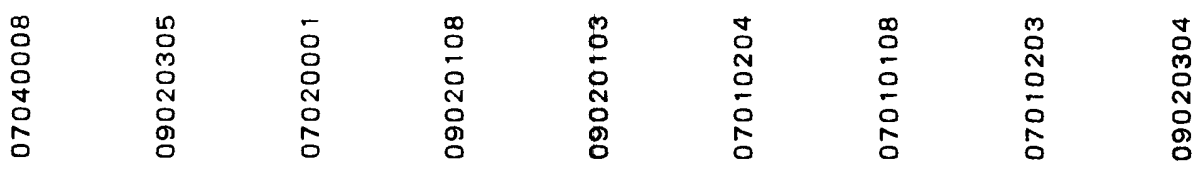

a

$\begin{array}{rl}0 & 0 \\ 0 & 0 \\ 0 & + \\ 0 & 0 \\ 0 & 0 \\ 0 & 0 \\ 0 & 0 \\ 0 & 0 \\ 0.0 \\ 0 \\ 0 \\ 0 \\ 0 \\ 0 \\ 0 \\ 0\end{array}$

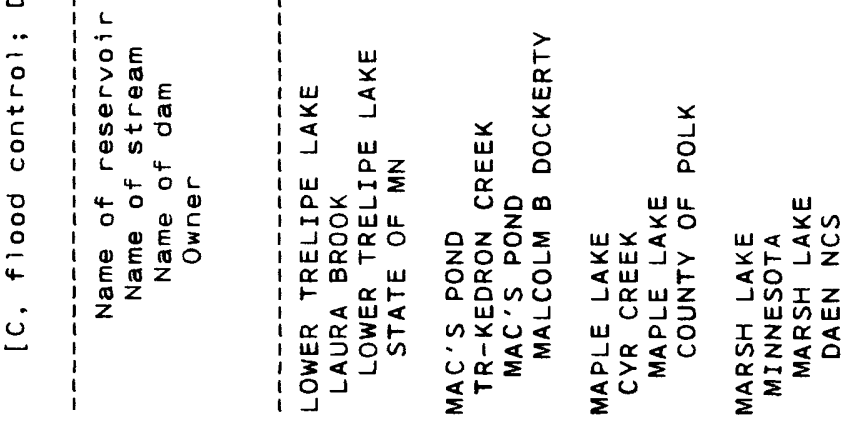

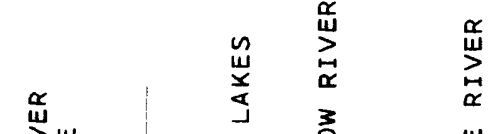

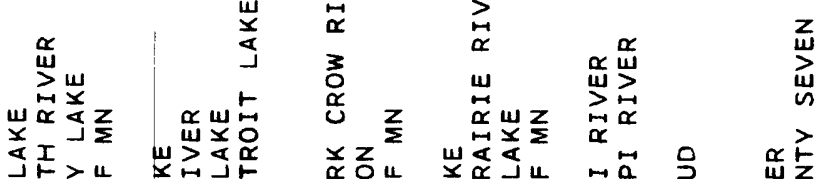

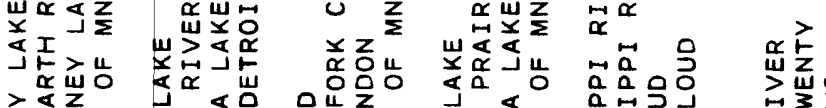

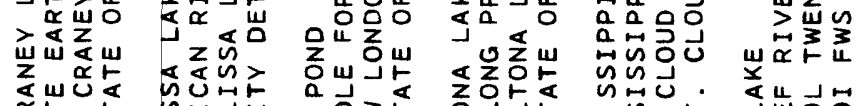

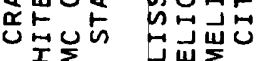

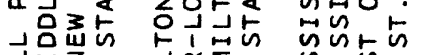
出용요 u夜

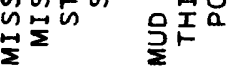




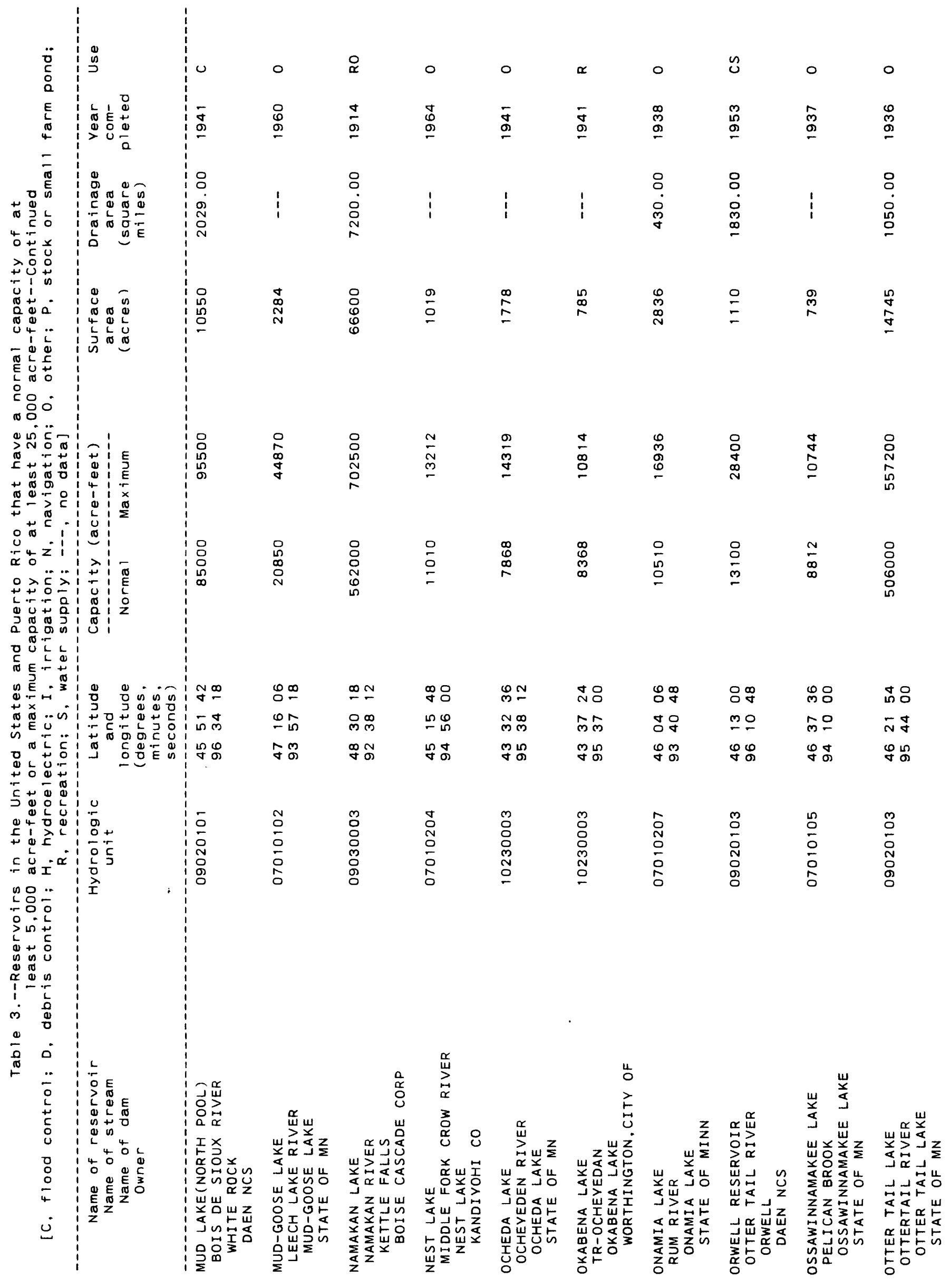




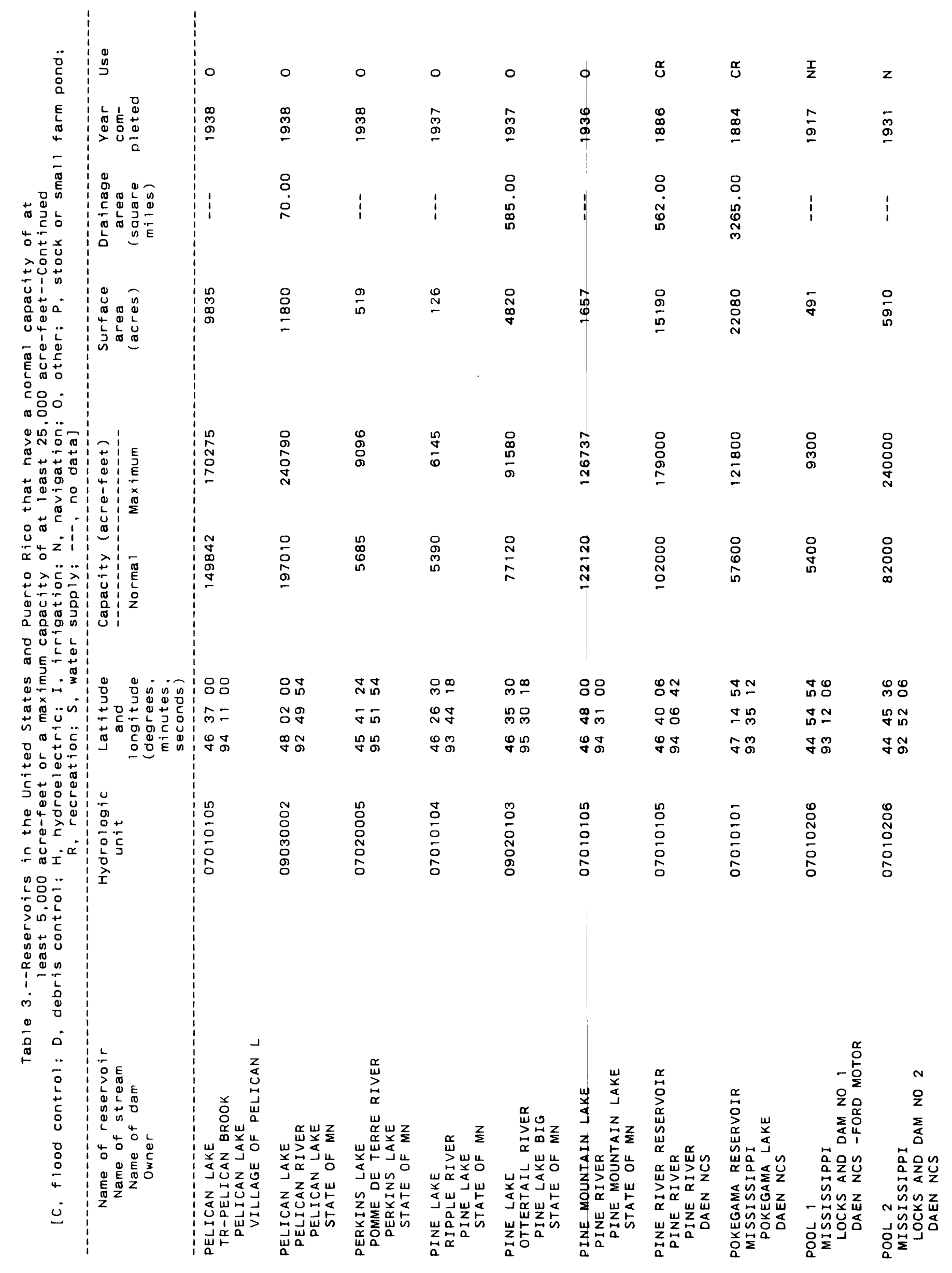




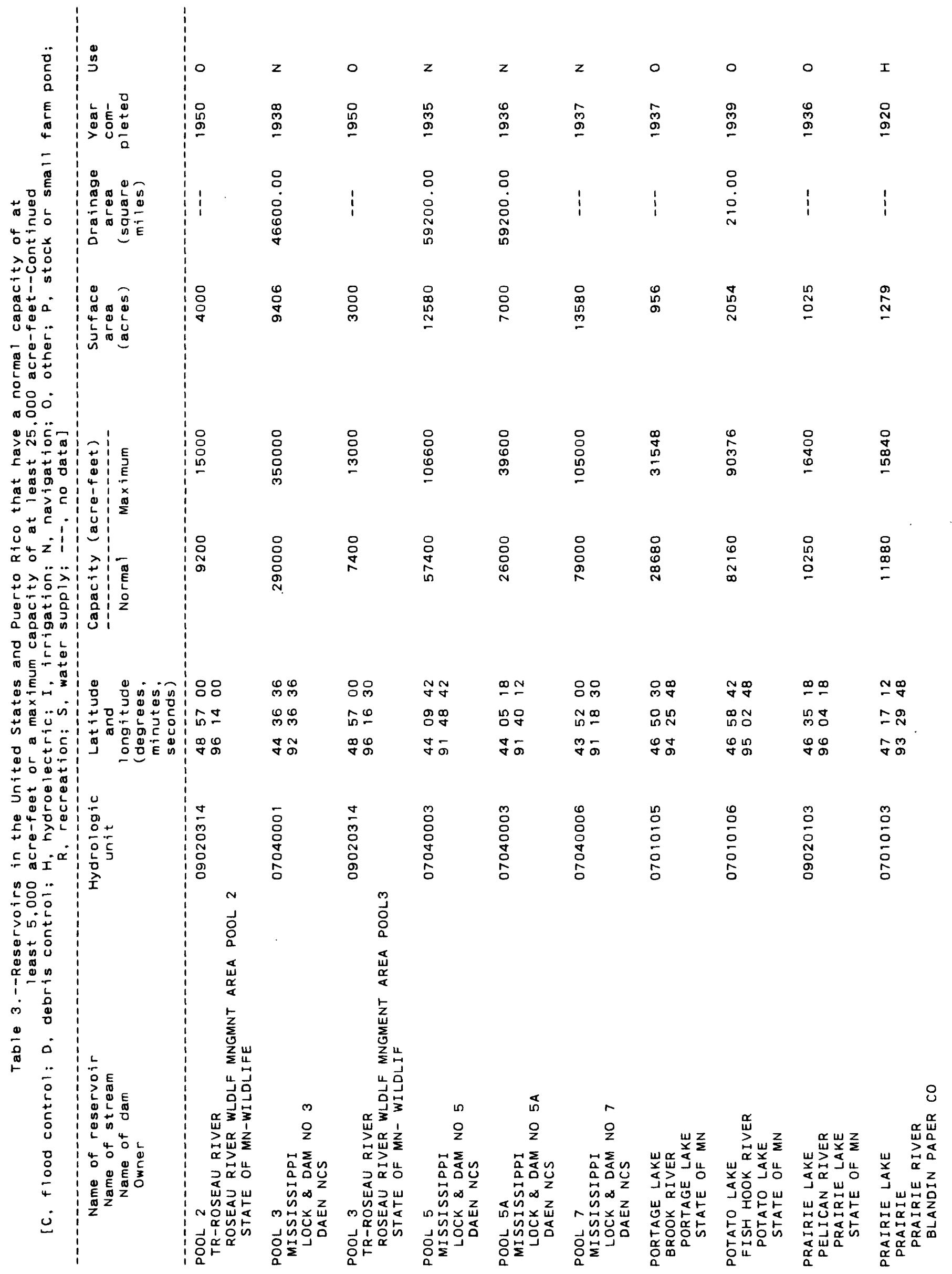




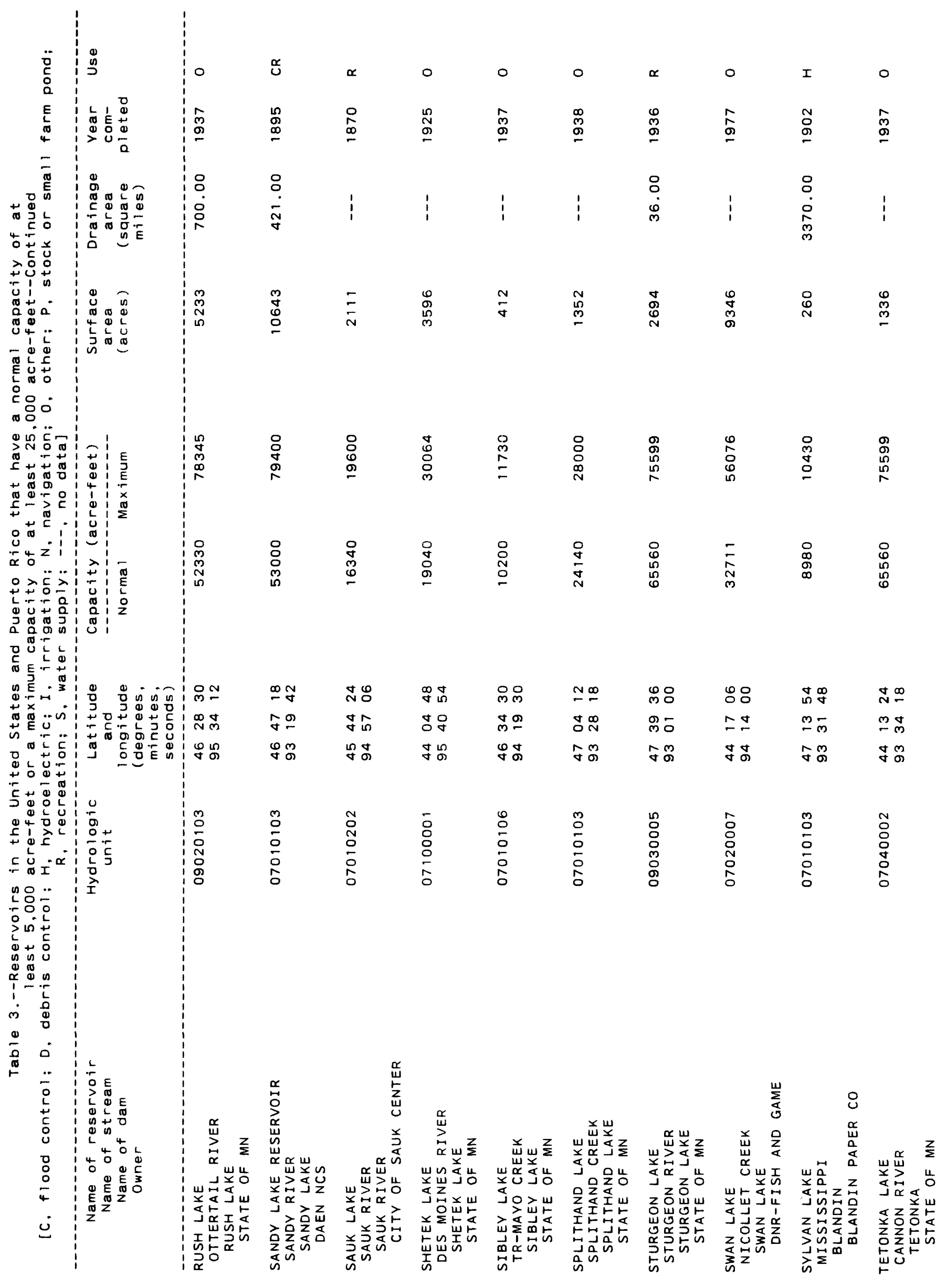




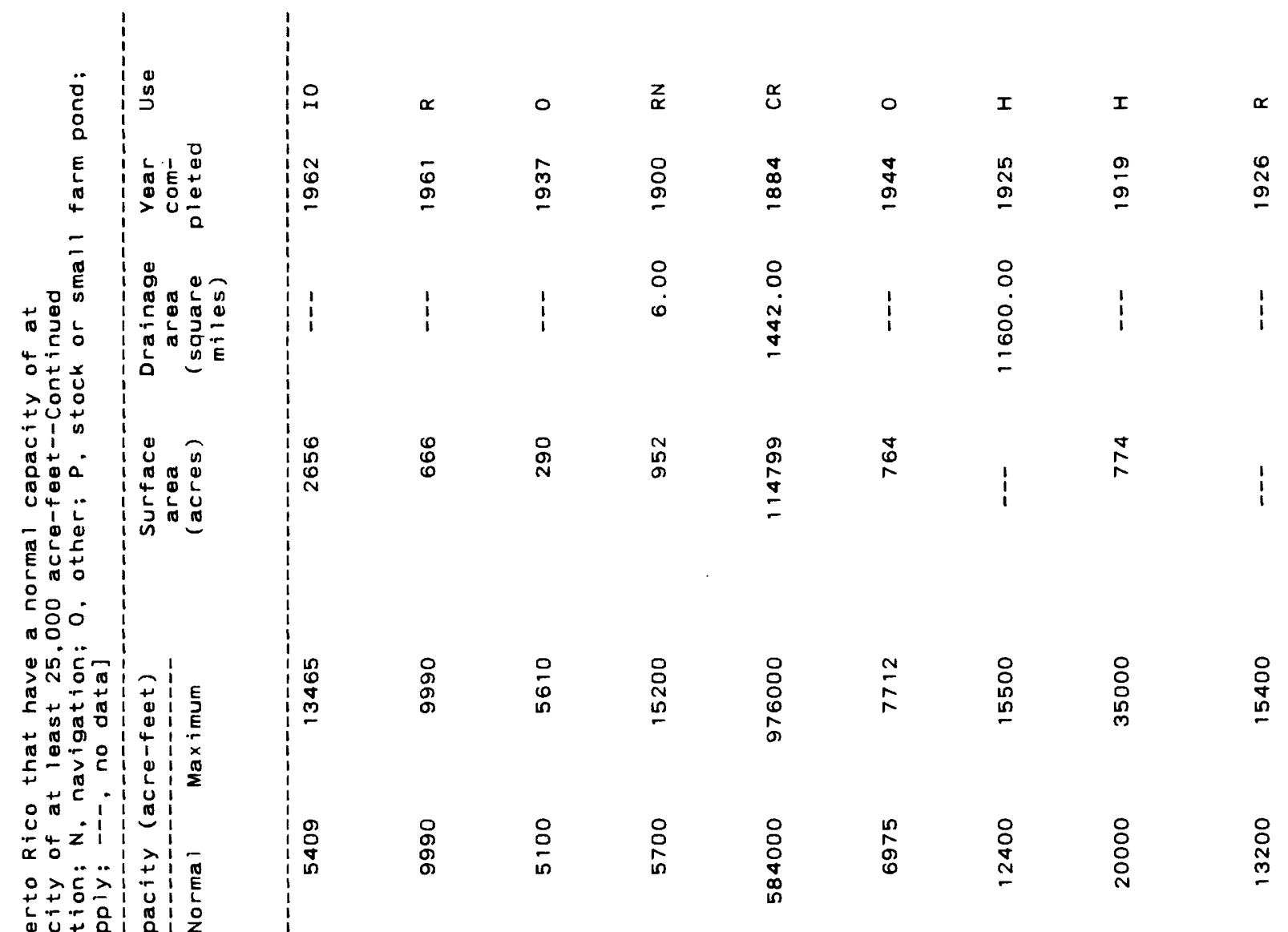

ฟำ

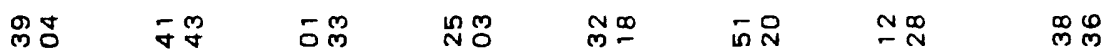

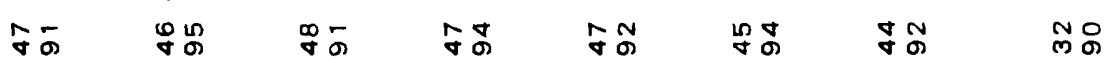

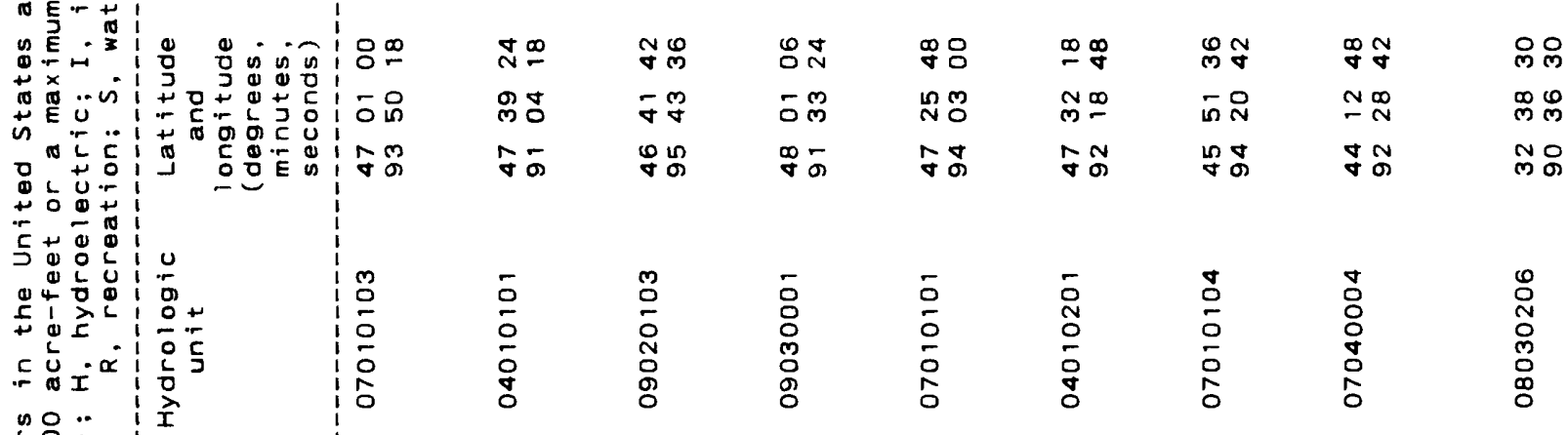

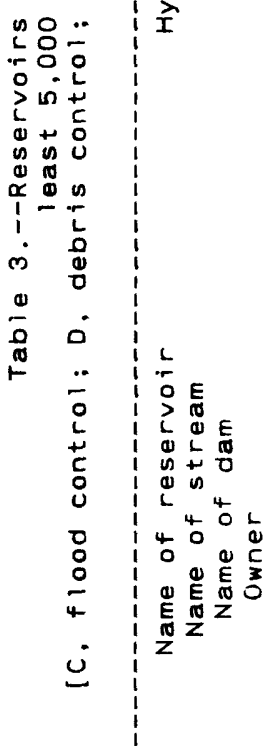

a

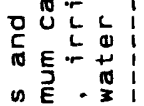

๑.

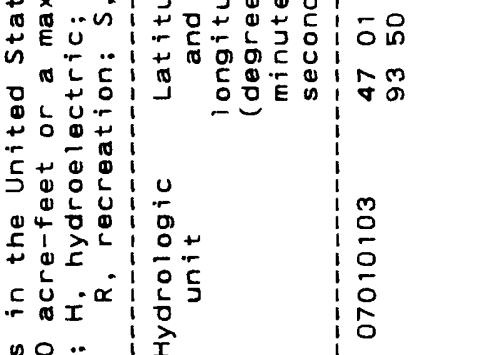




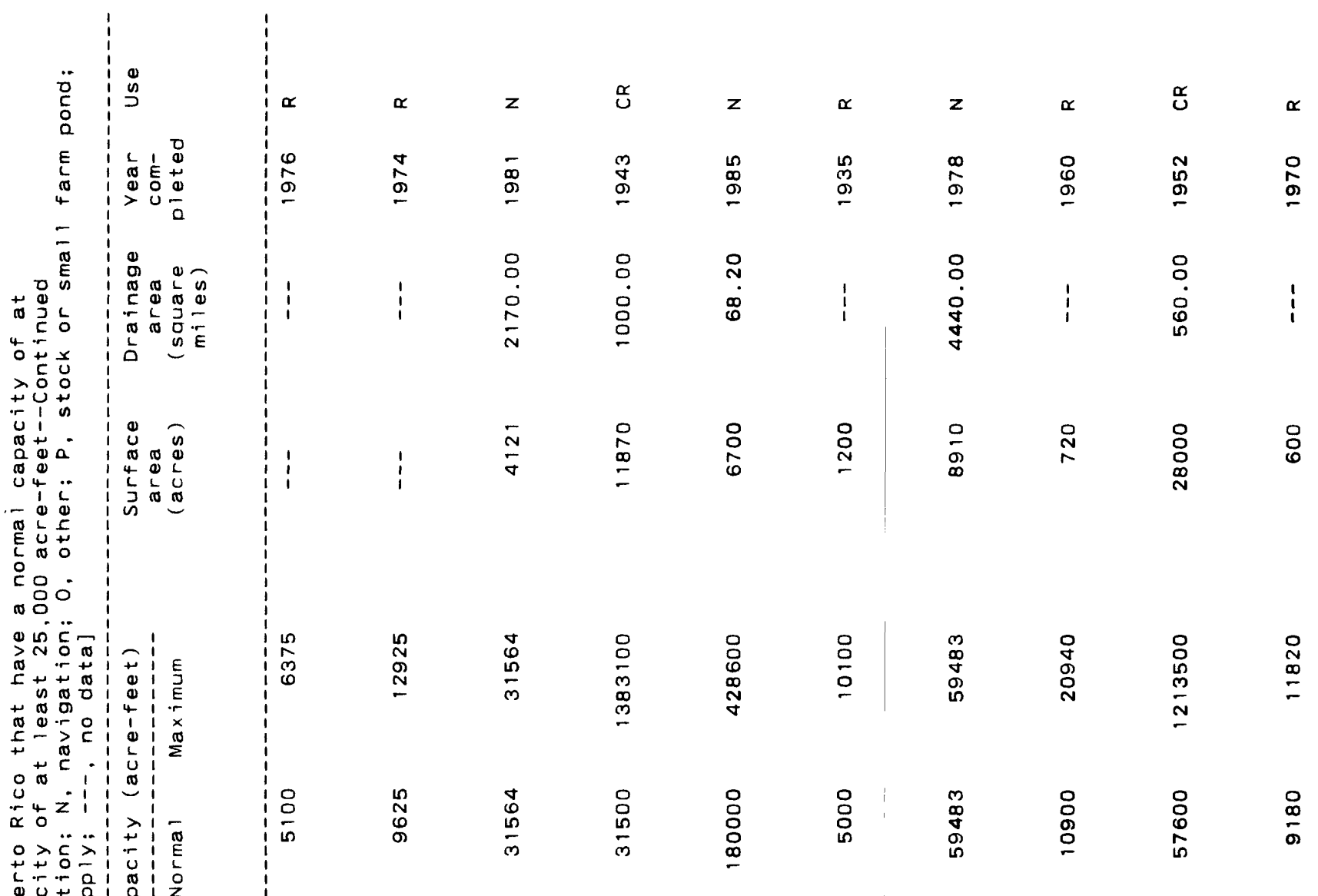

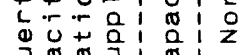

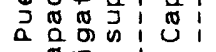

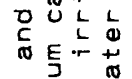

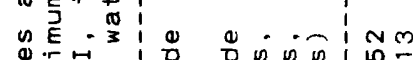

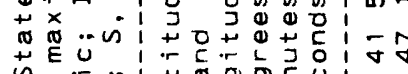

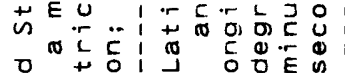

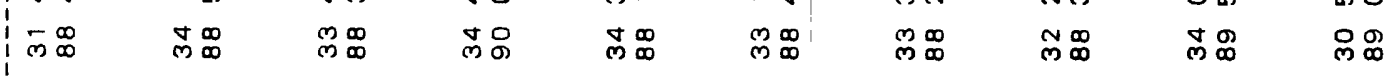

ه.

ᄃ+

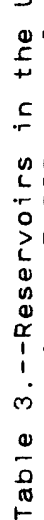

\begin{tabular}{c:c}
\hline & 0 \\
0 & -
\end{tabular}

m

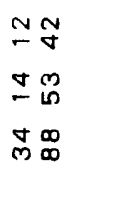

$\infty \sim$

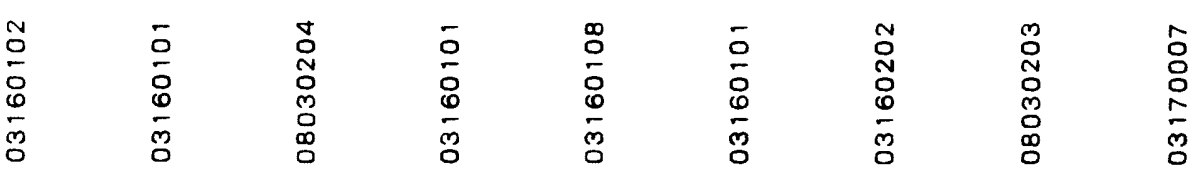

no..

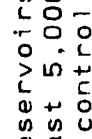

年

s
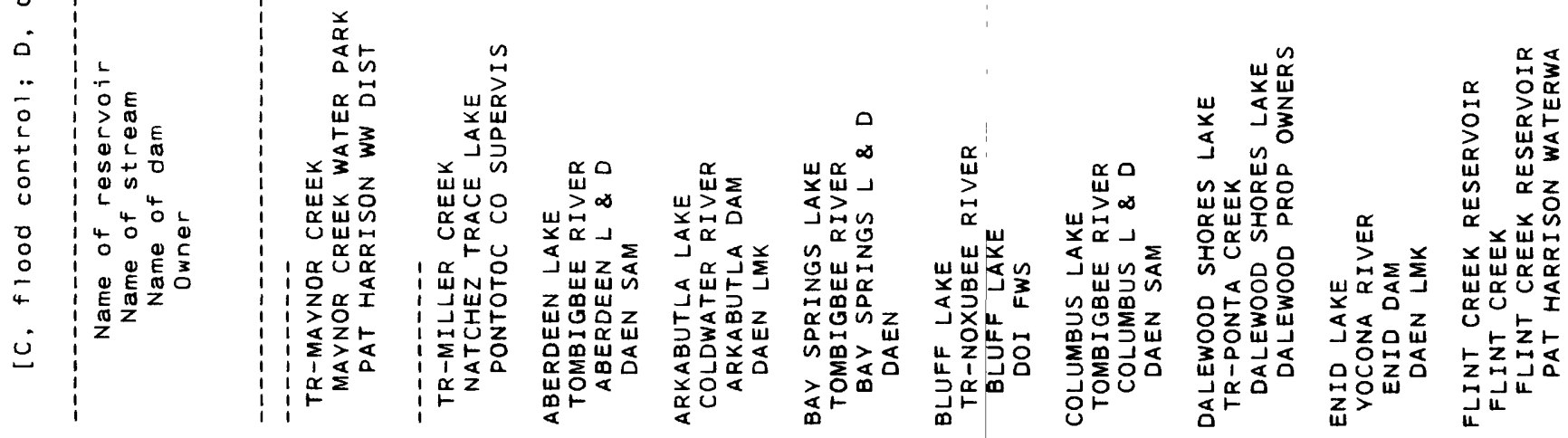


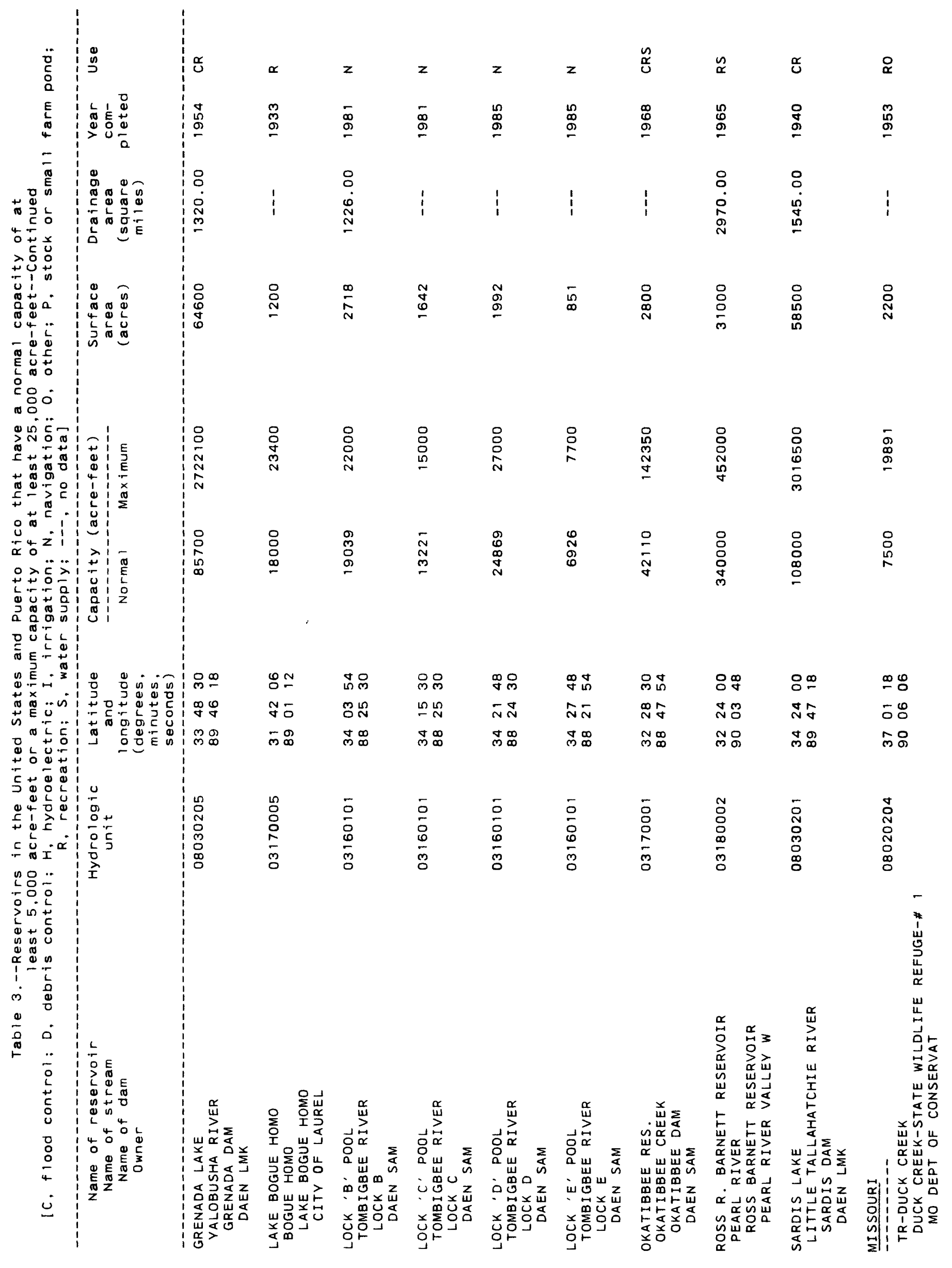




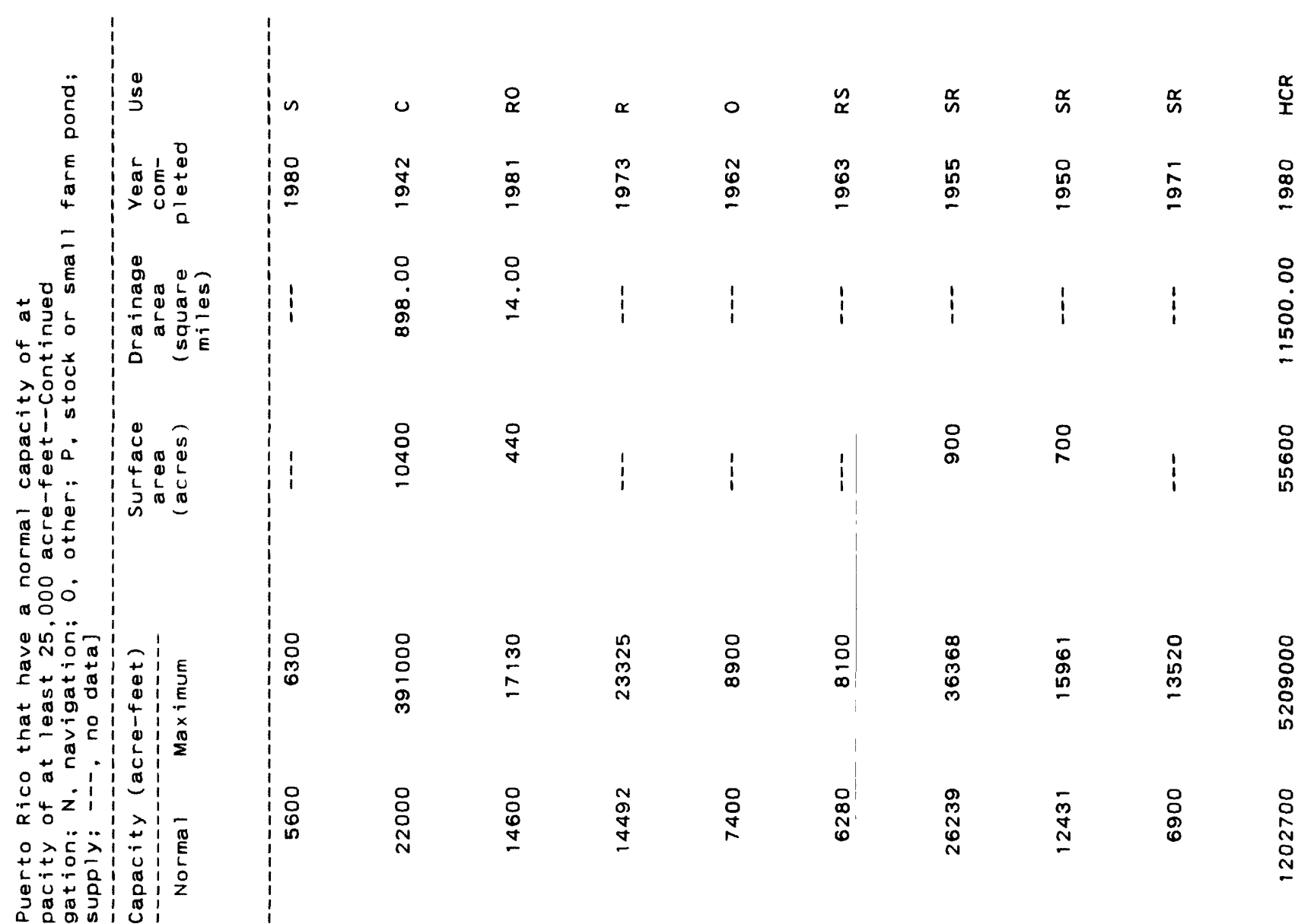

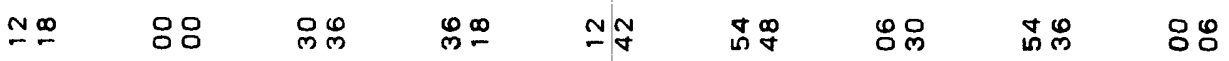

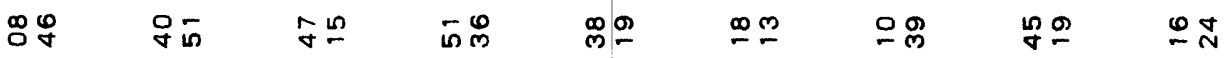
ॠ

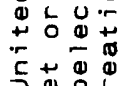

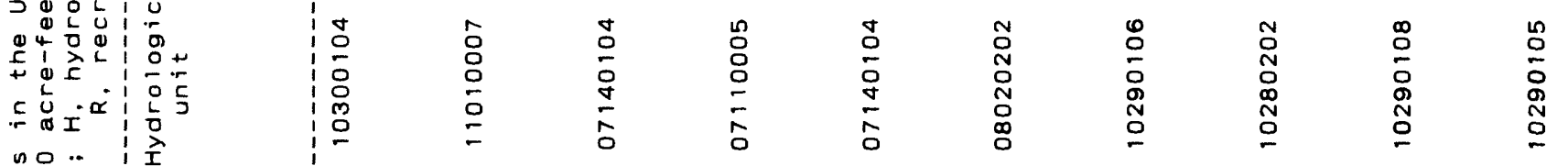
:

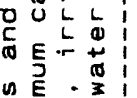

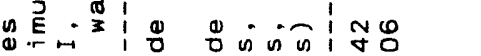

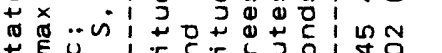<smiles></smiles>

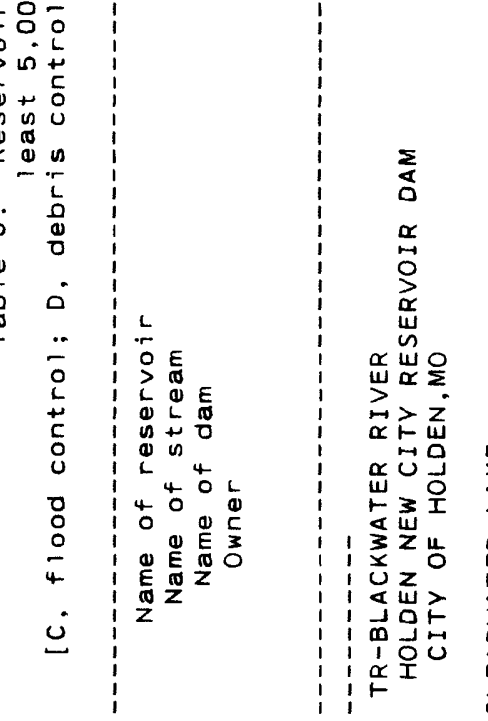<smiles>CC(C)(C)c1ccccc1</smiles> 


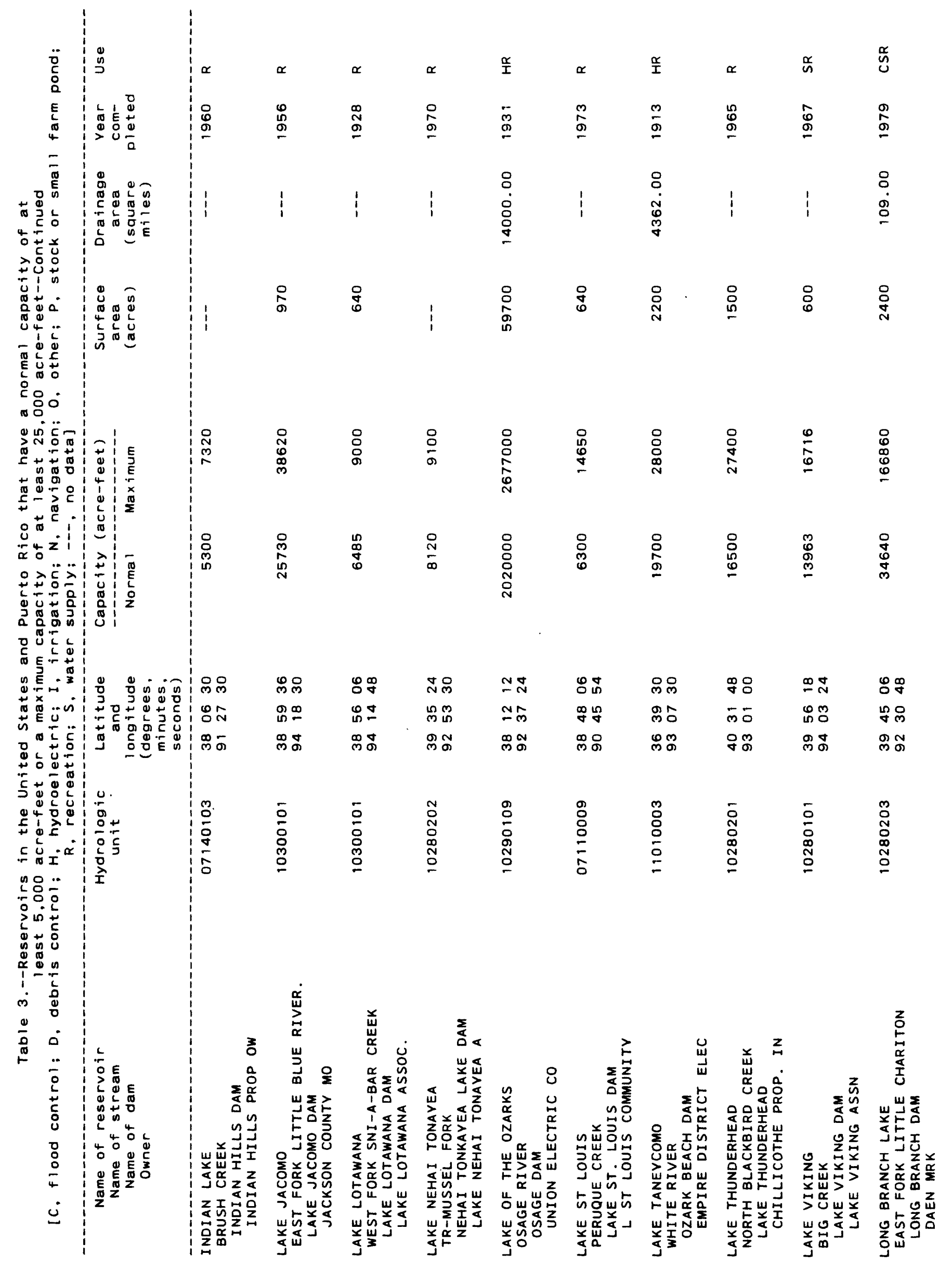




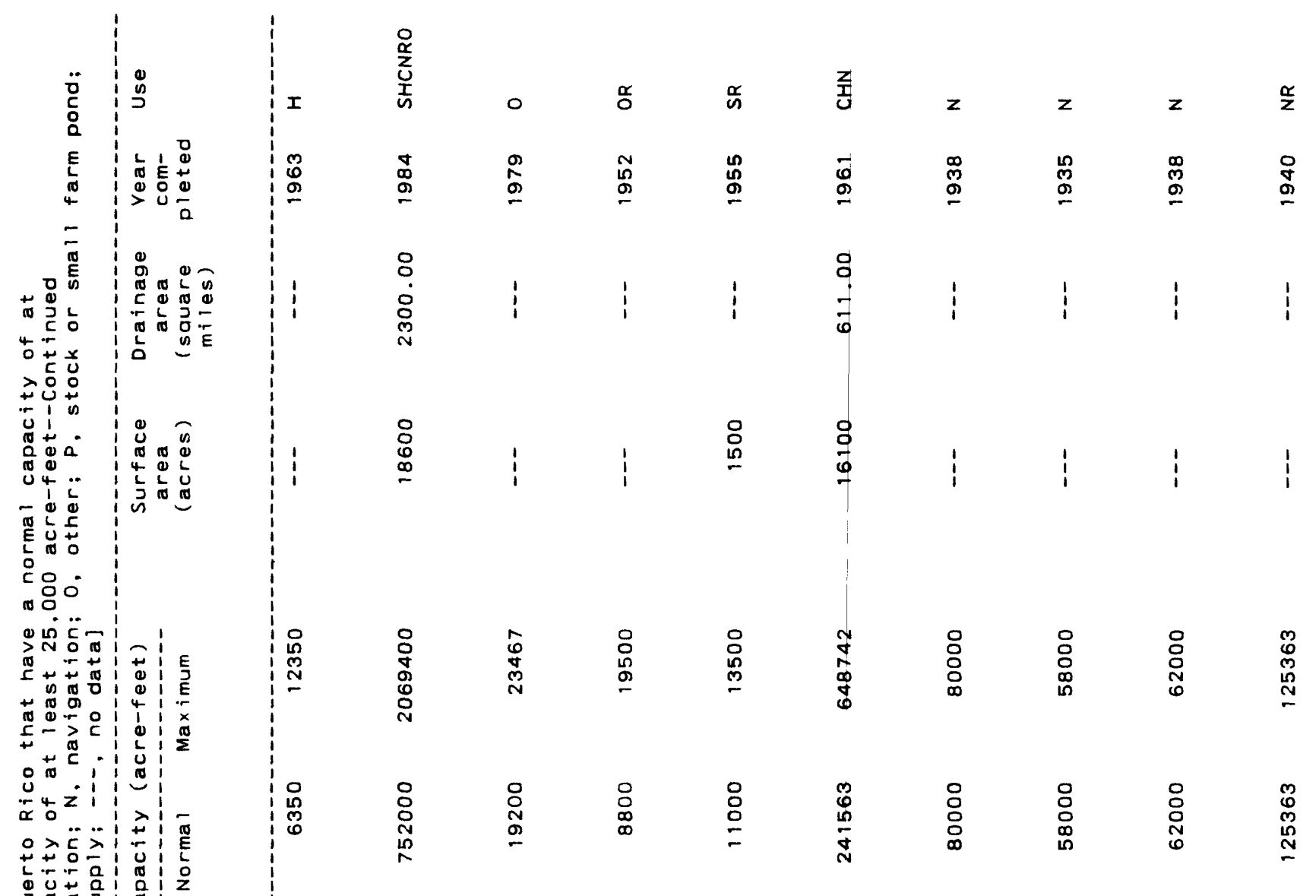

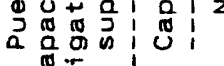

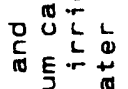

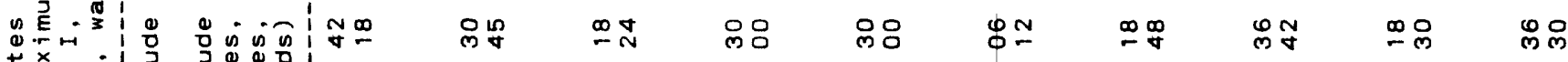

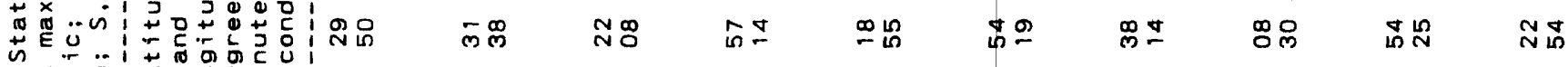

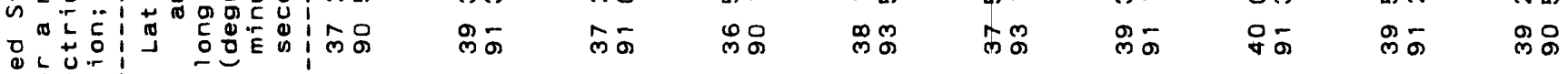

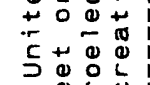

$\begin{array}{lllll}0 & 0 & 0 & 0 \\ 0 & 4 & 0 & 0\end{array}$

₹

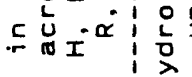

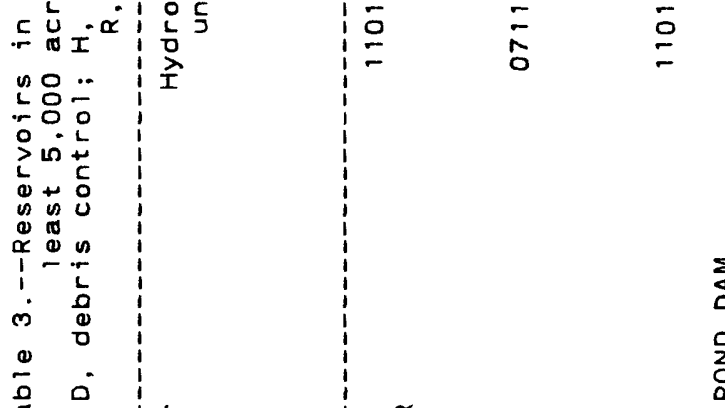

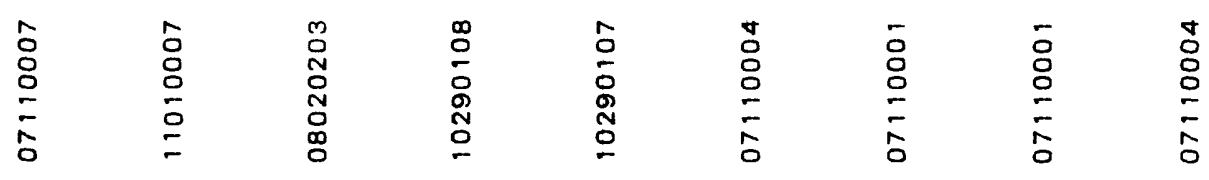

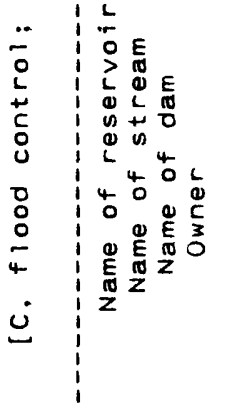

0
0
0
0

䍃文过

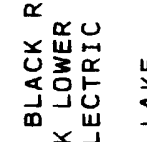

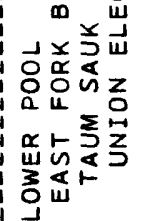
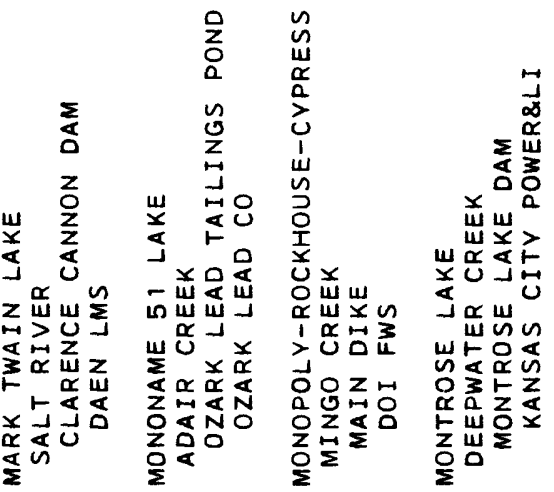

品

U

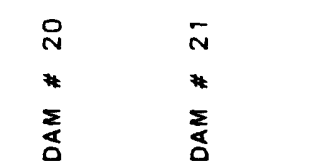





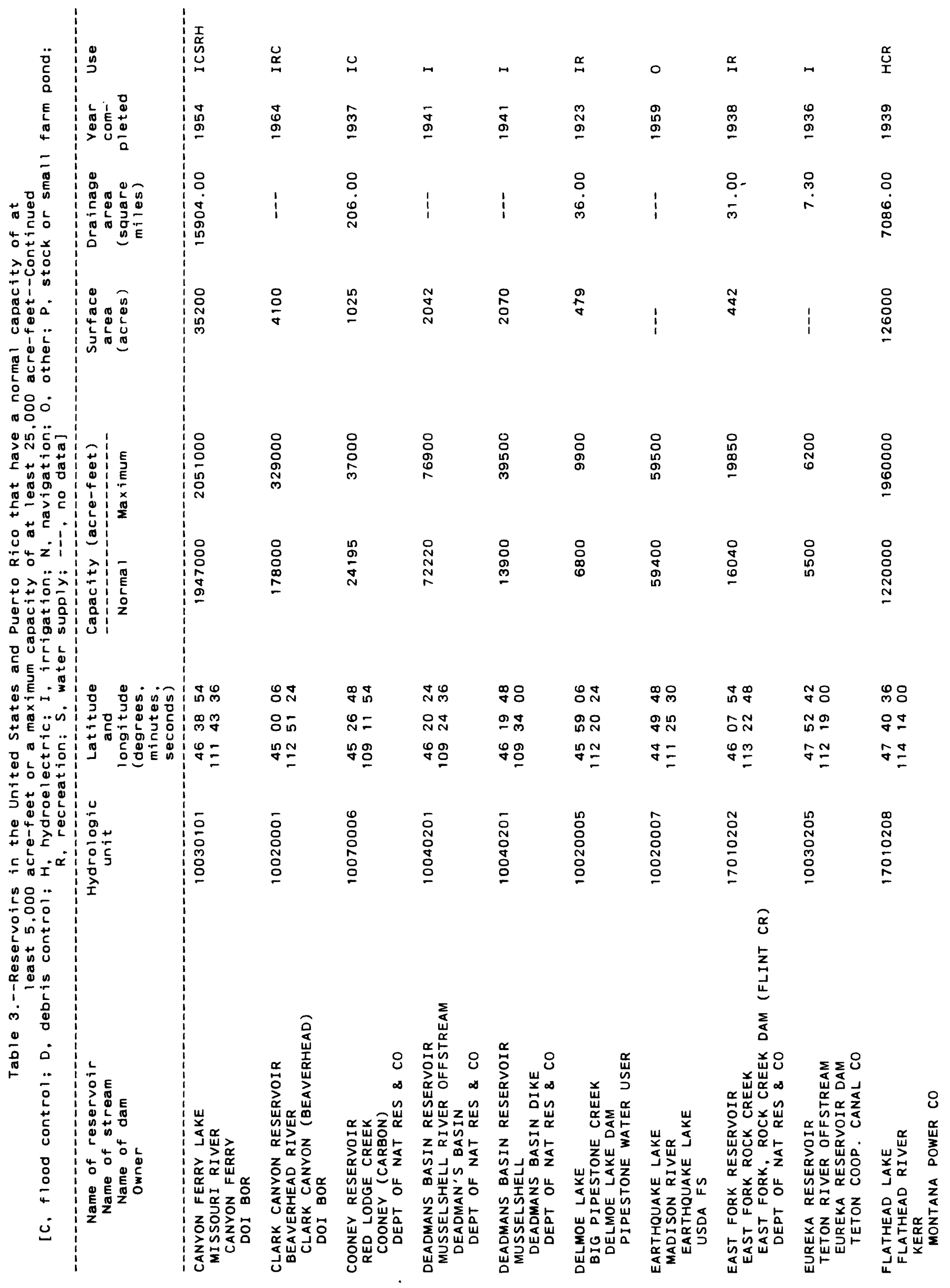




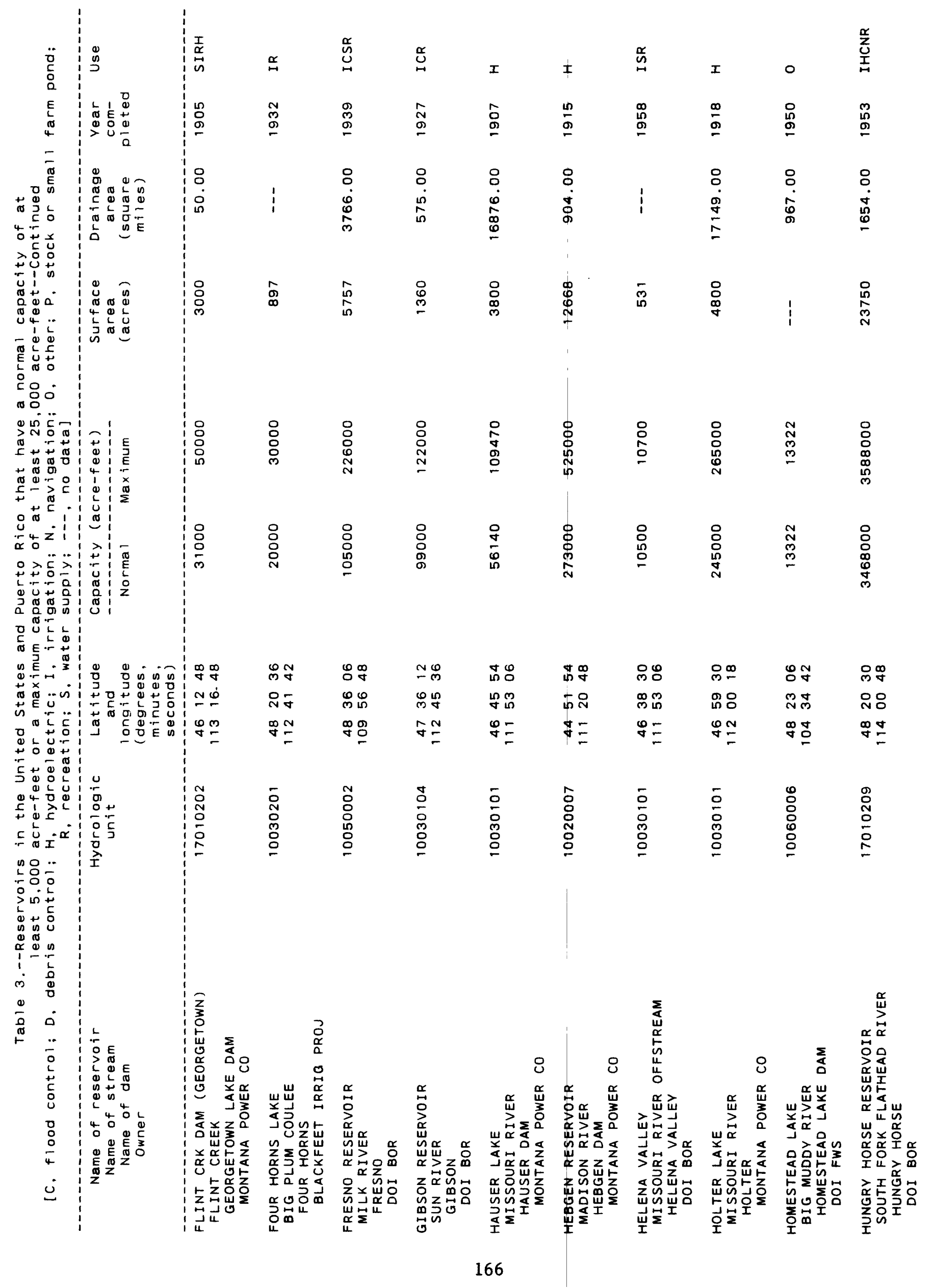




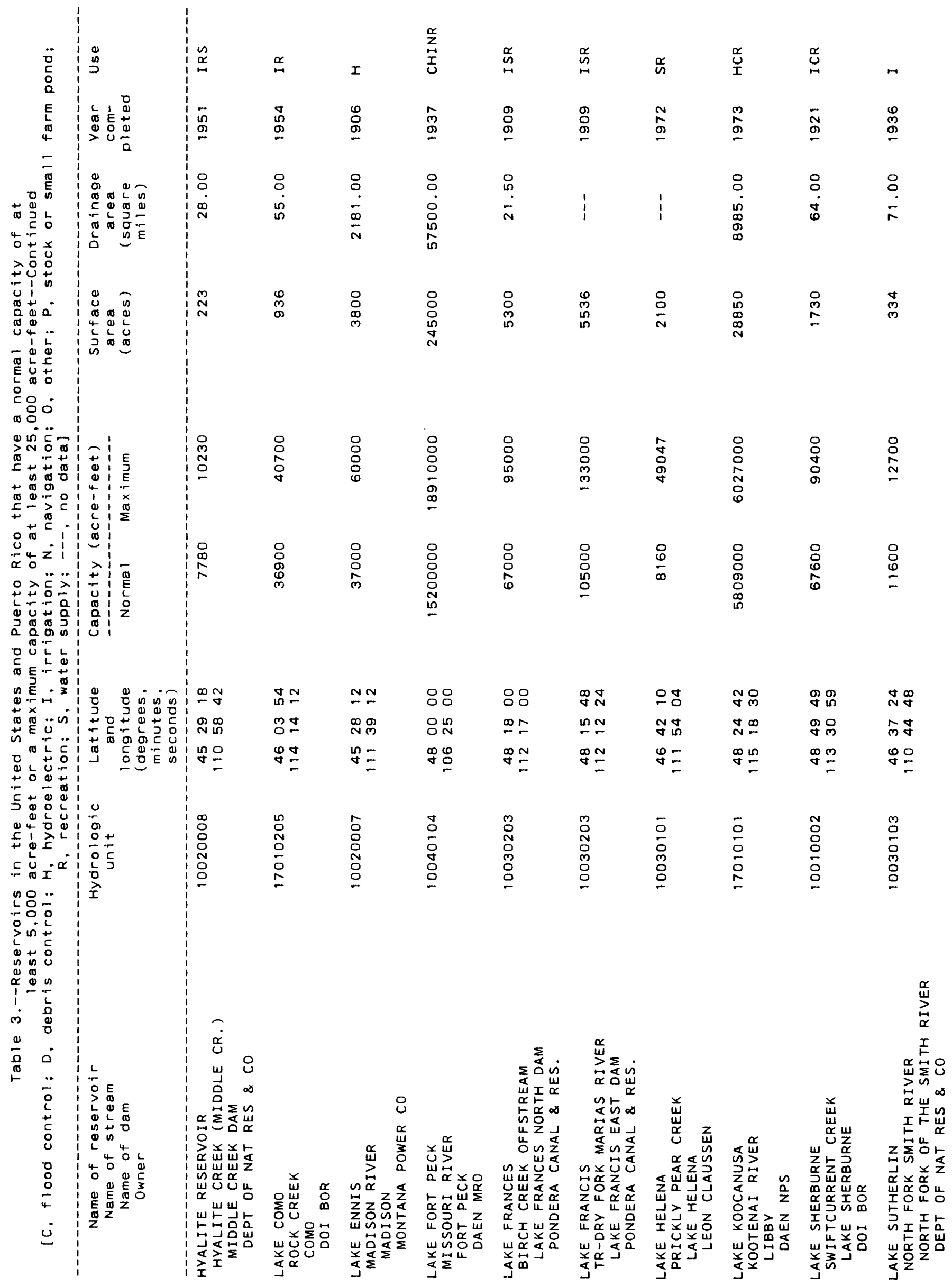




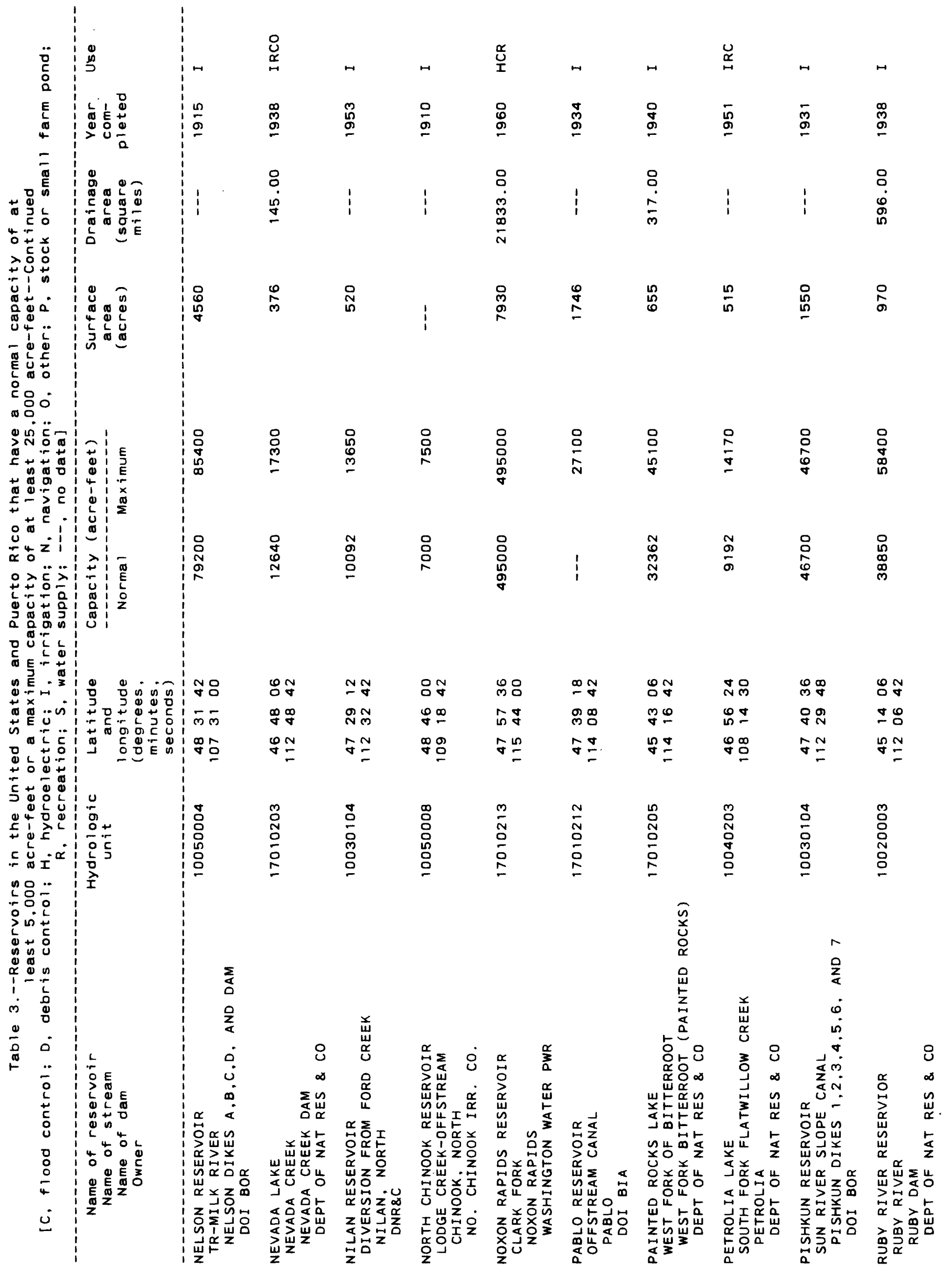




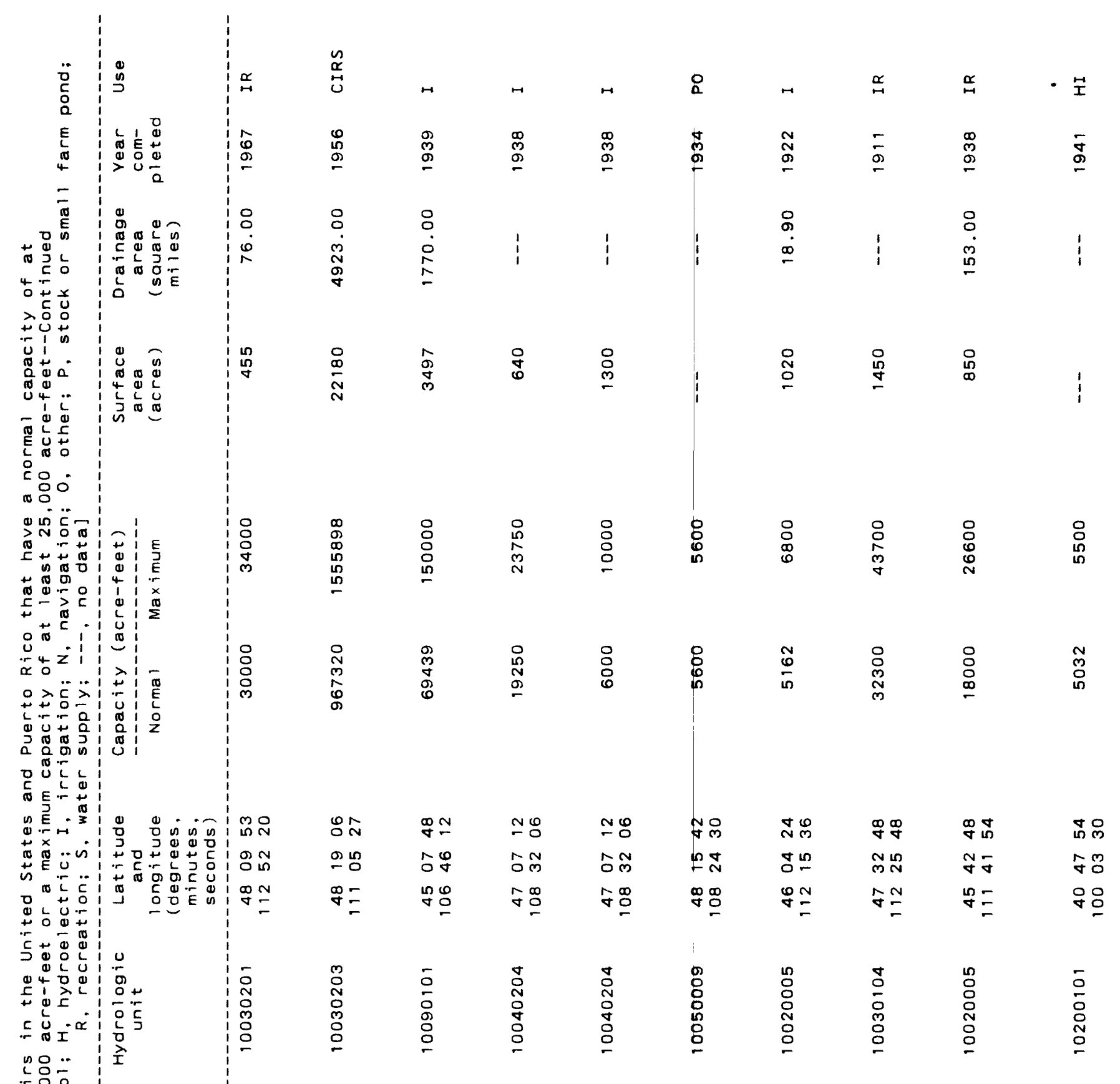




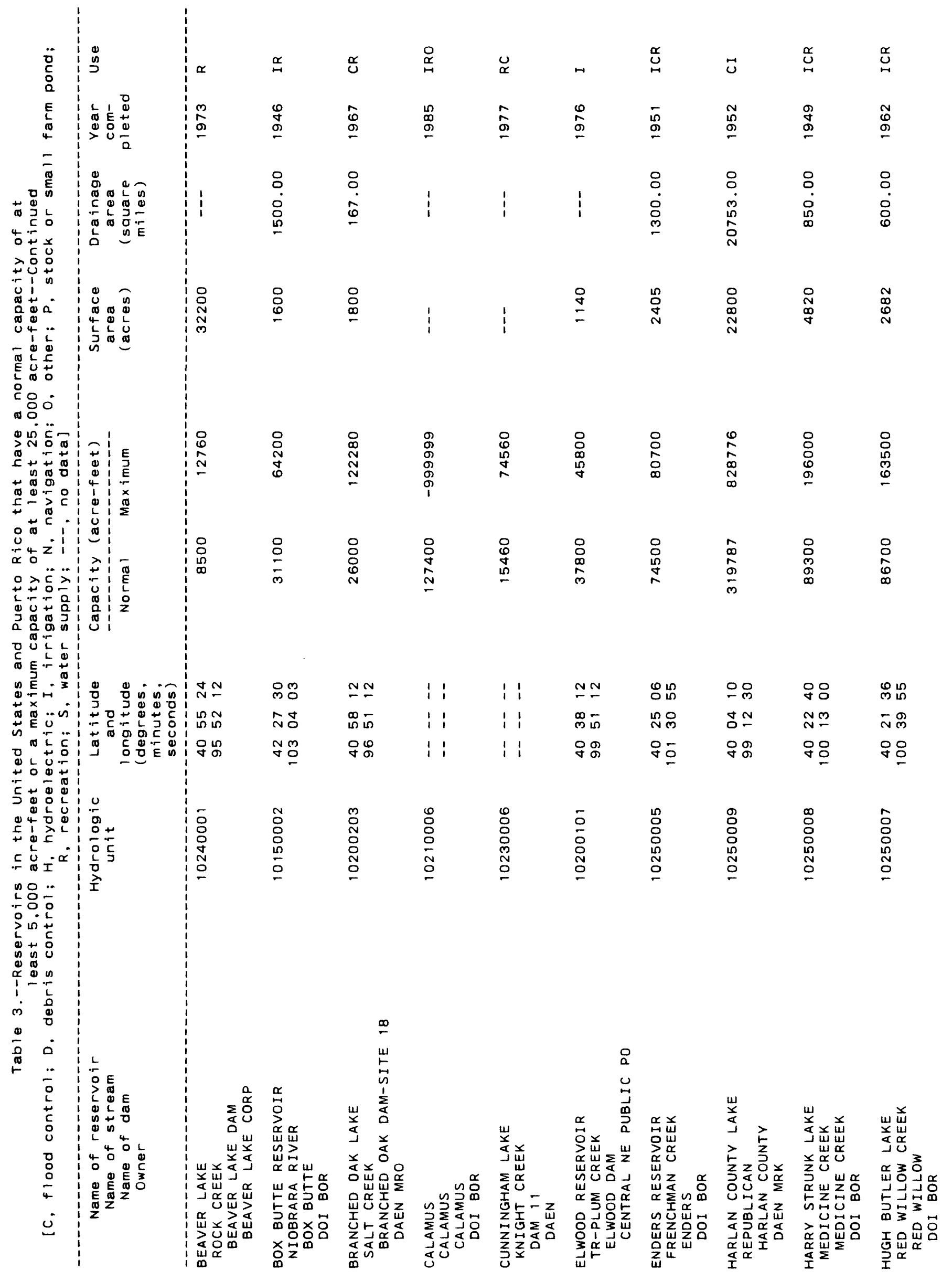




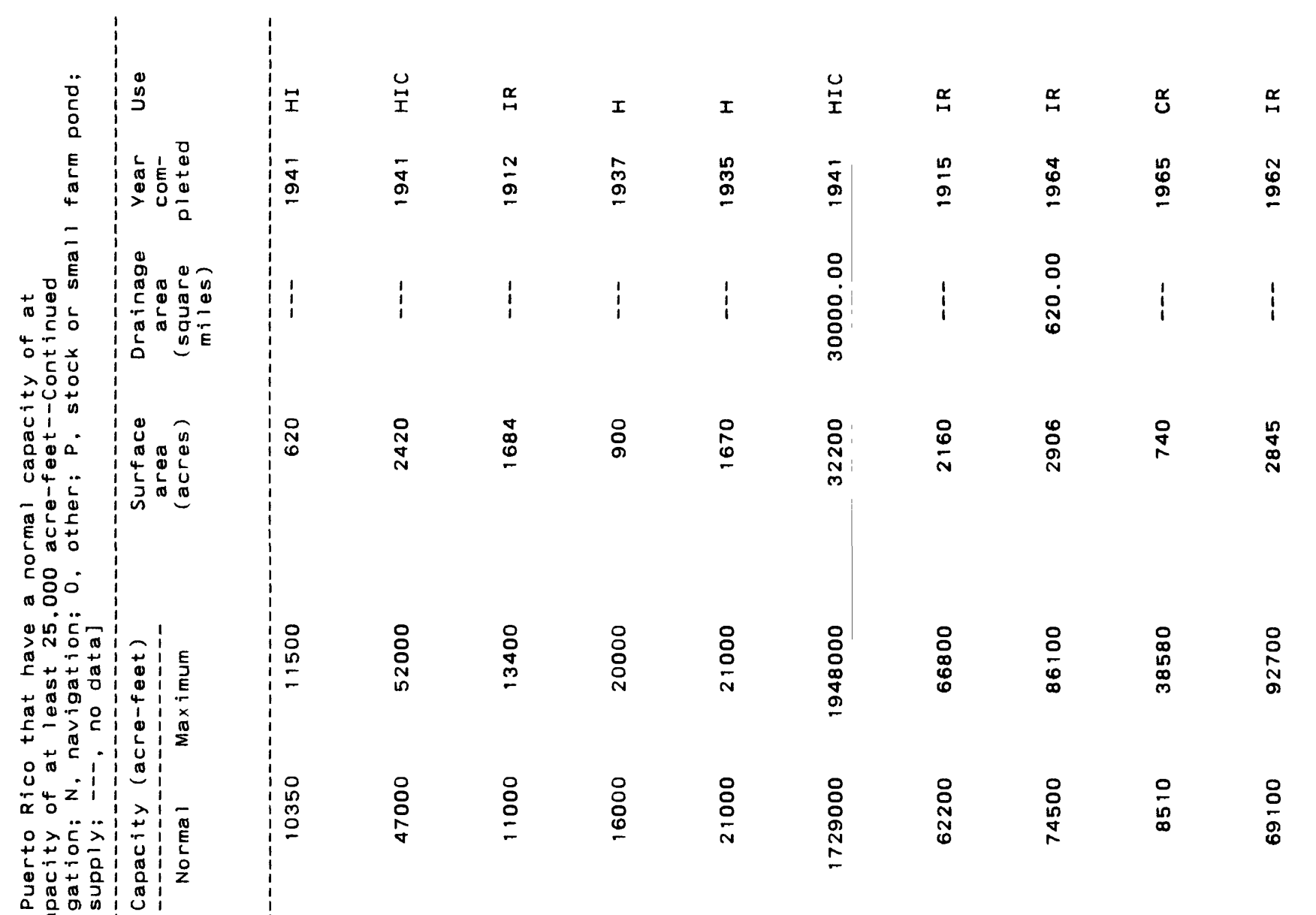

वण्.

T

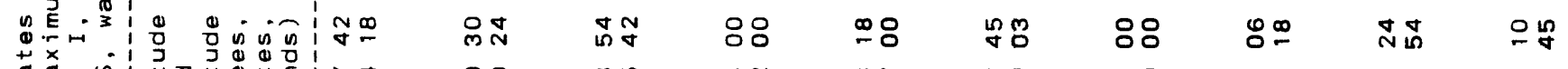

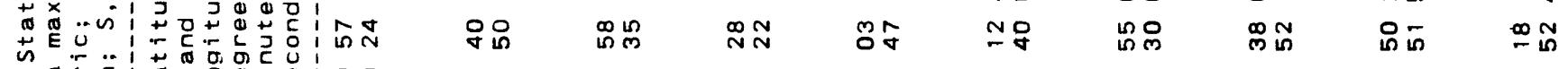

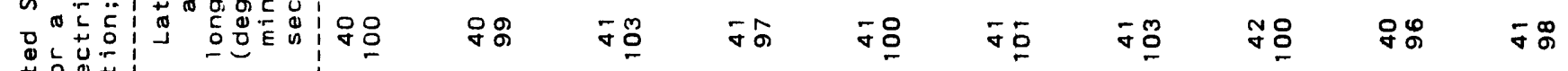

.

$\bar{c}+\overline{0}$

ᄃ원

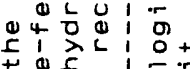

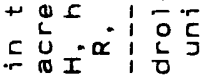

요요

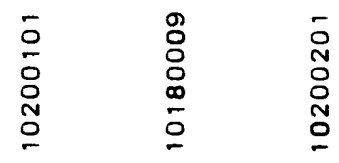

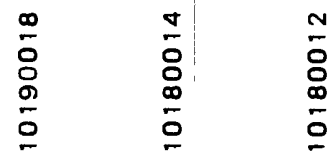

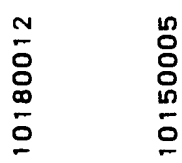

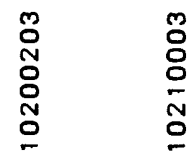

?

的点

艊的

定

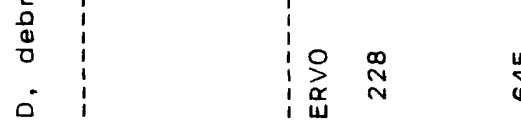

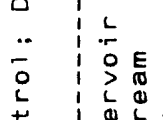

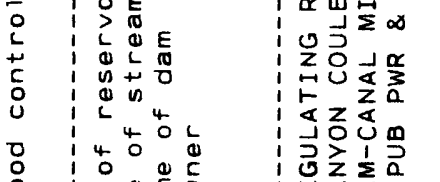

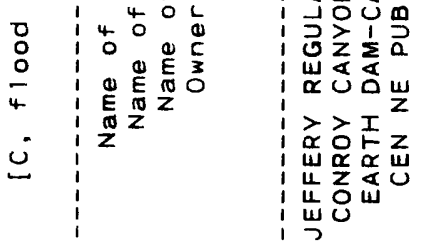

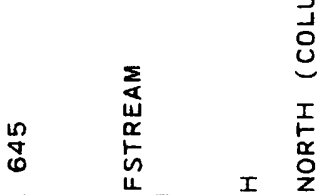

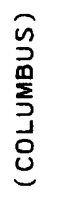




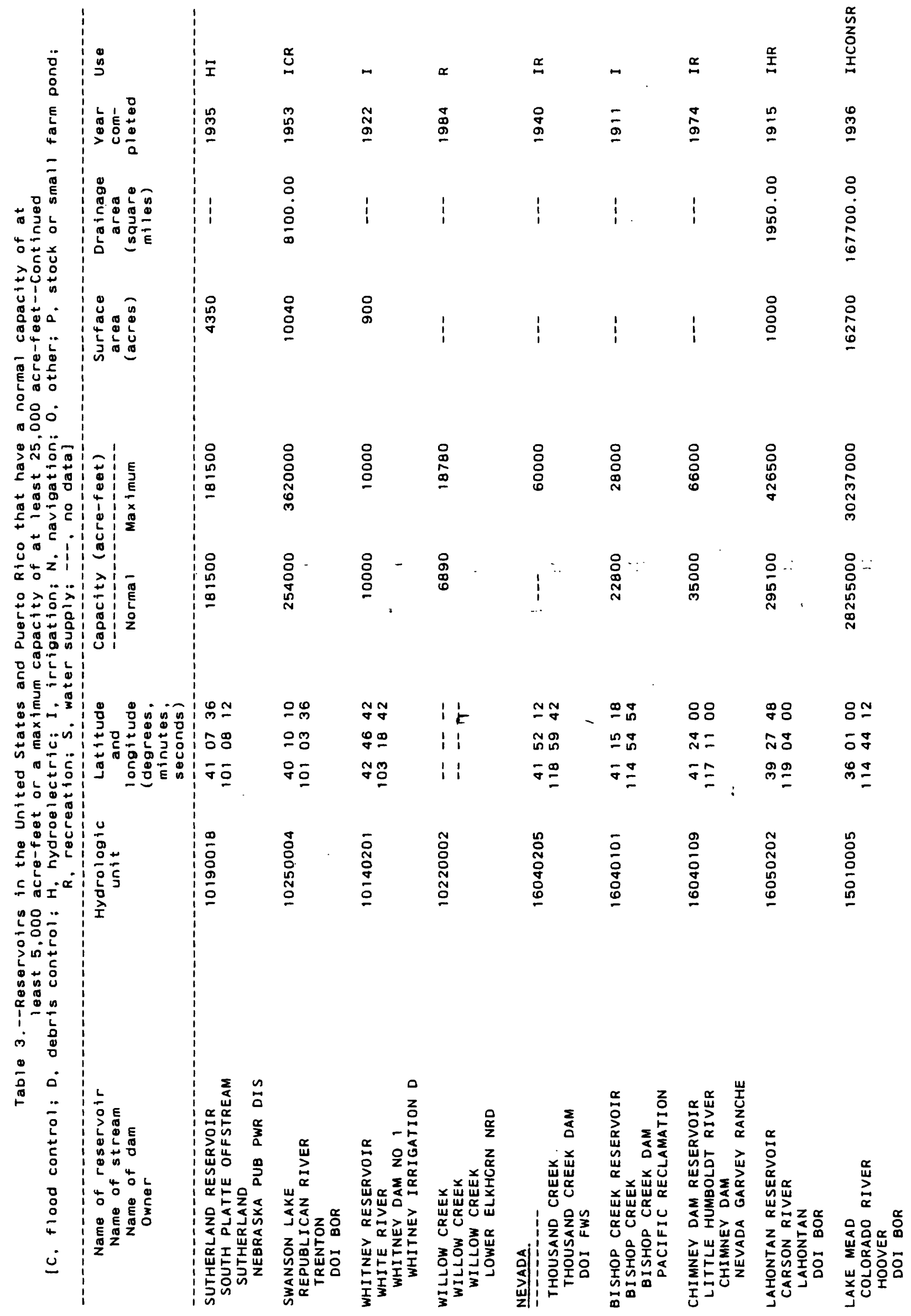




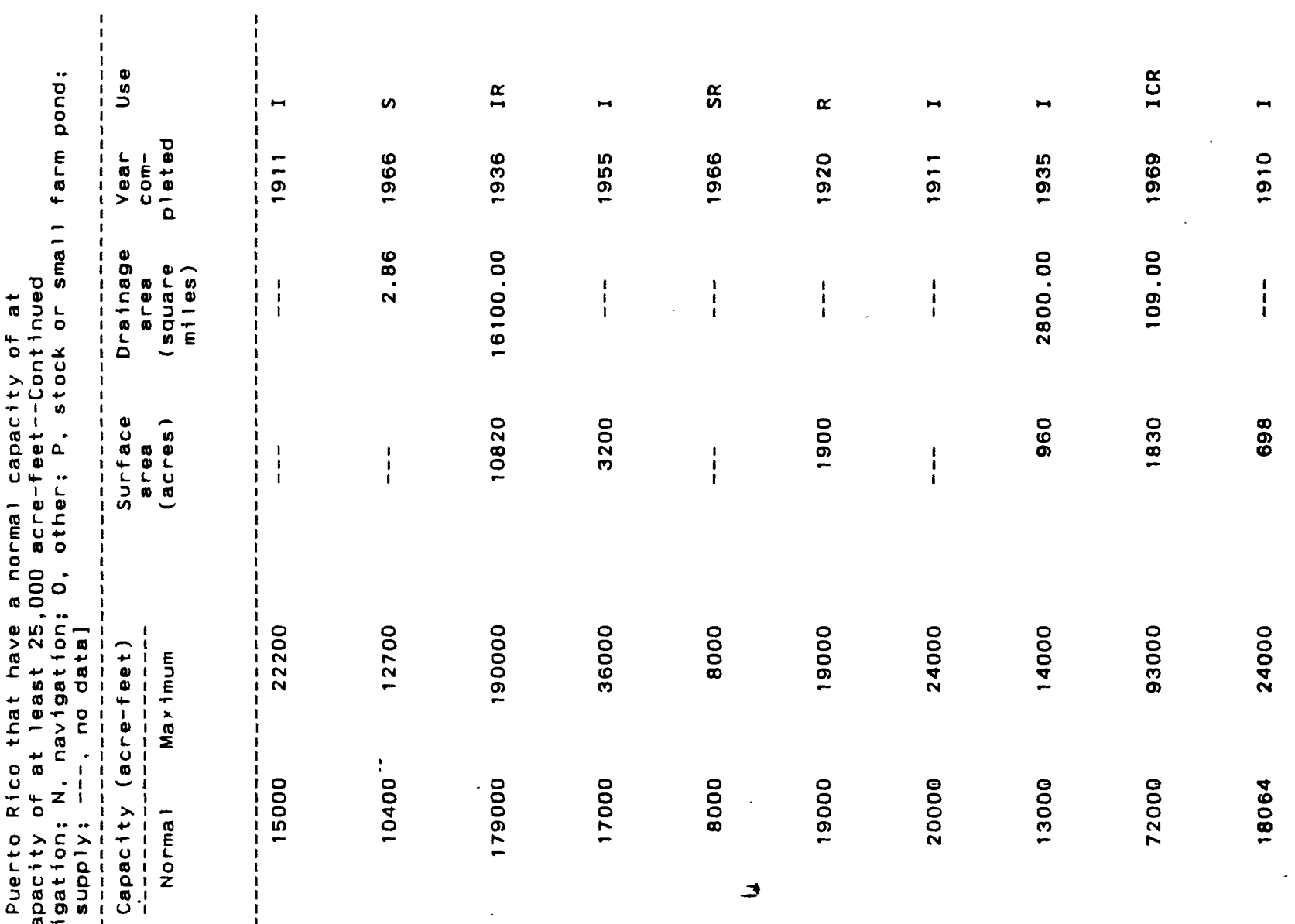

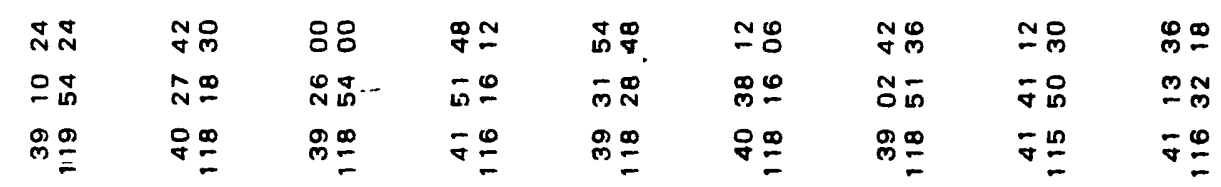

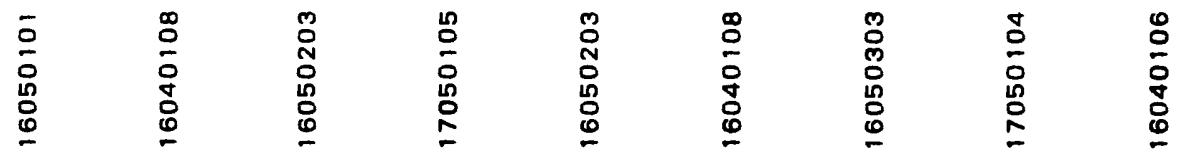
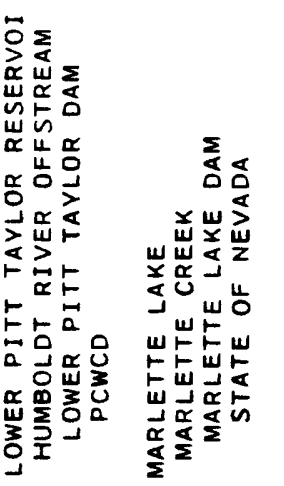

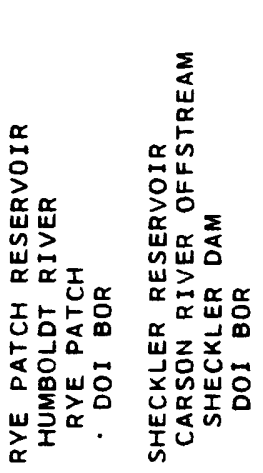

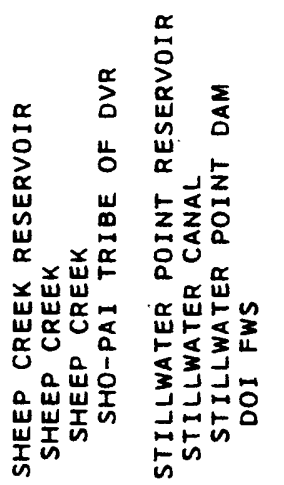

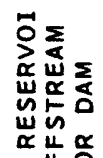

๔

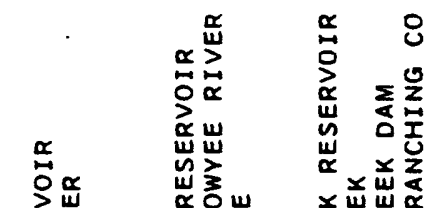

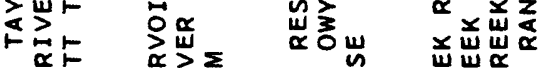

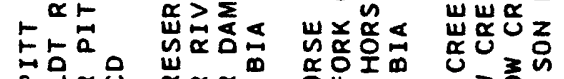

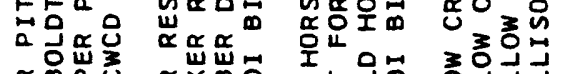

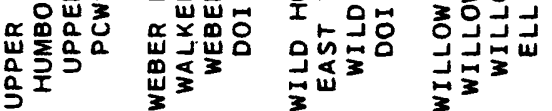




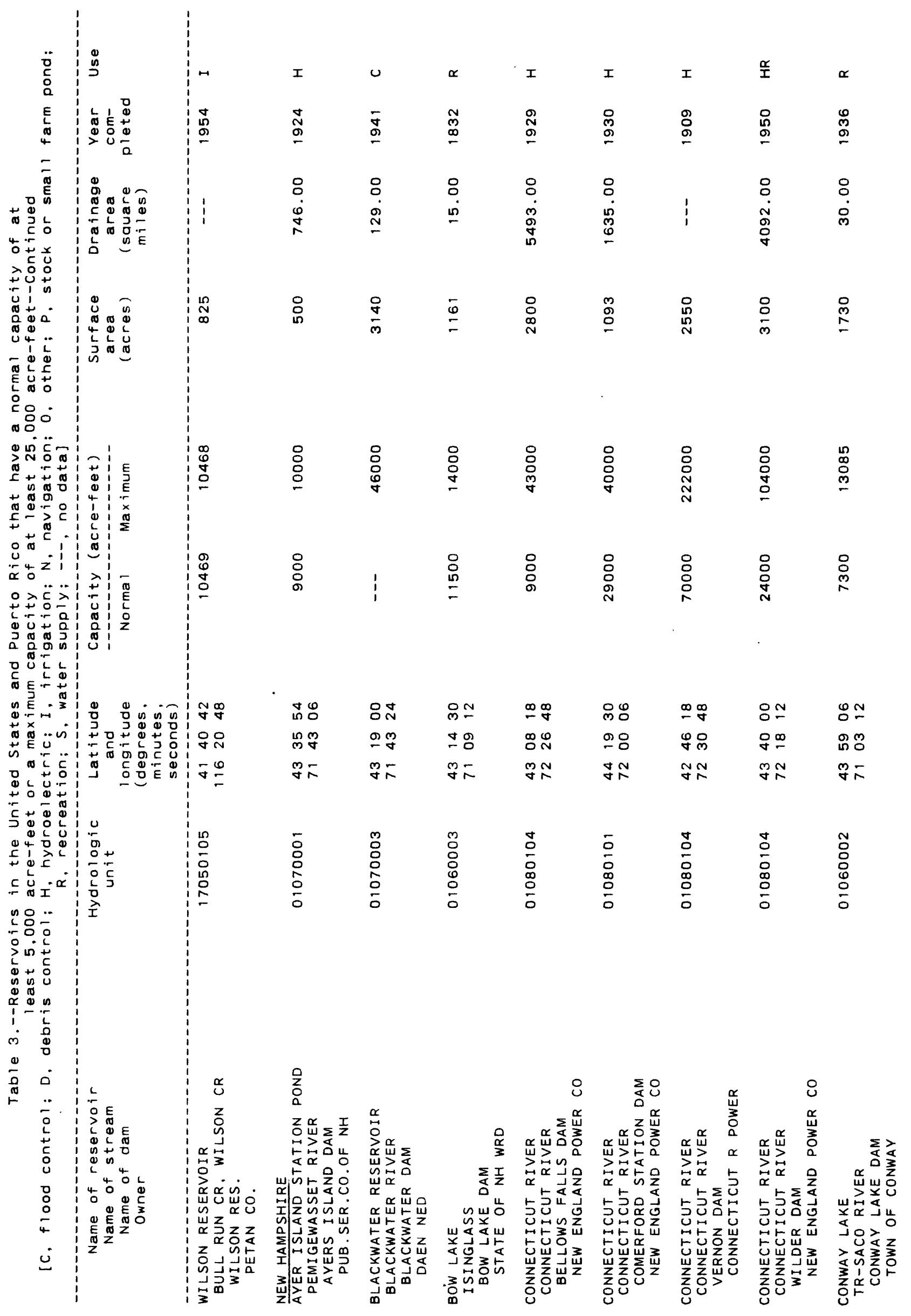




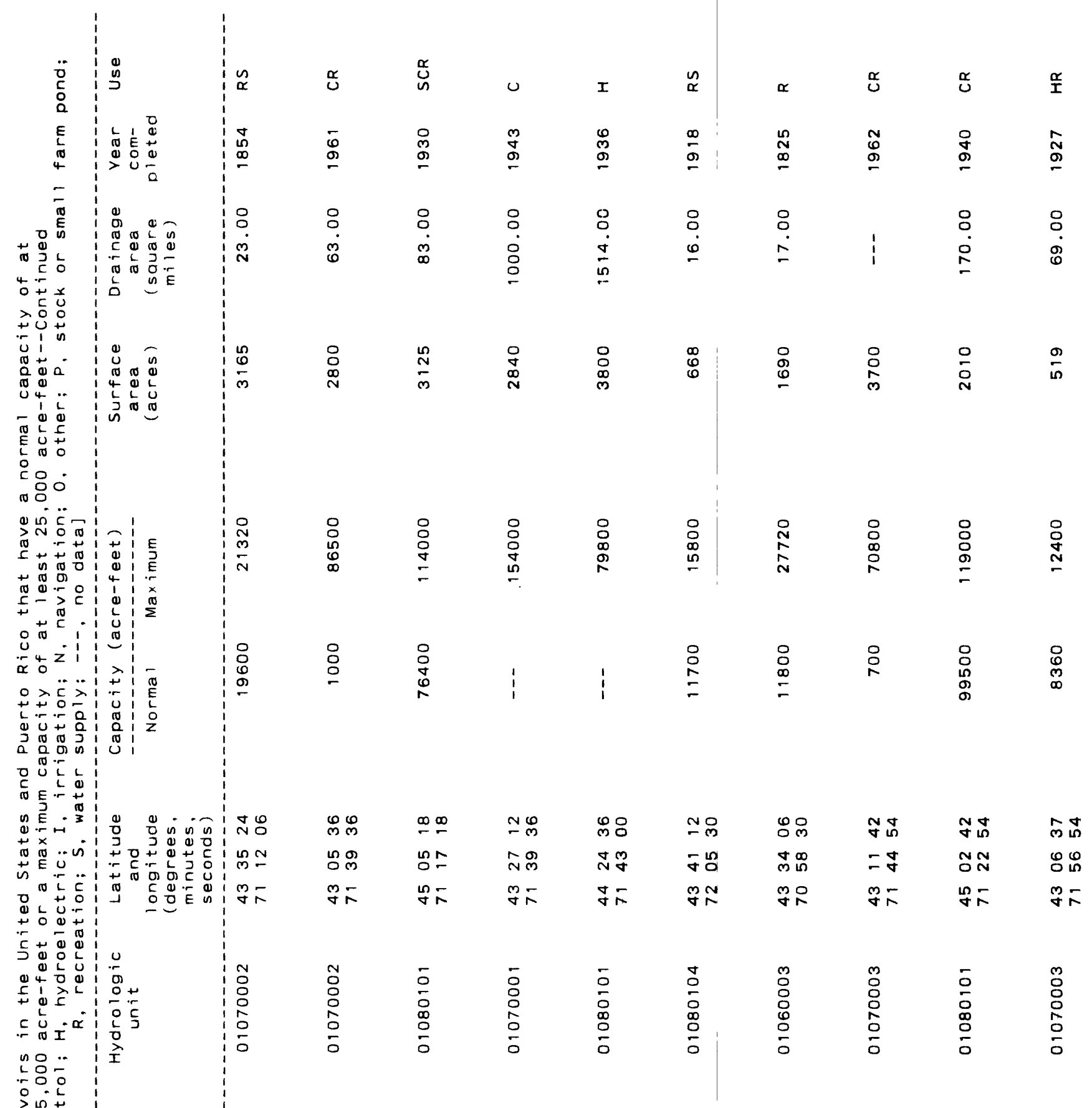




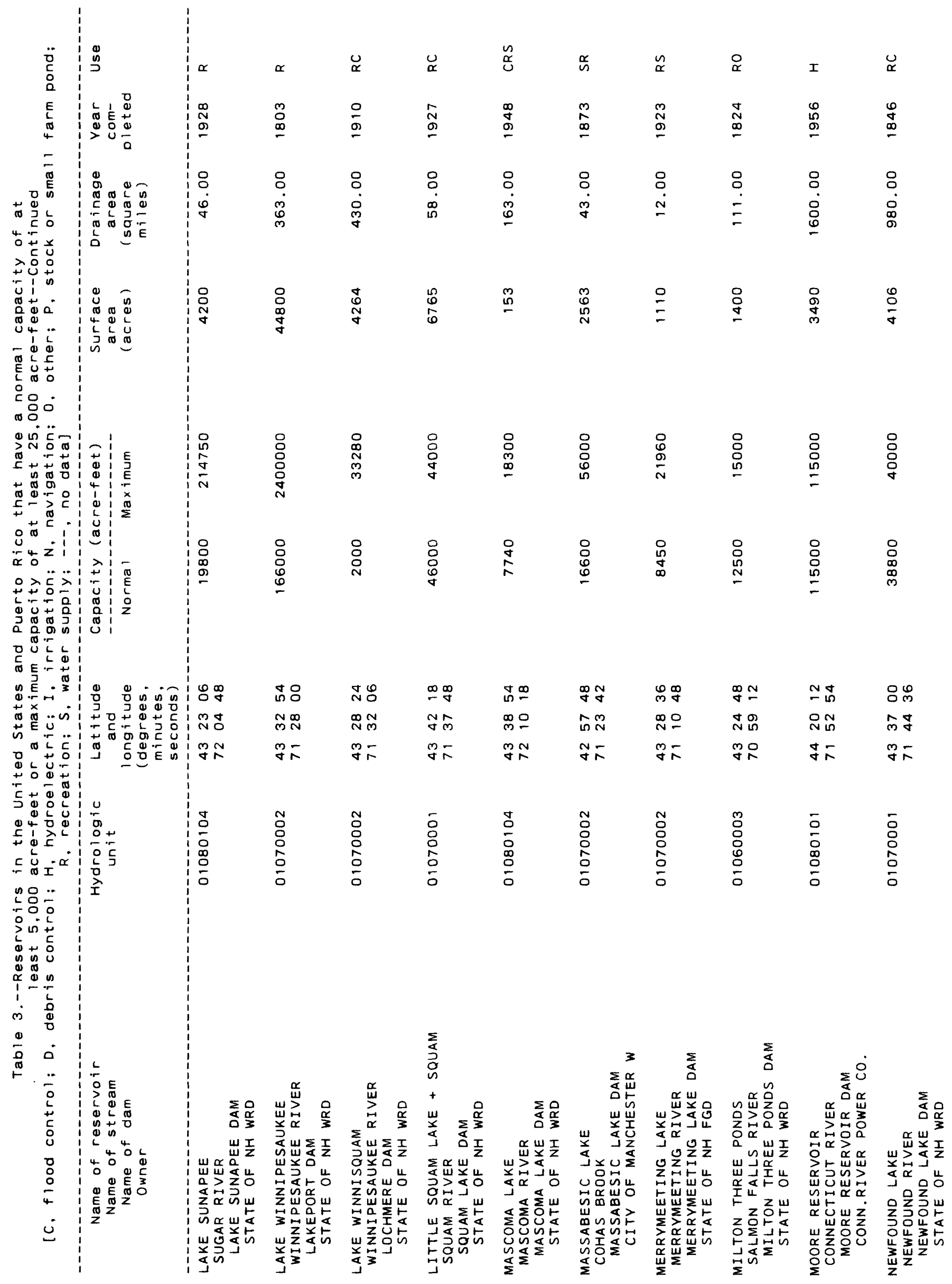




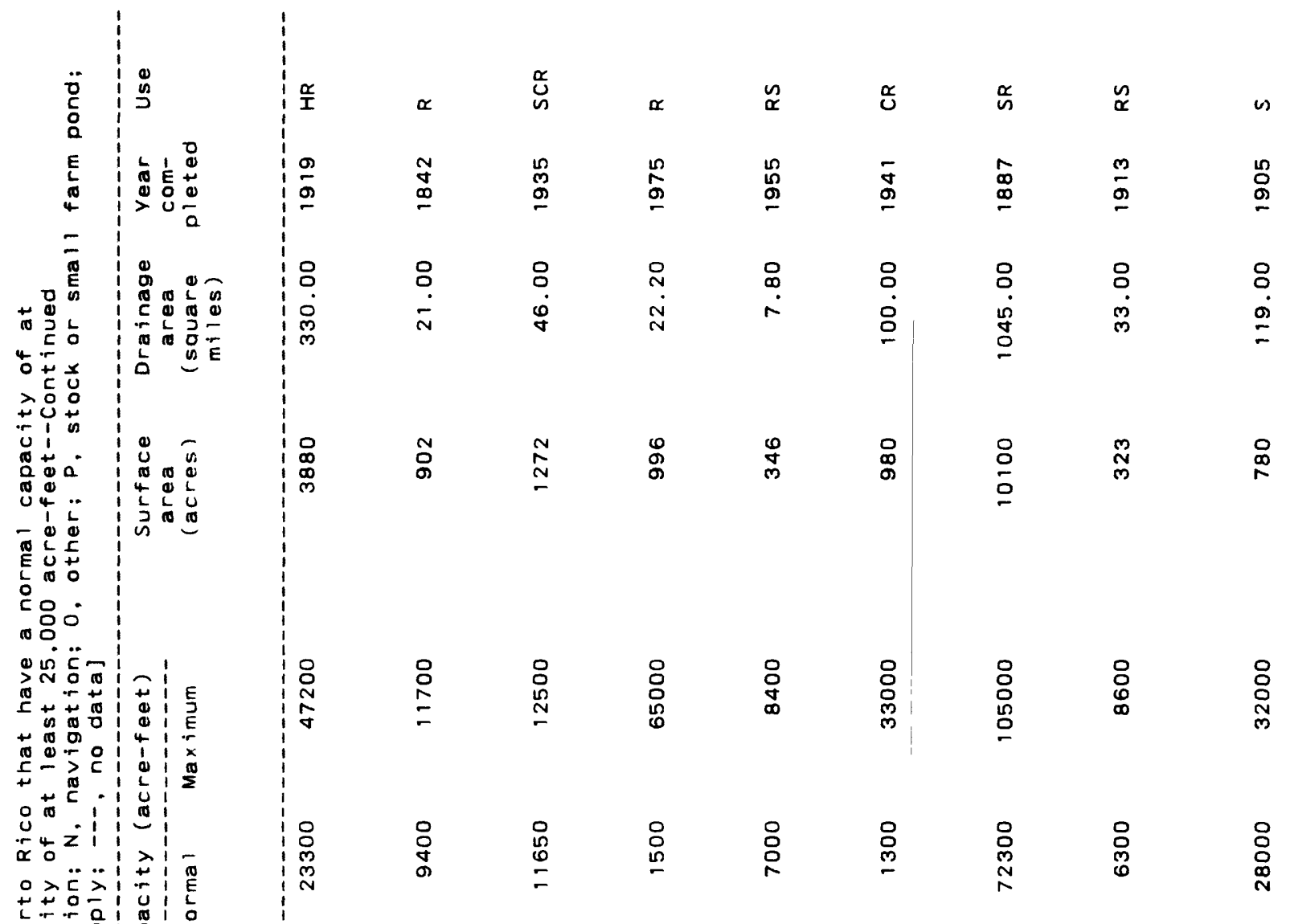

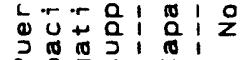

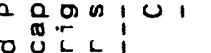

T

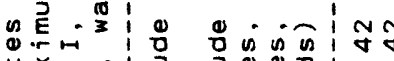

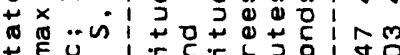

出 Eu

a

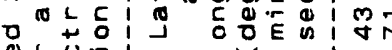

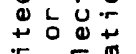

ᄃ년

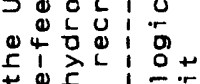

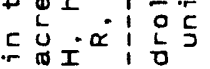

늠요

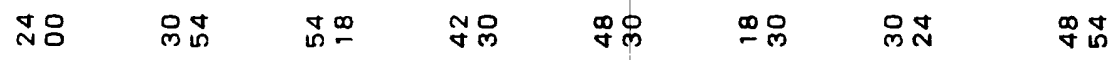

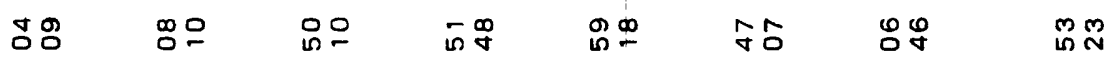

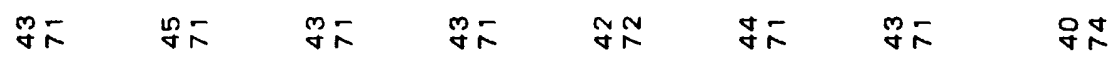

>is

a

0

$\begin{array}{ll:l}1-i & z & a\end{array}$

के

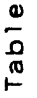

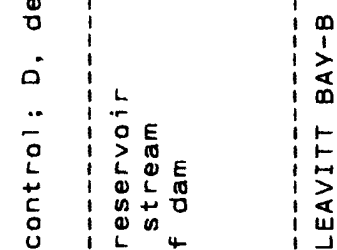

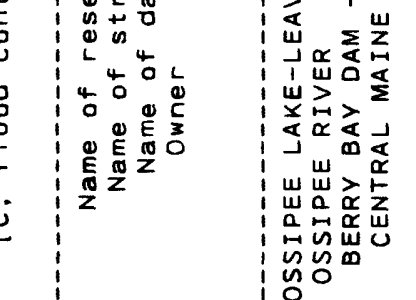

$\begin{array}{lll}0 & - & N \\ 0 & 0 & 0 \\ 0 & 0 & 0 \\ 0 & 0 & 0 \\ 0 & 0 & 0 \\ 0 & 0 & 0\end{array}$

$\overline{0}$
$\frac{0}{0}$
0
0

$\begin{array}{ll}\bar{D} & \overline{0} \\ 0 & 0 \\ 0 & 0 \\ 0 & 0 \\ 0 & 0\end{array}$

$\begin{array}{ll}\overline{8} & \text { : } \\ 0 & \frac{0}{0} \\ 0 & 0 \\ 0 & 0\end{array}$

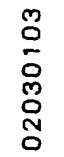
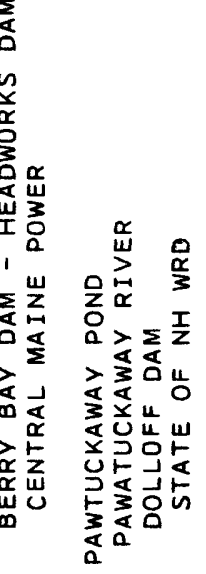

$\sum_{a}^{a}$

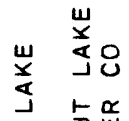

占点䍃

I

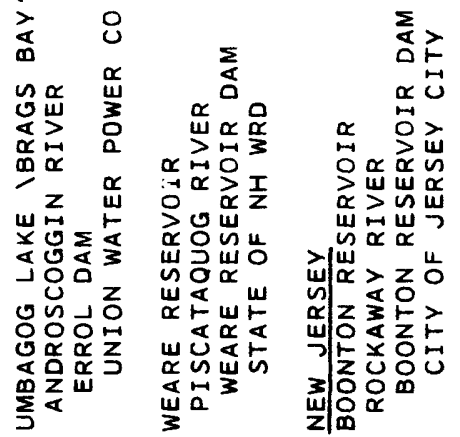



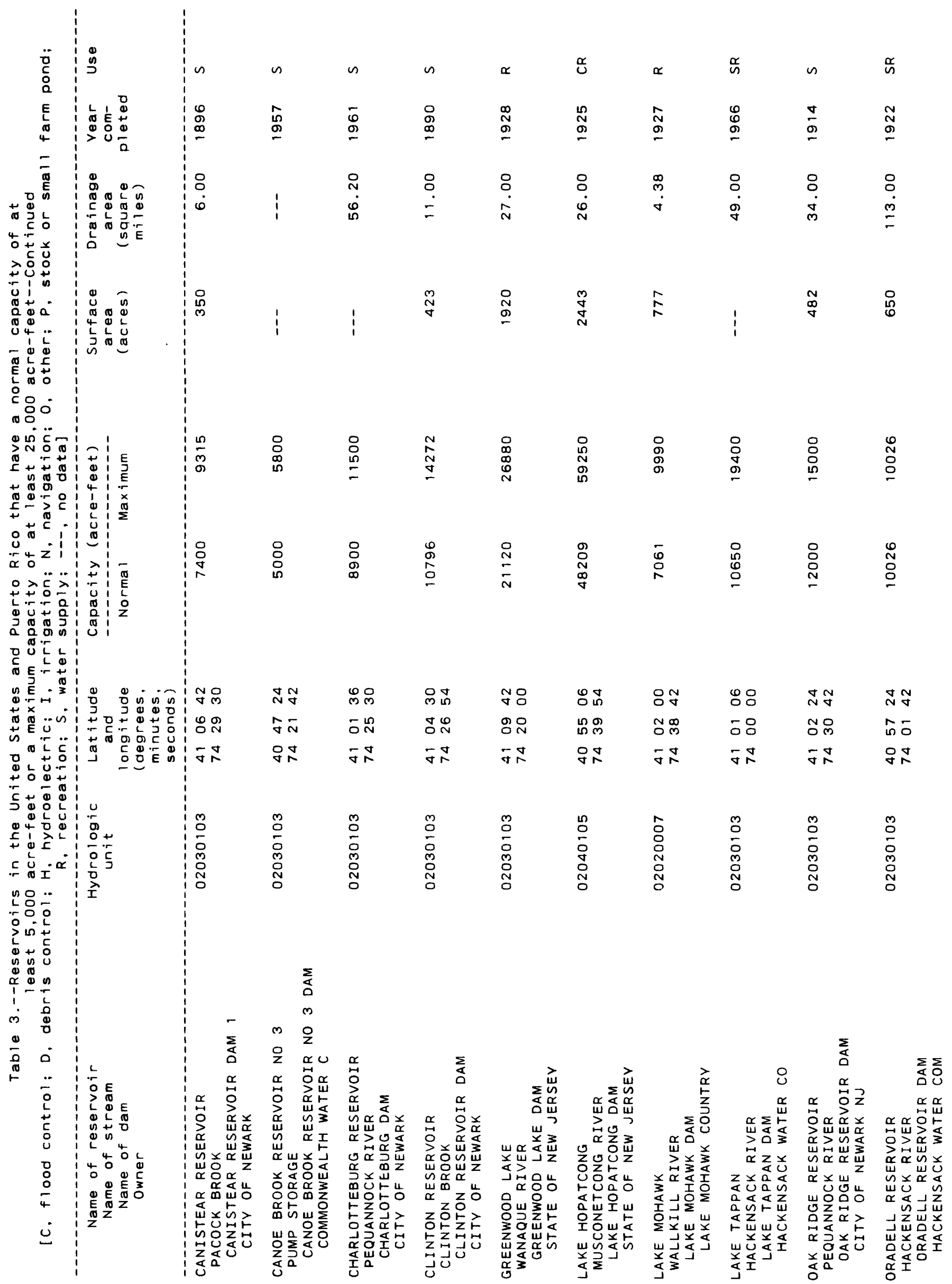


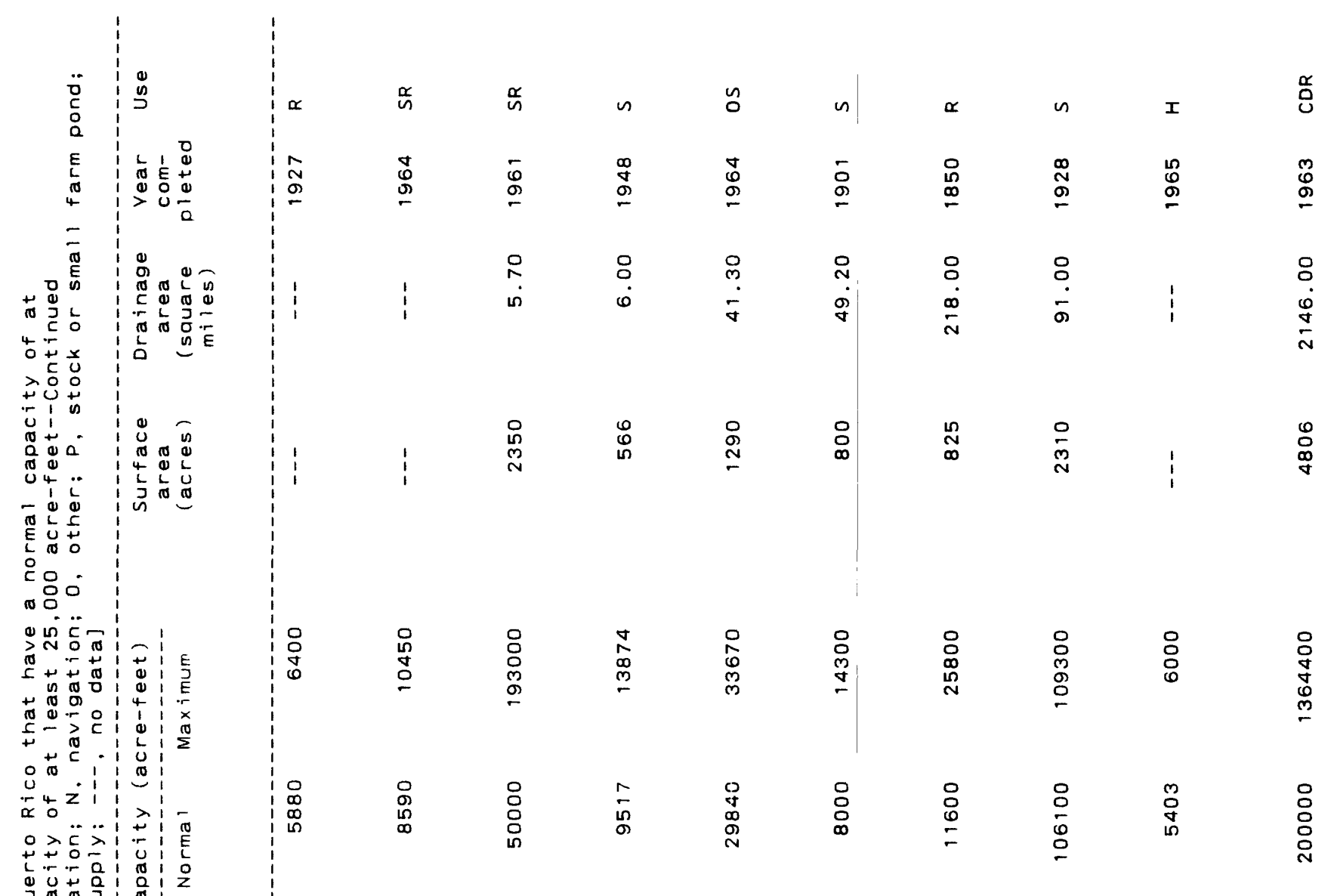

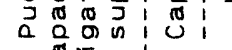

ช

(1) $E \div$

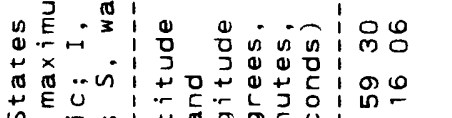

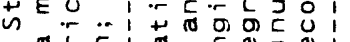

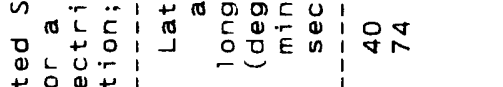

\begin{tabular}{|c|c|c|c|c|c|c|c|}
\hline$\stackrel{\infty}{\sim}$ & $\stackrel{\infty}{\sim}$ & $\stackrel{\infty}{\infty} \stackrel{\infty}{\sim}$ & 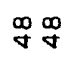 & ேㅗㅇ & $\because \cong$ & ్ㅣㅇㅇㅛ & $\underset{\sim}{\infty}$ \\
\hline 冓 & 电品 & in & 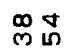 & 战 & No & NI & 呙๐ \\
\hline 守 & 宗界 & 宫界 & 守只 & 定走 & MN & 文圭 & 움 \\
\hline
\end{tabular}

$\bar{c}+\bar{a}$

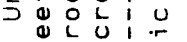

$\begin{array}{lll:l}0 & 0 \\ 0 & 0 & 0 \\ 0 & 0 & 0\end{array}$

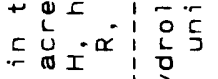

$\begin{array}{llll}\text { m } & \text { n } & \text { M } & \text { ! } \\ 0 & 0 & 0 & 0 \\ 0 & 0 & 0 & 0 \\ 0 & 0 & 0 & 0 \\ 0 & 0 & 0 & 0 \\ 0 & 0 & 0 & 0 \\ 0 & 0 & \text { D } & \text { D }\end{array}$

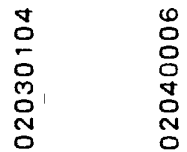

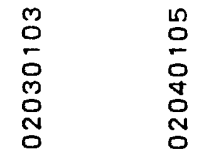

$\begin{array}{ll}n & \text { N } \\ & 0 \\ 0 & 0 \\ 0 & 0 \\ 0 & 0\end{array}$

0
0
0
2
0
0
0
0
0
1
0
0
0
0
0
0

$>$ in

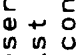

\&

1

m

8

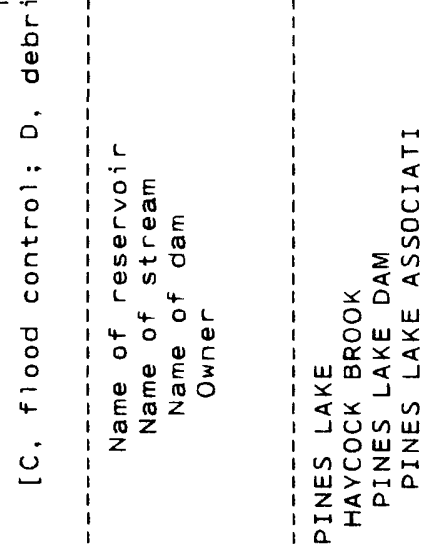

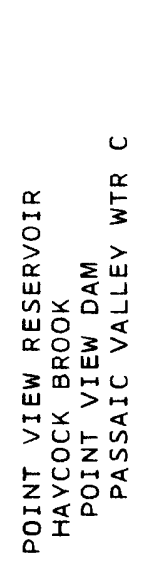
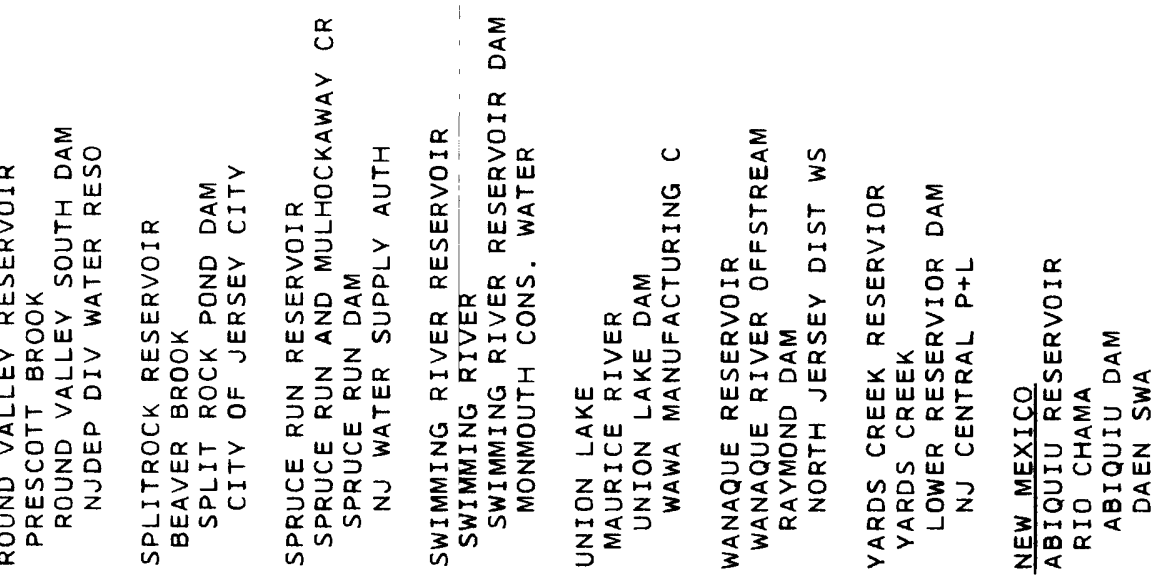


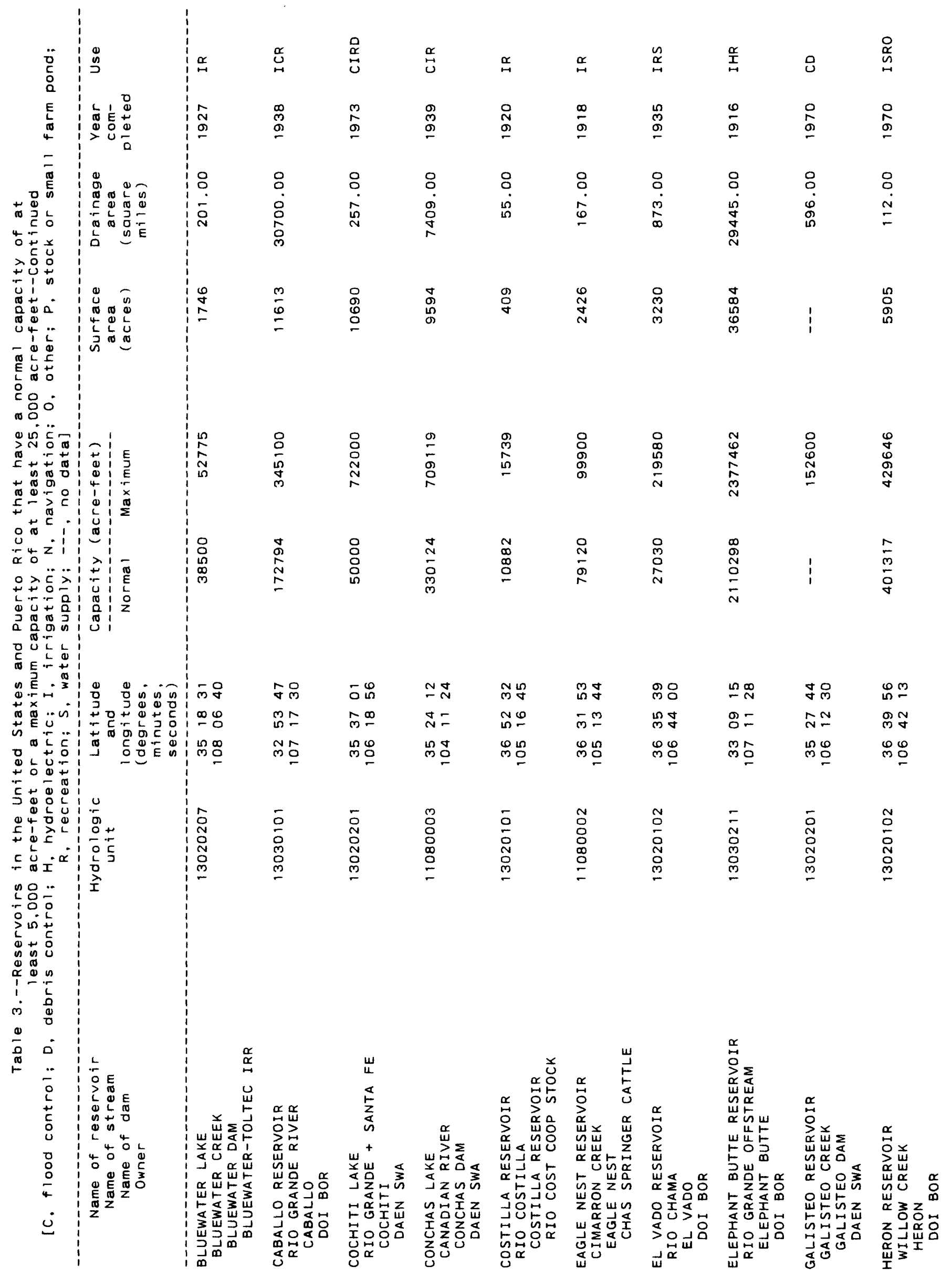




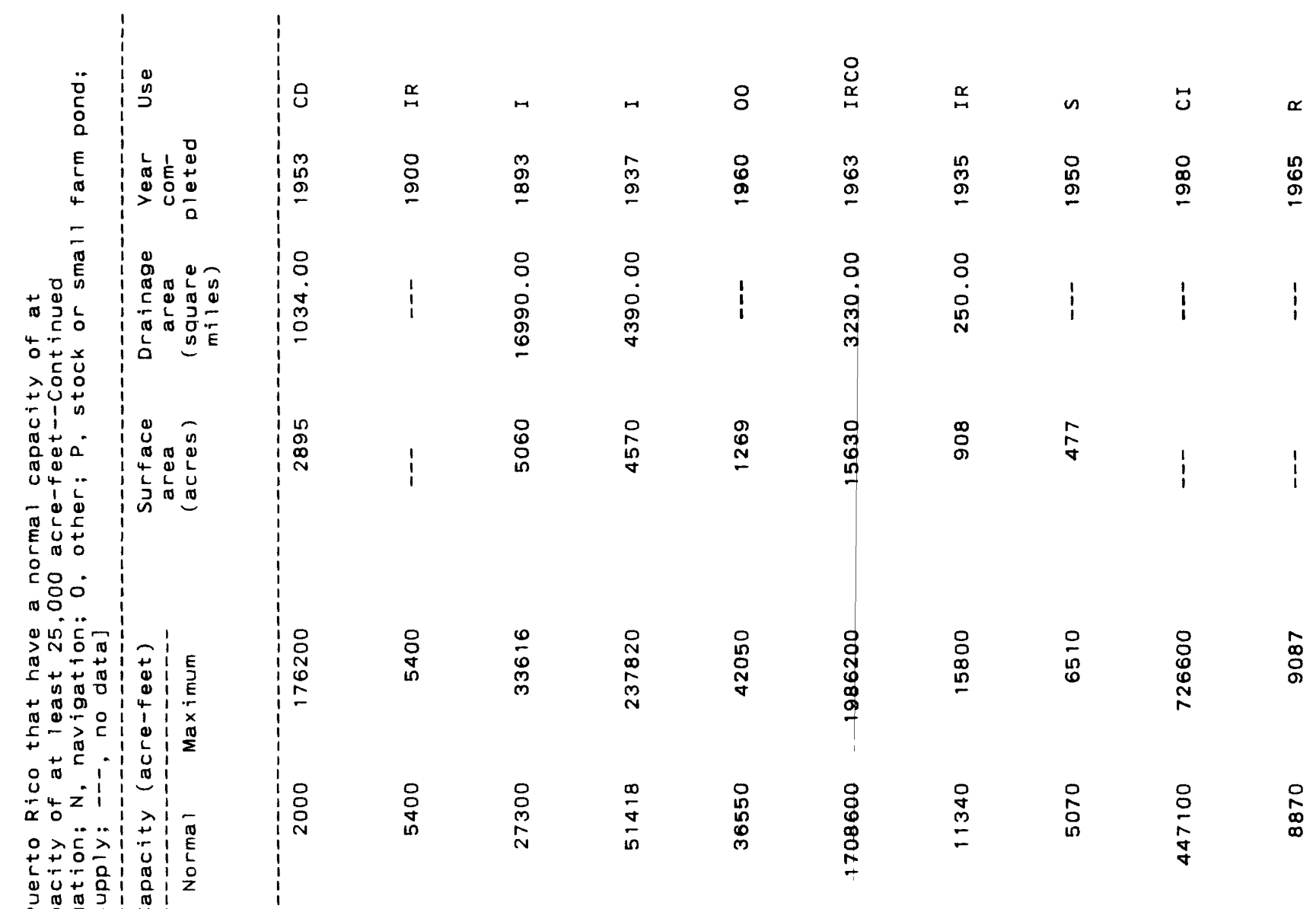

उ

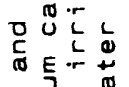

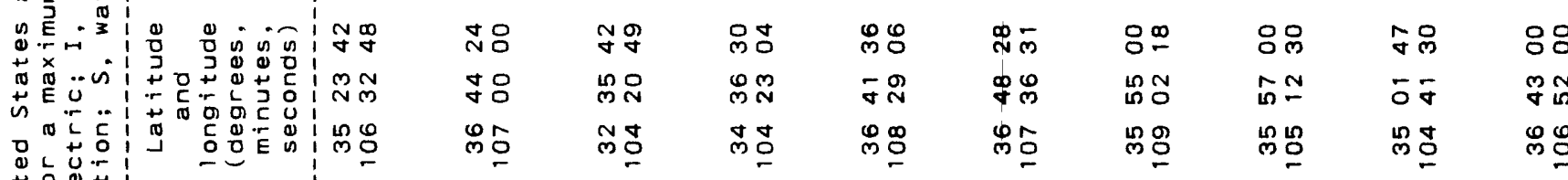

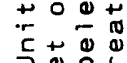

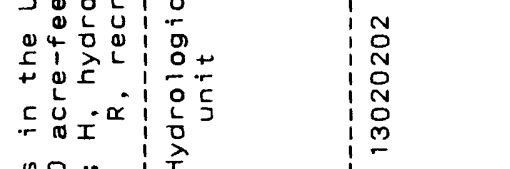

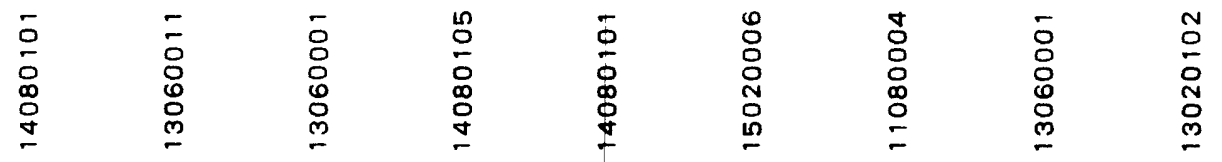

$$
\begin{gathered}
0 \\
2 \\
0 \\
2 \\
2 \\
0 \\
0 \\
0 \\
\alpha \\
1 \\
1 \\
m \\
0 \\
0 \\
0 \\
0 \\
1 \\
⺊
\end{gathered}
$$

임

>

峁茄。

年

i.

$\frac{10}{2}$

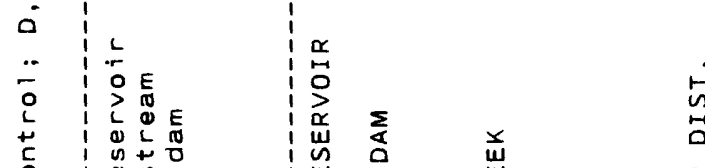

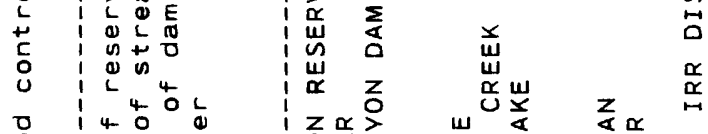

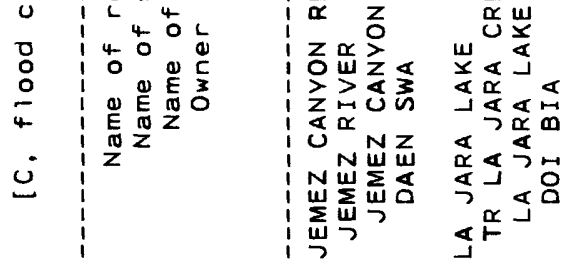

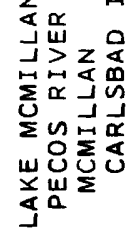

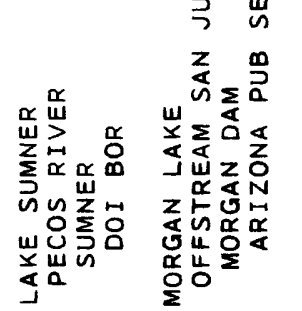

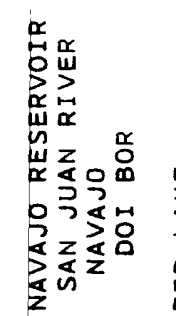

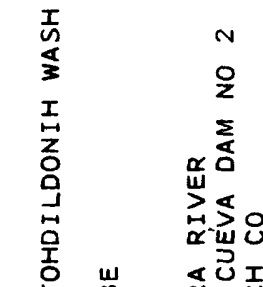




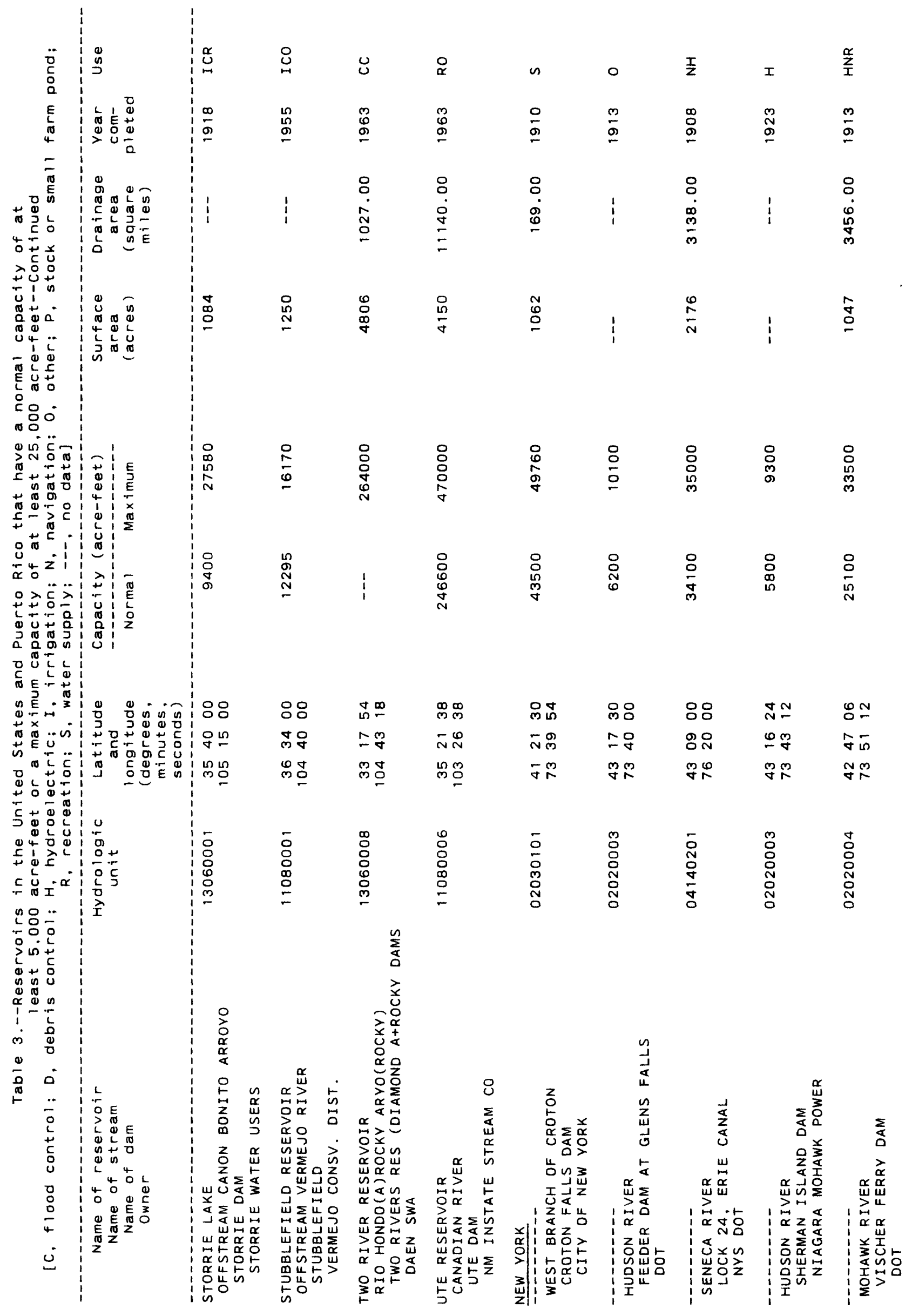




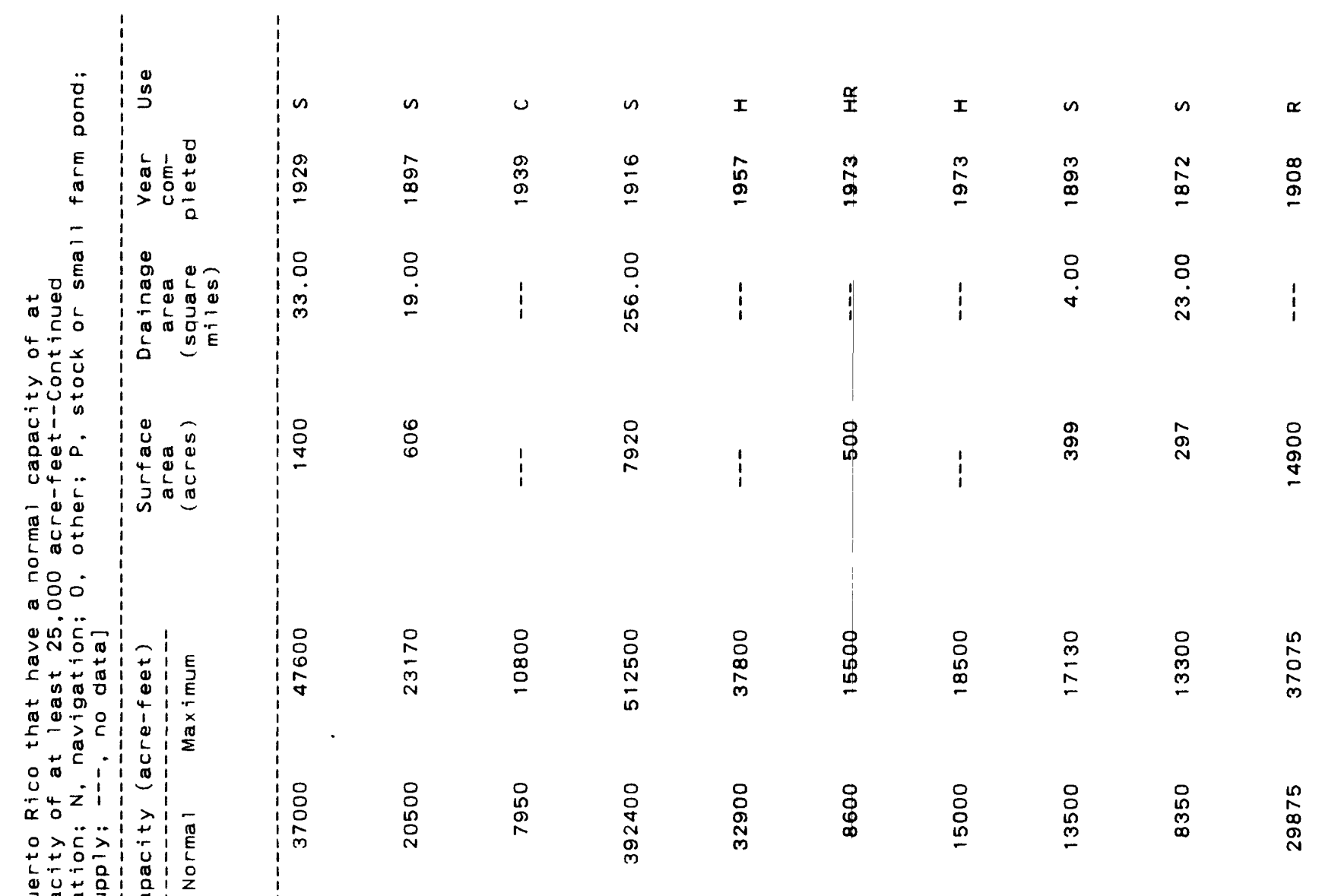

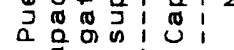

व

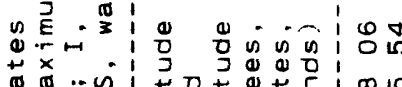

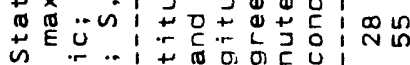

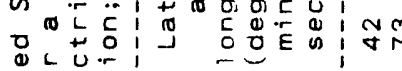

\begin{tabular}{|c|c|c|c|c|c|c|c|}
\hline$\stackrel{\infty}{-\infty}$ & 11 & $\stackrel{\infty}{-}$ & $\cong \stackrel{\infty}{q}$ & $\stackrel{\infty}{\mathbb{N}}$ & $\stackrel{\infty}{\sigma} \stackrel{\infty}{-}$ & $\stackrel{\infty}{\sim}$ & $\stackrel{\varphi}{0} \stackrel{0}{ }$ \\
\hline$=\stackrel{2}{q}$ & $i$ & $\stackrel{0}{\circ !}$ & 品 & $\hat{N}$ & $\stackrel{\mathscr{N}}{\sim} \stackrel{\mathscr{N}}{ }$ & N & $\hat{N}$ g \\
\hline $\bar{\sigma}$ & $i$ & $\dot{\nabla}$ & $\underset{R}{T}$ & GT & $\underset{T N}{N}$ & $=?$ & $-\frac{m}{2}$ \\
\hline
\end{tabular}

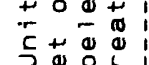

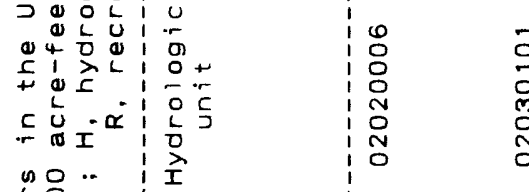

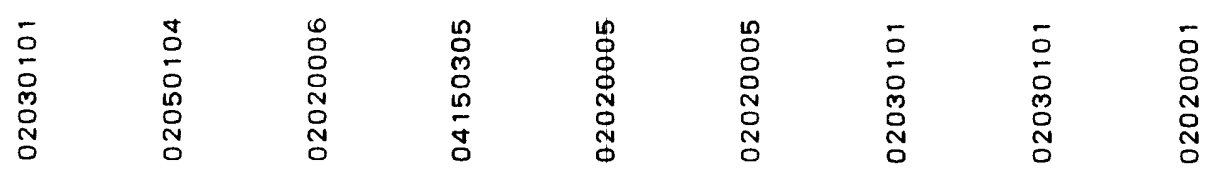

证

?

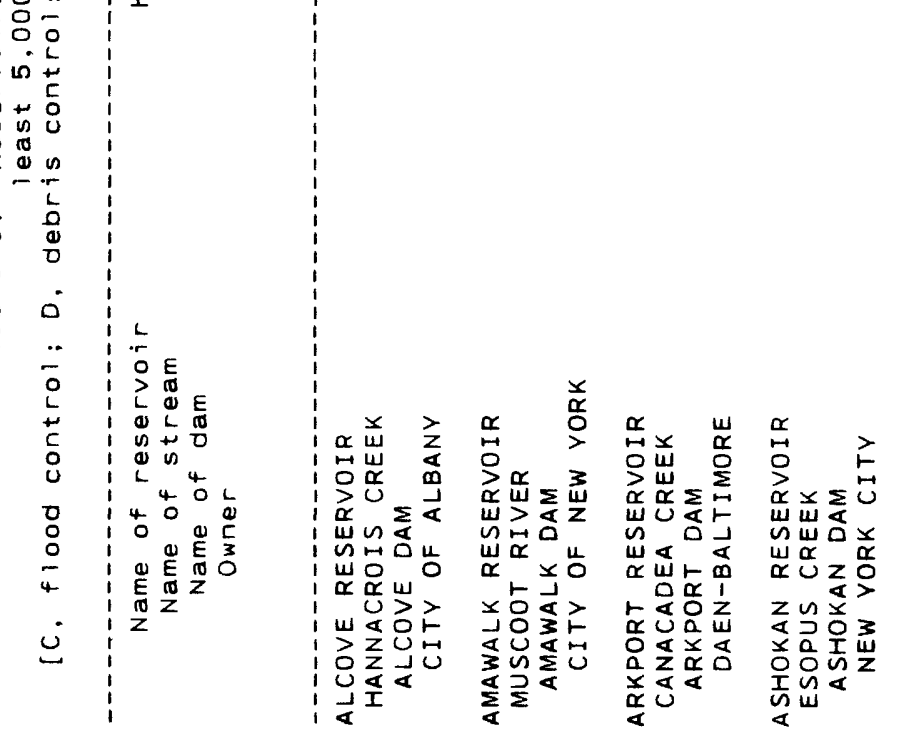




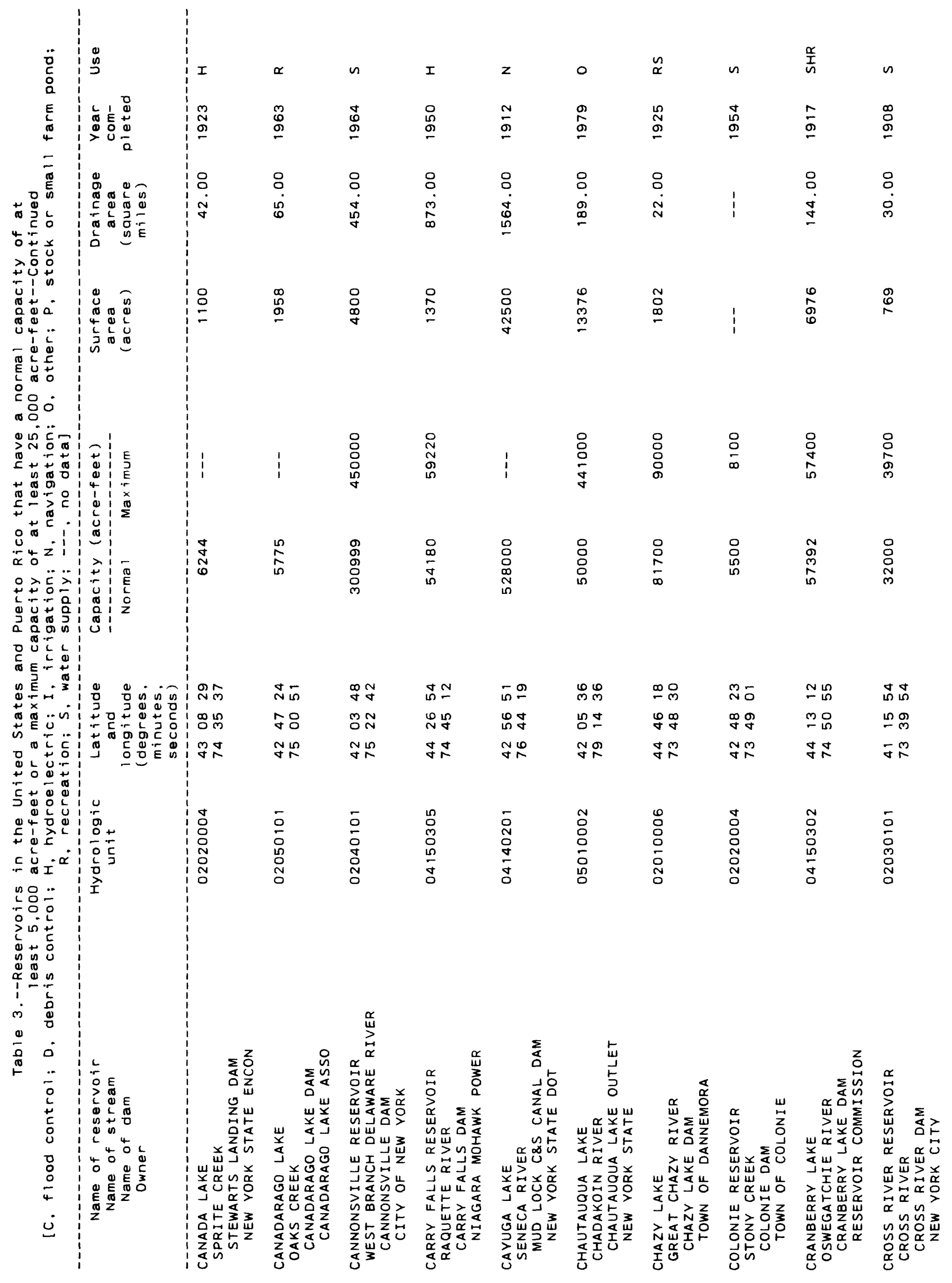




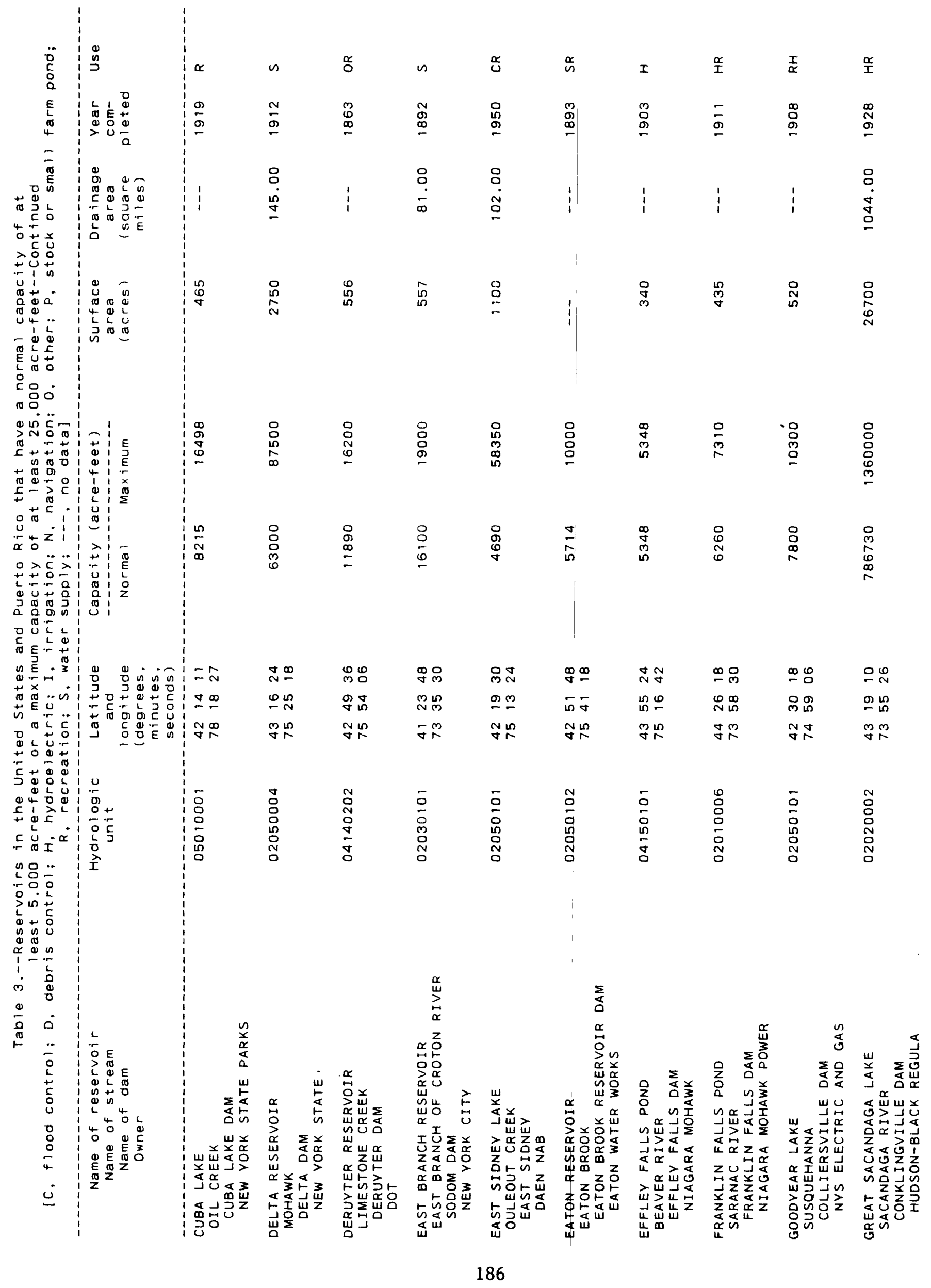




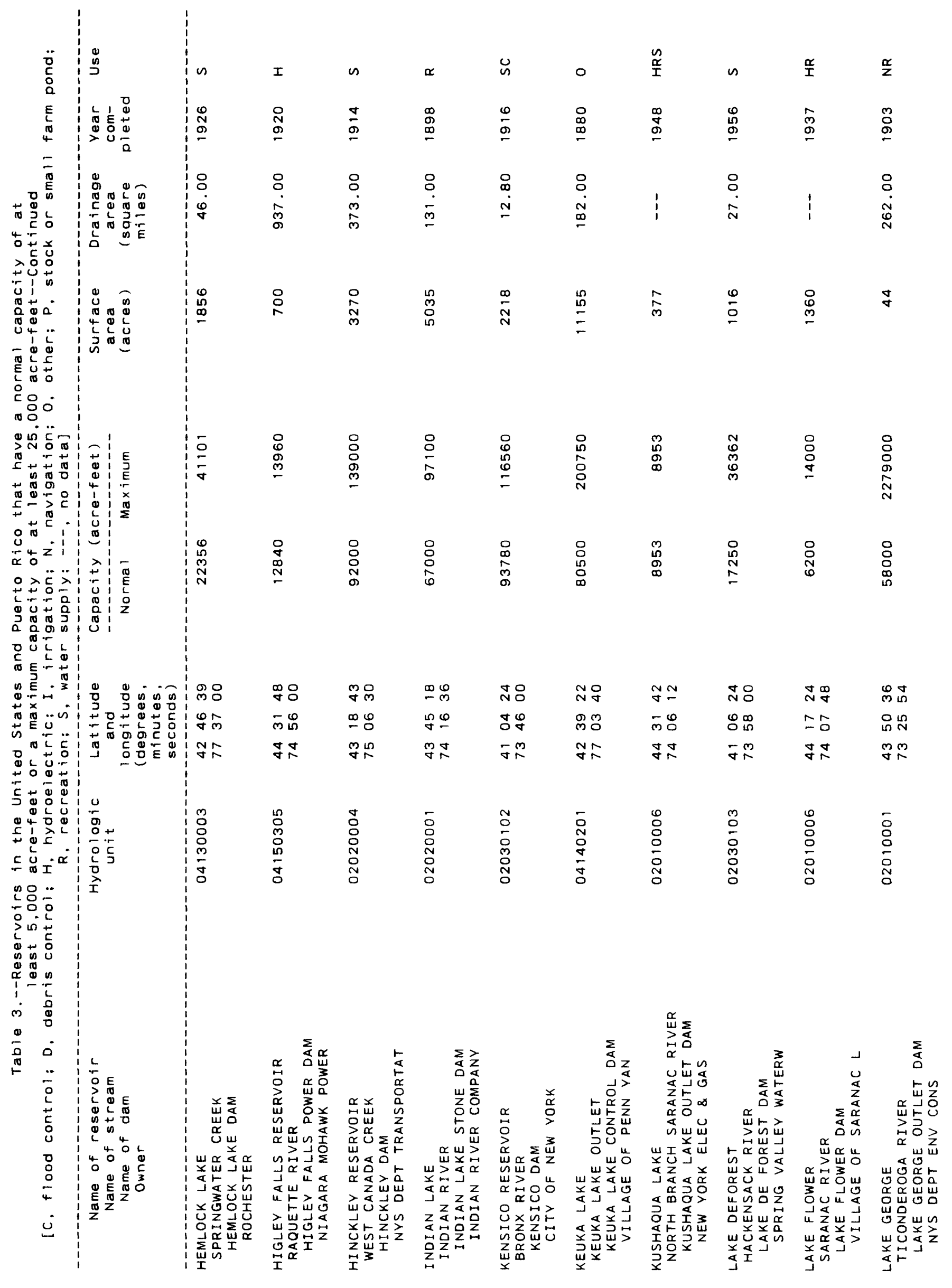




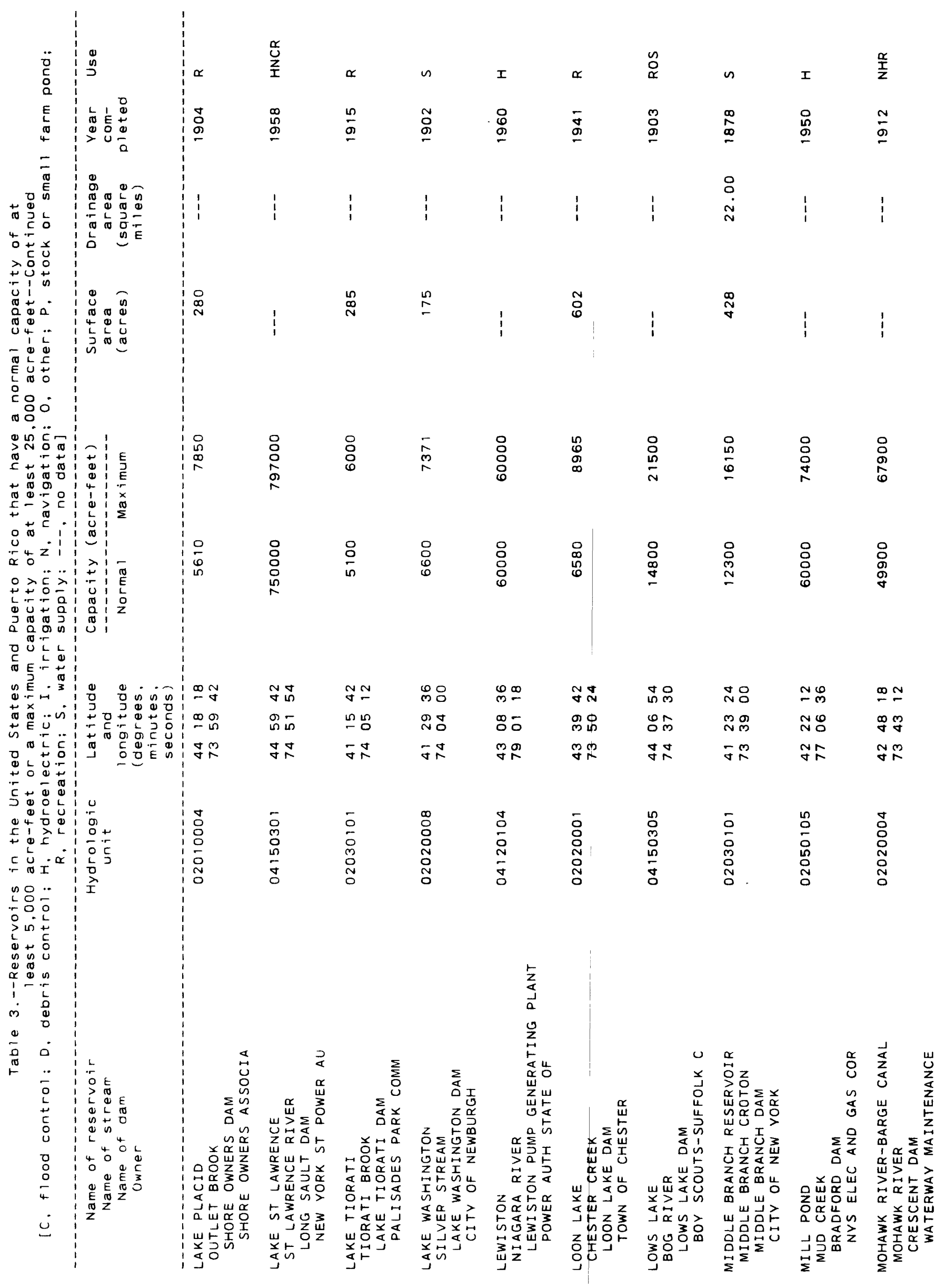




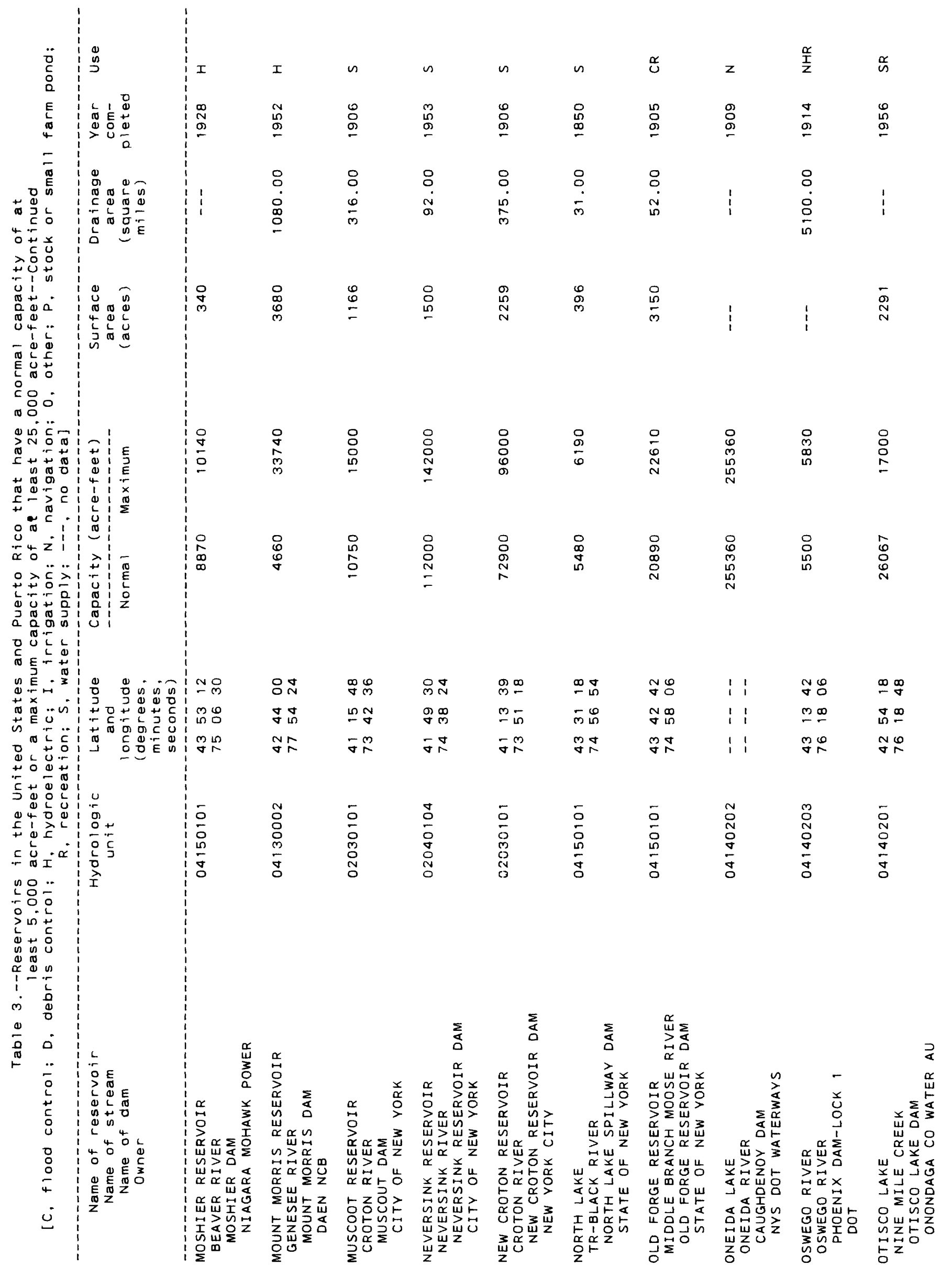




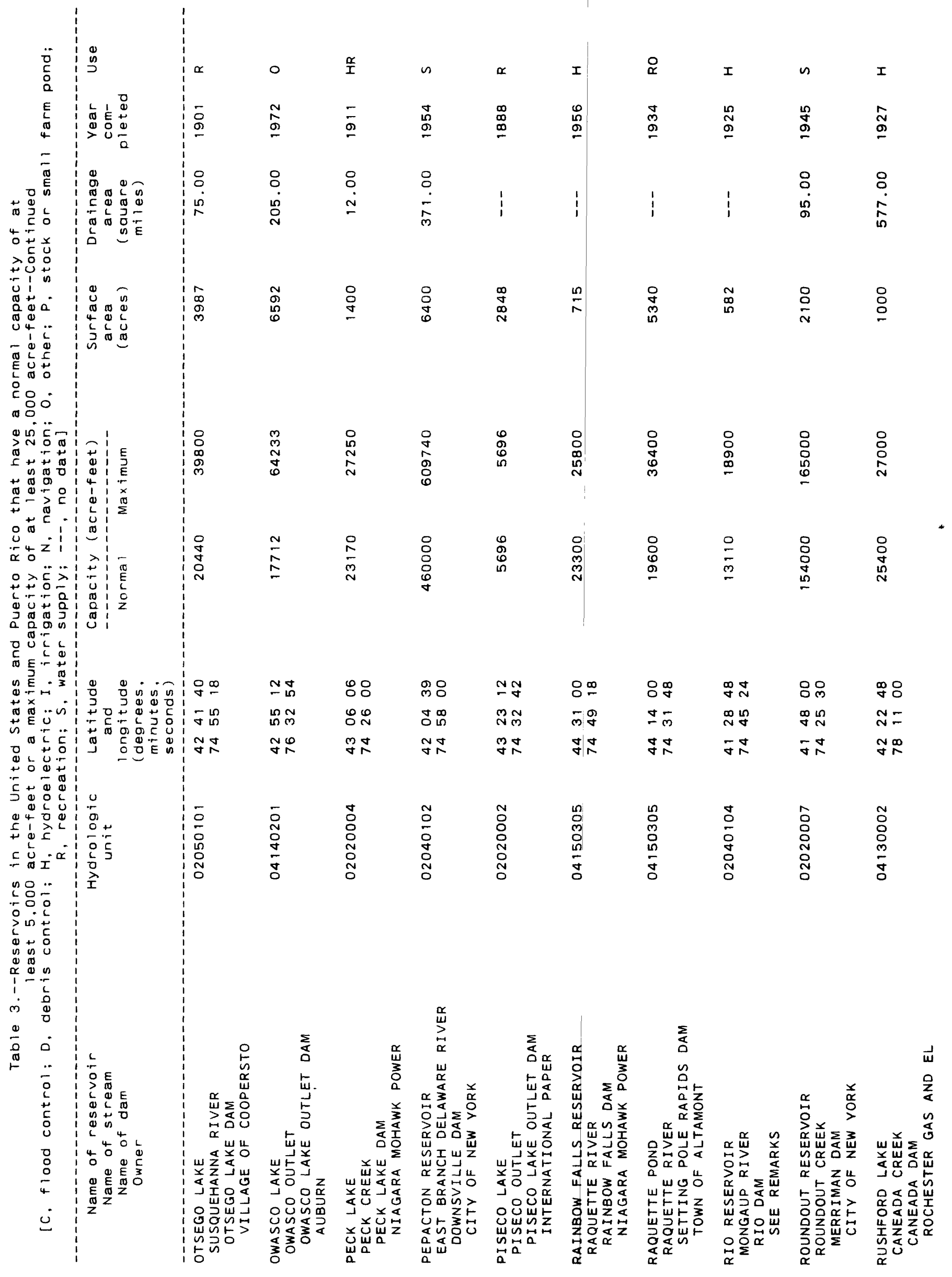




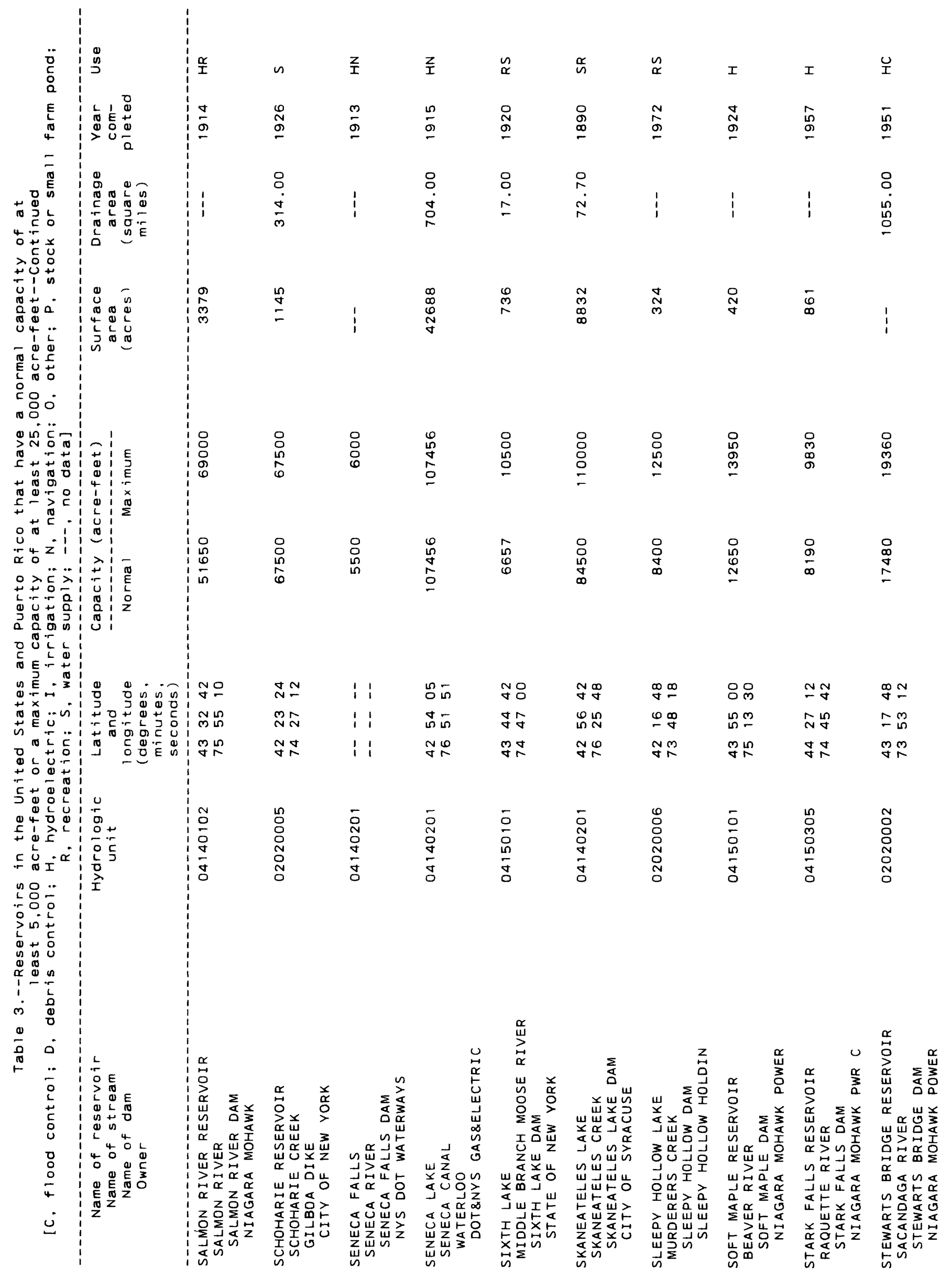




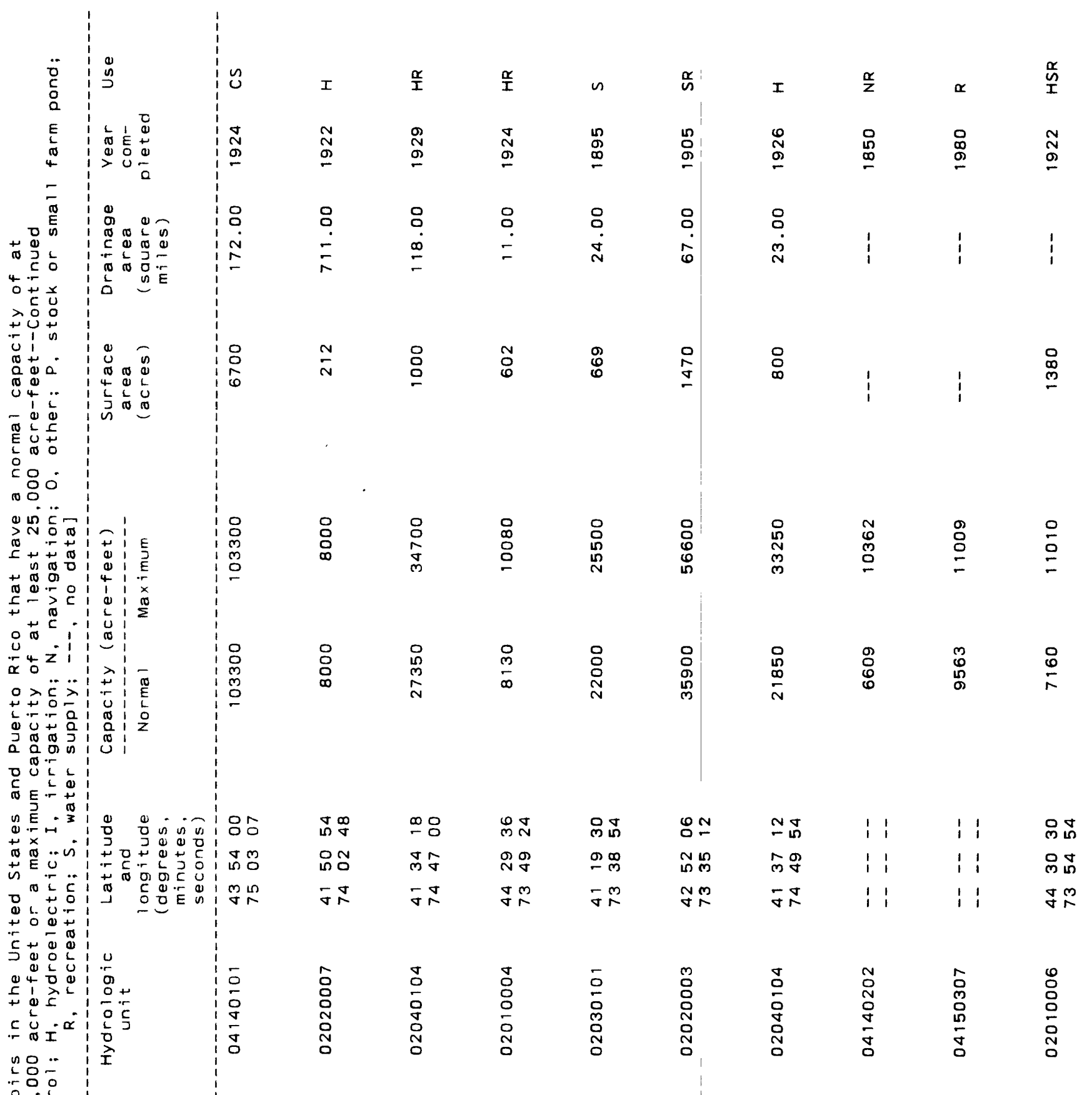




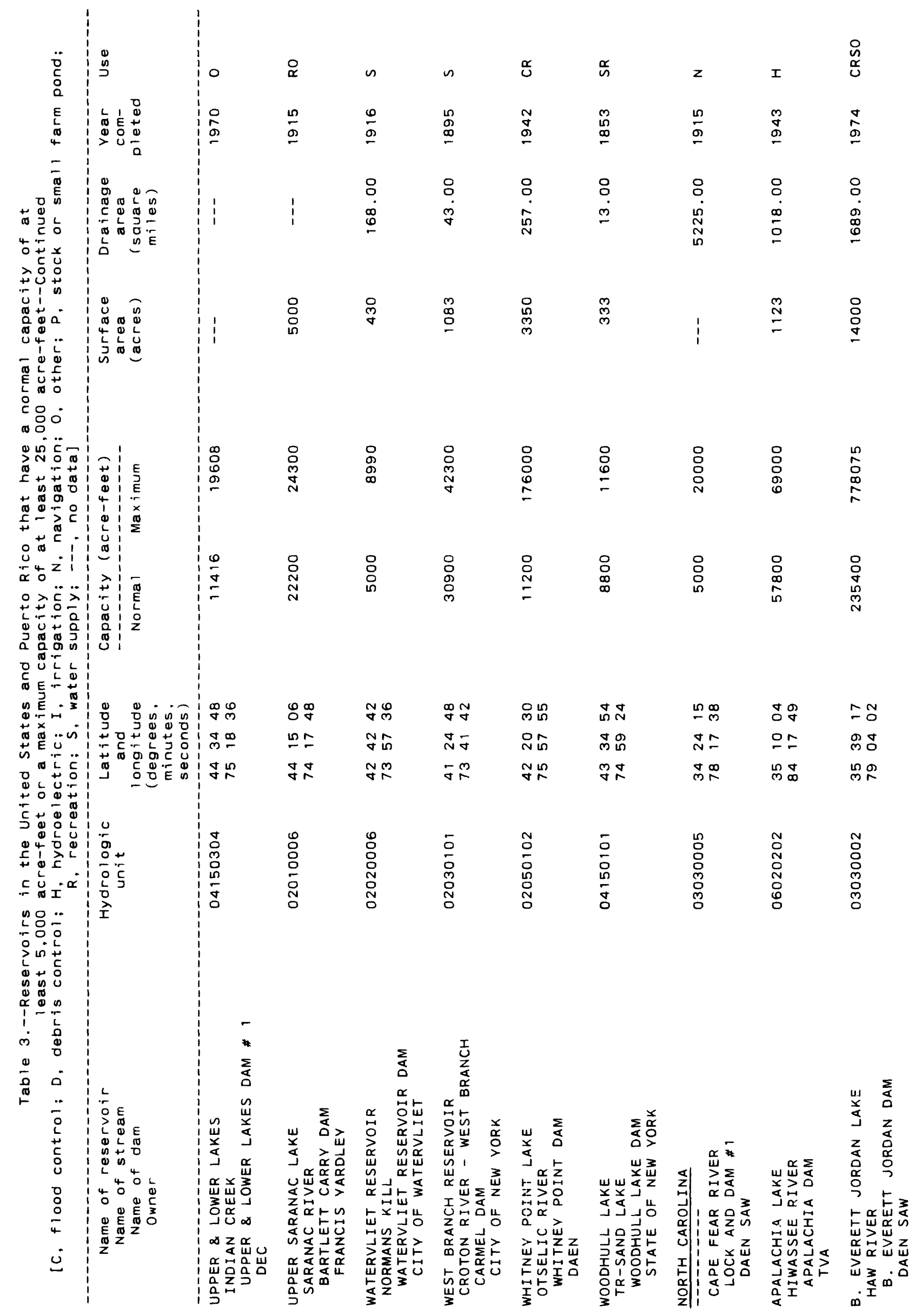




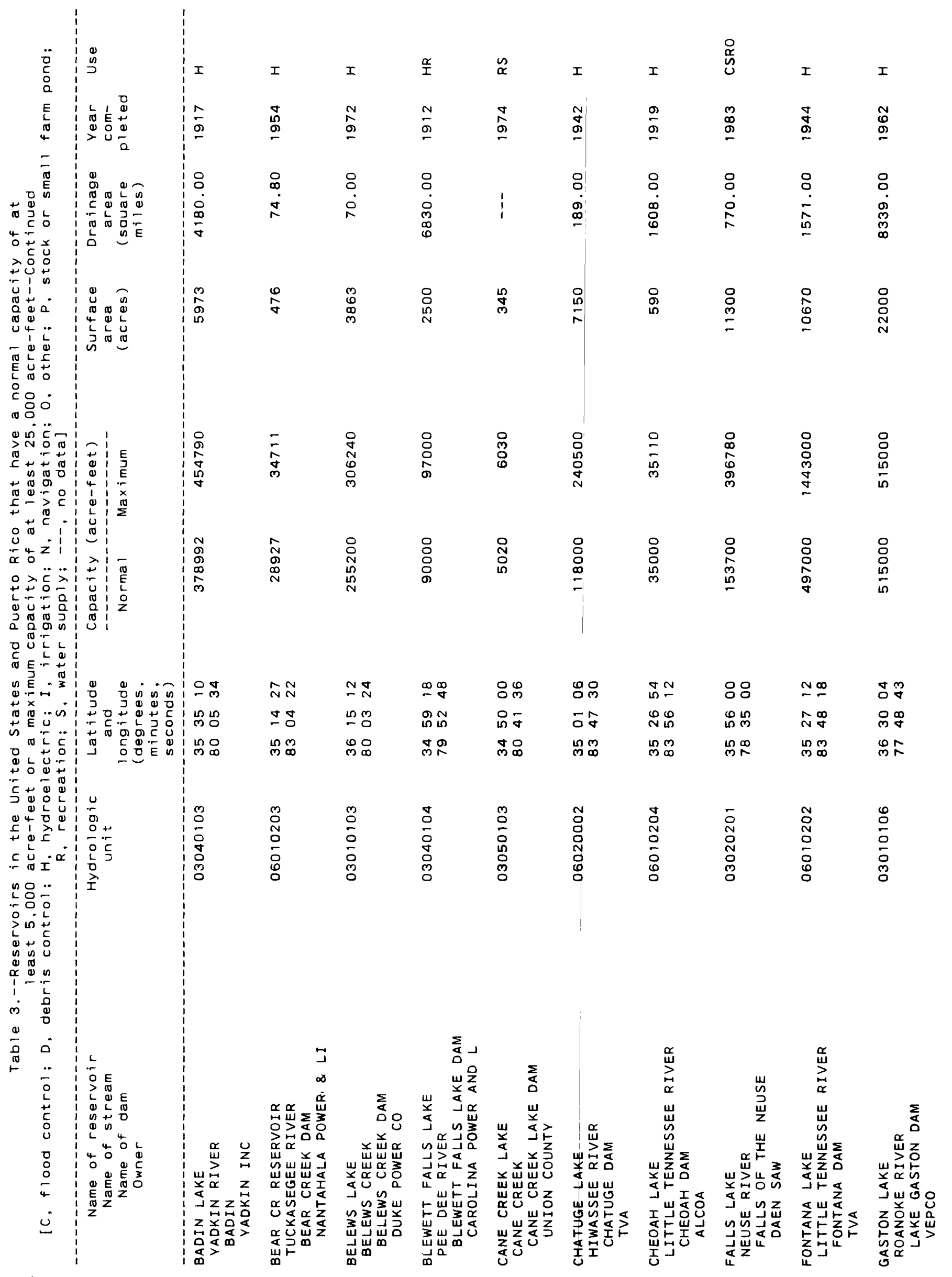




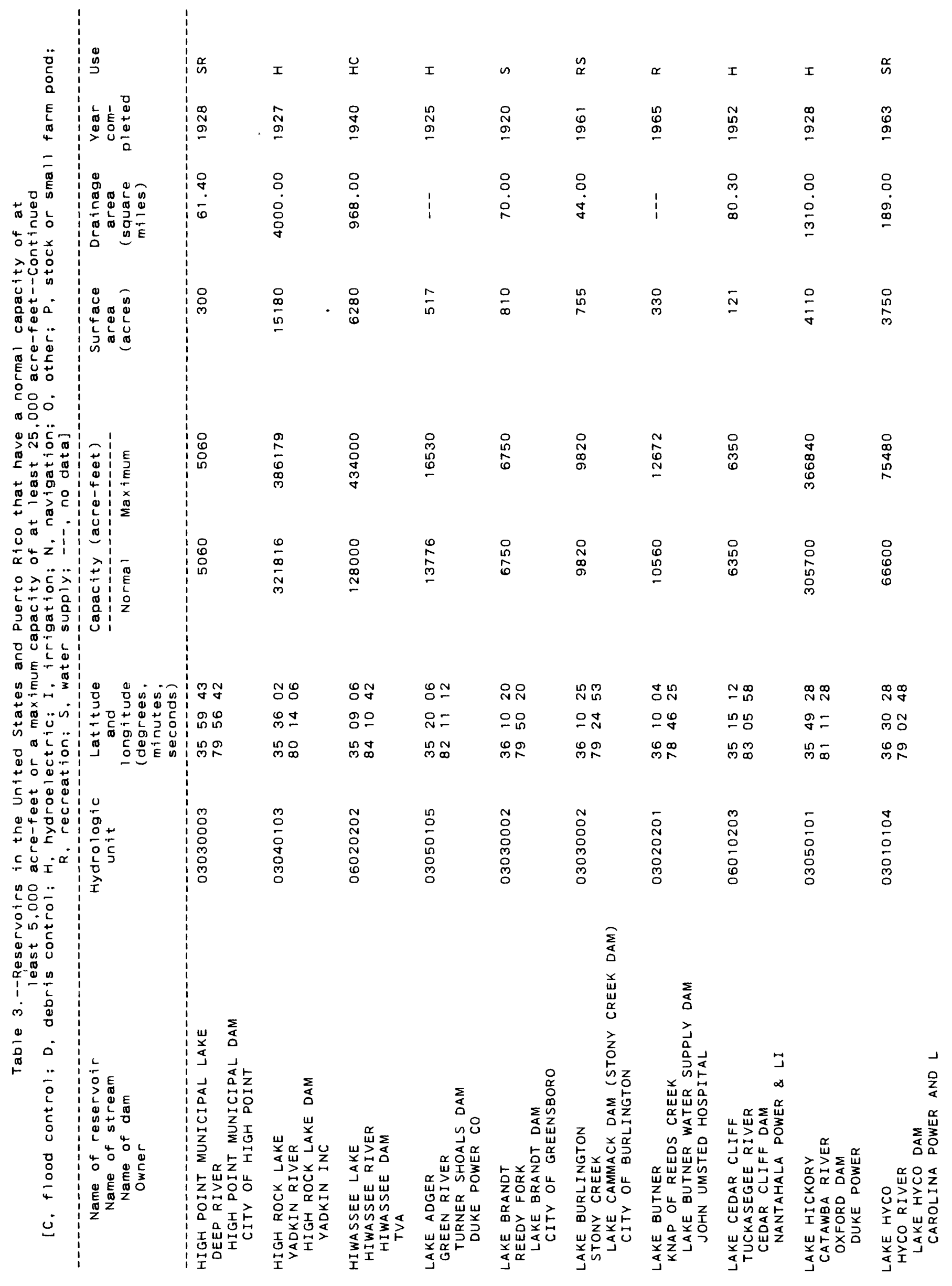




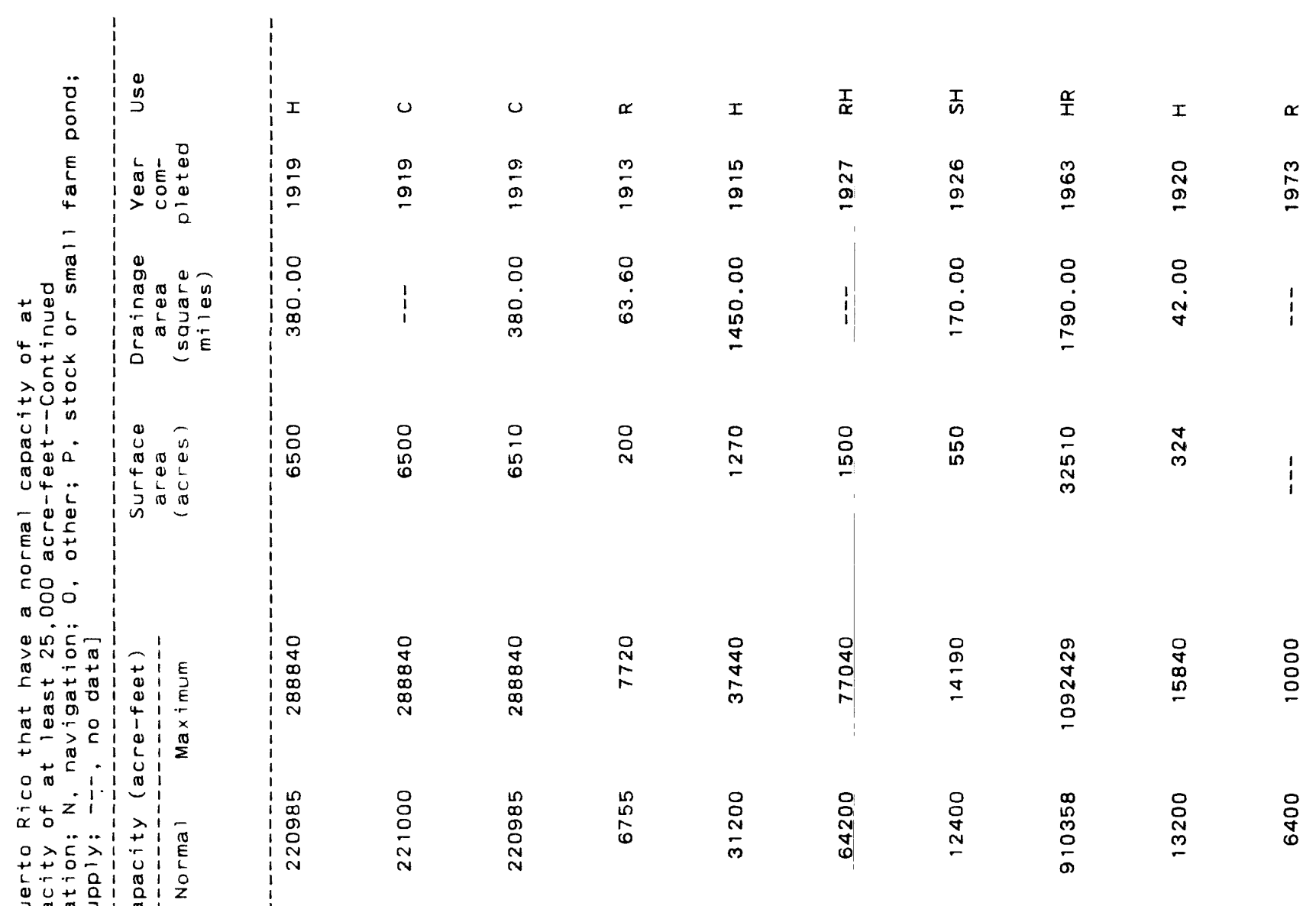

ग 0 \%

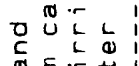

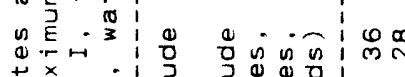

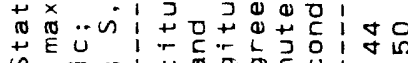

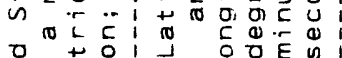

लिक

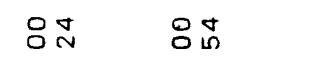

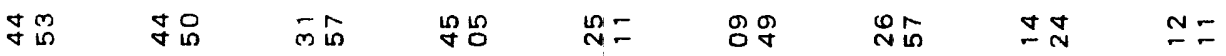

एँ

究

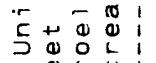

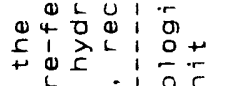

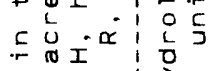

no..

(

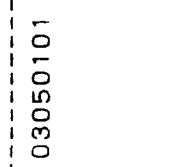

$\begin{array}{ll}5 & 5 \\ 0 & 0 \\ 0 & 0 \\ 10 & 0 \\ 0 & 0 \\ 9 & 0 \\ 0 & 0\end{array}$

$\begin{array}{ll}0 & 5 \\ 0 & 0 \\ 0 & 0 \\ 0 & 0 \\ 0 & 0 \\ 0 & 0 \\ 0 & 0\end{array}$

$\begin{array}{ll}10 & - \\ 0 & 0 \\ 0 & 0 \\ 1 & N \\ 0 & 0 \\ M & 0 \\ 0 & 0\end{array}$

5
0
0
10
0
0
0

$\begin{array}{ll}n & \text { I } \\ 0 & 8 \\ 0 & 0 \\ 0 & 0 \\ 0 & 0 \\ 0 & 0 \\ 0 & 0 \\ 0 & 0\end{array}$

- 0 O

a

路品

बֻ

1.

m
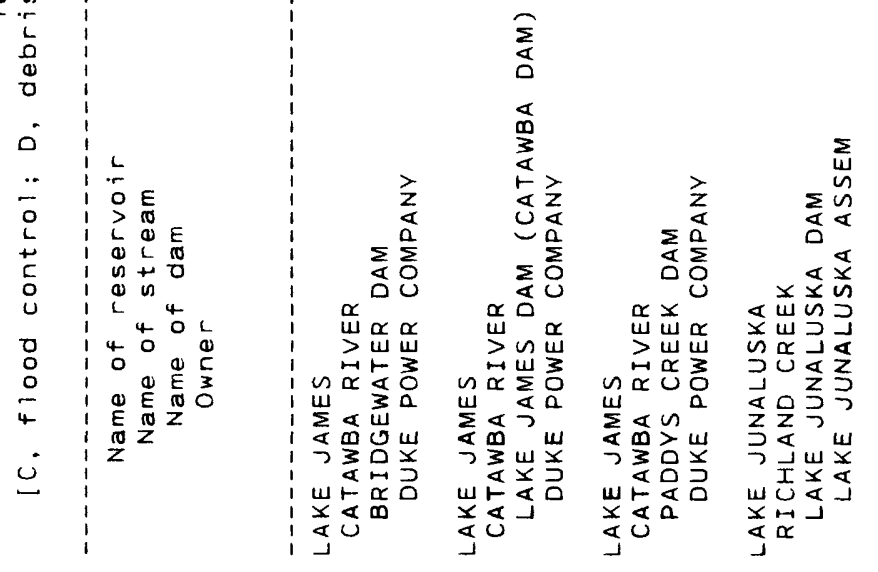

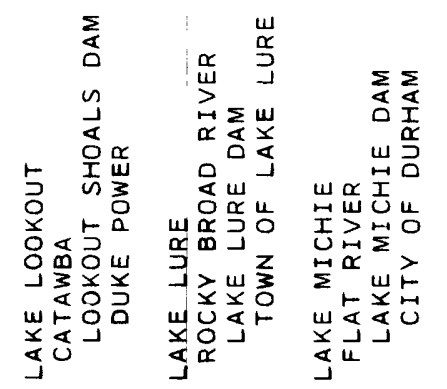
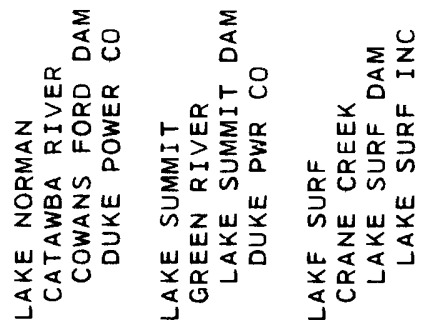


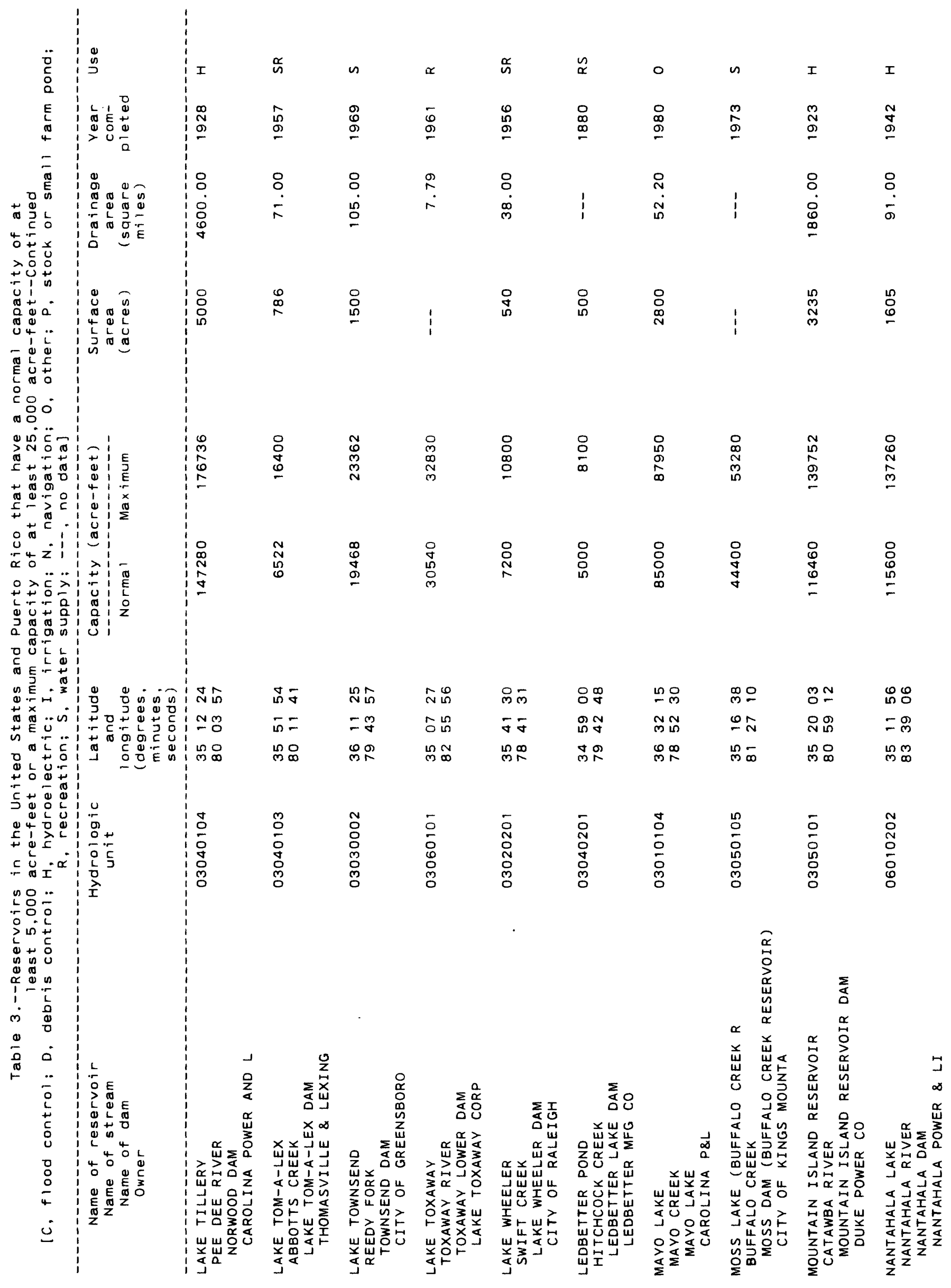




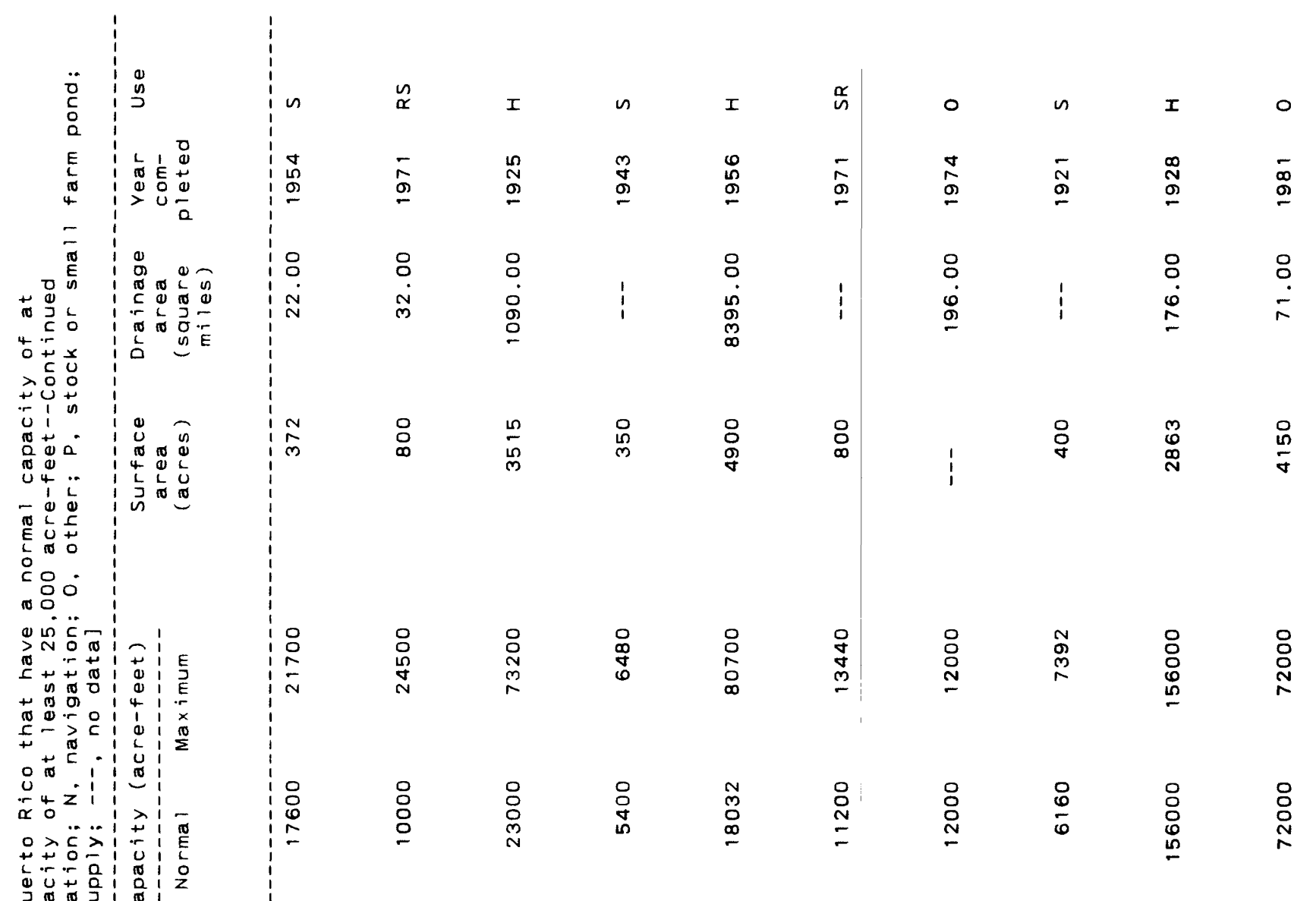

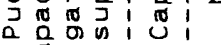

- 0 U $匚 5$

đo

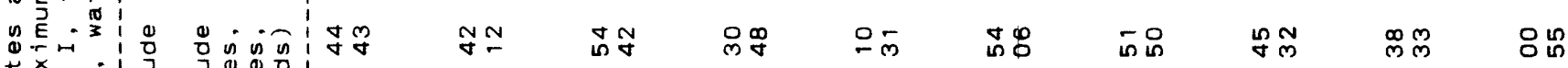

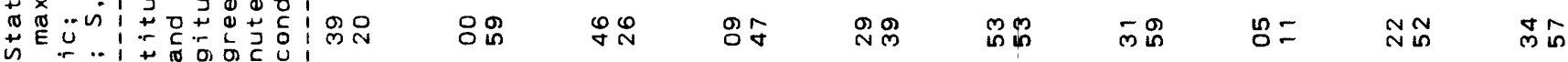

茫艺

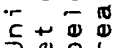

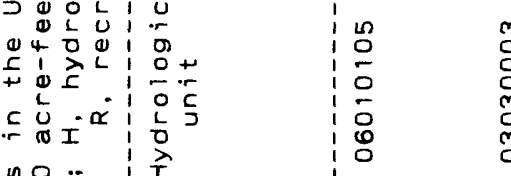

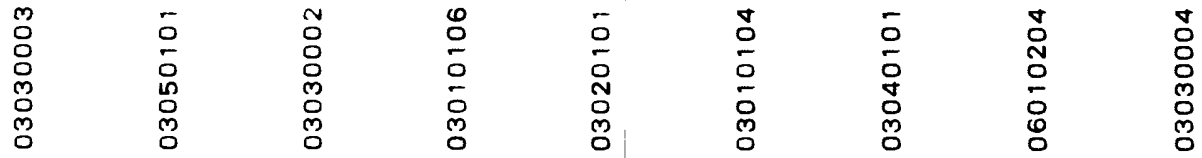

느묘

$>i n$

Do

in

$\underset{\alpha}{\infty}$

i.

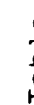

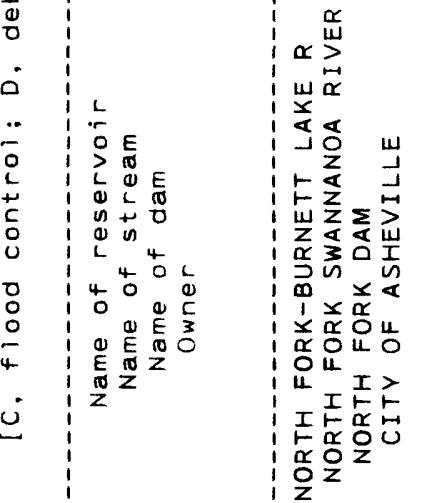

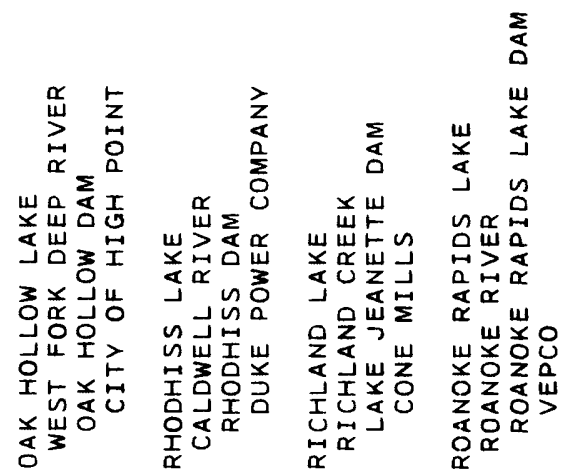

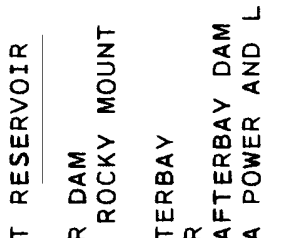

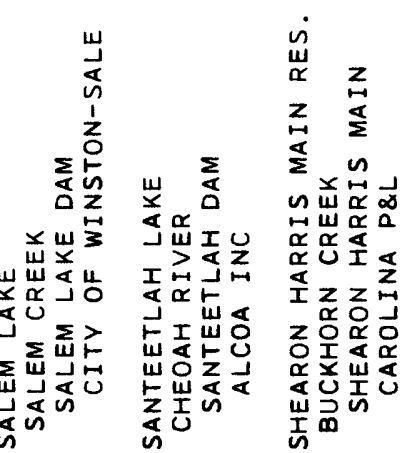




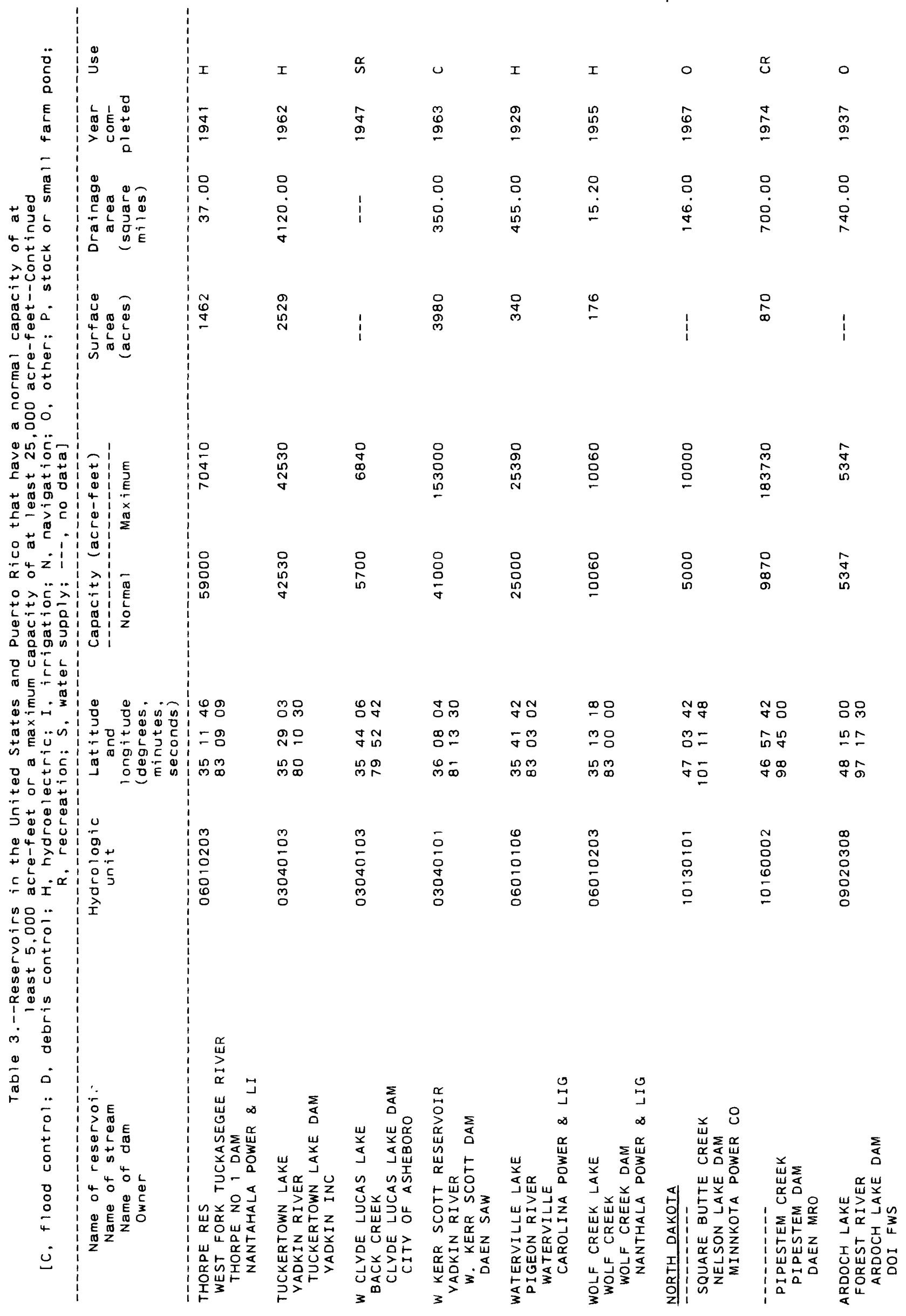




\begin{tabular}{|c|c|c|c|c|c|c|c|c|c|c|}
\hline $\begin{array}{l}\ddot{0} \\
\dot{c}\end{array}$ & 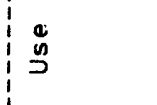 & 0 & 品 & $\stackrel{\alpha}{u}_{0}^{\alpha}$ & $\frac{\alpha}{\Delta}$ & $\alpha$ & 离 & $\circ$ & 0 & o \\
\hline$\frac{E}{\underline{E}}$ & 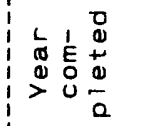 & $\begin{array}{l}\tilde{y} \\
0 \\
0\end{array}$ & 筸 & 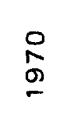 & $\begin{array}{l}\text { 品 } \\
\text { 资 }\end{array}$ & مొ & 吕 & 离 & $\begin{array}{l}\text { J } \\
\text { - }\end{array}$ & 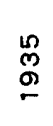 \\
\hline & 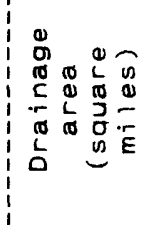 & $\begin{array}{l}\stackrel{\circ}{\circ} \\
\underset{\sim}{2}\end{array}$ & 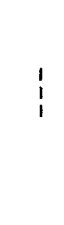 & $\begin{array}{l}\circ \\
\dot{q} \\
\dot{q}\end{array}$ & $\begin{array}{l}\stackrel{0}{0} \\
\dot{\square}\end{array}$ & $\vdots$ & 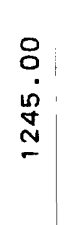 & 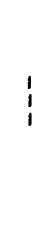 & $\begin{array}{l}: \\
\dot{0} \\
\dot{0} \\
\end{array}$ & $\begin{array}{l}: \\
\vdots \\
\vdots \\
0\end{array}$ \\
\hline 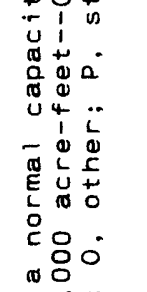 & 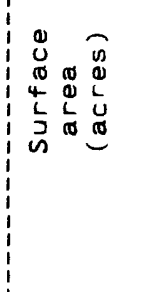 & 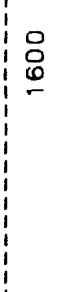 & $\vdots$ & 虽 & $\stackrel{\hat{o}}{\infty}$ & $\vdots$ & 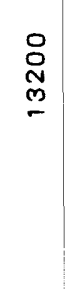 & 离 & đ̊ & 吕 \\
\hline 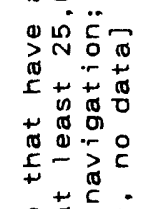 & 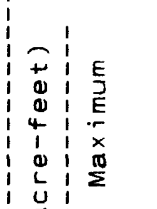 & 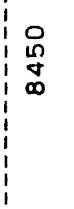 & $\begin{array}{l}\circ \\
\stackrel{0}{0} \\
\stackrel{0}{0}\end{array}$ & $\begin{array}{l}0 \\
0 \\
0 \\
0 \\
0\end{array}$ & $\underset{\substack{\text { D. } \\
\text { J }}}{ }$ & 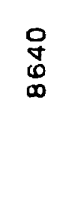 & 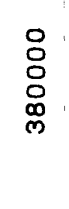 & 品 & ָี & \begin{tabular}{l}
$\stackrel{8}{0}$ \\
\multirow{2}{N}{}
\end{tabular} \\
\hline 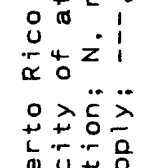 & 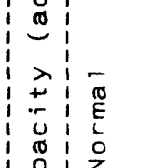 & 号 & 品 & $\begin{array}{l}\text { 总 } \\
\text { 足 }\end{array}$ & 苂 & 吕 & 总 & 然 & $\underset{\sim}{\mathbb{N}}$ & 5 \\
\hline
\end{tabular}

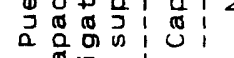

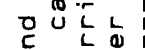

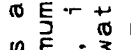

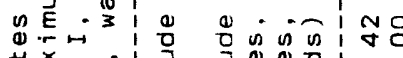

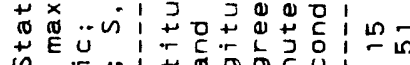

क

.

+

\&

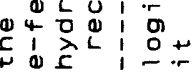

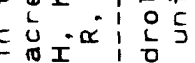

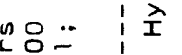

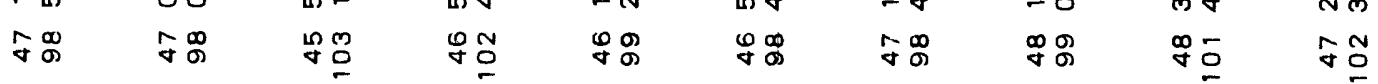

\begin{tabular}{|c|c|c|c|c|c|c|c|}
\hline$\cong \stackrel{\infty}{q}$ & ஸ் $\stackrel{\infty}{+}$ & $\simeq \mathscr{e}$ & $8_{0}^{\infty}$ & 옹류 & ৪৪ & $8:$ & ৪৪ \\
\hline NO & $\stackrel{\infty}{\infty}=$ & กิ & $\stackrel{\sim \infty}{\sim}$ & น็ N & 워 & $\Phi$ & 용ㅁ \\
\hline रे & $\stackrel{20}{8}$ & ON & சீ & $\stackrel{0}{\circ}$ & ऽั & \&̊ & ${ }_{\sigma}^{\infty}$ \\
\hline
\end{tabular}

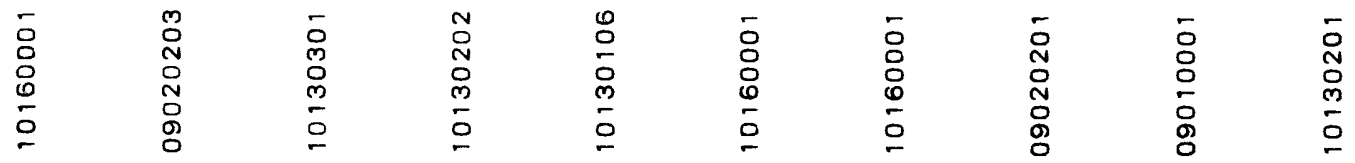

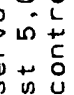

is 0

$x$

$\dot{0}$

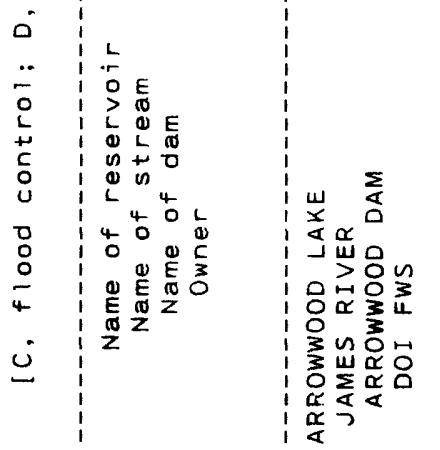

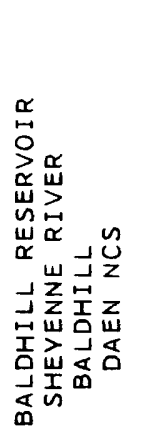
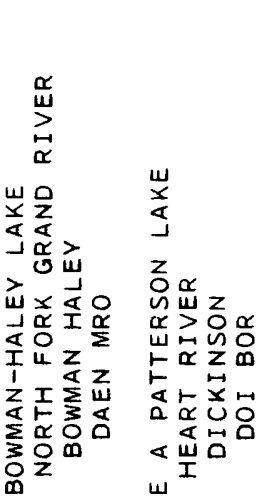

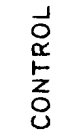

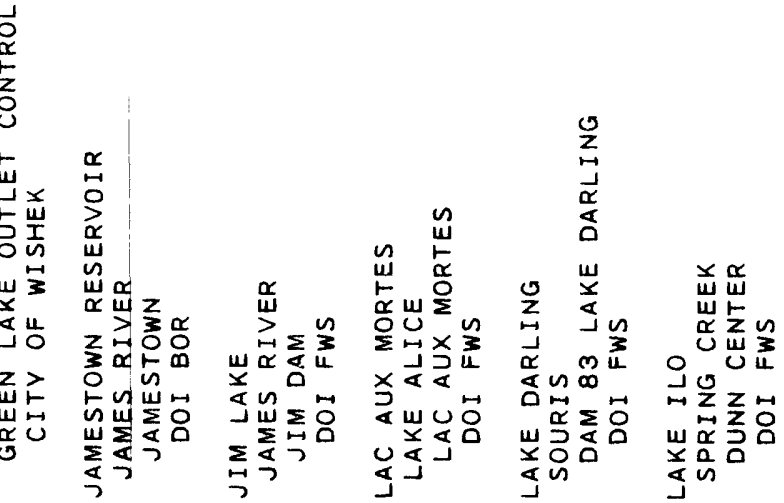




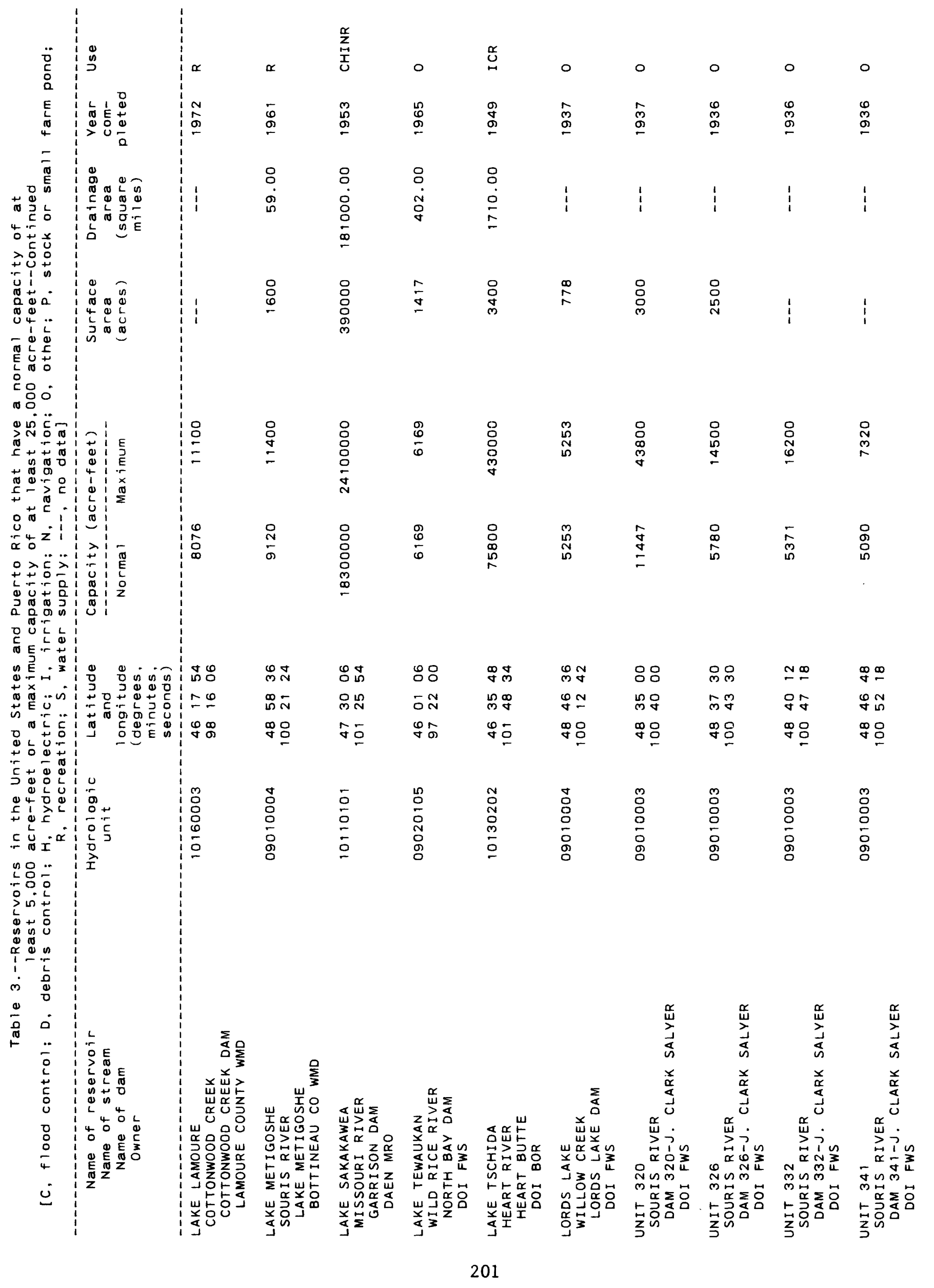




\begin{tabular}{|c|c|c|c|c|c|c|}
\hline$\alpha$ & 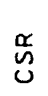 & $\alpha$ & 㸡 & 总 & $\begin{array}{l}\stackrel{0}{0} \\
\text { 品 }\end{array}$ & u \\
\hline 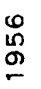 & 离 & ī & $\begin{array}{l}\hat{m} \\
0 \\
0\end{array}$ & $\begin{array}{l}\hat{0} \\
0 \\
0\end{array}$ & 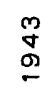 & $\begin{array}{l}\stackrel{\infty}{\infty} \\
\stackrel{2}{\Omega} \\
\stackrel{2}{2}\end{array}$ \\
\hline
\end{tabular}

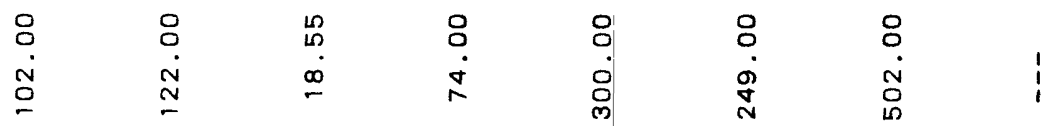

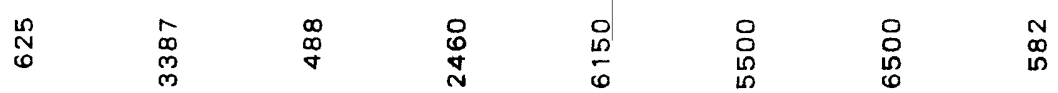

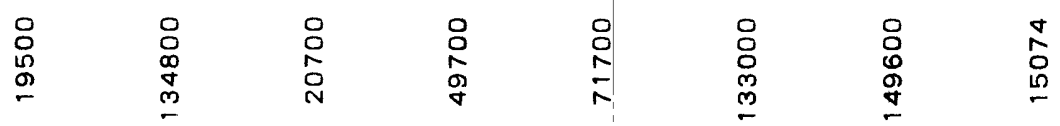

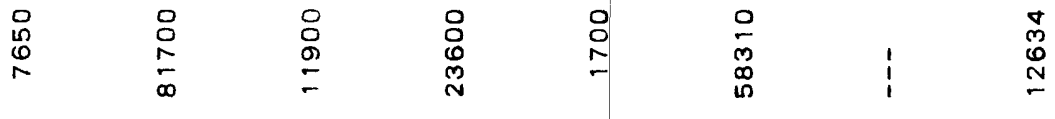

\begin{tabular}{|c|c|c|c|c|c|c|}
\hline 요 & MN & $\stackrel{\infty}{\circ}$ & ஜ요 & 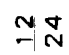 & $\underset{q}{\infty} \underset{-}{2}$ & $\stackrel{p}{m}_{\sigma}^{\infty}$ \\
\hline M्ल & 은 & $\stackrel{N O}{N}$ & mI & लू लె & № & $\stackrel{m}{\infty}$ \\
\hline ஓু & ơ & $O_{\infty}^{N}$ & $q_{\infty}$ & $g_{\infty}$ & $\bar{\sigma} \bar{\infty}$ & $q_{\infty}^{-\infty}$ \\
\hline
\end{tabular}

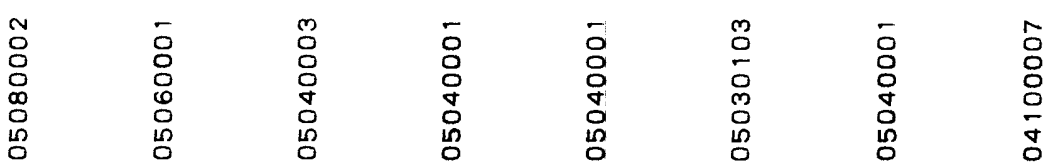

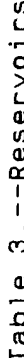

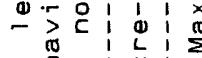

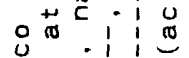

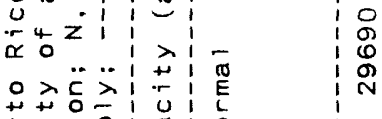

कu+

on

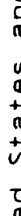

每

कृ

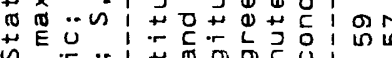

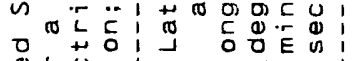

$\stackrel{\infty}{\rightarrow} 0$

舟宁
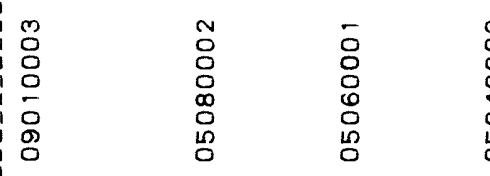

$\begin{array}{ll}0 \\ 8 \\ 8 & 8 \\ 0 & 0 \\ 0 & 0 \\ 0 & 0 \\ 0 & 0 \\ 0 & 0\end{array}$

음
व
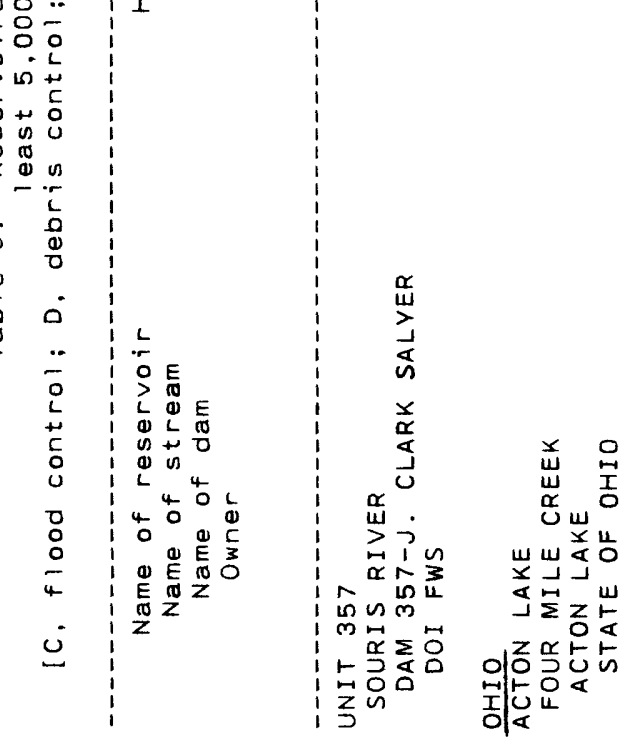

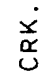

占

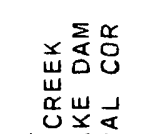

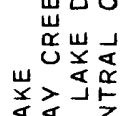

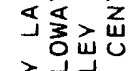

o

岃出势焉

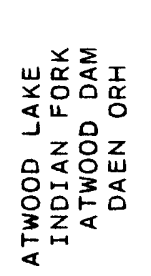

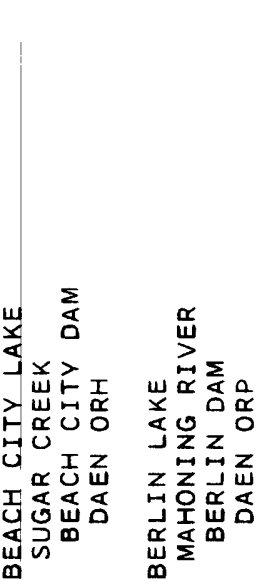

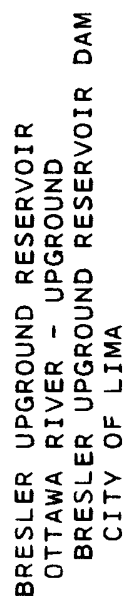




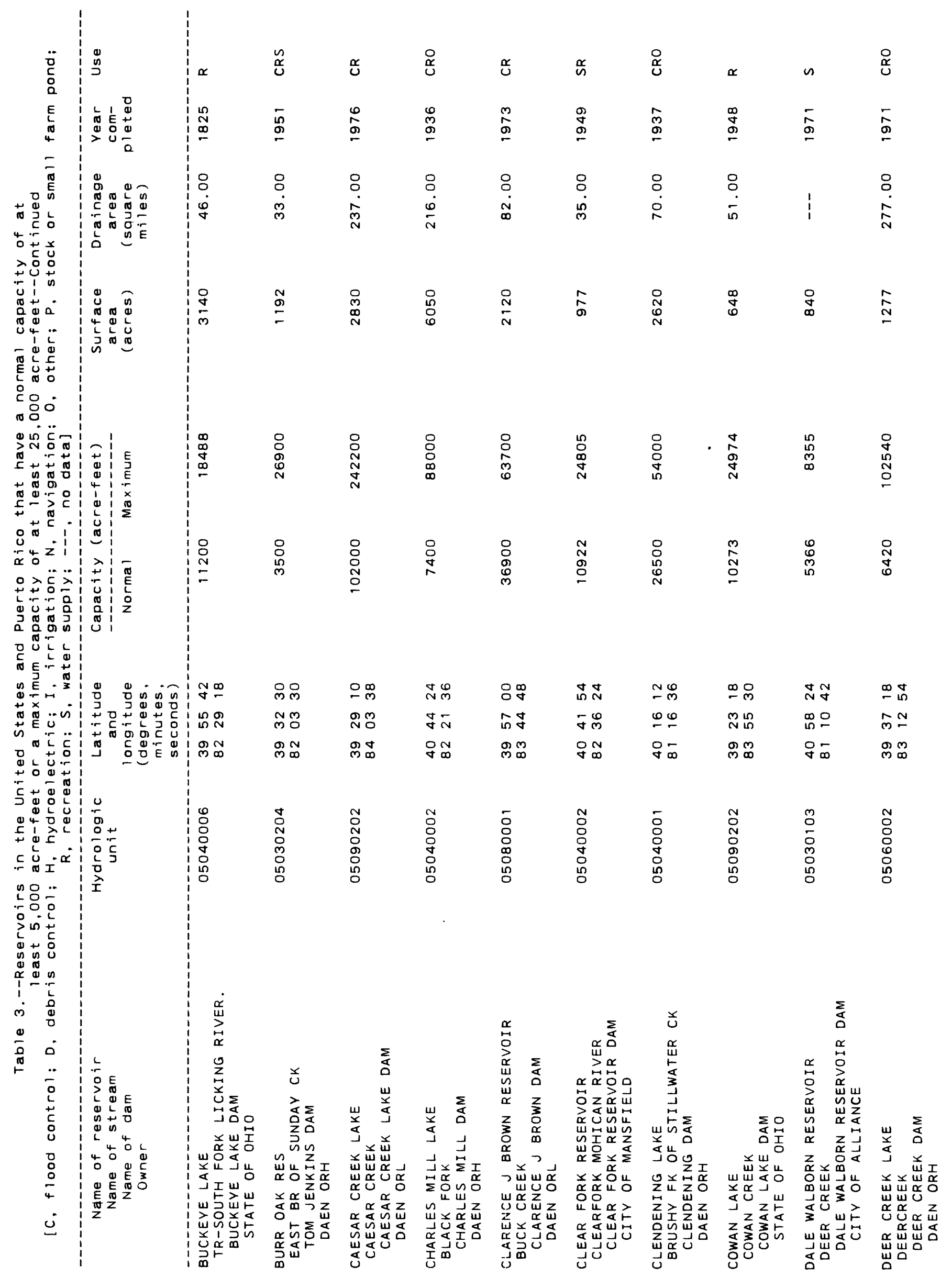




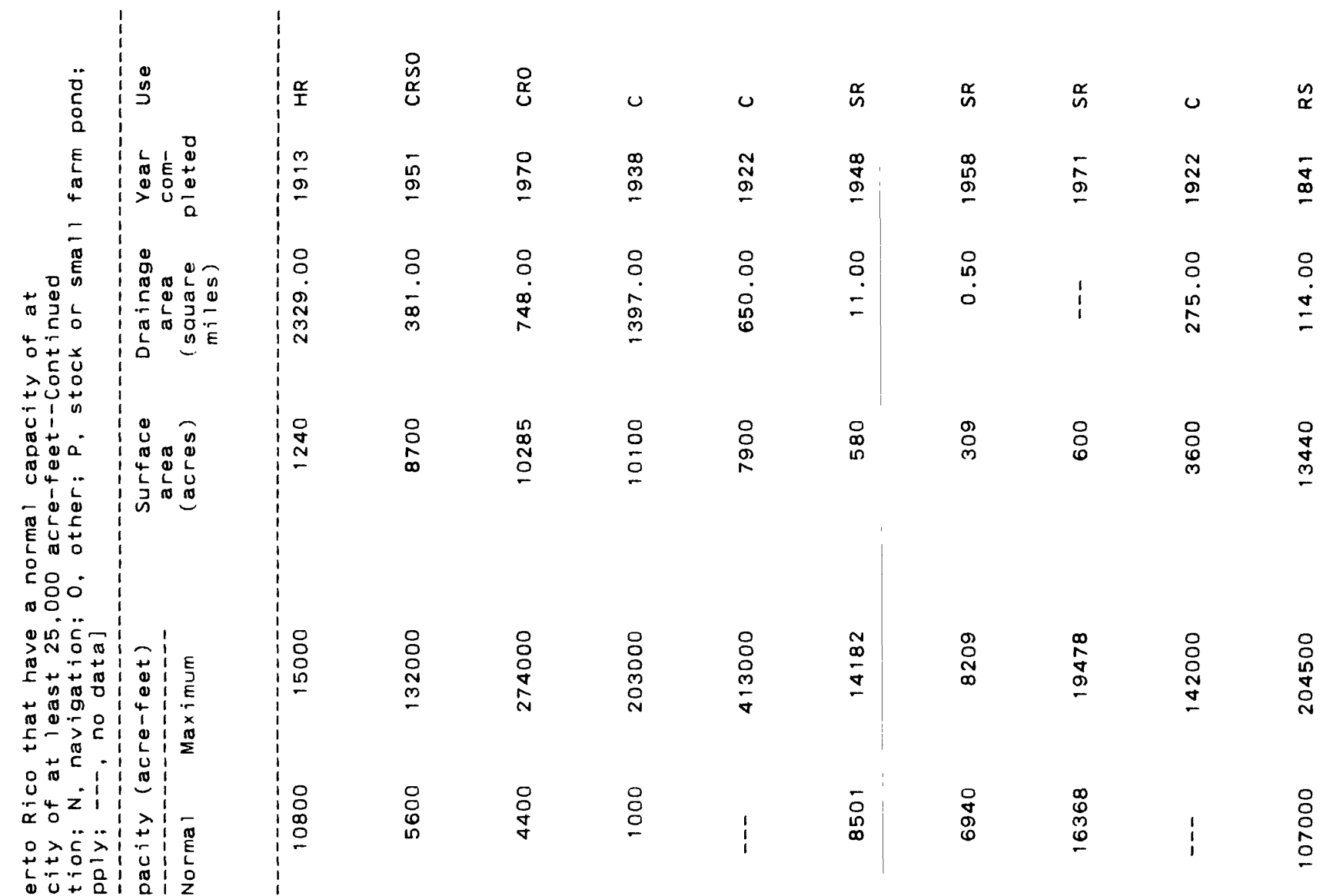

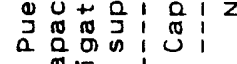

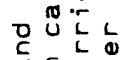

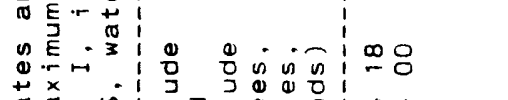

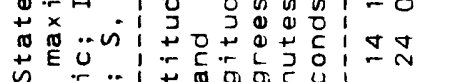

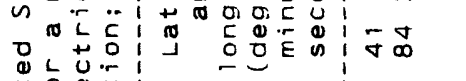

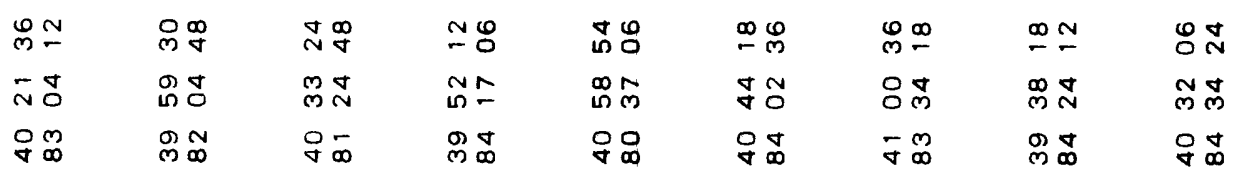

\section{-}

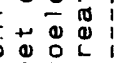

4 o

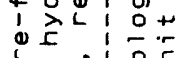

\begin{tabular}{c|c} 
\\
$c$
\end{tabular}

늠을

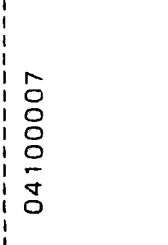

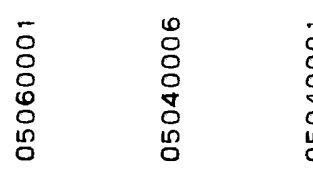

高高亳

高高总高

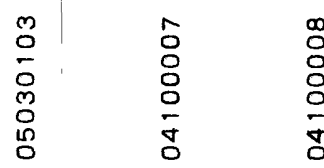

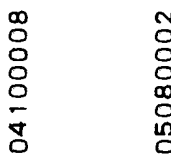

$\begin{array}{ll}2 & 0 \\ 0 & 0 \\ 0 & 0 \\ 0 & 0 \\ 0 & 0 \\ 0 & 0\end{array}$

in

$+8$

政

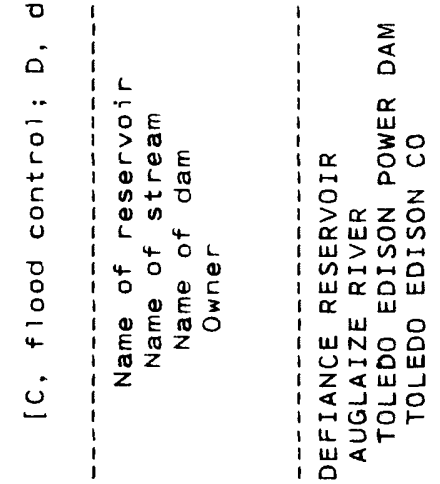

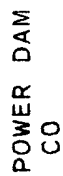

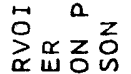

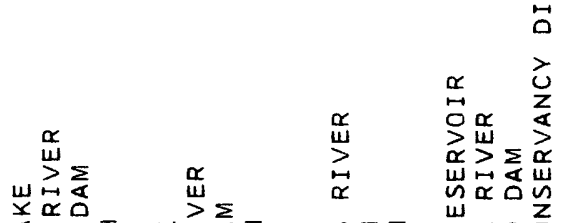
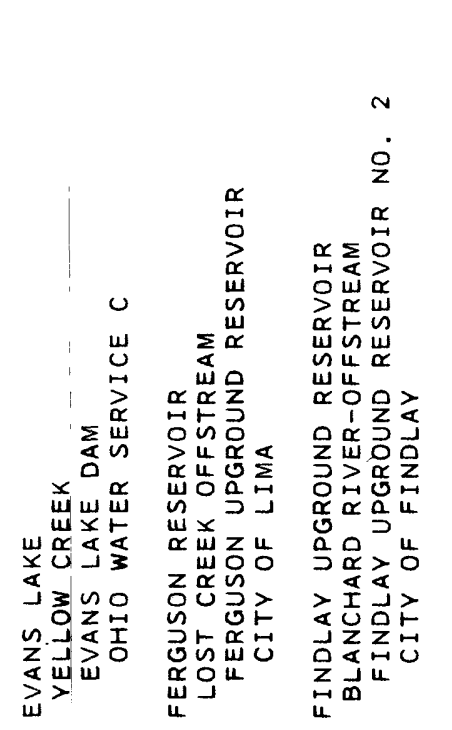

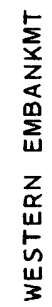

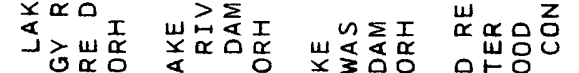

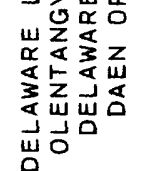
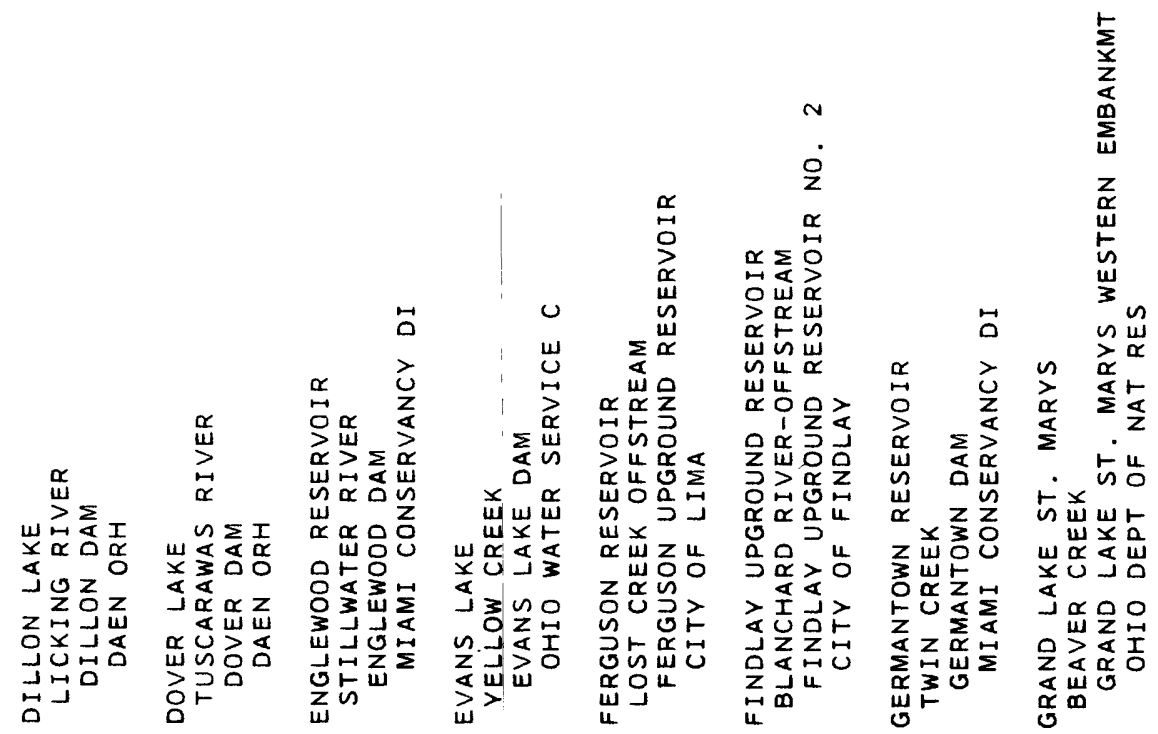


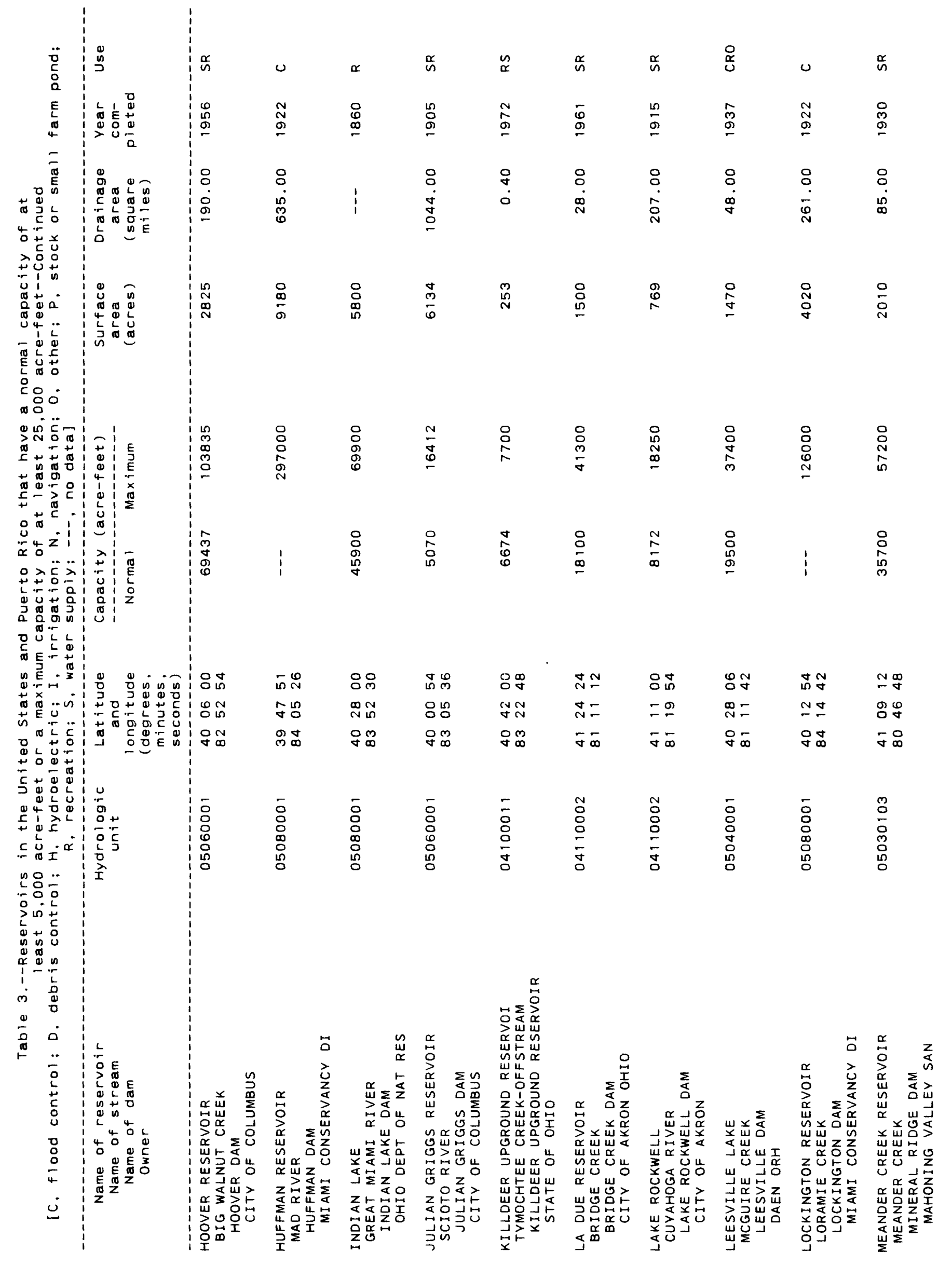




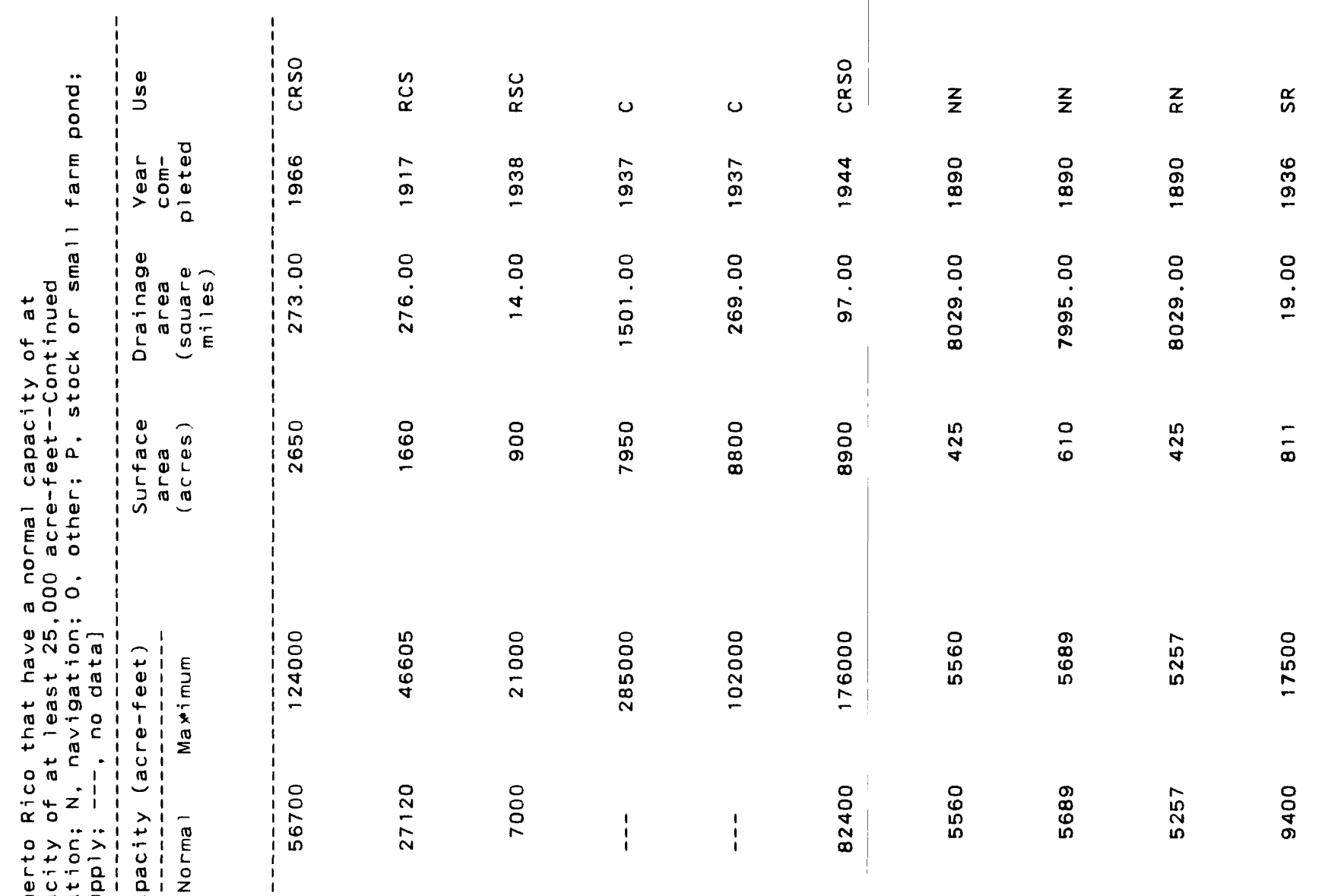

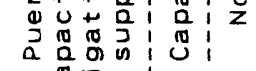

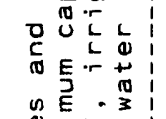

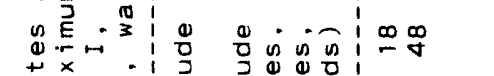

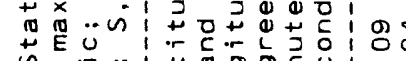

N

\section{.}

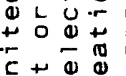

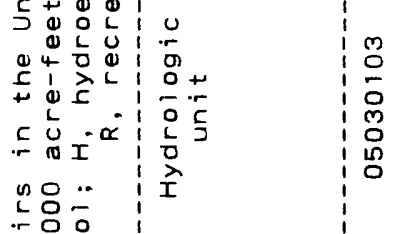

\begin{tabular}{|c|c|c|c|c|c|c|c|}
\hline$\stackrel{\mathscr{M}}{\sim} \underset{\sim}{ }$ & 品京 & $\stackrel{\sim}{\sim}$ & 요 & 옹 & $\simeq O$ & $\stackrel{\infty}{\sim}$ & 옹요 \\
\hline 周品 & $\stackrel{M N}{\alpha}$ & ำ & अ98 & $\stackrel{\infty}{q}$ & $\stackrel{\infty}{N} \stackrel{2}{N}$ & Б̄ & 守出 \\
\hline$\sigma_{\infty}$ & $\overline{-\infty}$ & PN & $O_{\infty}^{N}$ & - & gis & gi & ब্লা \\
\hline
\end{tabular}

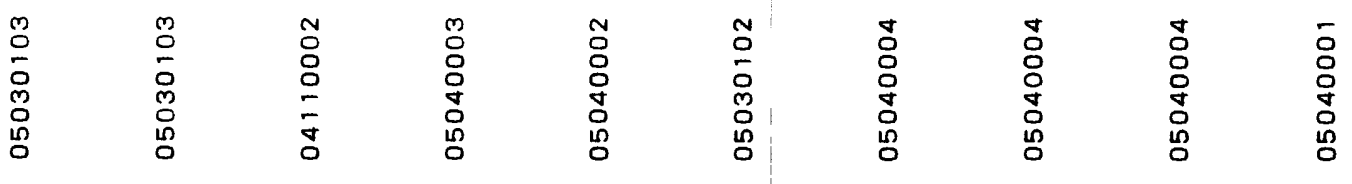

$>$ in

is o

ब

i.

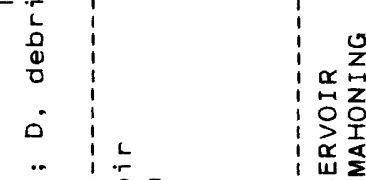

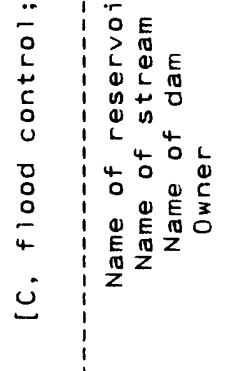

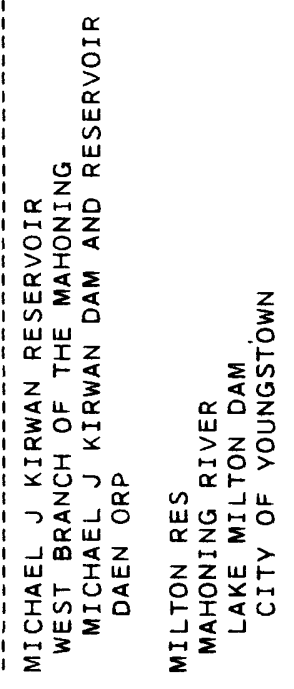

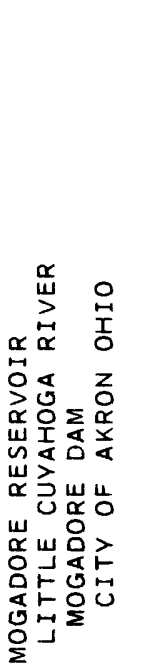

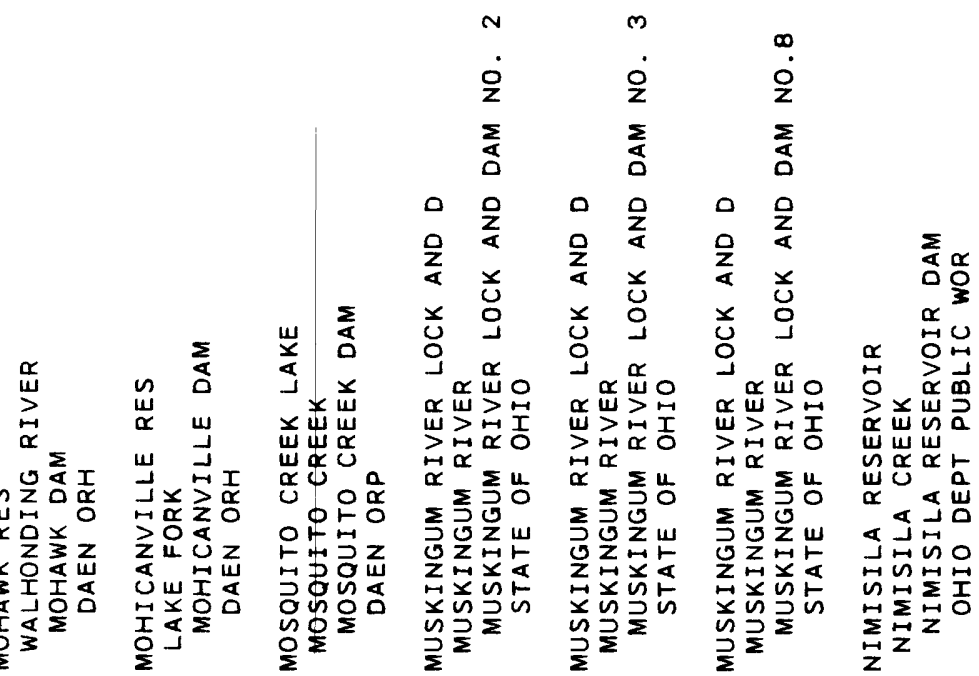




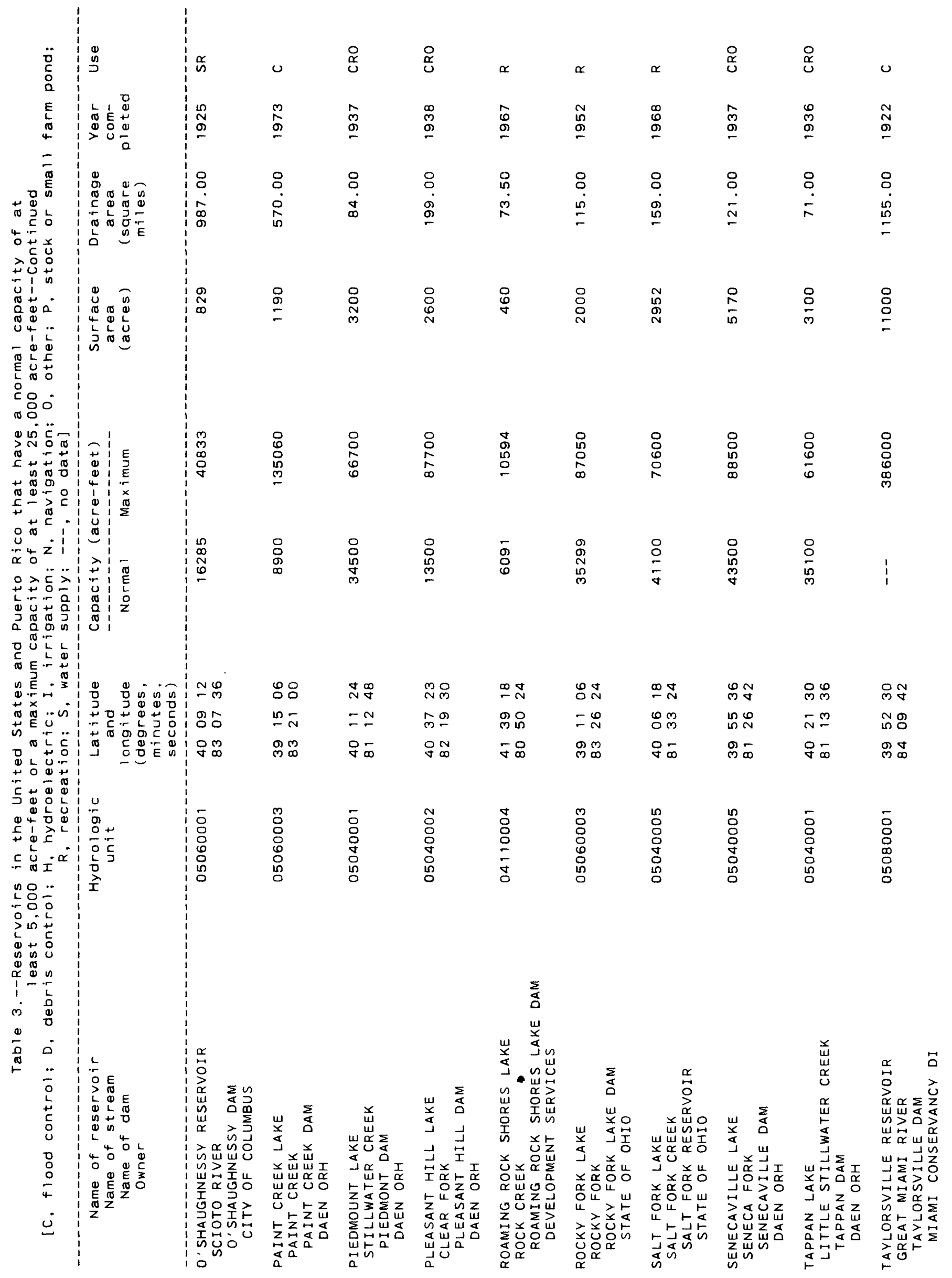




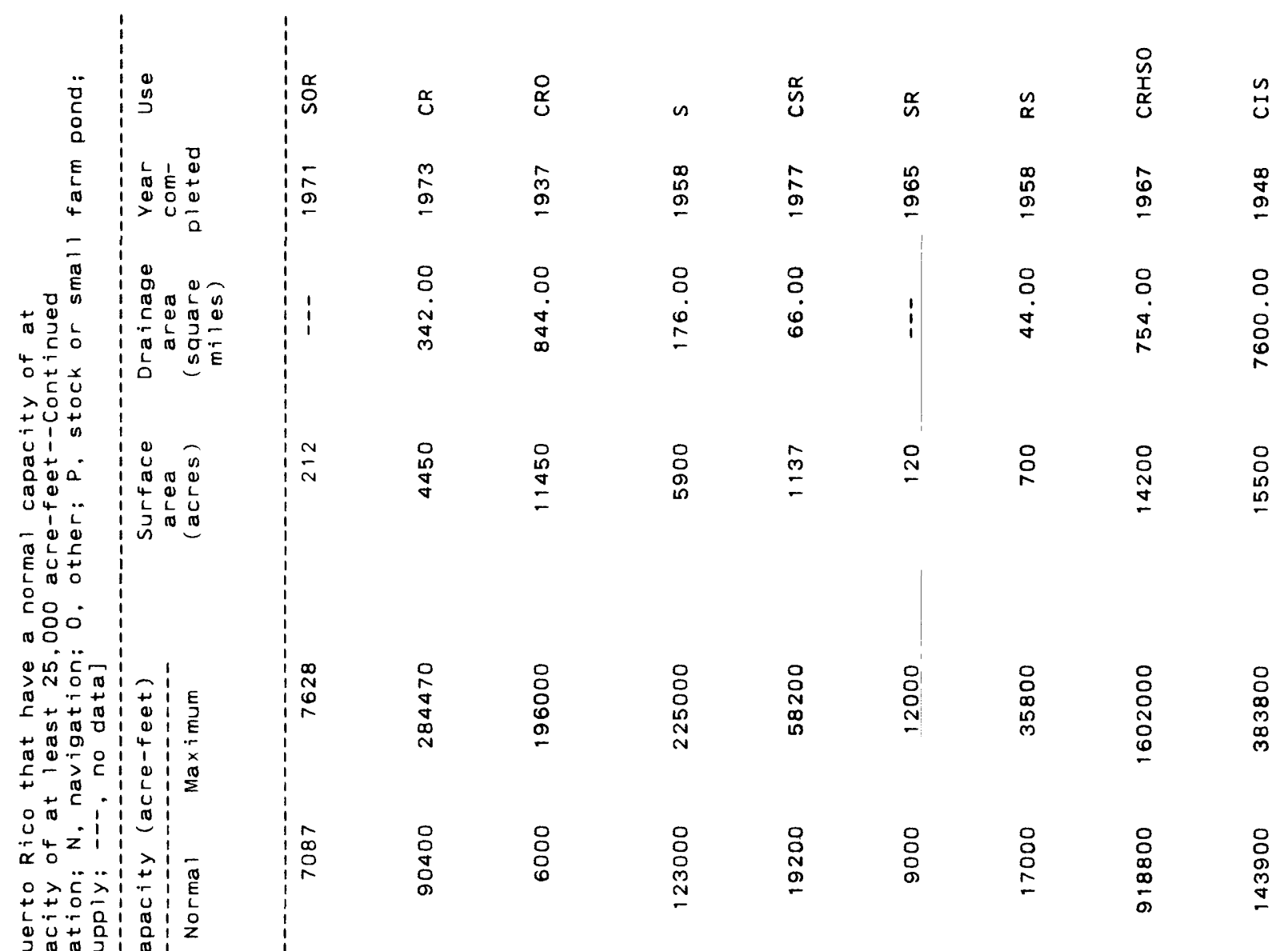

|

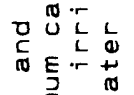

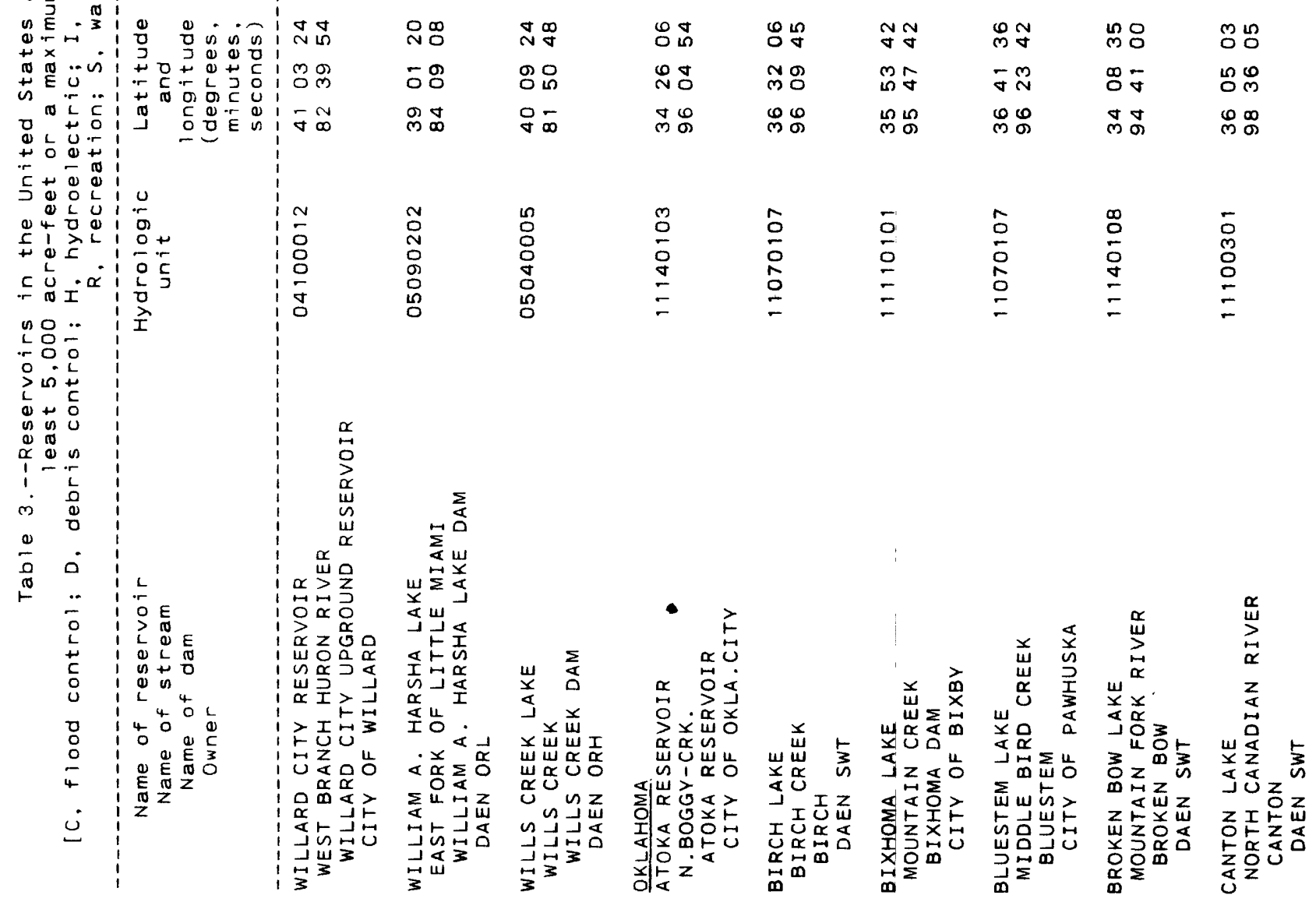




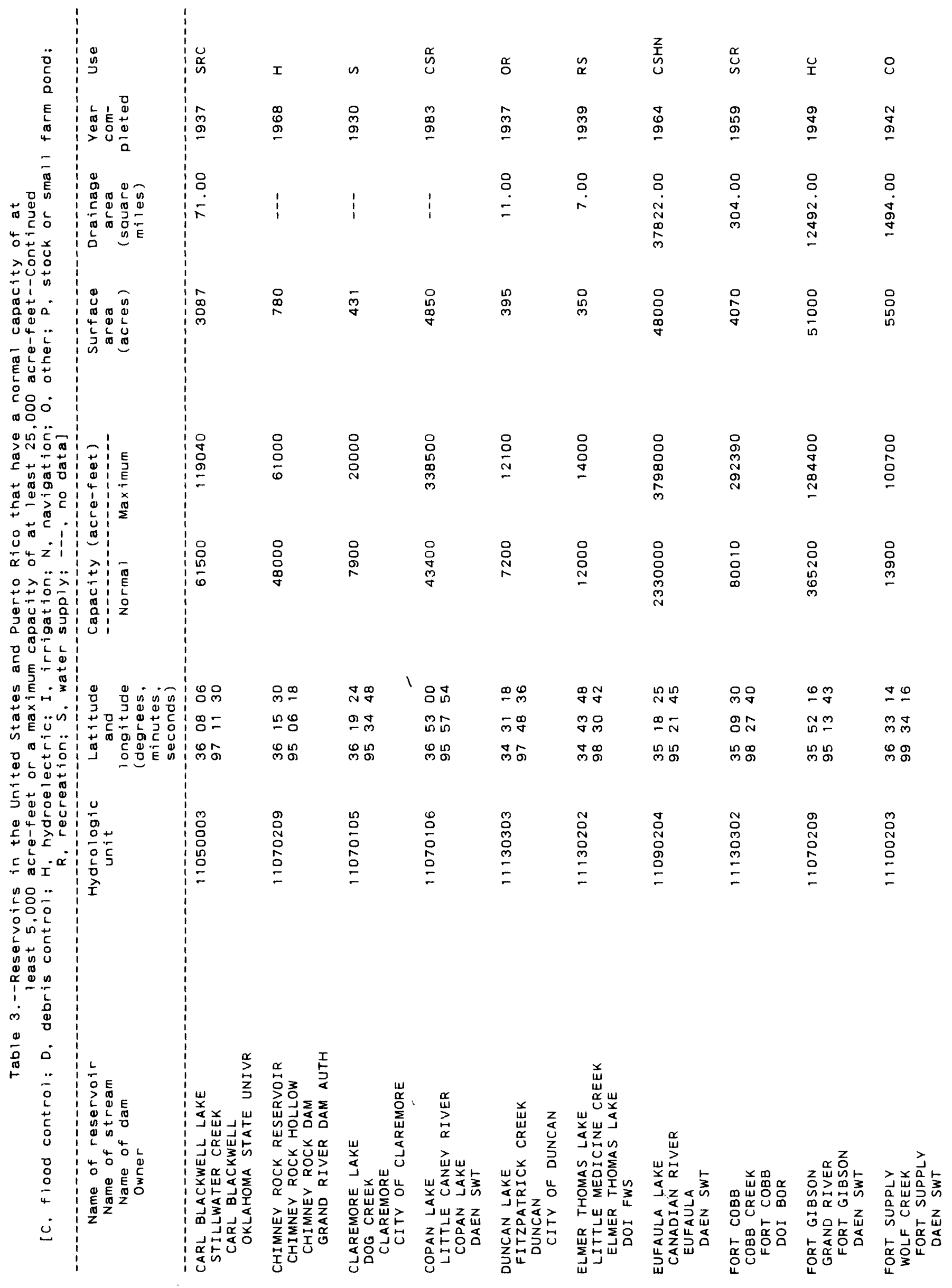




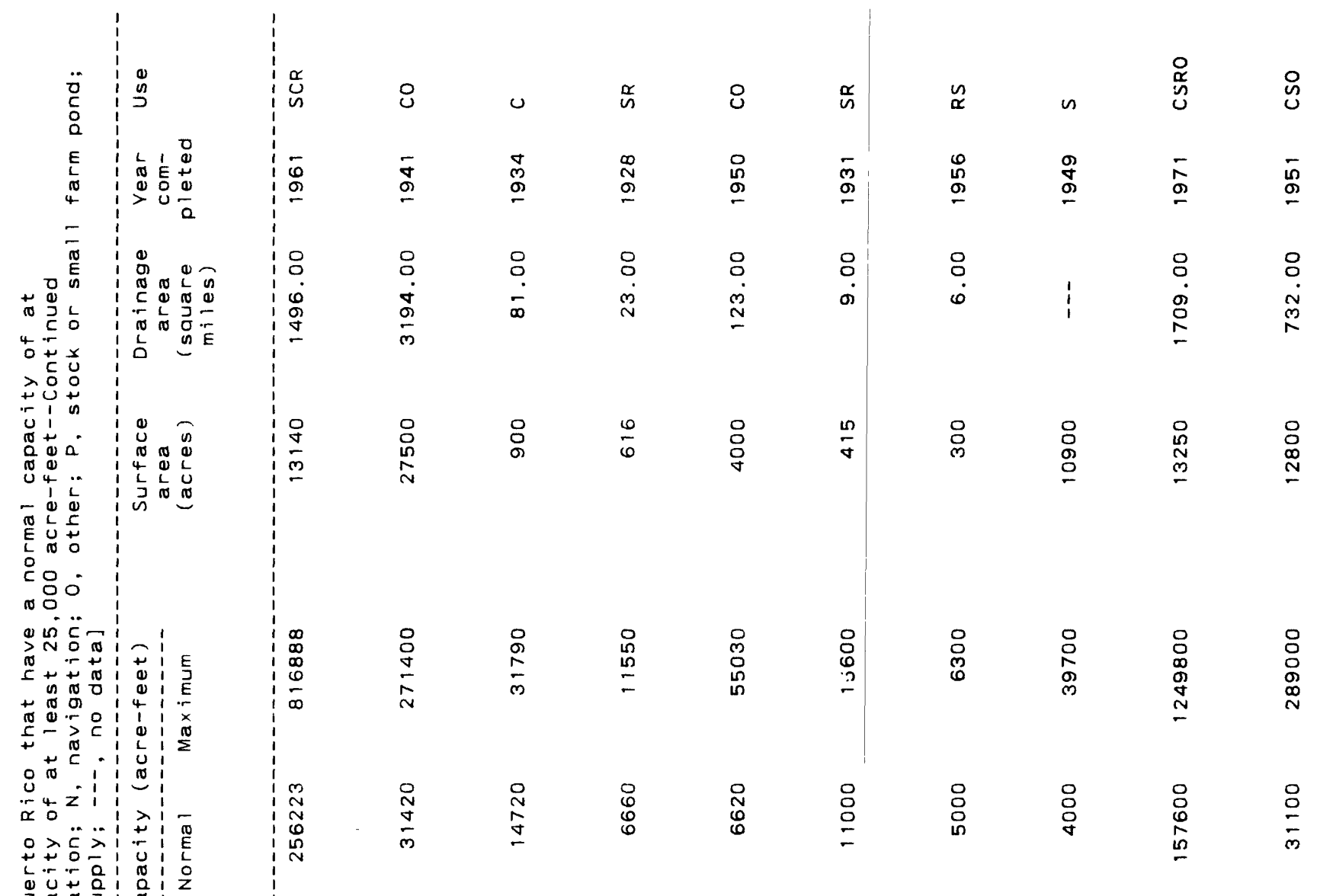

年

वण्:

N $E$ 的索

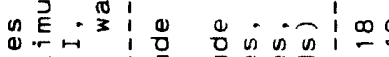

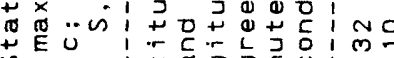

क स

O

年

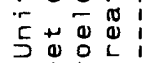

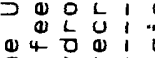$$
\stackrel{Ð}{+}
$$

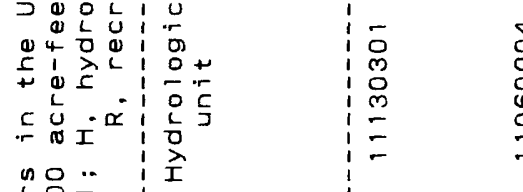

\begin{tabular}{|c|c|c|c|c|c|c|c|}
\hline 웅 & 잉요 & $\because \nsubseteq$ & กู & $\underset{\nabla}{\sim}$ & $\ddot{m} \stackrel{\infty}{+}$ & 응 & ㄱㅇ \\
\hline 寸ै & 삥으 & $\stackrel{2}{N} \stackrel{n}{n}$ & in: & -N & $\stackrel{\sim}{N}$ & \&̊ & $8 N$ \\
\hline లొ & "ूల్ & 只㔯 & "ू్ల & గूळ\% & 용 & 용 & 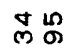 \\
\hline
\end{tabular}

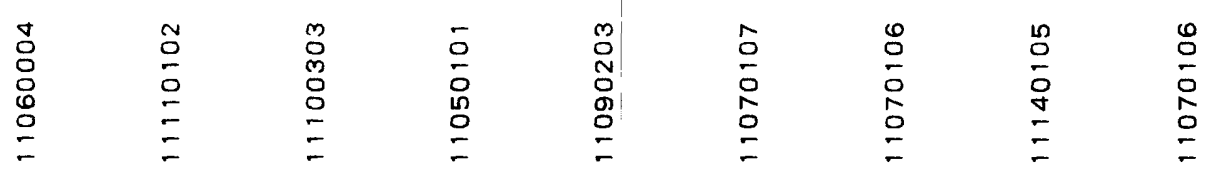

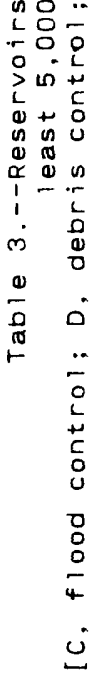
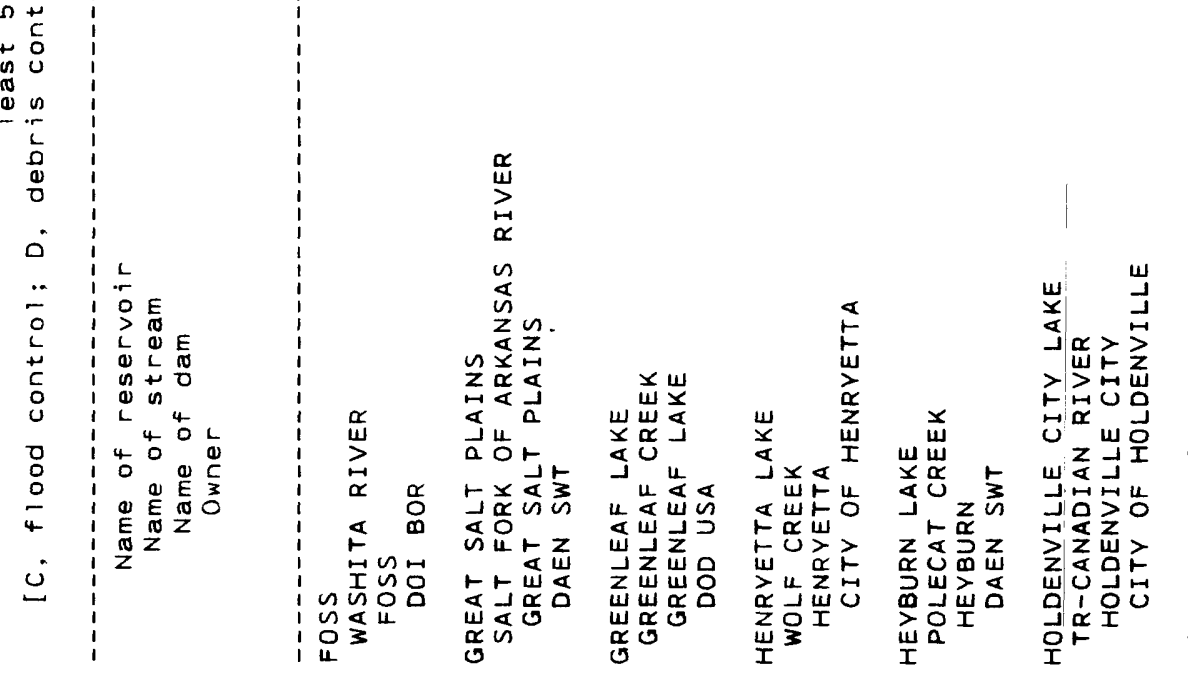

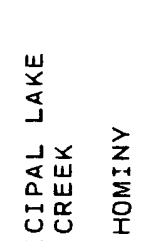

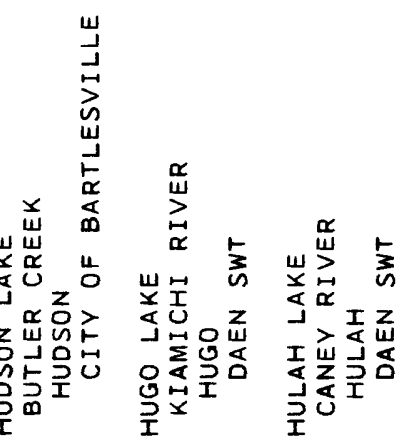




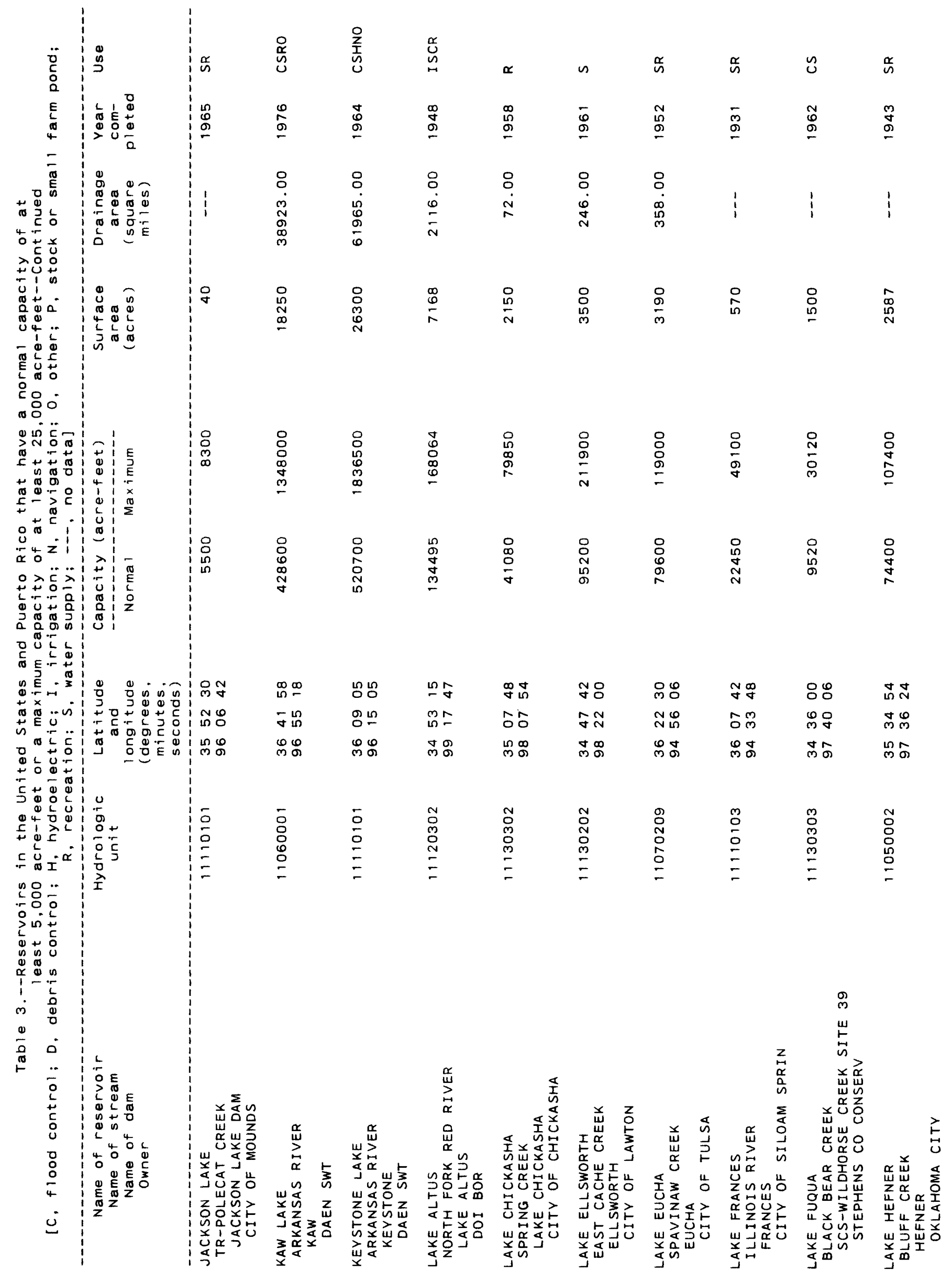




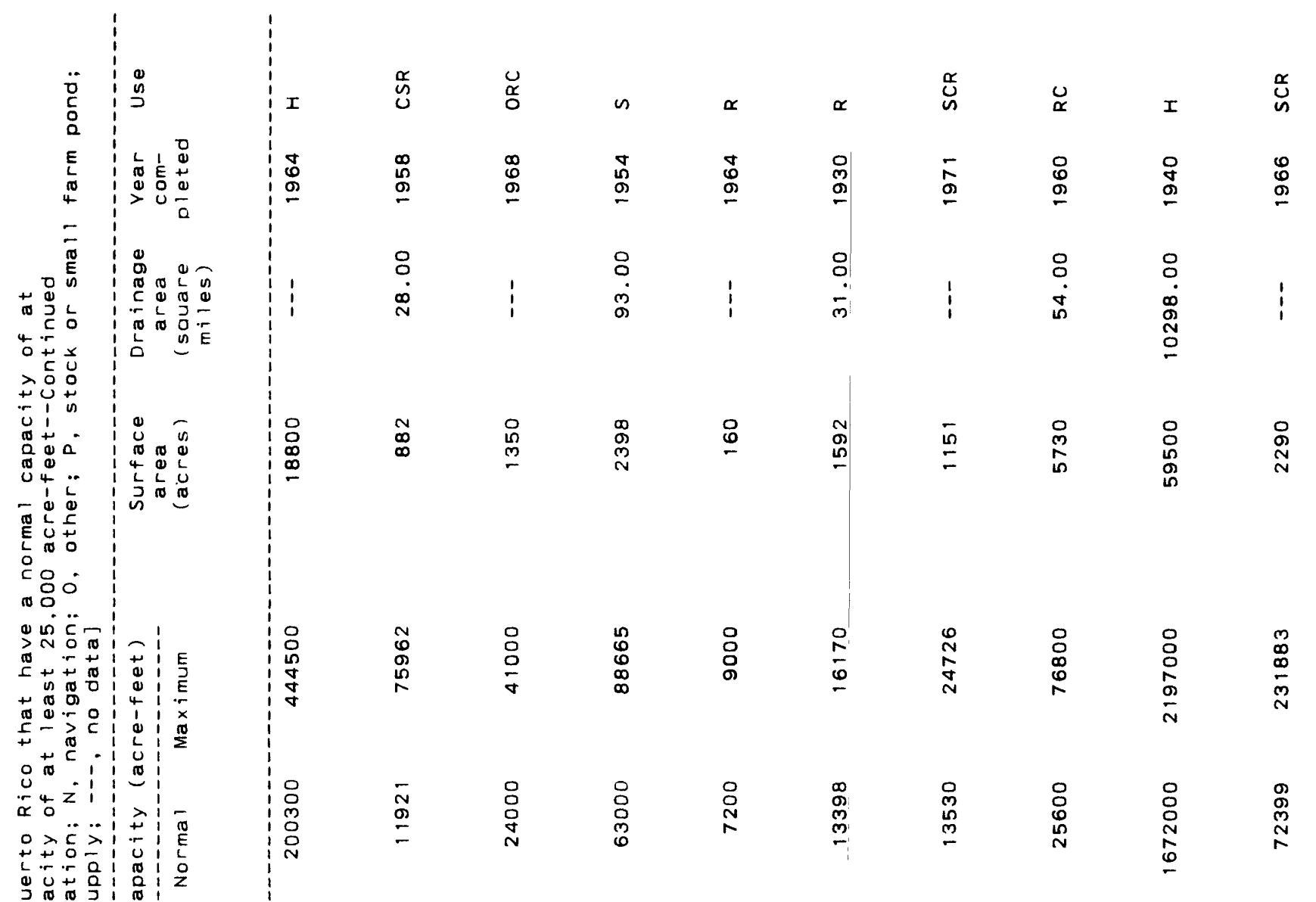

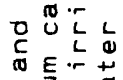

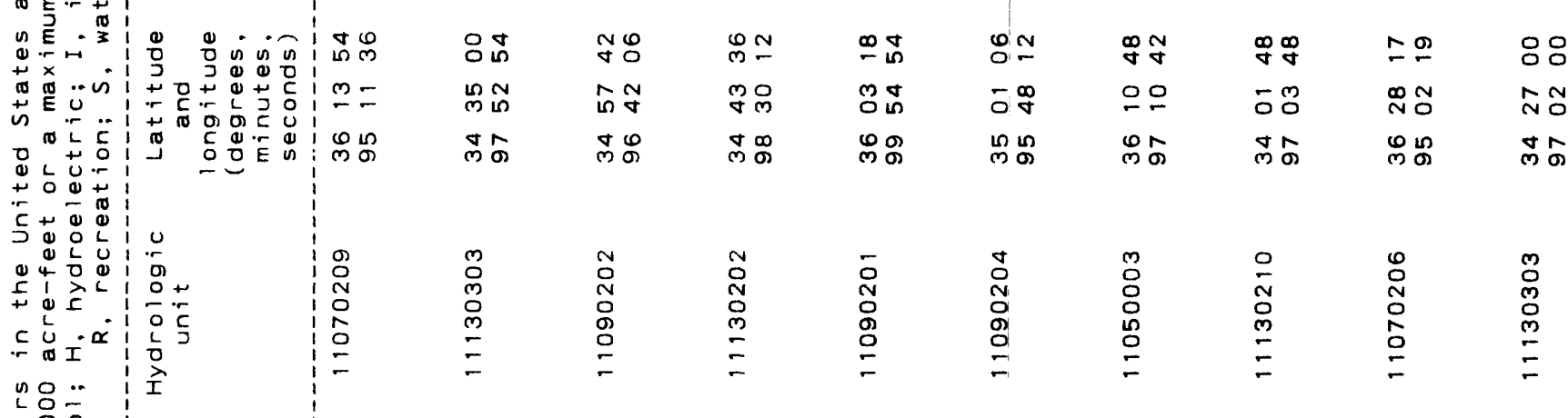

>

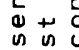

a

!.

i

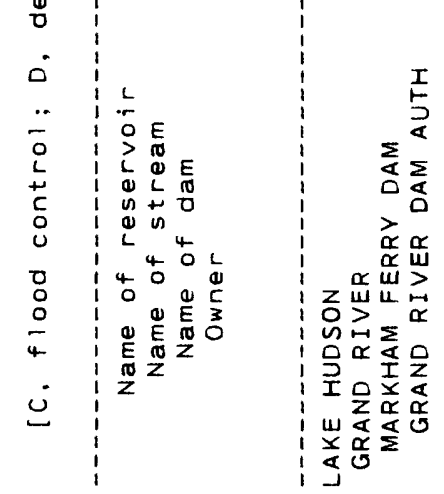

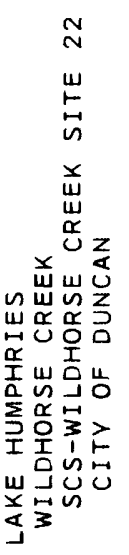

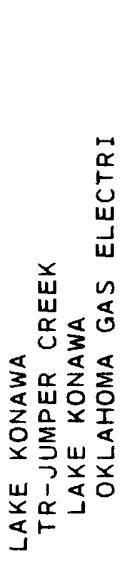

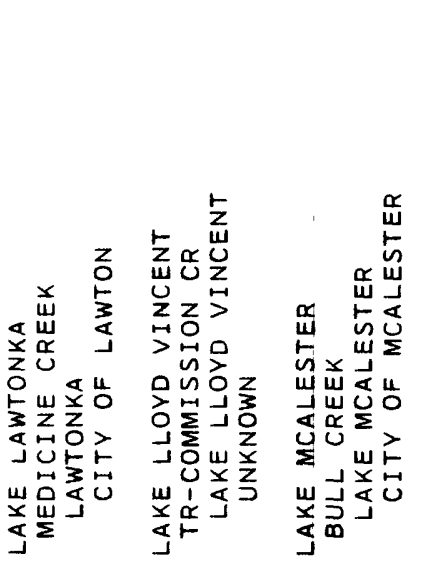
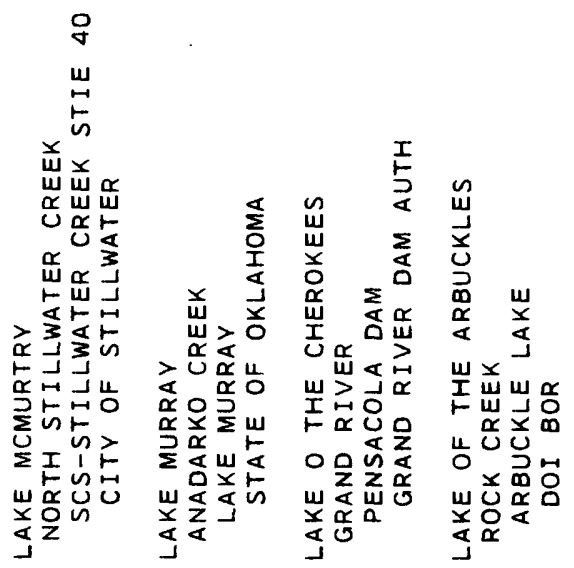


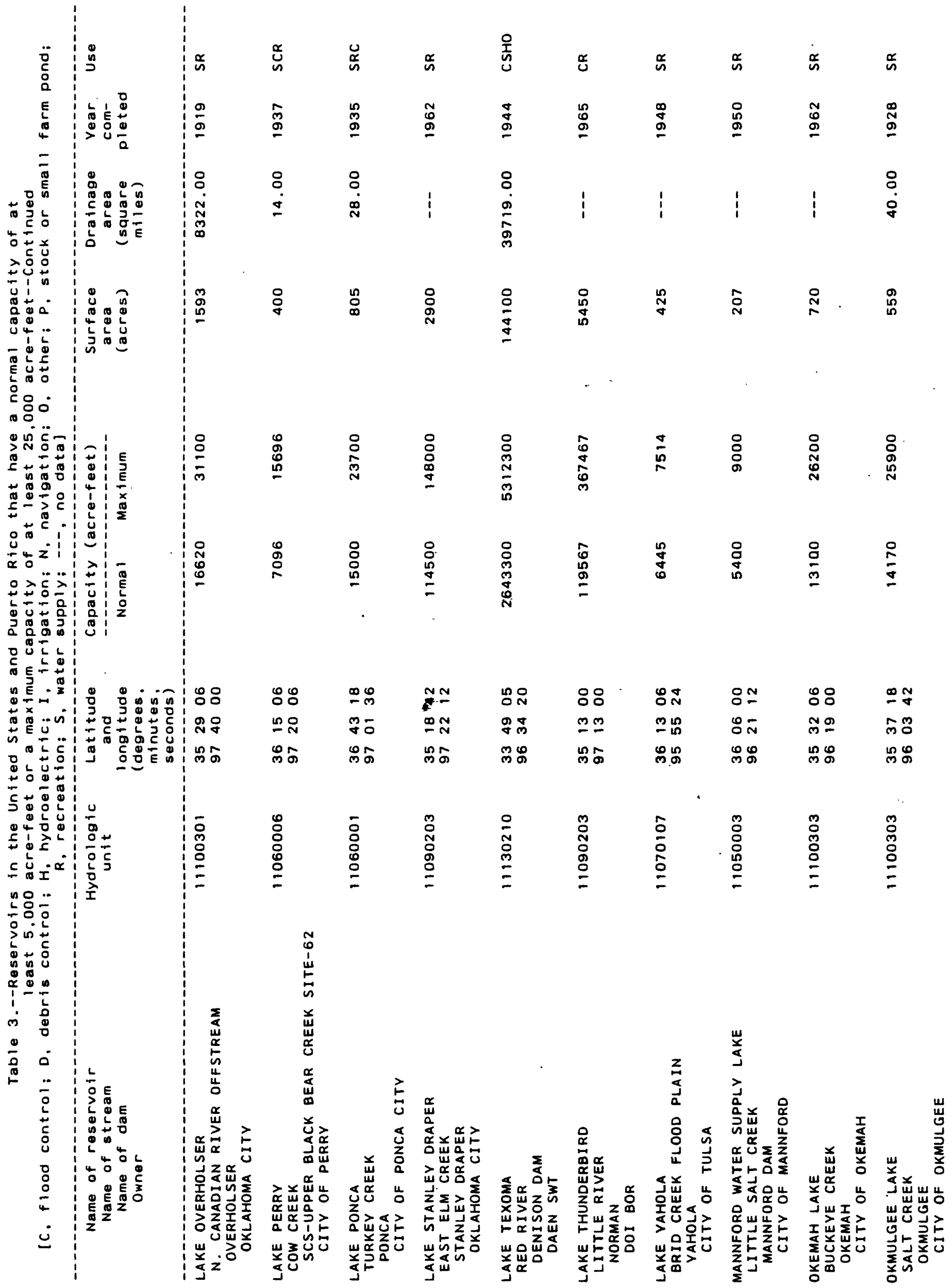




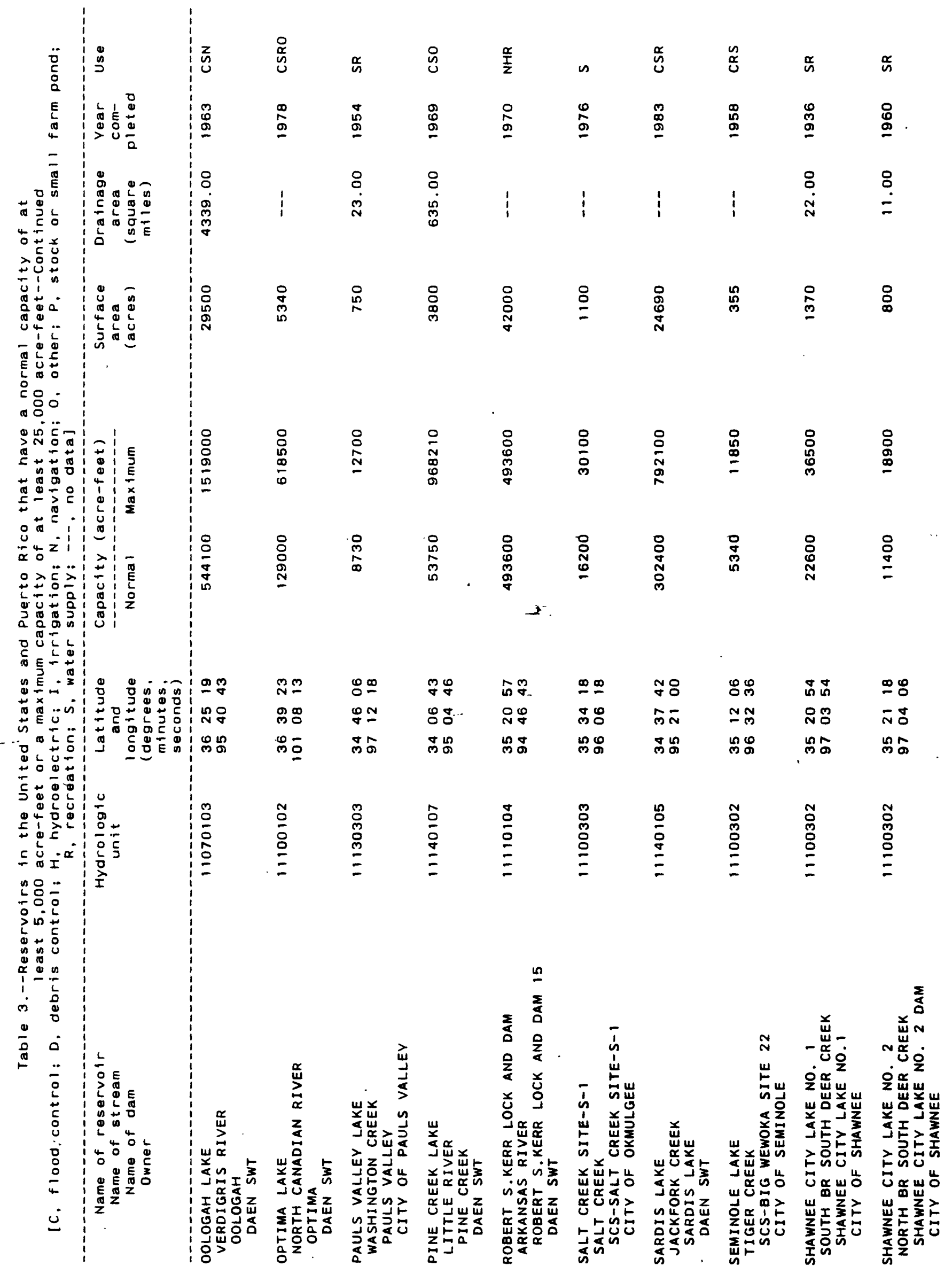




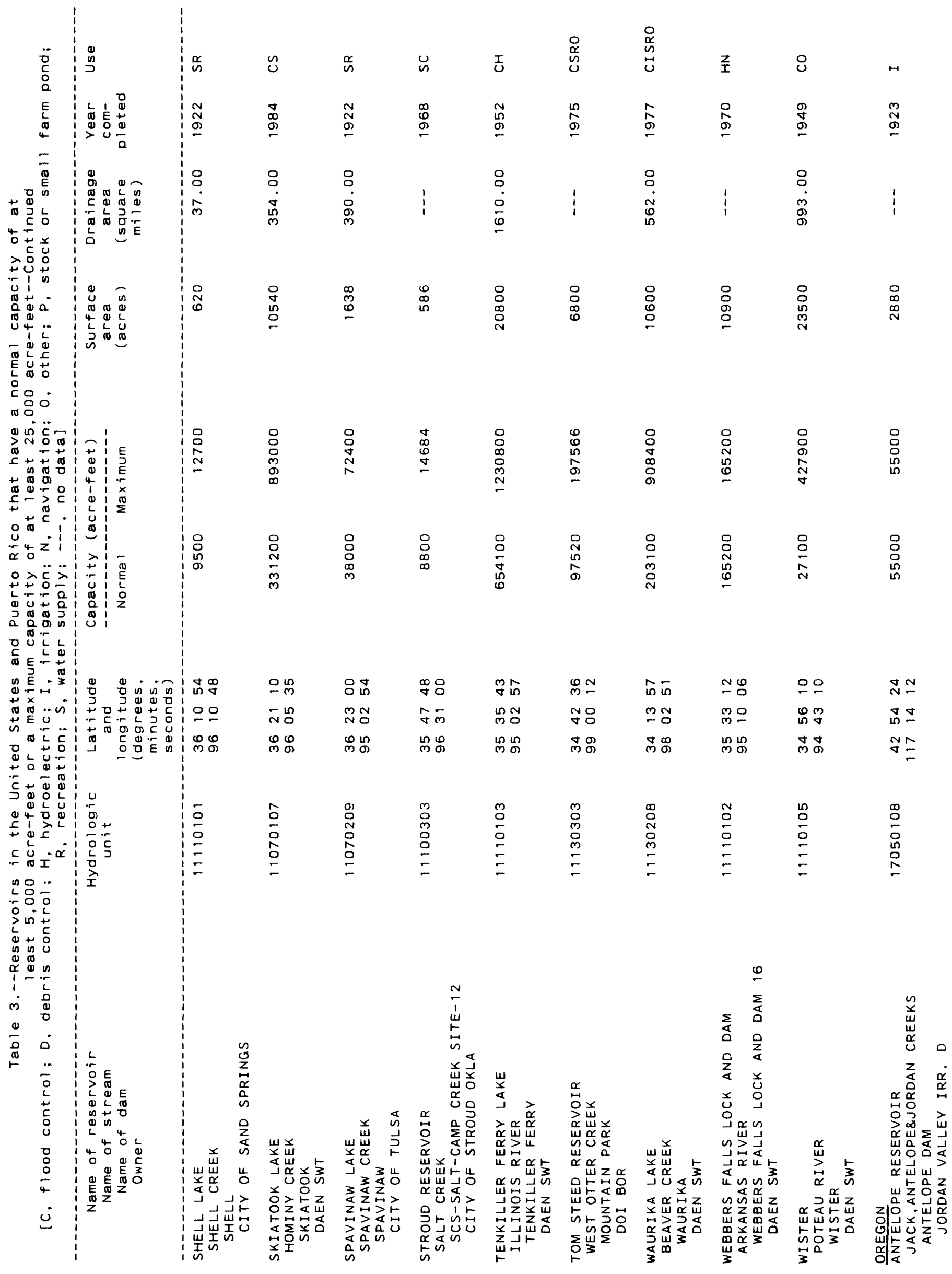




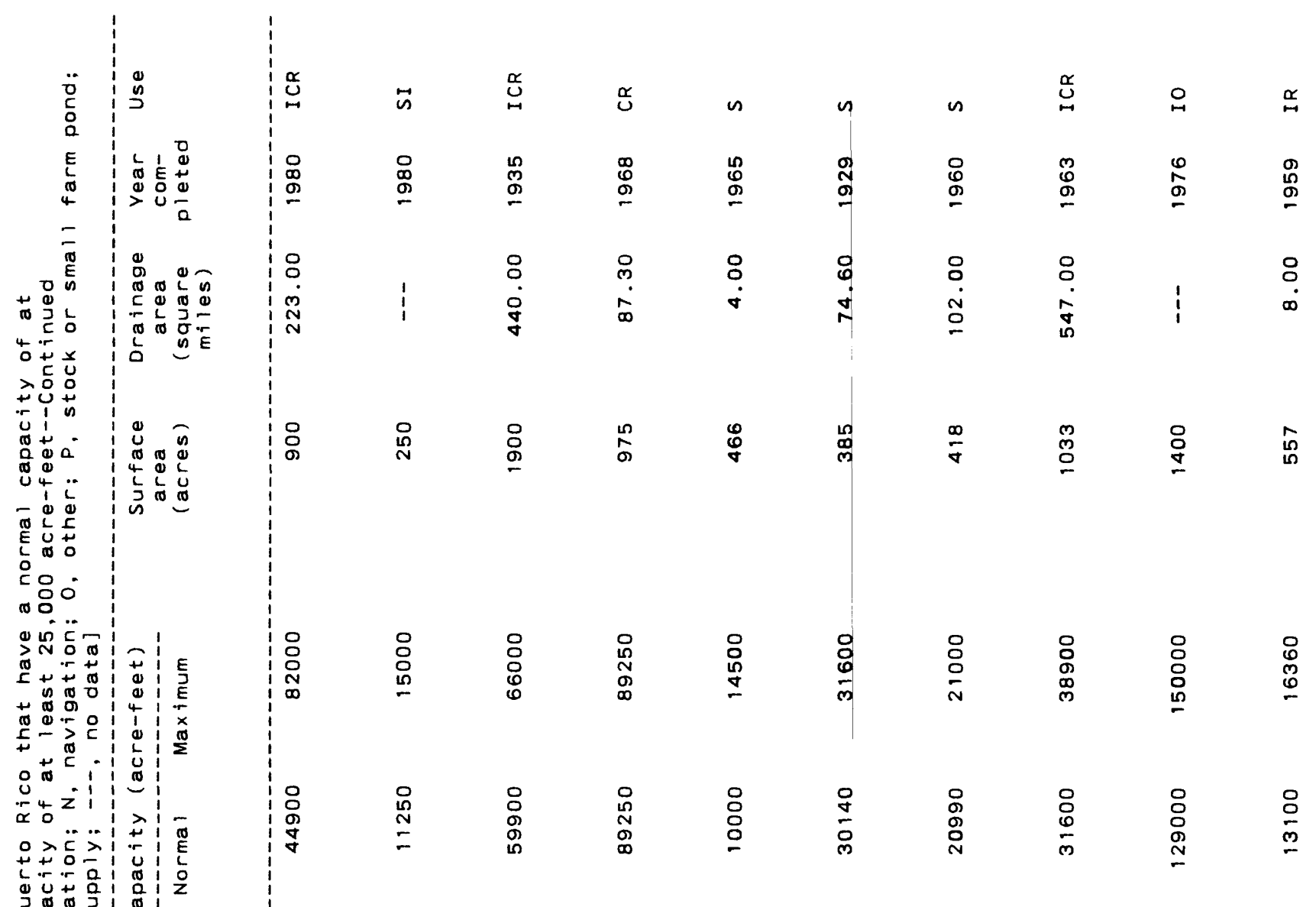

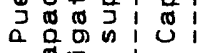

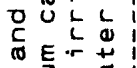

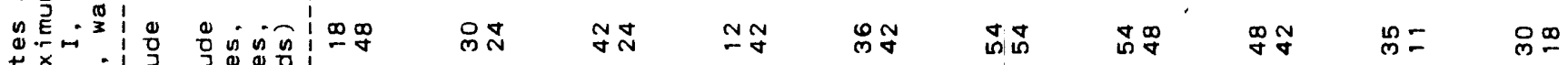

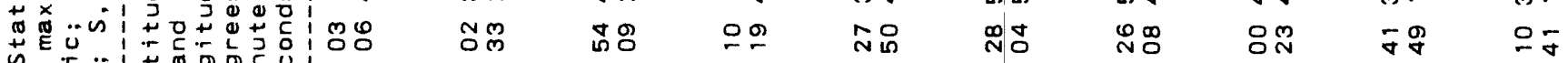

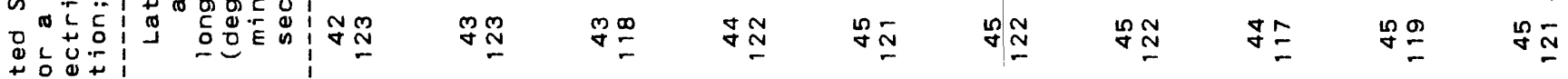

$+00$

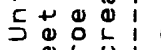

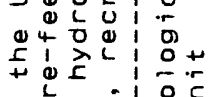

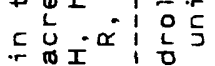

n으

$\therefore 0$

$>$ in

路

\& $\underset{\otimes}{\infty}$

(2)
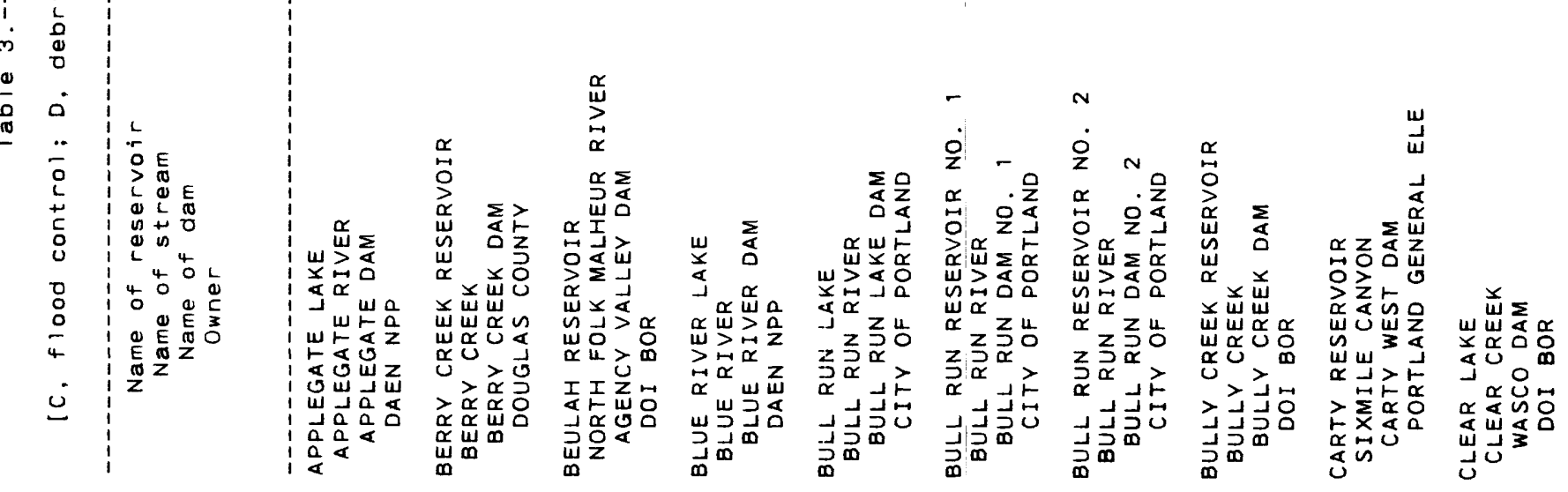

䍃

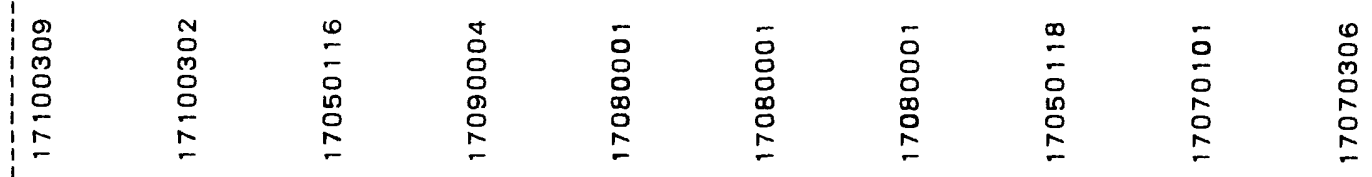




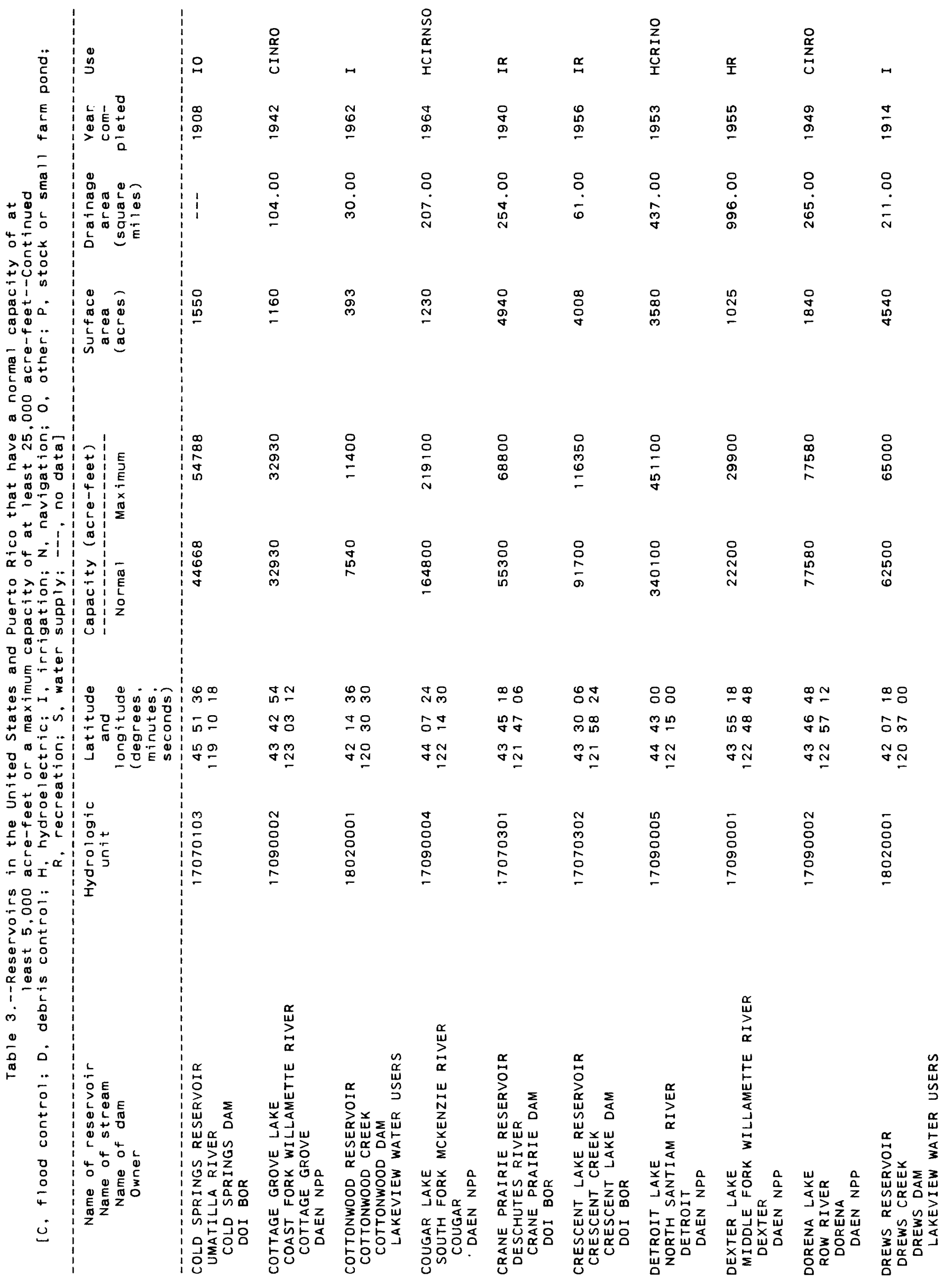




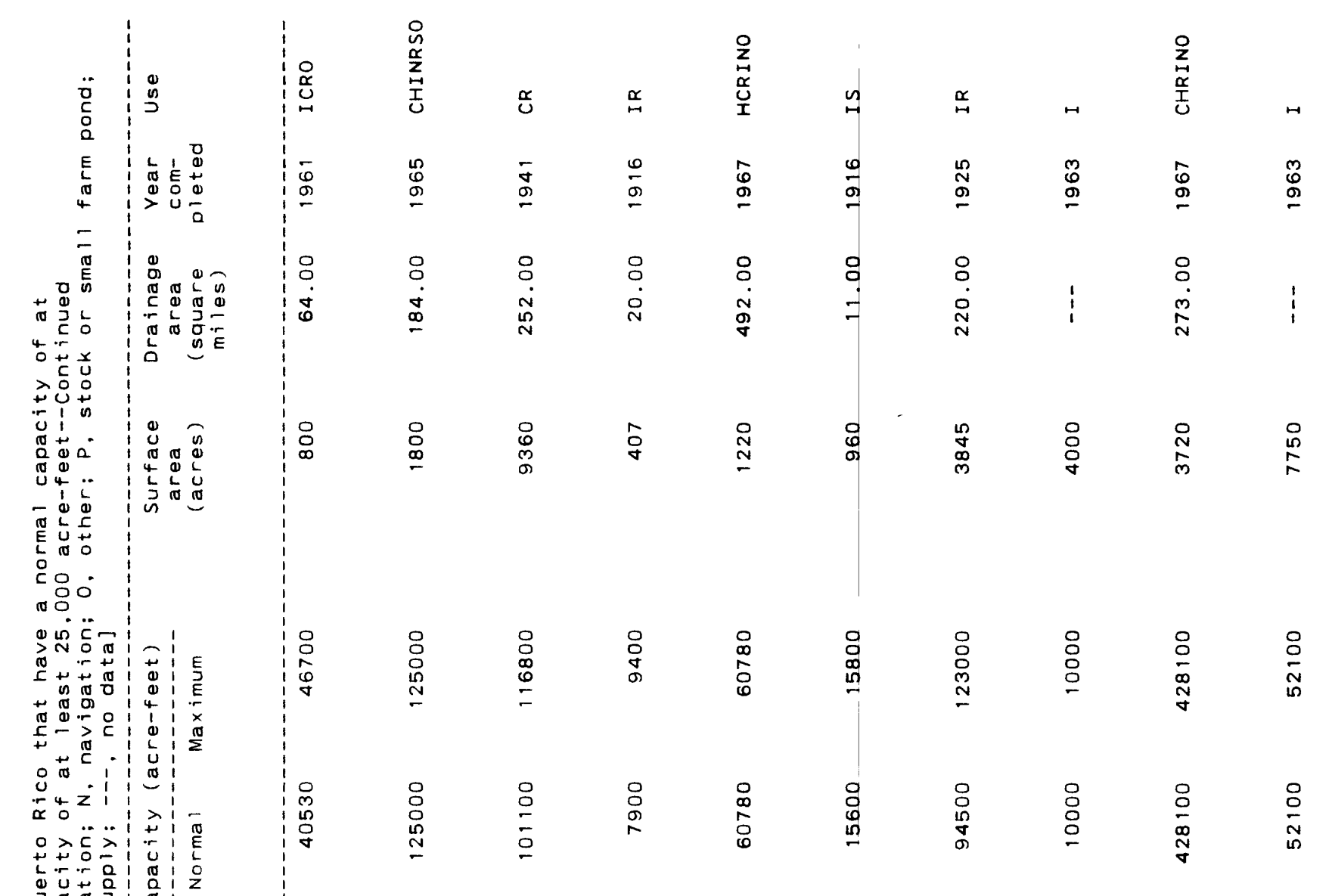

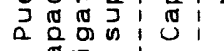

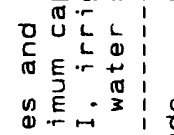

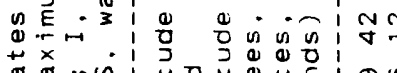

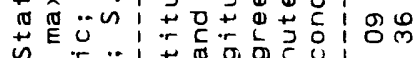

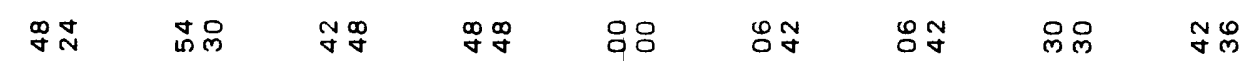

品

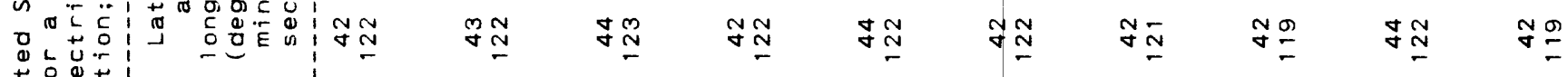

$+0 \underset{0}{\pi}$

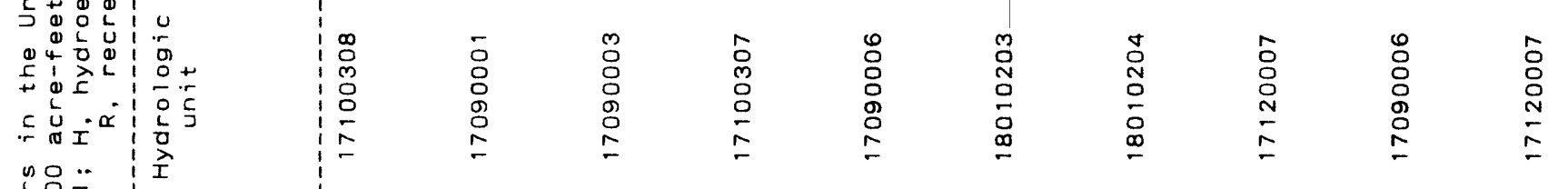

늠

$>$ in

a

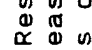

1.

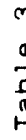

$\frac{0}{\pi}$

$\circ$

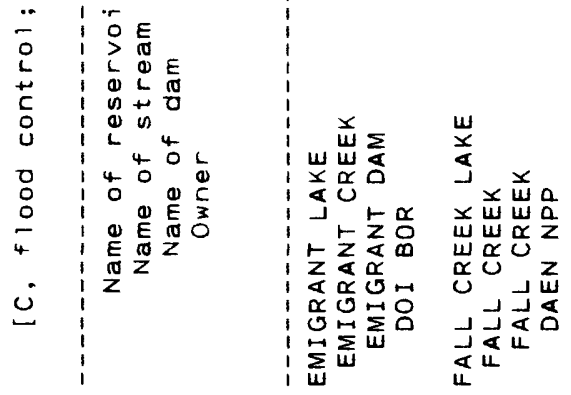

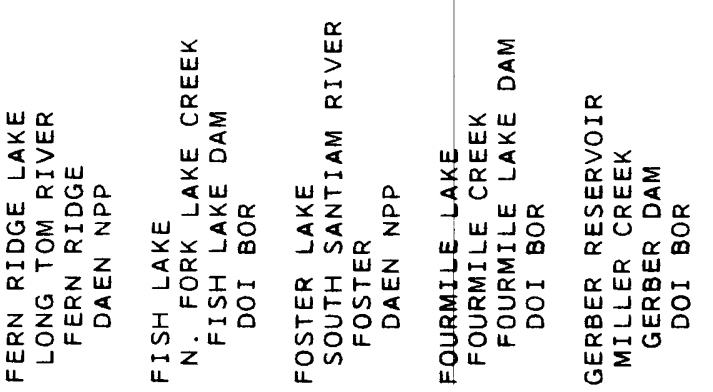

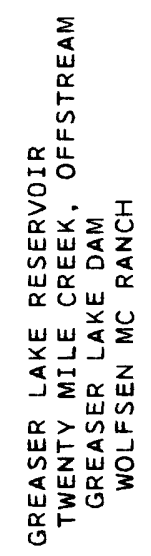

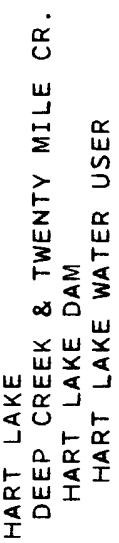




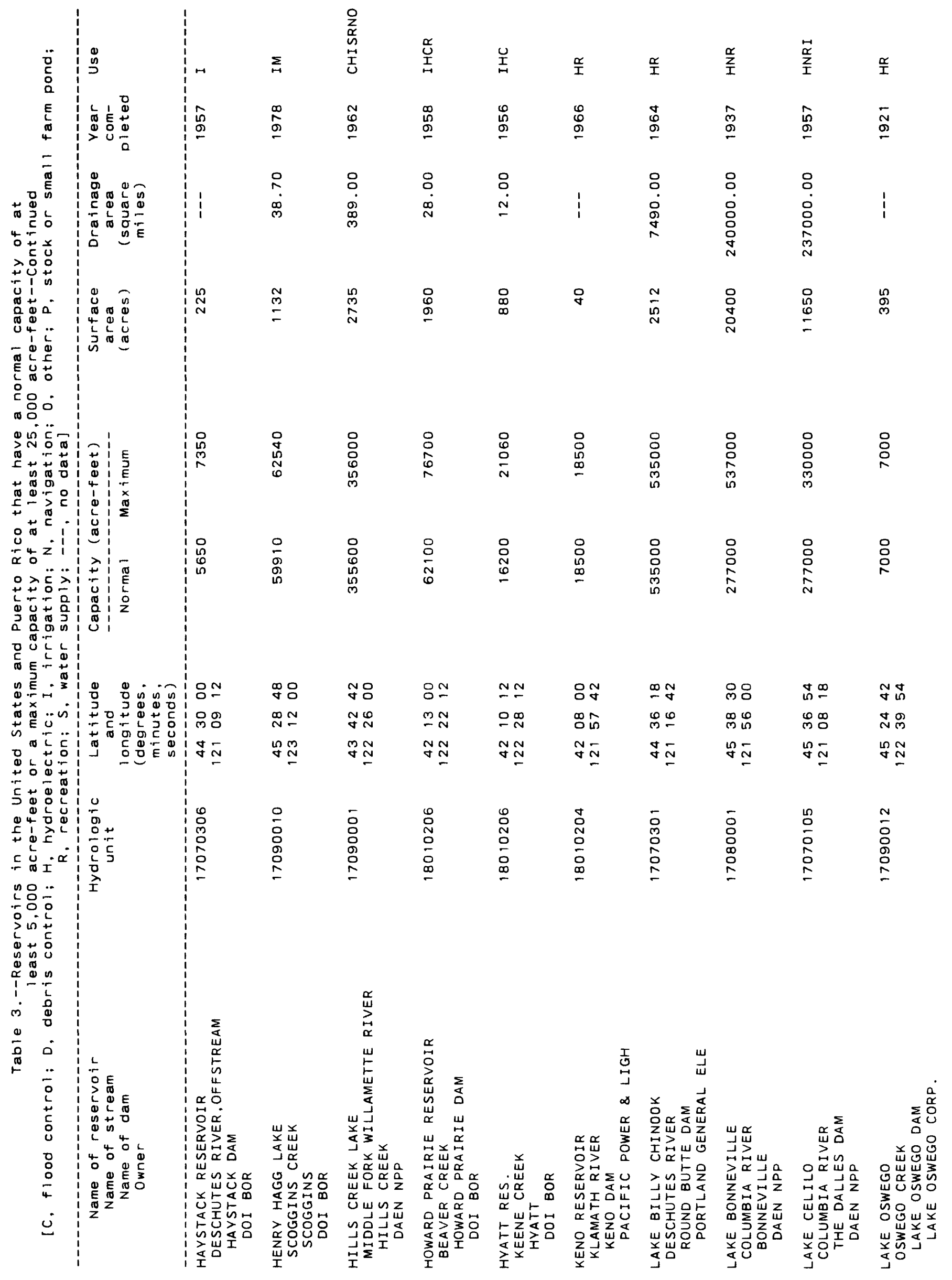




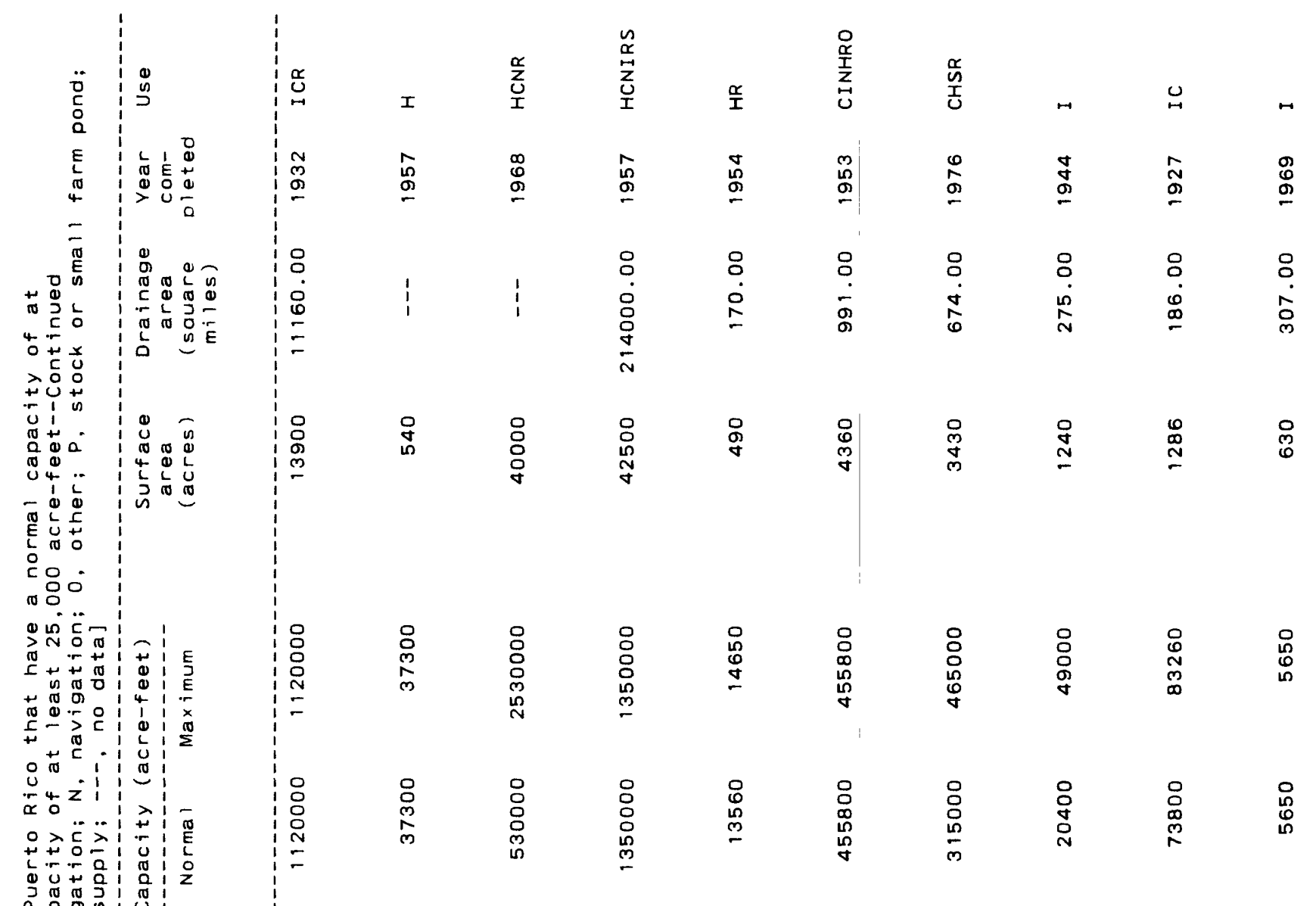

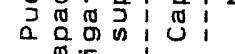

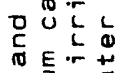

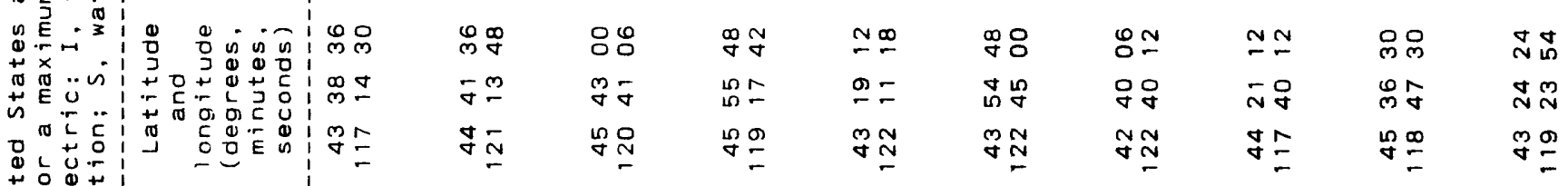

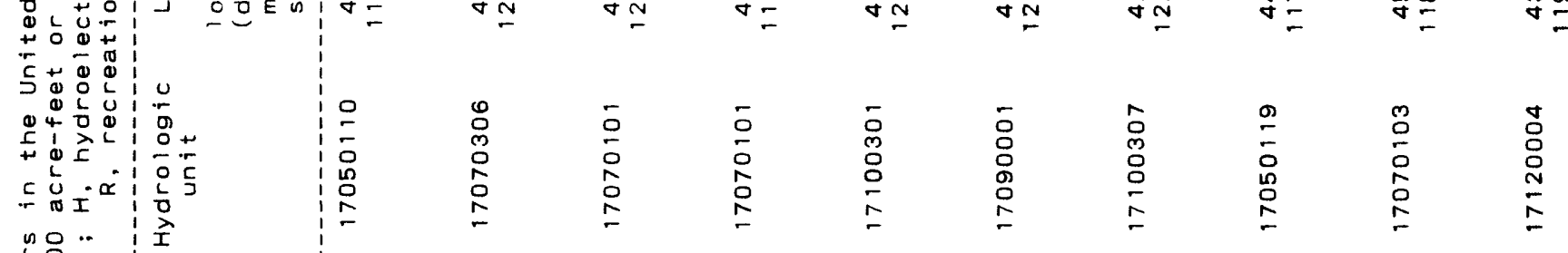

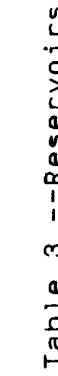
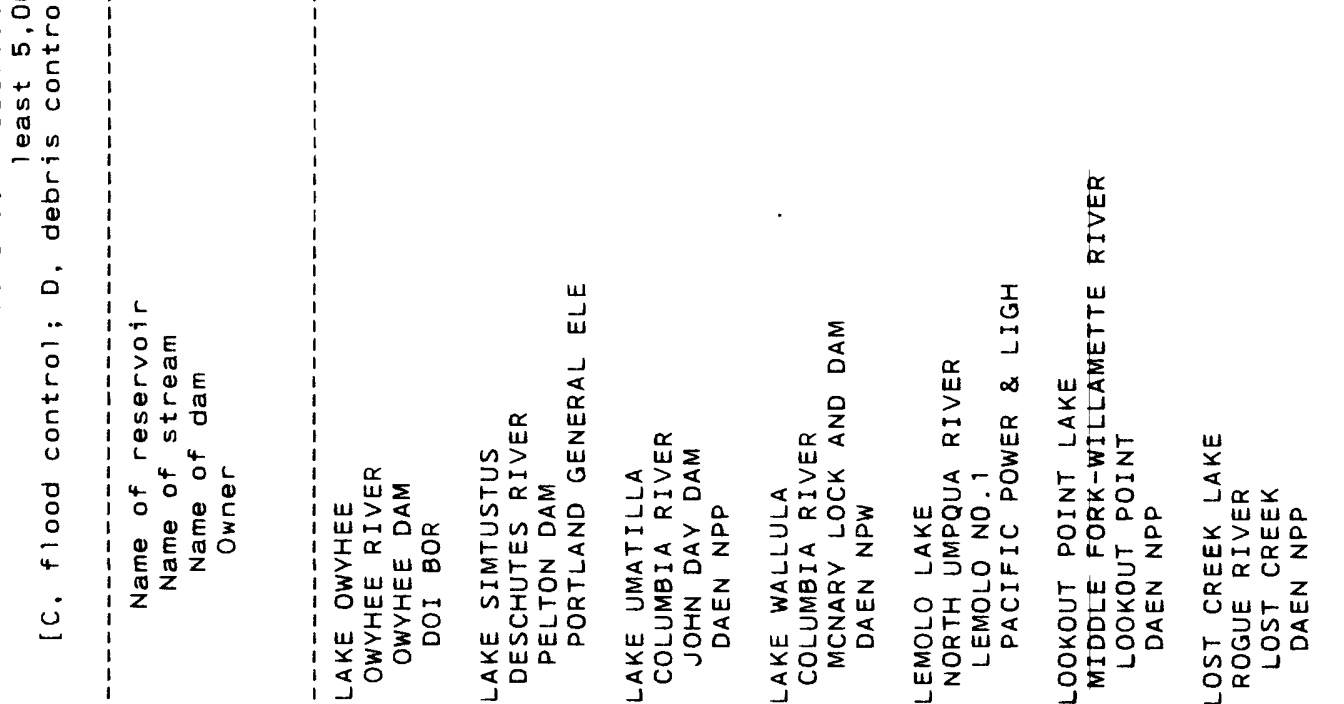

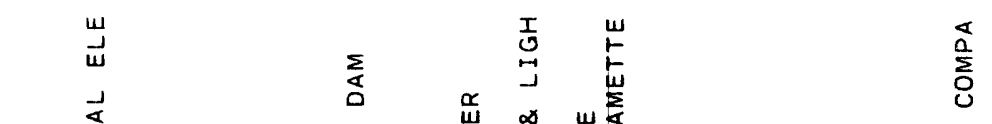

n

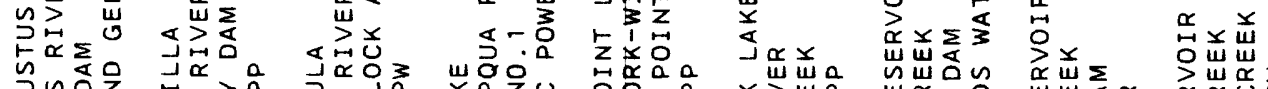

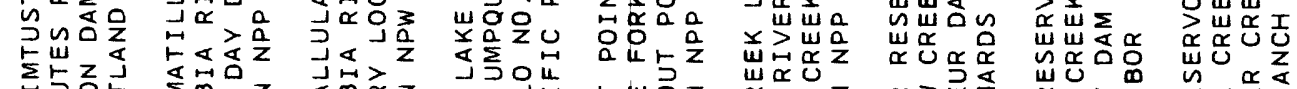

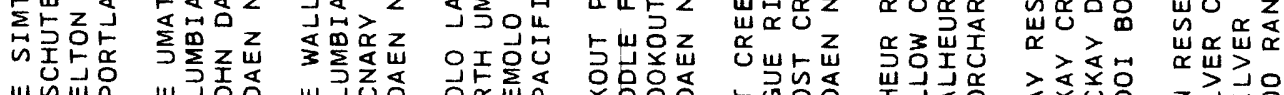

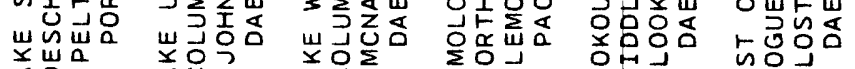




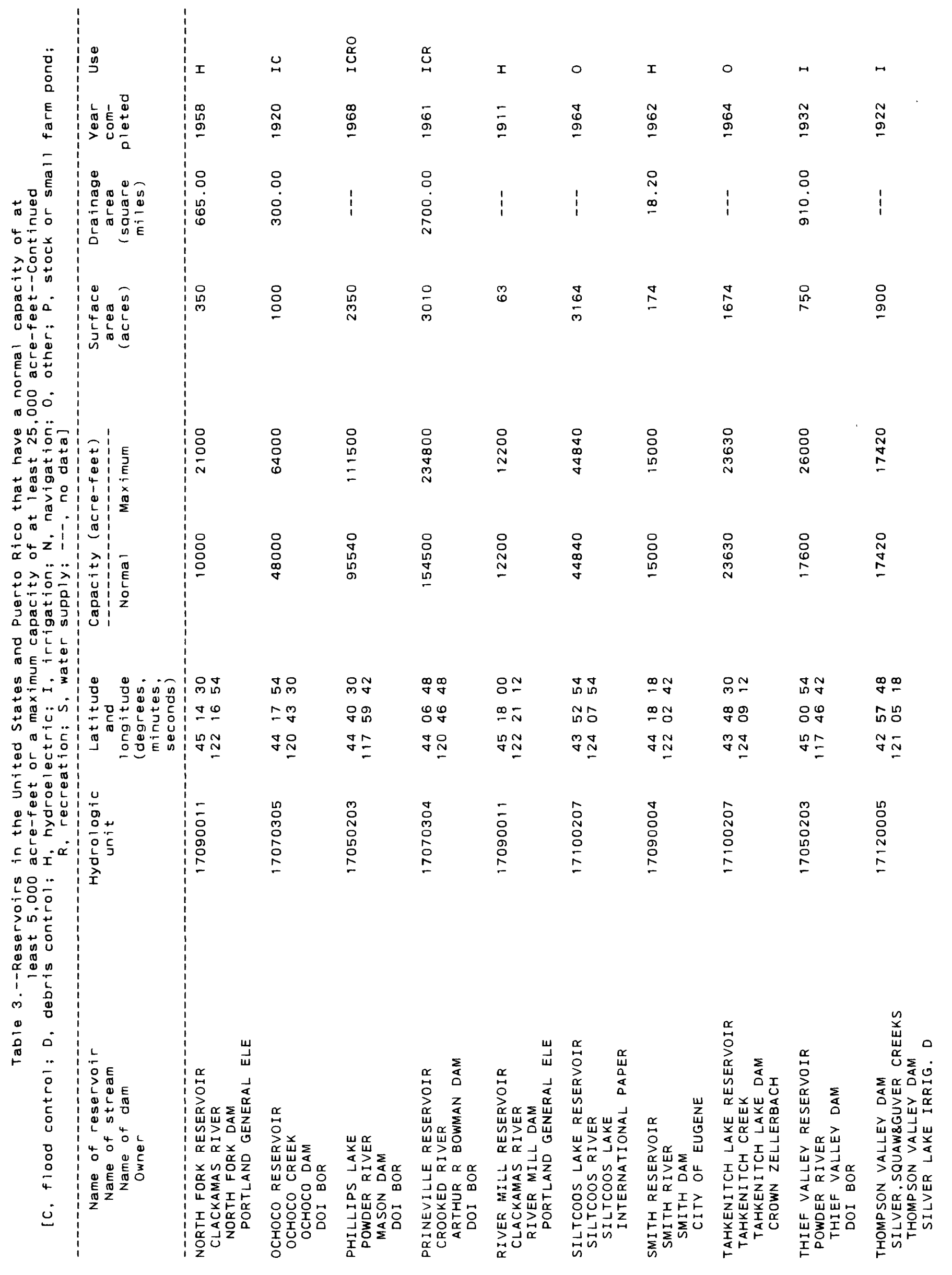



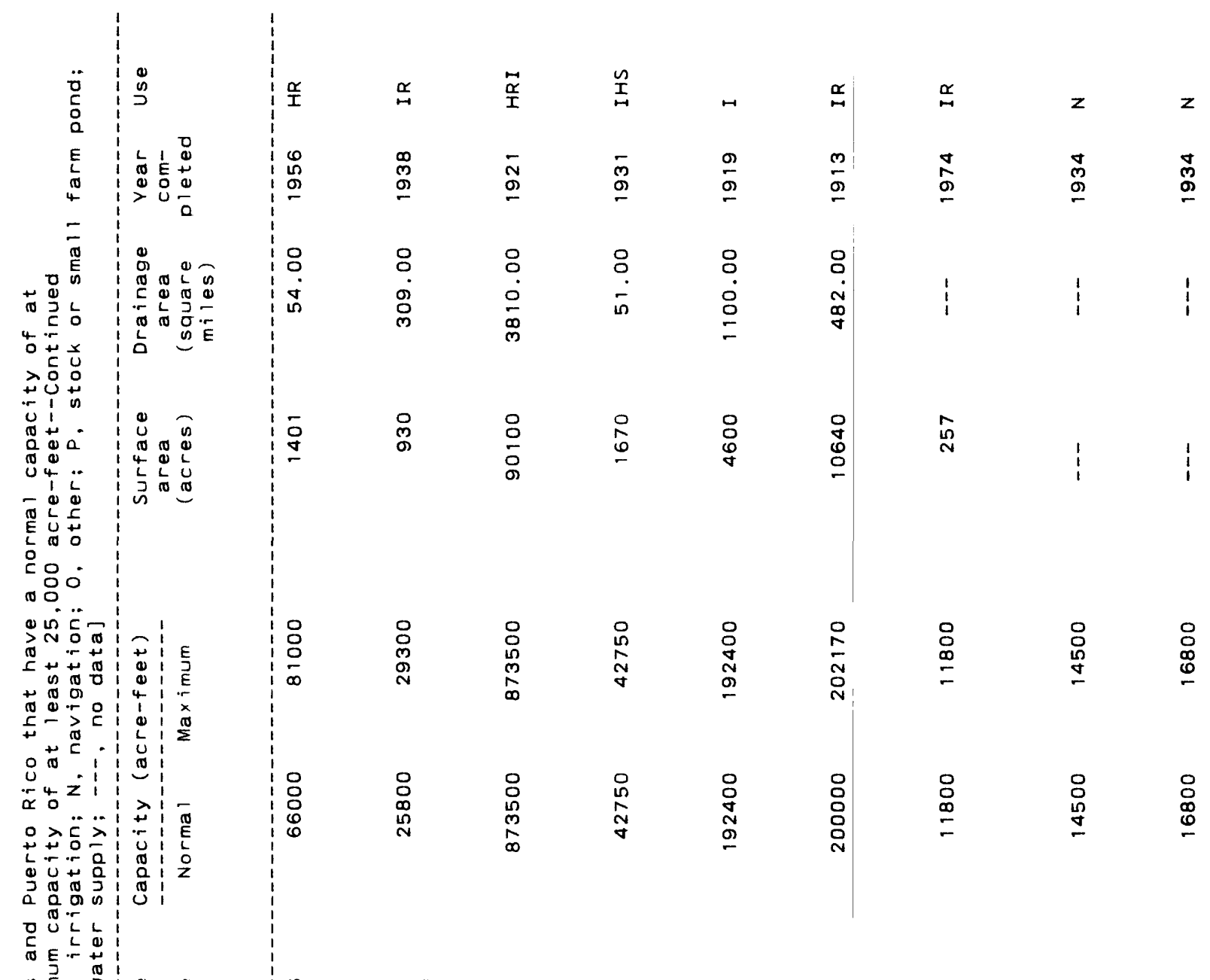

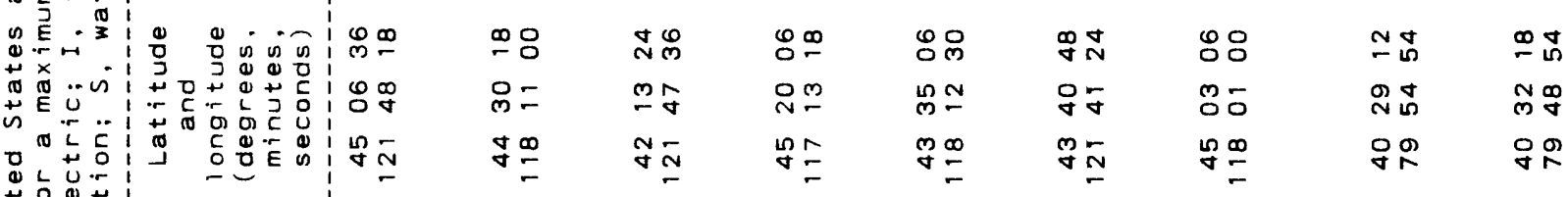

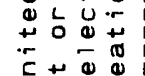

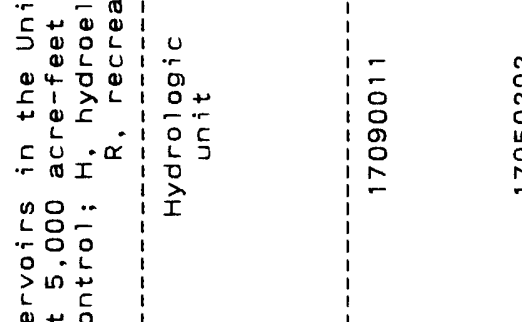

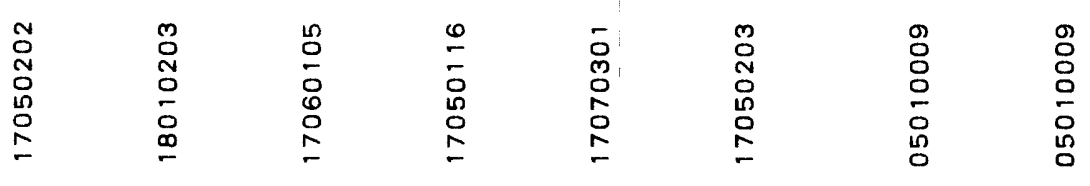

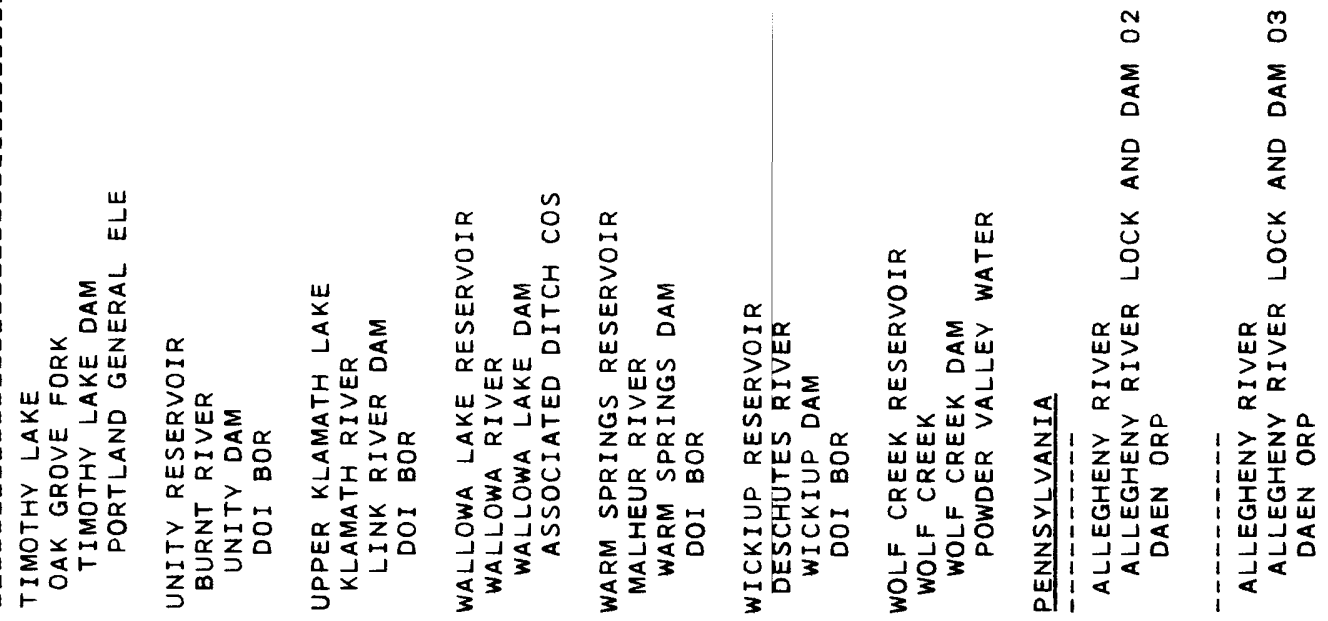




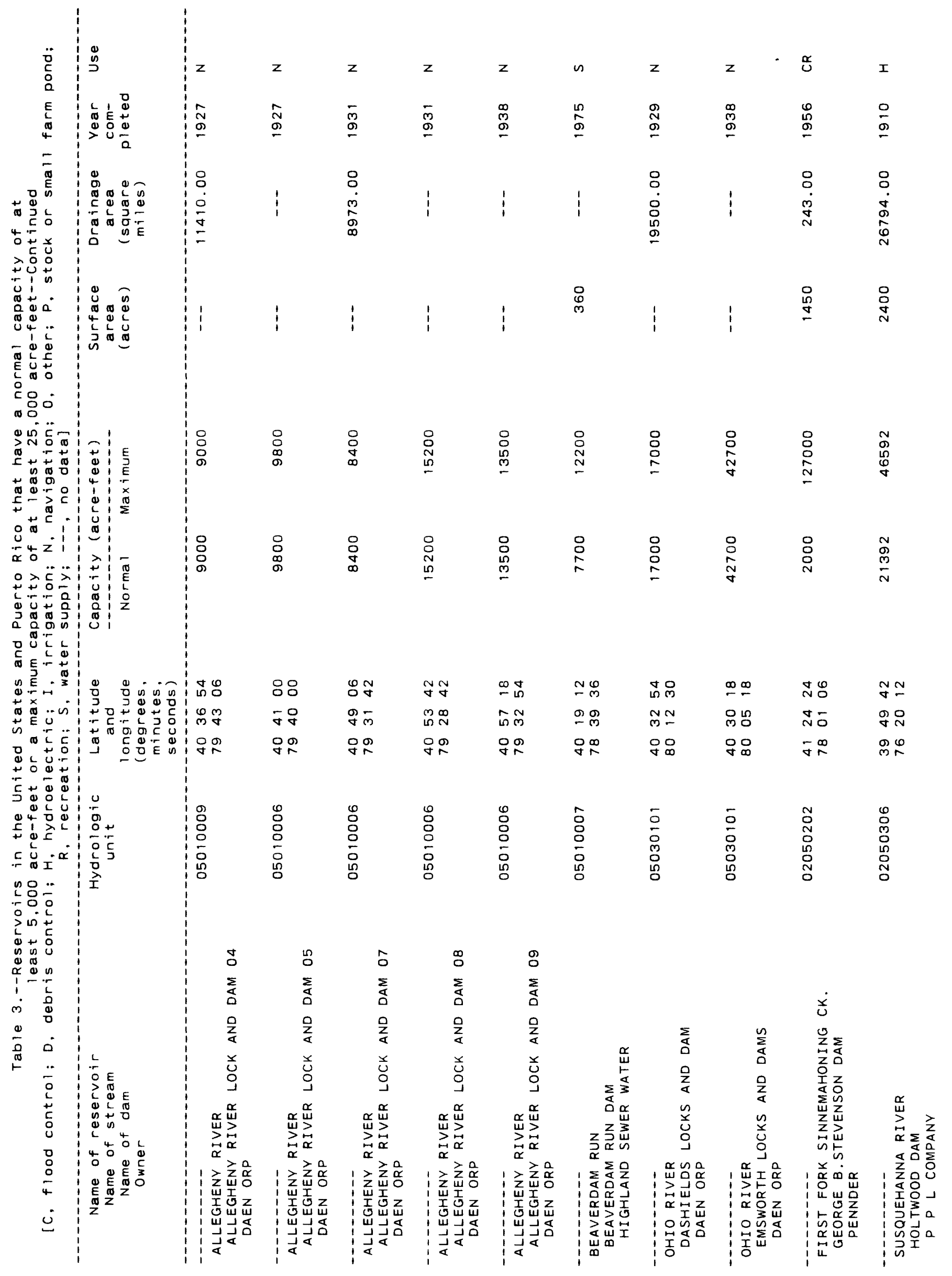




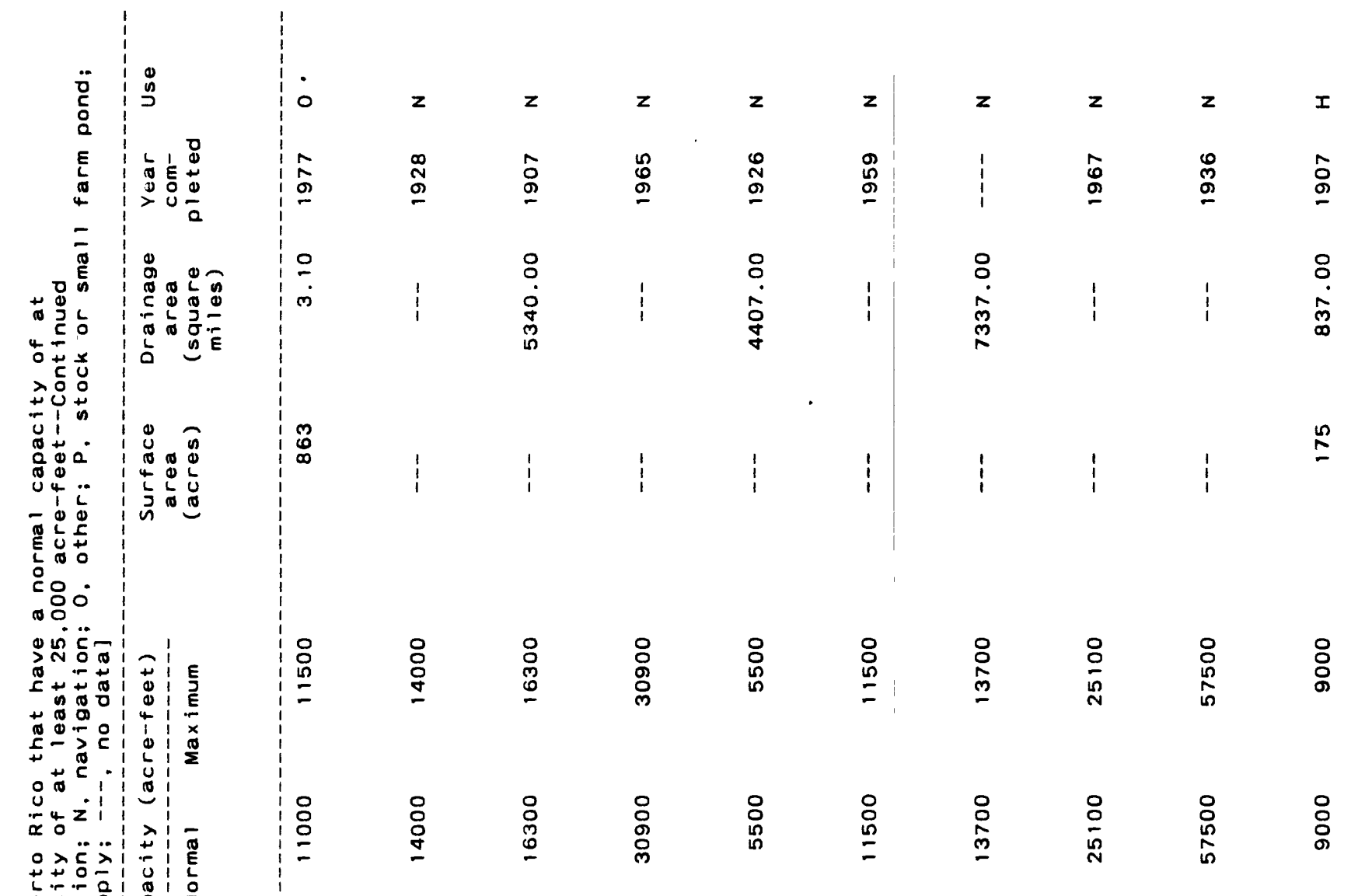

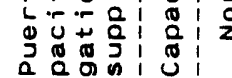

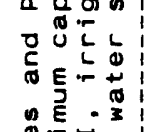

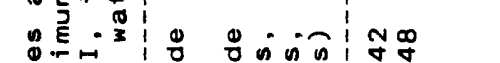

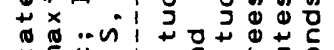

岁E:

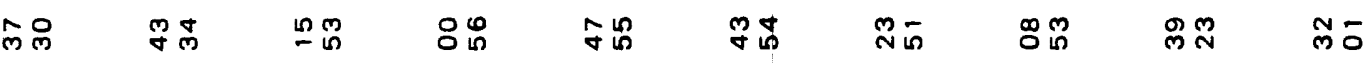

م

웅

웃 웃 웃 윰요

ल요

웅

우요

잉

ㅇำ

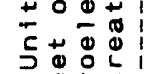

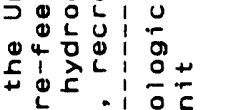

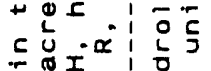

no $\cdots$ I

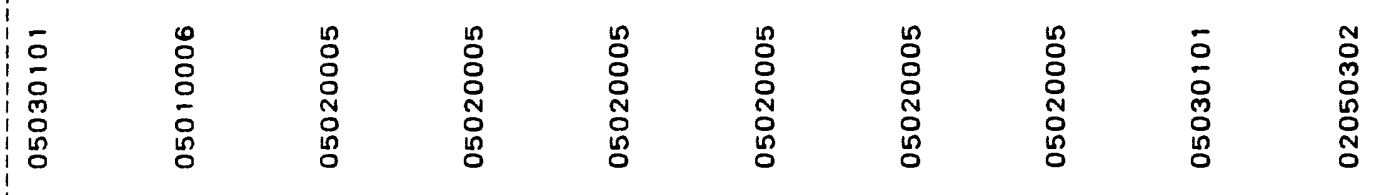

نे

政

舟

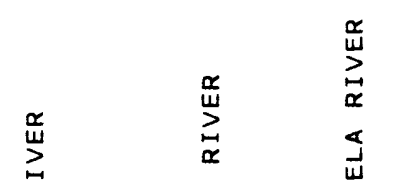

$\begin{array}{l:l:l}0 & & \\ . . & L & \end{array}$

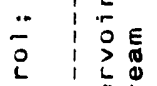

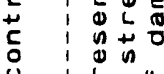

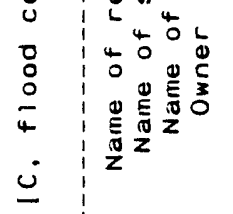

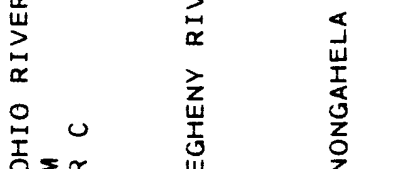

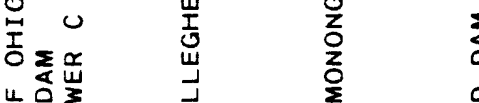

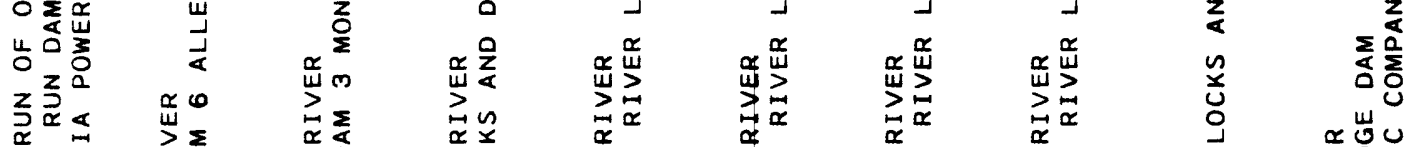

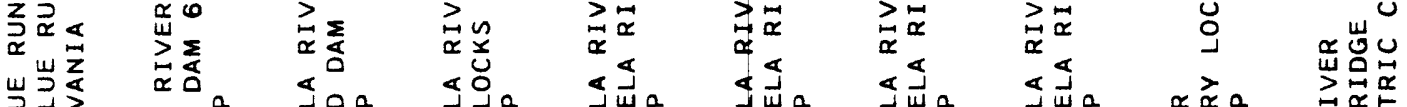

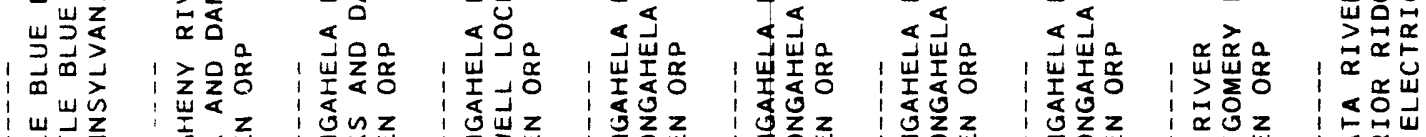

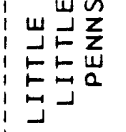

:

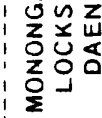

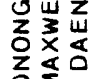

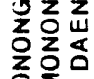

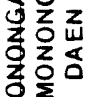

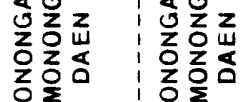

व过西

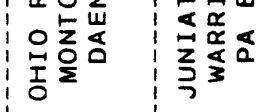




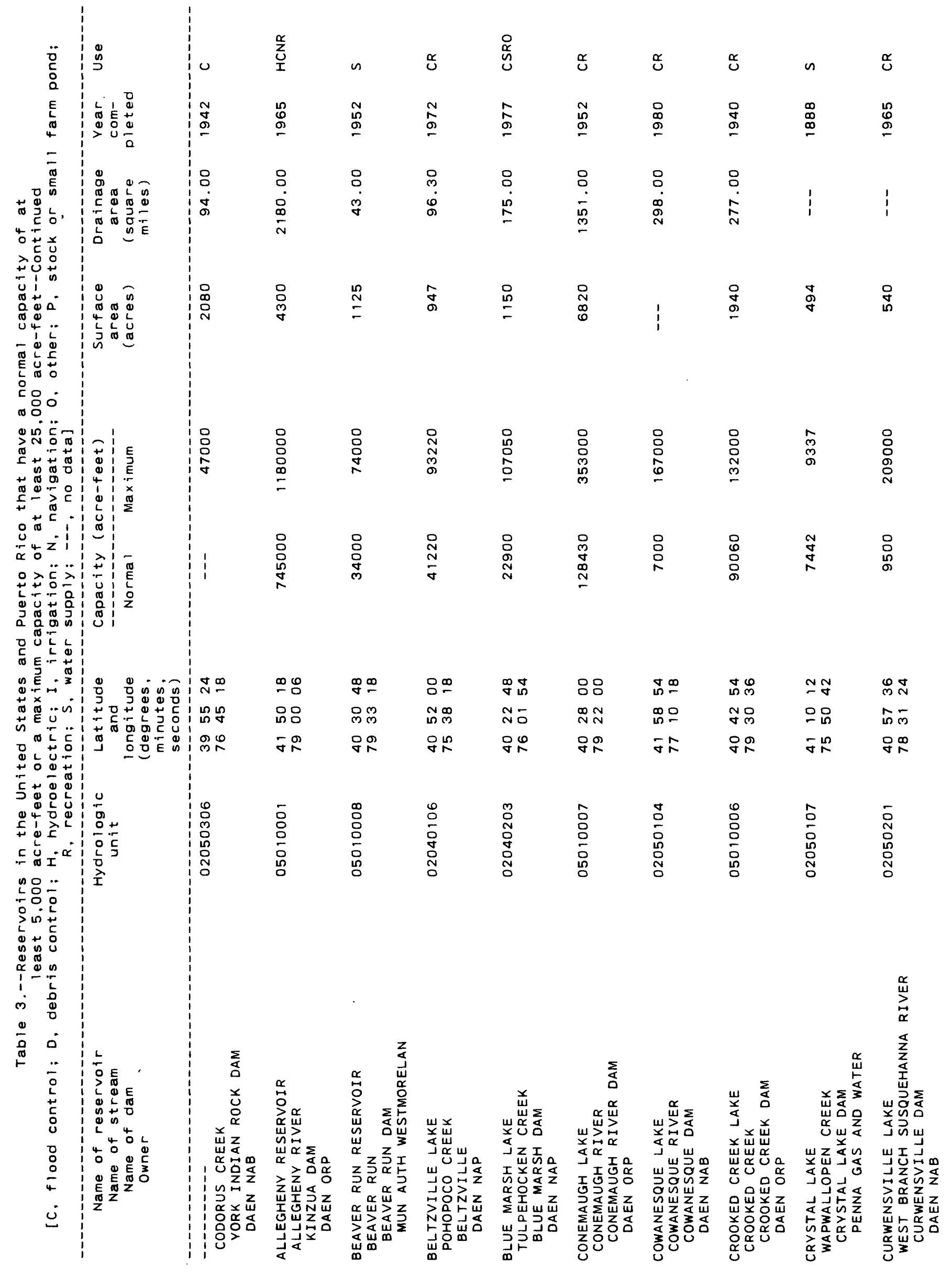




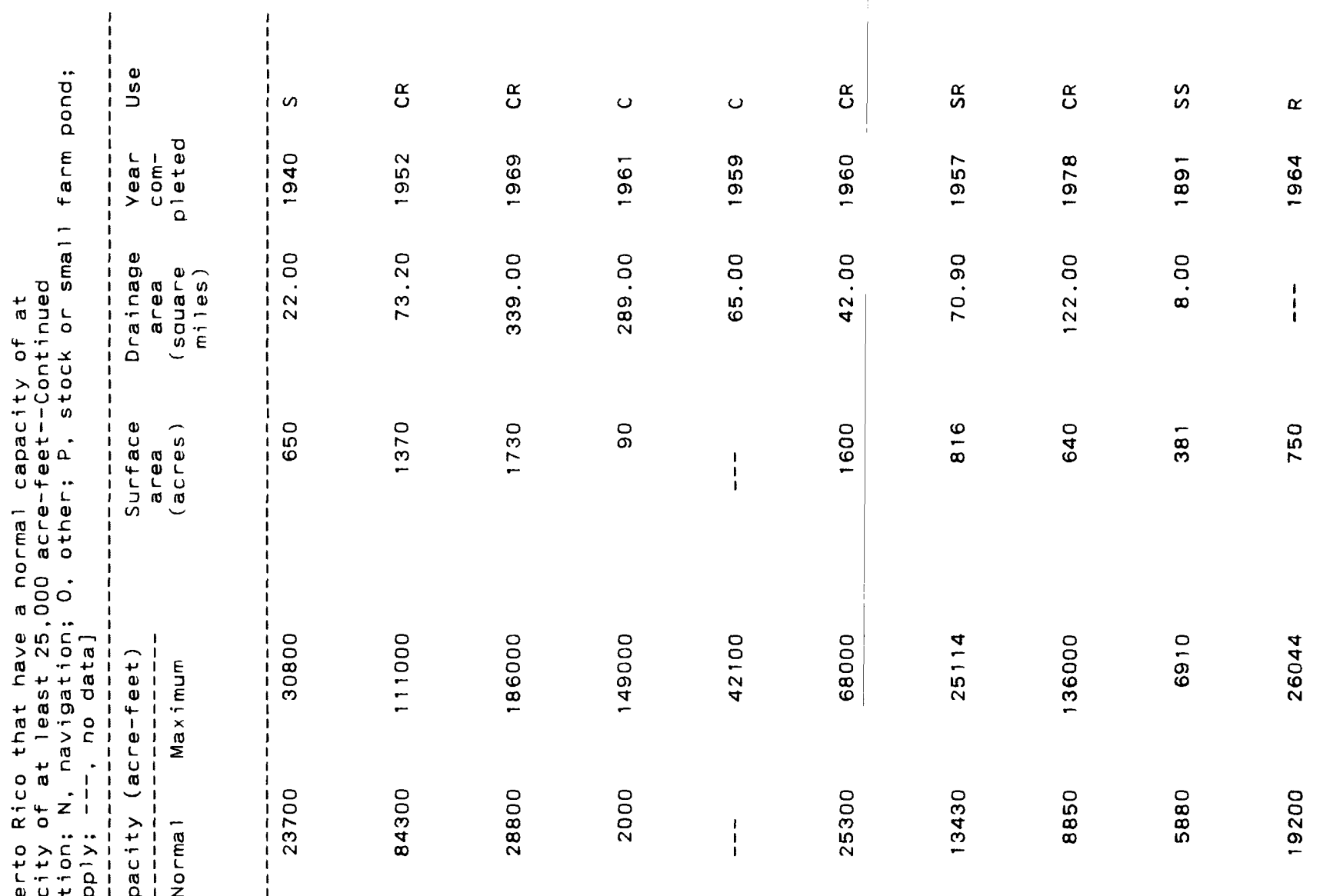

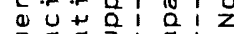

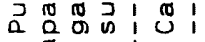

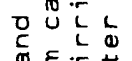

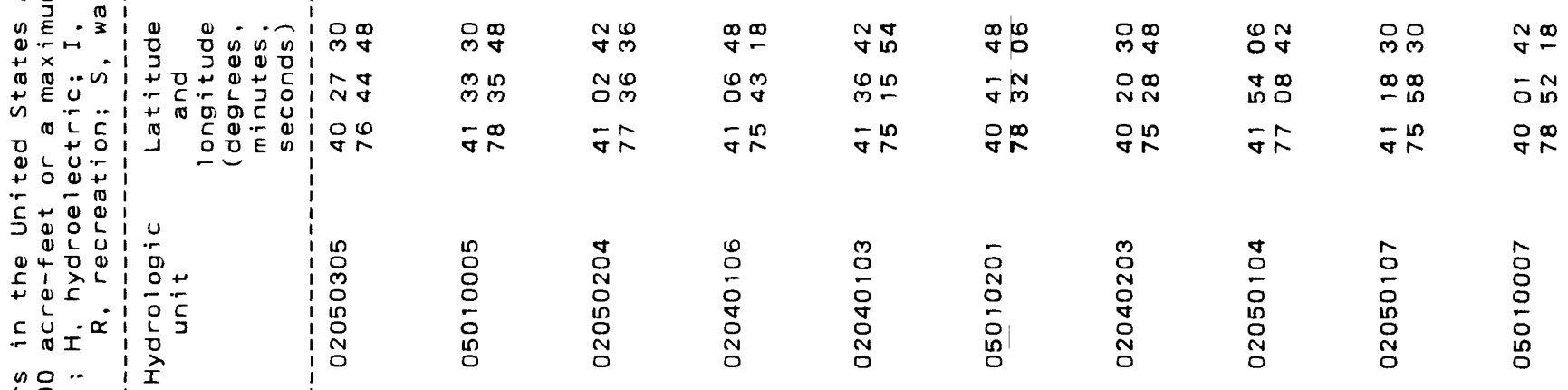

20

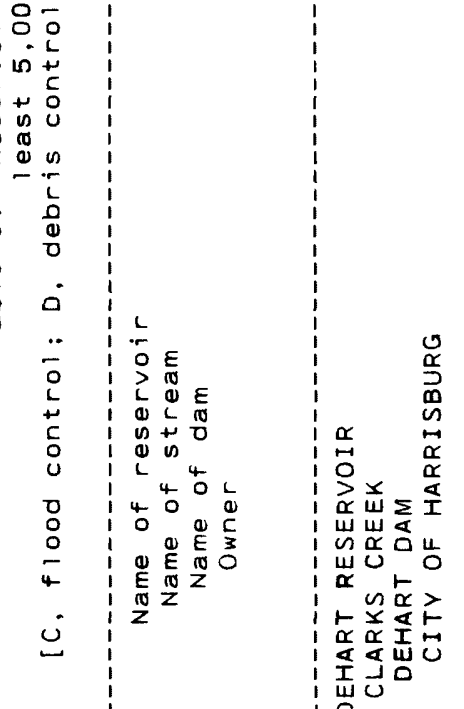

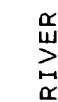

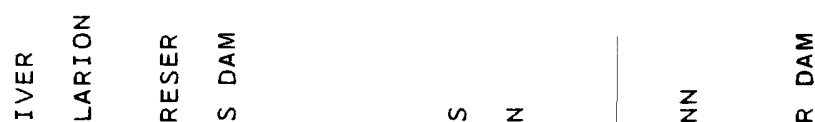

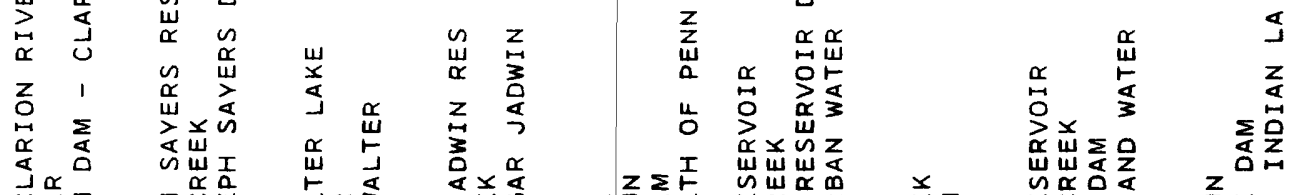

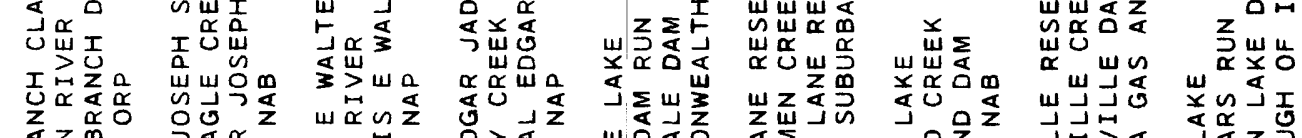

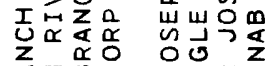

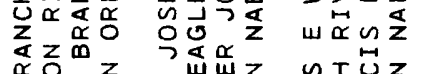
过接

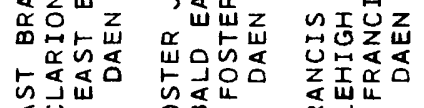

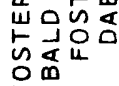

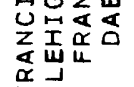

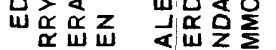

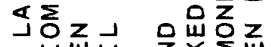

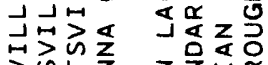

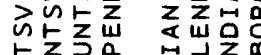

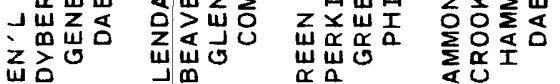

zํㅗㅁำ

员芯 


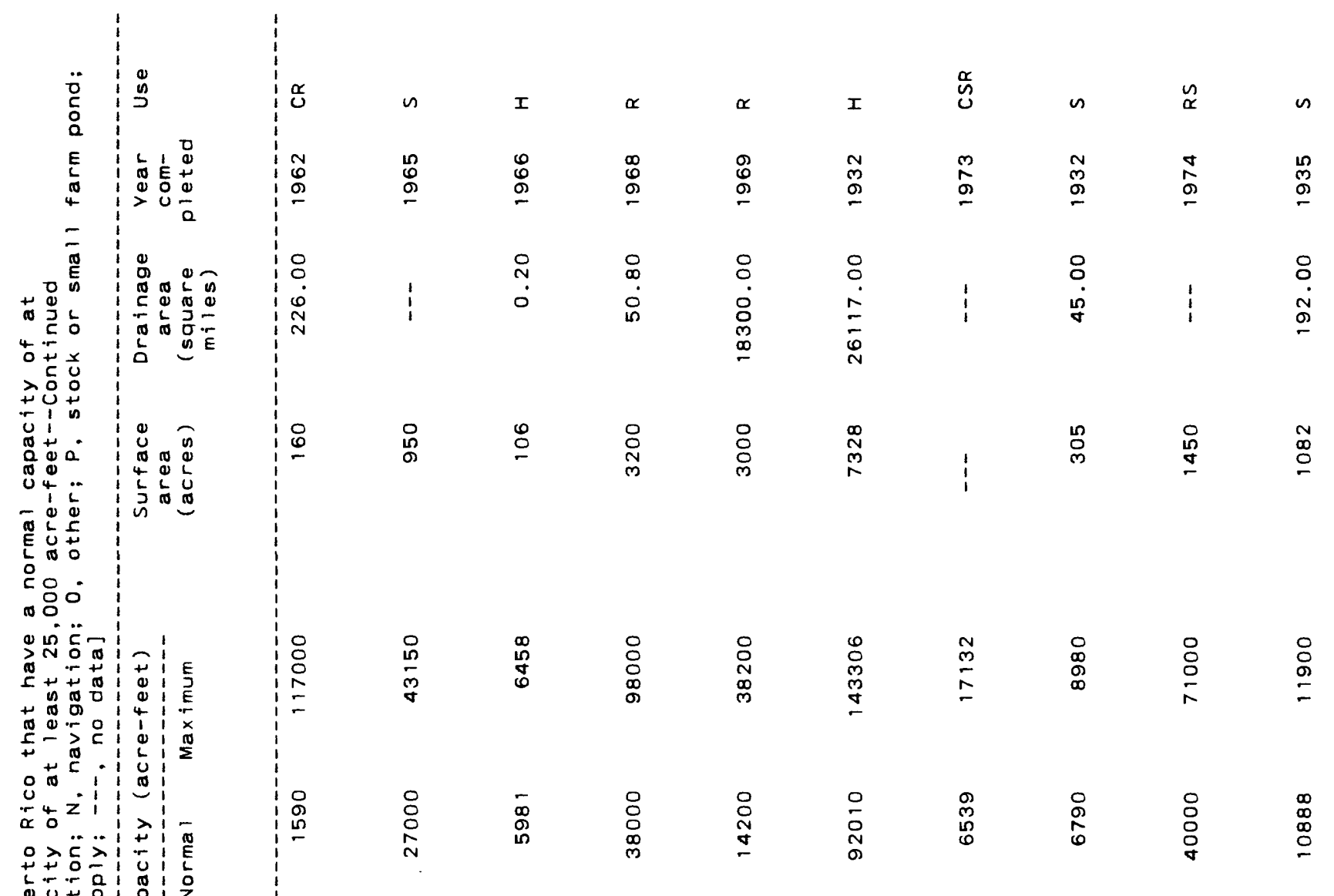

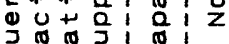

a o

वृ:

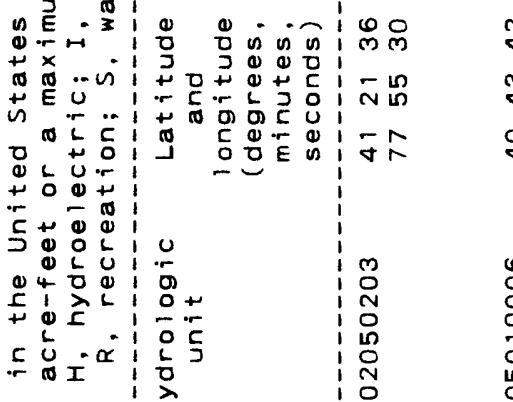

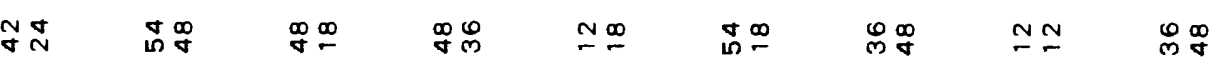

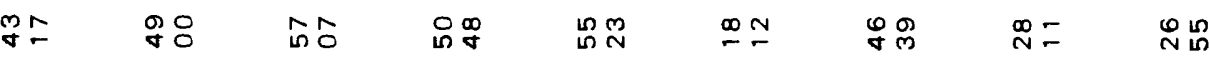

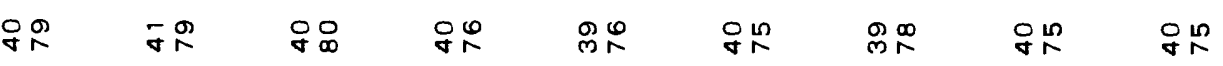

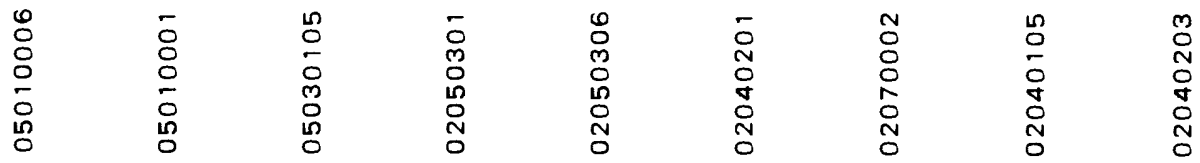

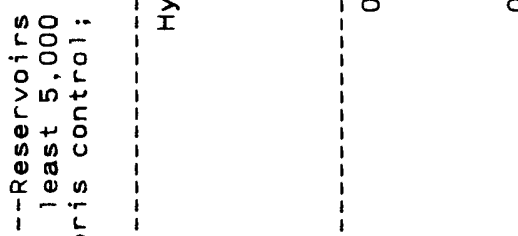

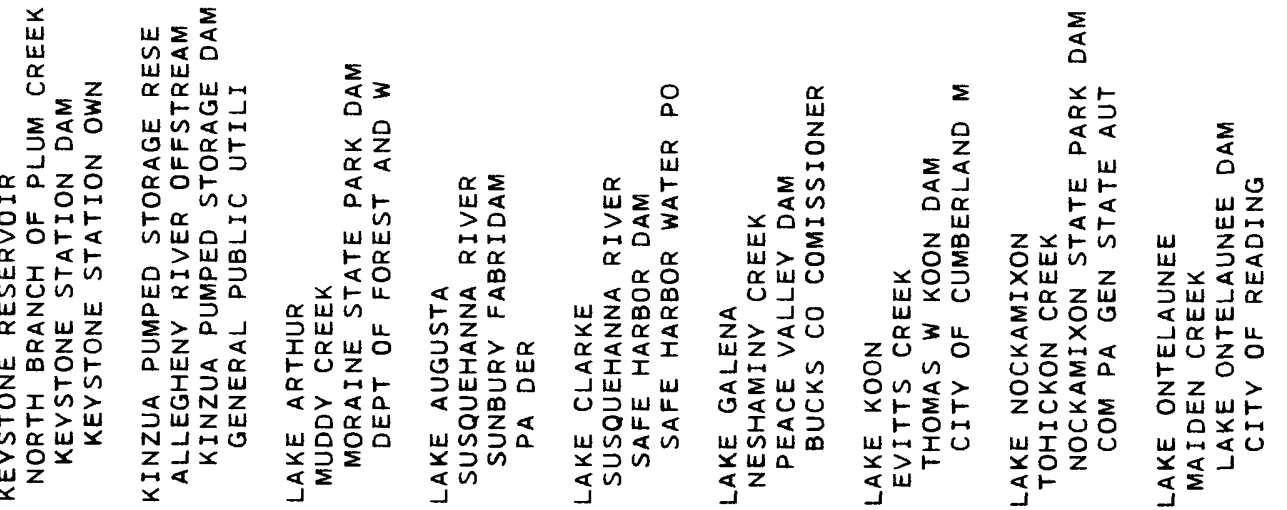




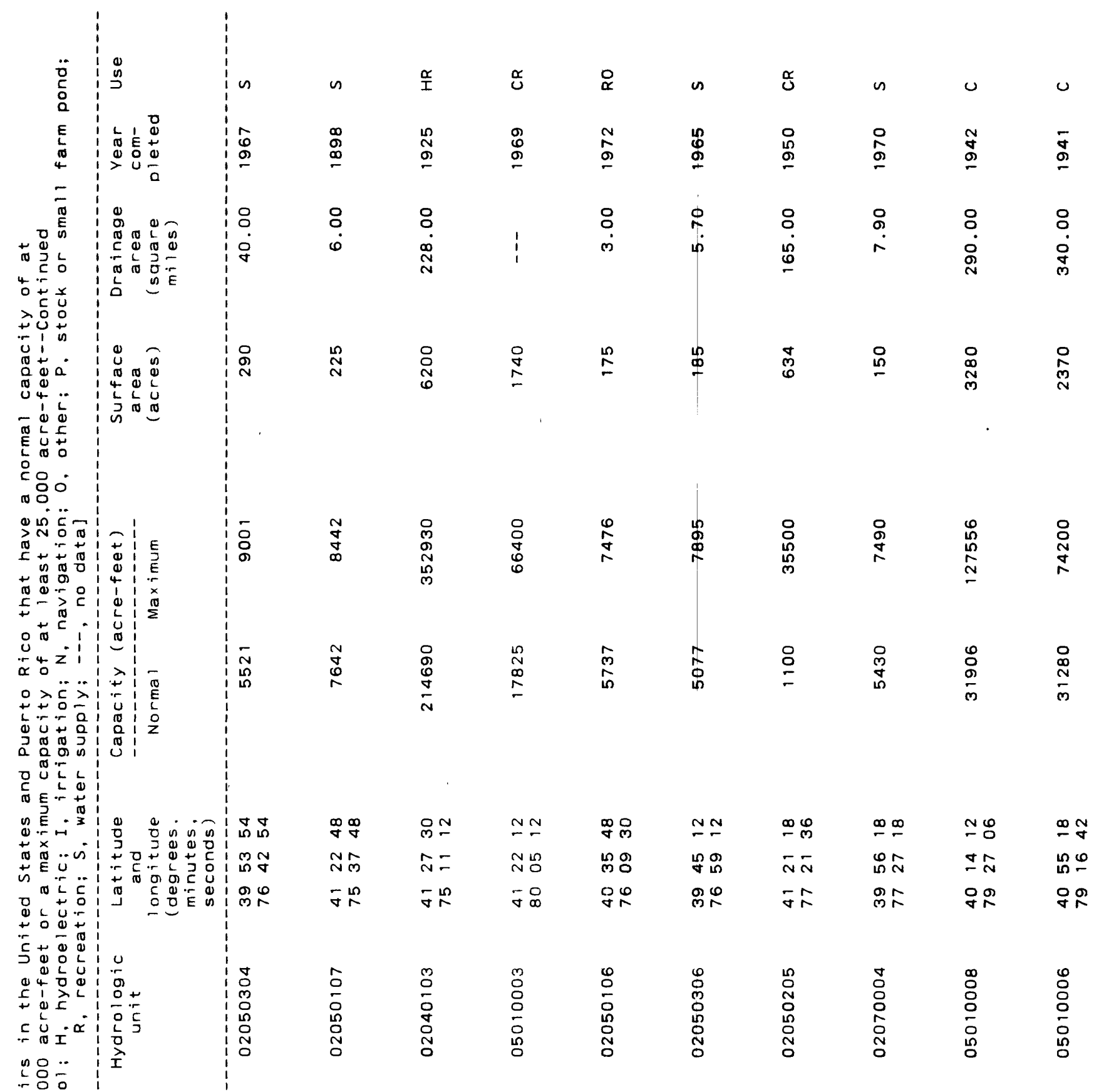




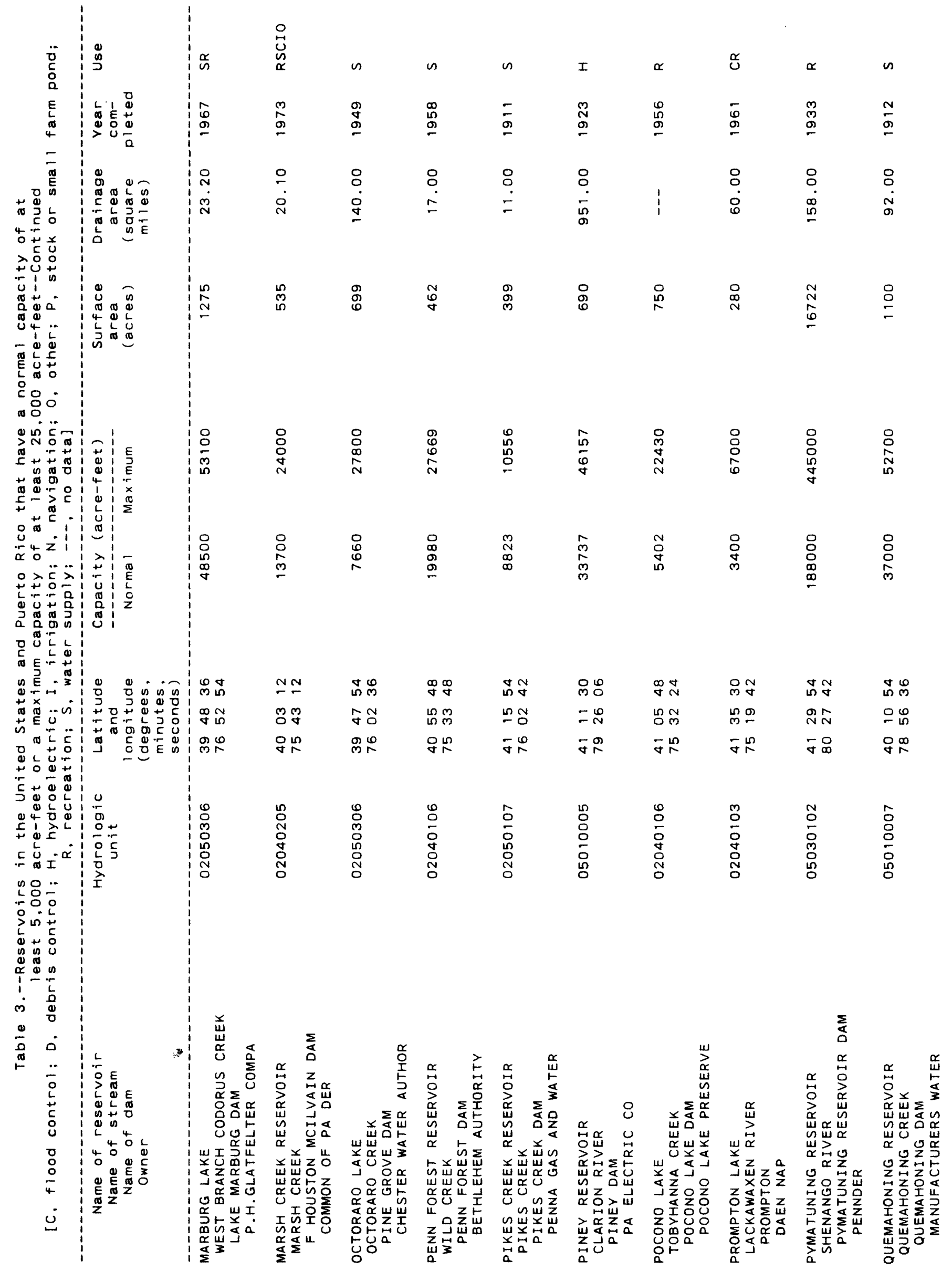




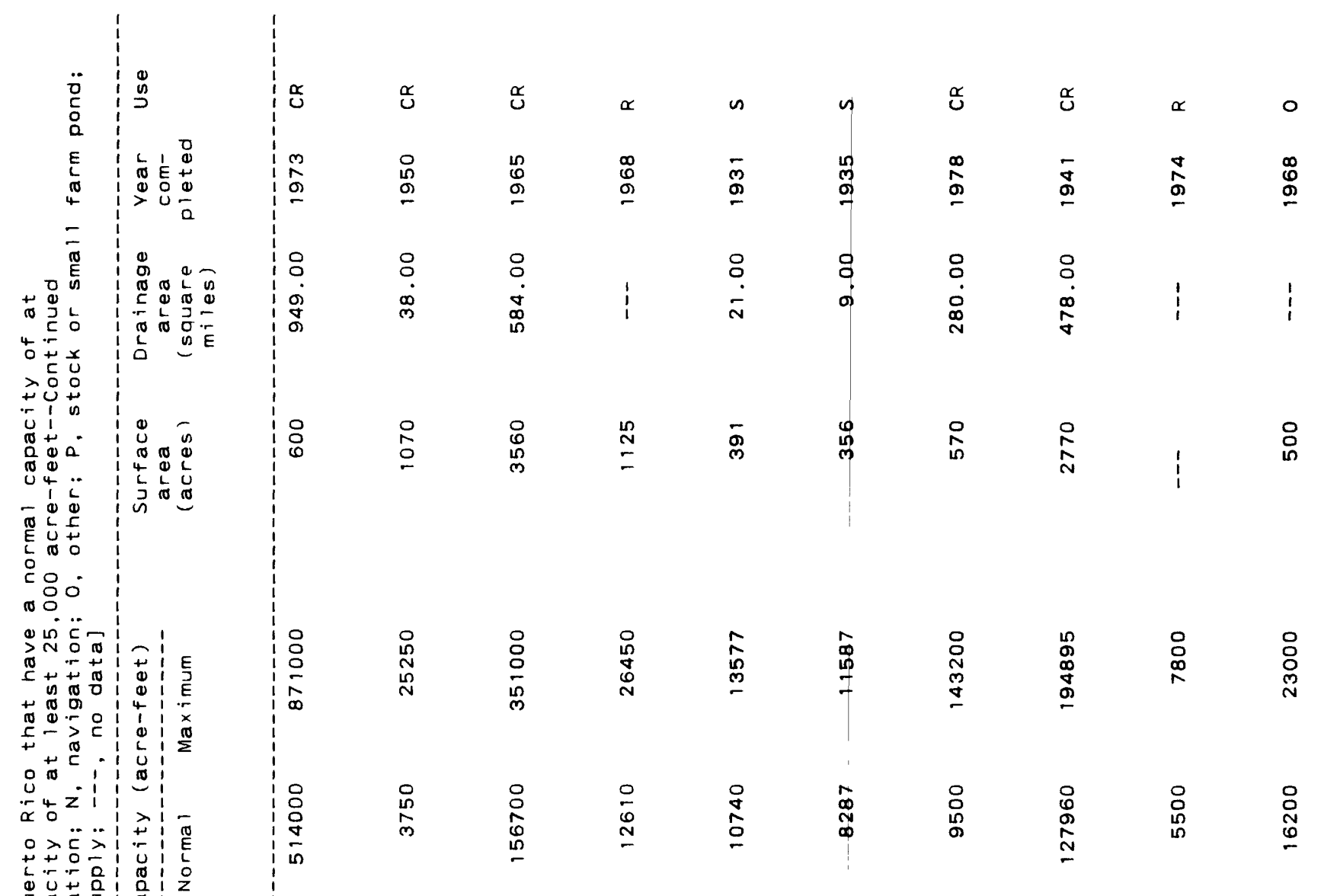

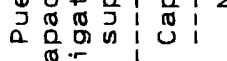

व

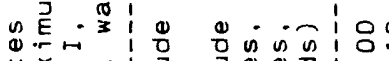

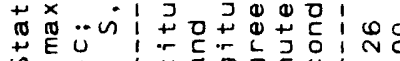

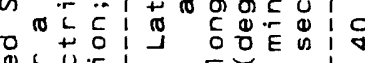

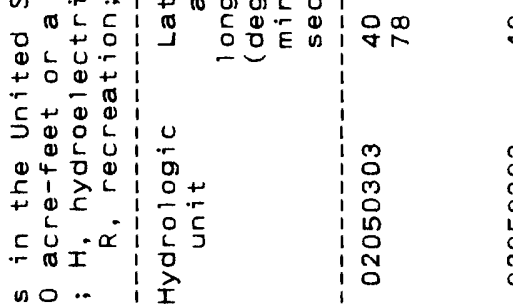

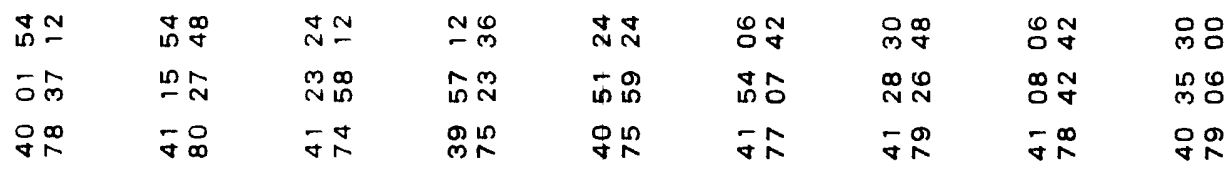

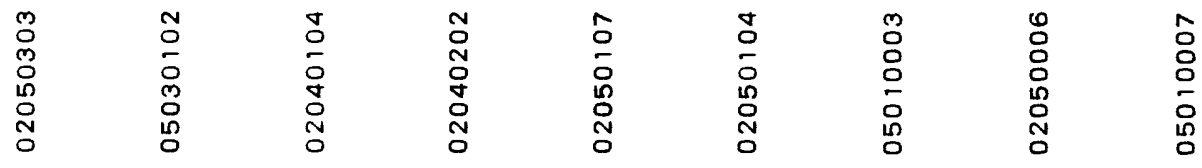

is

20.
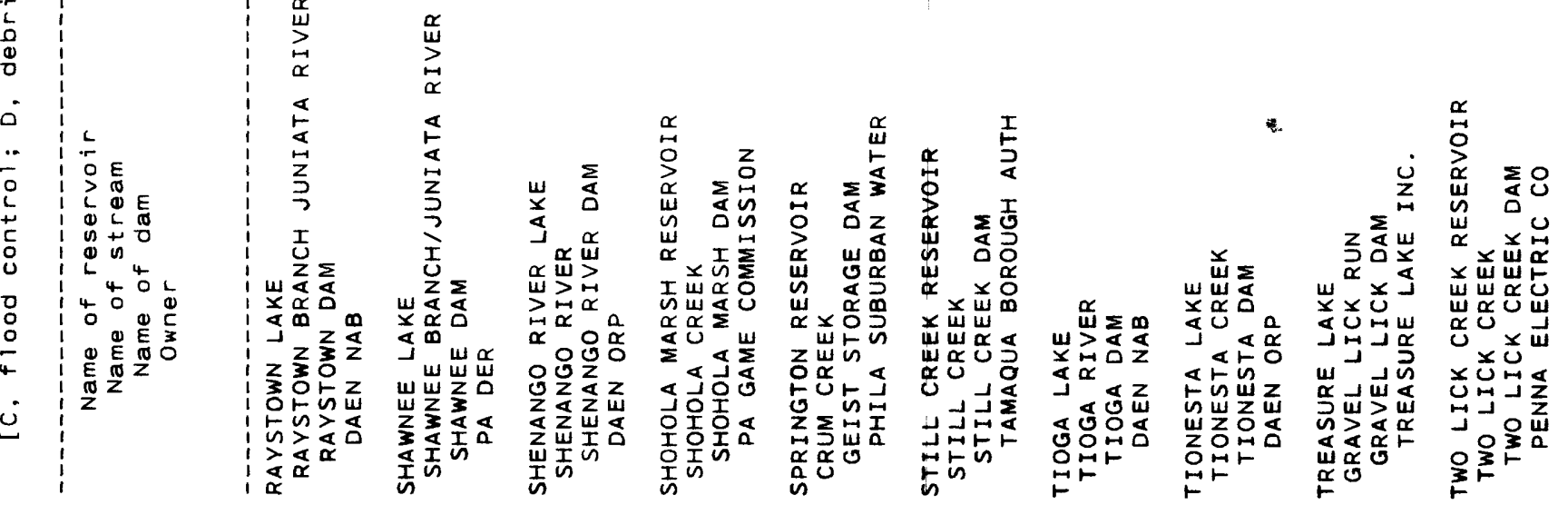


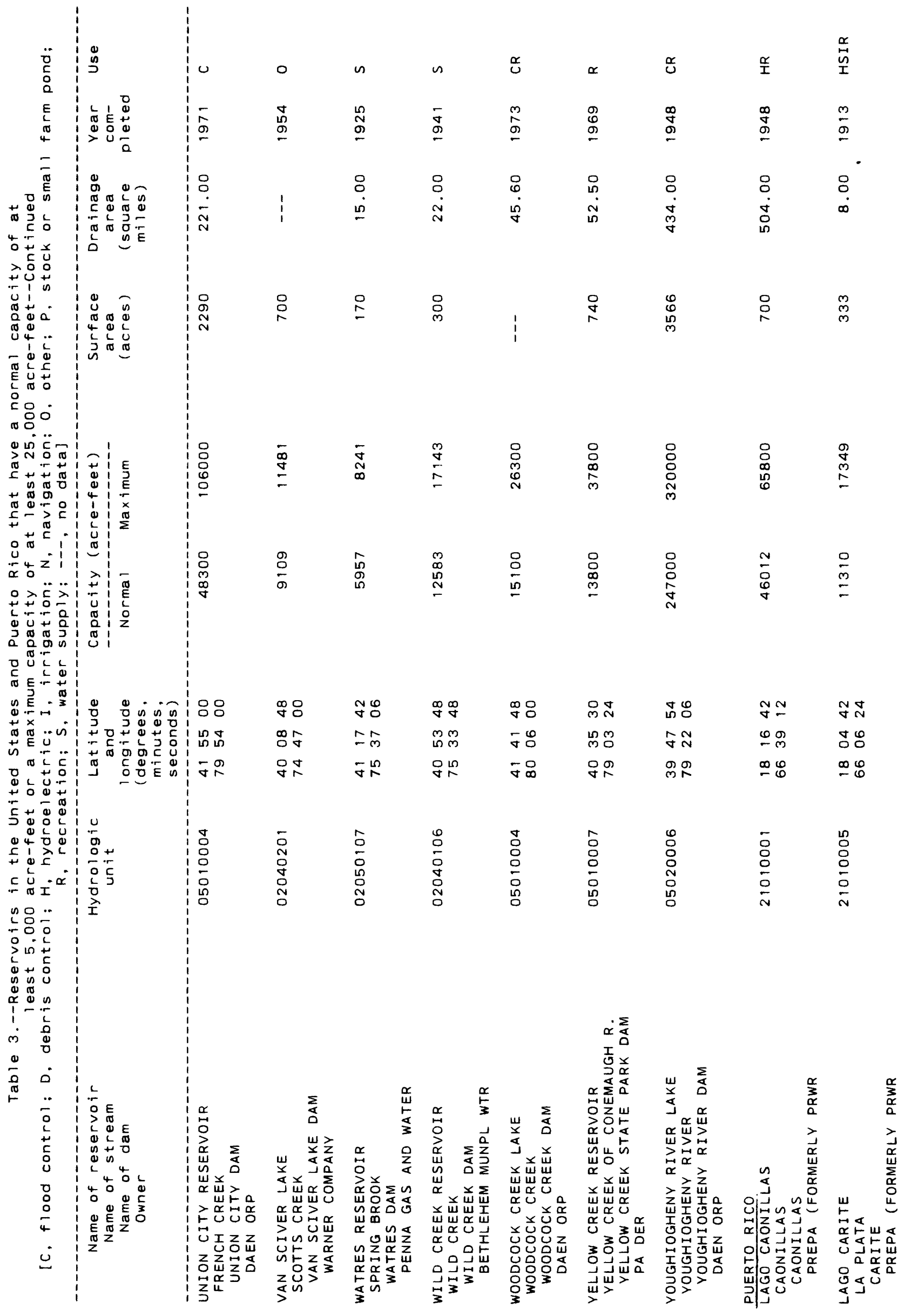




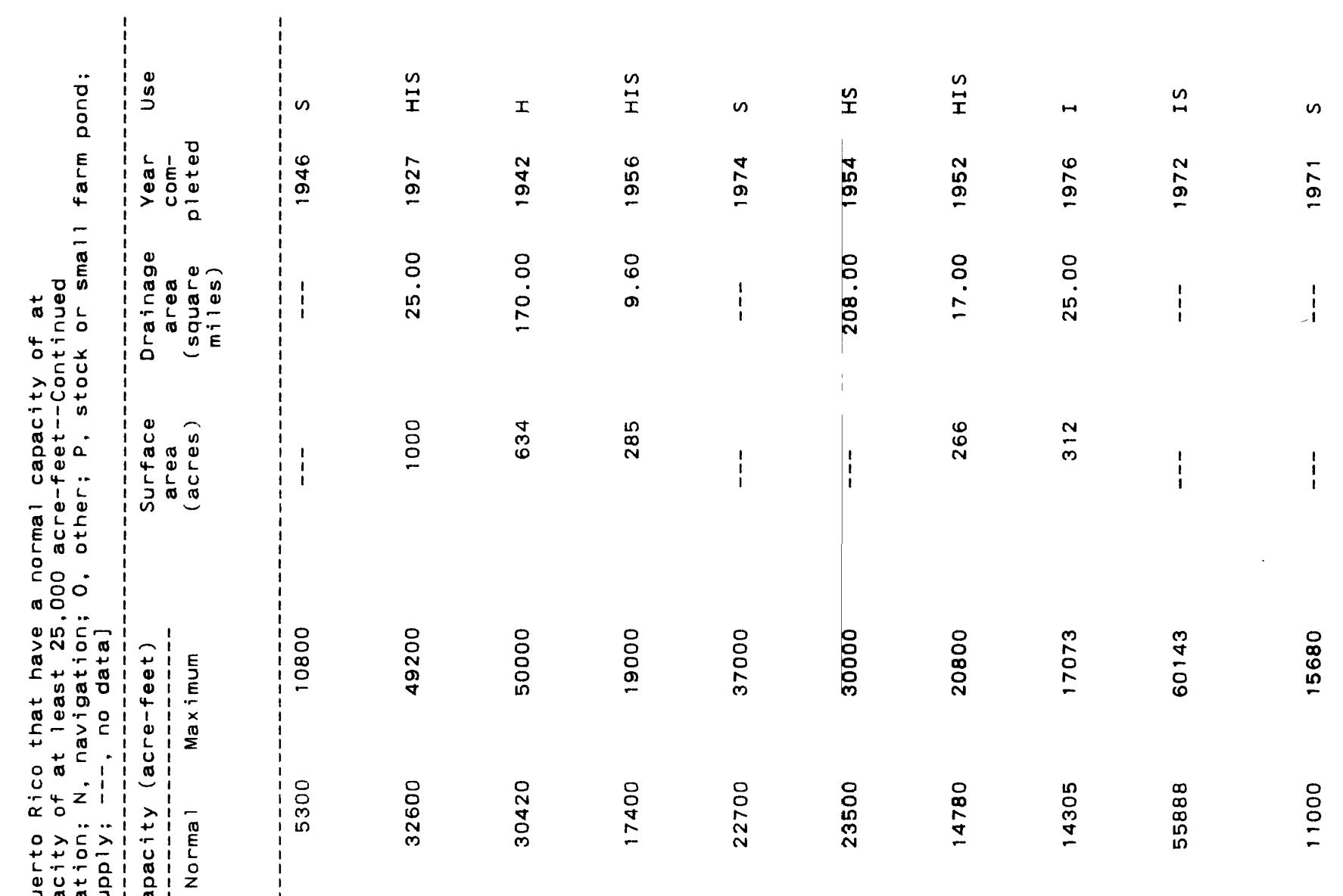

ㅇํ

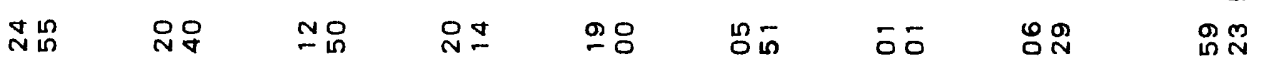

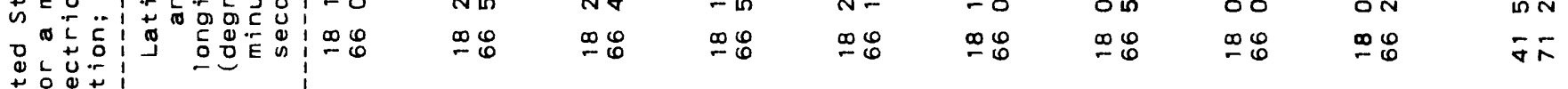

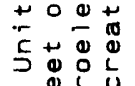

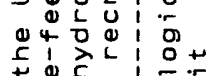

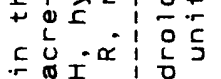

no‥

둥

> n⿺⿻一𠃋十

is

(4)

$1-\frac{\pi}{2}$

ले

$\underset{\frac{1}{0}}{\frac{0}{6}}$ $\stackrel{5}{\circ}$

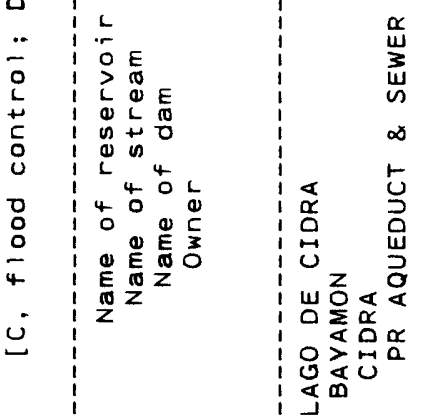

$\begin{array}{ll}\frac{0}{0} & \overline{0} \\ \frac{0}{2} & \frac{0}{N}\end{array}$

$\begin{array}{ll}0 & \stackrel{0}{0} \\ \frac{0}{0} & \frac{8}{0} \\ \frac{0}{2} & \frac{0}{2}\end{array}$

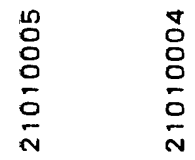

$\begin{array}{ll}\frac{D}{2} & \frac{D}{0} \\ \frac{0}{0} & \frac{0}{N}\end{array}$

3
8
8
8
0
0

妾

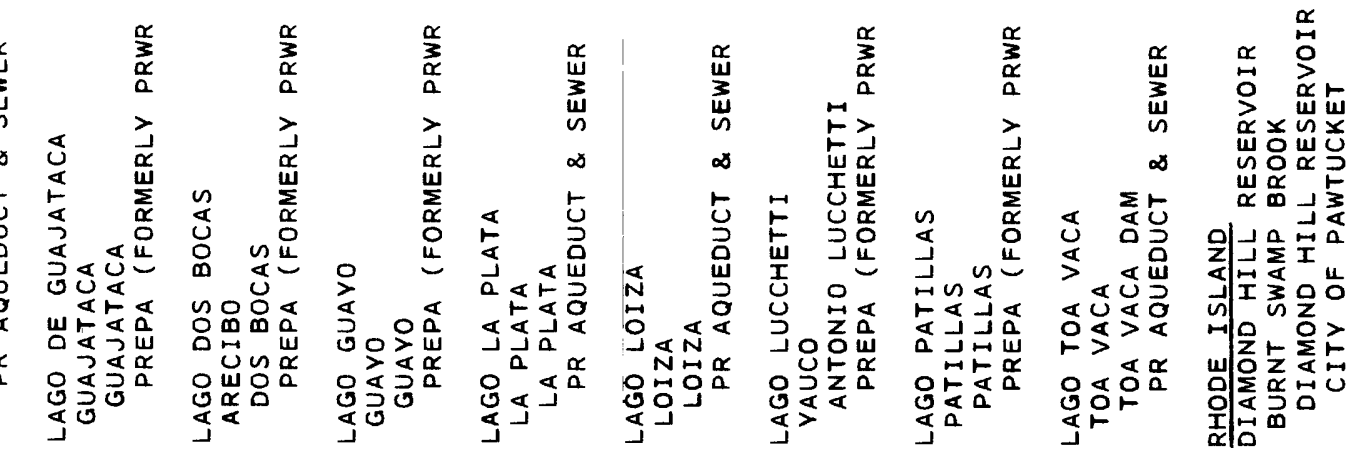




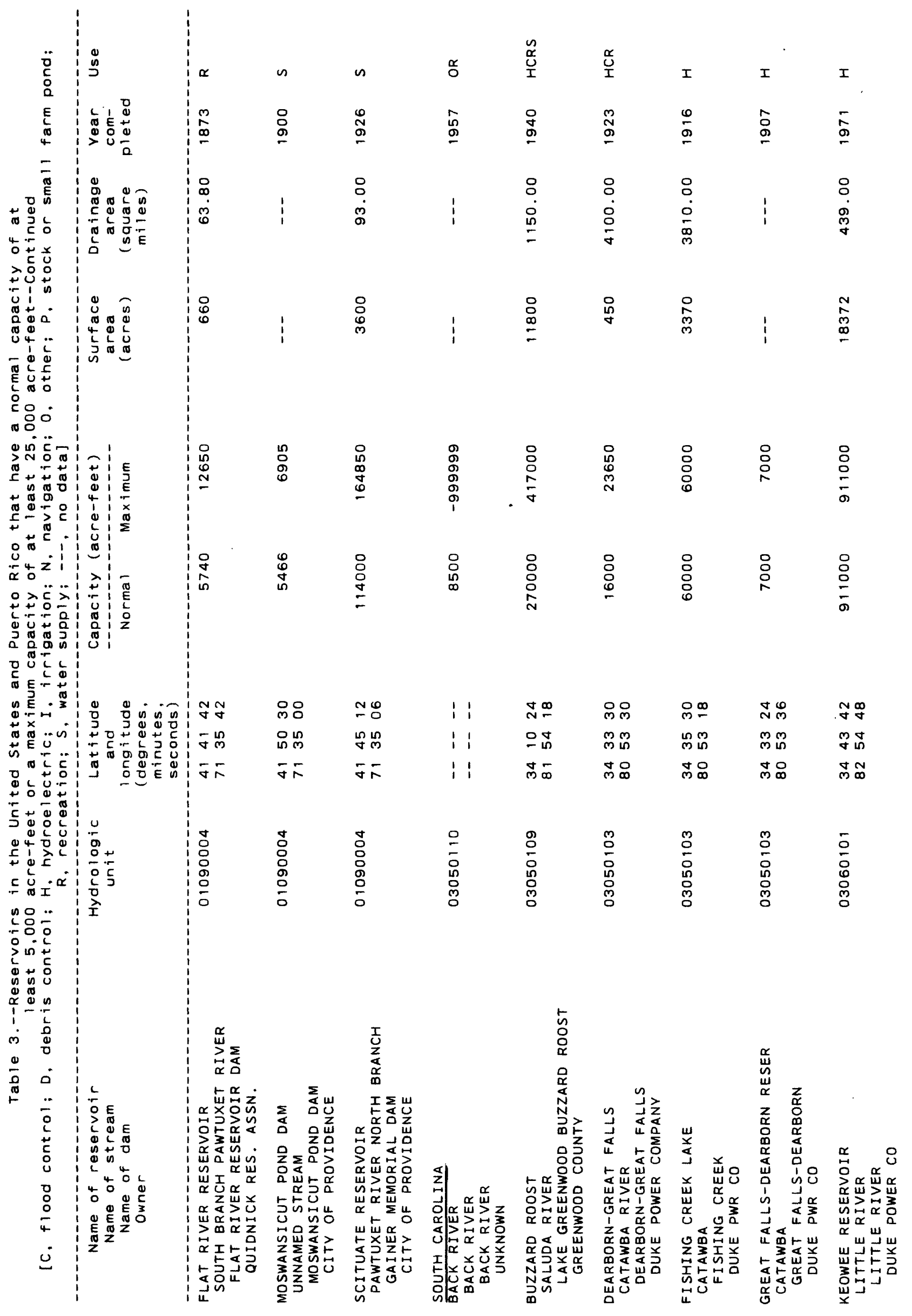




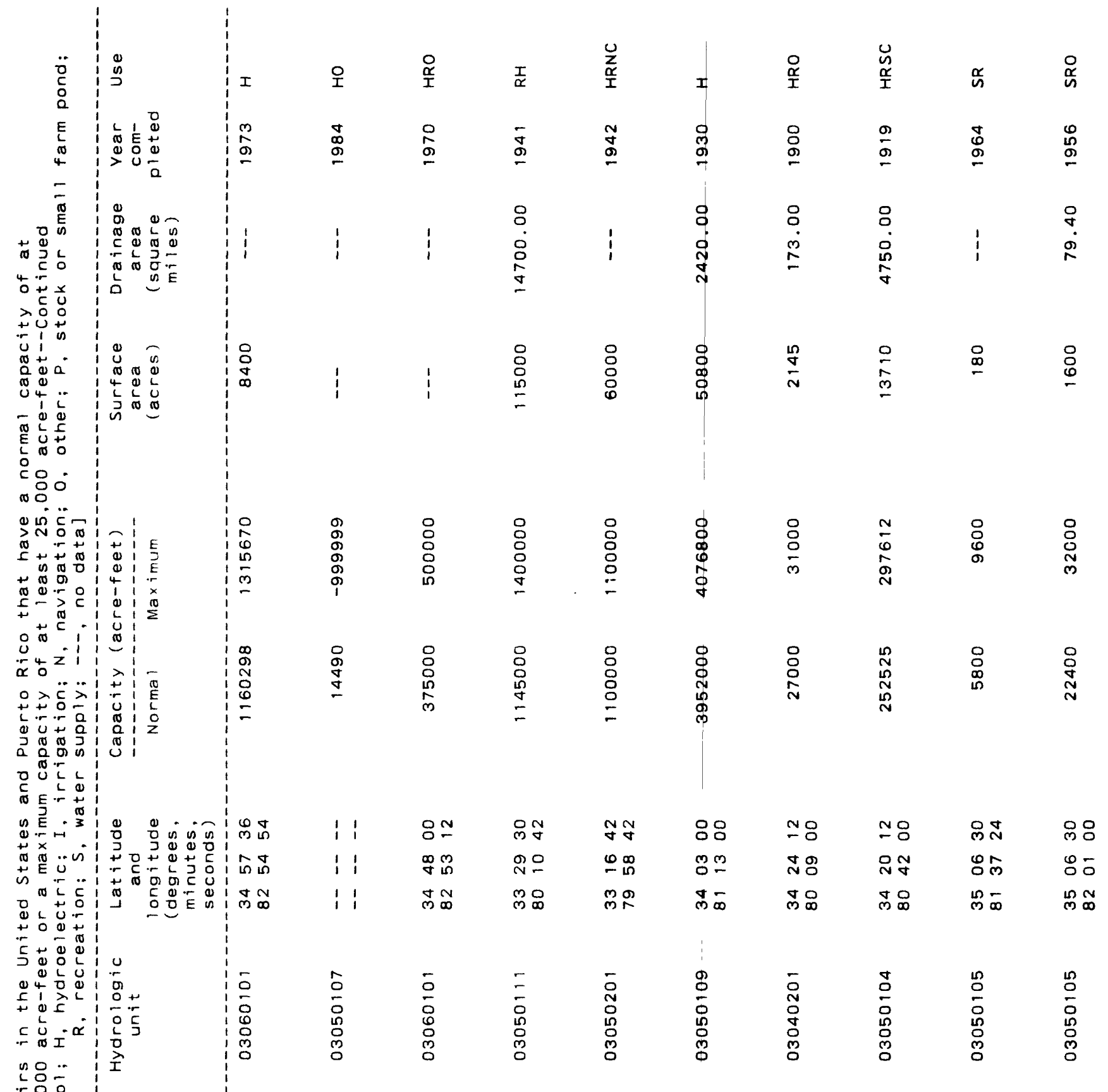




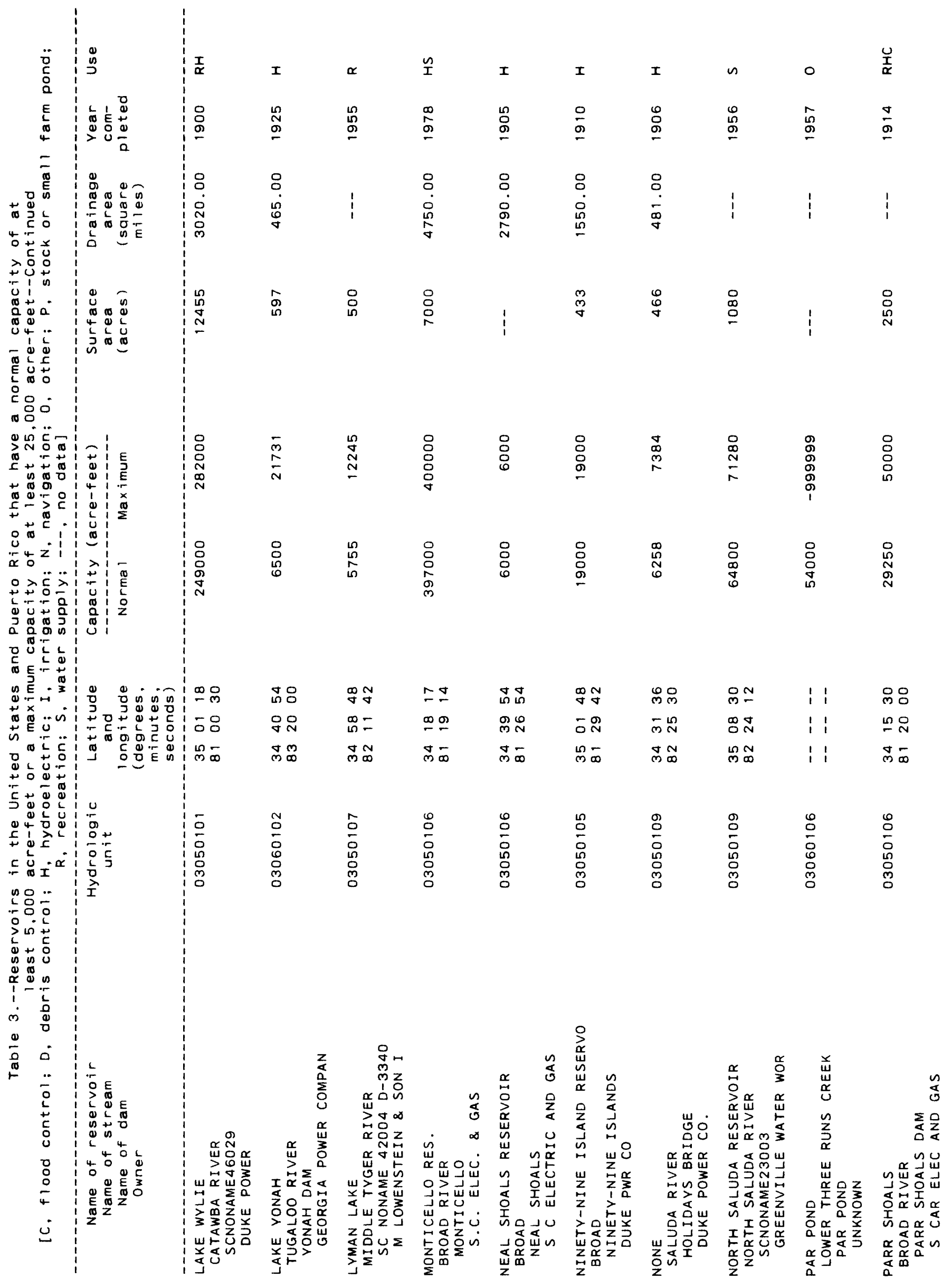




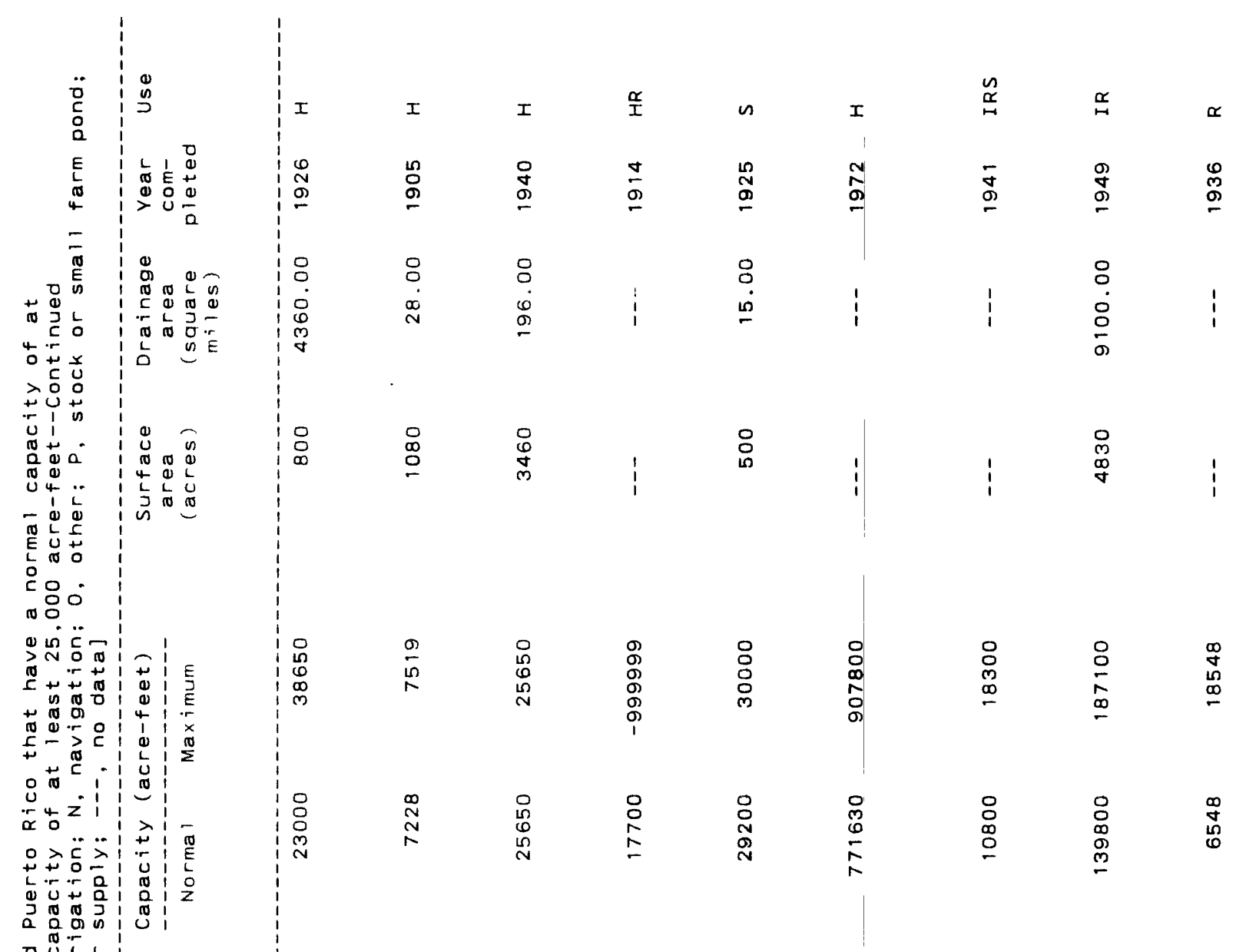

DU L

מ⿹

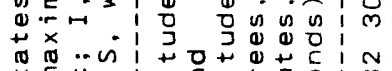

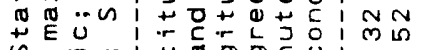

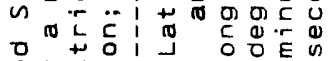

गे

\begin{tabular}{|c|c|c|c|c|c|c|c|}
\hline $\begin{array}{l}0 \\
00\end{array}$ & 이료 & 13 & $\stackrel{\infty}{\rightarrow} \stackrel{\infty}{-1}$ & $\check{m}^{\infty}$ & সD & $\stackrel{\infty}{\infty} \stackrel{\infty}{-1}$ & W F \\
\hline $\bar{~} \bar{N}$ & $\stackrel{n}{\infty}$ & i i & 웅ㅇ & กิ & 응 & $\stackrel{\sim}{N} \stackrel{0}{N}$ & $\underset{n}{\infty} \tilde{n}$ \\
\hline$\underset{m}{\sim}$ & সల్ల & i i & D్ & లెల & $\stackrel{m}{\rightarrow} \underset{0}{ }$ & m9 & m \\
\hline
\end{tabular}

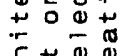

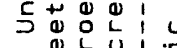

(1)

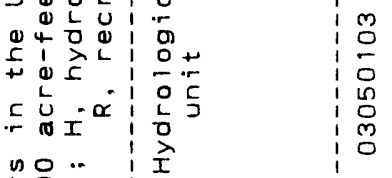

$\begin{array}{lll}0 & 0 \\ 0 & 0 \\ 0 & 0 \\ 0 & 0 \\ 0 & 0 \\ 0 & 0 \\ 0 & 0 \\ 0 & 0\end{array}$

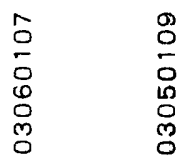

$\begin{array}{lll}5 & 0 & 0 \\ & 0 & 0 \\ 0 & 0 & 0 \\ 0 & 0 & 0\end{array}$

品

$>$

年

舟。

i.

\begin{tabular}{ll|l|l}
0 & 0 & 0 & 0 \\
0 & 0 & 0 & 0
\end{tabular}

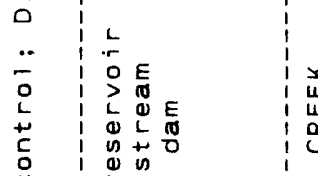

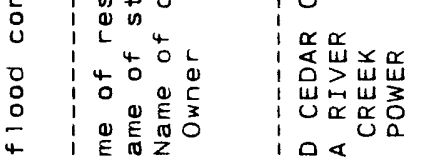

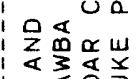

《造焉

过
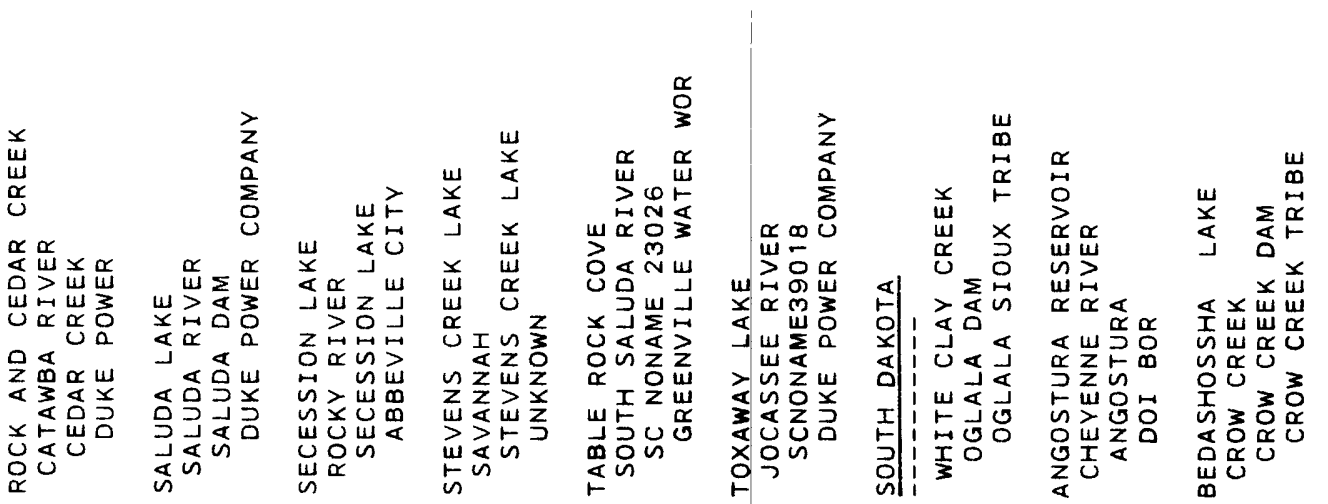


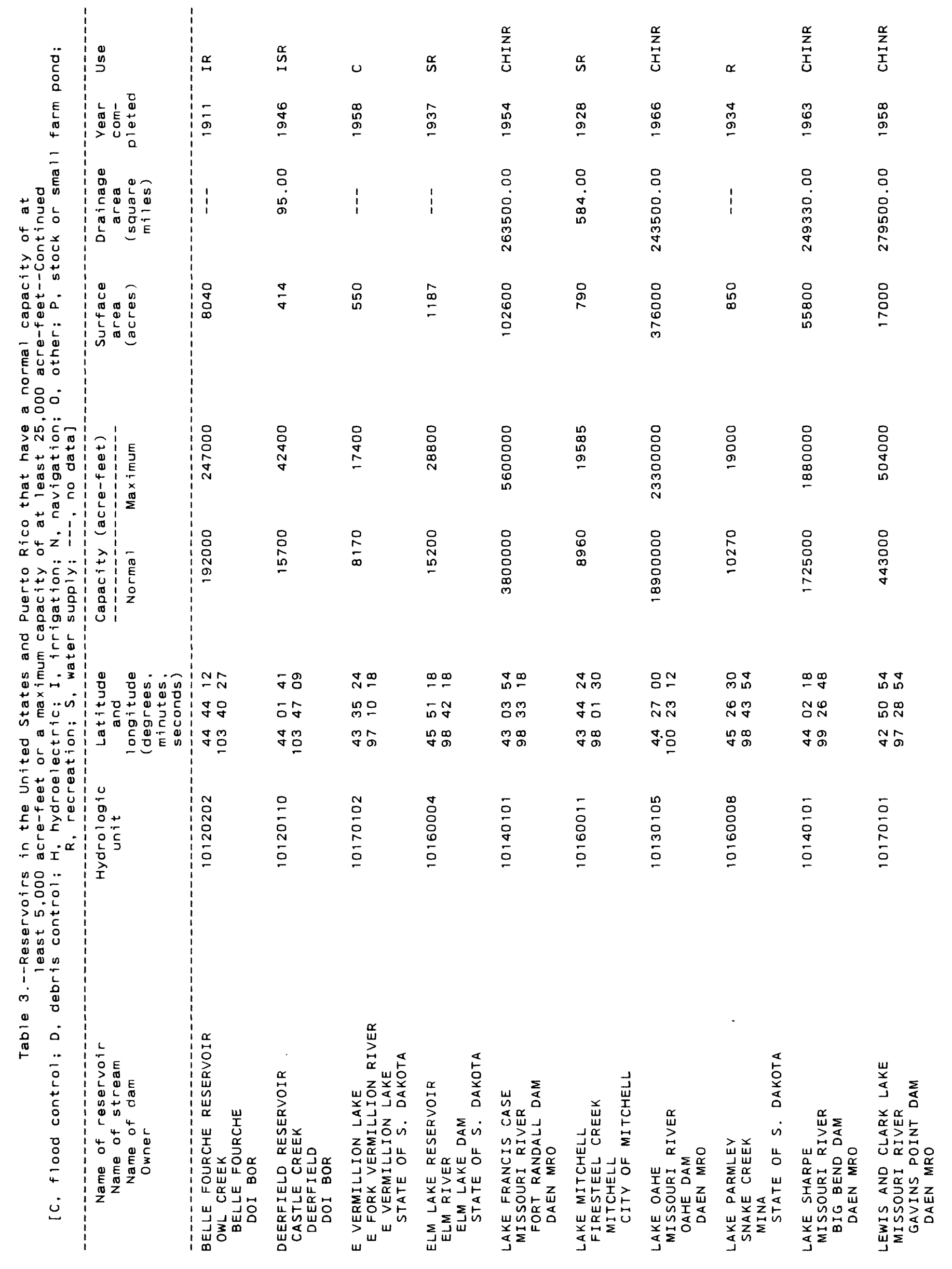




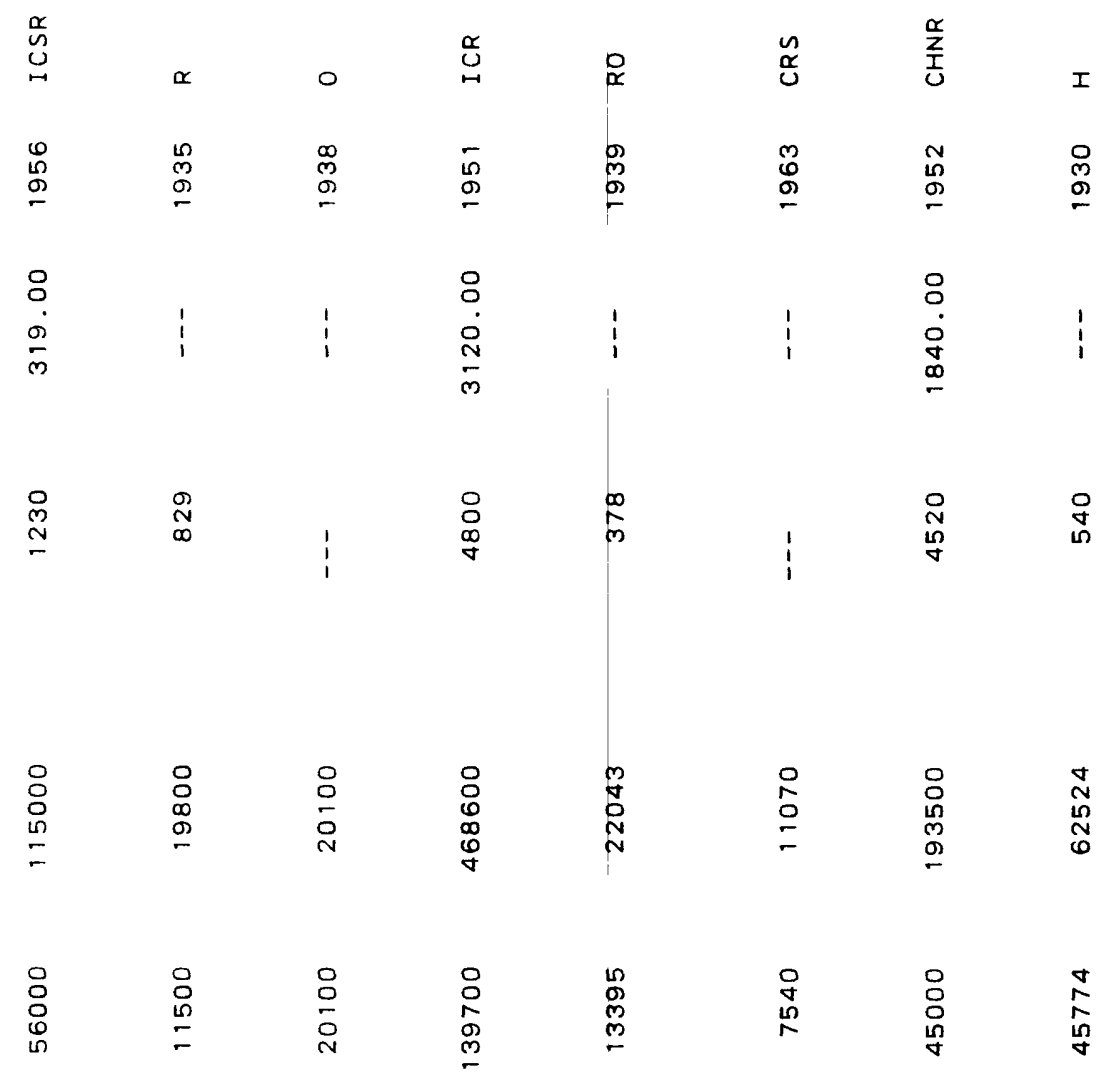

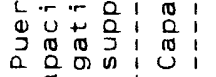

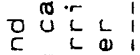

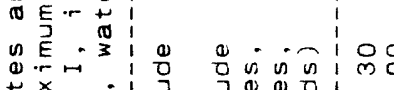

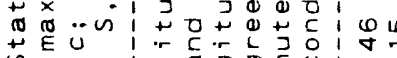

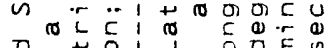

$\stackrel{\infty}{N}$

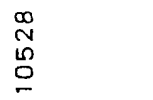

$\begin{array}{lll}0 & 0 & 0 \\ & 0 & 0 \\ 0 & 0 & 0\end{array}$

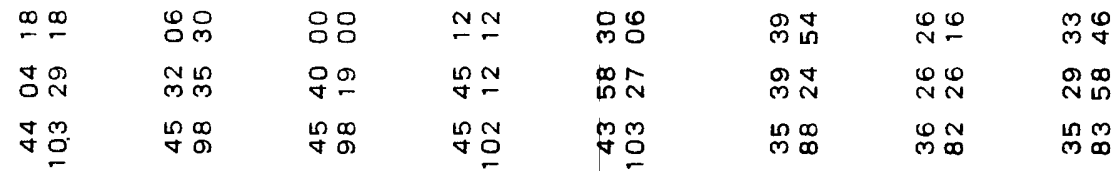

c+

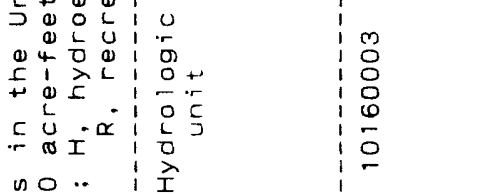

$\begin{array}{lllll}0 & m & m & 0 & 0 \\ 0 & 0 & 0 & 0 \\ 0 & 0 & 0 & 0 \\ & 0 & 0 & 0 & 0 \\ 0 & 0 & 0 & 0 \\ 0 & 0 & 0 & 0 & 0\end{array}$

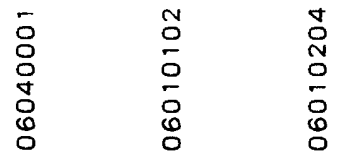
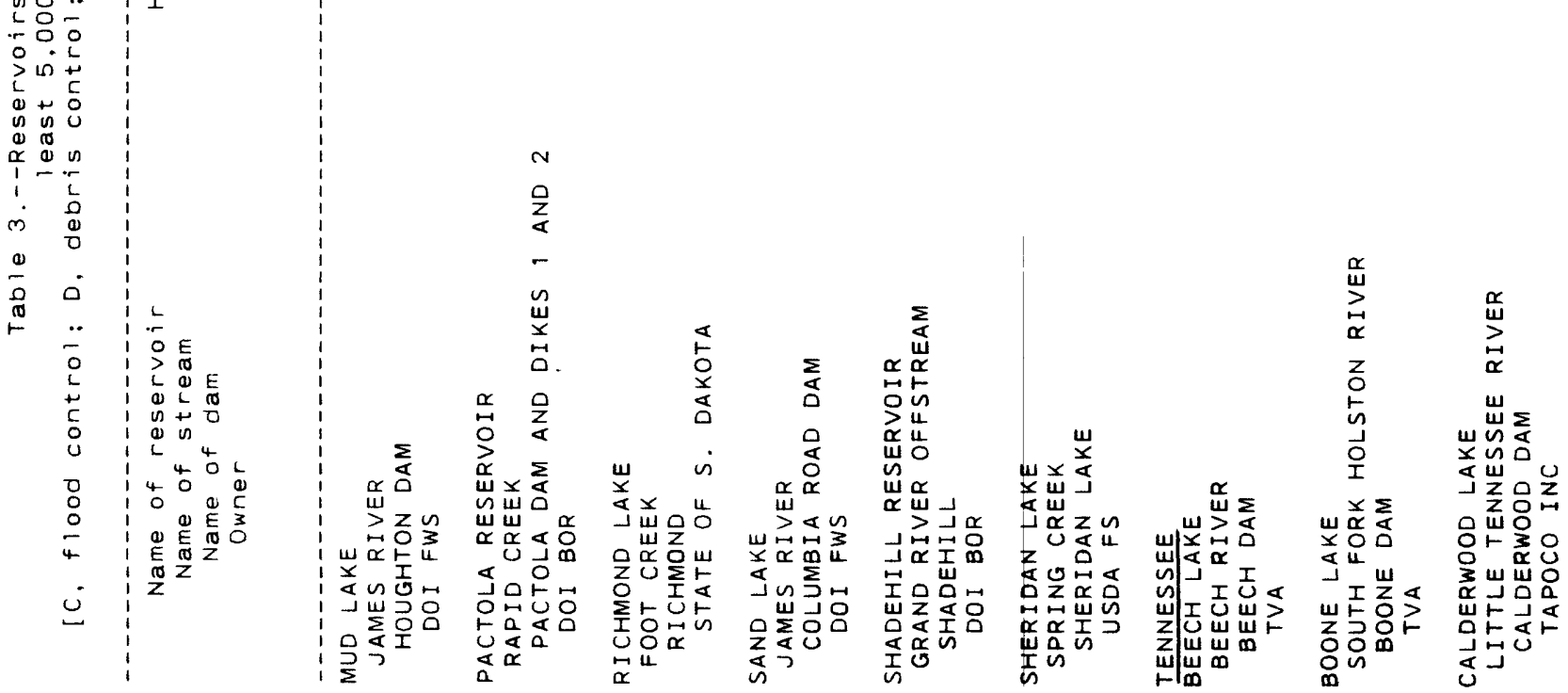


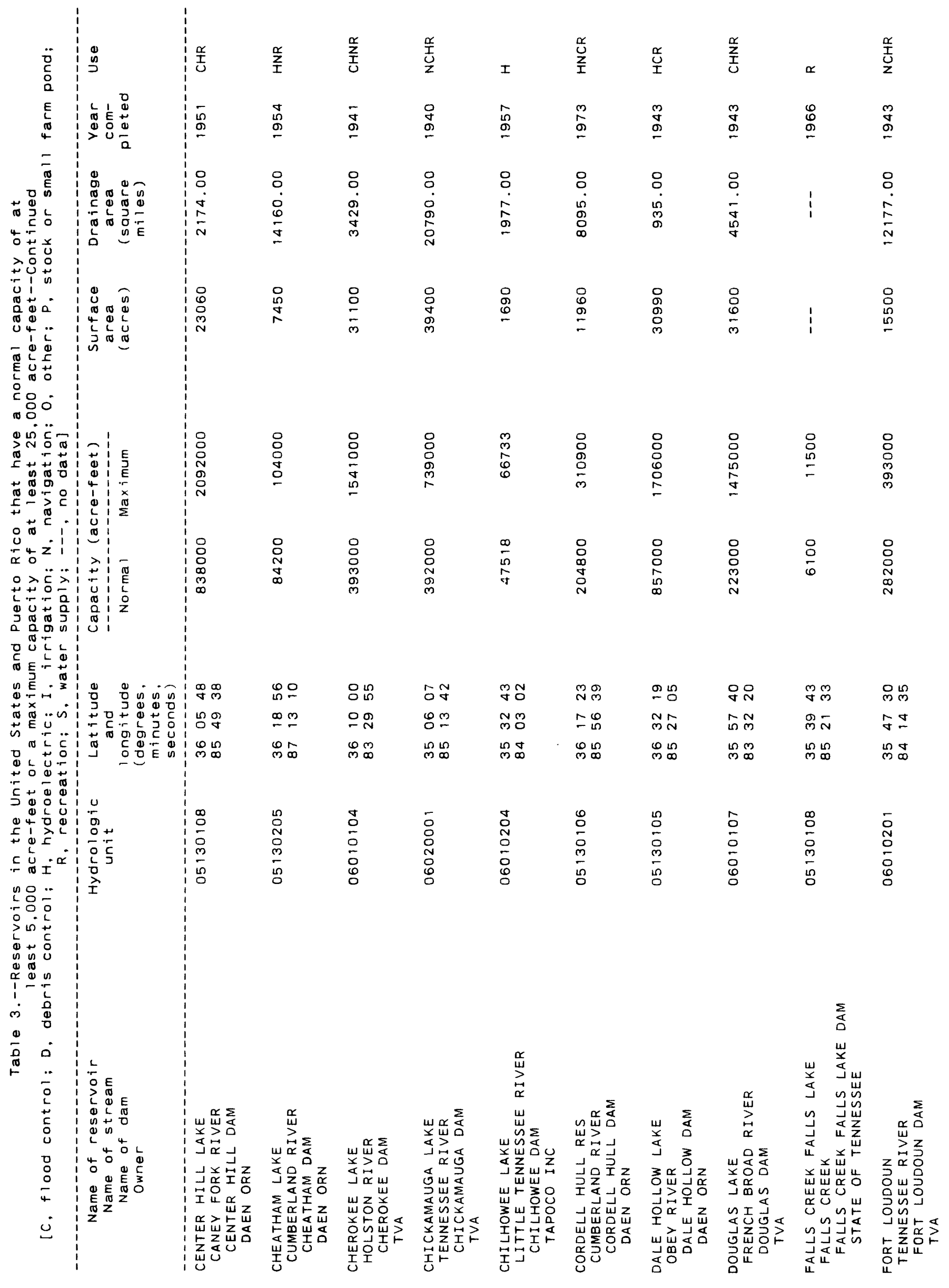




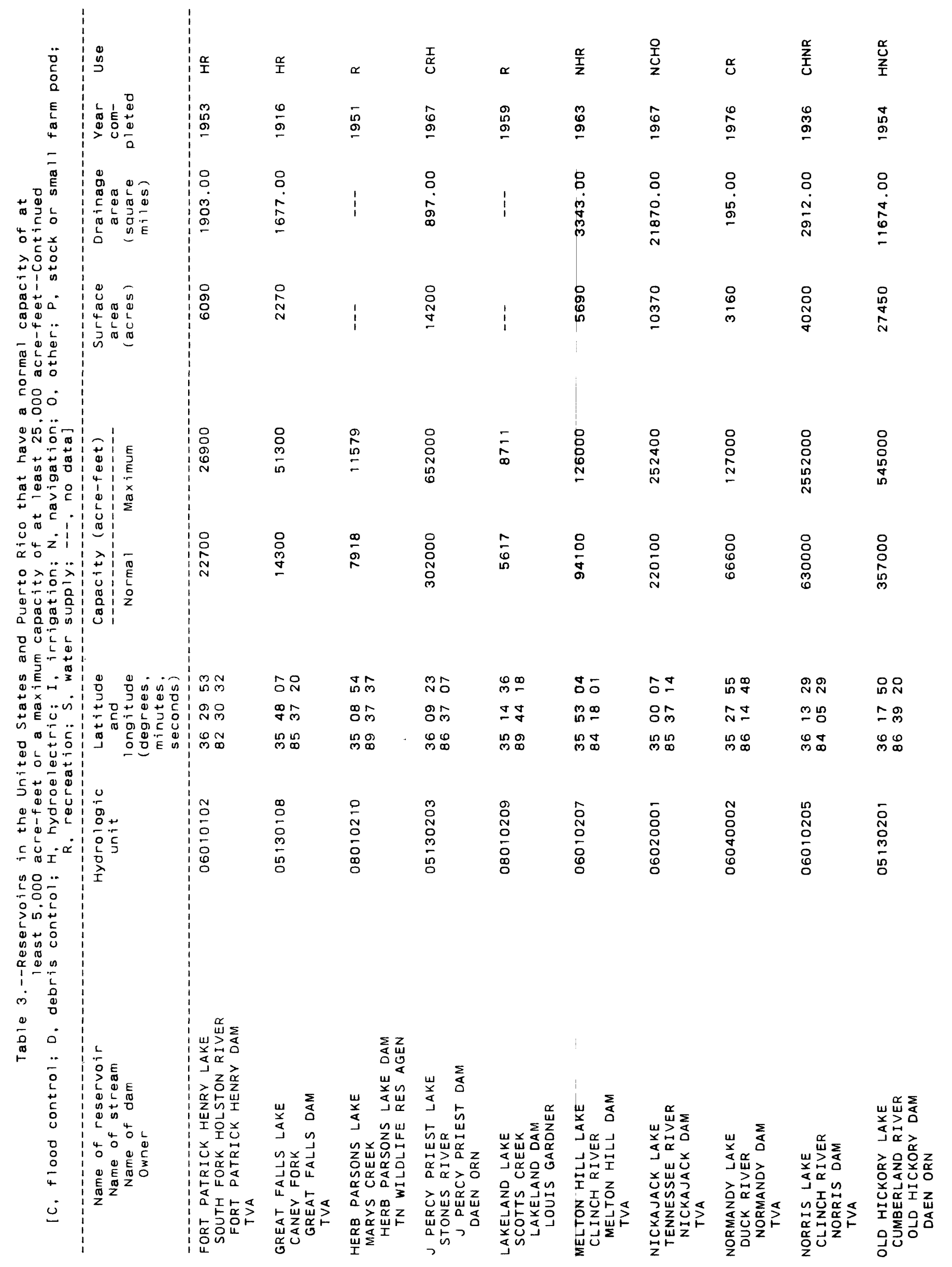




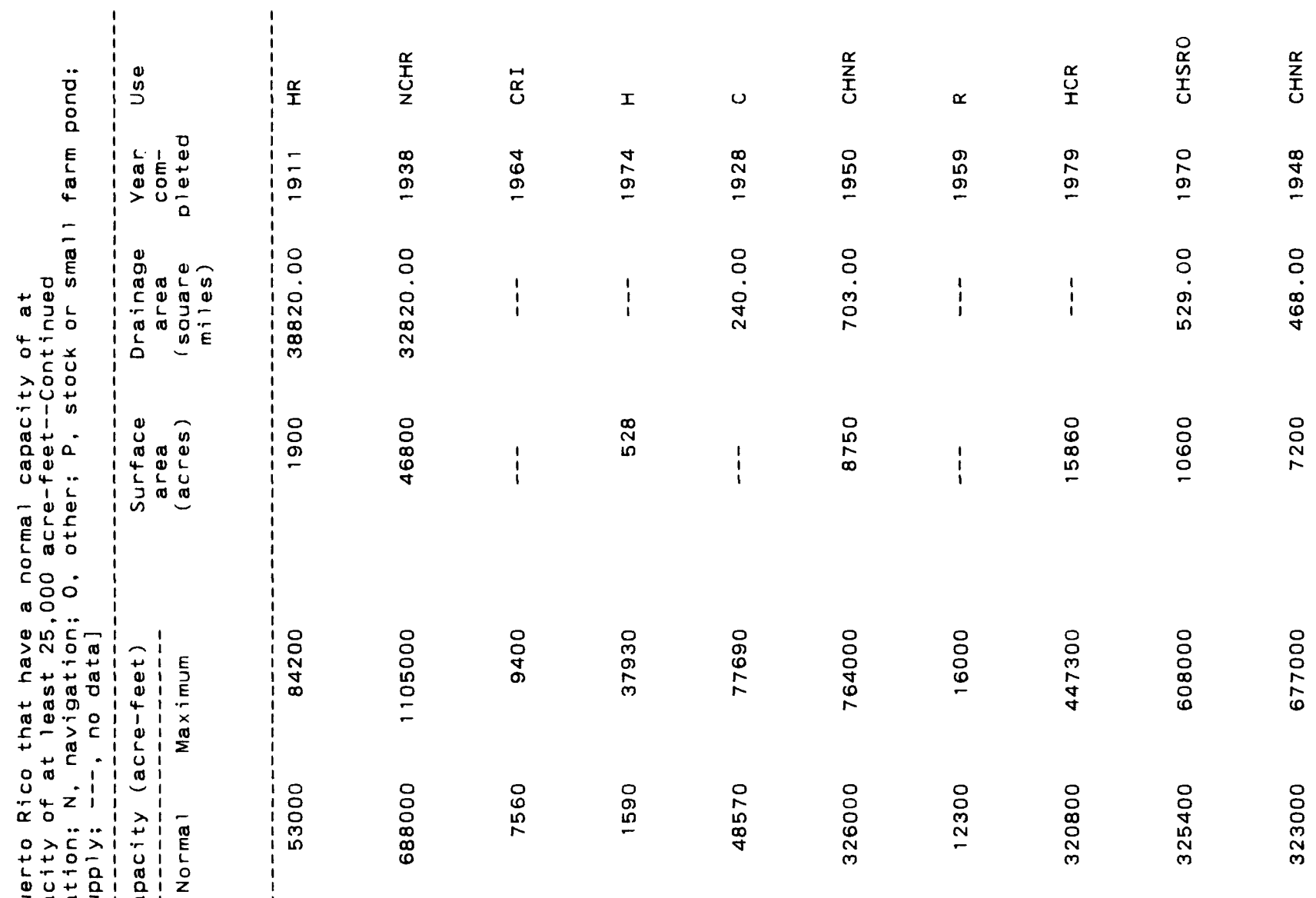

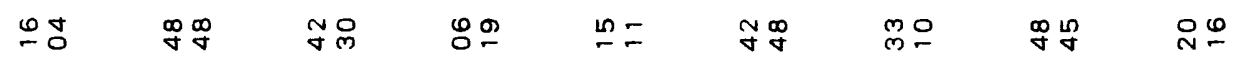

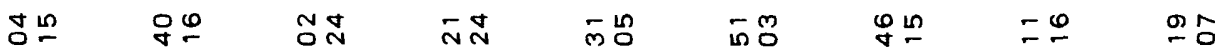

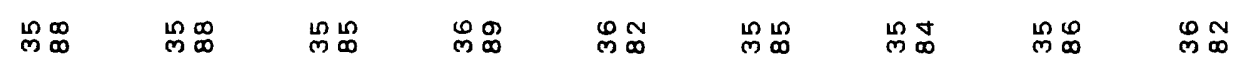
o

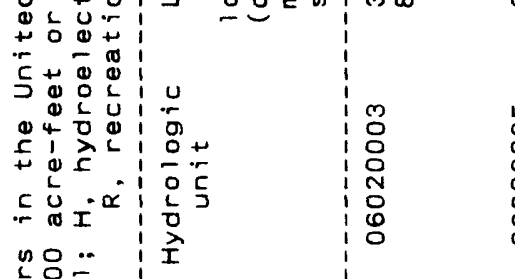

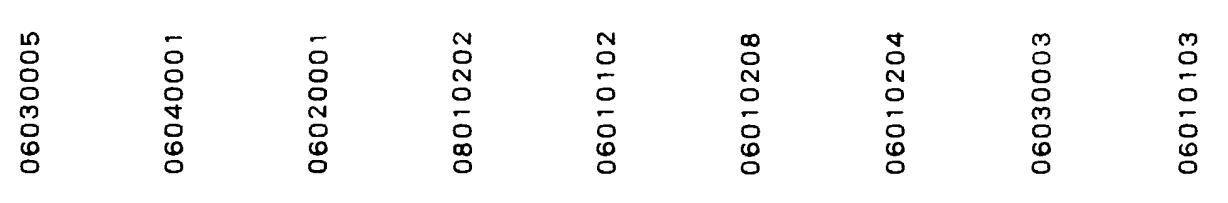
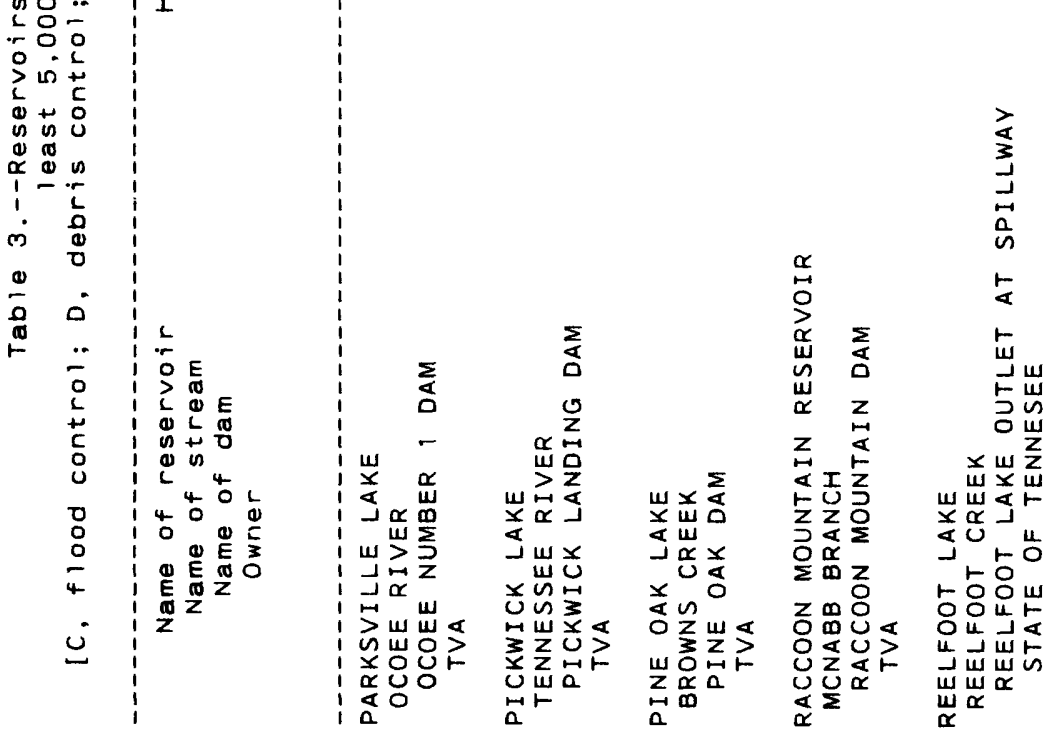


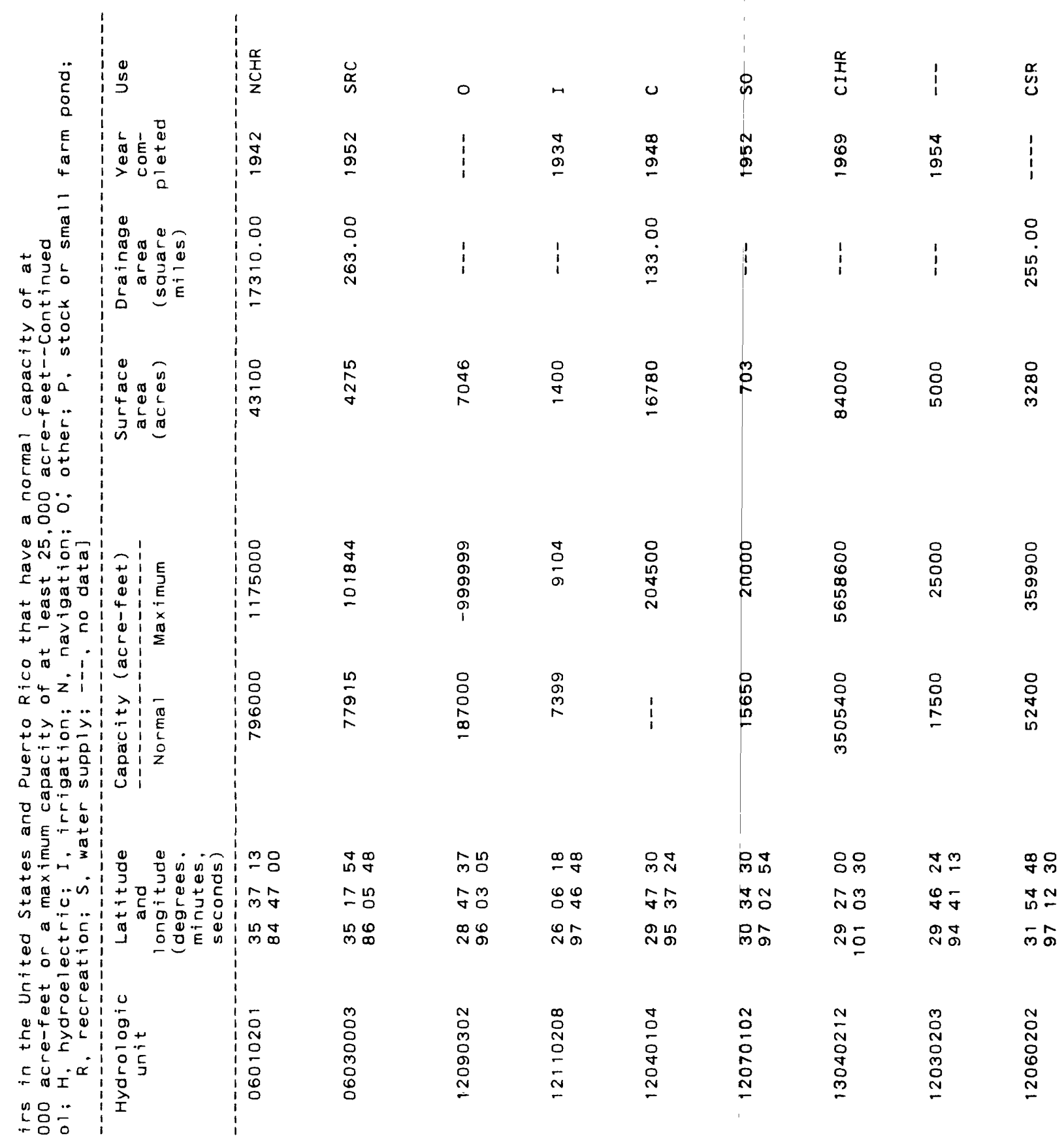




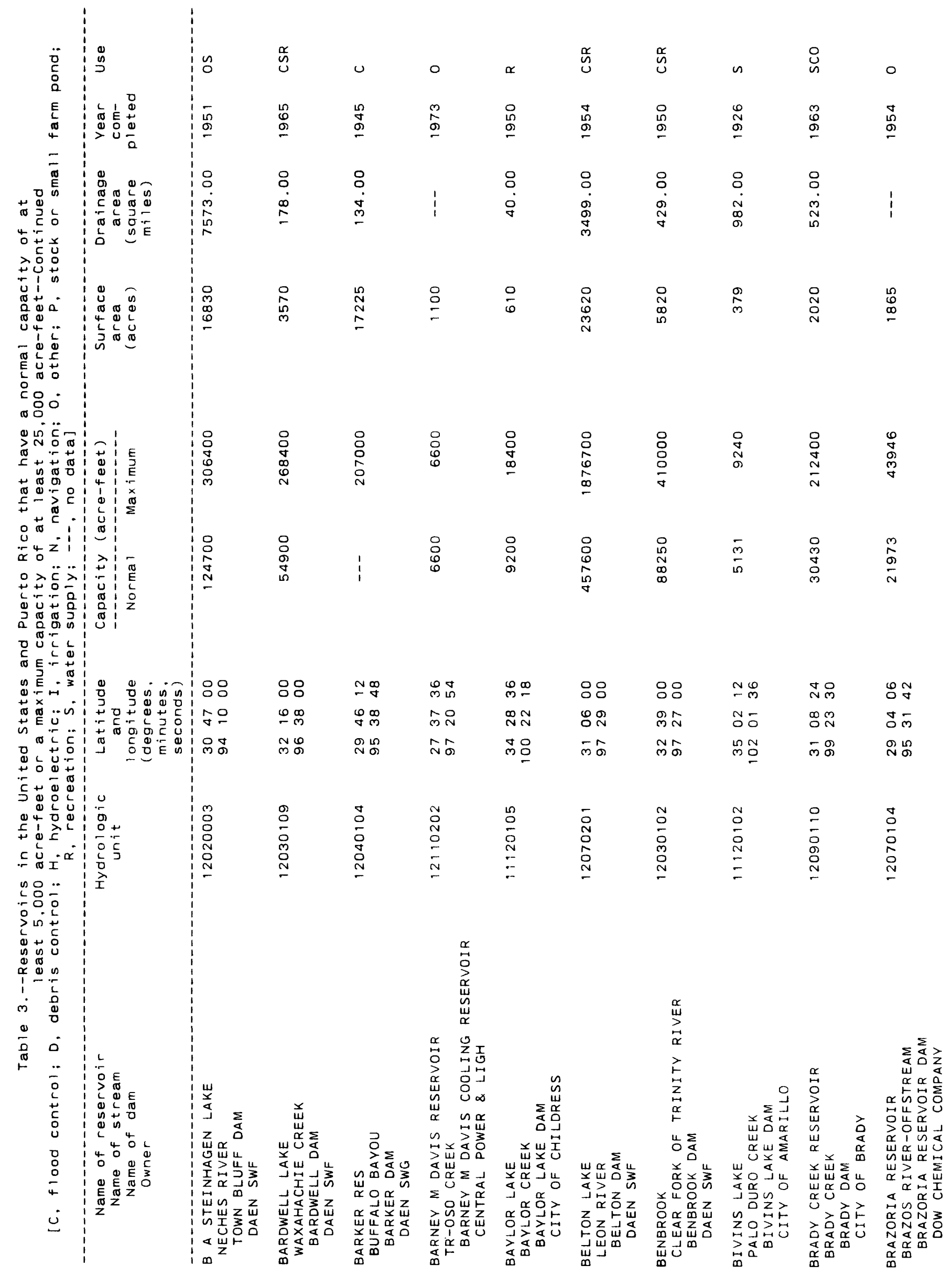




\begin{tabular}{|c|c|c|c|c|c|c|c|c|c|c|}
\hline $\begin{array}{l}\ddot{0} \\
\dot{0}\end{array}$ & $\stackrel{\circ}{n}$ & $\frac{\stackrel{\alpha}{a}}{n}$ & $\stackrel{\alpha}{\alpha}$ & $\alpha$ & $\circ$ & $\mathscr{\alpha}$ & 离 & $\stackrel{\alpha}{\mu}$ & o & $\circ$ \\
\hline 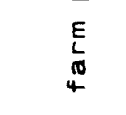 & 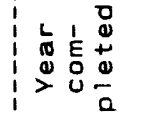 & \begin{tabular}{l}
\multirow{2}{\alpha}{} \\
$\stackrel{2}{2}$
\end{tabular} & $\begin{array}{l}\frac{n}{2} \\
\underline{a}\end{array}$ & 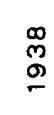 & $\stackrel{:}{\stackrel{\circ}{\circ}}$ & $\stackrel{g}{9}$ & 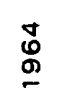 & 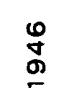 & 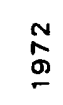 & 占 \\
\hline 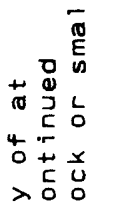 & 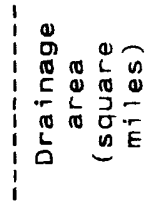 & \begin{tabular}{l}
0 \\
\hdashline \\
\hdashline \\
\hdashline
\end{tabular} & $\vdots$ & 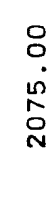 & $\vdots$ & $\begin{array}{l}: \\
\vdots \\
\dot{q}\end{array}$ & 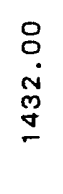 & $\begin{array}{l}\stackrel{\circ}{0} \\
\stackrel{0}{=}\end{array}$ & $\vdots$ & $\begin{array}{l}\circ \\
\vdots \\
\vdots \\
0\end{array}$ \\
\hline 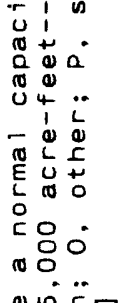 & 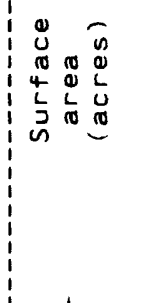 & 1 & 吉 & $\stackrel{\circ}{\circ}$ & 号 & 员 & $\underset{\infty}{\stackrel{D}{\infty}}$ & $\begin{array}{l}\text { : } \\
\stackrel{\leftrightarrow}{0}\end{array}$ & : & 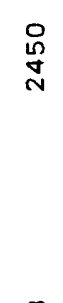 \\
\hline 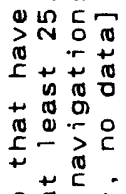 & 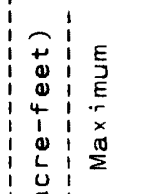 & $\begin{array}{l}\frac{\sigma}{\alpha} \\
\tilde{D}^{\prime} \\
\sigma \\
\sigma\end{array}$ & : & 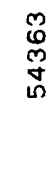 & $\frac{8}{\infty}$ & 总 & 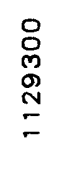 & 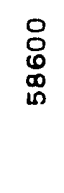 & : & 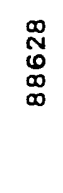 \\
\hline 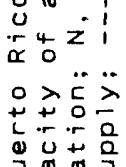 & 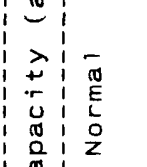 & $\begin{array}{l}0 \\
0 \\
0 \\
0 \\
0 \\
m\end{array}$ & î̃ & 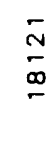 & $\begin{array}{l}\stackrel{8}{0} \\
\stackrel{\infty}{0} \\
0\end{array}$ & $\begin{array}{l}0 \\
\vdots \\
\infty\end{array}$ & 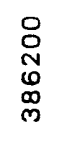 & : & 总 & $\underset{0}{\circ}$ \\
\hline
\end{tabular}

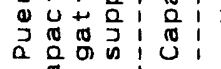

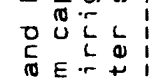

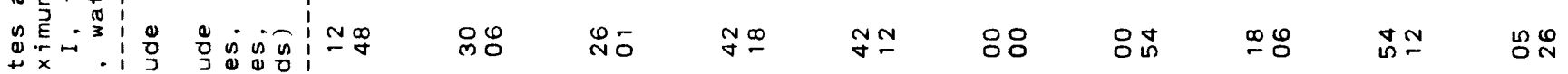

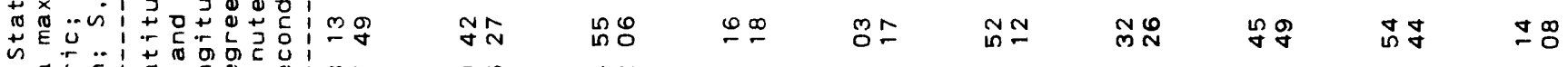

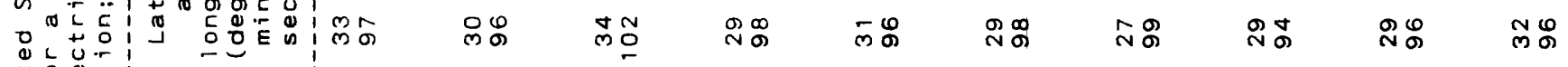

,

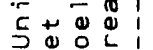

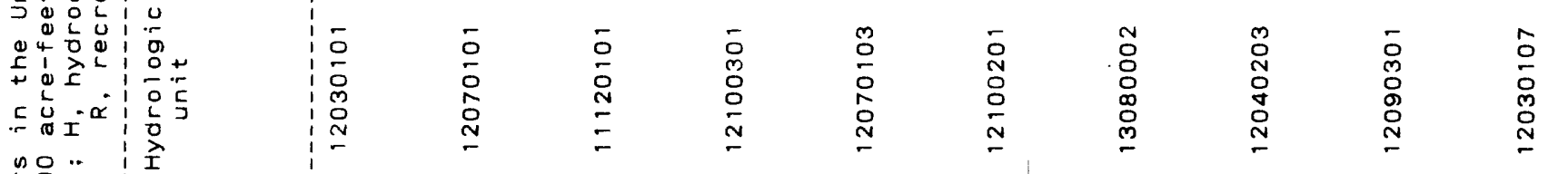

o-

L

(N)

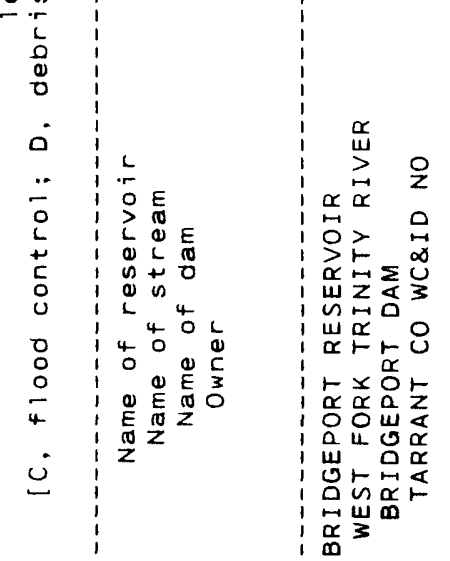

离

志出里

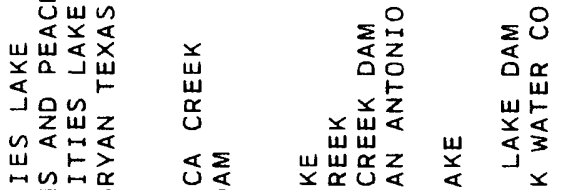

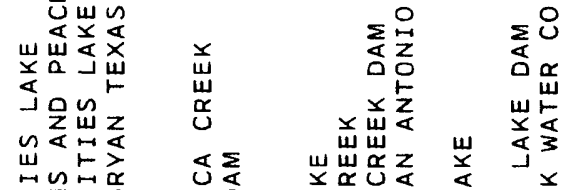

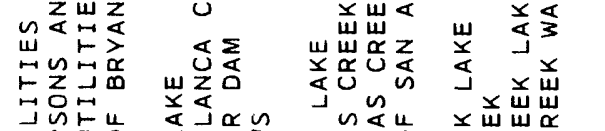

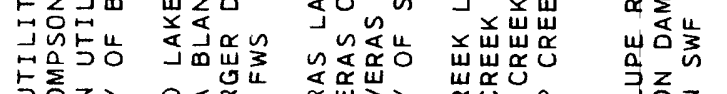

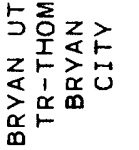

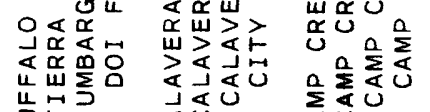

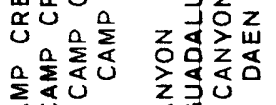

品

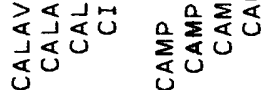

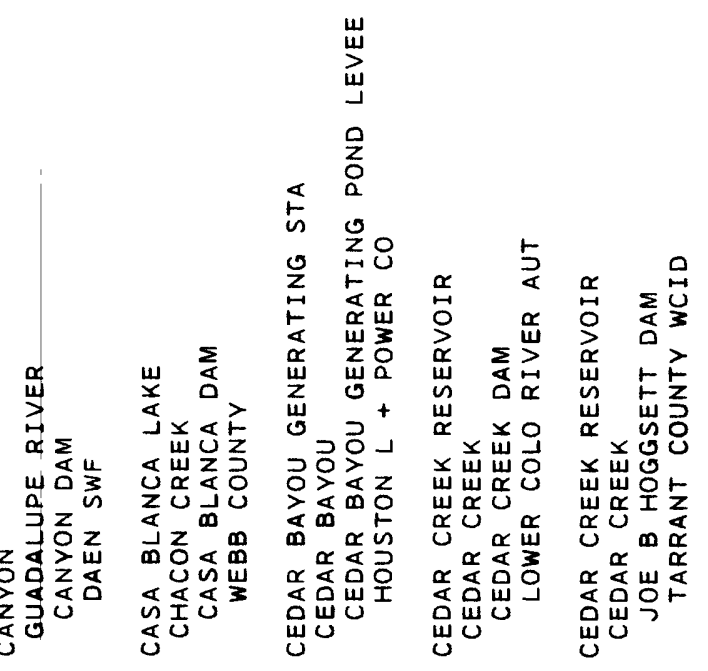




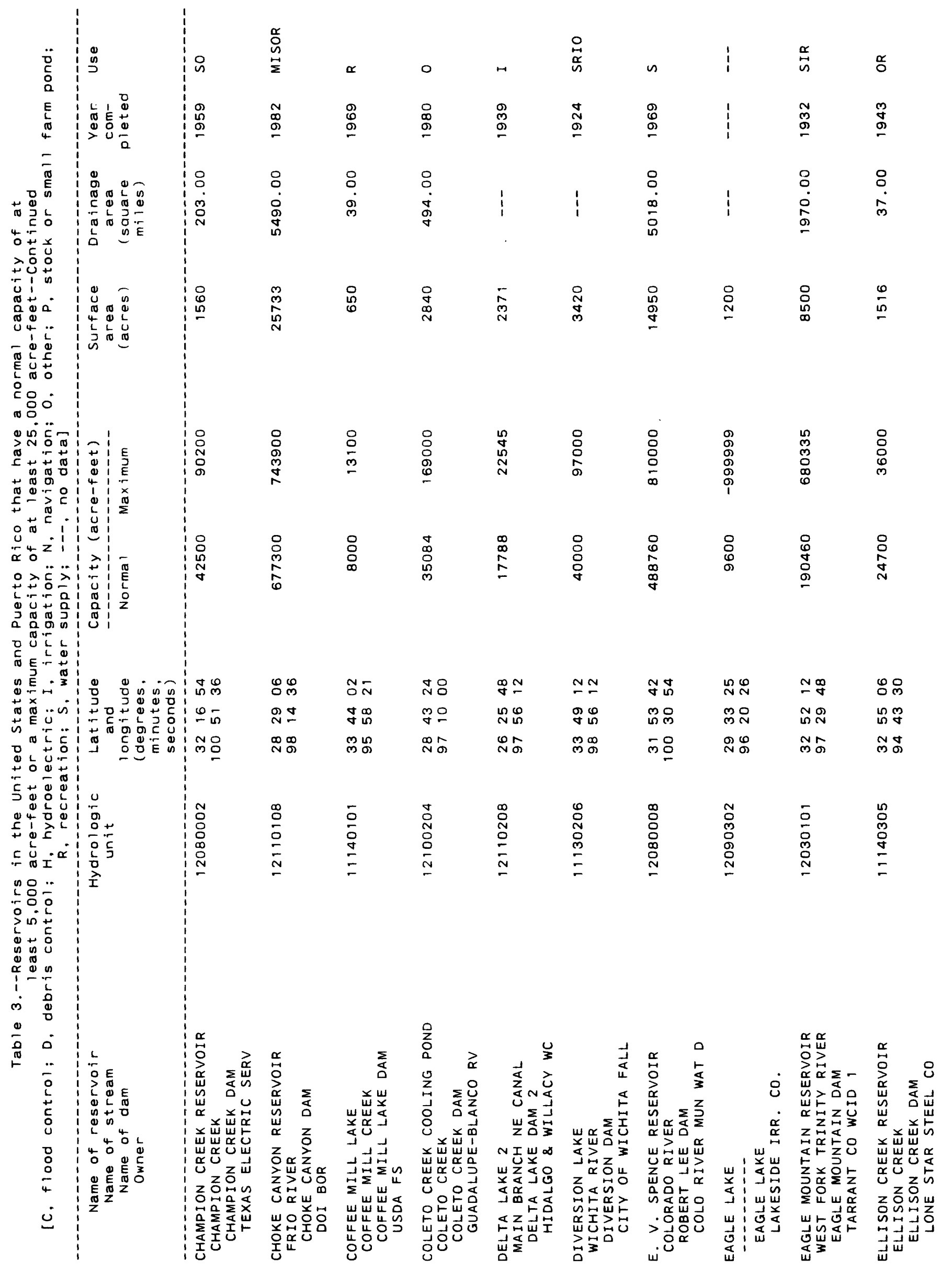




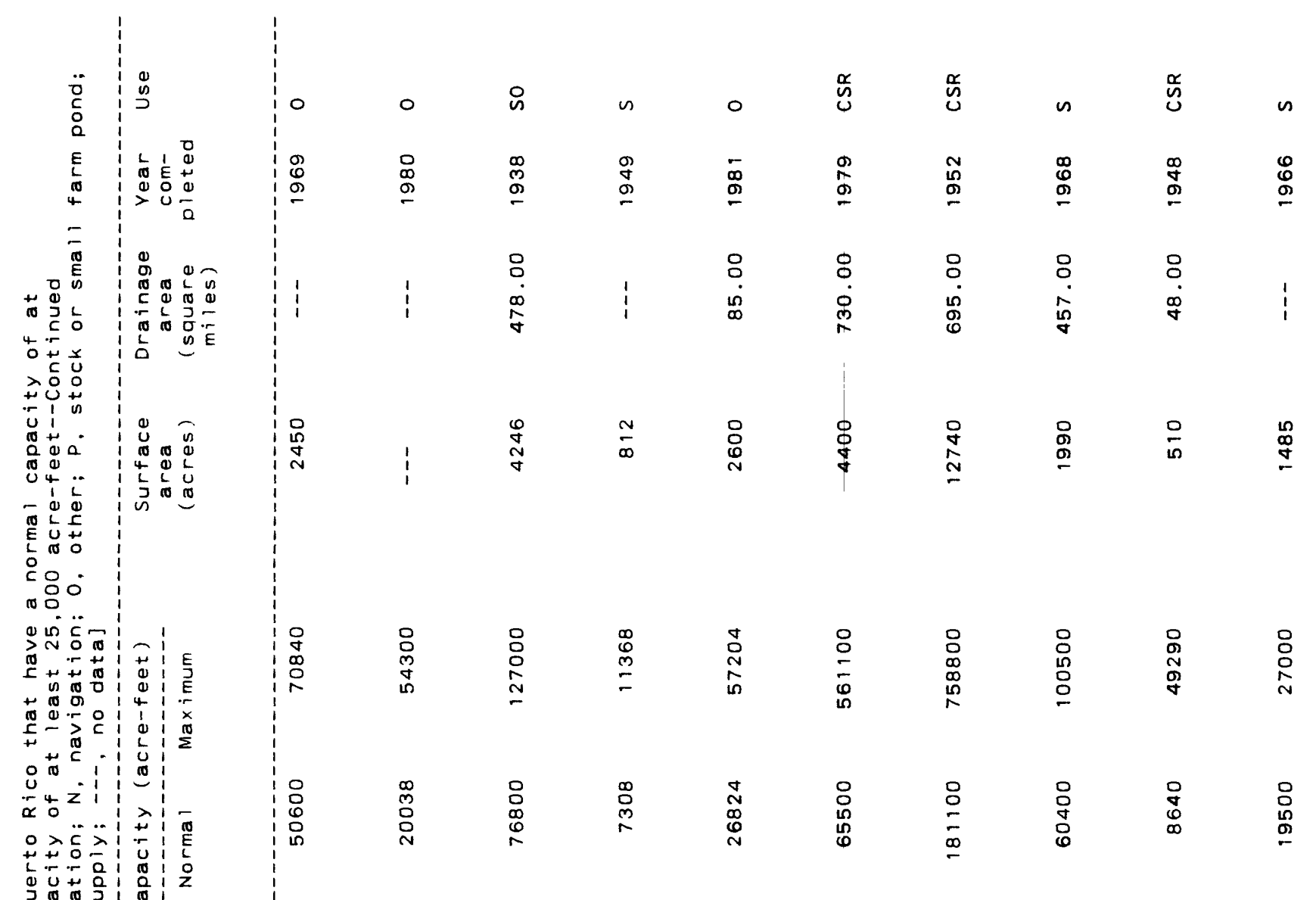

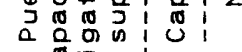

वU⿺辶一

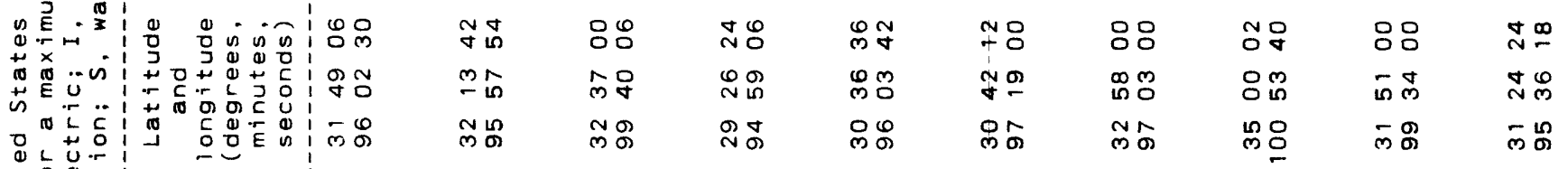

\begin{tabular}{|c|c|c|c|c|c|c|c|c|c|}
\hline 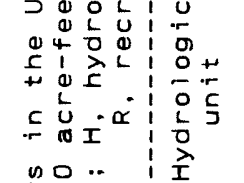 & 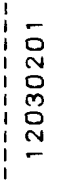 & $\begin{array}{l}\hat{0} \\
0 \\
0 \\
0 \\
0 \\
\end{array}$ & 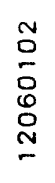 & 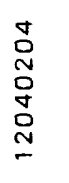 & $\begin{array}{l}3 \\
0 \\
0 \\
0 \\
0 \\
0 \\
\end{array}$ & 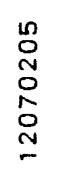 & 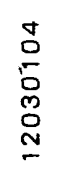 & $\begin{array}{l}\text { D } \\
\text { N } \\
\text { N } \\
\text { E } \\
\text { I }\end{array}$ & $\begin{array}{l}\infty \\
0 \\
0 \\
\stackrel{0}{\circ} \\
\stackrel{2}{ }\end{array}$ \\
\hline
\end{tabular}

.
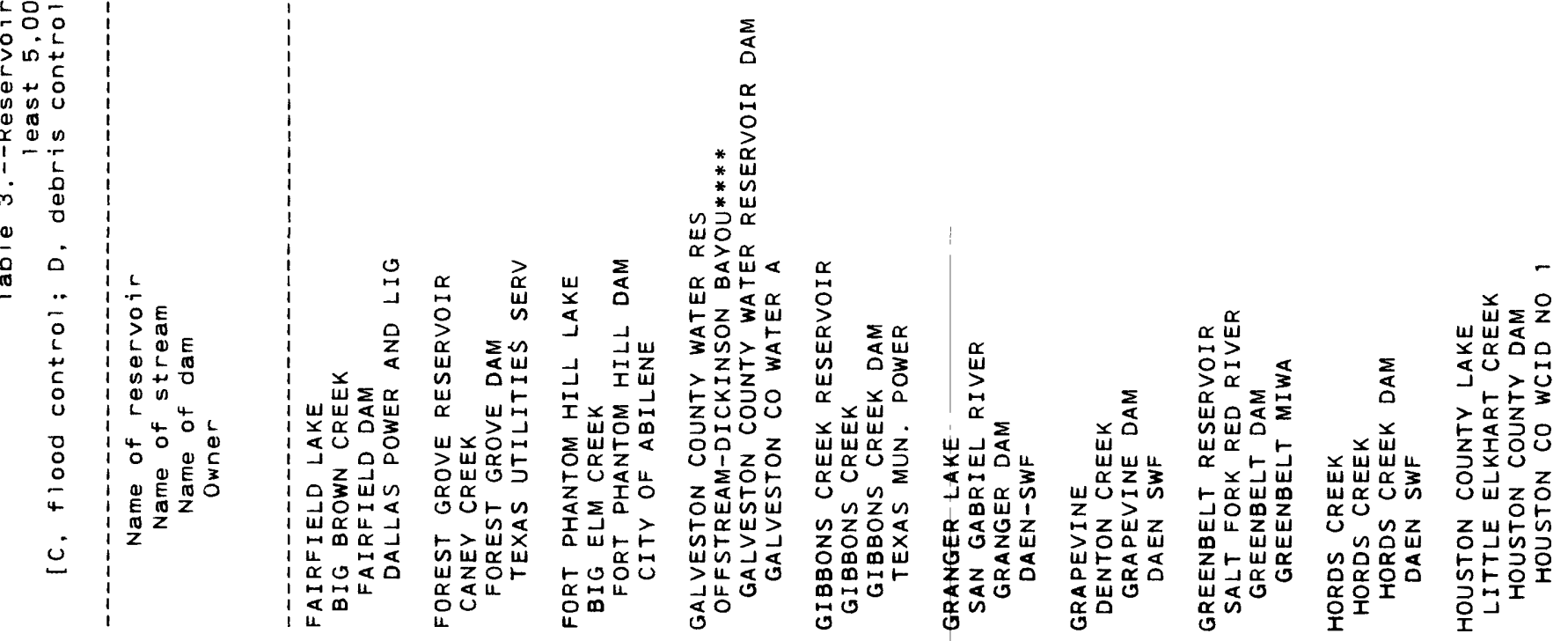


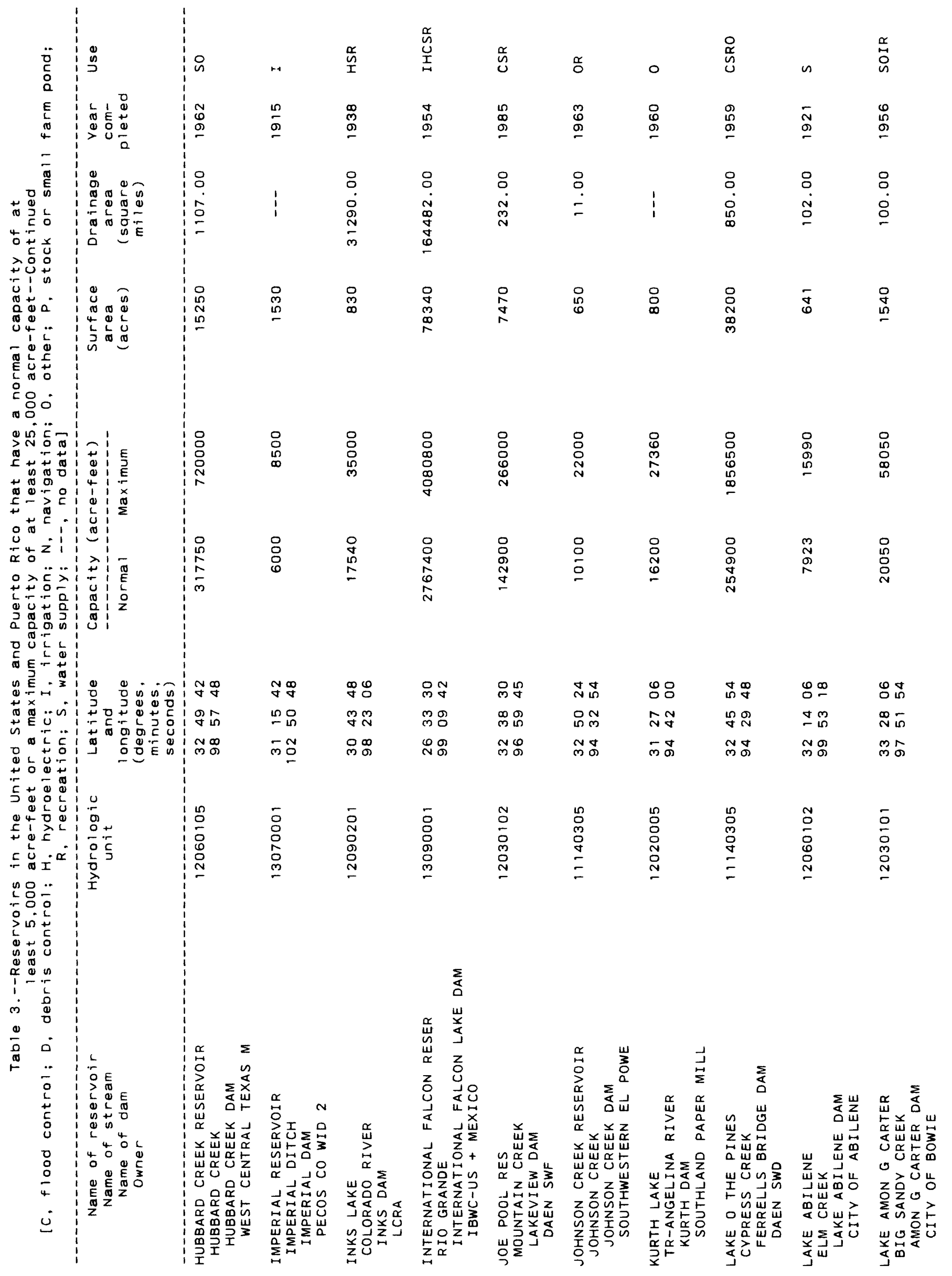




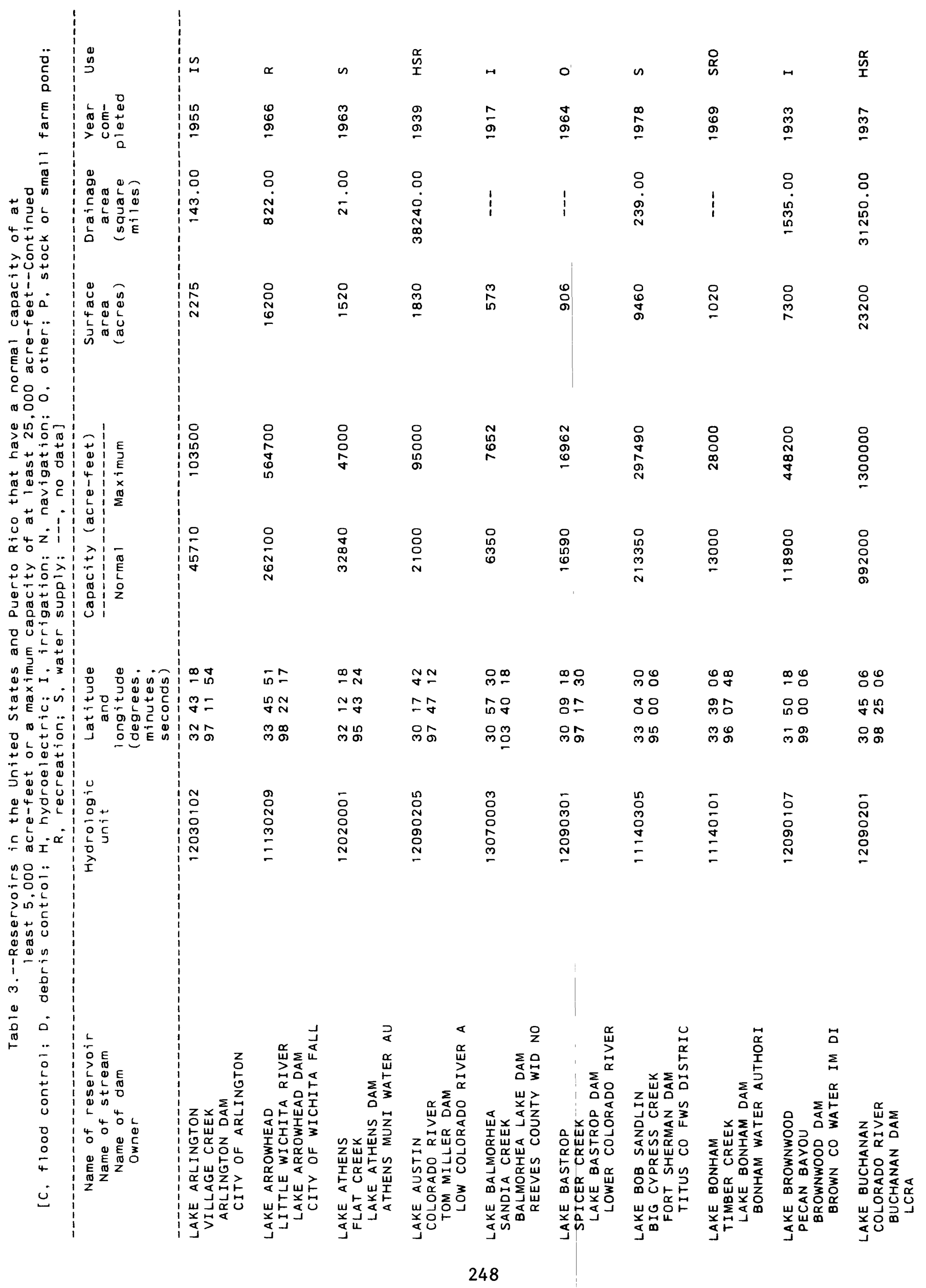




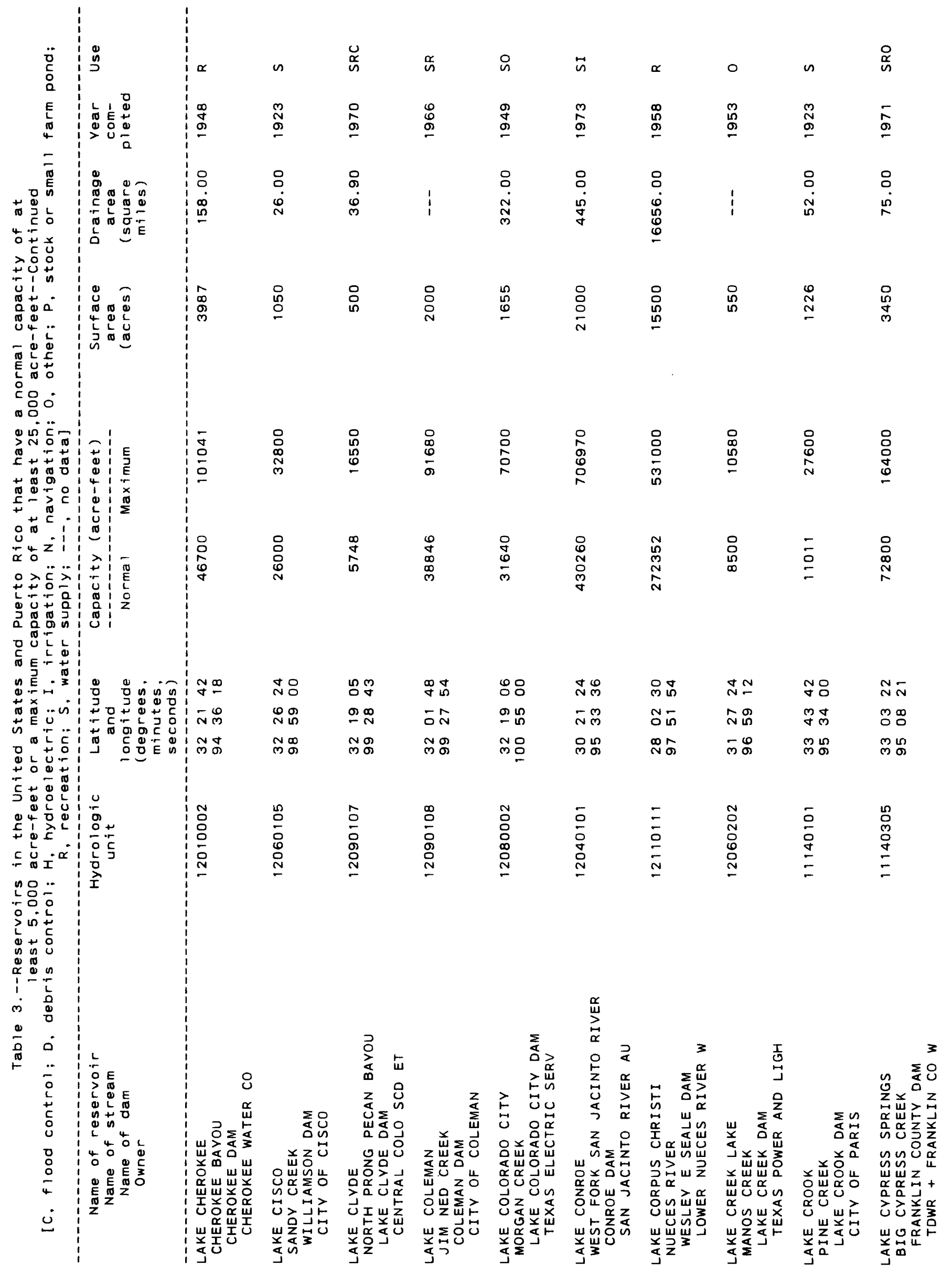




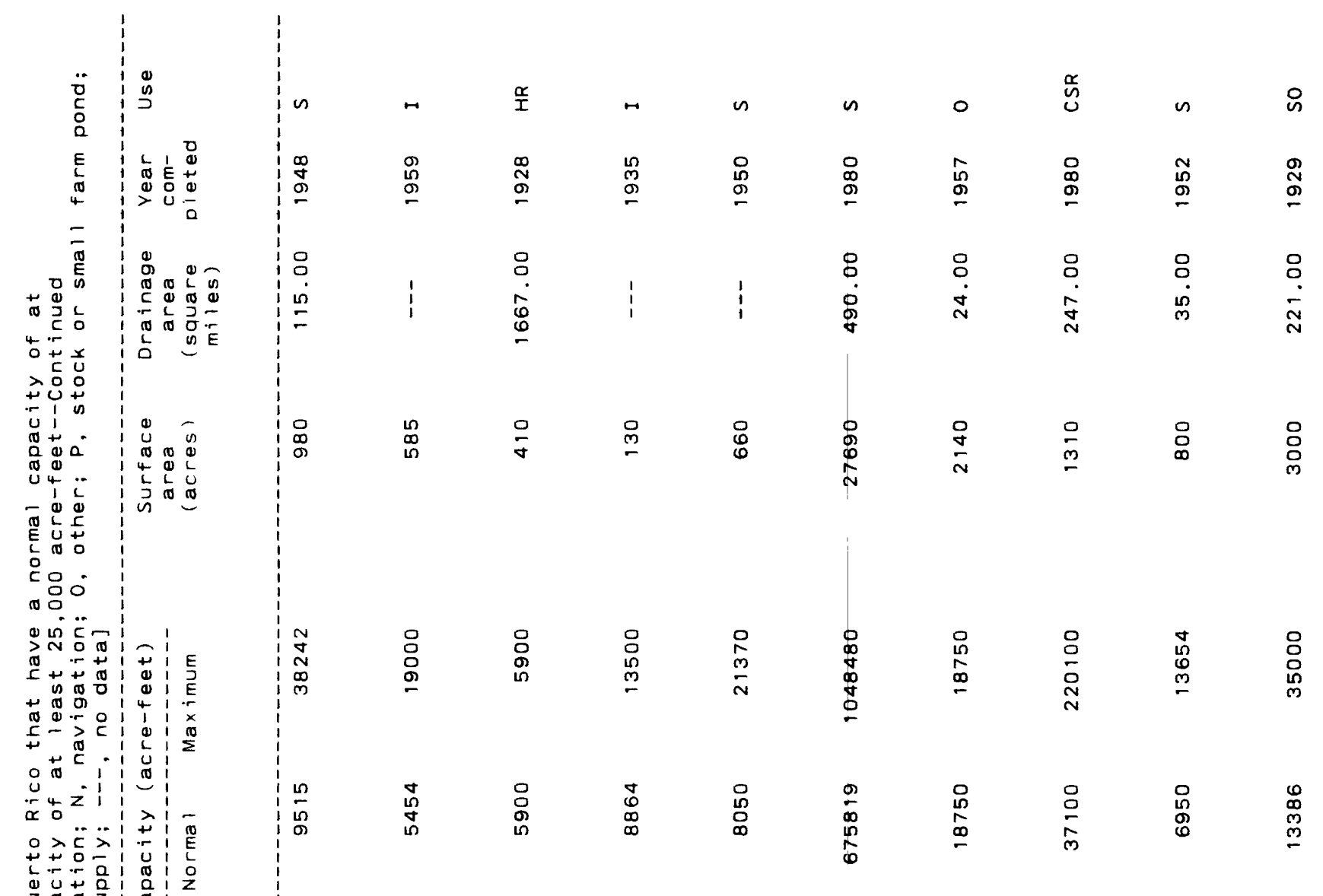

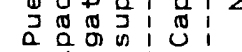

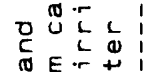

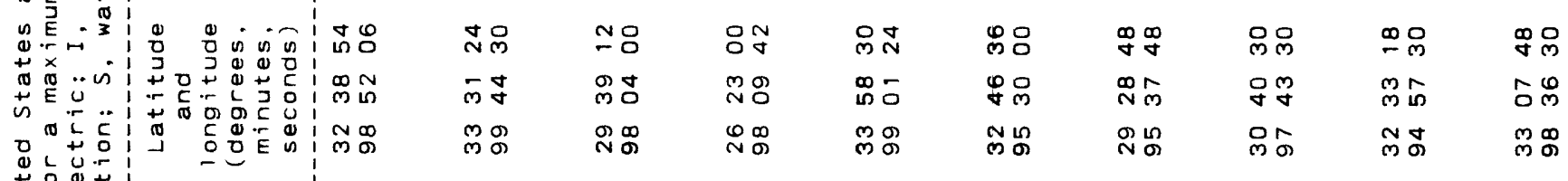

c

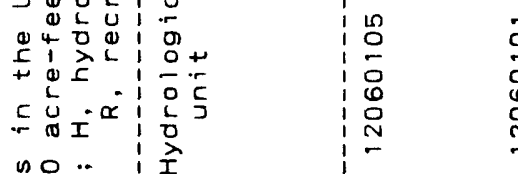

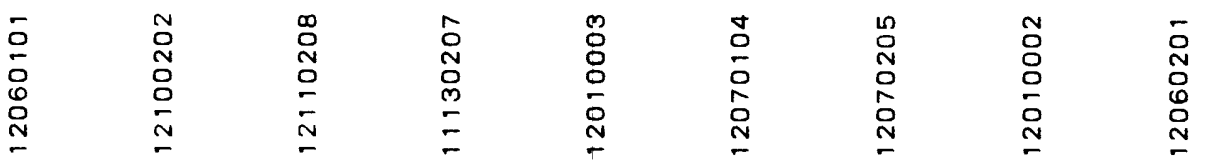

o
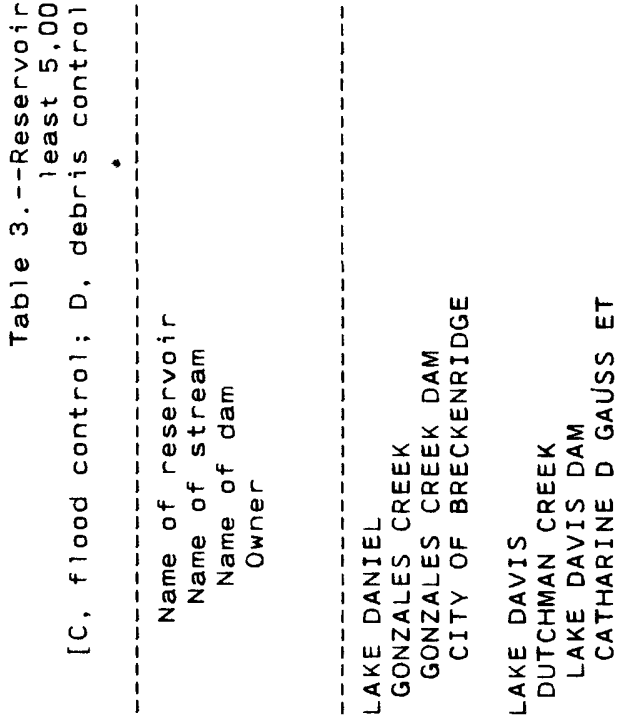

$\sum_{d}$

a

$\frac{I}{5}$ 


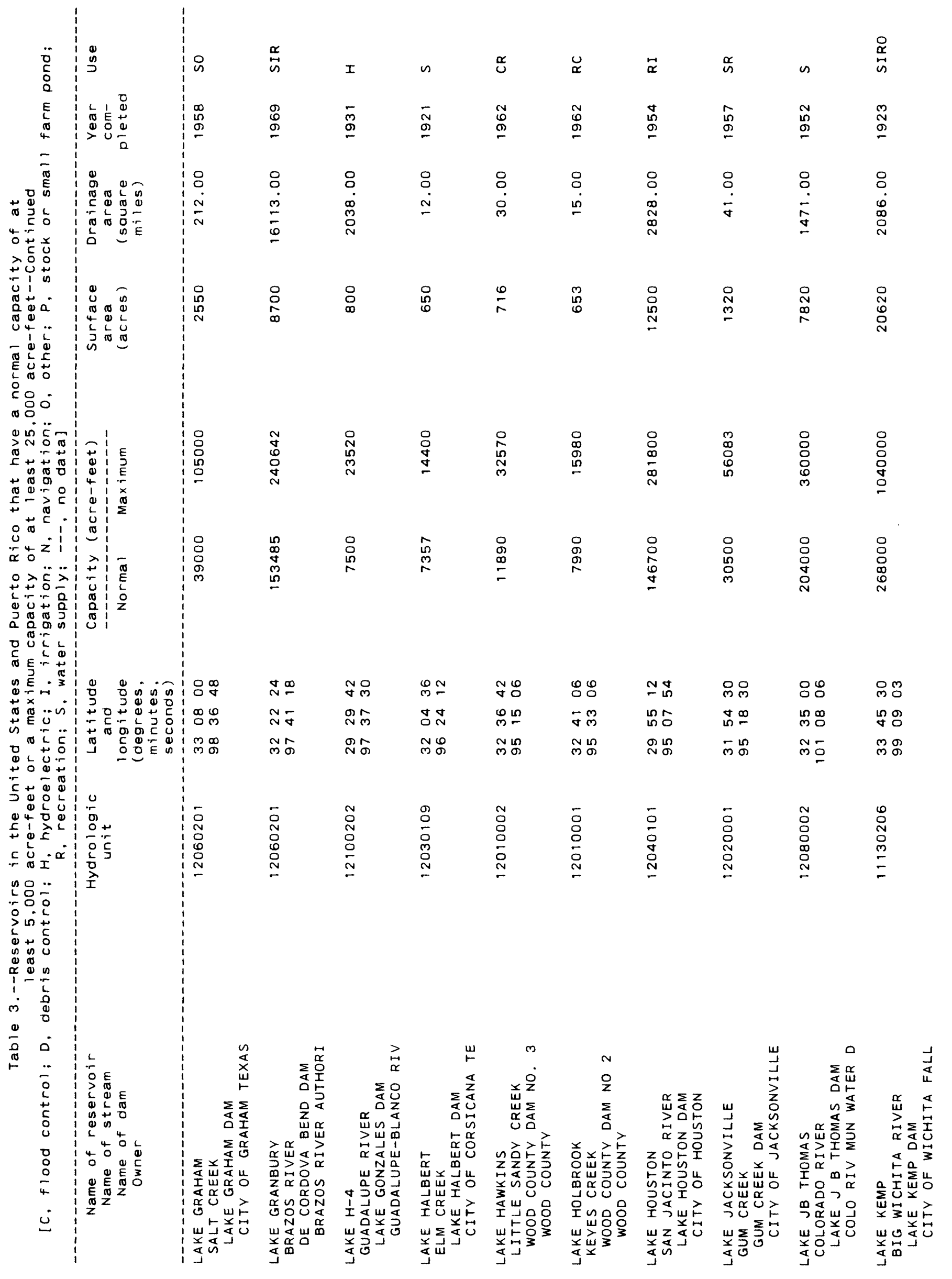




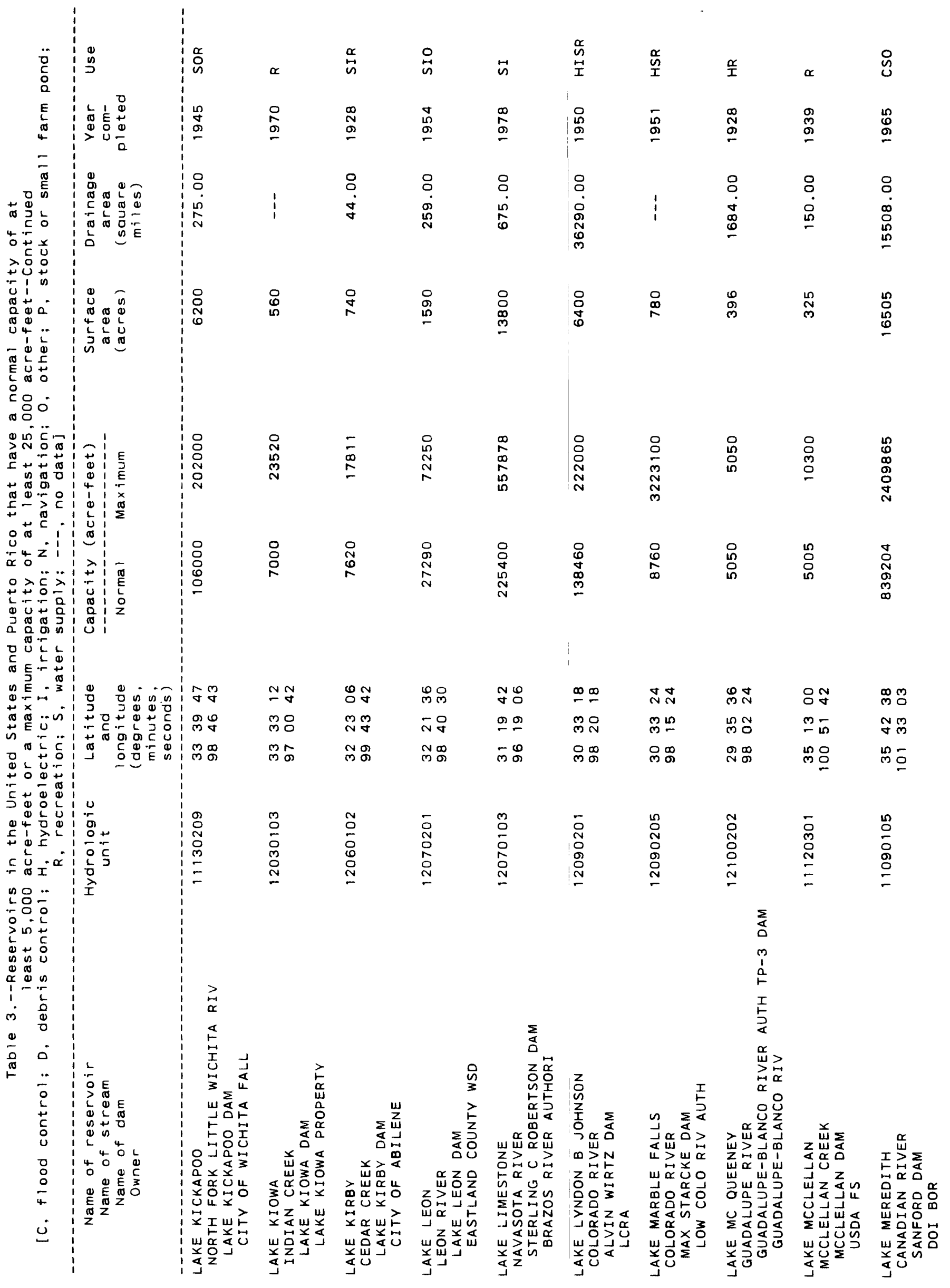




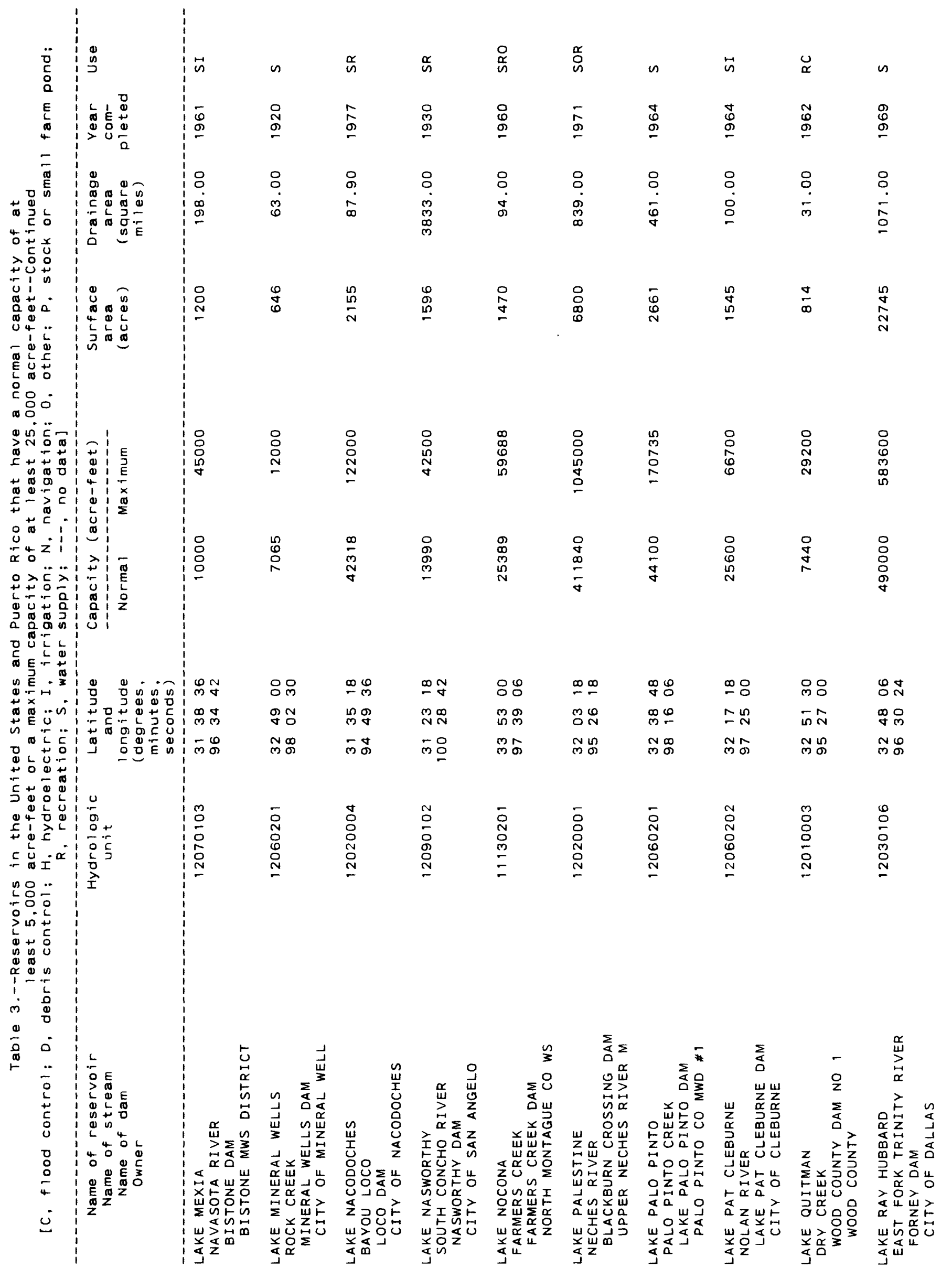




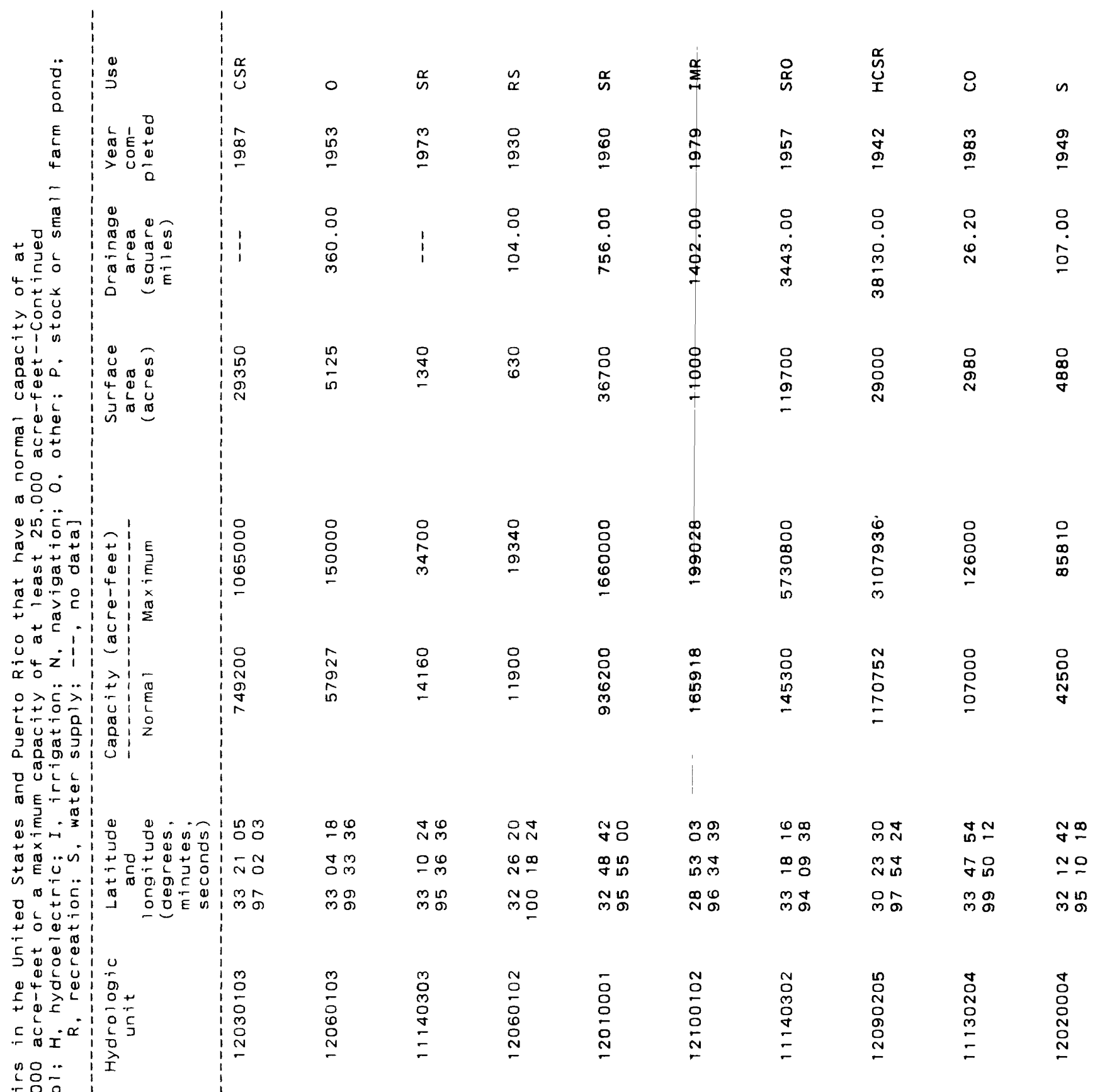




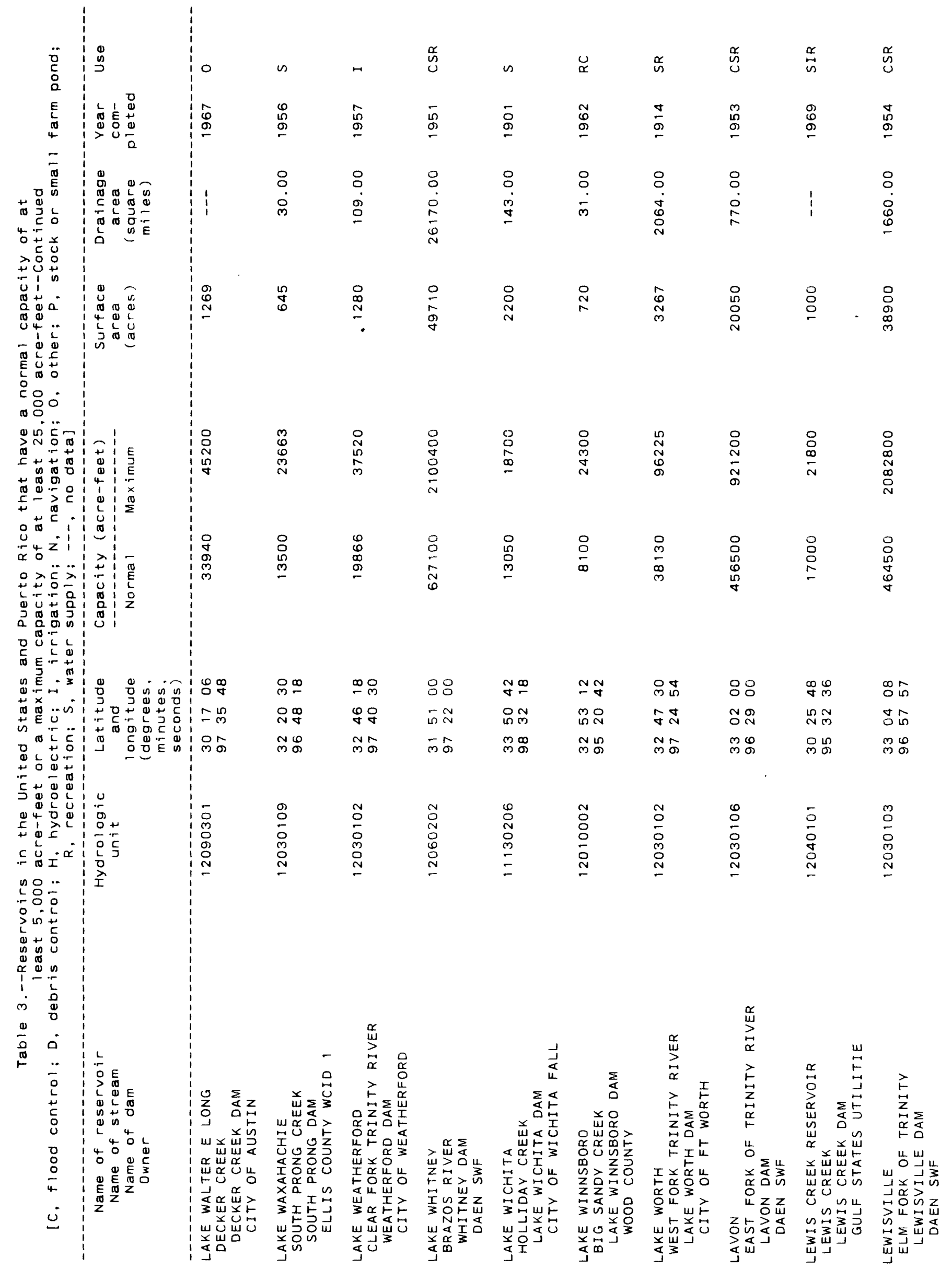




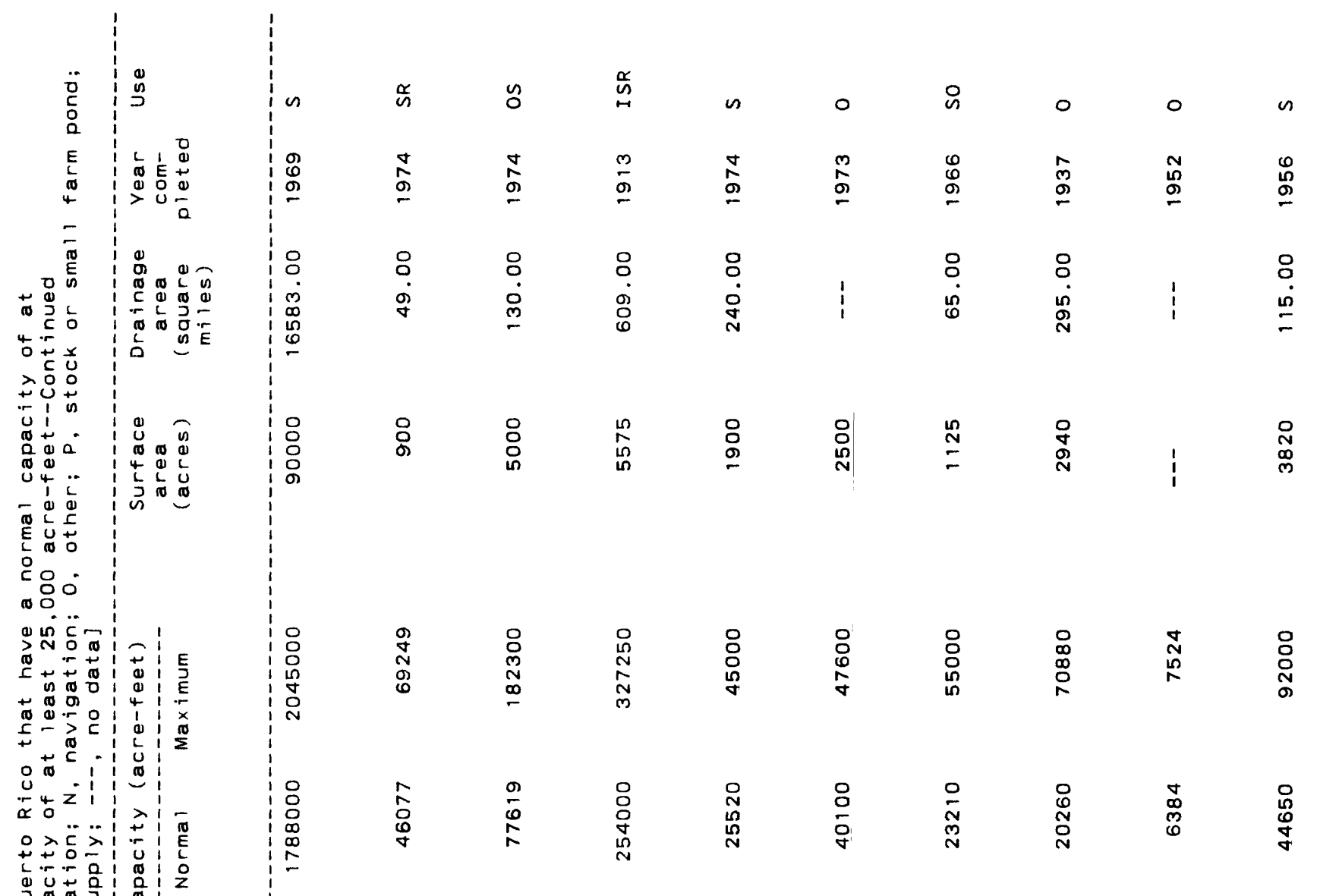

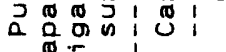

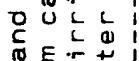

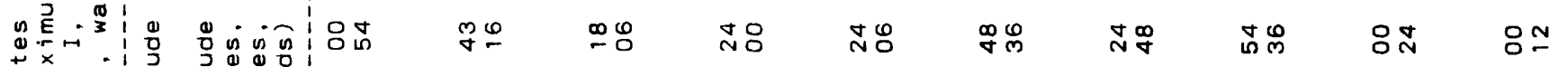

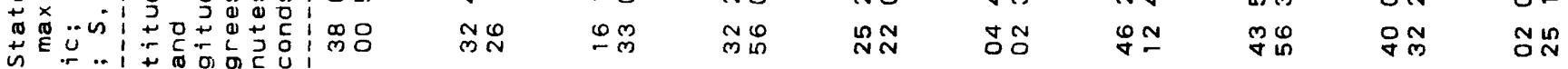

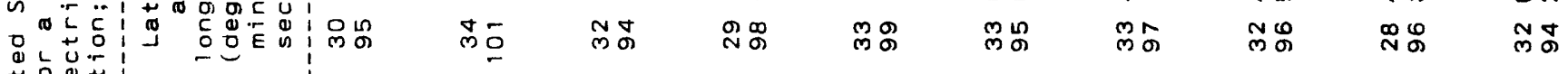

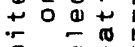

S 5

ब4 40 व

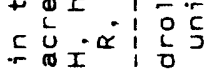

느욤

$>$ in

a 4 这

(n)

品

$\dot{m}$

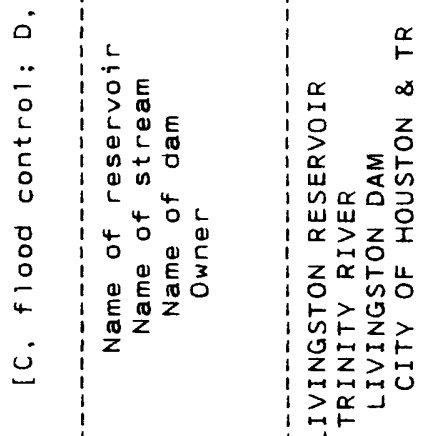

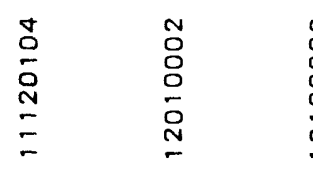

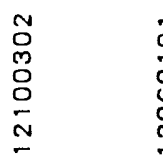

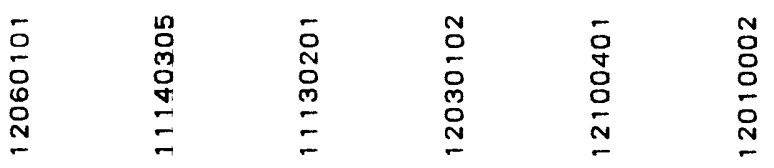

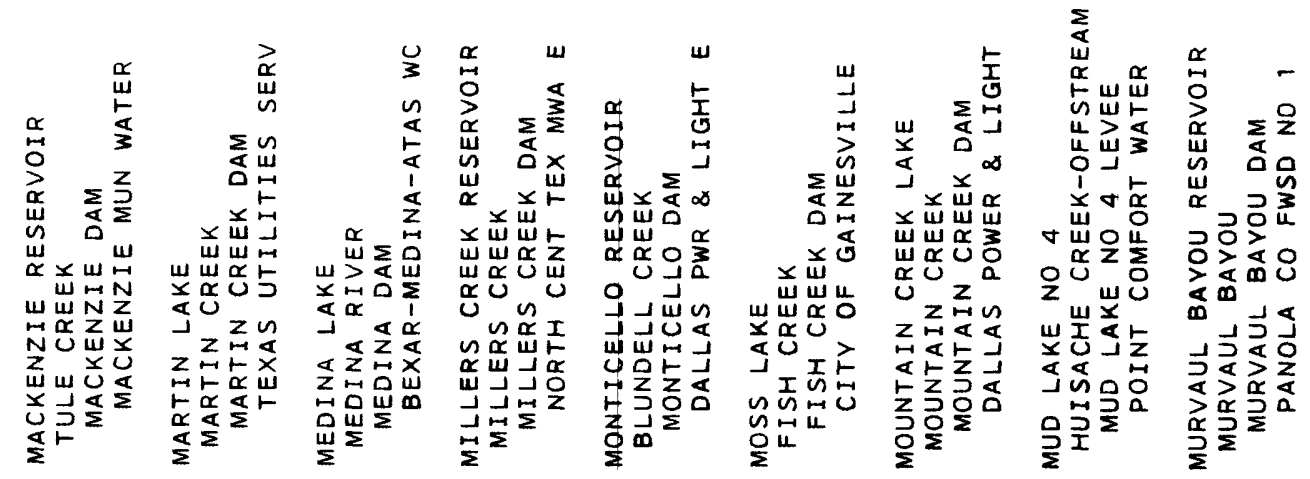




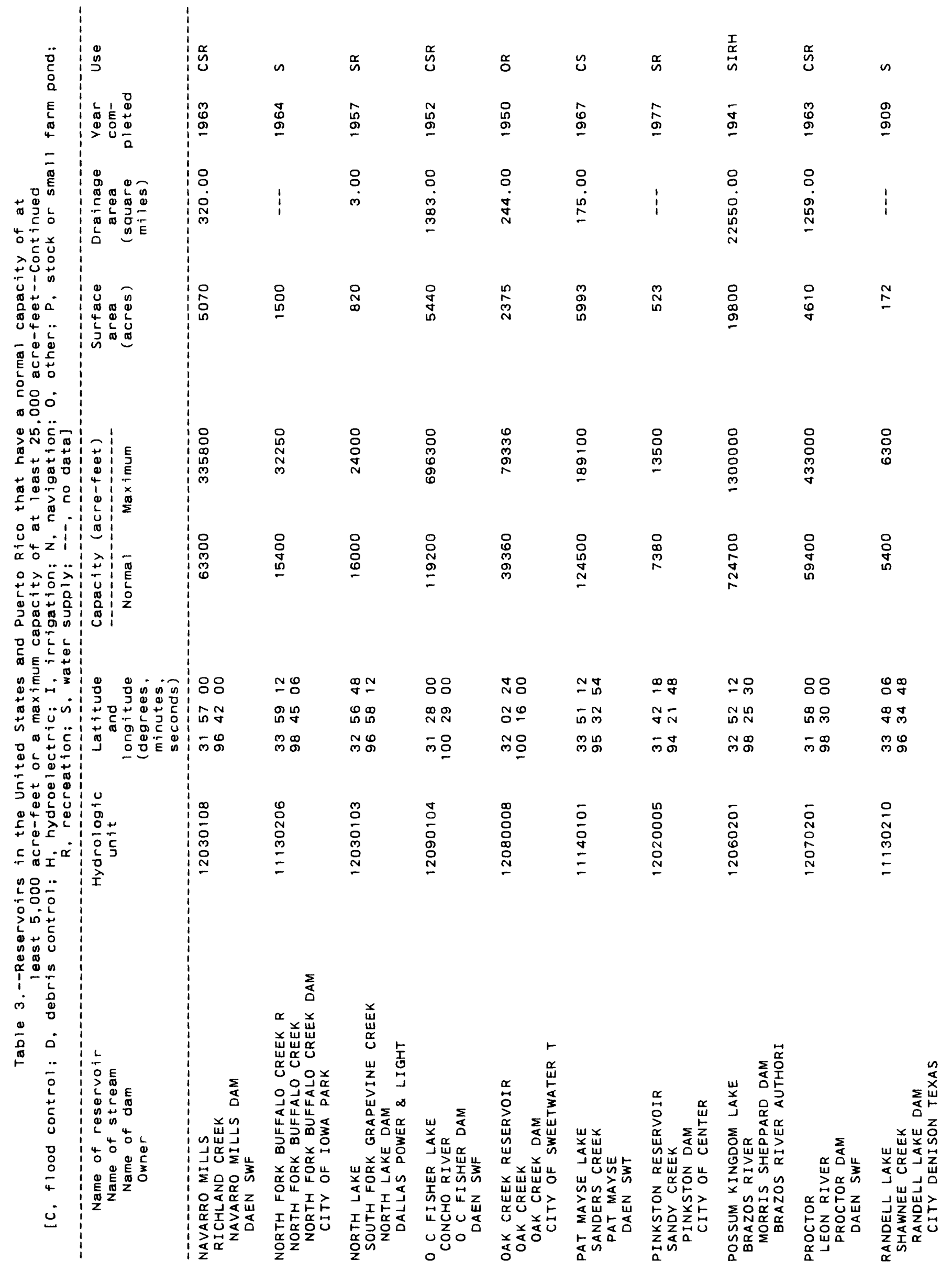




\begin{tabular}{|c|c|c|c|c|c|c|c|c|c|c|}
\hline $\begin{array}{l}\ddot{0} \\
\dot{c}\end{array}$ & 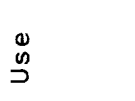 & $\stackrel{ }{1}$ & $\vdots$ & $\alpha$ & 0 & $\begin{array}{l}\frac{I}{\tilde{J}} \\
\tilde{U} \\
\mathcal{U}\end{array}$ & $n$ & $\tilde{u}$ & $\underset{\mathscr{\alpha}}{\mathscr{\alpha}}$ & $\stackrel{\alpha}{\tilde{s}}$ \\
\hline${ }_{0}^{E}$ & 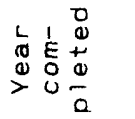 & $\begin{array}{l}0 \\
\stackrel{0}{0} \\
\stackrel{-}{-}\end{array}$ & 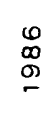 & $\begin{array}{l}\text { o } \\
\text { o }\end{array}$ & 兽 & 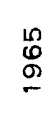 & $\underset{\stackrel{\sim}{\alpha}}{\stackrel{2}{2}}$ & 崩 & 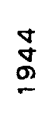 & 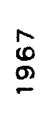 \\
\hline 焉 & $\stackrel{0}{0}_{\infty}^{\infty}$ & ㅇ. & 8 & : & & $\therefore$ & : & 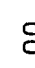 & : & $\therefore$ \\
\hline & & 兑 & : & $\begin{array}{l}\dot{\tilde{N}} \\
\stackrel{\Sigma}{2}\end{array}$ & 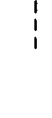 & 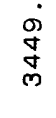 & 离 & 5 & $\dot{\sigma}$ & $\dot{0}$ \\
\hline & 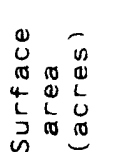 & $\begin{array}{l}\vdots \\
\vdots \\
\vdots\end{array}$ & 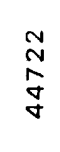 & $\underset{\sim}{\mathbb{N}}$ & $\begin{array}{l}8 \\
\text { in }\end{array}$ & $\begin{array}{l}\stackrel{8}{o} \\
\stackrel{8}{g} \\
=\end{array}$ & 总 & 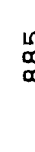 & 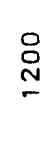 & $\begin{array}{l}0 \\
\stackrel{0}{0} \\
\vdots\end{array}$ \\
\hline 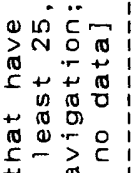 & 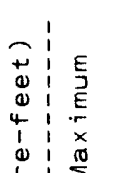 & $\begin{array}{l}\text { : } \\
\text { 吕 } \\
\text { on }\end{array}$ & $\begin{array}{l}\stackrel{8}{\circ} \\
: \\
0 \\
=\end{array}$ & $\begin{array}{l}8 \\
0 \\
0\end{array}$ & $\begin{array}{l}8 \\
0 \\
0\end{array}$ & $\begin{array}{l}8 \\
: \\
0 \\
0 \\
\circ\end{array}$ & $\begin{array}{l}8 \\
\text { o } \\
\text { on } \\
q\end{array}$ & $\frac{\pi}{0}$ & $\stackrel{\stackrel{\infty}{m}}{\frac{\sim}{\sim}}$ & 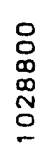 \\
\hline & 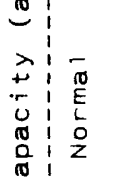 & $\begin{array}{l}\frac{8}{8} \\
\frac{8}{m}\end{array}$ & $\begin{array}{l}: \\
: \\
\stackrel{\circ}{0} \\
m \\
=\end{array}$ & 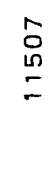 & $\frac{8}{1}$ & 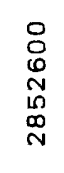 & $\begin{array}{l}\stackrel{8}{0} \\
\stackrel{0}{0} \\
=\end{array}$ & $\infty$ & 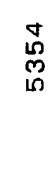 & $\begin{array}{l}\circ \\
\vdots \\
0 \\
0\end{array}$ \\
\hline
\end{tabular}

a o o o

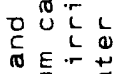

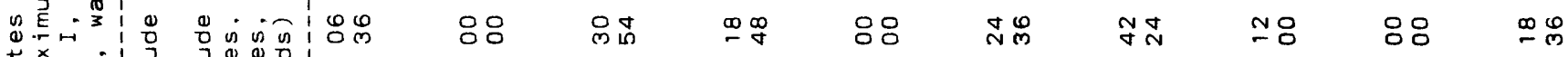

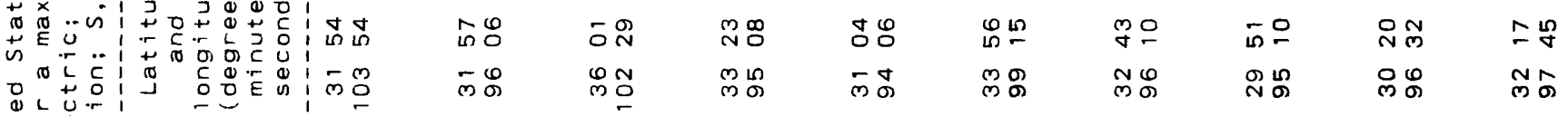
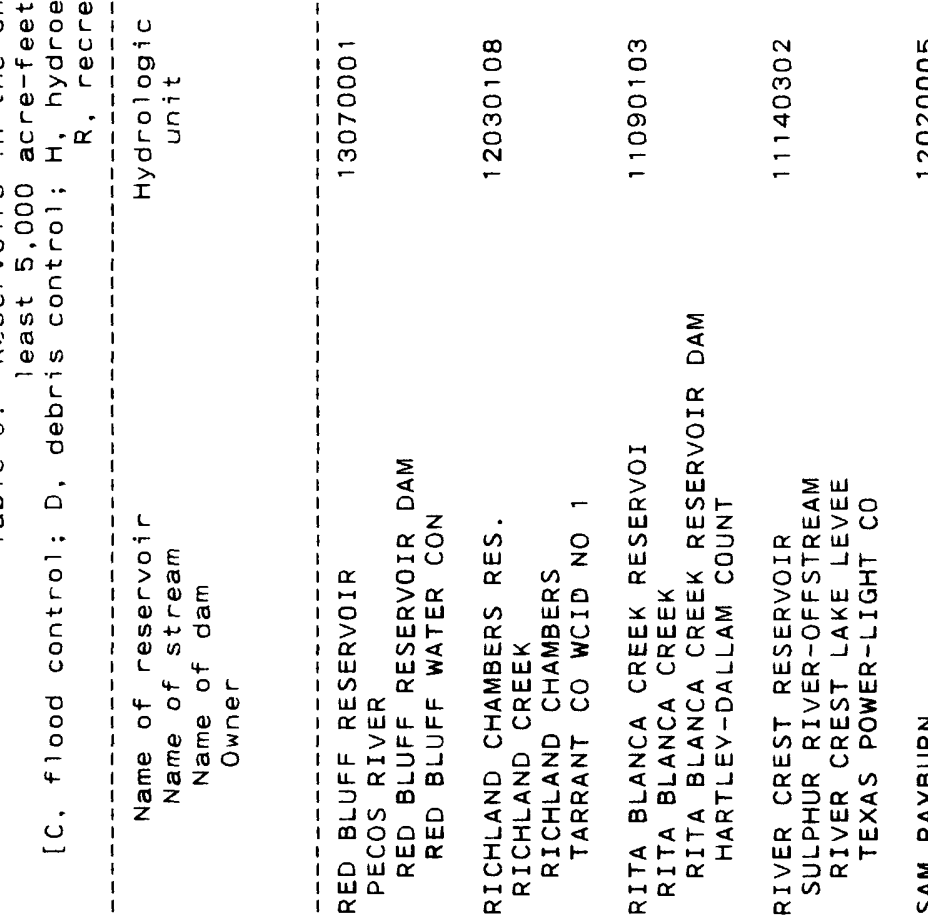

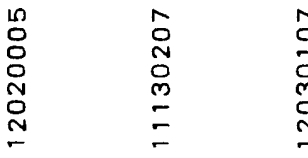

$\begin{array}{ll}1 & \text { J } \\ 0 & 0 \\ 0 & 0 \\ 0 & 0 \\ \text { N } & \text { N } \\ & \end{array}$

$\begin{array}{ll}N & N \\ 0 & \stackrel{N}{0} \\ 0 & O \\ N & 0 \\ & \stackrel{N}{N}\end{array}$

$N$
N
0
0
$O$
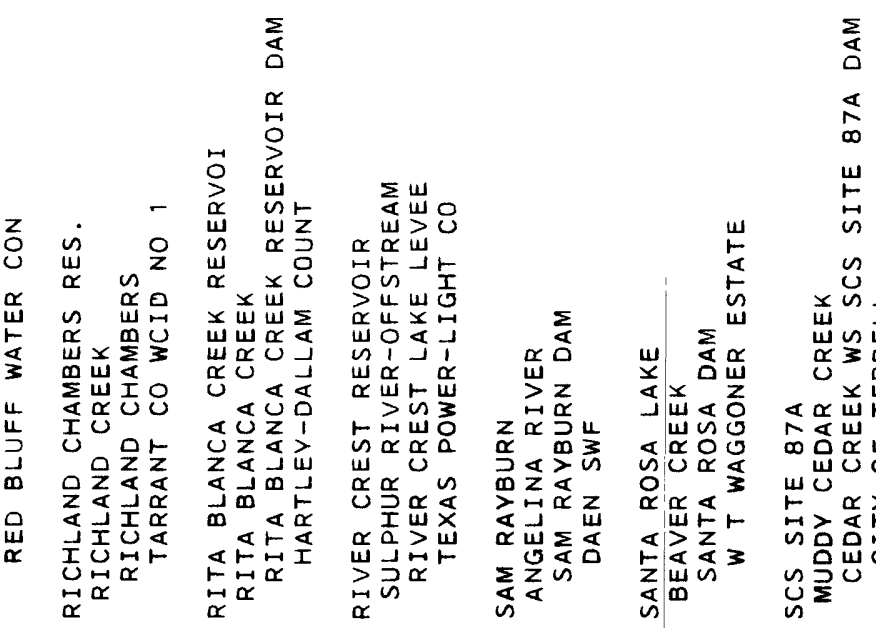

$\begin{array}{ll}0 & N \\ 0 & 0 \\ 0 & 0 \\ 0 & 0 \\ 0 & N\end{array}$
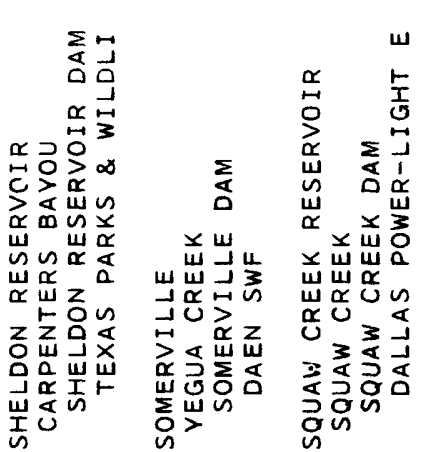


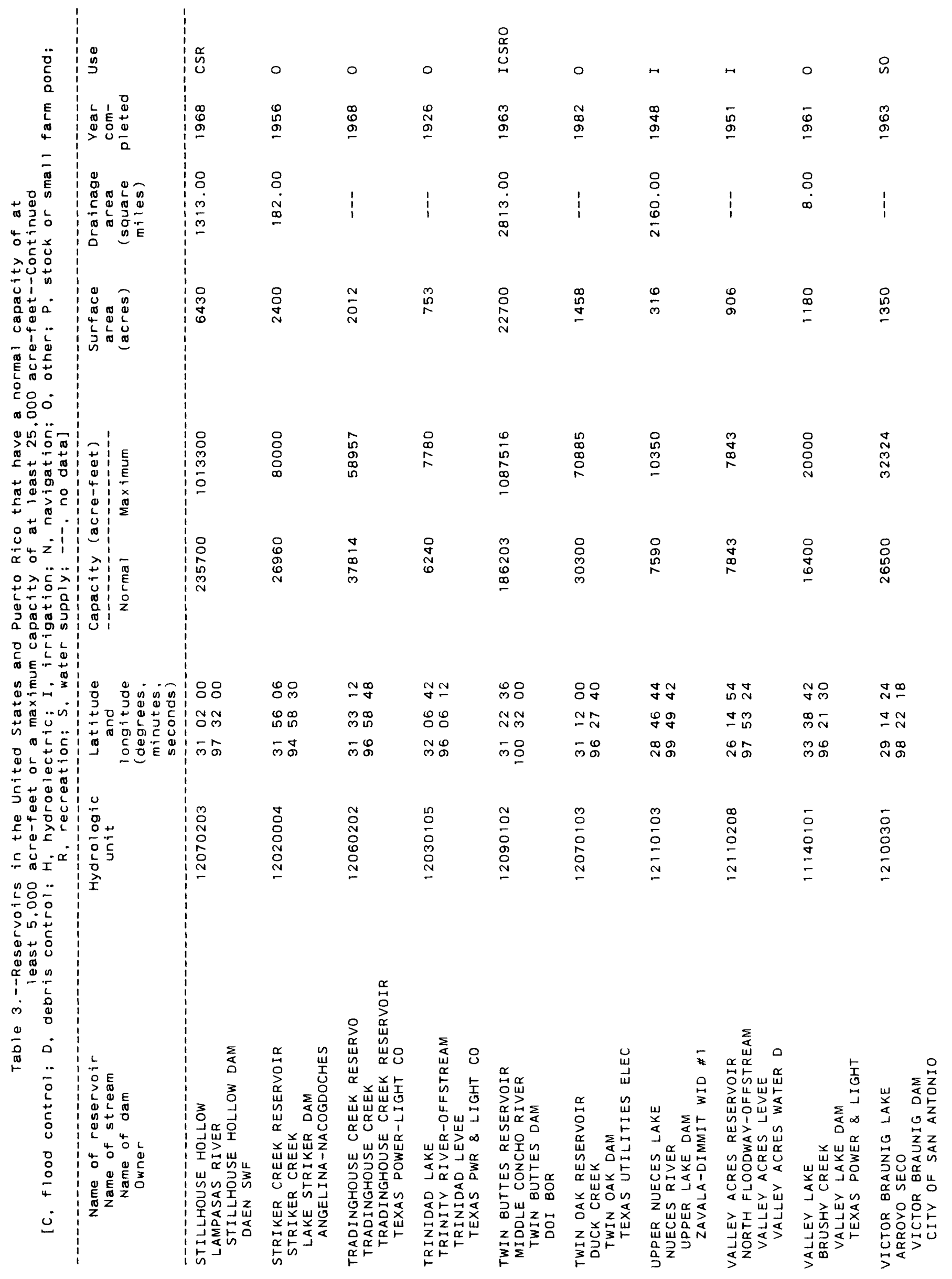




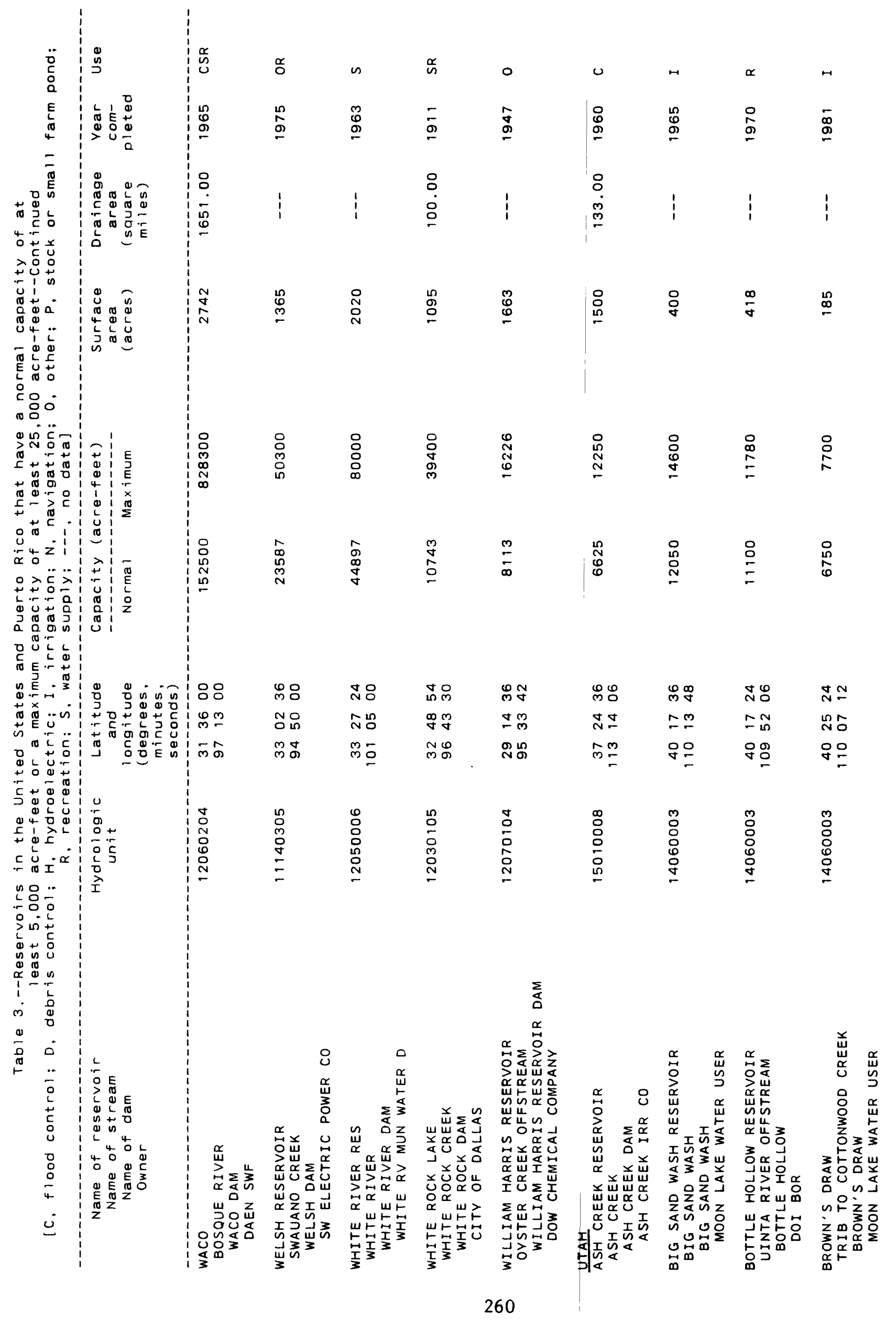




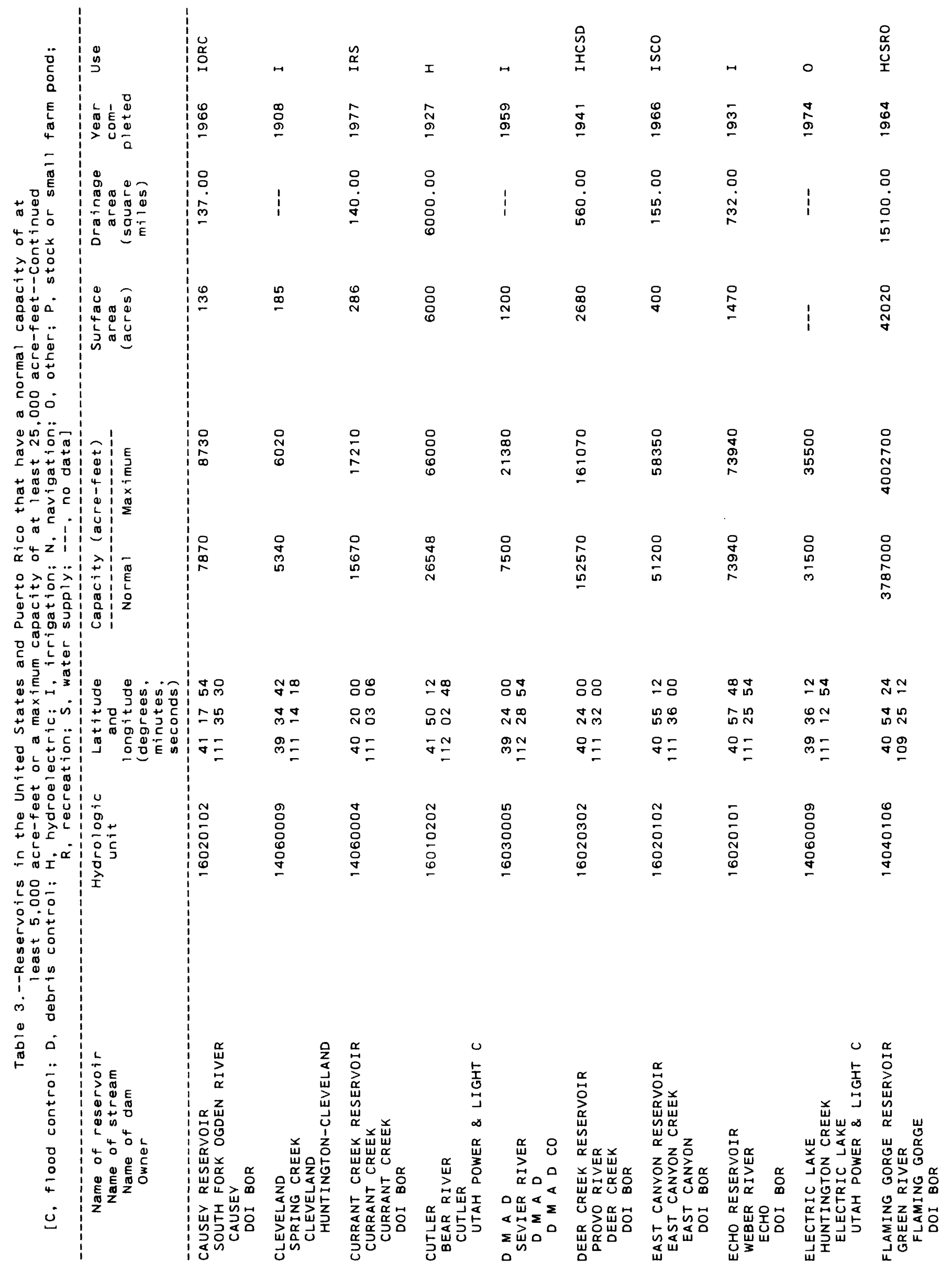




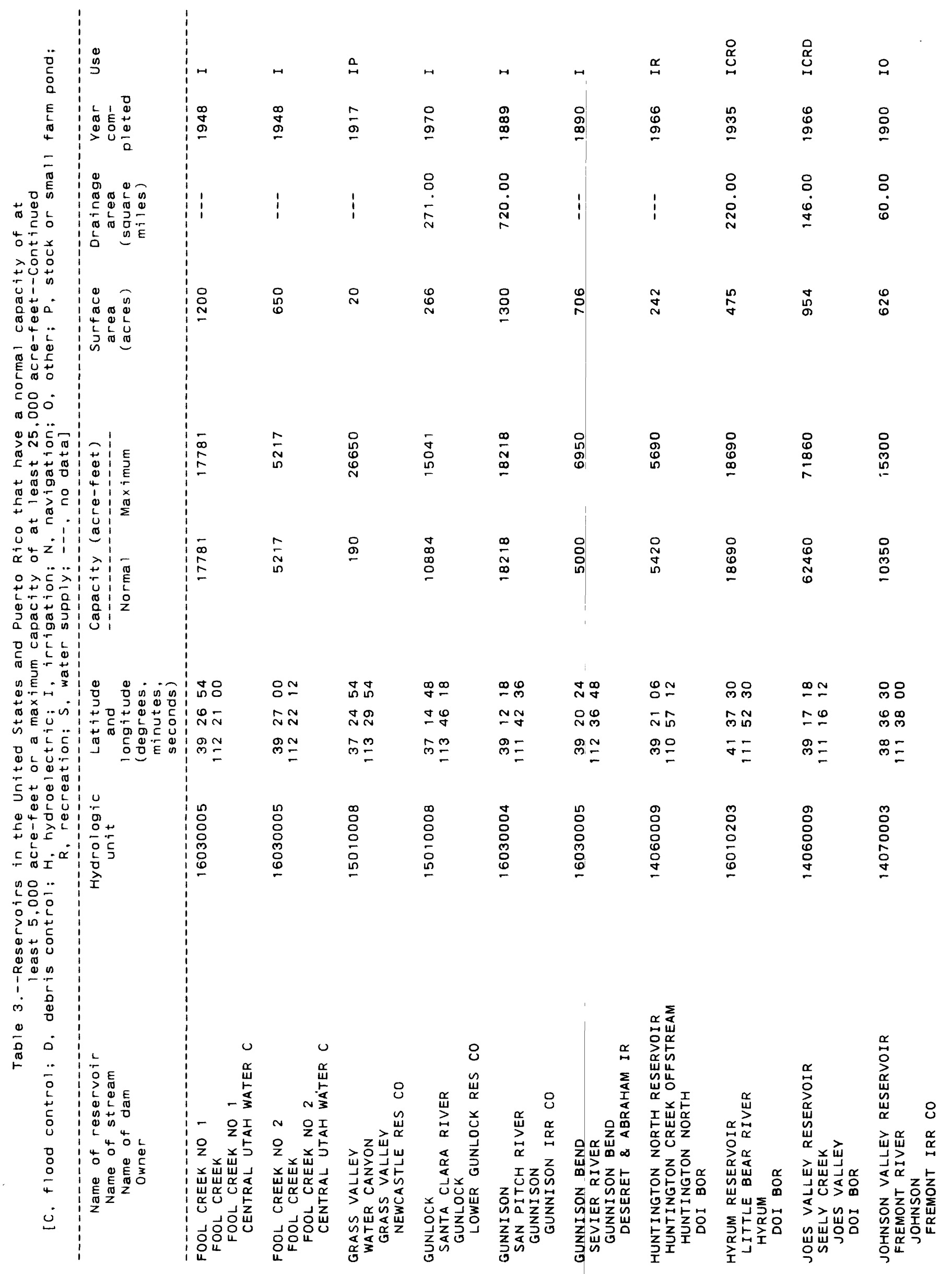




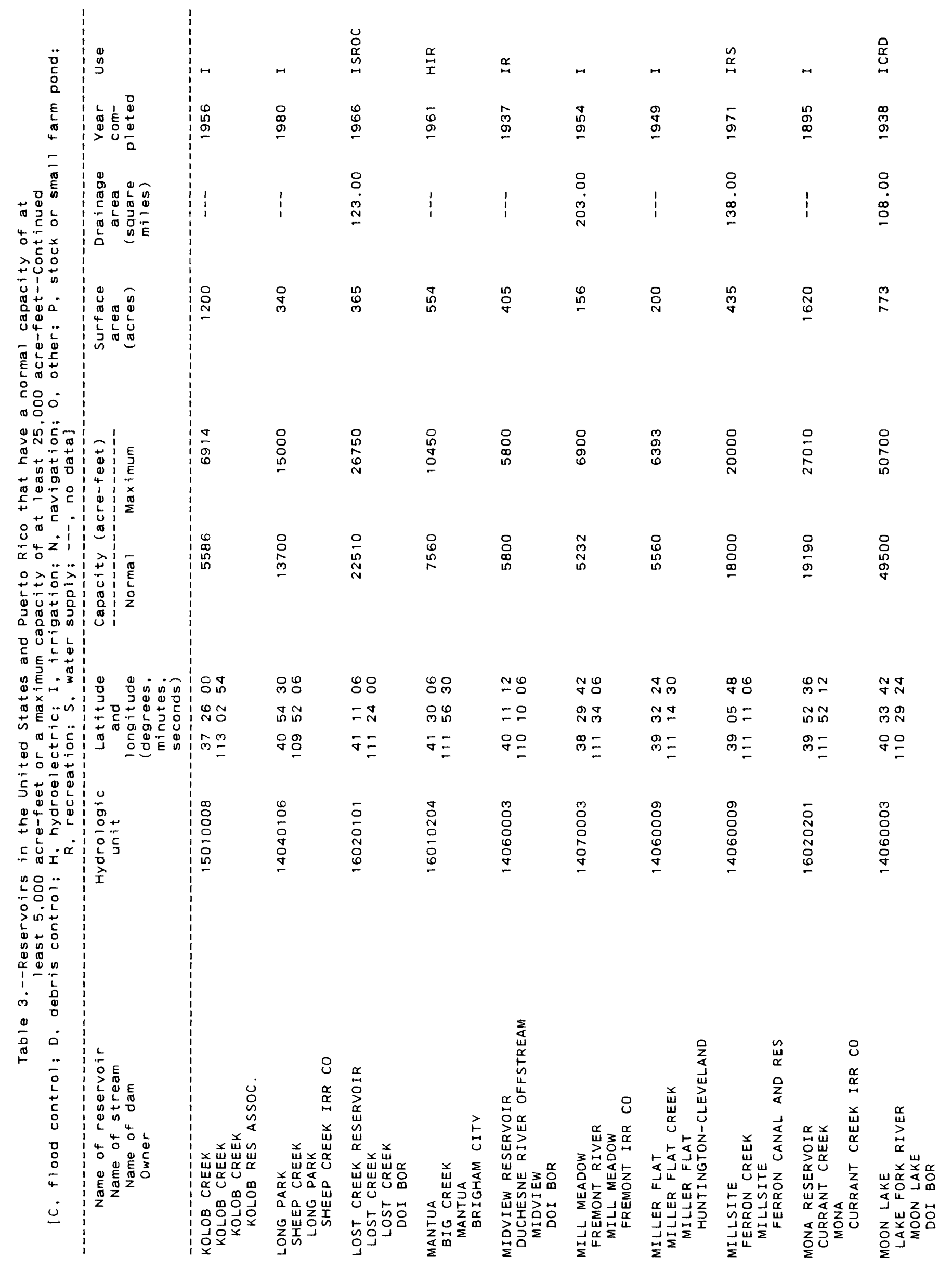




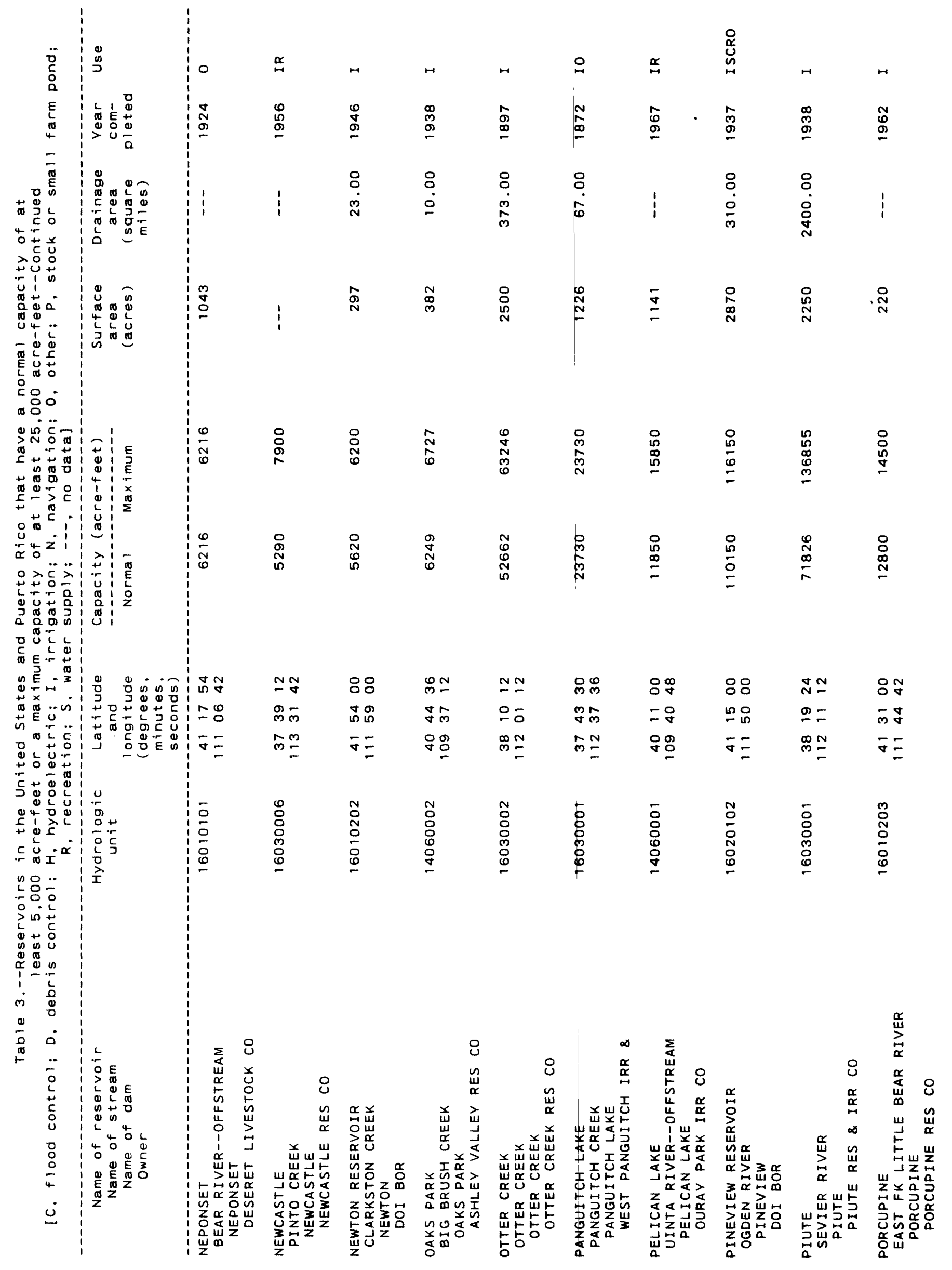




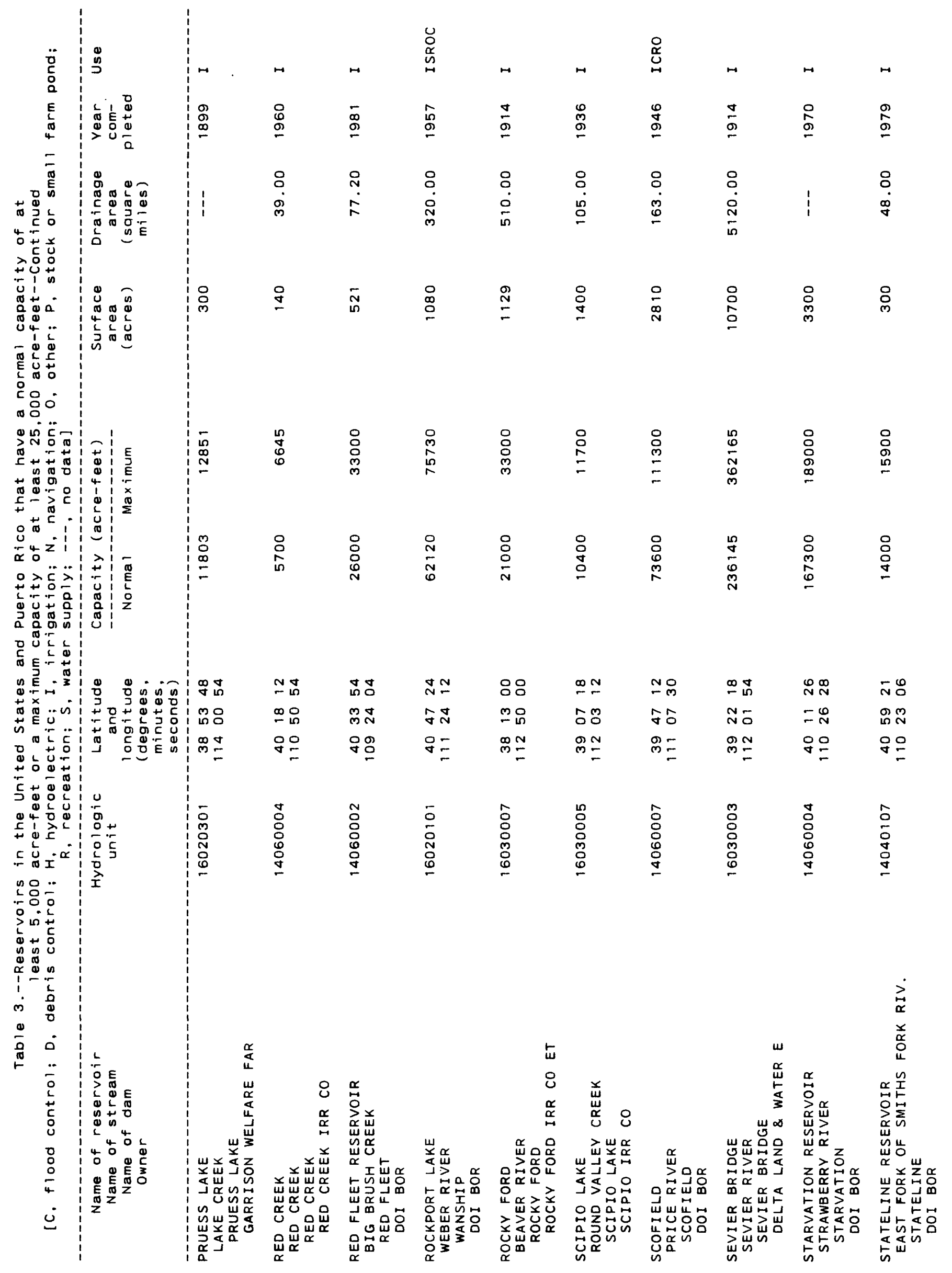




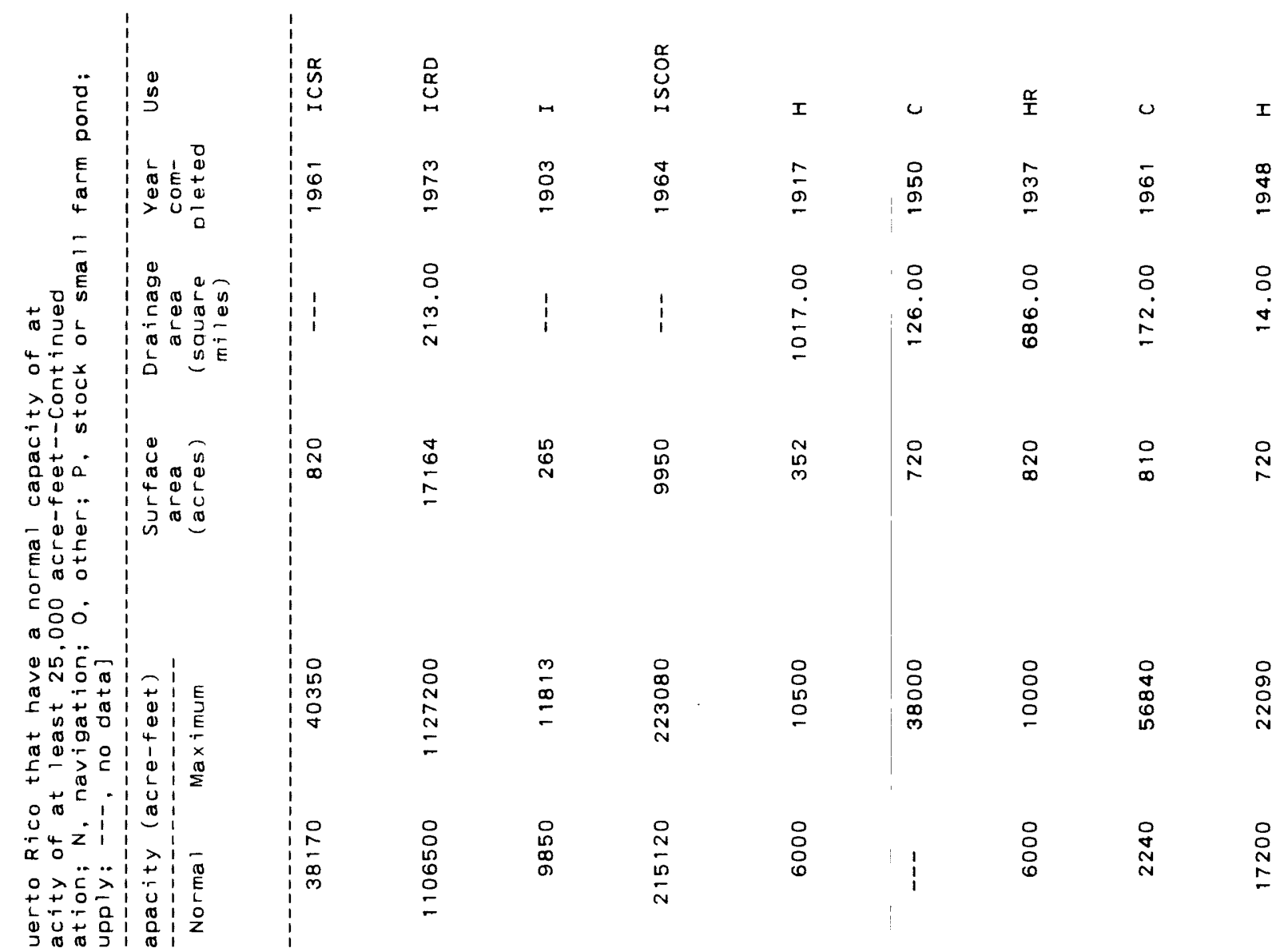

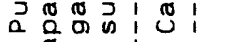

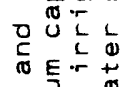

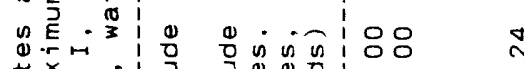

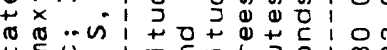

के E

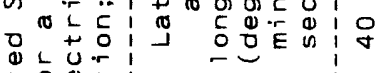

$\mathrm{m}$

\begin{tabular}{|c|c|c|c|c|c|}
\hline$\because \stackrel{\circ}{\circ}$ & $\mathcal{F} \underset{\nabla}{\mathcal{F}}$ & นึ่ & $\stackrel{\infty}{\sim}$ & 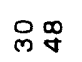 & $\stackrel{\infty}{\infty}$ \\
\hline $\bar{m} \bar{n}$ & N & $\stackrel{\infty}{N} \stackrel{0}{0}$ & $\underset{\sigma}{0}$ & ஹீ & $\because \mathscr{\sigma}$ \\
\hline$\hat{m} \stackrel{m}{=}$ & $\dot{\sigma} \stackrel{-}{-}$ & $\forall m$ & $\underset{\sigma}{M N}$ & $\forall \stackrel{m}{R}$ & $\underset{d}{M N}$ \\
\hline
\end{tabular}

$\bar{c}+\frac{\pi}{0}$

So

\begin{tabular}{rl|l}
$\Phi$ \\
+ \\
+
\end{tabular}

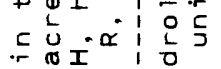

으ㅁㅛㅛ

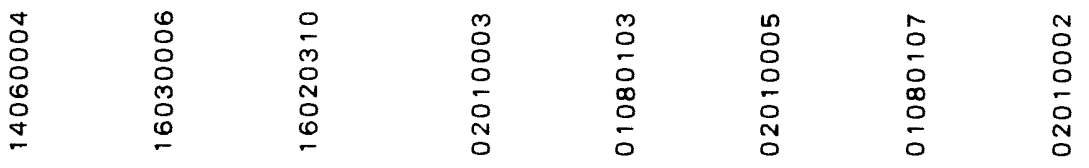

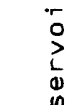

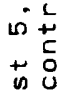

$\underset{10}{0} \stackrel{0}{0}$

ले

$\stackrel{\frac{1}{0}}{\circ}$
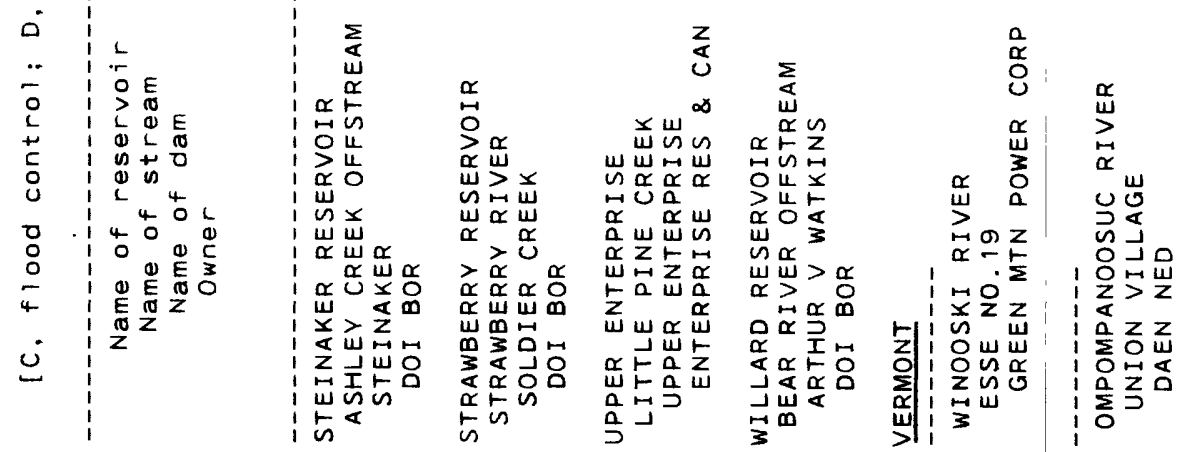

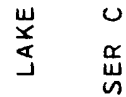

这

$\stackrel{\alpha}{a x}$

뭉 


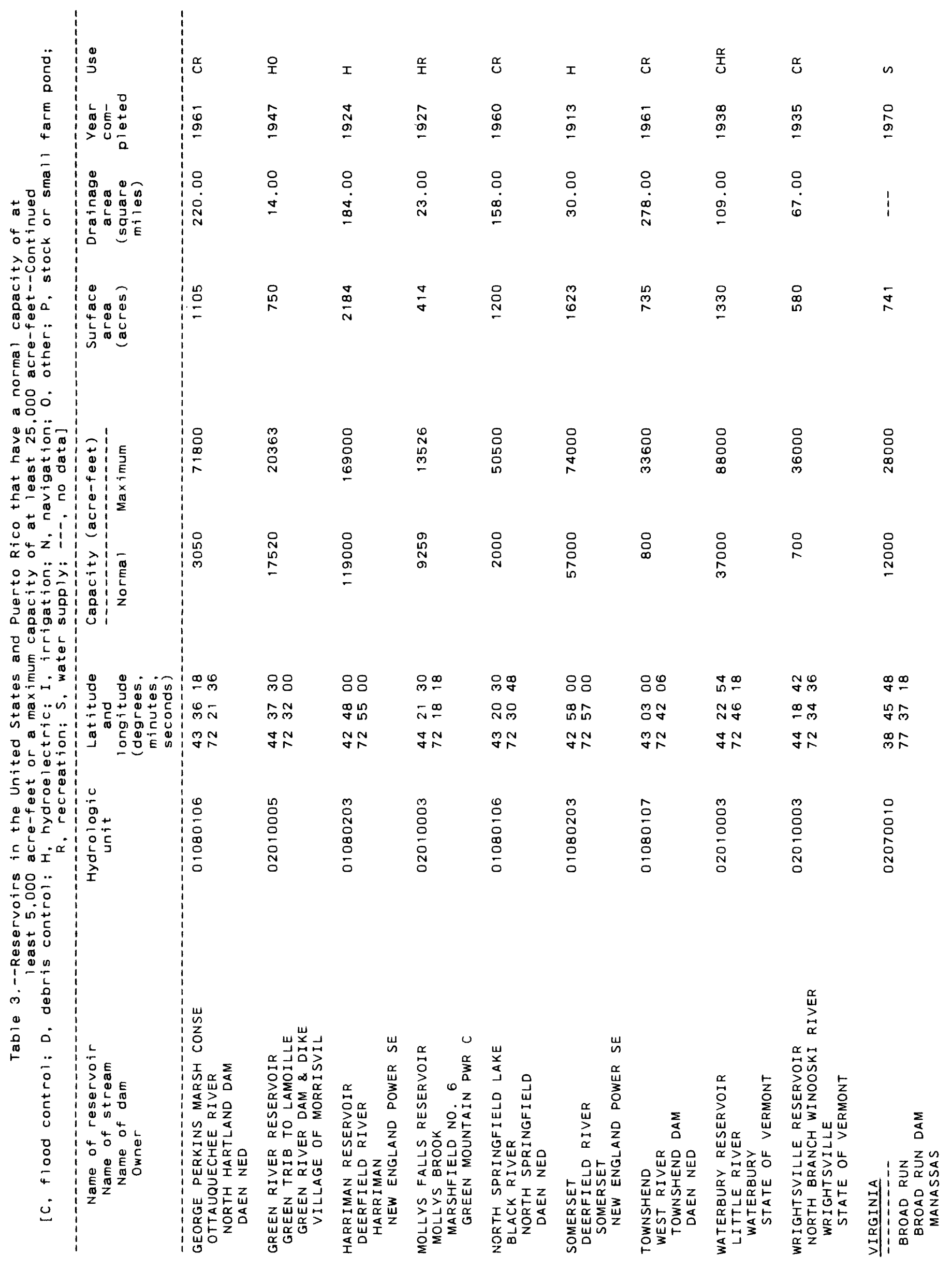




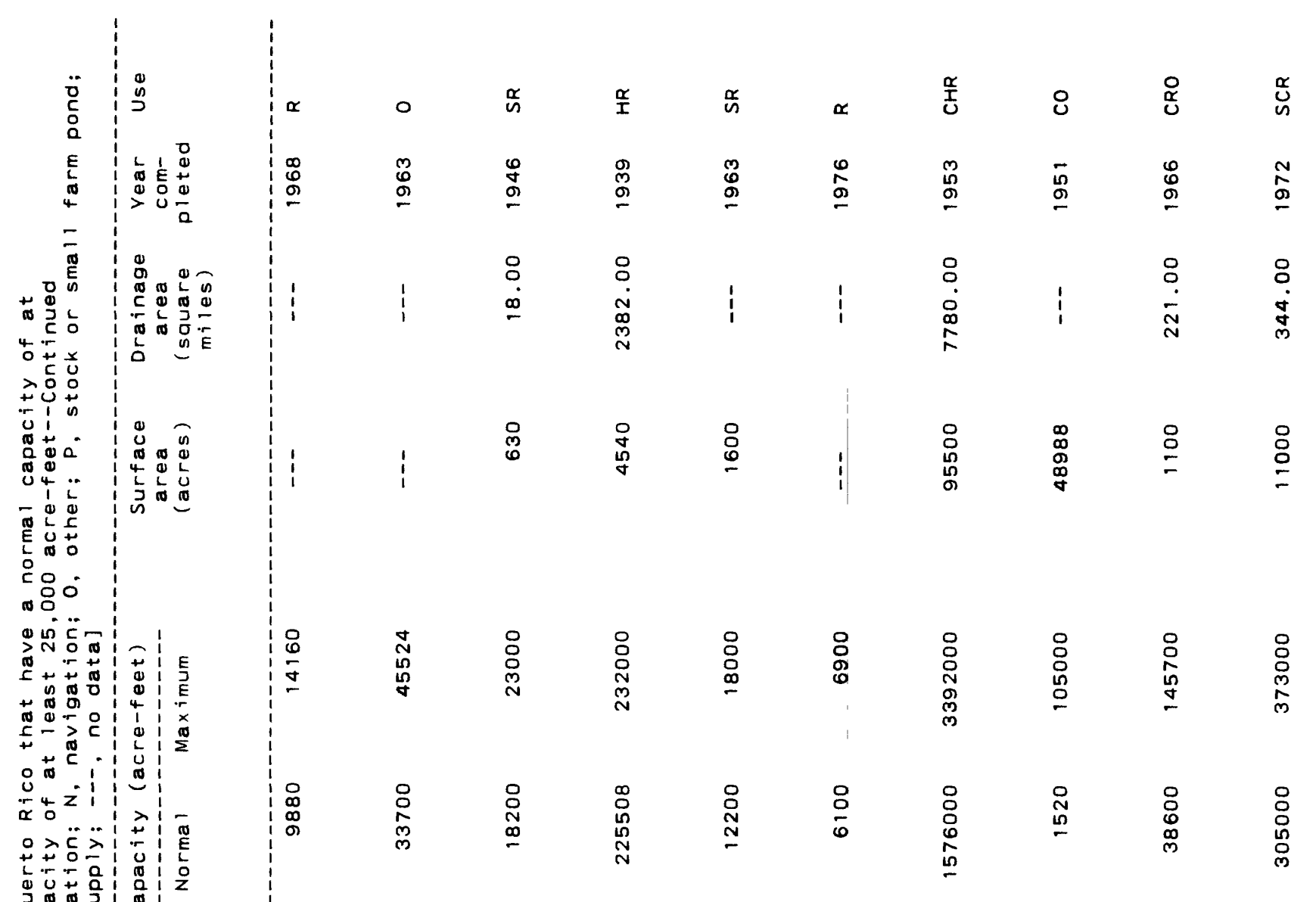

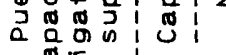

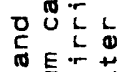

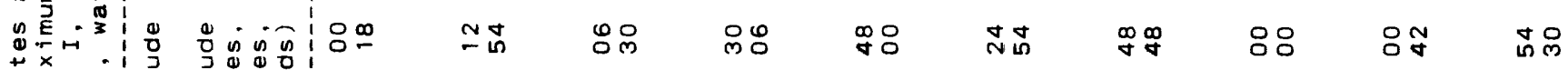

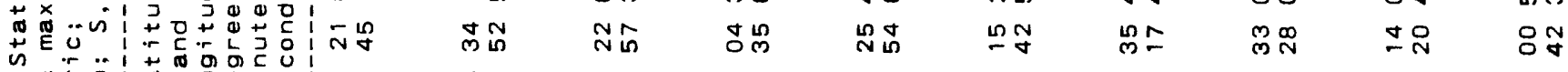
记落

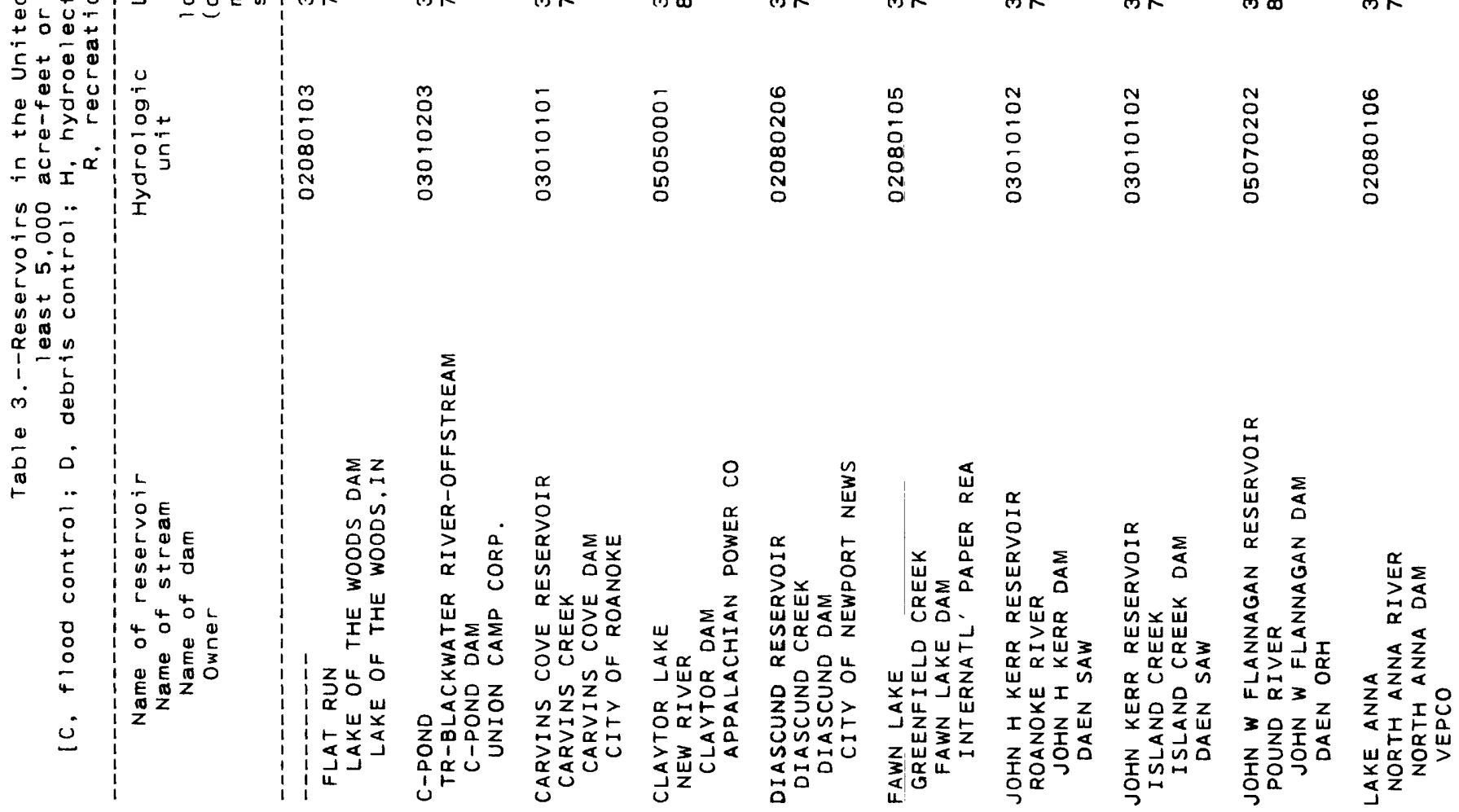




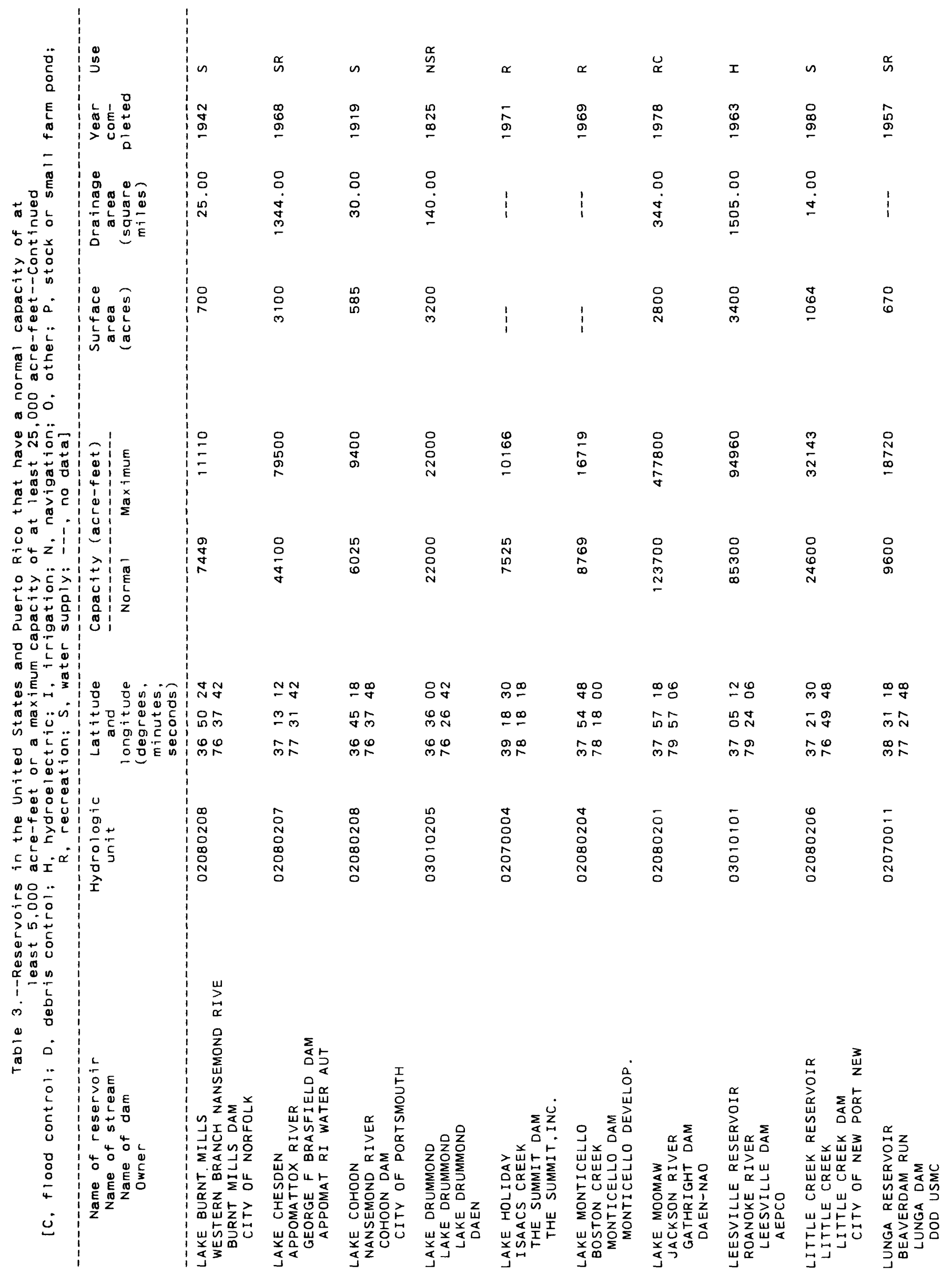




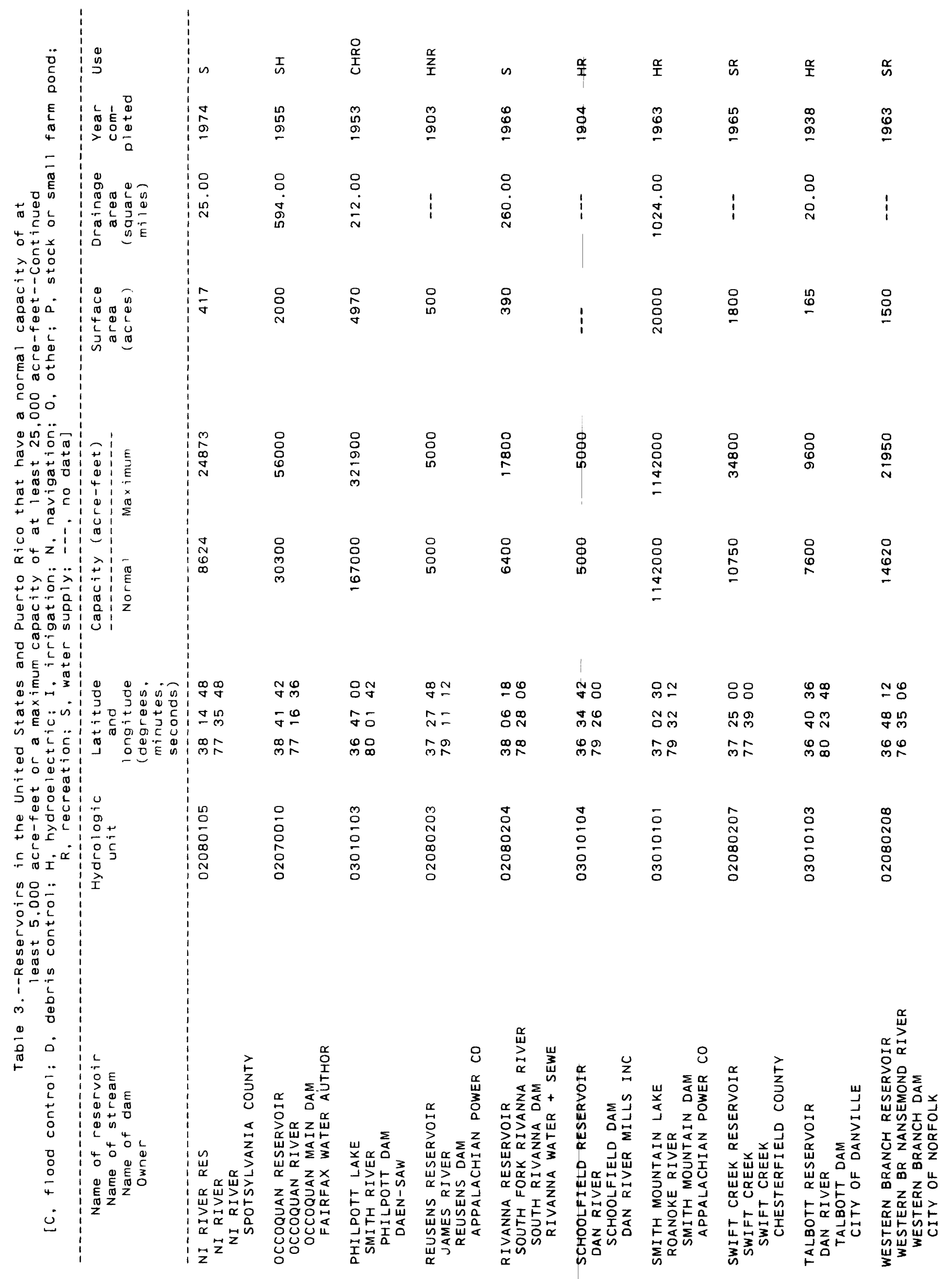




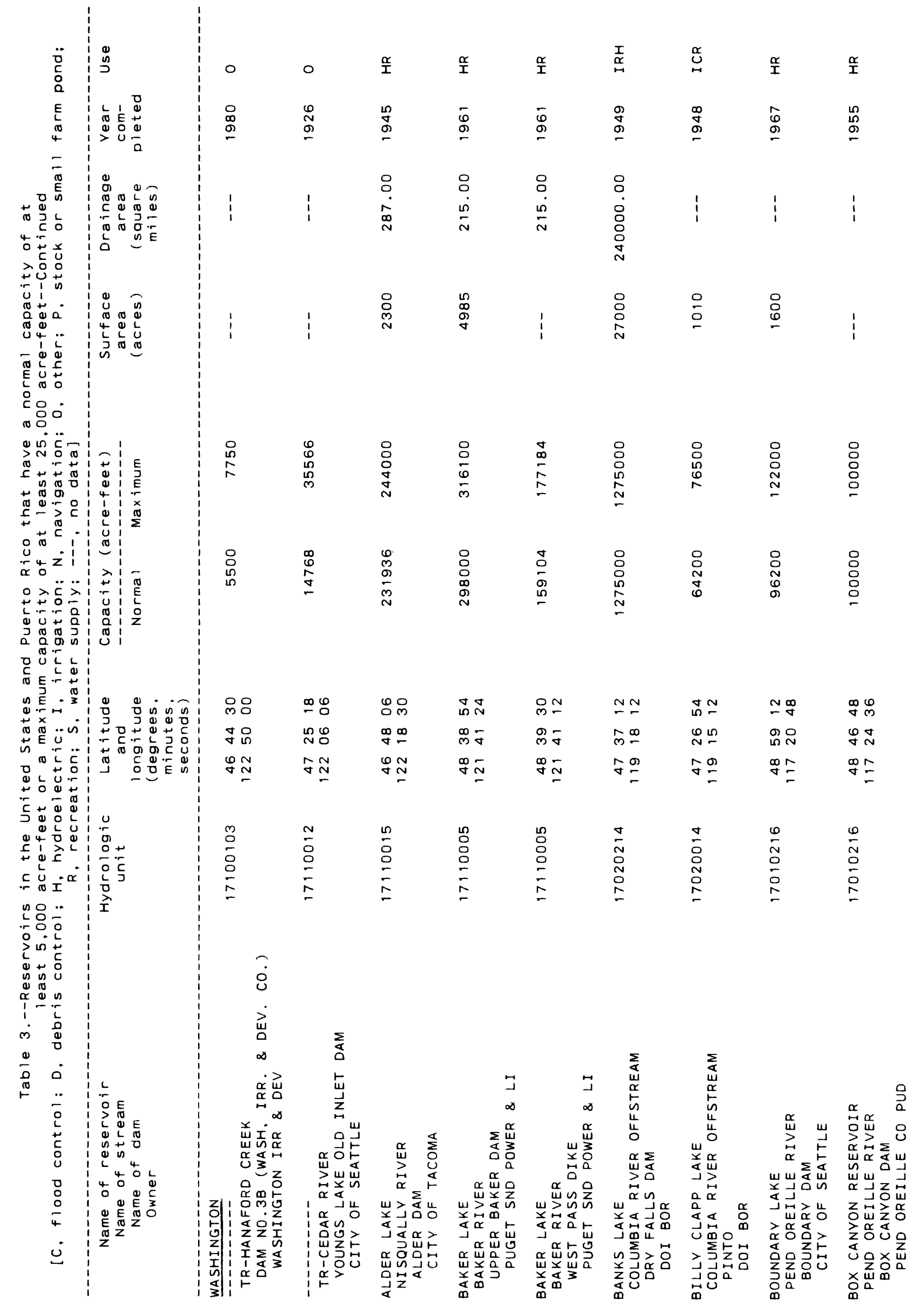




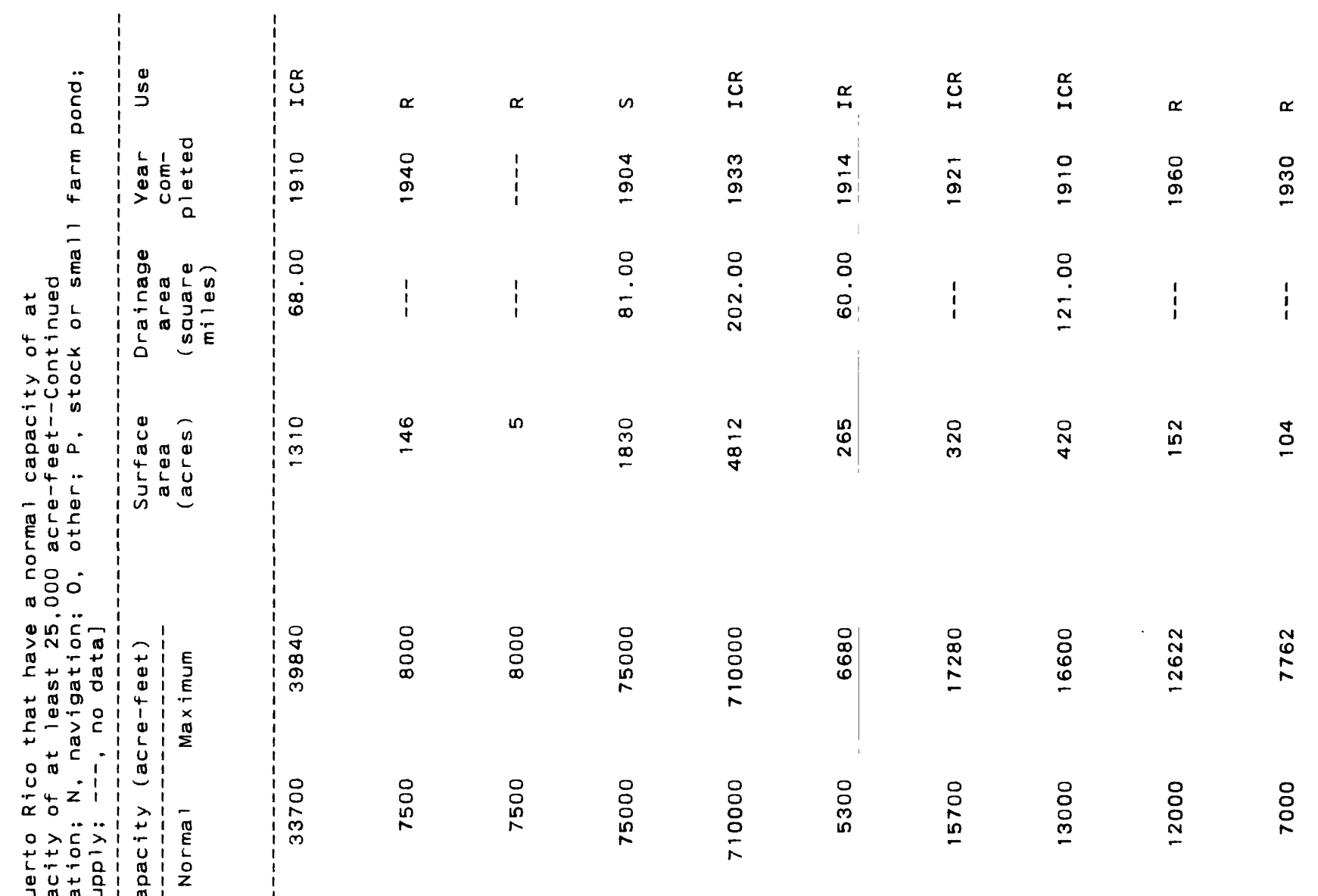

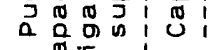

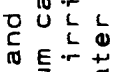

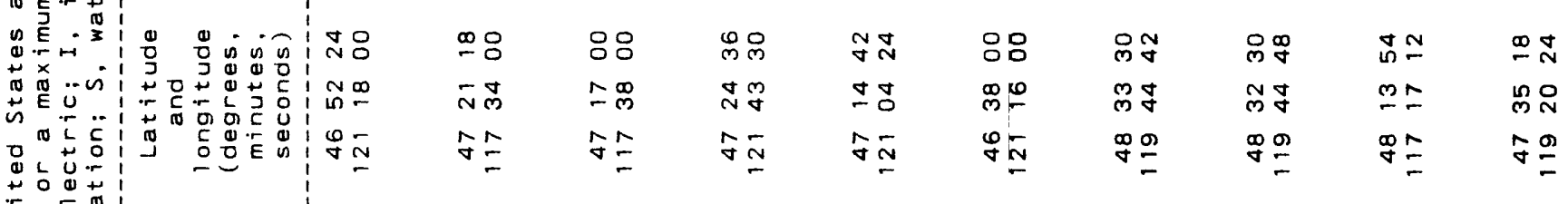

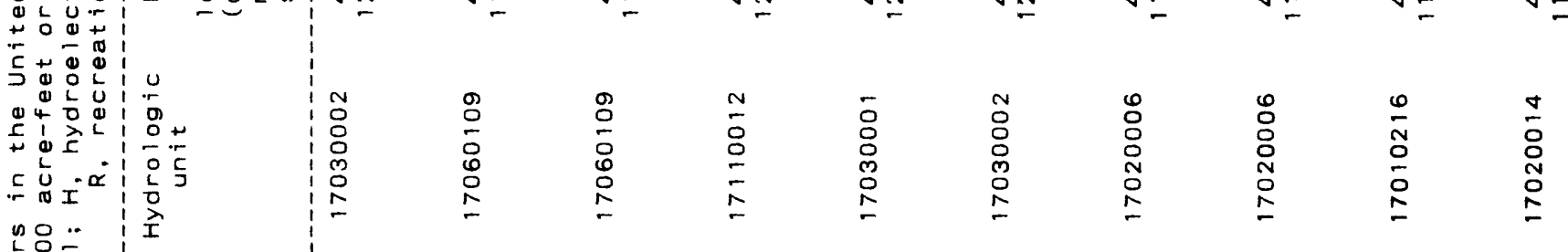

5

(1)

20

.

is

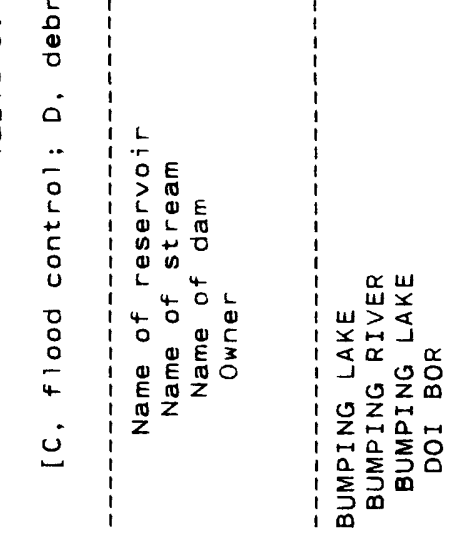

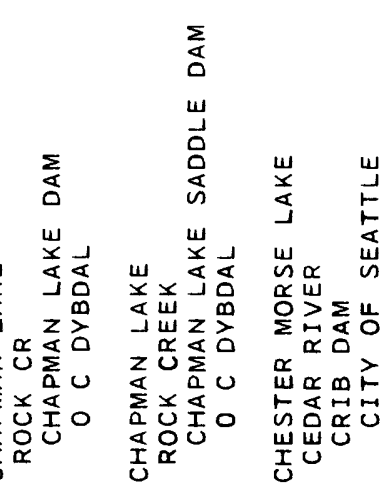

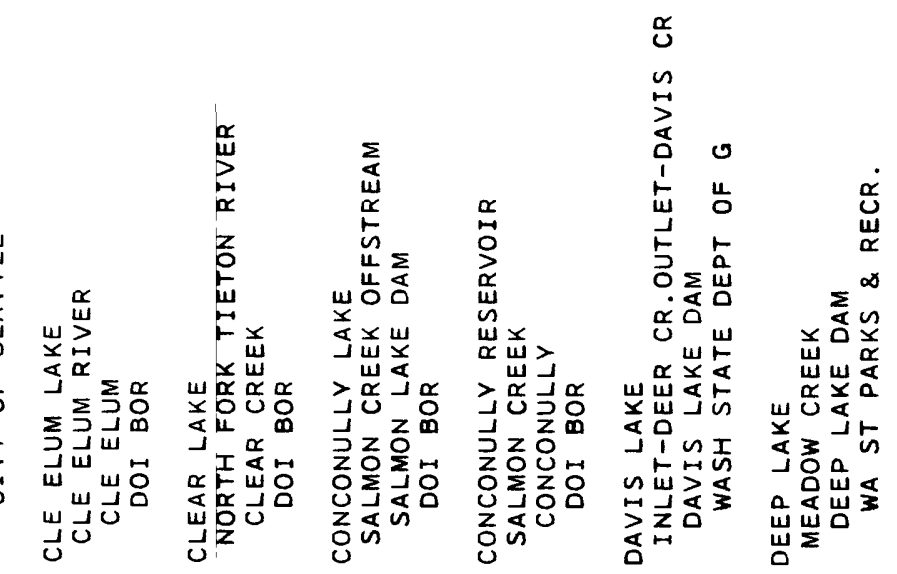




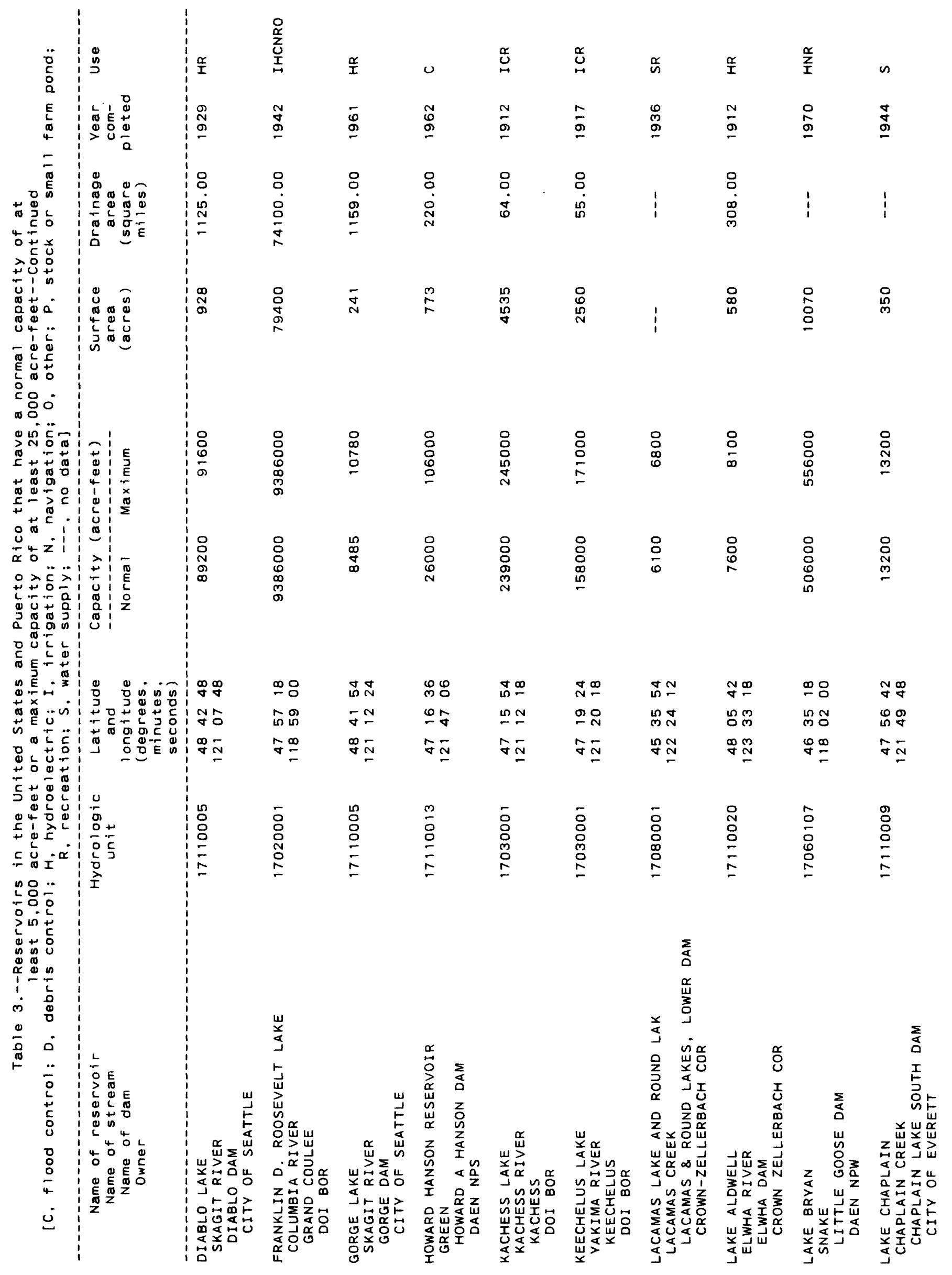



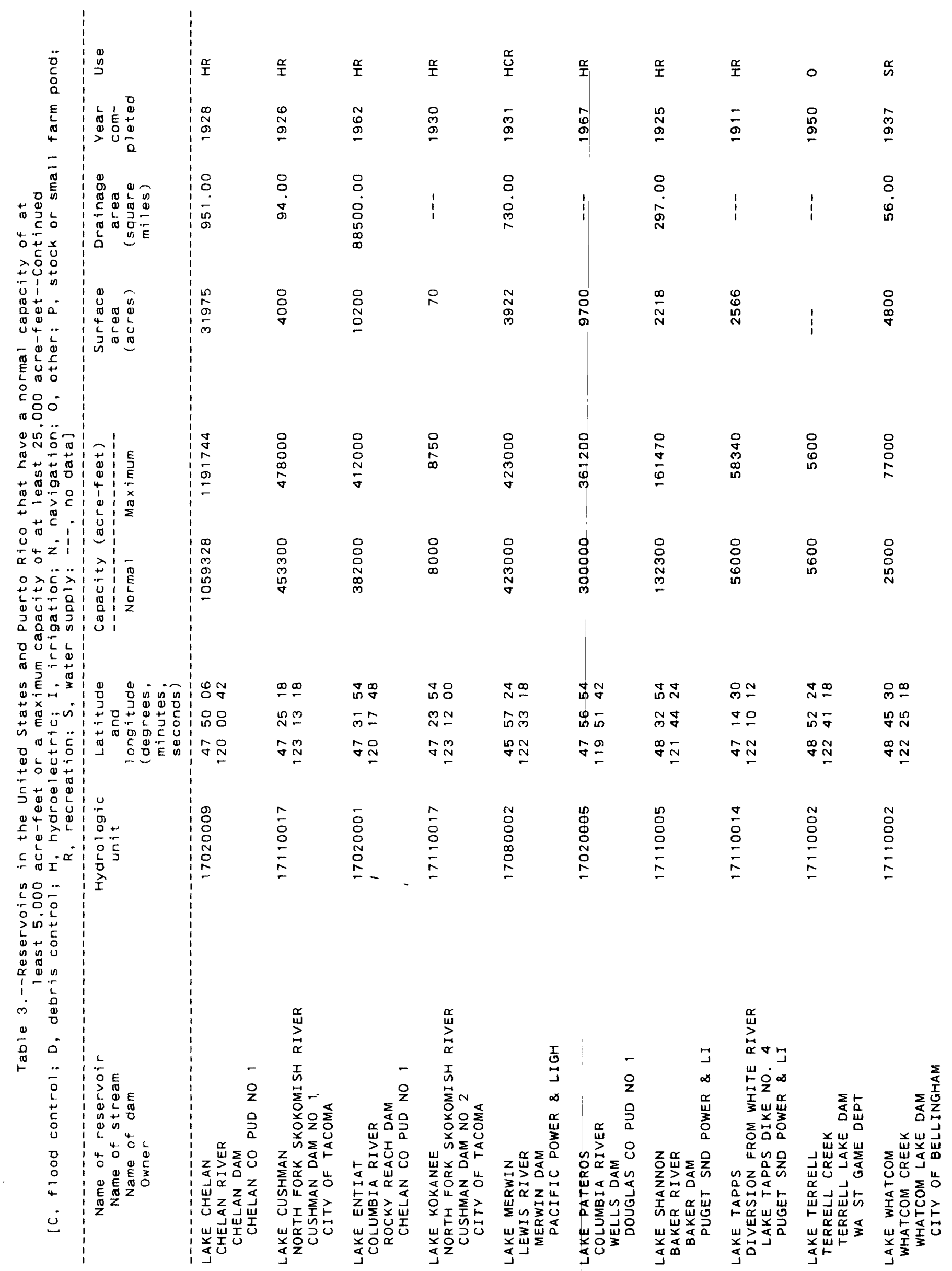


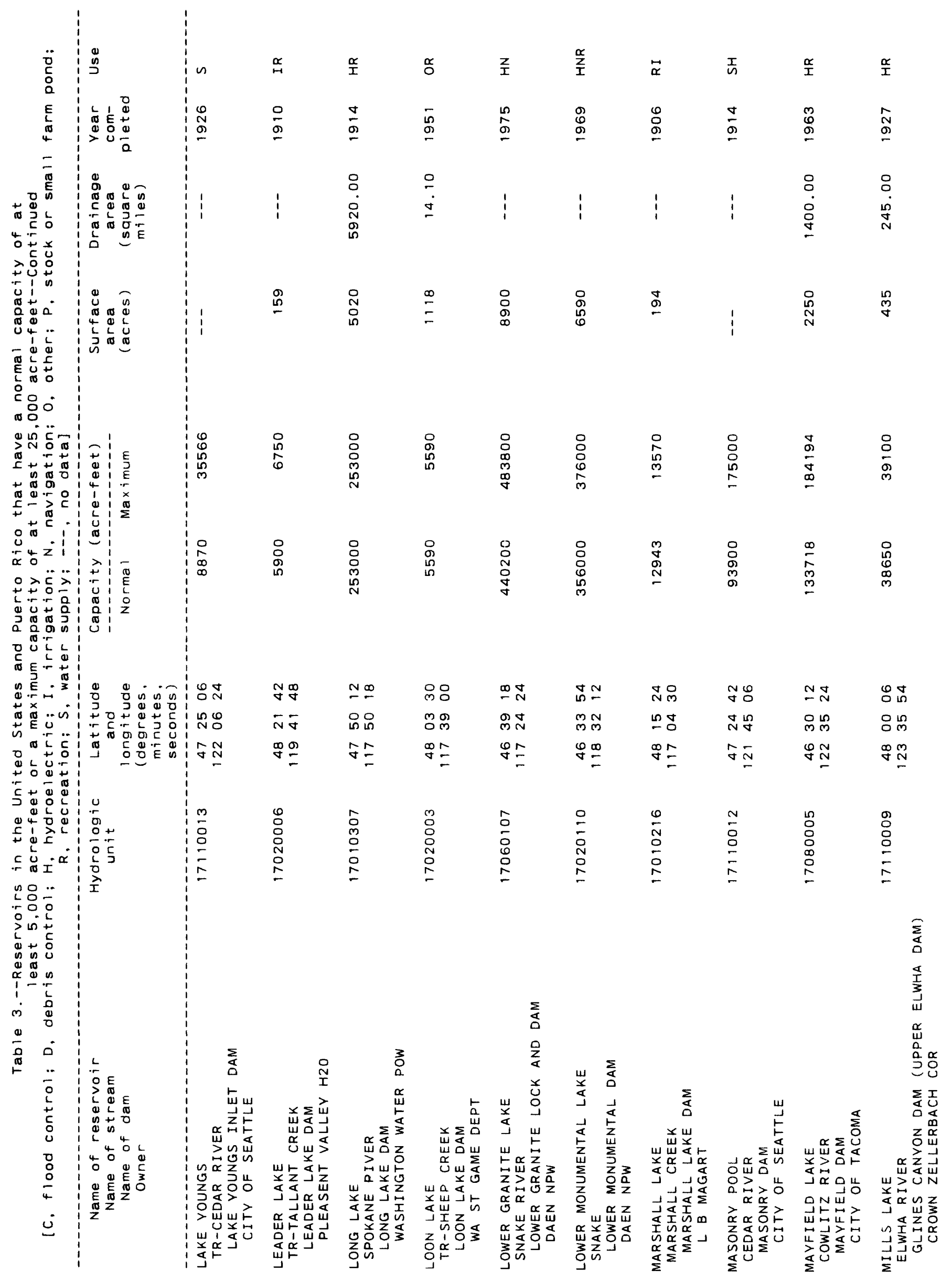




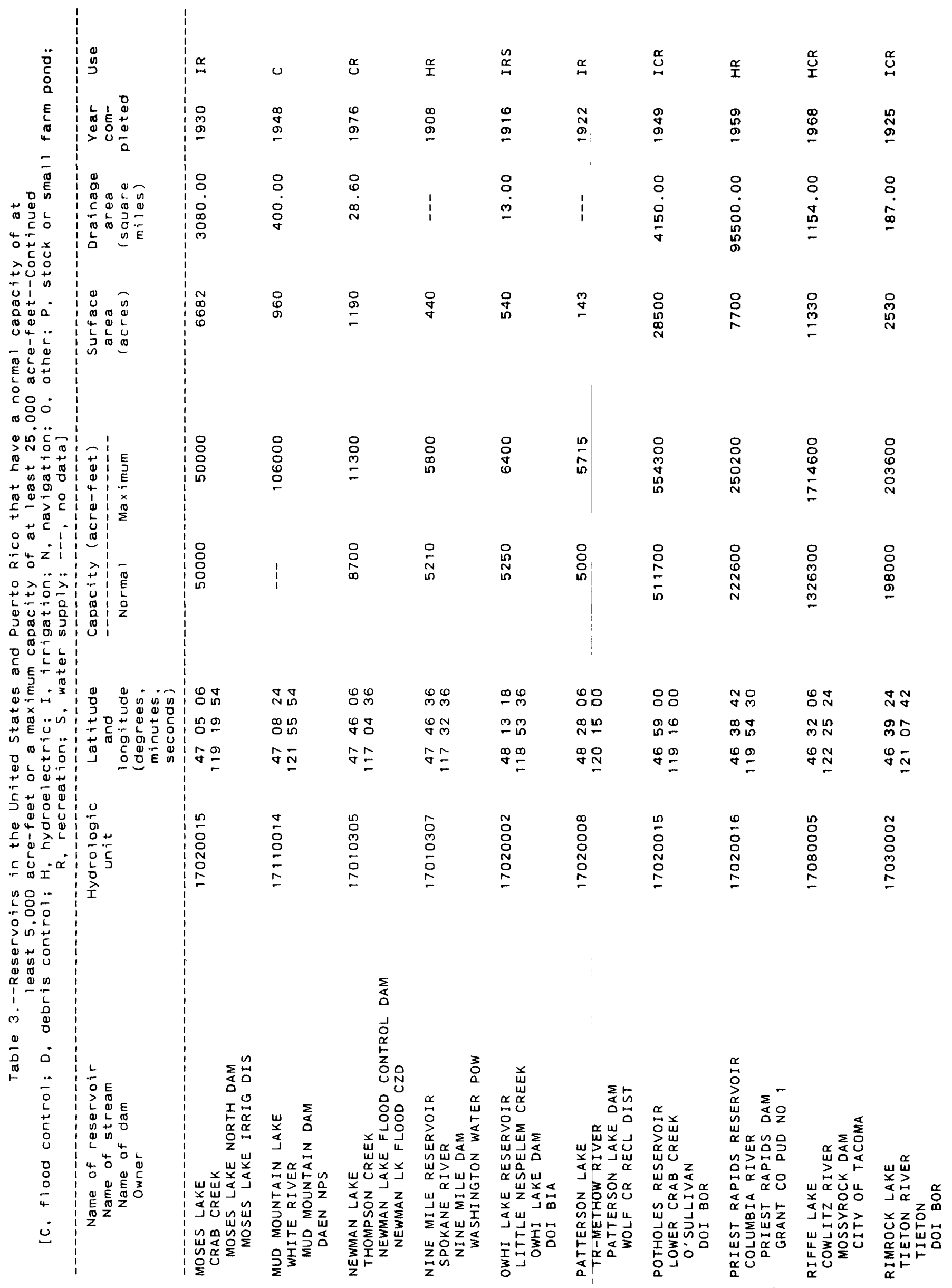




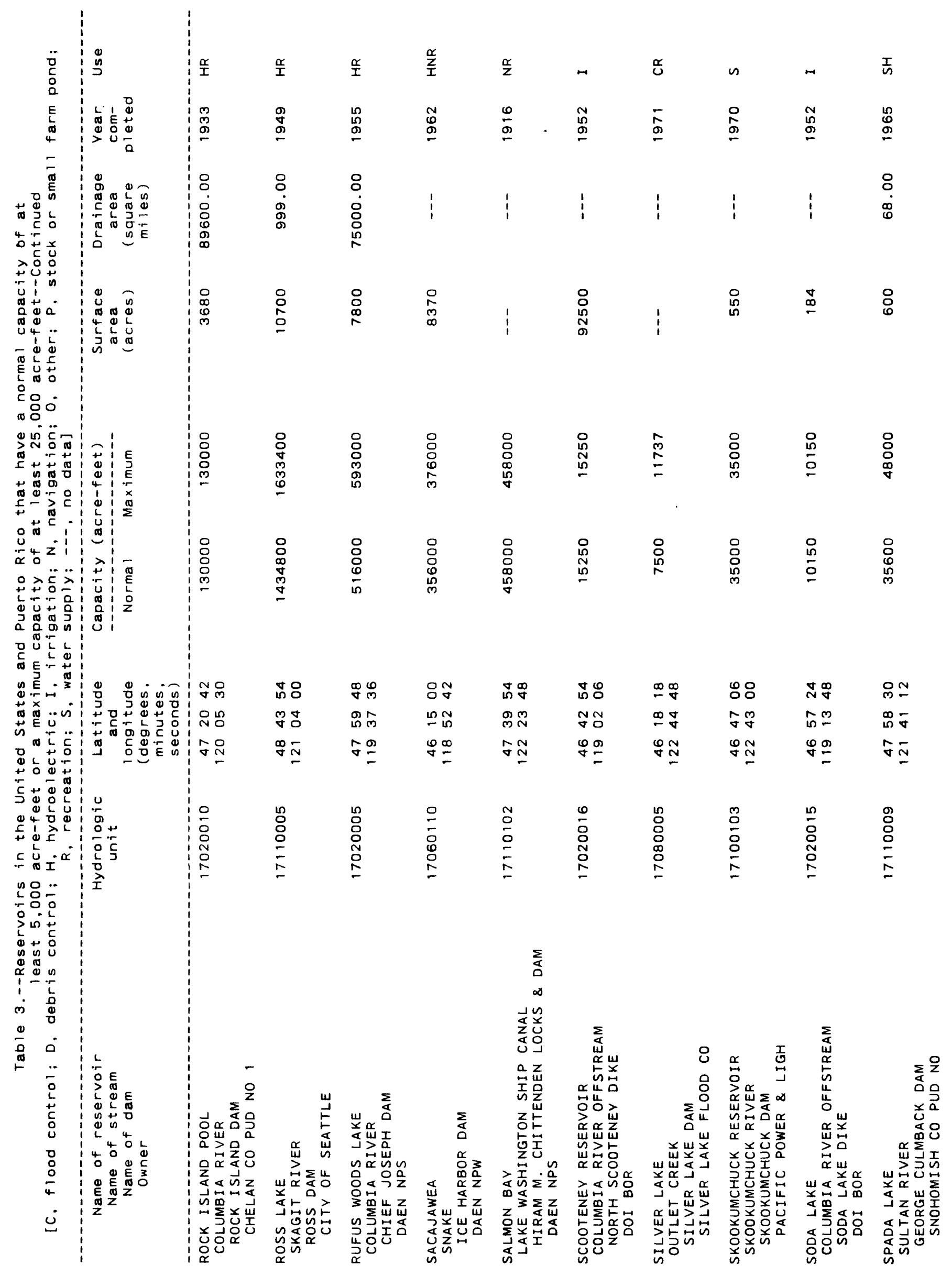



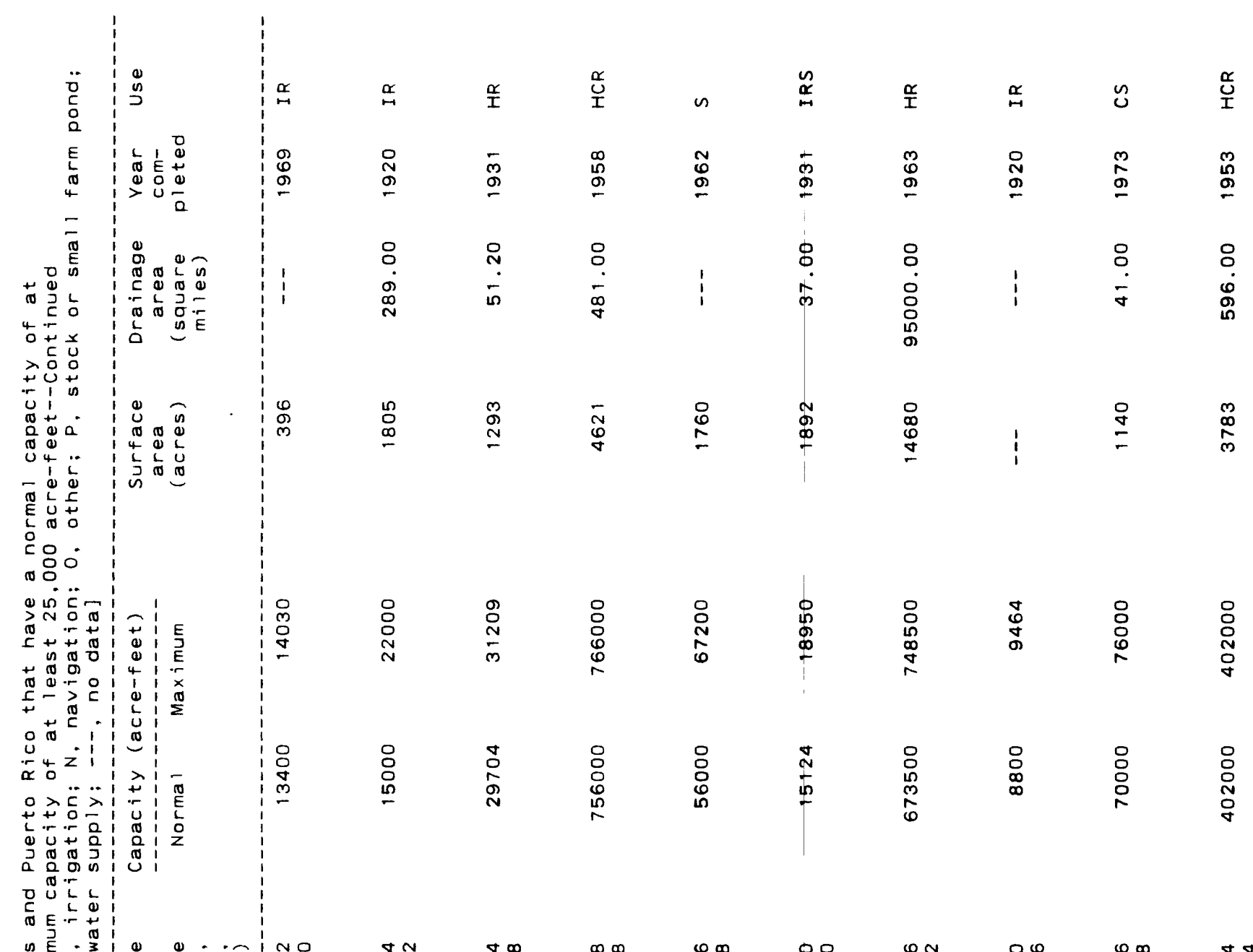

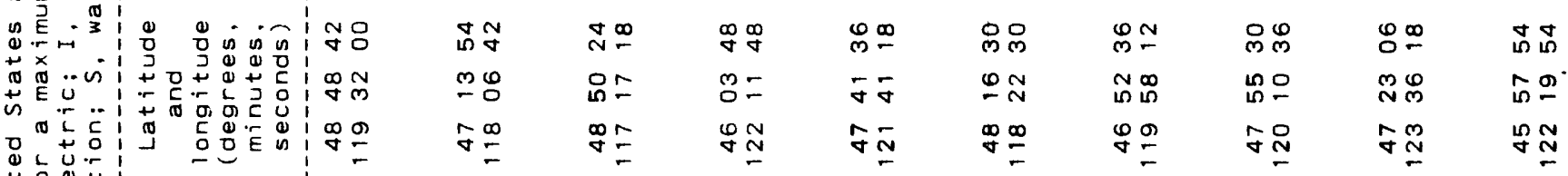
$\therefore 0$

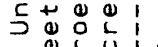

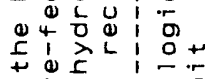

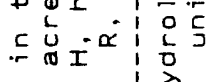

늠요뭉

웅

is is

x.

m

$\frac{1}{5}$

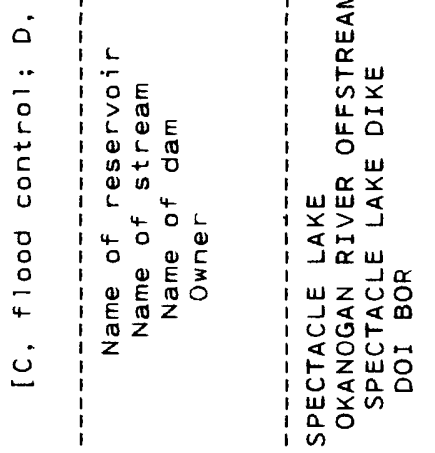

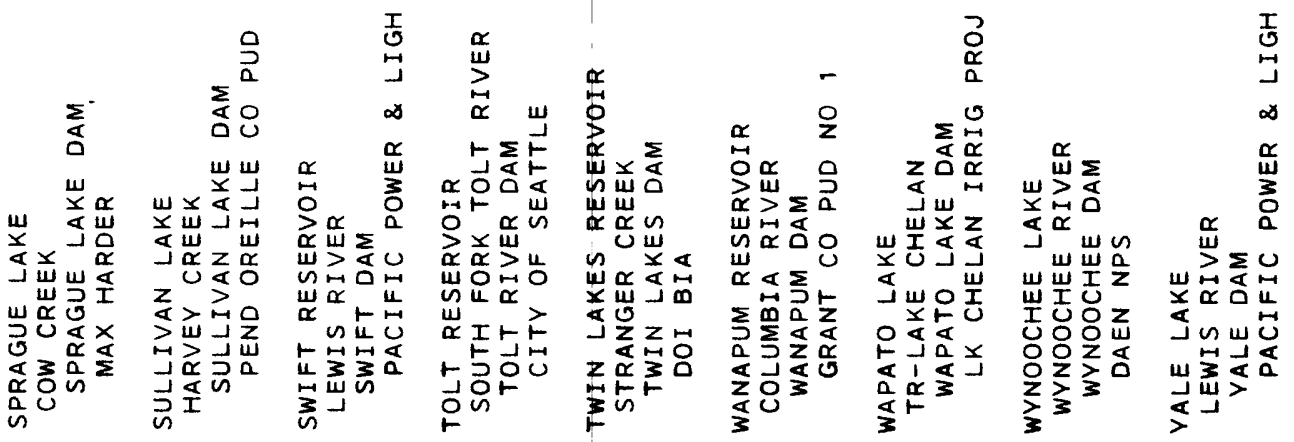




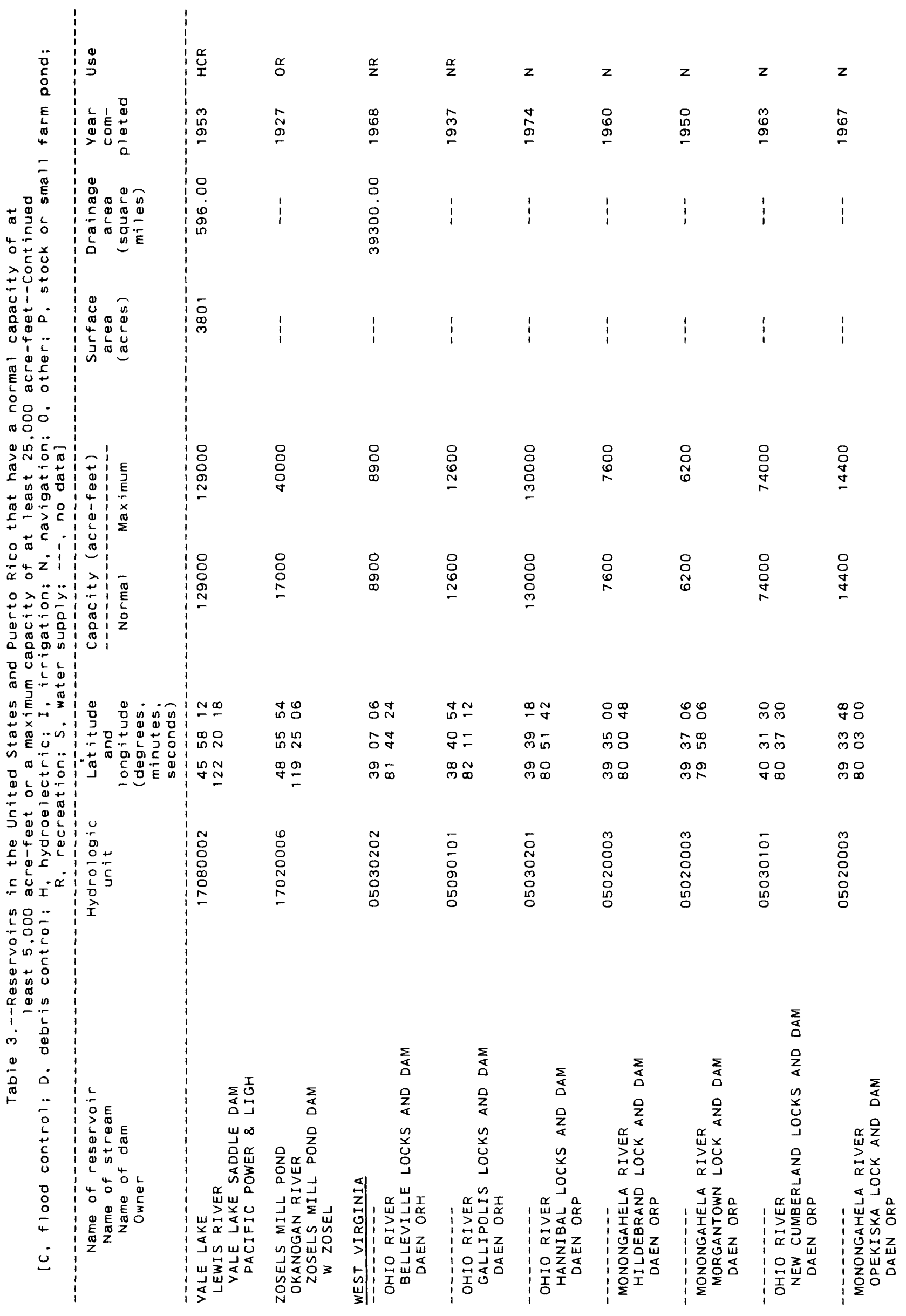




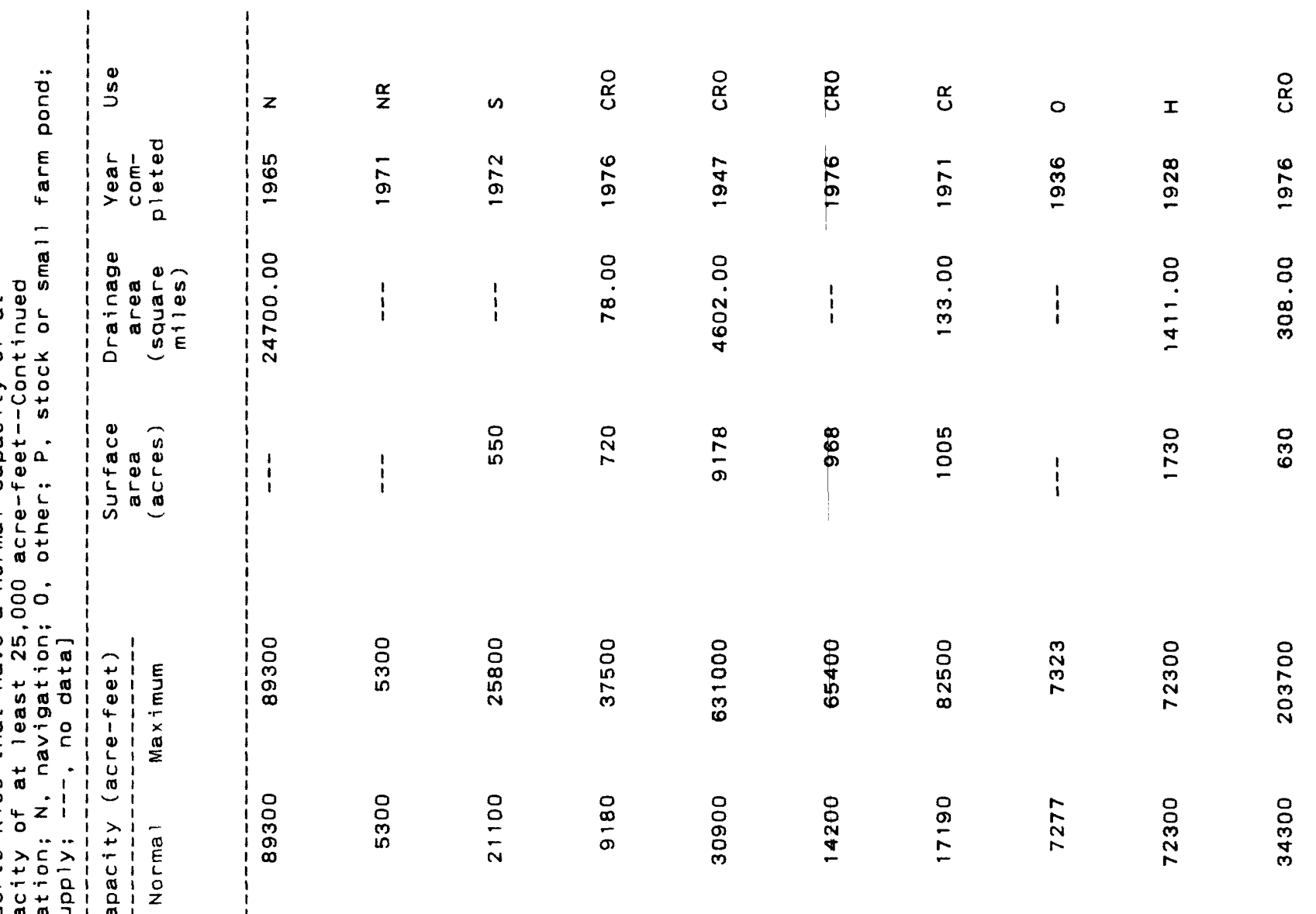

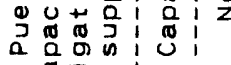

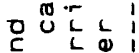

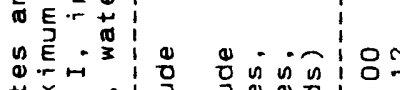

ॠ

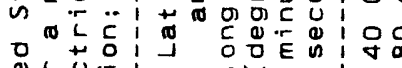

웅

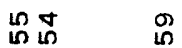

$\infty-\infty$

mo

$\begin{array}{ll}\text { N } & \text { N } \\ \text { N } & 0 \\ \text { N } & \text { D } \\ 0 & 0 \\ 0 & 0\end{array}$

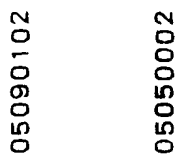

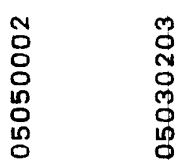

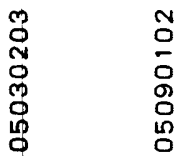

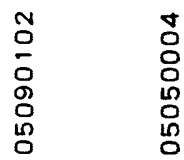

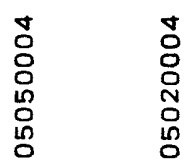

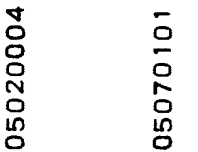

\section{$\stackrel{\oplus}{\stackrel{\infty}{\infty}}$}

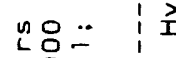

3 in

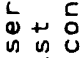

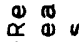

is

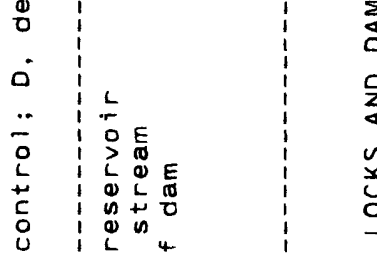

ำ

交

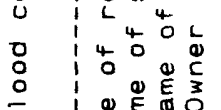

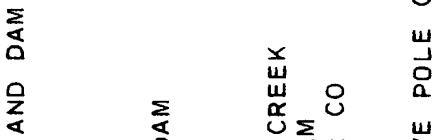

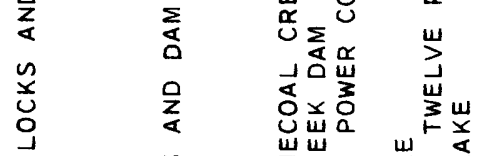

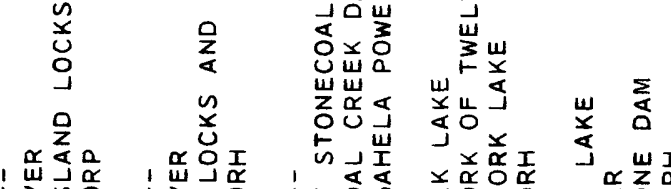

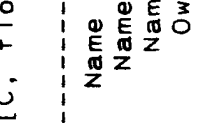

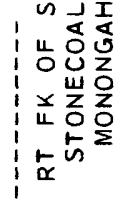

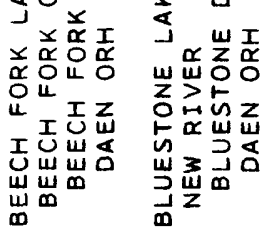
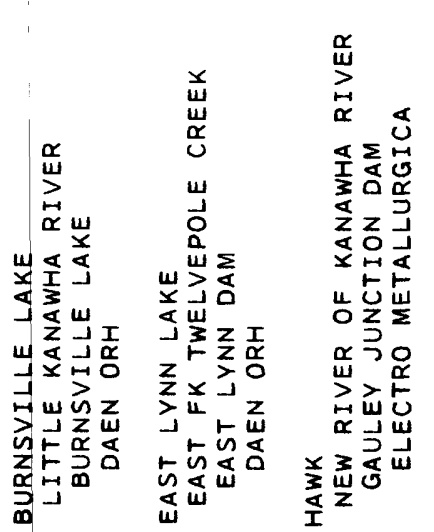

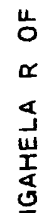




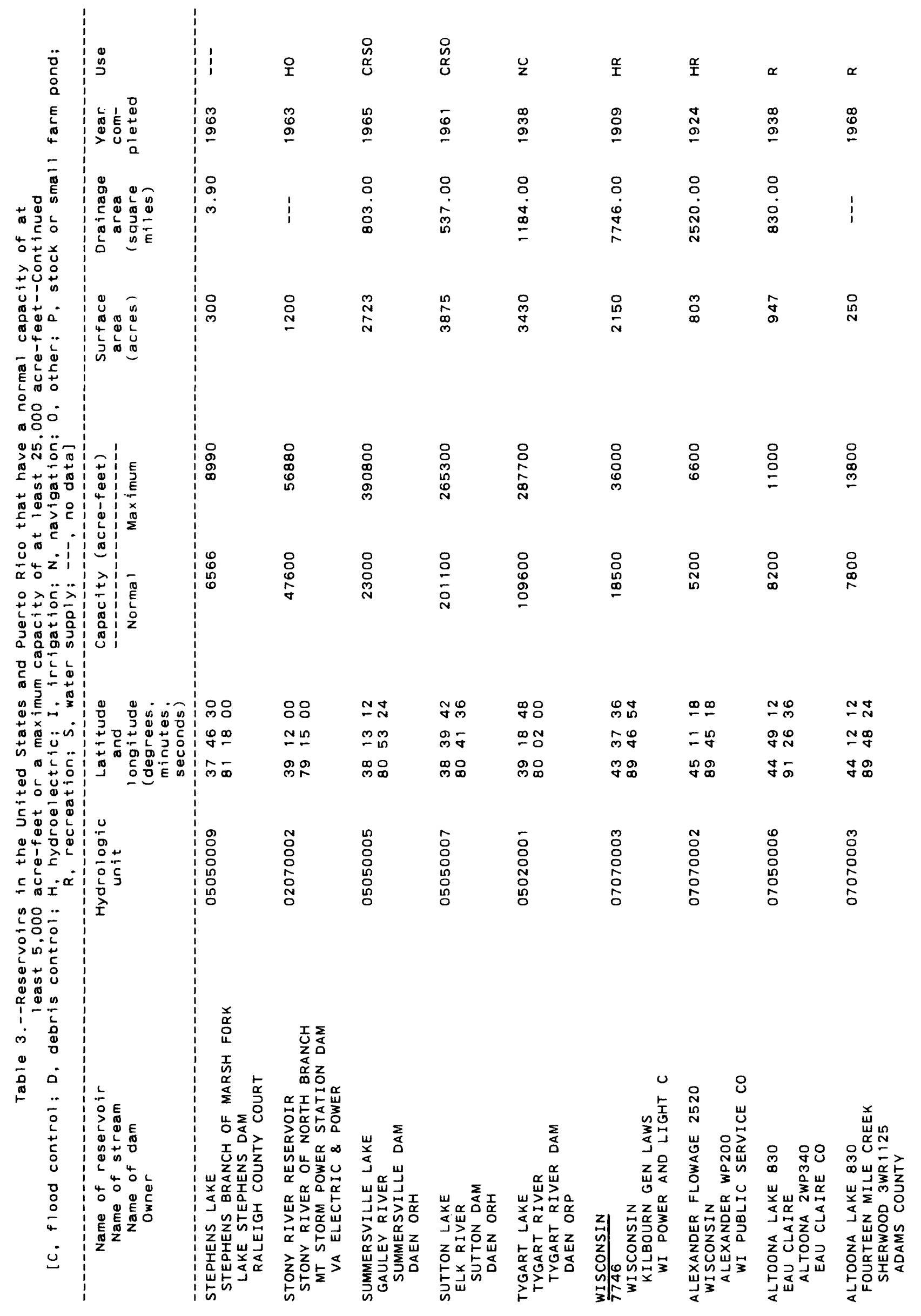




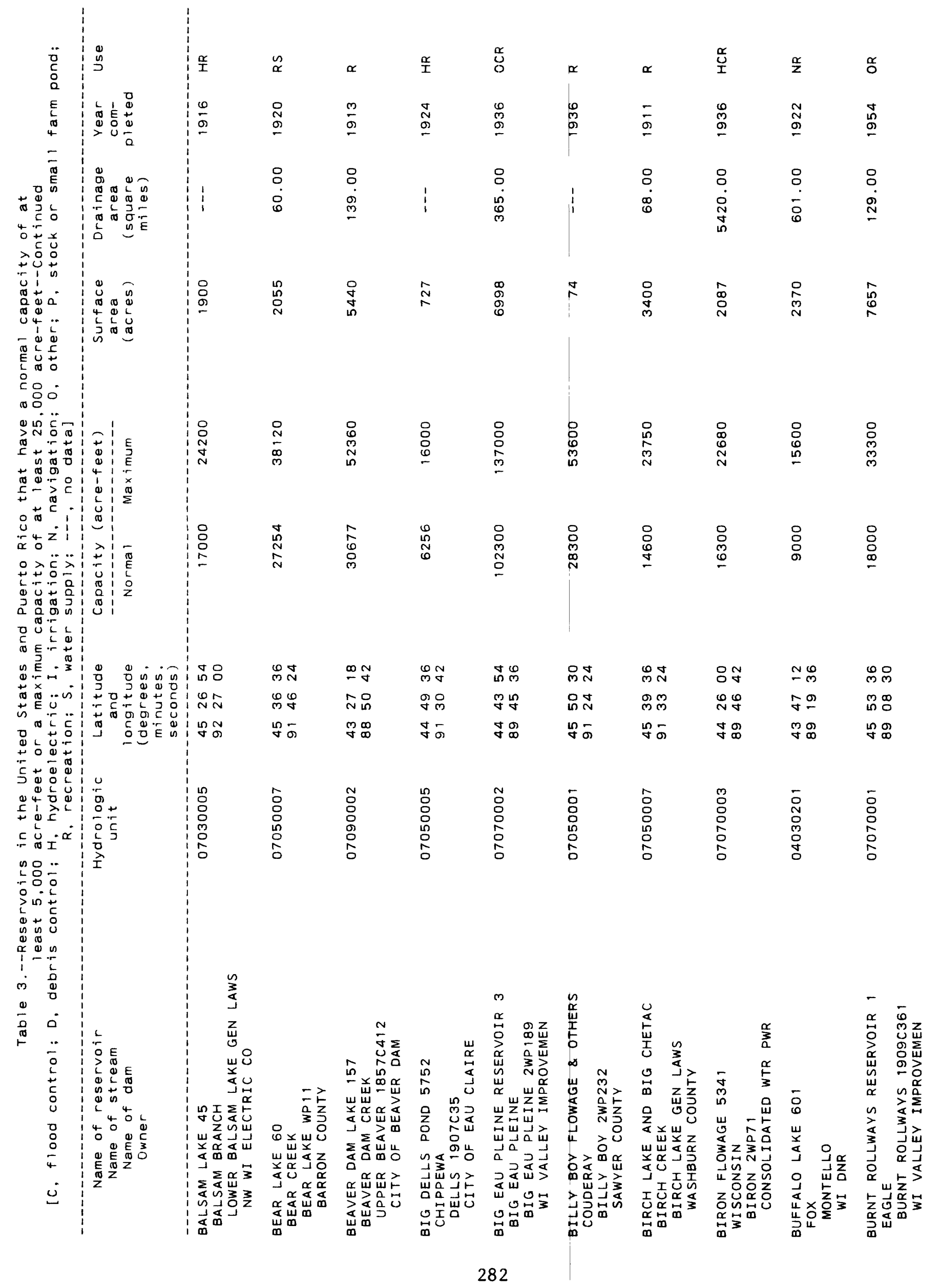




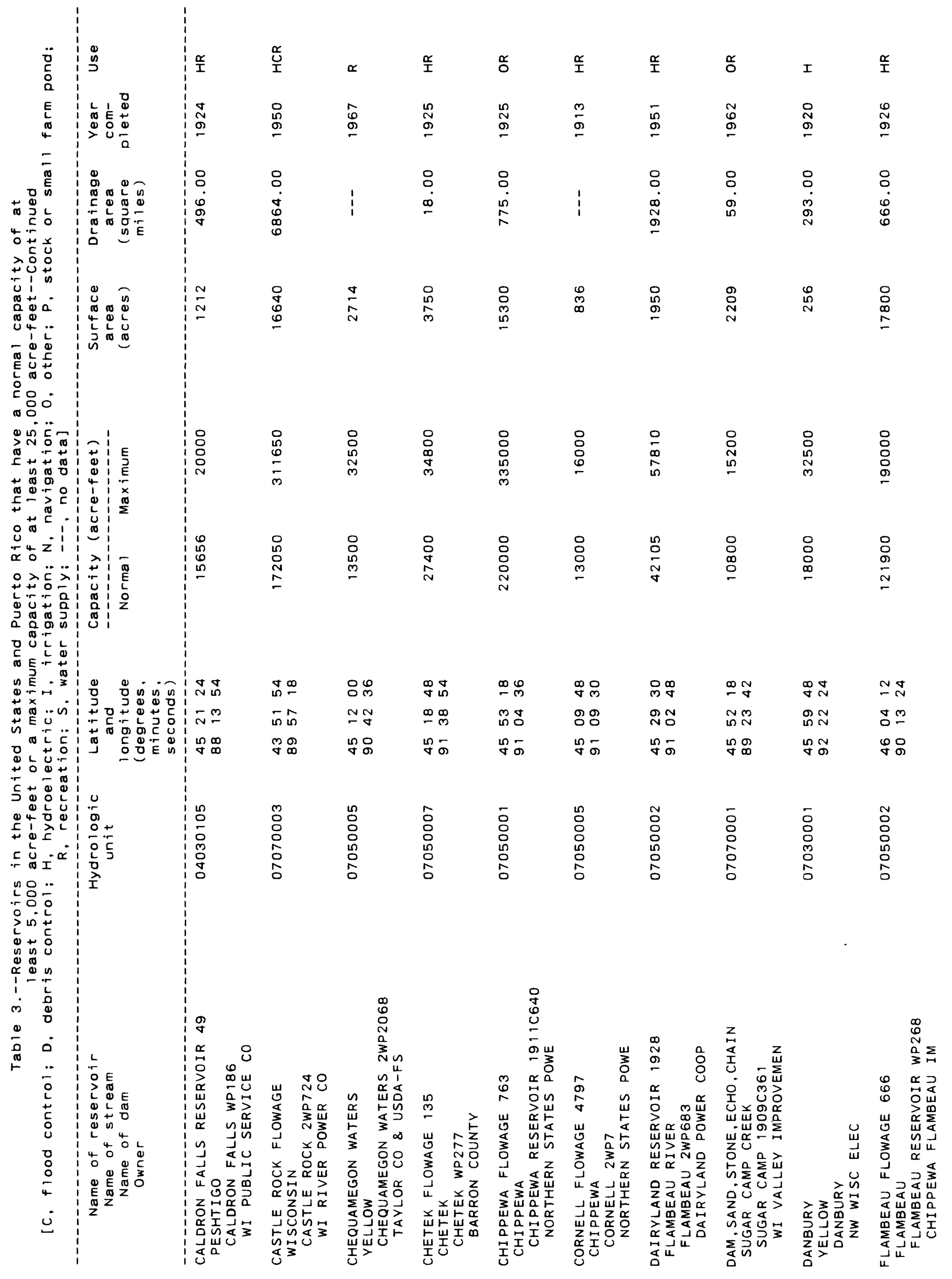




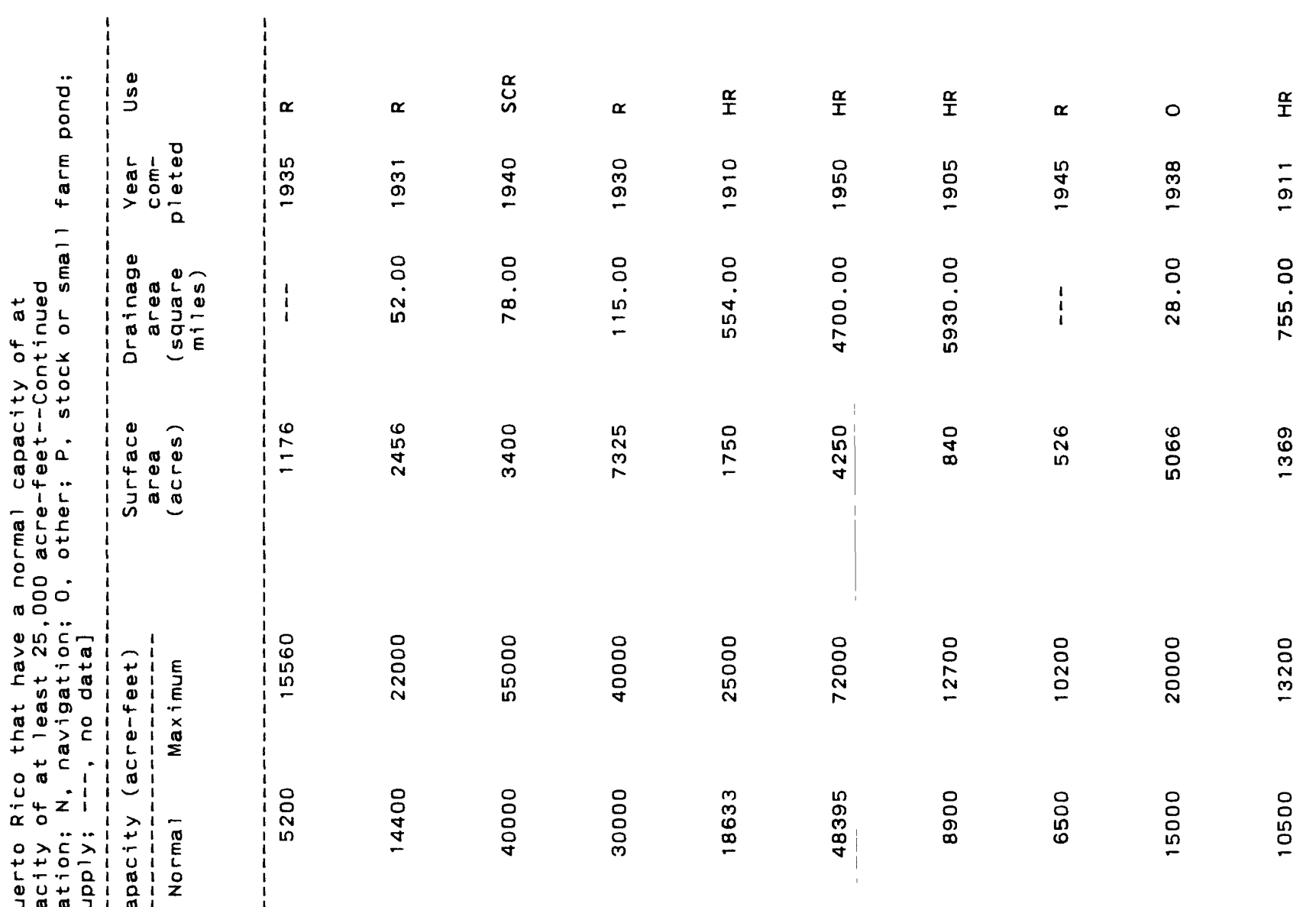

ว

a o on

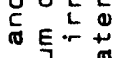

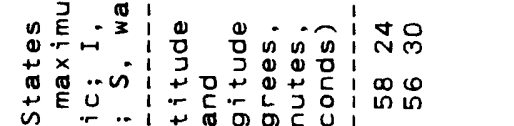

吃它

\begin{tabular}{|c|c|c|c|c|c|c|}
\hline ల్ల & ถับ พ & ஐ 욤 & 色 & F & $\simeq N$ & $\Phi \stackrel{0}{0}$ \\
\hline$\stackrel{\mathscr{L}}{N} \stackrel{m}{-2}$ & 은 & $\mathscr{O}$ & 95 & N & $\mathscr{N}$ & 동여 \\
\hline 웅 & $\mathfrak{O}_{+\infty}^{\infty}$ & $\ln _{\infty}^{\infty}$ & ஜூ & டீ & ஜी & \% \\
\hline
\end{tabular}

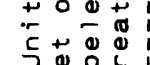

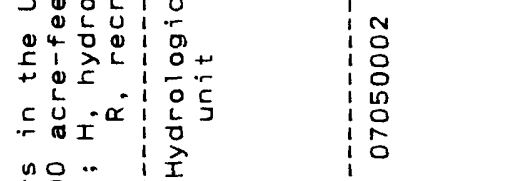

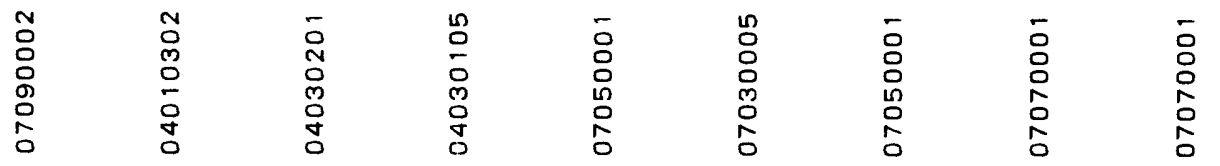

든 I

.

a

(5)

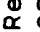
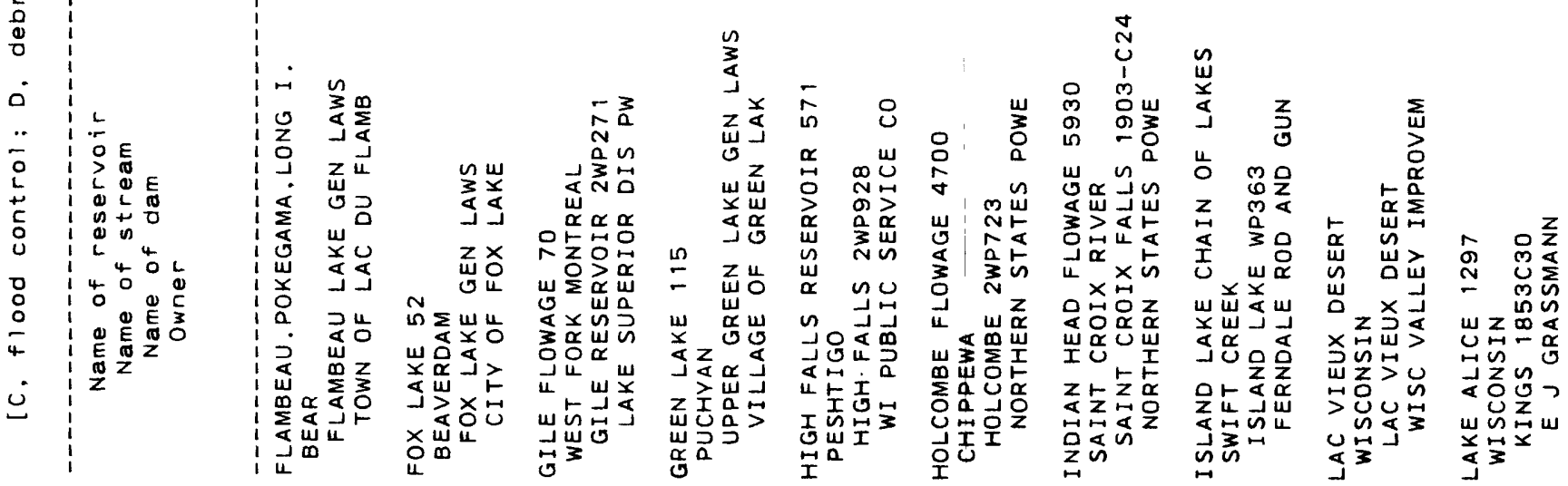


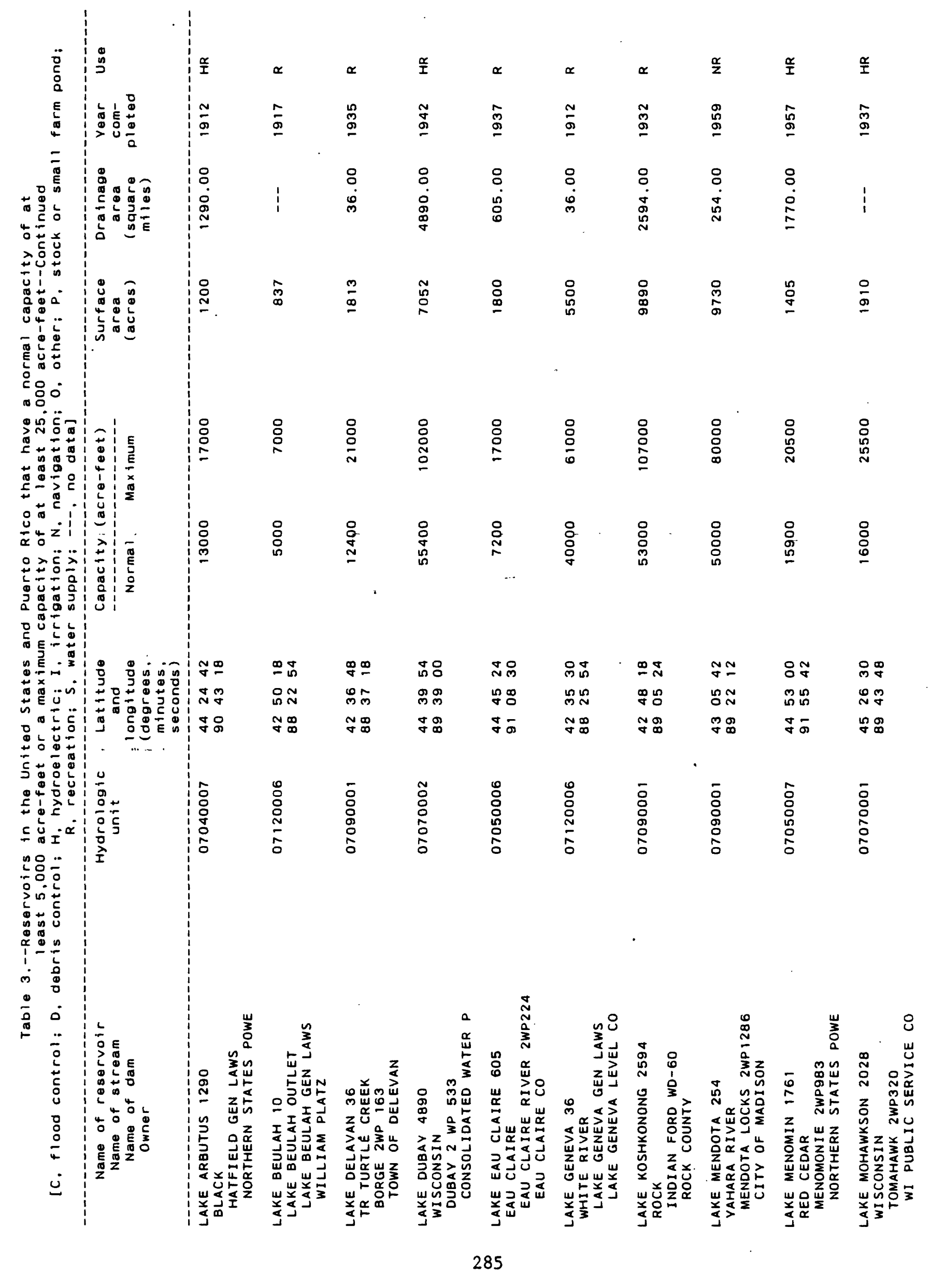




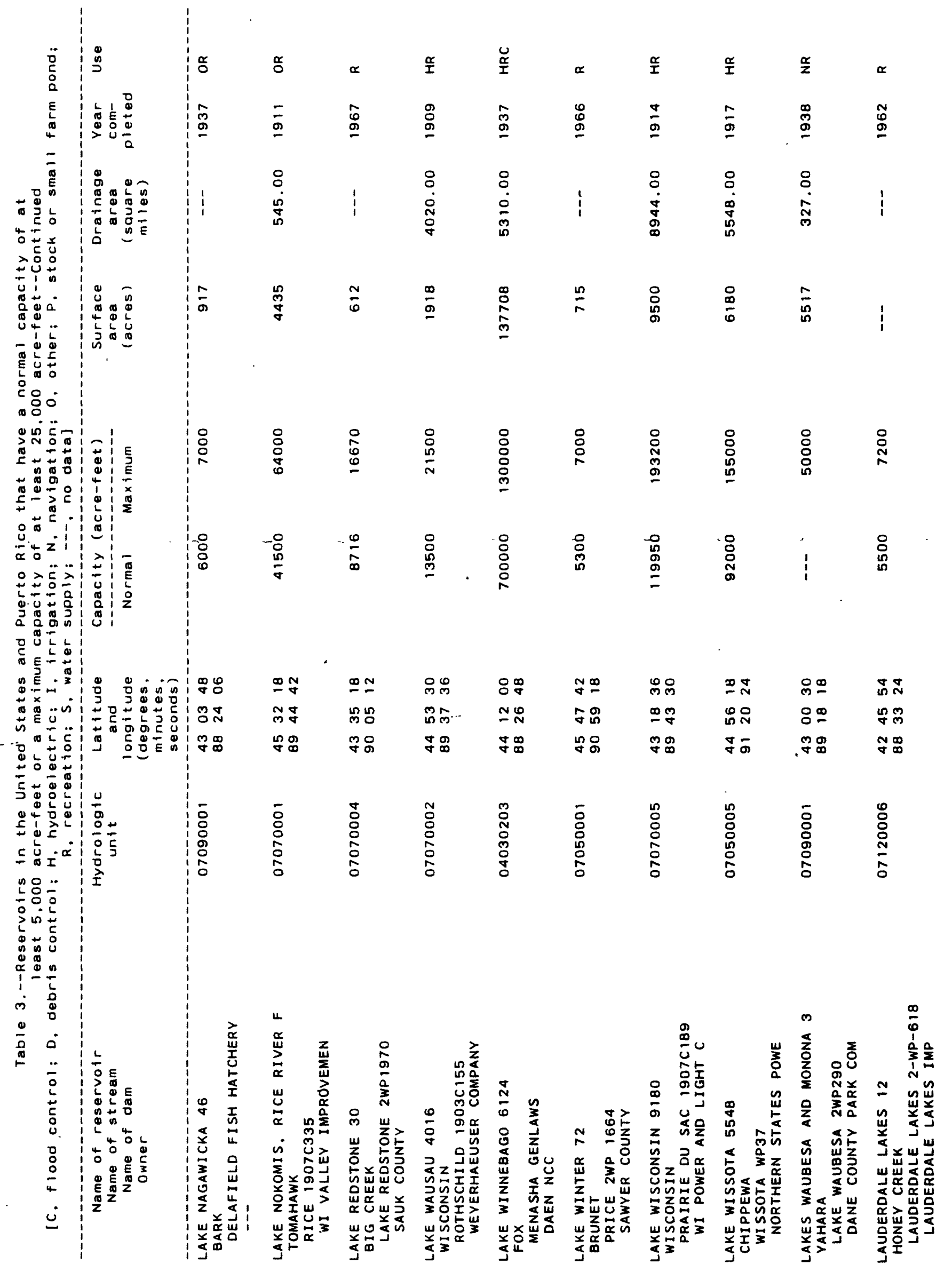




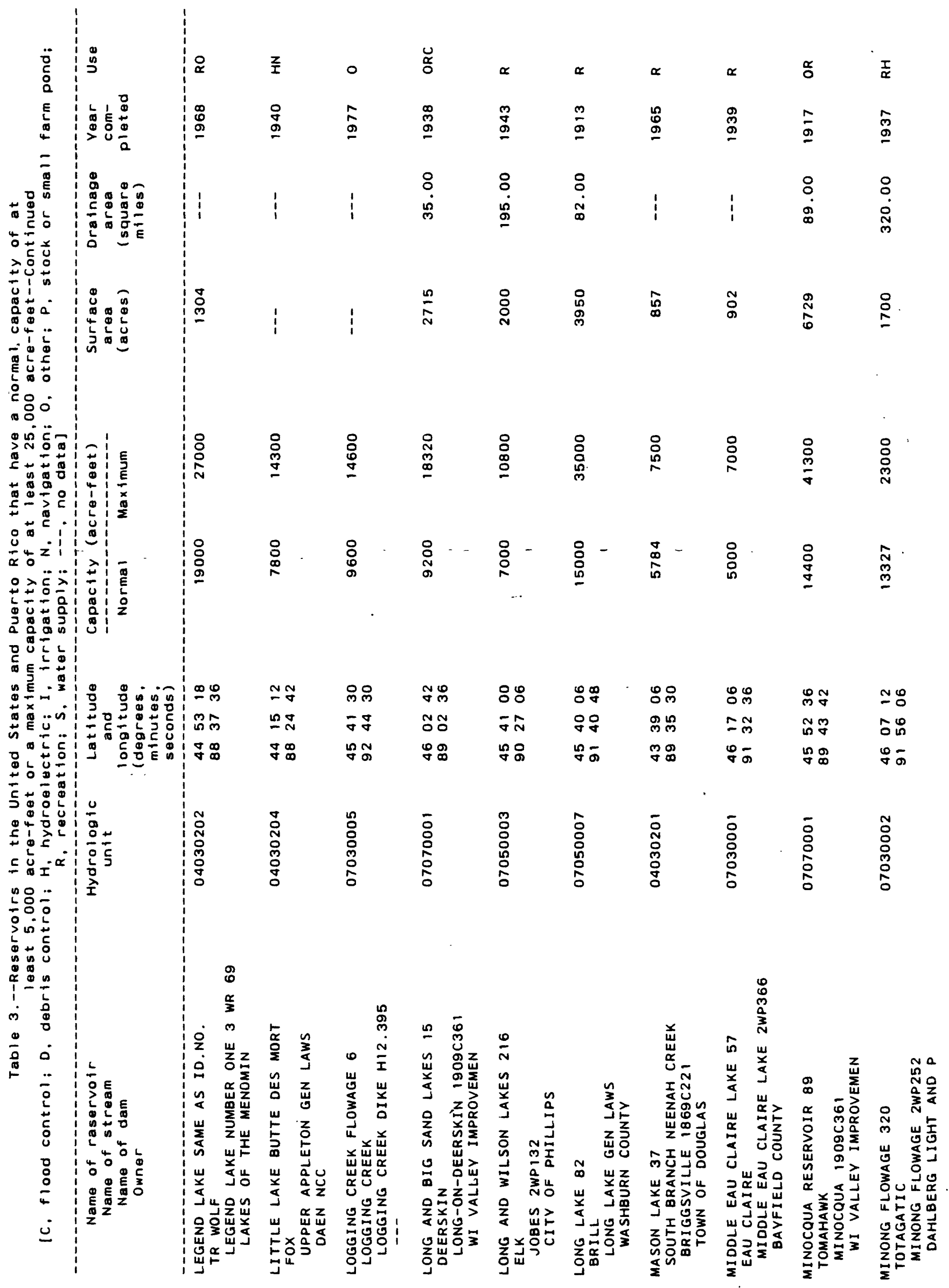




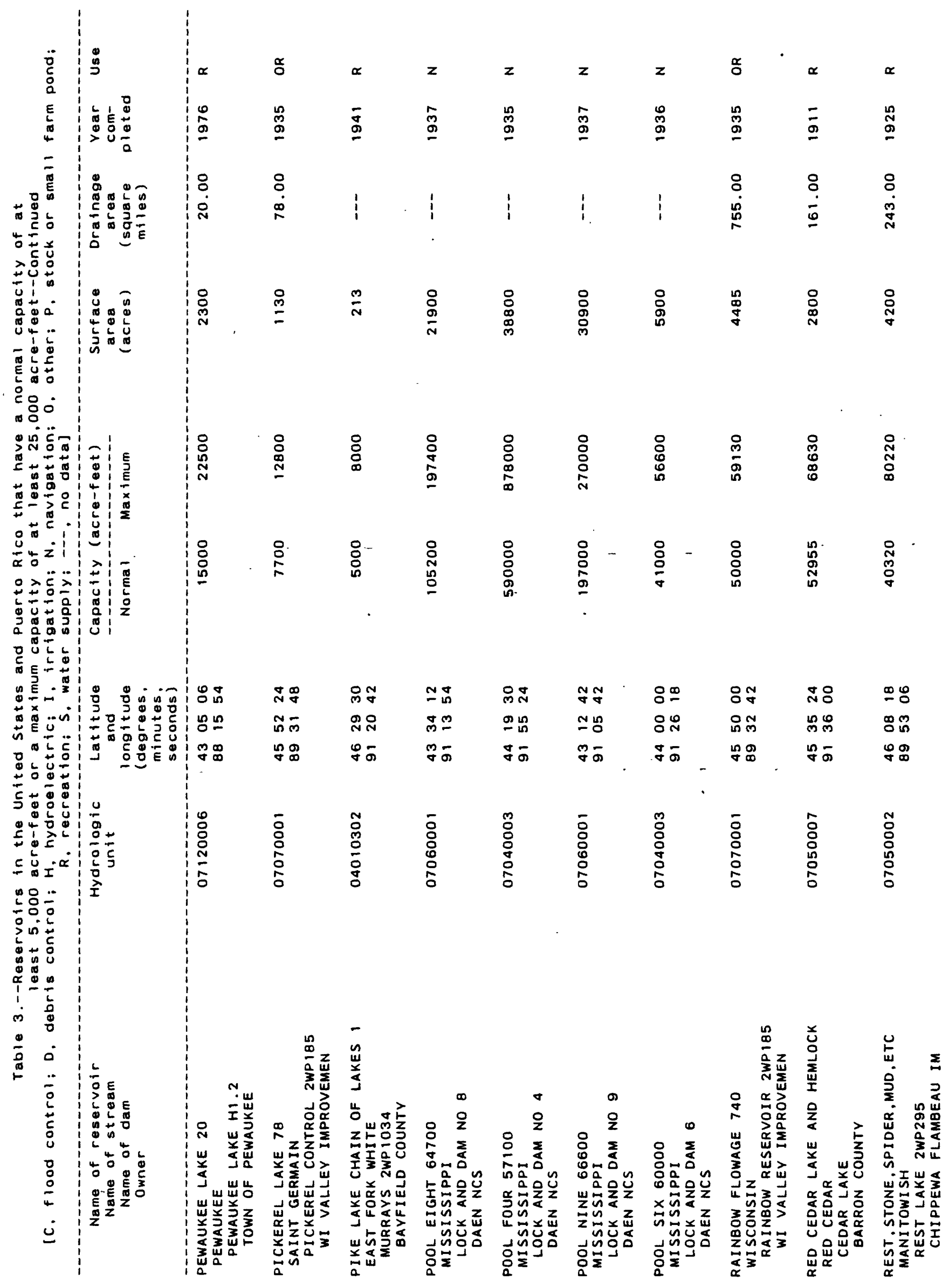




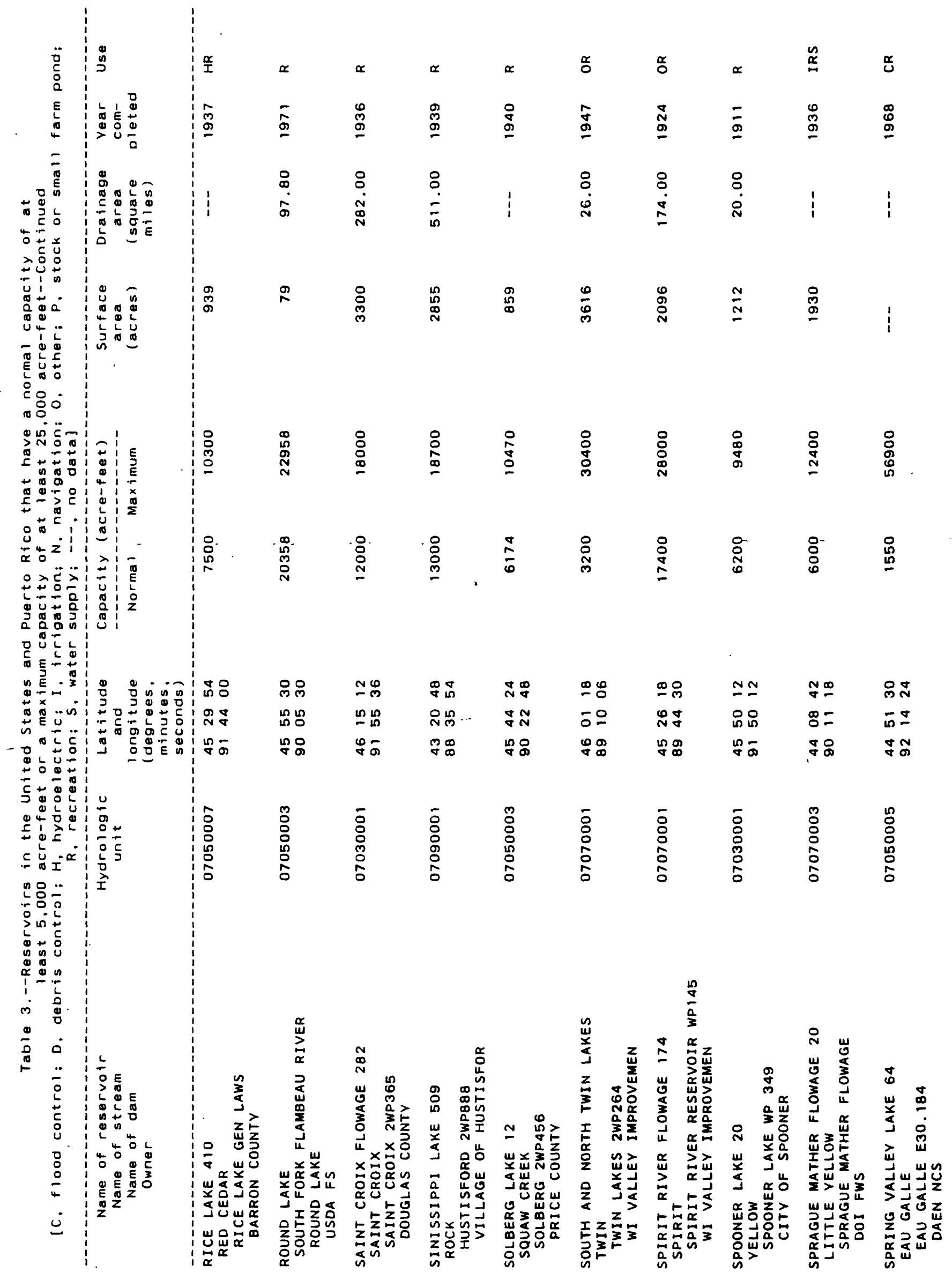




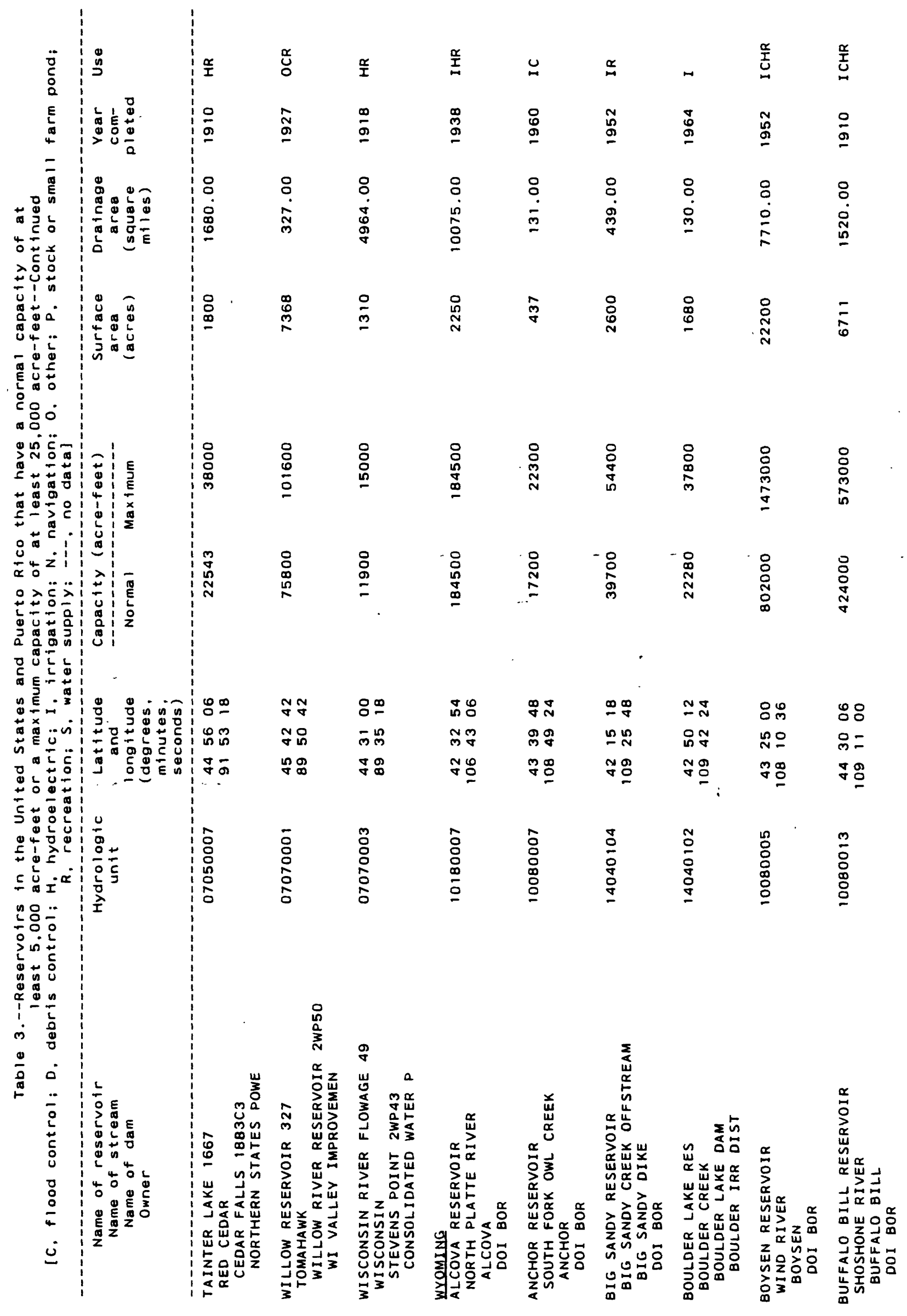




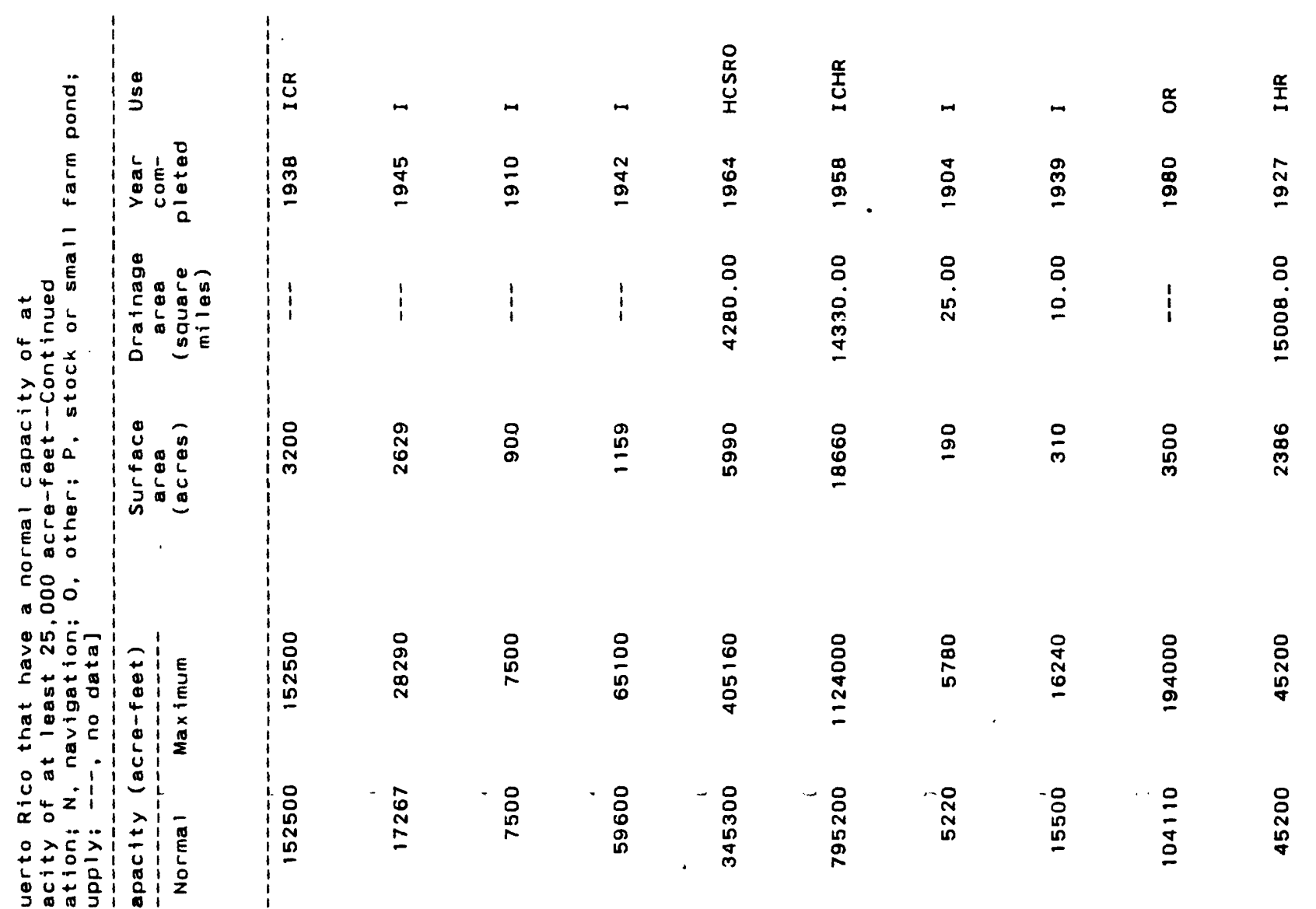

20ํำ

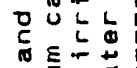

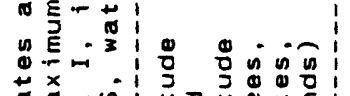

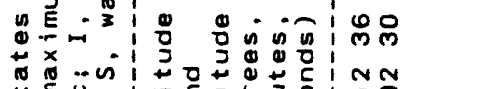

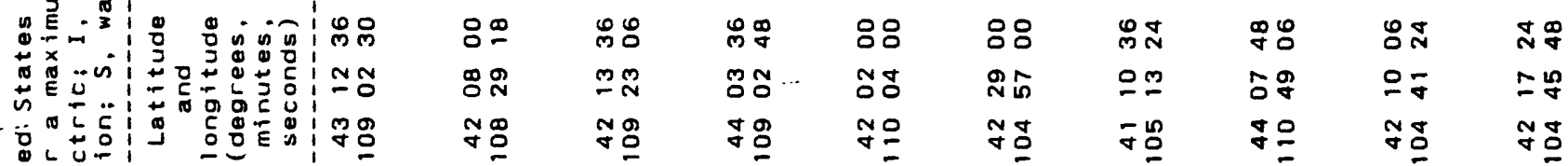

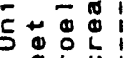

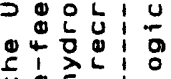

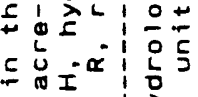

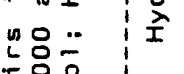

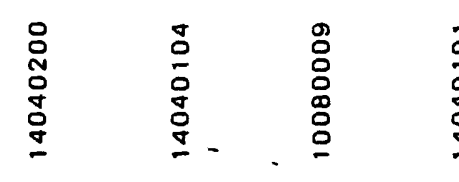

\begin{tabular}{lll}
0 & 0 \\
0 & 0 \\
\hline & 0 \\
0 & 0 \\
0 & 0
\end{tabular}

$\begin{array}{lll}0 & 0 \\ : & \stackrel{0}{2} \\ 0 & \stackrel{0}{+} \\ 0 & \stackrel{0}{0}\end{array}$

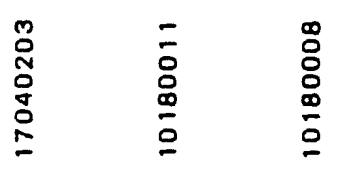

怔

$1-\frac{5}{2}$

ल

$\frac{0}{0} \div$

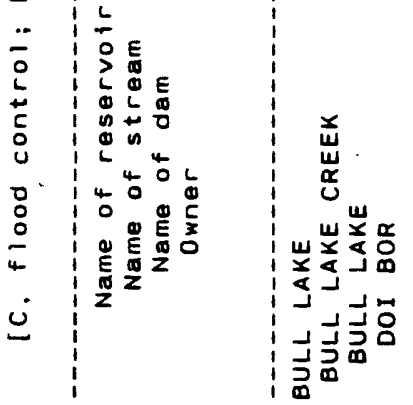

$\overline{0}$
0
0
0
0

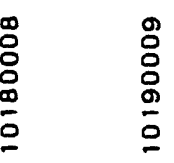

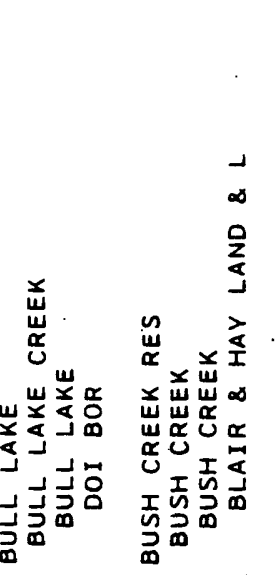

온

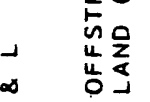

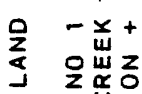

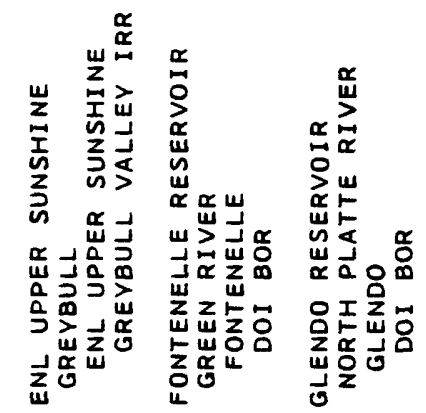

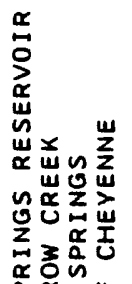

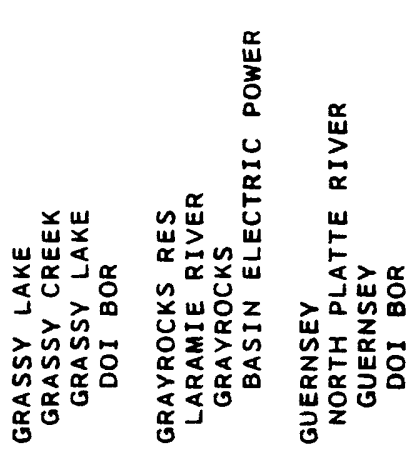




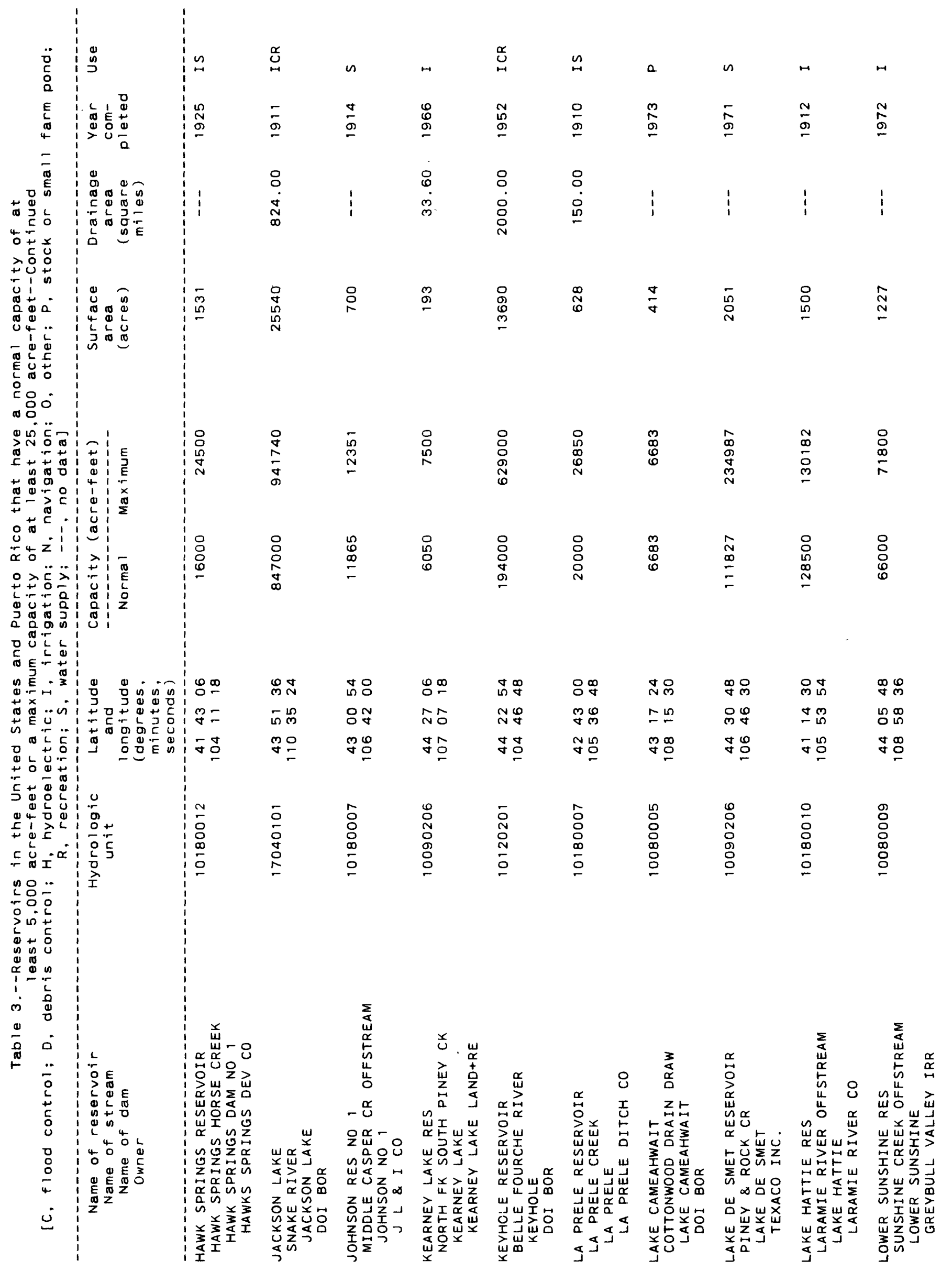




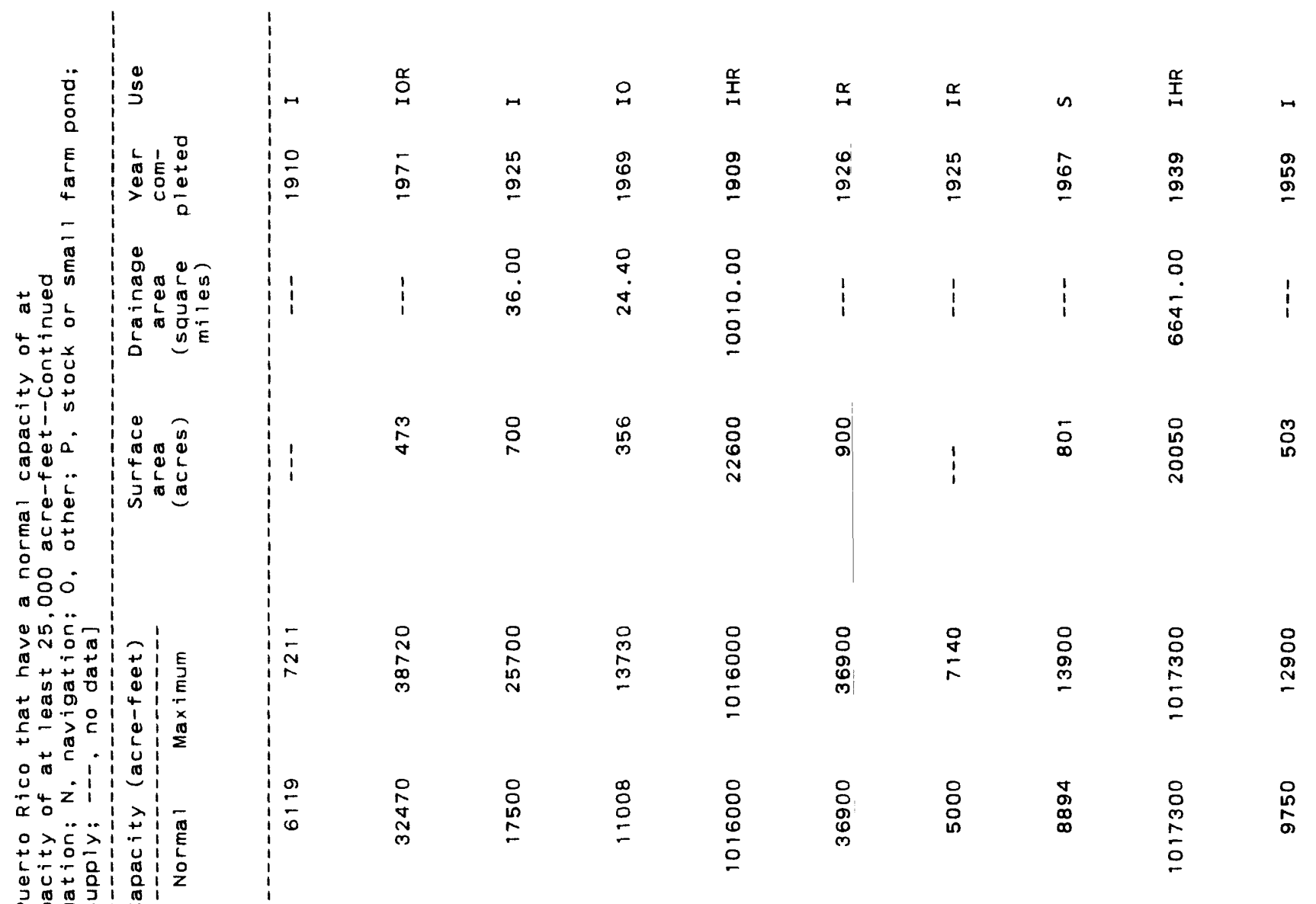

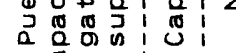

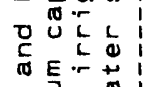

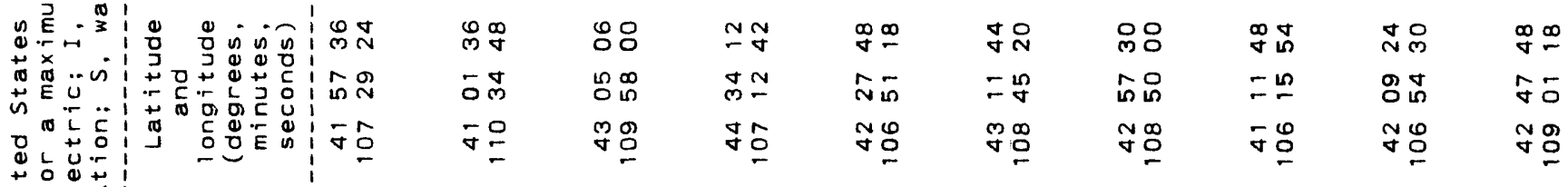

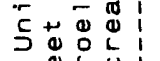

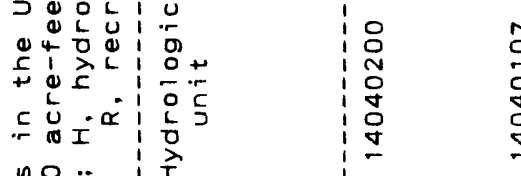

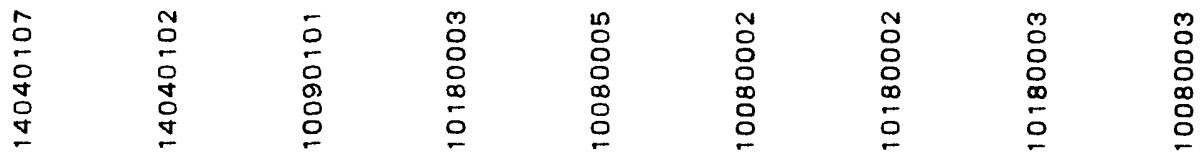

뇨으

$>$ in

ब

\&
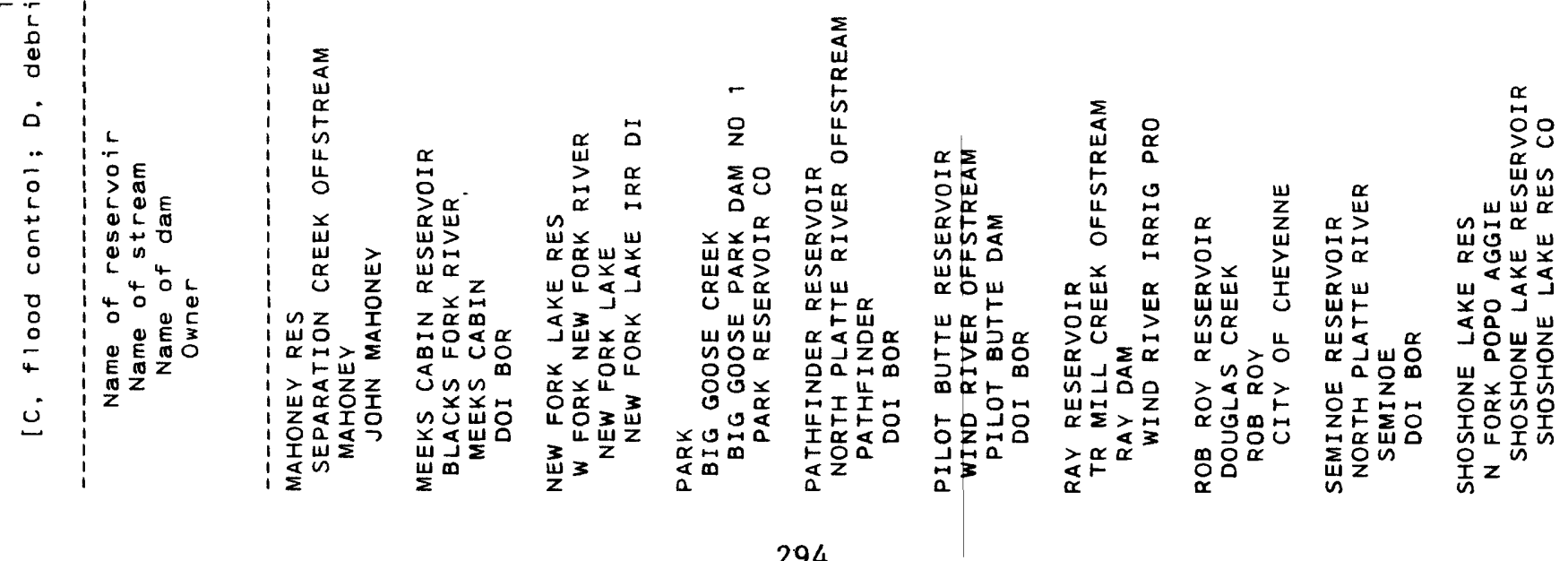


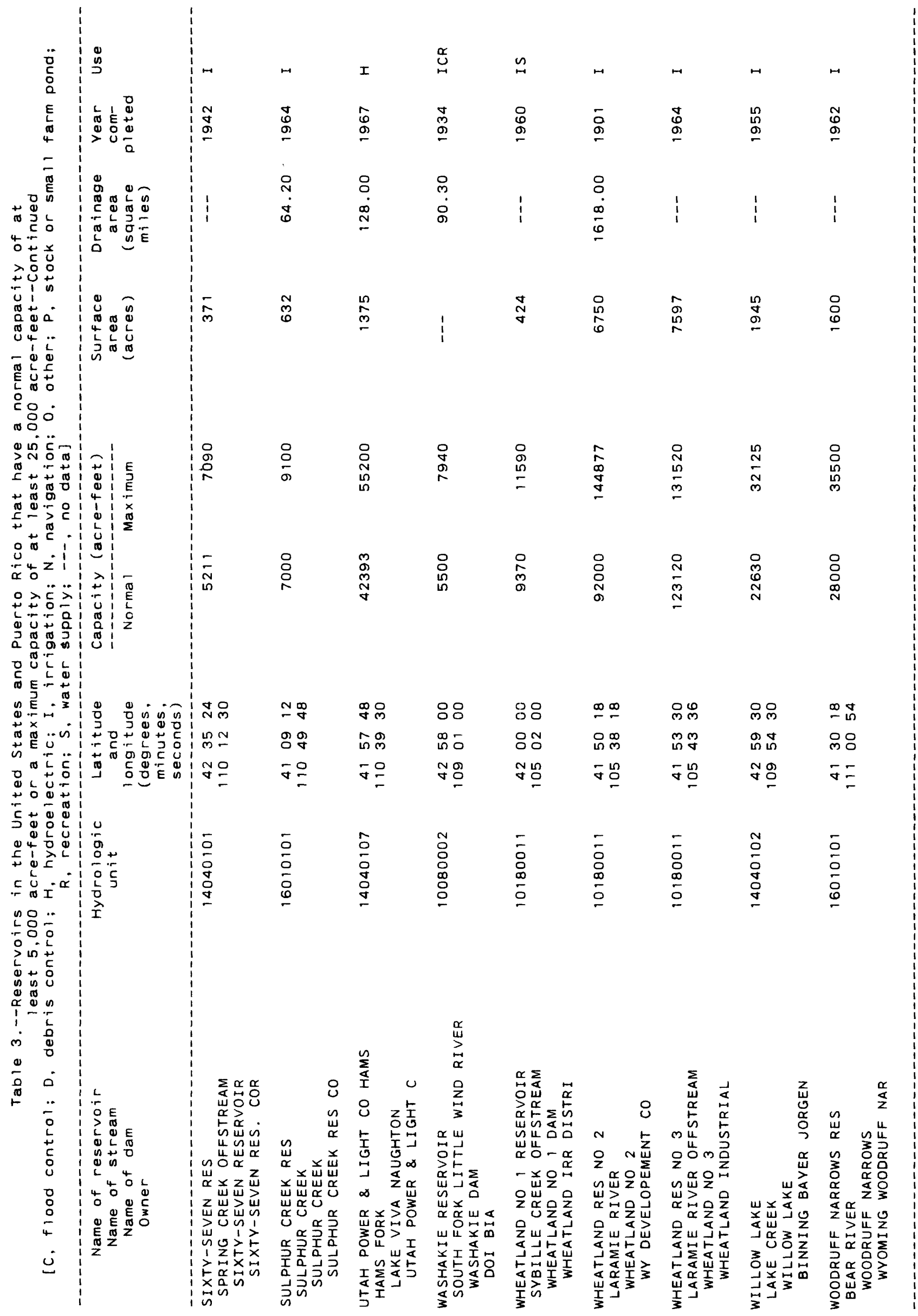

